Supporting Information For:

\title{
Enantioselective Synthesis of Indole-Fused Bicyclo[3.2.1]octanes via Palladium(II)-Catalyzed Cascade Reaction
}

Gang Wang, Jin-Chen Li, Yong-Gui Zhou and Zhi-Shi Ye*

\section{Table of Contents}

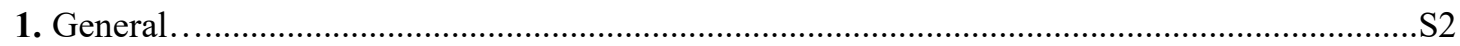

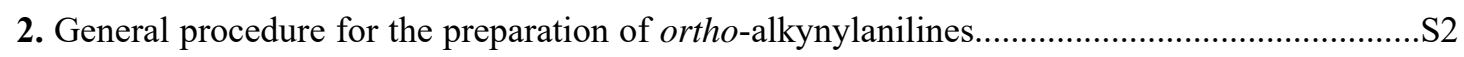

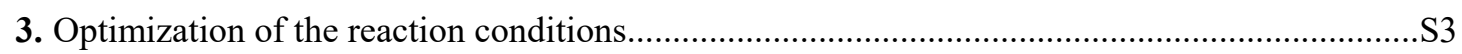

4. General procedure for enantioselective synthesis of indole-fused bicyclo[3.2.1]octane............S7

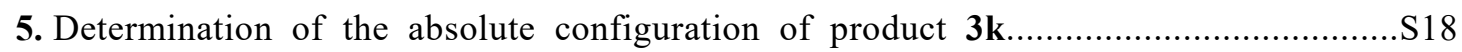

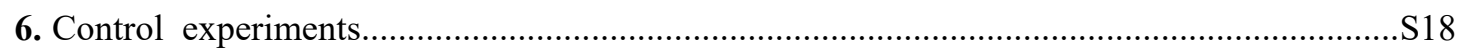

7. Scale-up experiment for enantioselective synthesis of indole-fused bicyclo[3.2.1]octane 3i...S19

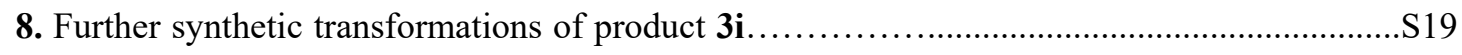

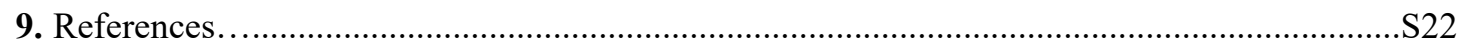

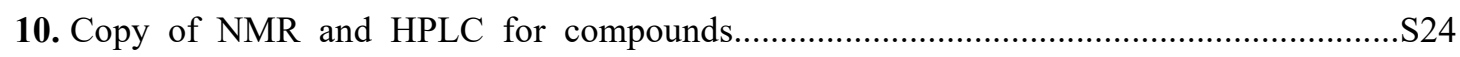




\section{General Motheds}

All reactions were carried out under an atmosphere of nitrogen using standard Schlenk techniques, unless otherwise noted. Anhydrous toluene and THF were distilled over sodium benzophenone ketyl under argon. All other solvents and reagents were used as obtained from commercial sources without further purification. ${ }^{1} \mathrm{H}$ NMR, ${ }^{13} \mathrm{C}$ NMR and ${ }^{19} \mathrm{~F}$ NMR spectra were recorded at room temperature in $\mathrm{CDCl}_{3}$ on $400 \mathrm{MHz}$ instrument with tetramethylsilane (TMS) as internal standard. Enantiomeric excess was determined by HPLC analysis, using chiral column described below in detail. Optical rotations were measured by polarimeter. Flash column chromatography was performed on silica gel (200-300 mesh). All reactions were monitored by TLC or NMR analysis.

\section{General procedure for the synthesis of ortho-alkynylanilines}
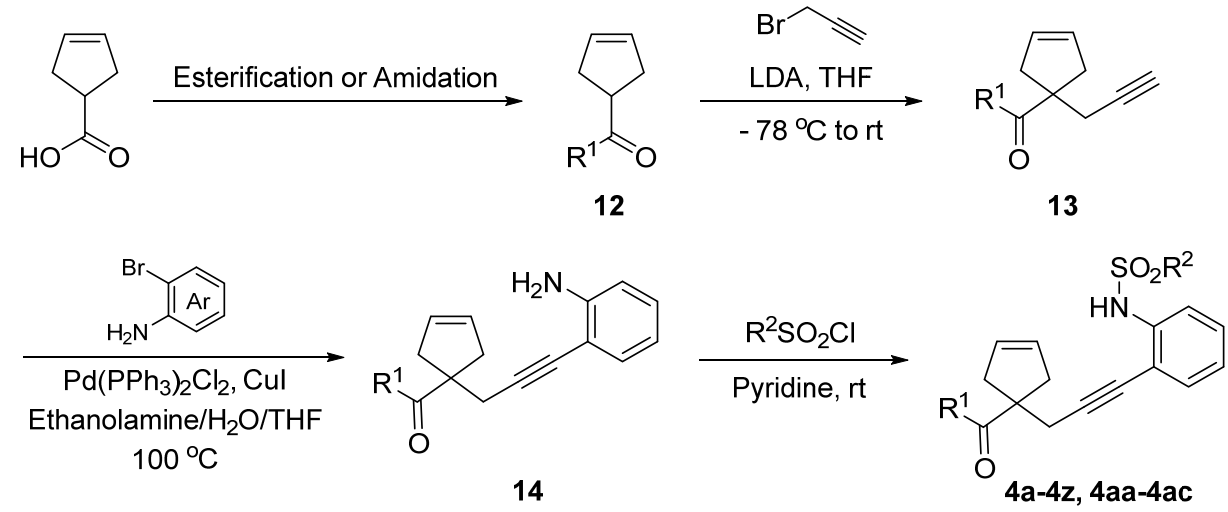

Compound 12 can be conveniently prepared from 3-Cyclopentene-1-carboxylic acid via esterification or amidation according to the known literature procedure. ${ }^{[1-3]}$

Synthesis of compound 13: ${ }^{[4]}$ In a nitrogen-filled Schlenk tube, a solution of 12 (50.00 mmol) in THF $(10.0 \mathrm{~mL})$ was added dropwise to the solution of LDA reagent $(55.00 \mathrm{mmol})$ in THF $(70.0$ $\mathrm{mL})$ at $-78{ }^{\circ} \mathrm{C}$. After two hours, the propargyl bromide $(2.6 \mathrm{~mL}, 50.00 \mathrm{mmol})$ was added dropwise, and the mixture was warmed to room temperature and stirred for overnight. After completion of the reaction (monitored by TLC), the mixture was quenched with aq. $\mathrm{NH}_{4} \mathrm{Cl}(5.0 \mathrm{~mL})$ and extracted with ethyl acetate $(3 \times 10.0 \mathrm{~mL})$. The combined organic layers were dried over sodium sulfate, filtered and concentrated under reduced pressure. The resulting crude material was purified by flash column chromatography (petroleum ether/ethyl acetate) to afford the desired compound 13.

Synthesis of compound $14:^{[5]}$ To a solution of the corresponding 2-bromoaniline $(3.0 \mathrm{mmol})$ in THF $(10.0 \mathrm{~mL})$ was added $\mathrm{Pd}\left(\mathrm{PPh}_{3}\right)_{2} \mathrm{Cl}_{2}(63.2 \mathrm{mg}, 0.09 \mathrm{mmol})$, CuI (11.4 mg, $\left.0.06 \mathrm{mmol}\right)$, ethanolamine ( $3 \mathrm{M}, 36.00 \mathrm{mmol})$ and the compound 13 (3.60 mmol). The mixture was stirred overnight in $100{ }^{\circ} \mathrm{C}$ oil bath. The reaction was cooled to room temperature and quenched with aq. $\mathrm{NH}_{4} \mathrm{Cl}(5.0 \mathrm{~mL})$. The aqueous layer was extracted with ethyl acetate $(3 \times 10.0 \mathrm{~mL})$. The combined organic alyers were dried over sodium sulfate, filtered and concentrated under reduced pressure. The resulting crude material was purified by flash column chromatography (petroleum ether/ethyl acetate) to afford the desired compound $\mathbf{1 4}$. 
Synthesis of compound $4:{ }^{[6]}$ Under nitrogen atmosphere, the mixture of corresponding sulfonyl chloride $(1.10 \mathrm{mmol})$ and compound $\mathbf{1 4}(1.00 \mathrm{mmol})$ in pyridine $(5.0 \mathrm{~mL})$ was stirred at room temperature for $24 \mathrm{~h}$. The mixture was treated with $\mathrm{HCl}(1 \mathrm{M})$, and extracted with ethyl acetate (3 $\mathrm{x} 10.0 \mathrm{~mL}$ ). The combined organic layers were dried over sodium sulfate, filtered and concentrated under reduced pressure. The resulting crude material was purified by flash column chromatography (petroleum ether/ethyl acetate) to afford the desired compound 4.

Benzyl 1-(3-(2-((4-methylphenyl)sulfonamido)phenyl)prop-2-yn-1-yl)cyclopent-3-ene-1carboxylate (4a): the reaction was conducted at $10 \mathrm{mmol}$ scale from 13, $2.0855 \mathrm{~g}, 43 \%$ yield for<smiles>CNc1ccccc1C#CCC1(C(=O)O)CC=CC1</smiles>
two steps, unknown compound, yellow oil, $\mathrm{R}_{f}=0.26$ (petroleum ether/ethyl acetate 10/1); ${ }^{1} \mathrm{H}$ NMR $\left(400 \mathrm{MHz}, \mathrm{CDCl}_{3}\right) \delta 7.65(\mathrm{~d}, J=8.3$ $\mathrm{Hz}, 2 \mathrm{H}), 7.50(\mathrm{~d}, J=8.3 \mathrm{~Hz}, 1 \mathrm{H}), 7.37(\mathrm{~s}, 1 \mathrm{H}), 7.31-7.20(\mathrm{~m}, 5 \mathrm{H})$, 7.16-7.08 (m, 4H), 6.90-6.83 (m, 1H), $5.64(\mathrm{~d}, J=8.2 \mathrm{~Hz}, 1 \mathrm{H}), 5.62(\mathrm{~d}, J$ $=8.2 \mathrm{~Hz}, 1 \mathrm{H}), 5.18(\mathrm{~s}, 2 \mathrm{H}), 2.93(\mathrm{~d}, J=15.0 \mathrm{~Hz}, 2 \mathrm{H}), 2.69(\mathrm{~s}, 2 \mathrm{H}), 2.42$ $(\mathrm{d}, J=15.0 \mathrm{~Hz}, 2 \mathrm{H}), 2.28(\mathrm{~s}, 3 \mathrm{H}) .{ }^{13} \mathrm{C} \mathrm{NMR}\left(100 \mathrm{MHz}, \mathrm{CDCl}_{3}\right) \delta 176.1,144.0,138.4,136.6$, 136.1, 132.2, 129.7, 129.3, 128.7, 128.6, 128.4, 128.3, 127.5, 123.9, 118.9, 113.9, 94.5, 77.6, 77.2, 77.1, 76.9, 67.2, 51.9, 42.8, 29.7, 21.8. HRMS (ESI-TOF) $\mathrm{m} / \mathrm{z}$ : [M+Na] ${ }^{+} \mathrm{C}_{29} \mathrm{H}_{27} \mathrm{NO}_{4} \mathrm{SNa}$ Calcd for 508.1553; found 508.1550.

Benzyl 1-(3-(2-(phenylsulfonamido)phenyl)prop-2-yn-1-yl)cyclopent-3-ene-1-carboxylate (4b): the reaction was conducted at $3.00 \mathrm{mmol}$ scale from 13, $0.5652 \mathrm{~g}, 40 \%$ yield for two steps,

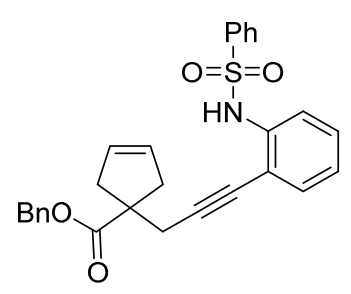
unknown compound, yellow oil, $\mathrm{R}_{f}=0.25$ (petroleum ether/ethyl acetate 10/1); ${ }^{1} \mathrm{H}$ NMR $\left(400 \mathrm{MHz}, \mathrm{CDCl}_{3}\right) \delta$ 7.86-7.80 (m, 2H), 7.61-7.55 (m, $1 \mathrm{H}), 7.52-7.45(\mathrm{~m}, 2 \mathrm{H}), 7.43-7.38(\mathrm{~m}, 2 \mathrm{H}), 7.37-7.33(\mathrm{~m}, 2 \mathrm{H})$, $7.33-7.25(\mathrm{~m}, 3 \mathrm{H}), 7.24-7.14(\mathrm{~m}, 2 \mathrm{H}), 6.97-6.93(\mathrm{~m}, 1 \mathrm{H}), 5.70(\mathrm{~d}, J=$ $8.2 \mathrm{~Hz}, 1 \mathrm{H}), 5.68(\mathrm{~d}, J=8.2 \mathrm{~Hz}, 1 \mathrm{H}), 5.25(\mathrm{~s}, 2 \mathrm{H}), 3.00(\mathrm{~d}, J=14.9 \mathrm{~Hz}$, 2H), $2.75(\mathrm{~s}, 2 \mathrm{H}), 2.48(\mathrm{~d}, J=15.2 \mathrm{~Hz}, 2 \mathrm{H}) .{ }^{13} \mathrm{C} \mathrm{NMR}(100 \mathrm{MHz}$, $\left.\mathrm{CDCl}_{3}\right) \delta 176.1,139.6,138.2,136.1,133.1,132.2,129.3,129.1,128.7,128.6,128.4,128.3,127.4$, 124.1, 119.2, 114.1, 94.5, 77.6, 77.2, 77.0, 76.9, 67.2, 52.0, 42.8, 29.6. HRMS (ESI-TOF) $\mathrm{m} / \mathrm{z}$ : $[\mathrm{M}+\mathrm{Na}]^{+} \mathrm{C}_{28} \mathrm{H}_{25} \mathrm{NO}_{4} \mathrm{SNa}$ Calcd for 494.1397; found 494.1392.

Benzyl 1-(3-(2-((4-methoxyphenyl)sulfonamido)phenyl)prop-2-yn-1-yl)cyclopent-3-ene-1carboxylate (4c): the reaction was conducted at $3.00 \mathrm{mmol}$ scale from 13, $0.6567 \mathrm{~g}, 46 \%$ yield for

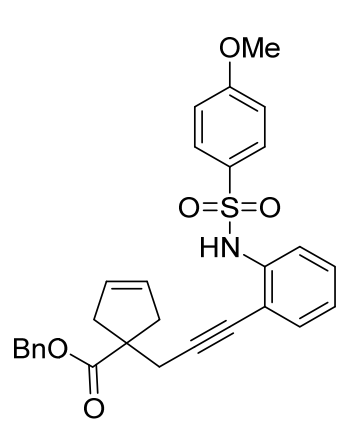
two steps, unknown compound, yellow oil, $\mathrm{R}_{f}=0.20$ (petroleum ether/ethyl acetate 10/1); ${ }^{1} \mathrm{H}$ NMR $\left(400 \mathrm{MHz}, \mathrm{CDCl}_{3}\right) \delta$ 7.79-7.73 (m, 2H), 7.60-7.54 (m, 1H), $7.40(\mathrm{~s}, 1 \mathrm{H}), 7.38-7.26(\mathrm{~m}, 5 \mathrm{H}), 7.23-7.15(\mathrm{~m}$, $2 \mathrm{H}), 6.97-6.91(\mathrm{~m}, 1 \mathrm{H}), 6.88-6.82(\mathrm{~m}, 2 \mathrm{H}), 5.70(\mathrm{~d}, J=8.2 \mathrm{~Hz}, 1 \mathrm{H})$, $5.68(\mathrm{~d}, J=8.2 \mathrm{~Hz}, 1 \mathrm{H}), 5.25(\mathrm{~s}, 2 \mathrm{H}), 3.79(\mathrm{~s}, 3 \mathrm{H}), 3.00(\mathrm{~d}, J=14.9 \mathrm{~Hz}$, $2 \mathrm{H}), 2.76(\mathrm{~s}, 2 \mathrm{H}), 2.49(\mathrm{~d}, J=15.1 \mathrm{~Hz}, 2 \mathrm{H}) .{ }^{13} \mathrm{C} \mathrm{NMR}(100 \mathrm{MHz}$, $\left.\mathrm{CDCl}_{3}\right) \delta 176.1,163.3,138.4,136.1,132.3,131.2,129.6,129.3,128.7$, $128.6,128.4,128.3,123.9,119.0,114.3,114.0,94.4,77.6,77.2,77.12$, 76.9, 67.2, 55.7, 52.0, 42.8, 29.7. HRMS (ESI-TOF) $\mathrm{m} / \mathrm{z}$ : $[\mathrm{M}+\mathrm{Na}]^{+} \mathrm{C}_{29} \mathrm{H}_{27} \mathrm{NO}_{5} \mathrm{SNa}$ Calcd for 524.1502; found 524.1505 . 
Benzyl 1-(3-(2-((phenylmethyl)sulfonamido)phenyl)prop-2-yn-1-yl)cyclopent-3-ene-1carboxylate (4d): the reaction was conducted at $3.00 \mathrm{mmol}$ scale from 13, $0.5714 \mathrm{~g}, 39 \%$ yield<smiles>O=C(OCc1ccccc1)C1(CC#Cc2ccccc2NS(=O)(=O)C2CCCC2)CCCC1</smiles>
for two steps, unknown compound, yellow oil, $\mathrm{R}_{f}=0.15$ (petroleum ether/ethyl acetate 20/1); ${ }^{1} \mathrm{H}$ NMR $\left(400 \mathrm{MHz}, \mathrm{CDCl}_{3}\right) \delta 7.61(\mathrm{~d}, J=8.3$ $\mathrm{Hz}, 1 \mathrm{H}), 7.36-7.25(\mathrm{~m}, 10 \mathrm{H}), 7.23-7.18(\mathrm{~m}, 2 \mathrm{H}), 7.10(\mathrm{~s}, 1 \mathrm{H})$, $7.05-6.99(\mathrm{~m}, 1 \mathrm{H}), 5.57(\mathrm{~d}, J=8.2 \mathrm{~Hz}, 1 \mathrm{H}), 5.55(\mathrm{~d}, J=8.2 \mathrm{~Hz}, 1 \mathrm{H})$, $5.16(\mathrm{~s}, 2 \mathrm{H}), 4.35(\mathrm{~s}, 2 \mathrm{H}), 2.89(\mathrm{~d}, J=14.9 \mathrm{~Hz}, 2 \mathrm{H}), 2.69(\mathrm{~s}, 2 \mathrm{H}), 2.32(\mathrm{~d}$, $J=15.1 \mathrm{~Hz}, 2 \mathrm{H}) .{ }^{13} \mathrm{C} \mathrm{NMR}\left(100 \mathrm{MHz}, \mathrm{CDCl}_{3}\right) \delta 176.1,138.8,136.1$, 132.5, 131.1, 129.6, 128.9, 128.9, 128.7, 128.7, 128.6, 128.4, 128.2, 123.8, 117.5, 113.2, 94.9, 77.6, 77.2, 76.9, 67.1, 57.8, 51.6, 42.7, 29.7. HRMS (ESI-TOF) $\mathrm{m} / \mathrm{z}$ : $[\mathrm{M}+\mathrm{Na}]^{+} \mathrm{C}_{29} \mathrm{H}_{27} \mathrm{NO}_{4} \mathrm{SNa}$ Calcd for 508.1553; found 508.1556 .

Benzyl 1-(3-(2-((4-nitrophenyl)sulfonamido)phenyl)prop-2-yn-1-yl)cyclopent-3-ene-1carboxylate (4e): the reaction was conducted at $3.00 \mathrm{mmol}$ scale from $13,0.5579 \mathrm{~g}, 36 \%$ yield for

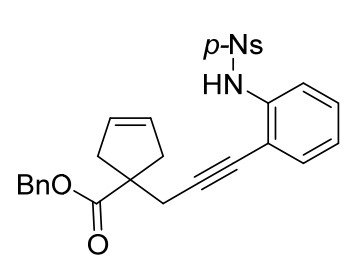
two steps, unknown compound, yellow oil, $\mathrm{R}_{f}=0.30$ (petroleum ether/ethyl acetate $1 / 1) ;{ }^{1} \mathrm{H}$ NMR $\left(400 \mathrm{MHz}, \mathrm{CDCl}_{3}\right) \delta 8.27-8.17(\mathrm{~m}$, $2 \mathrm{H}), 8.05-7.95(\mathrm{~m}, 2 \mathrm{H}), 7.64(\mathrm{~s}, 1 \mathrm{H}), 7.62(\mathrm{~d}, J=8.4 \mathrm{~Hz}, 1 \mathrm{H}), 7.39-7.25$ (m, 6H), 7.23-7.18 (m, 1H), 7.05-6.98 (m, 1H), 5.76-5.61 (m, 2H), 5.28 (d, $J=12.0 \mathrm{~Hz}, 2 \mathrm{H}), 3.00$ (d, $J=15.0 \mathrm{~Hz}, 2 \mathrm{H}), 2.72(\mathrm{~s}, 2 \mathrm{H}), 2.46$ (d, $J=$

$15.2 \mathrm{~Hz}, 2 \mathrm{H}) .{ }^{13} \mathrm{C}$ NMR $\left(100 \mathrm{MHz}, \mathrm{CDCl}_{3}\right) \delta 176.3,150.3,145.2,137.4,136.0,132.4,129.5$, $128.8,128.7,128.5,128.4,128.2,125.0,124.3,119.9,114.7,95.0,77.6,77.2,76.9,76.9,67.2$, 52.1, 42.7, 29.6. HRMS (ESI-TOF) $\mathrm{m} / \mathrm{z}$ : $[\mathrm{M}+\mathrm{Na}]^{+} \mathrm{C}_{28} \mathrm{H}_{24} \mathrm{~N}_{2} \mathrm{O}_{6} \mathrm{SNa}$ Calcd for 539.1247; found 539.1251 .

Benzyl 1-(3-(2-((tert-butoxycarbonyl)amino)phenyl)prop-2-yn-1-yl)cyclopent-3-ene-1carboxylate (4f): the reaction was conducted at $3.00 \mathrm{mmol}$ scale from $\mathbf{1 3}, 0.6214 \mathrm{~g}, 48 \%$ yield for<smiles>O=C(O)c1ccccc1C#CCC1(C(=O)OCc2ccccc2)CC=CC1</smiles>
two steps, unknown compound, yellow oil, $\mathrm{R}_{f}=0.20$ (petroleum ether/ethyl acetate 20/1); ${ }^{1} \mathrm{H}$ NMR $\left(400 \mathrm{MHz}, \mathrm{CDCl}_{3}\right) \delta 8.10(\mathrm{~d}, J=8.7$ $\mathrm{Hz}, 1 \mathrm{H}), 7.36-7.26(\mathrm{~m}, 7 \mathrm{H}), 7.35-7.26(\mathrm{~m}, 1 \mathrm{H}), 6.95-6.87(\mathrm{~m}, 1 \mathrm{H}), 5.70$ $(\mathrm{d}, J=8.3 \mathrm{~Hz}, 1 \mathrm{H}), 5.68(\mathrm{~d}, J=8.3 \mathrm{~Hz}, 1 \mathrm{H}), 5.20(\mathrm{~s}, 2 \mathrm{H}), 3.01(\mathrm{~d}, J=$ $15.1,2 \mathrm{H}), 2.85(\mathrm{~s}, 2 \mathrm{H}), 2.56(\mathrm{~d}, J=15.1 \mathrm{~Hz}, 2 \mathrm{H}), 1.53(\mathrm{~s}, 9 \mathrm{H}) .{ }^{13} \mathrm{C} \mathrm{NMR}$ $\left(100 \mathrm{MHz}, \mathrm{CDCl}_{3}\right) \delta 176.1,152.8,139.9,136.1,132.0,129.3,128.73,128.7,128.4,128.2,122.1$, 117.8, 111.6, 93.8, 80.8, 77.7, 77.6, 77.2, 76.9, 67.0, 51.9, 42.8, 29.8, 28.5. HRMS (ESI-TOF) $\mathrm{m} / \mathrm{z}$ : $[\mathrm{M}+\mathrm{Na}]^{+} \mathrm{C}_{27} \mathrm{H}_{29} \mathrm{NO}_{4} \mathrm{Na}$ Calcd for 454.1989; found 454.1986.

Benzyl 1-(3-(2-acetamidophenyl)prop-2-yn-1-yl)cyclopent-3-ene-1-carboxylate (4g): the reaction was conducted at $3.00 \mathrm{mmol}$ scale from 13, $0.5428 \mathrm{~g}, 49 \%$ yield for two steps, unknown<smiles>O=C(Nc1ccccc1C#CCC1(C(=O)O)CC=CC1)c1ccccc1</smiles>
compound, yellow oil, $\mathrm{R}_{f}=0.22$ (petroleum ether/ethyl acetate $10 / 1$ ); ${ }^{1} \mathrm{H}$ NMR $\left(400 \mathrm{MHz}, \mathrm{CDCl}_{3}\right) \delta 8.39(\mathrm{~d}, J=8.3 \mathrm{~Hz}, 1 \mathrm{H}), 8.08(\mathrm{~s}, 1 \mathrm{H})$, 7.44-7.16 (m, 7H), 7.02-6.92 (m, 1H), $5.67(\mathrm{~d}, J=8.2 \mathrm{~Hz}, 1 \mathrm{H}), 5.65(\mathrm{~d}$, $J=8.2 \mathrm{~Hz}, 1 \mathrm{H}), 5.18(\mathrm{~s}, 2 \mathrm{H}), 2.99(\mathrm{~d}, J=15.0 \mathrm{~Hz}, 2 \mathrm{H}), 2.81(\mathrm{~s}, 2 \mathrm{H})$, $2.53(\mathrm{~d}, J=15.2 \mathrm{~Hz}, 2 \mathrm{H}), 2.25(\mathrm{~s}, 3 \mathrm{H}) .{ }^{13} \mathrm{C}$ NMR $\left(100 \mathrm{MHz}, \mathrm{CDCl}_{3}\right) \delta$ 176.3, 169.3, 139.8, 135.9, 131.8, 129.3, 128.7, 128.5, 128.4, 128.1, 123.2, 119.6, 112.1, 94.0, 77.9, 77.6, 77.2, 76.9, 67.1, 52.5, 42.4, 29.9, 24.8. HRMS (ESI-TOF) $\mathrm{m} / \mathrm{z}:[\mathrm{M}+\mathrm{Na}]^{+} \mathrm{C}_{24} \mathrm{H}_{23} \mathrm{NO}_{3} \mathrm{Na}$ 
Benzyl 1-(3-(4-methyl-2-((4-methylphenyl)sulfonamido)phenyl)prop-2-yn-1-yl)cyclopent-3ene-1-carboxylate (4h): the reaction was conducted at $3.00 \mathrm{mmol}$ scale from 13, $0.5996 \mathrm{~g}, 40 \%$<smiles>Cc1ccc(C#CCC2(C(=O)O)CC=CC2)c(N)c1</smiles>
yield for two steps, unknown compound, yellow oil, $\mathrm{R}_{f}=0.2$ (petroleum ether/ethyl acetate 10/1); ${ }^{1} \mathrm{H}$ NMR (400 $\left.\mathrm{MHz}, \mathrm{CDCl}_{3}\right) \delta$ $7.71(\mathrm{~d}, J=8.3 \mathrm{~Hz}, 2 \mathrm{H}), 7.40$ (s, 1H), 7.38-7.27 (m, 6H), 7.19 (d, $J$ $=8.2 \mathrm{~Hz}, 2 \mathrm{H}), 7.06(\mathrm{~d}, J=7.8 \mathrm{~Hz}, 1 \mathrm{H}), 6.76(\mathrm{~d}, J=7.8 \mathrm{~Hz}, 1 \mathrm{H})$, $5.70(\mathrm{~d}, J=8.1 \mathrm{~Hz}, 1 \mathrm{H}), 5.68(\mathrm{~d}, J=8.0 \mathrm{~Hz}, 1 \mathrm{H}), 5.24(\mathrm{~s}, 2 \mathrm{H}), 2.99$ (d, $J=15.1 \mathrm{~Hz}, 2 \mathrm{H}), 2.74$ (s, 2H), $2.48(\mathrm{~d}, J=15.0 \mathrm{~Hz}, 2 \mathrm{H}), 2.35$ (s, 3H), 2.30 (s, 3H). ${ }^{13} \mathrm{C} \mathrm{NMR}$ $\left(100 \mathrm{MHz}, \mathrm{CDCl}_{3}\right) \delta 176.2,143.9,139.8,138.2,136.7,136.2,131.9,129.7,128.7,128.6,128.4$, 128.3, 127.5, 125.0, 119.7, 111.1, 93.6, 77.6, 77.2, 77.2, 76.9, 7.1, 52.0, 42.8, 29.7, 22.0, 21.8. HRMS (ESI-TOF) m/z: [M+Na] ${ }^{+} \mathrm{C}_{30} \mathrm{H}_{29} \mathrm{NO}_{4} \mathrm{SNa}$ Calcd for 522.1710; found 522.1706.

Benzyl 1-(3-(5-methyl-2-((4-methylphenyl)sulfonamido)phenyl)prop-2-yn-1-yl)cyclopent-3ene-1-carboxylate (4i): the reaction was conducted at $3.00 \mathrm{mmol}$ scale from $\mathbf{1 3}, 0.8271 \mathrm{~g}, 55 \%$<smiles>Cc1ccc(N)c(C#CCC2(C(=O)OCc3ccccc3)CC=CC2)c1</smiles>
yield for two steps, unknown compound, yellow oil, $\mathrm{R}_{f}=0.2$ (petroleum ether/ethyl acetate 10/1); ${ }^{1} \mathrm{H}$ NMR $\left(400 \mathrm{MHz}, \mathrm{CDCl}_{3}\right) \delta$ $7.68(\mathrm{~d}, J=8.3 \mathrm{~Hz}, 2 \mathrm{H}), 7.47(\mathrm{~d}, J=8.4 \mathrm{~Hz}, 1 \mathrm{H}), 7.40-7.26(\mathrm{~m}, 6 \mathrm{H})$, $7.17(\mathrm{~d}, J=8.0 \mathrm{~Hz}, 2 \mathrm{H}), 7.04-6.98(\mathrm{~m}, 1 \mathrm{H}), 6.98-6.93(\mathrm{~m}, 1 \mathrm{H})$, $5.70(\mathrm{~d}, J=8.2 \mathrm{~Hz}, 1 \mathrm{H}), 5.68(\mathrm{~d}, J=8.2 \mathrm{~Hz}, 1 \mathrm{H}), 5.24(\mathrm{~s}, 2 \mathrm{H}), 2.99$ (d, $J=15.0 \mathrm{~Hz}, 2 \mathrm{H}), 2.74(\mathrm{~s}, 2 \mathrm{H}), 2.47$ (d, $J=15.1 \mathrm{~Hz}, 2 \mathrm{H}), 2.33(\mathrm{~s}, 3 \mathrm{H}), 2.19$ (s, 3H). ${ }^{13} \mathrm{C} \mathrm{NMR}$ $\left(100 \mathrm{MHz}, \mathrm{CDCl}_{3}\right) \delta 176.1,143.8,136.6,136.1,135.8,133.8,132.5,130.1,129.6,128.7,128.6$, 128.3, 128.2, 127.4, 119.5, 114.1, 93.8, 77.6, 77.2, 76.9, 67.1, 51.9, 42.7, 29.6, 21.7, 20.7. HRMS (ESI-TOF) $m / z$ : $[\mathrm{M}+\mathrm{Na}]^{+} \mathrm{C}_{30} \mathrm{H}_{29} \mathrm{NO}_{4} \mathrm{SNa}$ Calcd for 522.1710; found 522.1708.

Benzyl 1-(3-(5-methoxy-2-((4-methylphenyl)sulfonamido)phenyl)prop-2-yn-1-yl)cyclopent-3ene-1-carboxylate (4g): the reaction was conducted at $3.00 \mathrm{mmol}$ scale from 13, $0.9032 \mathrm{~g}, 59 \%$<smiles>[3H]Nc1ccc(OC)cc1C#CCC1(C(=O)O)CC=CC1</smiles>
yield for two steps, unknown compound, yellow oil, $\mathrm{R}_{f}=0.2$ (petroleum ether/ethyl acetate 10/1); ${ }^{1} \mathrm{H}$ NMR $\left(400 \mathrm{MHz}, \mathrm{CDCl}_{3}\right)$ $\delta 7.62(\mathrm{~d}, J=8.3 \mathrm{~Hz}, 2 \mathrm{H}), 7.52(\mathrm{~d}, J=9.0 \mathrm{~Hz}, 1 \mathrm{H}), 7.36-7.25(\mathrm{~m}$, $5 \mathrm{H}), 7.16(\mathrm{~d}, J=8.2 \mathrm{~Hz}, 2 \mathrm{H}), 7.09(\mathrm{~s}, 1 \mathrm{H}), 6.82-6.77(\mathrm{~m}, 1 \mathrm{H})$, $6.68(\mathrm{~d}, J=2.9 \mathrm{~Hz}, 1 \mathrm{H}), 5.70(\mathrm{~d}, J=8.1 \mathrm{~Hz}, 1 \mathrm{H}), 5.68(\mathrm{~d}, J=8.1$ $\mathrm{Hz}, 1 \mathrm{H}), 5.24$ (s, 2H), 3.70 (s, 3H), 2.98 (d, J=15.0 Hz, 2H), 2.70 (s, 2H), 2.45 (d, J=15.0 Hz, $2 \mathrm{H}), 2.34(\mathrm{~s}, 3 \mathrm{H}) .{ }^{13} \mathrm{C}$ NMR $\left(100 \mathrm{MHz}, \mathrm{CDCl}_{3}\right) \delta 176.1,156.4,143.8,136.5,136.1,131.5,129.6$, 128.7, 128.6, 128.4, 128.2, 127.4, 122.5, 116.6, 116.3, 115.6, 93.9, 77.6, 77.2, 77.2, 76.9, 67.1, 55.6, 51.8, 42.8, 29.5, 21.7. HRMS (ESI-TOF) $\mathrm{m} / \mathrm{z}$ : $[\mathrm{M}+\mathrm{Na}]^{+} \mathrm{C}_{30} \mathrm{H}_{29} \mathrm{NO}_{5} \mathrm{SNa}$ Calcd for 538.1659; found 538.1662 . 
Benzyl 1-(3-(4-fluoro-2-((4-methylphenyl)sulfonamido)phenyl)prop-2-yn-1-yl)cyclopent-3-<smiles>[3H]Nc1cc(F)ccc1C#CCC1(C(=O)Cc2ccccc2)CC=CC1</smiles>

ene-1-carboxylate (4k): the reaction was conducted at $3.00 \mathrm{mmol}$ scale from 13, $0.7583 \mathrm{~g}, 75 \%$ yield for two steps, unknown compound, yellow solid, $\mathrm{R}_{f}=0.15$ (petroleum ether/ethyl acetate 15/1); ${ }^{1} \mathrm{H}$ NMR (400 MHz, $\left.\mathrm{CDCl}_{3}\right) \delta 7.77$ (d, $\left.J=8.3 \mathrm{~Hz}, 2 \mathrm{H}\right), 7.59$ (s, $1 \mathrm{H}), 7.38-7.26(\mathrm{~m}, 6 \mathrm{H}), 7.23(\mathrm{~d}, J=8.1 \mathrm{~Hz}, 2 \mathrm{H}), 7.18-7.07(\mathrm{~m}, 1 \mathrm{H})$, 6.69-6.57 (m, 1H), $5.70(\mathrm{~s}, 2 \mathrm{H}), 5.25(\mathrm{~s}, 2 \mathrm{H}), 3.01(\mathrm{~d}, J=15.0 \mathrm{~Hz}, 2 \mathrm{H}), 2.76(\mathrm{~s}, 2 \mathrm{H}), 2.49$ (d, $J=$ $15.1 \mathrm{~Hz}, 2 \mathrm{H}), 2.37(\mathrm{~s}, 3 \mathrm{H}) .{ }^{13} \mathrm{C} \mathrm{NMR}\left(100 \mathrm{MHz}, \mathrm{CDCl}_{3}\right) \delta 176.2,162.7\left(\mathrm{~d}, J_{\mathrm{C}-\mathrm{F}}=247.0 \mathrm{~Hz}\right)$, $144.3,140.2\left(\mathrm{~d}, J_{\mathrm{C}-\mathrm{F}}=12.0 \mathrm{~Hz}\right), 136.4,136.1,133.5\left(\mathrm{~d}, J_{\mathrm{C}-\mathrm{F}}=9.0 \mathrm{~Hz}\right), 129.9,128.7,128.6,128.4$, $128.3,127.5,110.9\left(\mathrm{~d}, J_{\mathrm{C}-\mathrm{F}}=22.0 \mathrm{~Hz}\right), 109.3\left(\mathrm{~d}, J_{\mathrm{C}-\mathrm{F}}=4.0 \mathrm{~Hz}\right), 106.0\left(\mathrm{~d}, J_{\mathrm{C}-\mathrm{F}}=28.0 \mathrm{~Hz}\right), 94.3$, 77.6, 77.2, 76.9, 76.2, 67.2, 52.0, 42.8, 29.7, 21.8. ${ }^{19} \mathrm{~F}$ NMR (376 MHz, $\left.\mathrm{CDCl}_{3}\right) \delta-107.86 . \mathrm{HRMS}$ (ESI-TOF) $\mathrm{m} / \mathrm{z}$ : $[\mathrm{M}+\mathrm{Na}]^{+} \mathrm{C}_{29} \mathrm{H}_{26} \mathrm{FNO}_{4} \mathrm{SNa}$ Calcd for 526.1459; found 526.1453.

Benzyl 1-(3-(5-fluoro-2-((4-methylphenyl)sulfonamido)phenyl)prop-2-yn-1-yl)cyclopent-3ene-1-carboxylate (4I): the reaction was conducted at $3.00 \mathrm{mmol}$ scale from 13, $1.0261 \mathrm{~g}, 68 \%$<smiles>O=C(Cc1ccccc1)C1(CC#Cc2cc(F)ccc2N[135I])CC=CC1</smiles>
yield for two steps, unknown compound, yellow solid, $\mathrm{R}_{f}=0.15$ (petroleum ether/ethyl acetate 15/1); ${ }^{1} \mathrm{H}$ NMR $\left(400 \mathrm{MHz}, \mathrm{CDCl}_{3}\right){ }^{1} \mathrm{H}$ $\operatorname{NMR}\left(400 \mathrm{MHz}, \mathrm{CDCl}_{3}\right) \delta 7.68(\mathrm{~d}, J=8.3 \mathrm{~Hz}, 2 \mathrm{H}), 7.58$ (dd, $J=9.1$, $5.0 \mathrm{~Hz}, 1 \mathrm{H}), 7.42-7.29(\mathrm{~m}, 6 \mathrm{H}), 7.22(\mathrm{~d}, J=8.3 \mathrm{~Hz}, 2 \mathrm{H}), 7.00-6.92$ (m, 1H), 6.88-6.82 (m, 1H), $5.73(\mathrm{~d}, J=8.2 \mathrm{~Hz}, 1 \mathrm{H}), 5.71(\mathrm{~d}, J=8.0$ $\mathrm{Hz}, 1 \mathrm{H}), 5.27(\mathrm{~s}, 2 \mathrm{H}), 3.01(\mathrm{~d}, J=15.0 \mathrm{~Hz}, 2 \mathrm{H}), 2.74(\mathrm{~s}, 2 \mathrm{H}), 2.47(\mathrm{~d}, J=15.0 \mathrm{~Hz}, 2 \mathrm{H}), 2.38(\mathrm{~s}$, $3 \mathrm{H}) .{ }^{13} \mathrm{C} \mathrm{NMR}\left(100 \mathrm{MHz}, \mathrm{CDCl}_{3}\right) \delta 175.8,158.8\left(\mathrm{~d}, J_{\mathrm{C}-\mathrm{F}}=243.0 \mathrm{~Hz}\right), 143.9,136.2,135.9,134.5$ $\left(\mathrm{d}, J_{\mathrm{C}-\mathrm{F}}=2.0 \mathrm{~Hz}\right), 129.5,128.5,128.4,128.2,128.1,127.2,121.6\left(\mathrm{~d}, J_{\mathrm{C}-\mathrm{F}}=9.0 \mathrm{~Hz}\right), 118.3\left(\mathrm{~d}, J_{\mathrm{C}-\mathrm{F}}\right.$ $=23.0 \mathrm{~Hz}), 116.3,116.2,116.1,95.3,77.6,77.2,76.9,76.1\left(\mathrm{~d}, J_{\mathrm{C}-\mathrm{F}}=3.0 \mathrm{~Hz}\right), 66.9,51.6,42.6$, 29.3, 21.5. ${ }^{19} \mathrm{~F}$ NMR (376 MHz, $\left.\mathrm{CDCl}_{3}\right) \quad \delta$-118.14. HRMS (ESI-TOF) $\mathrm{m} / \mathrm{z}:[\mathrm{M}+\mathrm{Na}]^{+}$ $\mathrm{C}_{29} \mathrm{H}_{26} \mathrm{FNO}_{4} \mathrm{SNa}$ Calcd for 526.1459; found 526.1456.

Benzyl 1-(3-(3-fluoro-2-((4-methylphenyl)sulfonamido)phenyl)prop-2-yn-1-yl)cyclopent-3ene-1-carboxylate (4m): the reaction was conducted at $3.00 \mathrm{mmol}$ scale from 13, $0.8450 \mathrm{~g}, 56 \%$<smiles>CNc1c(F)cccc1C#CCC1(C(=O)O)CC=CC1</smiles>
yield for two steps, unknown compound, yellow solid, $\mathrm{R}_{f}=0.2$ (petroleum ether/ethyl acetate 10/1); ${ }^{1} \mathrm{H}$ NMR $\left(400 \mathrm{MHz}, \mathrm{CDCl}_{3}\right) \delta 7.71$ (d, $J=8.2 \mathrm{~Hz}, 2 \mathrm{H}), 7.36-7.27(\mathrm{~m}, 4 \mathrm{H}), 7,27-7.23(\mathrm{~m}, 3 \mathrm{H}), 7.10-6.95(\mathrm{~m}$, $3 \mathrm{H}), 6.62$ (br s, 1H), $5.67(\mathrm{~d}, J=8.0 \mathrm{~Hz}, 1 \mathrm{H}), 5.65(\mathrm{~d}, J=7.5 \mathrm{~Hz}, 1 \mathrm{H})$, $5.21(\mathrm{~s}, 2 \mathrm{H}), 2.97(\mathrm{~d}, J=15.3 \mathrm{~Hz}, 2 \mathrm{H}), 2.65(\mathrm{~s}, 2 \mathrm{H}), 2.47(\mathrm{~d}, J=15.1 \mathrm{~Hz}$, $2 \mathrm{H}), 2.39(\mathrm{~s}, 3 \mathrm{H}) .{ }^{13} \mathrm{C} \mathrm{NMR}\left(100 \mathrm{MHz}, \mathrm{CDCl}_{3}\right) \delta 176.1,157.1\left(\mathrm{~d}, J_{\mathrm{C}-\mathrm{F}}=250.0 \mathrm{~Hz}\right), 143.8,137.6$, 136.1, 129.4, 128.7, 128.5, 128.3, 128.2, $127.9\left(\mathrm{~d}, J_{\mathrm{C}-\mathrm{F}}=3.0 \mathrm{~Hz}\right), 127.5,127.0\left(\mathrm{~d}, J_{\mathrm{C}-\mathrm{F}}=8.0 \mathrm{~Hz}\right)$, $126.2\left(\mathrm{~d}, J_{\mathrm{C}-\mathrm{F}}=13.0 \mathrm{~Hz}\right), 121.6\left(\mathrm{~d}, J_{\mathrm{C}-\mathrm{F}}=3.0 \mathrm{~Hz}\right), 116.9\left(\mathrm{~d}, J_{\mathrm{C}-\mathrm{F}}=20.0 \mathrm{~Hz}\right), 94.7,77.6,77.2,76.9$, 76.9, 67.1, 51.9, 42.7, 29.5, 21.7. ${ }^{19} \mathrm{~F}$ NMR $\left(376 \mathrm{MHz}, \mathrm{CDCl}_{3}\right) \delta-116.67$. HRMS (ESI-TOF) $\mathrm{m} / \mathrm{z}$ : $[\mathrm{M}+\mathrm{Na}]^{+} \mathrm{C}_{29} \mathrm{H}_{26} \mathrm{FNO}_{4} \mathrm{SNa}$ Calcd for 526.1459; found 526.1451 . 
Benzyl 1-(3-(4-chloro-2-((4-methylphenyl)sulfonamido)phenyl)prop-2-yn-1-yl)cyclopent-3ene-1-carboxylate (4n): the reaction was conducted at $3.00 \mathrm{mmol}$ scale from 13, $0.7502 \mathrm{~g}, 48 \%$

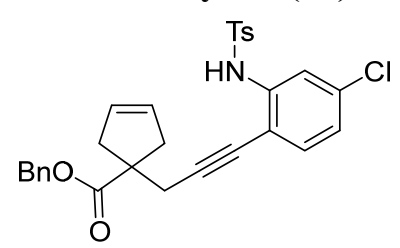
yield for two steps, unknown compound, yellow solid, $\mathrm{R}_{f}=0.25$ (petroleum ether/ethyl acetate 10/1); ${ }^{1} \mathrm{H}$ NMR $\left(400 \mathrm{MHz}, \mathrm{CDCl}_{3}\right) \delta$ $7.76(\mathrm{~d}, J=8.3 \mathrm{~Hz}, 2 \mathrm{H}), 7.61(\mathrm{~d}, J=2.0 \mathrm{~Hz}, 1 \mathrm{H}), 7.54(\mathrm{~s}, 1 \mathrm{H})$, $7.38-7.26(\mathrm{~m}, 5 \mathrm{H}), 7.22(\mathrm{~d}, J=8.1 \mathrm{~Hz}, 2 \mathrm{H}), 7.08(\mathrm{~d}, J=8.3 \mathrm{~Hz}, 1 \mathrm{H})$, 6.95-6.85 (m, 1H), $5.70(\mathrm{~d}, J=8.2 \mathrm{~Hz}, 1 \mathrm{H}), 5.68(\mathrm{~d}, J=8.1 \mathrm{~Hz}, 1 \mathrm{H})$, $5.25(\mathrm{~s}, 2 \mathrm{H}), 3.00(\mathrm{~d}, J=15.1 \mathrm{~Hz}, 2 \mathrm{H}), 2.75(\mathrm{~s}, 2 \mathrm{H}), 2.47(\mathrm{~d}, J=15.1 \mathrm{~Hz}, 2 \mathrm{H}), 2.36(\mathrm{~s}, 3 \mathrm{H}) .{ }^{13} \mathrm{C}$ NMR $\left(100 \mathrm{MHz}, \mathrm{CDCl}_{3}\right) \delta 176.1,144.3,139.5,136.4,136.0,135.0,132.9,129.9,128.7,128.6$, 128.4, 128.3, 127.5, 124.0, 118.6, 112.0, 95.5, 77.6, 77.2, 76.9, 76.3, 67.2, 51.9, 42.8, 29.7, 21.8. HRMS (ESI-TOF) $\mathrm{m} / z$ : $[\mathrm{M}+\mathrm{Na}]^{+} \mathrm{C}_{29} \mathrm{H}_{26} \mathrm{ClNO}_{4} \mathrm{SNa}$ Calcd for 542.1163; found 542.1157 .

Benzyl 1-(3-(5-chloro-2-((4-methylphenyl)sulfonamido)phenyl)prop-2-yn-1-yl)cyclopent-3ene-1-carboxylate (40): the reaction was conducted at $3.00 \mathrm{mmol}$ scale from 13, $0.8284 \mathrm{~g}, 53 \%$<smiles>O=C(Cc1ccccc1)C1(CC#Cc2cc(Cl)ccc2N[135I])CC=CC1</smiles>
yield for two steps, unknown compound, white solid, $\mathrm{R}_{f}=0.25$ (petroleum ether/ethyl acetate 10/1); ${ }^{1} \mathrm{H}$ NMR $\left(400 \mathrm{MHz}, \mathrm{CDCl}_{3}\right) \delta$ $7.73(\mathrm{~d}, J=8.3 \mathrm{~Hz}, 2 \mathrm{H}), 7.55(\mathrm{~d}, J=8.8 \mathrm{~Hz}, 1 \mathrm{H}), 7.51-7.29(\mathrm{~m}, 6 \mathrm{H})$, $7.26-7.21(\mathrm{~m}, 2 \mathrm{H}), 7.21-7.17(\mathrm{~m}, 1 \mathrm{H}), 7.14(\mathrm{~d}, J=2.4 \mathrm{~Hz}, 1 \mathrm{H}), 5.73$ $(\mathrm{d}, J=8.0 \mathrm{~Hz}, 1 \mathrm{H}), 5.71(\mathrm{~d}, J=8.2 \mathrm{~Hz}, 1 \mathrm{H}), 5.27(\mathrm{~s}, 2 \mathrm{H}), 3.02(\mathrm{~d}, J=$ $15.1 \mathrm{~Hz}, 2 \mathrm{H}), 2.77$ (s, 2H), 2.48 (d, $J=15.1 \mathrm{~Hz}, 2 \mathrm{H}), 2.39$ (s, 3H). ${ }^{13} \mathrm{C}$ NMR $\left(100 \mathrm{MHz}, \mathrm{CDCl}_{3}\right) \delta$ $176.1,144.3,137.1,136.4,136.0,131.7,129.8,129.4,129.2,128.8,128.6,128.5,128.3,127.5$, 120.3, 115.5, 95.7, 77.6, 77.2, 76.9, 76.0, 67.2, 51.9, 42.8, 29.6, 21.8. HRMS (ESI-TOF) $\mathrm{m} / \mathrm{z}$ : $[\mathrm{M}+\mathrm{Na}]^{+} \mathrm{C}_{29} \mathrm{H}_{26} \mathrm{ClNO}_{4} \mathrm{SNa}$ Calcd for 542.1163; found 542.1154.

Benzyl 1-(3-(2-((4-methylphenyl)sulfonamido)-5-(trifluoromethyl)phenyl)prop-2-yn-1-yl) cyclopent-3-ene-1-carboxylate (4p): the reaction was conducted at $3.00 \mathrm{mmol}$ scale from $\mathbf{1 3}$,<smiles>CNc1ccc(C(F)(F)F)cc1C#CCC1(C(=O)O)CC=CC1</smiles>
$0.7634 \mathrm{~g}, 46 \%$ yield for two steps, unknown compound, white solid, $\mathrm{R}_{f}=0.15$ (petroleum ether/ethyl acetate $15 / 1$ ); ${ }^{1} \mathrm{H}$ NMR $\left(400 \mathrm{MHz}, \mathrm{CDCl}_{3}\right) \delta$ 7.90-7.76 (m, 3H), $7.65(\mathrm{~d}, J=8.4 \mathrm{~Hz}, 1 \mathrm{H})$, $7.46-7.26(\mathrm{~m}, 7 \mathrm{H}), 7.25(\mathrm{~d}, J=8.9 \mathrm{~Hz}, 2 \mathrm{H}), 5.71(\mathrm{~s}, 2 \mathrm{H}), 5.26(\mathrm{~s}$, 2H), $3.02(\mathrm{~d}, J=15.1 \mathrm{~Hz}, 2 \mathrm{H}), 2.78(\mathrm{~s}, 2 \mathrm{H}), 2.49(\mathrm{~d}, J=15.0 \mathrm{~Hz}$, $2 \mathrm{H}), 2.38(\mathrm{~s}, 3 \mathrm{H}) .{ }^{13} \mathrm{C}$ NMR $\left(100 \mathrm{MHz}, \mathrm{CDCl}_{3}\right) \delta 176.2,144.5$, $141.5,136.4,136.0,130.0,129.3\left(\mathrm{q}, J_{\mathrm{C}-\mathrm{F}}=4.0 \mathrm{~Hz}\right), 128.7,128.6,128.5,128.4,127.5,126.1(\mathrm{q}$, $\left.J_{\mathrm{C}-\mathrm{F}}=4.0 \mathrm{~Hz}\right), 117.5,113.4,96.3,77.6,77.2,76.9,75.9,67.3,52.0,42.8,29.7,21.8 .{ }^{19} \mathrm{~F} \mathrm{NMR}$ $\left(376 \mathrm{MHz}, \mathrm{CDCl}_{3}\right.$ ) $\delta$-62.38. HRMS (ESI-TOF) $\mathrm{m} / \mathrm{z}$ : $[\mathrm{M}+\mathrm{Na}]^{+} \mathrm{C}_{30} \mathrm{H}_{26} \mathrm{~F}_{3} \mathrm{NO}_{4} \mathrm{SNa}$ Calcd for 576.1427; found 576.1426.

Benzyl 1-(3-(5-acetyl-2-((4-methylphenyl)sulfonamido)phenyl)prop-2-yn-1-yl)cyclopent-3 -ene-1-carboxylate (4q): the reaction was conducted at $3.00 \mathrm{mmol}$ scale from $\mathbf{1 3}, 0.5534 \mathrm{~g}, 35 \%$<smiles>CNc1ccc(C(C)=O)cc1C#CCC1(C(=O)OCc2ccccc2)CC=CC1</smiles>
yield for two steps, unknown compound, yellow oil, $\mathrm{R}_{f}=0.25$ (petroleum ether/ethyl acetate $5 / 1) ;{ }^{1} \mathrm{H}$ NMR $\left(400 \mathrm{MHz}, \mathrm{CDCl}_{3}\right) \delta$ $7.90-7.76(\mathrm{~m}, 5 \mathrm{H}), 7.62(\mathrm{~d}, J=8.7 \mathrm{~Hz}, 1 \mathrm{H}), 7.38-7.26(\mathrm{~m}, 5 \mathrm{H})$, $7.24(\mathrm{~d}, J=8.7 \mathrm{~Hz}, 2 \mathrm{H}), 5.72(\mathrm{~d}, J=8.1 \mathrm{~Hz}, 1 \mathrm{H}), 5.70(\mathrm{~d}, J=8.1$ $\mathrm{Hz}, 1 \mathrm{H}), 5.27$ (s, 2H), $3.03(\mathrm{~d}, J=15.1 \mathrm{~Hz}, 2 \mathrm{H}), 2.80(\mathrm{~s}, 2 \mathrm{H}), 2.51$ 
(d, $J=16.0 \mathrm{~Hz}, 2 \mathrm{H}), 2.49$ (s, 2H), 2.36 (s, 3H). ${ }^{13} \mathrm{C} \mathrm{NMR}\left(100 \mathrm{MHz}, \mathrm{CDCl}_{3} \delta\right.$ 196.3, 176.2, 144.5, 142.5, 136.4, 136.0, 132.6, 132.2, 130.0, 129.5, 128.7, 128.6, 128.4, 128.3, 127.5, 116.8, 113.0, 95.7, 77.6, 77.2, 76.9, 76.3, 67.2, 52.0, 42.8, 29.7, 26.5, 21.8. HRMS (ESI-TOF) $\mathrm{m} / \mathrm{z}$ : $[\mathrm{M}+\mathrm{Na}]^{+}$ $\mathrm{C}_{31} \mathrm{H}_{29} \mathrm{NO}_{5} \mathrm{SNa}$ Calcd for 550.1659; found 550.1662 .

Methyl 3-(3-(1-((benzyloxy)carbonyl)cyclopent-3-en-1-yl)prop-1-yn-1-yl)-4-((4-methylphen-<smiles>CNc1ccc(C(=O)OC)cc1C#CCC1(C(=O)OCc2ccccc2)CC=CC1</smilesyl)sulfonamido)benzoate (4r): the reaction was conducted at 3.00 mmol scale from 13, $0.7984 \mathrm{~g}, 49 \%$ yield for two steps, unknown compound, yellow oil, $\mathrm{R}_{f}=0.25$ (petroleum ether/ethyl acetate 5/1); ${ }^{1} \mathrm{H}$ NMR (400 MHz, $\left.\mathrm{CDCl}_{3}\right) \delta 7.89(\mathrm{~d}, J=2.0 \mathrm{~Hz}, 1 \mathrm{H})$, $7.88-7.82(\mathrm{~m}, 1 \mathrm{H}), 7.79(\mathrm{~d}, J=8.5 \mathrm{~Hz}, 3 \mathrm{H}), 7.61(\mathrm{~d}, J=8.7 \mathrm{~Hz}$, 1H), 7.41-7.26 (m, 5H), $7.23(\mathrm{~d}, J=8.0 \mathrm{~Hz}, 2 \mathrm{H}), 5.70(\mathrm{~s}, 2 \mathrm{H}), 5.26(\mathrm{~s}, 2 \mathrm{H}), 3.85(\mathrm{~s}, 3 \mathrm{H}), 3.02(\mathrm{~d}$, $J=15.1 \mathrm{~Hz}, 2 \mathrm{H}), 2.79(\mathrm{~s}, 2 \mathrm{H}), 2.51(\mathrm{~d}, J=15.1 \mathrm{~Hz}, 2 \mathrm{H}), 2.36(\mathrm{~s}, 3 \mathrm{H}) .{ }^{13} \mathrm{C}$ NMR $(100 \mathrm{MHz}$, $\left.\mathrm{CDCl}_{3}\right) \delta 176.2,166.1,144.5,142.4,136.3,136.0,133.7,130.6,129.9,128.7,128.6,128.4,128.3$, $127.5,125.2,116.9,113.0,95.6,77.6,77.2,76.9,76.2,67.2,52.3,51.9,42.8,29.7,21.8$. HRMS (ESI-TOF) $\mathrm{m} / \mathrm{z}$ : $[\mathrm{M}+\mathrm{Na}]^{+} \mathrm{C}_{31} \mathrm{H}_{29} \mathrm{NO}_{6} \mathrm{SNa}$ Calcd for 566.1608; found 566.1605 .

Benzyl 1-(3-(4,5-difluoro-2-((4-methylphenyl)sulfonamido)phenyl)prop-2-yn-1-yl)cyclopent3-ene-1-carboxylate (4s): the reaction was conducted at $3.00 \mathrm{mmol}$ scale from 13, $0.6097 \mathrm{~g}, 39 \%$<smiles>CNc1cc(F)c(F)cc1C#CCC1(C(=O)OCc2ccccc2)CC=CC1</smiles>
yield for two steps, unknown compound, yellow oil, $\mathrm{R}_{f}=0.20$ (petroleum ether/ethyl acetate 15/1); ${ }^{1} \mathrm{H}$ NMR $\left(400 \mathrm{MHz}, \mathrm{CDCl}_{3}\right) \delta$ $7.72(\mathrm{~d}, J=8.3 \mathrm{~Hz}, 2 \mathrm{H}), 7.53-7.41(\mathrm{~m}, 2 \mathrm{H}), 7.38-7.27(\mathrm{~m}, 5 \mathrm{H}), 7.23$ $(\mathrm{d}, J=8.2 \mathrm{~Hz}, 2 \mathrm{H}), 6.98-6.86(\mathrm{~m}, 1 \mathrm{H}), 5.70(\mathrm{~d}, J=8.1 \mathrm{~Hz}, 1 \mathrm{H}), 5.68$ (d, $J=8.0 \mathrm{~Hz}, 1 \mathrm{H}), 5.25(\mathrm{~s}, 2 \mathrm{H}), 3.00(\mathrm{~d}, J=15.1 \mathrm{~Hz}, 2 \mathrm{H}), 2.72$ (s, $2 \mathrm{H}), 2.45(\mathrm{~d}, J=15.1 \mathrm{~Hz}, 2 \mathrm{H}), 2.38(\mathrm{~s}, 3 \mathrm{H}) .{ }^{13} \mathrm{C} \mathrm{NMR}(100 \mathrm{MHz}$, $\left.\mathrm{CDCl}_{3}\right) \delta 176.1,150.4\left(\mathrm{dd}, J_{\mathrm{C}-\mathrm{F}}=249.0,13.0 \mathrm{~Hz}\right), 146.6\left(\mathrm{dd}, J_{\mathrm{C}-\mathrm{F}}=246.0,13.0 \mathrm{~Hz}\right), 144.4,136.2$, $136.0,135.5\left(\mathrm{dd}, J_{\mathrm{C}-\mathrm{F}}=9.0,2.0 \mathrm{~Hz}\right), 129.9,128.7,128.5,128.4,128.3,127.4,120.2\left(\mathrm{~d}, J_{\mathrm{C}-\mathrm{F}}=19.0\right.$ $\mathrm{Hz}), 110.2\left(\mathrm{dd}, J_{\mathrm{C}-\mathrm{F}}=7.0,4.0 \mathrm{~Hz}\right), 109.0\left(\mathrm{~d}, J_{\mathrm{C}-\mathrm{F}}=23.0 \mathrm{~Hz}\right), 95.2\left(\mathrm{~d}, J_{\mathrm{C}-\mathrm{F}}=2.0 \mathrm{~Hz}\right), 77.6,77.2$, $76.9,75.4\left(\mathrm{dd}, J_{\mathrm{C}-\mathrm{F}}=3.0,2.0 \mathrm{~Hz}\right), 67.2,51.9,42.8,29.5,21.7 .{ }^{19} \mathrm{~F} \mathrm{NMR}\left(376 \mathrm{MHz}, \mathrm{CDCl}_{3}\right) \delta$ $-132.37(\mathrm{~d}, J=22.2 \mathrm{~Hz}),-142.45(\mathrm{~d}, J=22.2 \mathrm{~Hz}) . \mathrm{HRMS}(\mathrm{ESI}-\mathrm{TOF}) \mathrm{m} / \mathrm{z}:[\mathrm{M}+\mathrm{Na}]^{+}$ $\mathrm{C}_{29} \mathrm{H}_{25} \mathrm{~F}_{2} \mathrm{NO}_{4} \mathrm{SNa}$ Calcd for 544.1365; found 544.1365.

Benzyl 1-(3-(5-fluoro-4-methyl-2-((4-methylphenyl)sulfonamido)phenyl)prop-2-yn-1-yl) cyclopent-3-ene-1-carboxylate (4t): the reaction was conducted at $3.00 \mathrm{mmol}$ scale from $\mathbf{1 3}$,<smiles>Cc1cc(N[12F])c(C#CCC2(C(=O)Cc3ccccc3)CC=CC2)cc1F</smiles>
$0.6516 \mathrm{~g}, 42 \%$ yield for two steps, unknown compound, yellow oil, $\mathrm{R}_{f}=0.20$ (petroleum ether/ethyl acetate 15/1); ${ }^{1} \mathrm{H}$ NMR $(400 \mathrm{MHz}$, $\left.\mathrm{CDCl}_{3}\right) \delta 7.65(\mathrm{~d}, J=8.3 \mathrm{~Hz}, 2 \mathrm{H}), 7.43(\mathrm{~d}, J=7.0 \mathrm{~Hz}, 1 \mathrm{H})$, 7.39-7.27 (m, 5H), 7.24-7.15 (m, 3H), 6.77 (d, $J=9.4 \mathrm{~Hz}, 1 \mathrm{H})$, $5.69(\mathrm{~d}, J=8.2 \mathrm{~Hz}, 1 \mathrm{H}), 5.67(\mathrm{~d}, J=8.2 \mathrm{~Hz}, 1 \mathrm{H}), 5.24(\mathrm{~s}, 2 \mathrm{H}), 2.98$ (d, $J=15.0 \mathrm{~Hz}, 2 \mathrm{H}), 2.70(\mathrm{~s}, 2 \mathrm{H}), 2.44(\mathrm{~d}, J=15.0 \mathrm{~Hz}, 2 \mathrm{H}), 2.36(\mathrm{~s}, 3 \mathrm{H}), 2.23$ (d, $J=1.7 \mathrm{~Hz}, 3 \mathrm{H})$. ${ }^{13} \mathrm{C}$ NMR $\left(100 \mathrm{MHz}, \mathrm{CDCl}_{3}\right) \delta 176.0,158.7\left(\mathrm{~d}, J_{\mathrm{C}-\mathrm{F}}=241.0 \mathrm{~Hz}\right), 144.0,136.4,136.1,134.2(\mathrm{~d}$, $\left.J_{\mathrm{C}-\mathrm{F}}=3.0 \mathrm{~Hz}\right), 129.7,128.7,128.6,128.4,128.3,127.4,126.8\left(\mathrm{~d}, J_{\mathrm{C}-\mathrm{F}}=19.0 \mathrm{~Hz}\right), 123.2\left(\mathrm{~d}, J_{\mathrm{C}-\mathrm{F}}=\right.$ $6.0 \mathrm{~Hz}), 118.0\left(\mathrm{~d}, J_{\mathrm{C}-\mathrm{F}}=25.0 \mathrm{~Hz}\right), 113.5\left(\mathrm{~d}, J_{\mathrm{C}-\mathrm{F}}=9.0 \mathrm{~Hz}\right), 94.3,77.6,77.2,76.9,76.4\left(\mathrm{~d}, J_{\mathrm{C}-\mathrm{F}}=\right.$ $3.0 \mathrm{~Hz}), 67.2,51.9,42.8,29.5,21.8,15.1\left(\mathrm{~d}, J_{\mathrm{C}-\mathrm{F}}=3.0 \mathrm{~Hz}\right) .{ }^{19} \mathrm{~F}$ NMR $\left(376 \mathrm{MHz}, \mathrm{CDCl}_{3}\right) \delta$ 
Benzyl 1-(3-(3-chloro-5-fluoro-2-((4-methylphenyl)sulfonamido)phenyl)prop-2-yn-1-yl) cyclopent-3-ene-1-carboxylate (4u): the reaction was conducted at $3.00 \mathrm{mmol}$ scale from $\mathbf{1 3}$,<smiles>[3H]Nc1c(Cl)cc(F)cc1C#CCC1(C(=O)OCc2ccccc2)CC=CC1</smiles>
$0.7573 \mathrm{~g}, 47 \%$ yield for two steps, unknown compound, yellow solid, $\mathrm{R}_{f}=0.20$ (petroleum ether/ethyl acetate $\left.15 / 1\right) ;{ }^{1} \mathrm{H}$ NMR $(400 \mathrm{MHz}$, $\left.\mathrm{CDCl}_{3}\right) \delta 7.64(\mathrm{~d}, J=8.2 \mathrm{~Hz}, 2 \mathrm{H}), 7.41-7.27(\mathrm{~m}, 5 \mathrm{H}), 7.22(\mathrm{~d}, J=8.1$ $\mathrm{Hz}, 2 \mathrm{H}), 7.12-7.00(\mathrm{~m}, 1 \mathrm{H}), 6.90-6.82(\mathrm{~m}, 1 \mathrm{H}), 6.38(\mathrm{~s}, 1 \mathrm{H}), 5.64(\mathrm{~s}$, 2H), 5.19 (s, 2H), 2.93 (d, $J=15.3 \mathrm{~Hz}, 2 \mathrm{H}), 2.57$ (s, 2H), 2.44 (d, $J=$ $15.1 \mathrm{~Hz}, 2 \mathrm{H}), 2.39(\mathrm{~s}, 3 \mathrm{H}) .{ }^{13} \mathrm{C} \mathrm{NMR}\left(100 \mathrm{MHz}, \mathrm{CDCl}_{3}\right) \delta 175.9,160.4\left(\mathrm{~d}, J_{\mathrm{C}-\mathrm{F}}=250.0 \mathrm{~Hz}\right)$, 143.9, 137.7, 136.2, $134.7\left(\mathrm{~d}, J_{\mathrm{C}-\mathrm{F}}=12.0 \mathrm{~Hz}\right), 131.3\left(\mathrm{~d}, J_{\mathrm{C}-\mathrm{F}}=4.0 \mathrm{~Hz}\right), 129.6,128.7,128.4,128.4$, $128.3,127.7,126.1\left(\mathrm{~d}, J_{\mathrm{C}-\mathrm{F}}=11.0 \mathrm{~Hz}\right), 118.4\left(\mathrm{~d}, J_{\mathrm{C}-\mathrm{F}}=13.0 \mathrm{~Hz}\right), 117.8\left(\mathrm{~d}, J_{\mathrm{C}-\mathrm{F}}=26.0 \mathrm{~Hz}\right), 95.2$, 77.6, $77.3\left(\mathrm{~d}, J_{\mathrm{C}-\mathrm{F}}=3.0 \mathrm{~Hz}\right), 77.2,76.9,67.0,51.8,42.8,29.3,21.7 .{ }^{19} \mathrm{~F} \mathrm{NMR}\left(376 \mathrm{MHz}, \mathrm{CDCl}_{3}\right)$ $\delta$-112.07. HRMS (ESI-TOF) $\mathrm{m} / \mathrm{z}$ : $[\mathrm{M}+\mathrm{Na}]^{+} \mathrm{C}_{29} \mathrm{H}_{25} \mathrm{ClFNO}_{4} \mathrm{SNa}$ Calcd for 560.1069; found 560.1071 .

Benzyl 1-(3-(5-chloro-3-fluoro-2-((4-methylphenyl)sulfonamido)phenyl)prop-2-yn-1-yl) cyclopent-3-ene-1-carboxylate (4v): the reaction was conducted at $3.00 \mathrm{mmol}$ scale from $\mathbf{1 3}$,<smiles>CNc1c(F)cc(Cl)cc1C#CCC1(C(=O)Cc2ccccc2)CC=CC1</smiles>
$0.6605 \mathrm{~g}, 41 \%$ yield for two steps, unknown compound, yellow solid, $\mathrm{R}_{f}=0.20$ (petroleum ether/ethyl acetate 15/1); ${ }^{1} \mathrm{H}$ NMR (400 MHz, $\left.\mathrm{CDCl}_{3}\right) \delta 7.71(\mathrm{~d}, J=8.2 \mathrm{~Hz}, 2 \mathrm{H}), 7.37-7.27(\mathrm{~m}, 5 \mathrm{H}), 7.25(\mathrm{~d}, J=$ $8.1 \mathrm{~Hz}, 2 \mathrm{H}), 7.08-7.02(\mathrm{~m}, 1 \mathrm{H}), 7.02-6.96(\mathrm{~m}, 1 \mathrm{H}), 6.64(\mathrm{~s}, 1 \mathrm{H})$, $5.67(\mathrm{~d}, J=8.1 \mathrm{~Hz}, 1 \mathrm{H}), 5.65(\mathrm{~d}, J=8.1 \mathrm{~Hz}, 1 \mathrm{H}), 5.21(\mathrm{~s}, 2 \mathrm{H}), 2.96$ (d, $J=15.2 \mathrm{~Hz}, 2 \mathrm{H}), 2.64(\mathrm{~s}, 2 \mathrm{H}), 2.44(\mathrm{~d}, J=15.2 \mathrm{~Hz}, 2 \mathrm{H}), 2.41$ (s, $3 \mathrm{H}) .{ }^{13} \mathrm{C}$ NMR $\left(100 \mathrm{MHz}, \mathrm{CDCl}_{3}\right) \delta 176.0,157.1\left(\mathrm{~d}, J_{\mathrm{C}-\mathrm{F}}=254.0 \mathrm{~Hz}\right), 144.0,137.4,136.0,132.1$ $\left(\mathrm{d}, J_{\mathrm{C}-\mathrm{F}}=10.0 \mathrm{~Hz}\right), 129.5,128.7,128.5,128.4,128.3,127.8\left(\mathrm{~d}, J_{\mathrm{C}-\mathrm{F}}=3.0 \mathrm{~Hz}\right), 127.5,125.1(\mathrm{~d}$, $\left.J_{\mathrm{C}-\mathrm{F}}=13.0 \mathrm{~Hz}\right), 122.8\left(\mathrm{~d}, J_{\mathrm{C}-\mathrm{F}}=4.0 \mathrm{~Hz}\right), 117.5\left(\mathrm{~d}, J_{\mathrm{C}-\mathrm{F}}=18.0 \mathrm{~Hz}\right), 96.1,77.6,77.2,76.9,75.9(\mathrm{~d}$, $J_{\mathrm{C}-\mathrm{F}}=4.0 \mathrm{~Hz}$ ), 67.1, 51.8, 42.7, 29.4, 21.7. ${ }^{19} \mathrm{~F} \mathrm{NMR}\left(376 \mathrm{MHz}, \mathrm{CDCl}_{3}\right) \delta-113.87 . \mathrm{HRMS}$ (ESI-TOF) $\mathrm{m} / \mathrm{z}$ : $[\mathrm{M}+\mathrm{Na}]^{+} \mathrm{C}_{29} \mathrm{H}_{25} \mathrm{ClFNO}_{4} \mathrm{SNa}$ Calcd for 560.1069; found 560.1070.

Benzyl 1-(3-(3,5-difluoro-2-((4-methylphenyl)sulfonamido)phenyl)prop-2-yn-1-yl)cyclopent -3-ene-1-carboxylate (4w): the reaction was conducted at $3.00 \mathrm{mmol}$ scale from 13, $1.001 \mathrm{~g}, 64 \%$

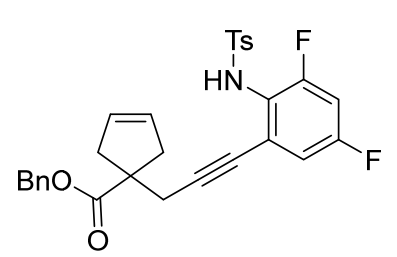
yield for two steps, unknown compound, yellow solid, $\mathrm{R}_{f}=0.20$ (petroleum ether/ethyl acetate 15/1); ${ }^{1} \mathrm{H} \mathrm{NMR}\left(400 \mathrm{MHz}, \mathrm{CDCl}_{3}\right) \delta=$ $7.68(\mathrm{~d}, J=8.3,2 \mathrm{H}), 7.57-7.27(\mathrm{~m}, 6 \mathrm{H}), 7.24(\mathrm{~d}, J=8.1,2 \mathrm{H})$, 6.85-6.76 (m, 1H), 6.76-6.68 (m, 1H), $5.67(\mathrm{~d}, J=8.1,1 \mathrm{H}), 5.65(\mathrm{~d}$, $J=8.1,1 \mathrm{H}), 5.21(\mathrm{~s}, 2 \mathrm{H}), 2.96(\mathrm{~d}, J=15.2,2 \mathrm{H}), 2.62(\mathrm{~s}, 2 \mathrm{H}), 2.43(\mathrm{~d}$, $J=15.4,2 \mathrm{H}), 2.40(\mathrm{~s}, 3 \mathrm{H}) .{ }^{13} \mathrm{C} \mathrm{NMR}\left(100 \mathrm{MHz}, \mathrm{CDCl}_{3}\right) \delta 176.0,160.4\left(\mathrm{dd}, J_{\mathrm{C}-\mathrm{F}}=228.0,14.0\right.$ $\mathrm{Hz}), 157.9\left(\mathrm{dd}, J_{\mathrm{C}-\mathrm{F}}=234.0,13.0 \mathrm{~Hz}\right), 144.0,137.4,136.1,129.6,128.7,128.5,128.4,128.3$, $127.6,123.4\left(\mathrm{dd}, J_{\mathrm{C}-\mathrm{F}}=12.0,4.0 \mathrm{~Hz}\right), 122.6\left(\mathrm{dd}, J_{\mathrm{C}-\mathrm{F}}=13.0,4.0 \mathrm{~Hz}\right), 114.8\left(\mathrm{dd}, J_{\mathrm{C}-\mathrm{F}}=23.0,4.0\right.$ $\mathrm{Hz}), 105.5\left(\mathrm{dd}, J_{\mathrm{C}-\mathrm{F}}=26.0,25.0 \mathrm{~Hz}\right), 95.8,77.6,77.2,76.9,76.3\left(\mathrm{dd}, J_{\mathrm{C}-\mathrm{F}}=4.0,4.0 \mathrm{~Hz}\right), 67.2$, 51.9, 42.8, 29.4, 21.8. ${ }^{19} \mathrm{~F}$ NMR (376 MHz, $\left.\mathrm{CDCl}_{3}\right) \delta-111.30$ (d, $\left.J=7.3 \mathrm{~Hz}\right),-112.02$ (d, $J=7.2$ Hz). HRMS (ESI-TOF) $\mathrm{m} / \mathrm{z}$ : [M+Na] ${ }^{+} \mathrm{C}_{29} \mathrm{H}_{25} \mathrm{~F}_{2} \mathrm{NO}_{4} \mathrm{SNa}$ Calcd for 544.1365; found 544.1358. 
Methyl 1-(3-(2-((4-methylphenyl)sulfonamido)phenyl)prop-2-yn-1-yl)cyclopent-3-ene-1carboxylate (4x): the reaction was conducted at $3.00 \mathrm{mmol}$ scale from 13, $0.4786 \mathrm{~g}, 39 \%$ yield<smiles>[3H]Nc1ccccc1C#CCC1(C(C)=O)CC=CC1</smiles>
for two steps, unknown compound, yellow oil, $\mathrm{R}_{f}=0.20$ (petroleum ether/ethyl acetate 10/1); ${ }^{1} \mathrm{H}$ NMR $\left(400 \mathrm{MHz}, \mathrm{CDCl}_{3}\right) \delta 7.73(\mathrm{~d}, J=8.3$ $\mathrm{Hz}, 2 \mathrm{H}), 7.57(\mathrm{~d}, J=7.9 \mathrm{~Hz}, 1 \mathrm{H}), 7.47$ (br s, 1H), 7.25-7.15 (m, 4H), $7.00-6.91(\mathrm{~m}, 1 \mathrm{H}), 5.71(\mathrm{~d}, J=8.3 \mathrm{~Hz}, 1 \mathrm{H}), 5.69(\mathrm{~d}, J=8.3 \mathrm{~Hz}, 1 \mathrm{H})$, $3.81(\mathrm{~s}, 3 \mathrm{H}), 2.98(\mathrm{~d}, J=14.9 \mathrm{~Hz}, 2 \mathrm{H}), 2.75(\mathrm{~s}, 2 \mathrm{H}), 2.48(\mathrm{~d}, J=15.2 \mathrm{~Hz}$, 2H), $2.36(\mathrm{~s}, 3 \mathrm{H}) .{ }^{13} \mathrm{C} \mathrm{NMR}\left(100 \mathrm{MHz}, \mathrm{CDCl}_{3}\right) \delta 176.9,144.0,138.4,136.6,132.2,129.7,129.3$, 128.6, 127.5, 123.9, 118.8, 113.9, 94.6, 77.6, 77.2, 77.0, 76.9, 52.8, 51.8, 42.8, 29.6, 21.7. HRMS (ESI-TOF) $\mathrm{m} / z$ : $[\mathrm{M}+\mathrm{Na}]^{+} \mathrm{C}_{23} \mathrm{H}_{23} \mathrm{NO}_{4} \mathrm{SNa}$ Calcd for 432.1240; found 432.1236 .

Ethyl 1-(3-(2-((4-methylphenyl)sulfonamido)phenyl)prop-2-yn-1-yl)cyclopent-3-ene-1carboxylate (4y): the reaction was conducted at $3.00 \mathrm{mmol}$ scale from 13, $0.8124 \mathrm{~g}, 64 \%$ yield<smiles>CCOC(=O)C1(CC#Cc2ccccc2NC)CC=CC1</smiles>
for two steps, unknown compound, yellow oil, $\mathrm{R}_{f}=0.20$ (petroleum ether/ethyl acetate 10/1); ${ }^{1} \mathrm{H}$ NMR $\left(400 \mathrm{MHz}, \mathrm{CDCl}_{3}\right) \delta 7.73(\mathrm{~d}, J=8.3$ $\mathrm{Hz}, 2 \mathrm{H}), 7.57(\mathrm{~d}, J=8.3 \mathrm{~Hz}, 1 \mathrm{H}), 7.48(\mathrm{~s}, 1 \mathrm{H}), 7.25-7.14(\mathrm{~m}, 4 \mathrm{H})$, $7.02-6.89(\mathrm{~m}, 1 \mathrm{H}), 5.71(\mathrm{~d}, J=8.2 \mathrm{~Hz}, 1 \mathrm{H}), 5.69(\mathrm{~d}, J=8.2 \mathrm{~Hz}, 1 \mathrm{H})$, 4.27 (q, $J=7.1 \mathrm{~Hz}, 2 \mathrm{H}), 2.98(\mathrm{~d}, J=15.0 \mathrm{~Hz}, 2 \mathrm{H}), 2.74(\mathrm{~s}, 2 \mathrm{H}), 2.48$ (d, $J=15.1 \mathrm{~Hz}, 2 \mathrm{H}), 2.36(\mathrm{~s}, 3 \mathrm{H}), 1.28(\mathrm{t}, J=7.1 \mathrm{~Hz}, 3 \mathrm{H}) .{ }^{13} \mathrm{C} \mathrm{NMR}\left(100 \mathrm{MHz}, \mathrm{CDCl}_{3}\right) \delta 176.3$, 144.0, 138.4, 136.6, 132.2, 129.7, 129.2, 128.6, 127.5, 123.9, 118.8, 113.9, 94.6, 77.6, 77.2, 76.9, 61.5, 51.8, 42.8, 29.7, 21.7, 14.5. HRMS (ESI-TOF) $\mathrm{m} / \mathrm{z}$ : $[\mathrm{M}+\mathrm{Na}]^{+} \mathrm{C}_{24} \mathrm{H}_{25} \mathrm{NO}_{4} \mathrm{SNa}$ Calcd for 446.1397; found 446.1389.

Isopropyl 1-(3-(2-((4-methylphenyl)sulfonamido)phenyl)prop-2-yn-1-yl)cyclopent-3-ene-1carboxylate (4z): the reaction was conducted at $3.00 \mathrm{mmol}$ scale from 13, $0.9049 \mathrm{~g}, 69 \%$ yield for<smiles>Nc1ccccc1C#CCC1(C(=O)O)CC=CC1</smiles>
two steps, unknown compound, yellow oil, $\mathrm{R}_{f}=0.20$ (petroleum ether/ethyl acetate 10/1); ${ }^{1} \mathrm{H}$ NMR $\left(400 \mathrm{MHz}, \mathrm{CDCl}_{3}\right) \delta 7.73(\mathrm{~d}, J=8.3$ $\mathrm{Hz}, 2 \mathrm{H}), 7.57(\mathrm{~d}, J=8.3 \mathrm{~Hz}, 1 \mathrm{H}), 7.49(\mathrm{~s}, 1 \mathrm{H}), 7.24-7.15(\mathrm{~m}, 4 \mathrm{H})$, $7.05-6.79(\mathrm{~m}, 1 \mathrm{H}), 5.70(\mathrm{~s}, 2 \mathrm{H}), 5.20-5.04(\mathrm{~m}, 1 \mathrm{H}), 2.97(\mathrm{~d}, J=14.9 \mathrm{~Hz}$, $2 \mathrm{H}), 2.73(\mathrm{~s}, 2 \mathrm{H}), 2.46(\mathrm{~d}, J=15.1 \mathrm{~Hz}, 2 \mathrm{H}), 2.36(\mathrm{~s}, 3 \mathrm{H}), 1.26(\mathrm{~s}, 3 \mathrm{H})$, $1.25(\mathrm{~s}, 3 \mathrm{H}) .{ }^{13} \mathrm{C} \mathrm{NMR}\left(100 \mathrm{MHz}, \mathrm{CDCl}_{3}\right) \delta 175.8,144.0,138.4,136.6,132.2,129.7,129.2,128.7$, $127.5,123.9,118.8,114.0,94.7,77.6,77.2,76.9,68.7,51.8,42.7,29.7,21.9,21.8$. HRMS (ESI-TOF) $m / z$ : $[\mathrm{M}+\mathrm{Na}]^{+} \mathrm{C}_{25} \mathrm{H}_{27} \mathrm{NO}_{4} \mathrm{SNa}$ Calcd for 460.1553; found 460.1549 .

tert-Butyl 1-(3-(2-((4-methylphenyl)sulfonamido)phenyl)prop-2-yn-1-yl)cyclopent-3-ene-1carboxylate (4aa): the reaction was conducted at $3.00 \mathrm{mmol}$ scale from 13, $0.9742 \mathrm{~g}, 72 \%$ yield

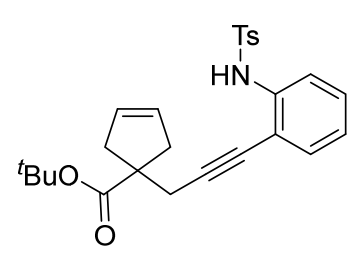
for two steps, unknown compound, yellow oil, $\mathrm{R}_{f}=0.20$ (petroleum ether/ethyl acetate 10/1); ${ }^{1} \mathrm{H}$ NMR $\left(400 \mathrm{MHz}, \mathrm{CDCl}_{3}\right) \delta 7.73(\mathrm{~d}, J=8.3$, $2 \mathrm{H}), 7.57(\mathrm{~d}, J=8.2,1 \mathrm{H}), 7.49(\mathrm{~s}, 1 \mathrm{H}), 7.21(\mathrm{~d}, J=8.7,4 \mathrm{H}), 6.95(\mathrm{t}, J=$ $7.5,1 \mathrm{H}), 5.69(\mathrm{~s}, 2 \mathrm{H}), 2.95(\mathrm{~d}, J=15.1,2 \mathrm{H}), 2.71(\mathrm{~s}, 2 \mathrm{H}), 2.42(\mathrm{~d}, J=$ $14.9,2 \mathrm{H}), 2.36(\mathrm{~s}, 3 \mathrm{H}), 1.47$ (s, 9H). ${ }^{13} \mathrm{C}$ NMR $\left(100 \mathrm{MHz}, \mathrm{CDCl}_{3}\right) \delta$ 175.4, 144.0, 138.3, 136.6, 132.2, 129.7, 129.2, 128.7, 127.5, 123.9, 118.9, 114.0, 94.8, 81.2, 77.6, 77.2, 76.9, 76.8, 52.4, 42.6, 29.9, 28.2, 21.7. HRMS (ESI-TOF) $\mathrm{m} / \mathrm{z}$ : $[\mathrm{M}+\mathrm{Na}]^{+} \mathrm{C}_{26} \mathrm{H}_{29} \mathrm{NO}_{4} \mathrm{SNa}$ Calcd for 474.1710; found 474.1706. 
4-methyl- $N$-(2-(3-(1-(morpholine-4-carbonyl)cyclopent-3-en-1-yl)prop-1-yn-1-yl)phenyl)benz enesulfonamide (4ab): the reaction was conducted at $3.00 \mathrm{mmol}$ scale from 13, $0.8630 \mathrm{~g}, 62 \%$

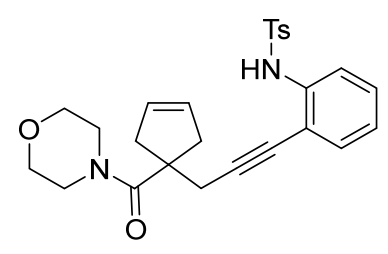
yield for two steps, unknown compound, white solid, $\mathrm{R}_{f}=0.50$ (petroleum ether/ethyl acetate 1/1); ${ }^{1} \mathrm{H}$ NMR $\left(400 \mathrm{MHz}, \mathrm{CDCl}_{3}\right) \delta$ 7.98 (s, 1H), 7.78 (d, $J=7.3 \mathrm{~Hz}, 2 \mathrm{H}), 7.59$ (d, $J=8.3 \mathrm{~Hz}, 1 \mathrm{H})$, 7.27-7.10 (m, 4H), 7.00-6.85 (m, 1H), $5.82(\mathrm{~s}, 2 \mathrm{H}), 3.69(\mathrm{~s}, 4 \mathrm{H})$, $3.40($ br s, 4H), $2.82(\mathrm{~d}, J=16.3 \mathrm{~Hz}, 2 \mathrm{H}), 2.71(\mathrm{~s}, 2 \mathrm{H}), 2.62(\mathrm{~d}, J=$ $17.0 \mathrm{~Hz}, 2 \mathrm{H}), 2.36(\mathrm{~d}, J=2.2 \mathrm{~Hz}, 3 \mathrm{H}) .{ }^{13} \mathrm{C}$ NMR $\left(100 \mathrm{MHz}, \mathrm{CDCl}_{3}\right) \delta 174.6,143.7,139.3,136.9$, 131.7, 129.6, 129.4, 129.0, 127.4, 123.6, 118.8, 114.0, 96.2, 77.6, 77.2, 76.9, 76.1, 50.6, 44.6, 31.9, 21.7. HRMS (ESI-TOF) $m / z$ : $[\mathrm{M}+\mathrm{Na}]^{+} \mathrm{C}_{26} \mathrm{H}_{28} \mathrm{~N}_{2} \mathrm{O}_{4} \mathrm{SNa}$ Calcd for 487.1662 ; found 487.1657 .

$N, N$-diethyl-1-(3-(2-((4-methylphenyl)sulfonamido)phenyl)prop-2-yn-1-yl)cyclopent-3-ene-1carboxamide (4ac): the reaction was conducted at $3.00 \mathrm{mmol}$ scale from $\mathbf{1 3}, 0.7698 \mathrm{~g}, 57 \%$ yield<smiles>CCN(CC)C(=O)C1(CC#Cc2ccccc2NC)CC=CC1</smiles>
for two steps, unknown compound, white solid, $\mathrm{R}_{f}=0.20$ (petroleum ether/ethyl acetate 4/1); ${ }^{1} \mathrm{H}$ NMR $\left(400 \mathrm{MHz}, \mathrm{CDCl}_{3}\right) \delta 8.14(\mathrm{~s}, 1 \mathrm{H})$, $7.80(\mathrm{~d}, J=8.0 \mathrm{~Hz}, 2 \mathrm{H}), 7.58(\mathrm{~d}, J=8.2 \mathrm{~Hz}, 1 \mathrm{H}), 7.30-7.04(\mathrm{~m}, 4 \mathrm{H})$, 6.97-6.83 (m, 1H), $5.79(\mathrm{~s}, 2 \mathrm{H}), 3.44(\mathrm{~s}, 2 \mathrm{H}), 3.23(\mathrm{~s}, 2 \mathrm{H}), 2.89(\mathrm{~d}, J=$ $16.1 \mathrm{~Hz}, 2 \mathrm{H}), 2.69$ (s, 2H), $2.56(\mathrm{~d}, J=15.7 \mathrm{~Hz}, 2 \mathrm{H}), 2.33$ (s, 3H), 1.18

$(\mathrm{s}, 3 \mathrm{H}), 1.11(\mathrm{~s}, 3 \mathrm{H}) .{ }^{13} \mathrm{C} \mathrm{NMR}\left(100 \mathrm{MHz}, \mathrm{CDCl}_{3}\right) \delta 174.8,143.5,139.0,137.0,131.7,129.5$, 129.2, 128.8, 127.3, 123.5, 118.8, 114.0, 95.6, 77.6, 77.2, 76.9, 76.3, 51.2, 44.2, 41.0, 40.6, 31.6, 21.6, 13.9, 12.7. HRMS (ESI-TOF) $\mathrm{m} / \mathrm{z}$ : $[\mathrm{M}+\mathrm{Na}]^{+} \mathrm{C}_{26} \mathrm{H}_{30} \mathrm{~N}_{2} \mathrm{O}_{3} \mathrm{SNa}$ Calcd for 473.1869; found 473.1871 .

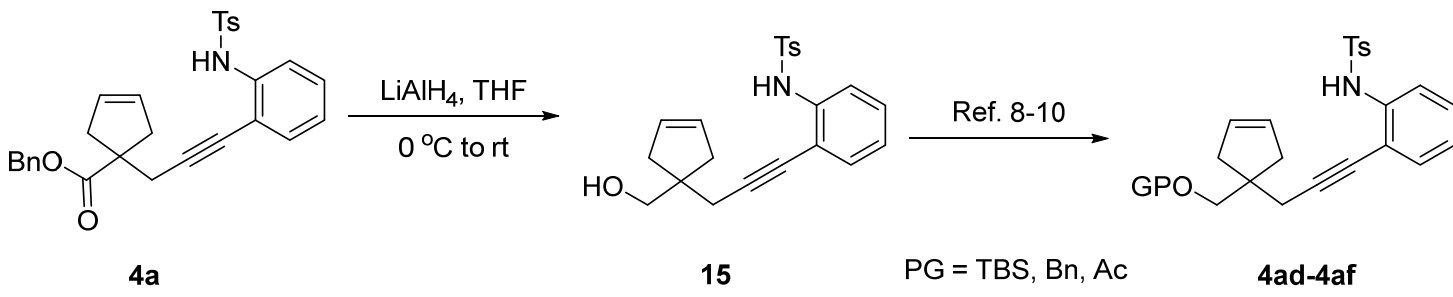

Synthesis of compounds 4ad-4af: ${ }^{[7]}$ In a nitrogen-filled Schlenk tube, to a solution of the $4 \mathbf{a}$ (242.5 mg, $0.50 \mathrm{mmol})$ in THF $(8.0 \mathrm{~mL})$ was slowly added $\mathrm{LiAlH}_{4}(28.5 \mathrm{mg}, 0.75 \mathrm{mmol})$ at $0{ }^{\circ} \mathrm{C}$. The mixture was stirred at room temperature for $2 \mathrm{~h}$. Subsequently, the reaction was quenched by a saturated aqueous solution of sodium tartrate $(5.0 \mathrm{~mL})$ and extracted with ethyl acetate $(3 \times 10.0$ $\mathrm{mL}$ ). The combined organic layers were dried over sodium sulfate, filtered and concentrated under reduced pressure. The resulting crude material was purified by flash column chromatography (petroleum ether/ethyl acetate) to afford the desired compound 15 (yellow oil, $169.3 \mathrm{mg}, \mathbf{9 1 \%}$ yield). After obtaining the reduced product 15, the hydroxyl groups were protected with TBS, ${ }^{[8]}$ $\mathrm{Bn}^{[9]}$ and $\mathrm{Ac}^{[10]}$ to give the desired compounds 4ad-4af, respectively.

\section{(1-(3-(2-((4-methylphenyl)sulfonamido)phenyl)prop-2-yn-1-yl)cyclopent-3-en-1-yl)methyl}

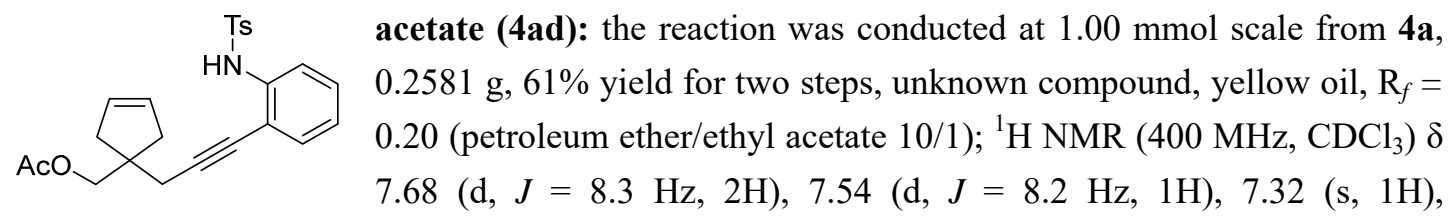


7.27-7.16 (m, 4H), 7.03-6.92 (m, 1H), $5.70(\mathrm{~s}, 2 \mathrm{H}), 4.09$ (s, 2H), 2.59 (s, 2H), 2.43-2.06 (m, 7H), $2.12(\mathrm{~s}, 3 \mathrm{H}) .{ }^{13} \mathrm{C} \mathrm{NMR}\left(100 \mathrm{MHz}, \mathrm{CDCl}_{3}\right) \delta 171.4,144.1,138.0,136.5,132.4,129.8,129.2,129.2$, 127.4, 124.2, 119.1, 114.3, 95.0, 77.6, 77.2, 77.1, 76.9, 69.5, 44.9, 41.6, 28.4, 21.7, 21.2. HRMS (ESI-TOF) $m / z$ : $[\mathrm{M}+\mathrm{Na}]^{+} \mathrm{C}_{24} \mathrm{H}_{25} \mathrm{NO}_{4} \mathrm{SNa}$ Calcd for 446.1397; found 446.1400 .

\section{$N$-(2-(3-(1-((benzyloxy)methyl)cyclopent-3-en-1-yl)prop-1-yn-1-yl)phenyl)-4-methylbenzene}

sulfonamide (4ae): the reaction was conducted at $1.00 \mathrm{mmol}$ scale from $\mathbf{4 a}, 0.1955 \mathrm{~g}, 41 \%$ yield

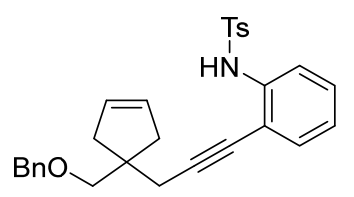
for two steps, unknown compound, yellow oil, $\mathrm{R}_{f}=0.20$ (petroleum ether/ethyl acetate 10/1); ${ }^{1} \mathrm{H}$ NMR $\left(400 \mathrm{MHz}, \mathrm{CDCl}_{3}\right) \delta 7.65(\mathrm{~d}, J=8.3$ $\mathrm{Hz}, 2 \mathrm{H}), 7.59-7.52(\mathrm{~m}, 1 \mathrm{H}), 7.38-7.30(\mathrm{~m}, 4 \mathrm{H}), 7.25(\mathrm{~d}, J=3.0 \mathrm{~Hz}, 2 \mathrm{H})$, $7.23-7.15(\mathrm{~m}, 4 \mathrm{H}), 7.02-6.92(\mathrm{~m}, 1 \mathrm{H}), 5.69(\mathrm{~d}, J=6.3 \mathrm{~Hz}, 1 \mathrm{H}), 5.68(\mathrm{~d}$, $J=6.7 \mathrm{~Hz}, 1 \mathrm{H}), 4.58(\mathrm{~s}, 2 \mathrm{H}), 3.42(\mathrm{~s}, 2 \mathrm{H}), 2.64(\mathrm{~s}, 2 \mathrm{H}), 2.42-2.29$ (m, 7H). ${ }^{13} \mathrm{C} \mathrm{NMR}(100 \mathrm{MHz}$, $\left.\mathrm{CDCl}_{3}\right) \delta 144.1,138.7,137.9,136.4,132.3,129.7,129.4,129.0,128.5,127.7,127.4,124.1,119.1$, 114.7, 96.3, 77.6, 77.2, 76.9, 76.5, 76.3, 73.5, 45.7, 41.9, 28.7, 21.7. HRMS (ESI-TOF) $\mathrm{m} / \mathrm{z}$ : $[\mathrm{M}+\mathrm{Na}]^{+} \mathrm{C}_{29} \mathrm{H}_{29} \mathrm{NO}_{3} \mathrm{SNa}$ Calcd for 494.1760; found 494.1760.

$N$-(2-(3-(1-(((tert-butyldimethylsilyl)oxy)methyl)cyclopent-3-en-1-yl)prop-1-yn-1-yl)phenyl)-4 -methylbenzenesulfonamide (4af): the reaction was conducted at $1.00 \mathrm{mmol}$ scale from $\mathbf{4 a}$,

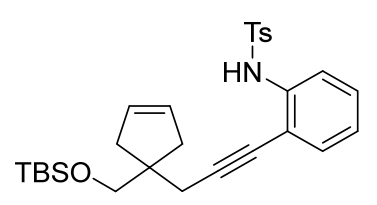
$0.1981 \mathrm{~g}, 40 \%$ yield for two steps, unknown compound, yellow oil, $\mathrm{R}_{f}$ $=0.20$ (petroleum ether/ethyl acetate $10 / 1) ;{ }^{1} \mathrm{H}$ NMR $(400 \mathrm{MHz}$, $\left.\mathrm{CDCl}_{3}\right) \delta 7.69-7.63(\mathrm{~m}, 2 \mathrm{H}), 7.55(\mathrm{~d}, J=8.2 \mathrm{~Hz}, 1 \mathrm{H}), 7.25-7.16(\mathrm{~m}$, $5 \mathrm{H}), 6.99-6.94(\mathrm{~m}, 1 \mathrm{H}), 5.69(\mathrm{~s}, 2 \mathrm{H}), 3.49(\mathrm{~s}, 2 \mathrm{H}), 2.58(\mathrm{~s}, 2 \mathrm{H}), 2.36(\mathrm{~s}$, $3 \mathrm{H}), 2.29$ (s, 4H), 0.93-0.89 (m, 9H), 0.09-0.03 (m, 6H). $\left.{ }^{13} \mathrm{C} \mathrm{NMR} \mathrm{(100} \mathrm{MHz,} \mathrm{CDCl}_{3}\right) \delta 144.1$, $137.8,136.4,132.3,129.8,129.5,129.0,127.4,124.2$, 119.0, 114.7, 96.6, 77.6, 77.2, 76.9, 76.4, $68.8,46.9,41.2,28.1,26.1,21.7,18.5,-5.3$. HRMS (ESI-TOF) $\mathrm{m} / \mathrm{z}$ : $[\mathrm{M}+\mathrm{Na}]^{+} \mathrm{C}_{28} \mathrm{H}_{37} \mathrm{NO}_{3} \mathrm{SSiNa}$ Calcd for 518.2156; found 518.2151.

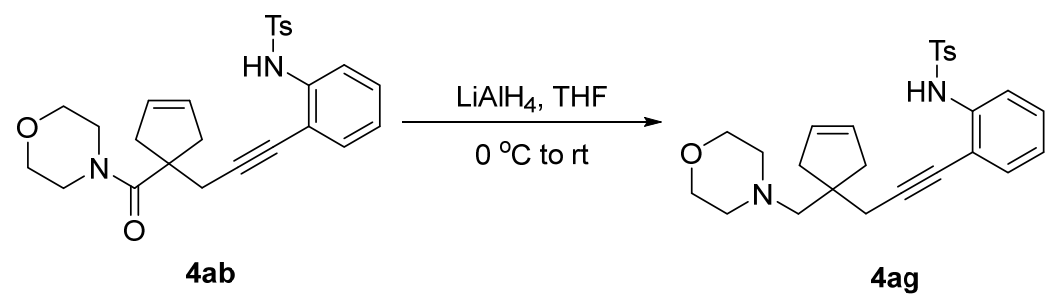

Synthesis of compounds $4 \mathbf{a g}:{ }^{[7]}$ In a nitrogen-filled Schlenk tube, to a solution of the 4ab (139.2 $\mathrm{mg}, 0.30 \mathrm{mmol})$ in THF $(5.0 \mathrm{~mL})$ was slowly added $\mathrm{LiAlH}_{4}(19.0 \mathrm{mg}, 0.5 \mathrm{mmol})$ at $0{ }^{\circ} \mathrm{C}$. The mixture was stirred at room temperature for $2 \mathrm{~h}$. Subsequently, the reaction was quenched by a saturated aqueous solution of sodium tartrate $(3.0 \mathrm{~mL})$ and extracted with ethyl acetate $(3 \times 10.0$ $\mathrm{mL}$ ). The combined organic layers were dried over sodium sulfate, filtered and concentrated under reduced pressure. The resulting crude material was purified by flash column chromatography (petroleum ether/ethyl acetate) to afford the desired compound $\mathbf{4 a g}$ (white solid, $112.1 \mathrm{mg}, 83 \%$ yield). $\mathrm{R}_{f}=0.15$ (petroleum ether/ethyl acetate 10/1); ${ }^{1} \mathrm{H} \mathrm{NMR}\left(400 \mathrm{MHz}, \mathrm{CDCl}_{3}\right) \delta 7.67(\mathrm{~d}, J=$ $8.2 \mathrm{~Hz}, 2 \mathrm{H}), 7.55(\mathrm{~d}, J=8.2 \mathrm{~Hz}, 1 \mathrm{H}), 7.34-7.14(\mathrm{~m}, 5 \mathrm{H}), 6.96(\mathrm{t}, J=7.5 \mathrm{~Hz}, 1 \mathrm{H}), 5.69(\mathrm{~s}, 2 \mathrm{H})$, 3.79-3.62 (m, 4H), 2.60 (s, 2H), 2.58-2.50 (m, 4H), 2.44 (s, 2H), $2.36(\mathrm{~s}, 3 \mathrm{H}), 2.29$ (s, 4H). ${ }^{13} \mathrm{C}$ NMR $\left(100 \mathrm{MHz}, \mathrm{CDCl}_{3}\right) \delta 144.1,137.9,136.4,132.2,129.8,129.5,129.0,127.4,124.1,118.8$, 
114.5, 96.9, 77.6, 77.2, 76.9, 76.6, 67.4, 66.2, 55.9, 46.7, 43.3, 29.2, 21.7. HRMS (ESI-TOF) m/z: $[\mathrm{M}+\mathrm{Na}]^{+} \mathrm{C}_{26} \mathrm{H}_{30} \mathrm{~N}_{2} \mathrm{O}_{3} \mathrm{SNa}$ Calcd for 473.1869; found 473.1875.

\section{Optimization of the Conditions}

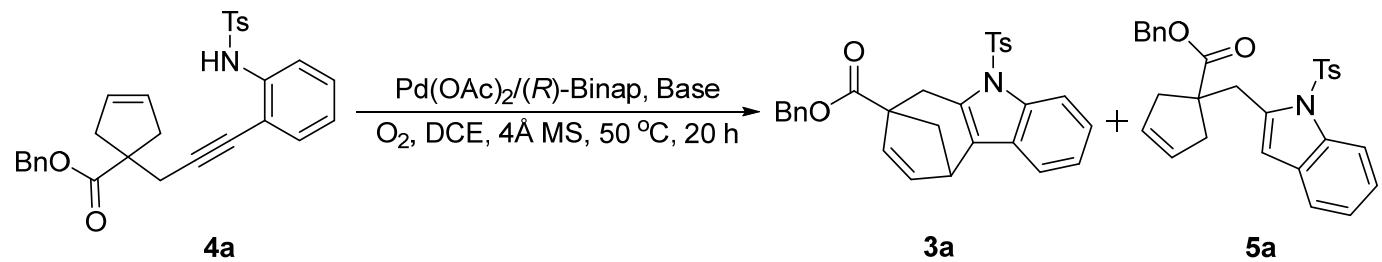

\begin{tabular}{|c|c|c|c|}
\hline Entry & Base (x eq.) & 3a/5a Yield $(\%)^{[b]}$ & 3a $\operatorname{Ee}(\%)^{[\mathrm{c}]}$ \\
\hline 1 & $\mathrm{Na}_{2} \mathrm{CO}_{3}(1.0)$ & $16 / 16$ & 24 \\
\hline 2 & $\mathrm{~K}_{2} \mathrm{CO}_{3}(1.0)$ & $26 / 10$ & 50 \\
\hline 3 & $\mathrm{Cs}_{2} \mathrm{CO}_{3}(1.0)$ & $29 / 11$ & 48 \\
\hline 4 & $\mathrm{Li}_{2} \mathrm{CO}_{3}(1.0)$ & $10 / 14$ & 12 \\
\hline 5 & KOAc (1.0) & $9 /<5$ & 12 \\
\hline 6 & CsOAc (1.0) & $8 /<5$ & 22 \\
\hline 7 & $\mathrm{Et}_{3} \mathrm{~N}(1.0)$ & $13 /<5$ & 48 \\
\hline 8 & ${ }^{i} \mathrm{Pr}_{2} \mathrm{NH}(1.0)$ & $5 / 9$ & 32 \\
\hline 9 & Pyridine (1.0) & $6 /<5$ & 30 \\
\hline 10 & EtONa (1.0) & $32 / 29$ & 44 \\
\hline 11 & ${ }^{t} \mathrm{BuOK}(1.0)$ & $41 / 22$ & 46 \\
\hline 12 & ${ }^{t} \mathrm{BuOLi}(1.0)$ & $26 / 17$ & 36 \\
\hline 13 & ${ }^{t} \mathrm{BuONa}(1.0)$ & $28 / 21$ & 48 \\
\hline 14 & $\mathrm{~K}_{3} \mathrm{PO}_{4}(1.0)$ & $38 / 21$ & 50 \\
\hline 15 & $\mathrm{NaOH}(1.0)$ & $31 / 15$ & 42 \\
\hline 16 & $\mathrm{KOH}(1.0)$ & $30 / 14$ & 36 \\
\hline 17 & $\mathrm{~K}_{3} \mathrm{PO}_{4}(\mathbf{1 . 5})$ & $46 / 23$ & 54 \\
\hline 18 & $\mathrm{~K}_{3} \mathrm{PO}_{4}(2.0)$ & $47 / 24$ & 50 \\
\hline 19 & $\mathrm{~K}_{3} \mathrm{PO}_{4}(3.0)$ & $52 / 46$ & 42 \\
\hline
\end{tabular}

[a] 4a (48.5 g, $0.10 \mathrm{mmol}), \mathrm{Pd}(\mathrm{OAc})_{2}(2.2 \mathrm{mg}, 0.01 \mathrm{mmol}),(R)$-Binap $(6.8 \mathrm{mg}, 0.011 \mathrm{mmol})$, base (x eq.), DCE $(2.0 \mathrm{~mL}), 4 \AA$ molecular sieve $(100 \mathrm{mg}), \mathrm{O}_{2}$ balloon, $50{ }^{\circ} \mathrm{C}, 20 \mathrm{~h}$. [b] Isolated yield. [c] Determined by HPLC. 


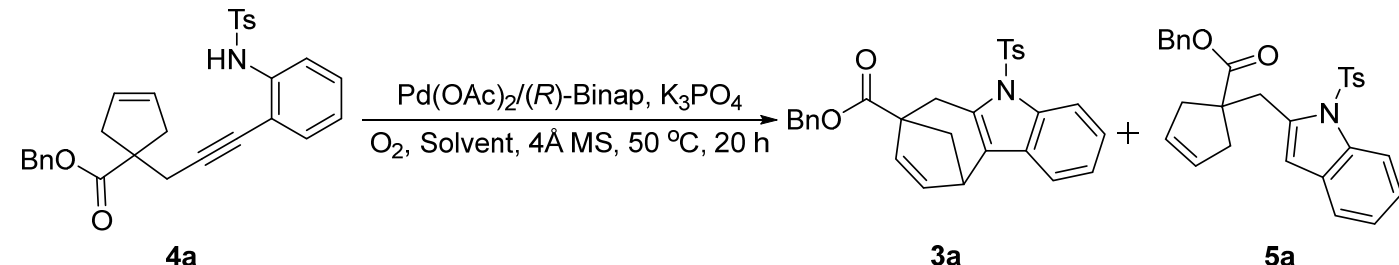

\begin{tabular}{|c|c|c|c|}
\hline Entry & Solvent & 3a/5a Yield $(\%)^{[b]}$ & 3a $\operatorname{Ee}(\%)^{[\mathrm{c}]}$ \\
\hline 1 & DCE & $46 / 23$ & 54 \\
\hline 2 & $\mathrm{DCM}$ & $19 / 14$ & 62 \\
\hline 3 & DMSO & $<5 /-$ & - \\
\hline 4 & DMF & $<5 /-$ & - \\
\hline 5 & $\mathrm{MeCN}$ & $11 / 37$ & 46 \\
\hline 6 & THF & $24 / 35$ & 62 \\
\hline 7 & 1,4-dioxane & $32 / 17$ & 54 \\
\hline 8 & ethylacetate & $37 / 33$ & 56 \\
\hline 9 & $\mathrm{Et}_{2} \mathrm{O}$ & $24 /<5$ & 68 \\
\hline 10 & $\mathrm{PhMe}$ & $27 / 11$ & 62 \\
\hline 11 & $o$-xylene & $36 / 10$ & 64 \\
\hline 12 & $\mathrm{PhH}$ & $35 / 13$ & 64 \\
\hline 13 & $\mathrm{PhCF}_{3}$ & $64 / 21$ & 74 \\
\hline 14 & mesitylene & $28 / 24$ & 56 \\
\hline 15 & $p$-xylene & $36 / 18$ & 56 \\
\hline
\end{tabular}

[a] 4a (48.5 g, $0.10 \mathrm{mmol}), \mathrm{Pd}(\mathrm{OAc})_{2}(2.2 \mathrm{mg}, 0.01 \mathrm{mmol}),(R)$-Binap (6.8 $\left.\mathrm{mg}, 0.011 \mathrm{mmol}\right)$, $\mathrm{K}_{3} \mathrm{PO}_{4}(31.8 \mathrm{~g}, 0.15 \mathrm{mmol})$, solvent $(2.0 \mathrm{~mL}), 4 \AA$ molecular sieve $(100 \mathrm{mg}), \mathrm{O}_{2}$ balloon, $50{ }^{\circ} \mathrm{C}, 20$ h. [b] Isolated yield. [c] Determined by HPLC.
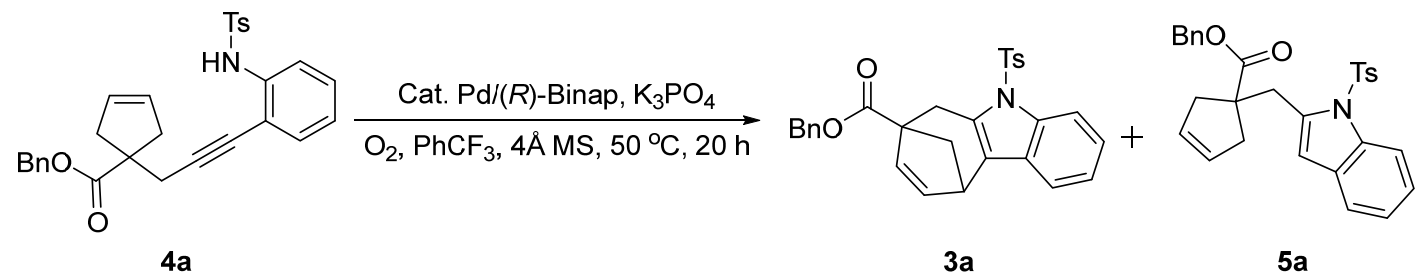

\begin{tabular}{cccc}
\hline Entry & Cat. Pd & 3a/5a Yield $(\%)^{[\mathrm{b}]}$ & 3a Ee $(\%)^{[\mathrm{c}]}$ \\
\hline 1 & $\mathrm{Pd}(\mathrm{OAc})_{2}$ & $64 / 21$ & 74 \\
2 & $\mathrm{Pd}\left(\mathrm{CF}_{3} \mathrm{CO}_{2}\right)_{2}$ & $40 / 25$ & 74 \\
3 & $\mathrm{Pd}\left(\mathrm{PPh}_{3}\right)_{2} \mathrm{Cl}_{2}$ & $76 / 11$ & 64 \\
4 & $\mathrm{Pd}(\mathrm{acac})_{2}$ & $27 / 25$ & 78 \\
5 & $\mathrm{Pd}(\mathrm{dppf}) \mathrm{Cl}_{2}$ & $50 / 14$ & 64 \\
6 & $\mathrm{Pd}(\mathrm{MeCN})_{2} \mathrm{Cl}_{2}$ & $34 / 28$ & 64 \\
7 & $\mathrm{Pd}(\mathrm{cod}) \mathrm{Cl}_{2}$ & $24 / 26$ & 70 \\
8 & {$\left[\mathrm{Pd}(\mathrm{allyl}) \mathrm{Cl}_{2}\right.$} & $31 / 19$ & 62 \\
9 & $\mathrm{Pd}(\mathrm{hfac})_{2}$ & $22 / 22$ & 60
\end{tabular}


[a] 4a (48.5 g, $0.10 \mathrm{mmol})$, Cat. $\mathrm{Pd}(0.01 \mathrm{mmol}),(R)$-Binap ( $6.8 \mathrm{mg}, 0.011 \mathrm{mmol}), \mathrm{K}_{3} \mathrm{PO}_{4}(31.8 \mathrm{~g}$, $0.15 \mathrm{mmol})$, solvent $(2.0 \mathrm{~mL}), 4 \AA$ molecular sieve $(100 \mathrm{mg}), \mathrm{O}_{2}$ balloon, $50{ }^{\circ} \mathrm{C}, 20 \mathrm{~h}$. [b] Isolated yield. [c] Determined by HPLC.

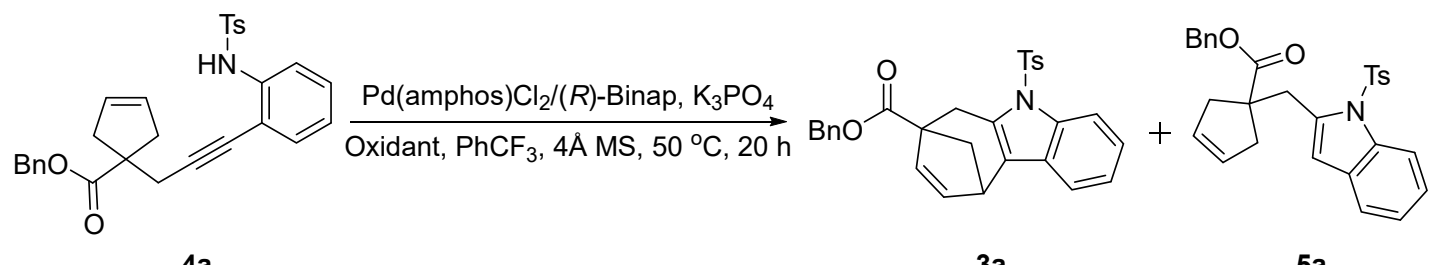

4a

$3 a$

$5 a$

\begin{tabular}{cccc}
\hline Entry & Oxidant & 3a/5a Yield $(\%)^{[\mathrm{b}]}$ & 3a Ee $(\%)^{[\mathrm{c}]}$ \\
\hline $\mathbf{1}$ & $\mathbf{O}_{2}$ & $\mathbf{6 3} / 7$ & $\mathbf{8 4}$ \\
2 & $\mathrm{Cu}(\mathrm{OAc})_{2}$ & $14 / 83$ & 68 \\
3 & $\mathrm{AgNO}_{3}$ & $17 / 74$ & 52 \\
4 & $p$-Benzoquinone & $19 / 23$ & 70 \\
\hline
\end{tabular}

[a] $4 \mathrm{a}(48.5 \mathrm{~g}, 0.10 \mathrm{mmol}), \mathrm{Pd}(\mathrm{amphos}) \mathrm{Cl}_{2}(0.0071 \mathrm{~g}, 0.01 \mathrm{mmol}),(R)$-Binap $(6.8 \mathrm{mg}, 0.011$ mmol), $\mathrm{K}_{3} \mathrm{PO}_{4}(31.8 \mathrm{~g}, 0.15 \mathrm{mmol})$, solvent $(2.0 \mathrm{~mL}), 4 \AA$ molecular sieve $(100 \mathrm{mg}), \mathrm{O}_{2}$ balloon or oxidant $(0.15 \mathrm{mmol})), 50{ }^{\circ} \mathrm{C}, 20 \mathrm{~h}$. [b] Isolated yield. [c] Determined by HPLC.<smiles>CNc1ccccc1C#CCC1(C(=O)O)CC=CC1</smiles>

$4 a$

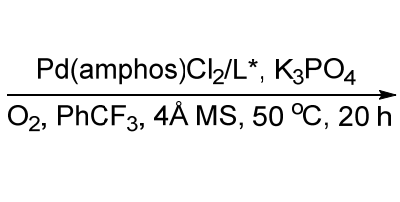<smiles>Cn1c2c(c3ccccc31)C1(C(=O)OCc3ccccc3)C=CC2CC1</smiles>

$3 a$<smiles>[Y9]n1c(CC2(C(=O)O[Ga])CC=CC2)cc2ccccc21</smiles>

$5 a$

\begin{tabular}{cccc}
\hline Entry & $\mathrm{L}^{*}$ & 3a/5a Yield $(\%)^{[\mathrm{b}]}$ & 3a Ee $(\%)^{[\mathrm{c}]}$ \\
\hline 1 & $(R)$-Binap & $63 / 7$ & 84 \\
2 & $(R, R)$-DIOP & $67 /<5$ & 6 \\
& $(R)$-Me-Bophoz & $55 / 23$ & 28 \\
3 & $(R)-$ MeO-Biphep & $74 / 7$ & 84 \\
4 & $\left(R_{a x}, S, S\right)-\mathrm{C}_{3}{ }^{*}-$ Tunephos & $52 / 10$ & 70 \\
5 & $(R)-\mathrm{DM}$-Segphos & $<5 / 10$ & 50 \\
6 & $(R)$-H8-Binap & $52 /<5$ & 78 \\
7 & $(R)-$ P-Phos & $45 / 16$ & 82 \\
8 & $(R)-2$-furyl-MeOBiphep & $52 / 16$ & 16 \\
9 & $(R)-$ Cl-MeO-Biphep & $52 / 17$ & 74 \\
$\mathbf{1 0}$ & $(S)-$ Synphos & $\mathbf{6 1} /<\mathbf{5}$ & $\mathbf{9 0}$ \\
11 & $(R)-\mathrm{C}_{3}$-Tunephos & $66 /<5$ & 82 \\
\hline
\end{tabular}

[a] 4a (48.5 g, $0.10 \mathrm{mmol}), \mathrm{Pd}(\mathrm{amphos}) \mathrm{Cl}_{2}(0.0071 \mathrm{~g}, 0.01 \mathrm{mmol}), \mathrm{L}^{*}(0.011 \mathrm{mmol}), \mathrm{K}_{3} \mathrm{PO}_{4}(31.8$ $\mathrm{g}, 0.15 \mathrm{mmol})$, solvent $(2.0 \mathrm{~mL}), 4 \AA$ molecular sieve $(100 \mathrm{mg}), \mathrm{O}_{2}$ balloon, $50{ }^{\circ} \mathrm{C}, 20 \mathrm{~h} .[\mathrm{b}]$ 
Isolated yield. [c] Determined by HPLC.

\begin{tabular}{ccccc} 
& & & \\
\\
Entry & $\mathrm{T} /{ }^{\circ} \mathrm{C}$ & $\mathbf{3 a} / \mathbf{h a ~ Y i e l d ~}(\%)^{[\mathrm{b}]}$ & $\mathbf{3 a ~ E e ~}(\%)^{[\mathrm{cc}]}$ \\
\hline 1 & 40 & 60 & $67 / 3$ & 84 \\
$\mathbf{2}$ & $\mathbf{4 5}$ & $\mathbf{4 8}$ & $\mathbf{7 5 / 1 3}$ & $\mathbf{9 2}$ \\
3 & 50 & 20 & $60 /<5$ & 90 \\
4 & 80 & 11 & $72 / 7$ & 78 \\
\hline
\end{tabular}

[a] 4a (48.5 g, $0.10 \mathrm{mmol}), \mathrm{Pd}(\mathrm{amphos}) \mathrm{Cl}_{2}(0.0071 \mathrm{~g}, 0.01 \mathrm{mmol})$, (S)-Synphos $(0.0070 \mathrm{~g}, 0.011$ $\mathrm{mmol}), \mathrm{K}_{3} \mathrm{PO}_{4}(31.8 \mathrm{~g}, 0.15 \mathrm{mmol})$, solvent $(2.0 \mathrm{~mL}), 4 \AA$ molecular sieve $(100 \mathrm{mg}), \mathrm{O}_{2}$ balloon, [b] Isolated yield. [c] Determined by HPLC. 


\section{General procedure for enantioselective synthesis of indole-fused bicyclo[3.2.1]octane.}

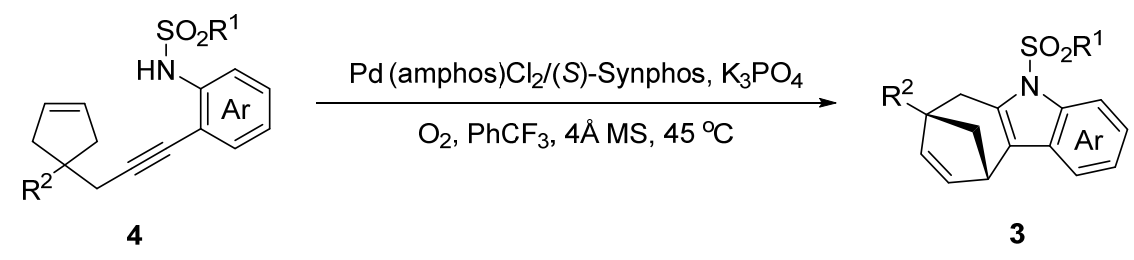

In the glovebox, a Schlenk tube was charged with 4 ( $0.10 \mathrm{mmol}), \mathrm{Pd}(\mathrm{amphos}) \mathrm{Cl}_{2}(7.1 \mathrm{mg}, 0.01$ mmol), (S)-Synphos (7.0 mg, $0.011 \mathrm{mmol}), \mathrm{K}_{3} \mathrm{PO}_{4}(31.8 \mathrm{mg}, 0.15 \mathrm{mmol})$, and $4 \AA \mathrm{MS}(100 \mathrm{mg})$ in the $\mathrm{PhCF}_{3}(2.0 \mathrm{~mL})$. Then, the Schlenk tube was removed from glovebox and the nitrogen atmosphere was replaced with the oxygen using an oxygen balloon. The mixture was stirred in 45 ${ }^{\circ} \mathrm{C}$ oil bath. After completion of the reaction (monitored by TLC), the resulting mixture was concentrated under reduced pressure. The resulting crude material was purified by flash column chromatography to afford the desired products. The enantiomeric excess of the products was determined by HPLC with chiral columns.

Benzyl (7R,10S)-5-tosyl-6,10-dihydro-7,10-methanocyclohepta[b]indole-7(5H)-carboxylate (3a): the reaction was conducted at $0.10 \mathrm{mmol}$ scale, $0.0362 \mathrm{~g}, 75 \%$ yield, $92 \%$ ee, unknown

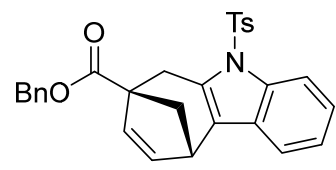
compound, white foam solid, $[\alpha]^{20}{ }_{\mathrm{D}}=-155.77\left(\mathrm{c} 0.64, \mathrm{CHCl}_{3}\right), \mathrm{R}_{f}=0.13$ (petroleum ether/ethyl acetate 20/1); ${ }^{1} \mathrm{H}$ NMR (400 $\left.\mathrm{MHz}, \mathrm{CDCl}_{3}\right) \delta$ 8.15-8.05 (m, 1H), $7.61(\mathrm{~d}, J=8.4 \mathrm{~Hz}, 2 \mathrm{H}), 7.46-7.30(\mathrm{~m}, 6 \mathrm{H})$, $7.25-7.20(\mathrm{~m}, 2 \mathrm{H}), 7.15(\mathrm{~d}, J=8.1 \mathrm{~Hz}, 2 \mathrm{H}), 6.34(\mathrm{dd}, J=5.4,2.7 \mathrm{~Hz}$, $1 \mathrm{H}), 5.82(\mathrm{~d}, J=5.5 \mathrm{~Hz}, 1 \mathrm{H}), 5.28(\mathrm{~d}, J=12.5 \mathrm{~Hz}, 1 \mathrm{H}), 5.25(\mathrm{~d}, J=12.5 \mathrm{~Hz}, 1 \mathrm{H}), 3.72-3.66(\mathrm{~m}$, $1 \mathrm{H}), 3.56(\mathrm{~d}, J=18.2 \mathrm{~Hz}, 1 \mathrm{H}), 3.23(\mathrm{~d}, J=18.2 \mathrm{~Hz}, 1 \mathrm{H}), 2.43$ (dd, $J=9.9,4.6 \mathrm{~Hz}, 1 \mathrm{H}), 2.31(\mathrm{~s}$, $3 \mathrm{H}), 1.99(\mathrm{~d}, J=9.9 \mathrm{~Hz}, 1 \mathrm{H}) .{ }^{13} \mathrm{C} \mathrm{NMR}\left(100 \mathrm{MHz}, \mathrm{CDCl}_{3}\right) \delta 174.7,144.9,140.9,136.1,135.9$, 135.7, 131.6, 130.0, 129.8, 128.8, 128.4, 128.2, 128.1, 126.5, 124.4, 124.0, 123.5, 117.9, 114.6, 66.8, 54.8, 44.6, 36.5, 32.3, 21.7. HPLC: Chiracel IC column, elute: $n$-hexane/i-propanol $=90 / 10$, detector: $254 \mathrm{~nm}, 30{ }^{\circ} \mathrm{C}$, flow rate: $0.8 \mathrm{~mL} / \mathrm{min}$, retention time $20.9 \mathrm{~min}$ (major) and $23.1 \mathrm{~min}$; HRMS (EI-TOF) m/z: [M] $]^{+}$Calcd for $\mathrm{C}_{29} \mathrm{H}_{25} \mathrm{NO}_{4} \mathrm{~S}$ 483.1504; Found 483.1509.

Benzyl (7R,10S)-5-(phenylsulfonyl)-6,10-dihydro-7,10-methanocyclohepta[b]indole-7(5H)carboxylate (3b): the reaction was conducted at $0.10 \mathrm{mmol}$ scale, $0.0240 \mathrm{~g}, 51 \%$ yield, $88 \%$ ee,<smiles>O=C(Cc1ccccc1)C12CCC(CC1)C1CCCC12c1cn(S(=O)(=O)c2ccccc2)c2ccccc12</smiles>
unknown compound, white foam solid, $[\alpha]^{20}{ }_{\mathrm{D}}=-128.23$ (c 0.68, $\left.\mathrm{CHCl}_{3}\right)$, $\mathrm{R}_{f}=0.25$ (petroleum ether/ethyl acetate 10/1). ${ }^{1} \mathrm{H}$ NMR $(400 \mathrm{MHz}$, $\left.\mathrm{CDCl}_{3}\right) \delta 8.14-8.07(\mathrm{~m} \mathrm{1H}), 7.72(\mathrm{~d}, J=7.5 \mathrm{~Hz}, 2 \mathrm{H}), 7.51-7.34(\mathrm{~m}, 9 \mathrm{H})$, $7.25-7.20(\mathrm{~m}, 2 \mathrm{H}), 6.35(\mathrm{dd}, J=5.2,2.6 \mathrm{~Hz}, 1 \mathrm{H}), 5.82(\mathrm{~d}, J=5.5 \mathrm{~Hz}$, $1 \mathrm{H}), 5.33-5.20(\mathrm{~m}, 2 \mathrm{H}), 3.73-3.66(\mathrm{~m}, 1 \mathrm{H}), 3.57(\mathrm{~d}, J=18.2 \mathrm{~Hz}, 1 \mathrm{H}), 3.23(\mathrm{~d}, J=18.2 \mathrm{~Hz}, 1 \mathrm{H})$, $2.44(\mathrm{dd}, J=9.8,4.5 \mathrm{~Hz}, 1 \mathrm{H}), 1.98(\mathrm{~d}, J=9.9 \mathrm{~Hz}, 1 \mathrm{H}) .{ }^{13} \mathrm{C} \mathrm{NMR}\left(100 \mathrm{MHz}, \mathrm{CDCl}_{3}\right) \delta 174.7$, $140.9,138.9,136.1,135.8,133.9,131.7,129.9,129.4,128.8,128.5,128.3,128.1,126.5,124.7$, 124.2, 123.6, 117.9, 114.7, 66.9, 54.8, 44.7, 36.5, 32.3. HPLC: Chiracel IC column, elute: $n$-hexane $/$-propanol $=90 / 10$, detector: $254 \mathrm{~nm}, 30{ }^{\circ} \mathrm{C}$, flow rate: $0.8 \mathrm{~mL} / \mathrm{min}$, retention time 20.3 min (major) and $21.9 \mathrm{~min}$; HRMS (EI-TOF) $\mathrm{m} / \mathrm{z}$ : [M] ${ }^{+}$Calcd for $\mathrm{C}_{28} \mathrm{H}_{23} \mathrm{NO}_{4} \mathrm{~S}$ 469.1348; found 469.1353 . 
Benzyl (7R,10S)-5-((4-methoxyphenyl)sulfonyl)-6,10-dihydro-7,10-methanocyclohepta[b]indole-7(5H)-carboxylate $(3 \mathrm{c})$ : the reaction was conducted at $0.10 \mathrm{mmol}$ scale, $0.0310 \mathrm{~g}, 62 \%$ (1) yield, $90 \%$ ee, unknown compound, white foam solid, $[\alpha]^{20}{ }_{D}=-168.58(c$ $0.58, \mathrm{CHCl}_{3}$ ), $\mathrm{R}_{f}=0.20$ (petroleum ether/ethyl acetate 10/1); ${ }^{1} \mathrm{H}$ NMR $\left(400 \mathrm{MHz}, \mathrm{CDCl}_{3}\right) \delta 8.12-8.08(\mathrm{~m}, 1 \mathrm{H}), 7.68(\mathrm{~d}, J=9.0 \mathrm{~Hz}, 2 \mathrm{H})$, 7.44-7.34 (m, 6H), 7.26-7.21 (m, 2H), $6.82(\mathrm{~d}, J=9.0 \mathrm{~Hz}, 2 \mathrm{H}), 6.35(\mathrm{dd}$, $J=5.5,2.8 \mathrm{~Hz}, 1 \mathrm{H}), 5.82(\mathrm{~d}, J=5.5 \mathrm{~Hz}, 1 \mathrm{H}), 5.29(\mathrm{~d}, J=12.5 \mathrm{~Hz}, 1 \mathrm{H})$, $5.25(\mathrm{~d}, J=12.5 \mathrm{~Hz}, 1 \mathrm{H}), 3.77(\mathrm{~s}, 3 \mathrm{H}), 3.73-3.66(\mathrm{~m}, 1 \mathrm{H}), 3.56(\mathrm{~d}, J=$ $18.3 \mathrm{~Hz}, 1 \mathrm{H}), 3.23(\mathrm{~d}, J=18.2 \mathrm{~Hz}, 1 \mathrm{H}), 2.43(\mathrm{dd}, J=9.9,4.6 \mathrm{~Hz}, 1 \mathrm{H})$, $1.99(\mathrm{~d}, J=9.9 \mathrm{~Hz}, 1 \mathrm{H}) .{ }^{13} \mathrm{C} \mathrm{NMR}\left(100 \mathrm{MHz}, \mathrm{CDCl}_{3}\right) \delta 174.8,163.8,140.9,136.2,135.7,131.6$, $130.5,129.8,128.8,128.8,128.5,128.3,128.2,124.3,124.0,123.5,117.9,114.7,114.6,66.9$, 55.8, 54.8, 44.7, 36.5, 32.4. HPLC: Chiracel IC column, elute: $n$-hexane/i-propanol $=90 / 10$, detector: $254 \mathrm{~nm}, 30{ }^{\circ} \mathrm{C}$, flow rate: $0.8 \mathrm{~mL} / \mathrm{min}$, retention time $31.7 \mathrm{~min}$ (major) and $35.8 \mathrm{~min}$; HRMS (EI-TOF) $\mathrm{m} / \mathrm{z}$ : [M] ${ }^{+} \mathrm{C}_{29} \mathrm{H}_{25} \mathrm{NO}_{5} \mathrm{~S}$ Calcd for 499.1453; found 499.1456.

Benzyl (7R,10S)-5-(benzylsulfonyl)-6,10-dihydro-7,10-methanocyclohepta[b]indole-7(5H)carboxylate (3d): the reaction was conducted at $0.10 \mathrm{mmol}$ scale, $0.0332 \mathrm{~g}, 69 \%$ yield, $90 \%$ ee,

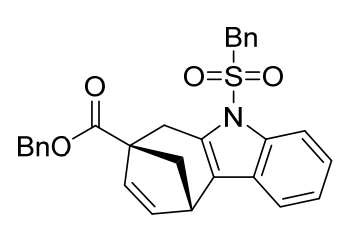
unknown compound, white foam solid, $[\alpha]^{20}{ }_{\mathrm{D}}=-115.29$ (c 0.66, $\left.\mathrm{CHCl}_{3}\right)$, $\mathrm{R}_{f}=0.20$ (petroleum ether/ethyl acetate $\left.15 / 1\right) ;{ }^{1} \mathrm{H}$ NMR $(400 \mathrm{MHz}$, $\left.\mathrm{CDCl}_{3}\right) \delta 7.99-7.91(\mathrm{~m}, 1 \mathrm{H}), 7.55-7.46(\mathrm{~m}, 1 \mathrm{H}), 7.44-7.32(\mathrm{~m}, 5 \mathrm{H})$, 7.34-7.27 (m, 2H), 7.22-7.14 (m, 1H), 7.12-7.03 (m, 2H), 6.75-6.65 (m, 2H), $6.26(\mathrm{dd}, J=5.0,2.5 \mathrm{~Hz}, 1 \mathrm{H}), 5.58(\mathrm{~d}, J=5.0 \mathrm{~Hz}, 1 \mathrm{H}), 5.18(\mathrm{~d}, J=12.7 \mathrm{~Hz}, 1 \mathrm{H}), 5.14(\mathrm{~d}, J$ $=12.7 \mathrm{~Hz}, 1 \mathrm{H}), 4.43(\mathrm{~s}, 2 \mathrm{H}), 3.69-3.59(\mathrm{~m}, 1 \mathrm{H}), 2.65-2.35(\mathrm{~m}, 2 \mathrm{H}), 2.26(\mathrm{dd}, J=9.7,4.4 \mathrm{~Hz}, 1 \mathrm{H})$, $1.68(\mathrm{~d}, J=9.7 \mathrm{~Hz}, 1 \mathrm{H}) .{ }^{13} \mathrm{C} \mathrm{NMR}\left(100 \mathrm{MHz}, \mathrm{CDCl}_{3}\right) \delta 174.4,140.3,136.2,135.2,133.7,130.7$, 130.2, 129.4, 128.9, 128.8, 128.4, 128.1, 127.9, 127.0, 124.0, 123.5, 121.6, 118.1, 113.8, 66.7, 59.3, 54.5, 44.2, 36.3, 31.3. HPLC: Chiracel IC column, elute: $n$-hexane/i-propanol $=90 / 10$, detector: $254 \mathrm{~nm}, 30{ }^{\circ} \mathrm{C}$, flow rate: $0.8 \mathrm{~mL} / \mathrm{min}$, retention time $34.3 \mathrm{~min}$ and $39.6 \mathrm{~min}$ (major); HRMS (EI-TOF) $\mathrm{m} / \mathrm{z}$ : [M] ${ }^{+} \mathrm{C}_{29} \mathrm{H}_{25} \mathrm{NO}_{4} \mathrm{~S}$ Calcd for 483.1504; found 483.1512.

Benzyl (7R,10S)-5-((4-nitrophenyl)sulfonyl)-6,10-dihydro-7,10-methanocyclohepta[b]indole7(5H)-carboxylate (3e): the reaction was conducted at $0.10 \mathrm{mmol}$ scale, $0.0299 \mathrm{~g}, 58 \%$ yield,

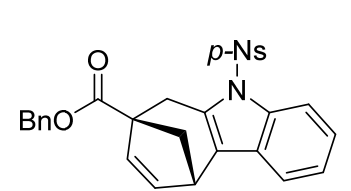
$87 \%$ ee, unknown compound, yellow foam solid, $[\alpha]^{20}{ }_{D}=-143.49$ (c 0.60 , $\left.\mathrm{CHCl}_{3}\right), \mathrm{R}_{f}=0.20$ (petroleum ether/ethyl acetate 10/1); ${ }^{1} \mathrm{H}$ NMR $(400$ $\left.\mathrm{MHz}, \mathrm{CDCl}_{3}\right) \delta 8.20(\mathrm{~d}, J=8.9 \mathrm{~Hz}, 2 \mathrm{H}), 8.09-8.04(\mathrm{~m}, 1 \mathrm{H}), 7.87(\mathrm{~d}, J=$ $8.9 \mathrm{~Hz}, 2 \mathrm{H}), 7.47-7.35(\mathrm{~m}, 6 \mathrm{H}), 7.31-7.26(\mathrm{~m}, 2 \mathrm{H}), 6.36(\mathrm{dd}, J=5.5,2.8$ $\mathrm{Hz}, 1 \mathrm{H}), 5.84(\mathrm{~d}, J=5.5 \mathrm{~Hz}, 1 \mathrm{H}), 5.29(\mathrm{~d}, J=12.4 \mathrm{~Hz}, 1 \mathrm{H}), 5.25(\mathrm{~d}, J=12.4 \mathrm{~Hz}, 1 \mathrm{H}), 3.75-3.66$ (m, 1H), 3.55 (d, $J=18.3 \mathrm{~Hz}, 1 \mathrm{H}), 3.19$ (d, $J=18.3 \mathrm{~Hz}, 1 \mathrm{H}), 2.46$ (dd, $J=10.0,4.6 \mathrm{~Hz}, 1 \mathrm{H}), 1.97$ $(\mathrm{d}, J=10.0 \mathrm{~Hz}, 1 \mathrm{H}) .{ }^{13} \mathrm{C} \mathrm{NMR}\left(100 \mathrm{MHz}, \mathrm{CDCl}_{3}\right) \delta 174.5,150.7,143.9,140.9,136.0,135.6$, 131.4, 130.1, 128.9, 128.6, 128.2, 127.8, 126.3, 124.8, 124.7, 124.4, 118.4, 114.6, 67.0, 54.7, 44.7, 36.5, 32.4. HPLC: Chiracel IG column, elute: $n$-hexane/i-propanol $=80 / 20$, detector: $254 \mathrm{~nm}, 30$ ${ }^{\circ} \mathrm{C}$, flow rate: $0.7 \mathrm{~mL} / \mathrm{min}$, retention time $28.8 \mathrm{~min}$ (major) and $35.6 \mathrm{~min}$; HRMS (EI-TOF) $\mathrm{m} / \mathrm{z}$ : $[\mathrm{M}]^{+} \mathrm{C}_{28} \mathrm{H}_{22} \mathrm{~N}_{2} \mathrm{O}_{6} \mathrm{~S}$ Calcd for 514.1199; found 514.1196. 
Benzyl (7R,10S)-3-methyl-5-tosyl-6,10-dihydro-7,10-methanocyclohepta[b]indole-7(5H)carboxylate (3h): the reaction was conducted at $0.10 \mathrm{mmol}$ scale, $0.0322 \mathrm{~g}, 65 \%$ yield, $93 \%$ ee,<smiles></smiles>
unknown compound, white foam solid, $[\alpha]^{20}{ }_{\mathrm{D}}=-180.17$ (c 0.56, $\mathrm{CHCl}_{3}$ ), $\mathrm{R}_{f}=0.13$ (petroleum ether/ethyl acetate 20/1); ${ }^{1} \mathrm{H}$ NMR $\left(400 \mathrm{MHz}, \mathrm{CDCl}_{3}\right) \delta 7.91(\mathrm{~s}, 1 \mathrm{H}), 7.59(\mathrm{~d}, J=8.4 \mathrm{~Hz}, 2 \mathrm{H})$, 7.42-7.27 (m, 6H), $7.15(\mathrm{~d}, J=8.1 \mathrm{~Hz}, 2 \mathrm{H}), 7.06-7.02(\mathrm{~m}, 1 \mathrm{H})$, $6.33(\mathrm{dd}, J=5.5,2.7 \mathrm{~Hz}, 1 \mathrm{H}), 5.80(\mathrm{~d}, J=5.5 \mathrm{~Hz}, 1 \mathrm{H}), 5.28(\mathrm{~d}, J=12.5 \mathrm{~Hz}, 1 \mathrm{H}), 5.24(\mathrm{~d}, J=12.5$ Hz, 1H), 3.69-3.62 (m, 1H), 3.53 (d, $J=18.2 \mathrm{~Hz}, 1 \mathrm{H}), 3.19$ (d, $J=18.1 \mathrm{~Hz}, 1 \mathrm{H}), 2.46(\mathrm{~s}, 3 \mathrm{H})$, $2.41(\mathrm{dd}, J=9.9,4.6 \mathrm{~Hz}, 1 \mathrm{H}), 2.32(\mathrm{~s}, 3 \mathrm{H}), 1.97(\mathrm{~d}, J=9.9 \mathrm{~Hz}, 1 \mathrm{H}) .{ }^{13} \mathrm{C}$ NMR $\left(100 \mathrm{MHz}, \mathrm{CDCl}_{3}\right)$ $\delta$ 174. 8, 144.8, 140.9, 136.2, 136.2, 136.1, 134.1, 130.8, 130.0, 129.8, 128.8, 128.4, 128.1, 126.5, 126.1, 124.9, 124.4, 117.5, 114.9, 66.8, 54.8, 44.6, 36.6, 32.6, 22.2, 21.7. HPLC: Chiracel IG column, elute: $n$-hexane/i-propanol $=90 / 10$, detector: $254 \mathrm{~nm}, 30{ }^{\circ} \mathrm{C}$, flow rate: $0.8 \mathrm{~mL} / \mathrm{min}$, retention time $30.8 \mathrm{~min}$ (major) and $38.1 \mathrm{~min}$; HRMS (EI-TOF) $\mathrm{m} / \mathrm{z}$ : $[\mathrm{M}]^{+} \mathrm{C}_{30} \mathrm{H}_{27} \mathrm{NO}_{4} \mathrm{~S}$ Calcd for 497.1661; found 497.1671.

Benzyl (7R,10S)-3-methyl-5-tosyl-6,10-dihydro-7,10-methanocyclohepta[b]indole-7(5H)carboxylate (3i): the reaction was conducted at $0.10 \mathrm{mmol}$ scale, $0.0365 \mathrm{~g}, 73 \%$ yield, $93 \%$ ee,

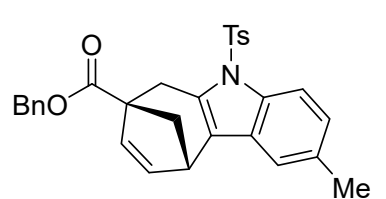
unknown compound, white foam solid, $[\alpha]^{20}{ }_{\mathrm{D}}=-152.86$ (c 0.80 , $\left.\mathrm{CHCl}_{3}\right), \mathrm{R}_{f}=0.13$ (petroleum ether/ethyl acetate 20/1); ${ }^{1} \mathrm{H}$ NMR (400 $\left.\mathrm{MHz}, \mathrm{CDCl}_{3}\right) \delta 7.96(\mathrm{~d}, J=8.5 \mathrm{~Hz}, 1 \mathrm{H}), 7.59(\mathrm{~d}, J=8.3 \mathrm{~Hz}, 2 \mathrm{H})$, $7.42-7.32(\mathrm{~m}, 5 \mathrm{H}), 7.21(\mathrm{~s}, 1 \mathrm{H}), 7.14(\mathrm{~d}, J=8.2 \mathrm{~Hz}, 2 \mathrm{H}), 7.05(\mathrm{~d}, J=$ $8.5 \mathrm{~Hz}, 1 \mathrm{H}), 6.33(\mathrm{dd}, J=5.3,2.7 \mathrm{~Hz}, 1 \mathrm{H}), 5.81(\mathrm{~d}, J=5.5 \mathrm{~Hz}, 1 \mathrm{H}), 5.28(\mathrm{~d}, J=12.6 \mathrm{~Hz}, 1 \mathrm{H})$, $5.24(\mathrm{~d}, J=12.6 \mathrm{~Hz}, 1 \mathrm{H}), 3.69-3.62(\mathrm{~m}, 1 \mathrm{H}), 3.54(\mathrm{~d}, J=18.2 \mathrm{~Hz}, 1 \mathrm{H}), 3.21(\mathrm{~d}, J=18.2 \mathrm{~Hz}, 1 \mathrm{H})$, 2.45-2.40 (m, 1H), $2.40(\mathrm{~s}, 3 \mathrm{H}), 2.30(\mathrm{~s}, 3 \mathrm{H}), 1.97(\mathrm{~d}, J=9.9 \mathrm{~Hz}, 1 \mathrm{H}) .{ }^{13} \mathrm{C}$ NMR $(100 \mathrm{MHz}$, $\left.\mathrm{CDCl}_{3}\right) \delta 174.8,144.8,140.9,136.1,136.0,134.0,133.2,131.7,130.0,129.8,128.8,128.5,128.4$, 128.1, 126.5, 125.4, 124.4, 117.9, 114.4, 66.9, 54.8, 44.7, 36.5, 32.4, 21.7, 21.5. HPLC: Chiracel IG column, elute: $n$-hexane/i-propanol $=90 / 10$, detector: $254 \mathrm{~nm}, 30^{\circ} \mathrm{C}$, flow rate: $0.8 \mathrm{~mL} / \mathrm{min}$, retention time $31.3 \mathrm{~min}$ (major) and $40.9 \mathrm{~min}$; HRMS (EI-TOF) $\mathrm{m} / \mathrm{z}$ : [M] ${ }^{+} \mathrm{C}_{30} \mathrm{H}_{27} \mathrm{NO}_{4} \mathrm{~S}$ Calcd for 497.1661; found 497.1653.

Benzyl (7R,10S)-2-methoxy-5-tosyl-6,10-dihydro-7,10-methanocyclohepta[b]indole-7(5H)carboxylate (3j): the reaction was conducted at $0.10 \mathrm{mmol}$ scale, $0.0422 \mathrm{~g}, 82 \%$ yield, $94 \%$ ee,

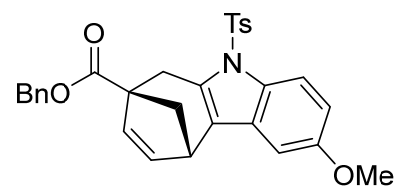
unknown compound, white foam solid, $[\alpha]_{\mathrm{D}}^{20}=-173.99$ (c 0.80 , $\mathrm{CHCl}_{3}$ ), $\mathrm{R}_{f}=0.20$ (petroleum ether/ethyl acetate 10/1); ${ }^{1} \mathrm{H}$ NMR $\left(400 \mathrm{MHz}, \mathrm{CDCl}_{3}\right) \delta 7.98(\mathrm{~d}, J=8.8 \mathrm{~Hz}, 1 \mathrm{H}), 7.57(\mathrm{~d}, J=8.3 \mathrm{~Hz}$, $2 \mathrm{H}), 7.45-7.31(\mathrm{~m}, 5 \mathrm{H}), 7.14(\mathrm{~d}, J=8.2 \mathrm{~Hz}, 2 \mathrm{H}), 6.89-6.79(\mathrm{~m}, 2 \mathrm{H})$, $6.34(\mathrm{dd}, J=5.4,2.7 \mathrm{~Hz}, 1 \mathrm{H}), 5.82$ (d, $J=5.5 \mathrm{~Hz}, 1 \mathrm{H}), 5.28$ (d, $J=12.5 \mathrm{~Hz}, 1 \mathrm{H}), 5.25$ (d, $J=12.5$ $\mathrm{Hz}, 1 \mathrm{H}), 3.82$ (s, 3H), 3.68-3.61 (m, 1H), 3.54 (d, $J=18.3 \mathrm{~Hz}, 1 \mathrm{H}), 3.20$ (d, $J=18.3 \mathrm{~Hz}, 1 \mathrm{H})$, $2.42(\mathrm{dd}, J=9.9,4.6 \mathrm{~Hz}, 1 \mathrm{H}), 2.31(\mathrm{~s}, 3 \mathrm{H}), 1.97$ (d, $J=9.9 \mathrm{~Hz}, 1 \mathrm{H}) .{ }^{13} \mathrm{C}$ NMR $\left(100 \mathrm{MHz}, \mathrm{CDCl}_{3}\right)$ $\delta 174.7,156.7,144.8,140.8,136.1,135.8,132.5,130.3,130.0,129.9,129.3,128.8,128.5,128.1$, 126.5, 124.7, 115.6, 112.5, 100.8, 66.9, 55.8, 54.8, 44.7, 36.6, 32.4, 21.7. HPLC: Chiracel IG column, elute: $n$-hexane/i-propanol $=90 / 10$, detector: $254 \mathrm{~nm}, 30{ }^{\circ} \mathrm{C}$, flow rate: $0.8 \mathrm{~mL} / \mathrm{min}$, retention time $60.8 \mathrm{~min}$ (major) and $71.0 \mathrm{~min}$; HRMS (EI-TOF) $\mathrm{m} / \mathrm{z}$ : $[\mathrm{M}]^{+} \mathrm{C}_{30} \mathrm{H}_{27} \mathrm{NO}_{5} \mathrm{~S}$ Calcd for 513.1610; found 513.1617. 
carboxylate (3k): the reaction was conducted at $0.10 \mathrm{mmol}$ scale, $0.0380 \mathrm{~g}, 76 \%$ yield, $85 \%$ ee,<smiles>CN1c2cc(F)ccc2C2CC1C=CC2(C)C(=O)OCc1ccccc1</smiles>
unknown compound, white solid, $[\alpha]^{20}{ }_{\mathrm{D}}=-151.68$ (c $\left.0.65, \mathrm{CHCl}_{3}\right), \mathrm{R}_{f}$ $=0.13$ (petroleum ether/ethyl acetate 20/1); ${ }^{1} \mathrm{H}$ NMR $(400 \mathrm{MHz}$, $\left.\mathrm{CDCl}_{3}\right) \delta 7.91-7.78(\mathrm{~m}, 1 \mathrm{H}), 7.62(\mathrm{~d}, J=7.3 \mathrm{~Hz}, 2 \mathrm{H}), 7.45-7.30(\mathrm{~m}$, $6 \mathrm{H}), 7.19(\mathrm{~d}, J=7.3 \mathrm{~Hz}, 2 \mathrm{H}), 7.07-6.90(\mathrm{~m}, 1 \mathrm{H}), 6.34(\mathrm{~s}, 1 \mathrm{H})$, 5.91-5.72 (m, 1H), $5.27(\mathrm{~s}, 2 \mathrm{H}), 3.72-3.60(\mathrm{~m}, 1 \mathrm{H}), 3.53(\mathrm{~d}, J=18.0 \mathrm{~Hz}, 1 \mathrm{H}), 3.20(\mathrm{~d}, J=18.0$ $\mathrm{Hz}, 1 \mathrm{H}), 2.50-2.37(\mathrm{~m}, 1 \mathrm{H}), 2.34(\mathrm{~s}, 3 \mathrm{H}), 1.97(\mathrm{~d}, J=9.6 \mathrm{~Hz}, 1 \mathrm{H}) .{ }^{13} \mathrm{C} \mathrm{NMR}\left(100 \mathrm{MHz}, \mathrm{CDCl}_{3}\right) \delta$ $174.6,160.7\left(\mathrm{~d}, J_{\mathrm{C}-\mathrm{F}}=239.0 \mathrm{~Hz}\right), 145.2,140.8,136.1,135.9,135.8,131.8\left(\mathrm{~d}, J_{\mathrm{C}-\mathrm{F}}=5.0 \mathrm{~Hz}\right), 130.2$, $130.0,128.8,128.5,128.2,126.6,124.6,124.1,118.4\left(\mathrm{~d}, J_{\mathrm{C}-\mathrm{F}}=10.0 \mathrm{~Hz}\right), 111.7\left(\mathrm{~d}, J_{\mathrm{C}-\mathrm{F}}=24.0 \mathrm{~Hz}\right)$, $102.3\left(\mathrm{~d}, J_{\mathrm{C}-\mathrm{F}}=28.0 \mathrm{~Hz}\right), 66.9,54.7,44.6,36.5,32.3,21.8 .{ }^{19} \mathrm{~F} \mathrm{NMR}\left(376 \mathrm{MHz}, \mathrm{CDCl}_{3}\right) \delta-117.40$. HPLC: Chiracel IG column, elute: $n$-hexane/i-propanol $=90 / 10$, detector: $254 \mathrm{~nm}, 30{ }^{\circ} \mathrm{C}$, flow rate: $0.8 \mathrm{~mL} / \mathrm{min}$, retention time $31.9 \mathrm{~min}$ (major) and $38.7 \mathrm{~min}$; HRMS (EI-TOF) $\mathrm{m} / \mathrm{z}:[\mathrm{M}]^{+}$ $\mathrm{C}_{29} \mathrm{H}_{24} \mathrm{FNO}_{4} \mathrm{~S}$ Calcd for 501.1410; found 501.1416.

Benzyl (7R,10S)-2-fluoro-5-tosyl-6,10-dihydro-7,10-methanocyclohepta[b]indole-7(5H)carboxylate (31): the reaction was conducted at $0.10 \mathrm{mmol}$ scale, $0.0325 \mathrm{~g}, 65 \%$ yield, $90 \%$ ee,

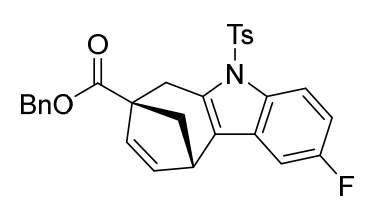
unknown compound, white foam solid, $[\alpha]_{\mathrm{D}}^{20}=-159.83$ (c 0.65 , $\left.\mathrm{CHCl}_{3}\right), \mathrm{R}_{f}=0.23$ (petroleum ether/ethyl acetate 15/1); ${ }^{1} \mathrm{H}$ NMR $(400$ $\left.\mathrm{MHz}, \mathrm{CDCl}_{3}\right) \delta$ 8.15-7.95 (m, 1H), $7.59(\mathrm{~d}, J=8.3 \mathrm{~Hz}, 2 \mathrm{H}), 7.46-7.29$ F $\quad(\mathrm{m}, 5 \mathrm{H}), 7.17(\mathrm{~d}, J=8.1 \mathrm{~Hz}, 2 \mathrm{H}), 7.12-7.02(\mathrm{~m}, 1 \mathrm{H}), 7.00-6.90(\mathrm{~m}$, $1 \mathrm{H}), 6.33(\mathrm{dd}, J=5.2,2.6 \mathrm{~Hz}, 1 \mathrm{H}), 5.82(\mathrm{~d}, J=5.5 \mathrm{~Hz}, 1 \mathrm{H}), 5.31-5.21(\mathrm{~m}, 2 \mathrm{H}), 3.65-3.59(\mathrm{~m}$, $1 \mathrm{H}), 3.55(\mathrm{~d}, J=18.4 \mathrm{~Hz}, 1 \mathrm{H}), 3.22(\mathrm{~d}, J=18.4 \mathrm{~Hz}, 1 \mathrm{H}), 2.44$ (dd, $J=9.9,4.6 \mathrm{~Hz}, 1 \mathrm{H}), 2.33$ (s, $3 \mathrm{H}), 1.97(\mathrm{~d}, J=9.9 \mathrm{~Hz}, 1 \mathrm{H}) .{ }^{13} \mathrm{C}$ NMR $\left(100 \mathrm{MHz}, \mathrm{CDCl}_{3}\right) \delta 174.6,160.0\left(\mathrm{~d}, J_{\mathrm{C}-\mathrm{F}}=239.0 \mathrm{~Hz}\right)$, $145.1,140.8,136.1,135.7,133.6,132.0,130.1,130.0,129.3\left(\mathrm{~d}, J_{\mathrm{C}-\mathrm{F}}=10.0 \mathrm{~Hz}\right), 128.8,128.5$, $128.2,126.5,124.4\left(\mathrm{~d}, J_{\mathrm{C}-\mathrm{F}}=4.0 \mathrm{~Hz}\right) 115.7\left(\mathrm{~d}, J_{\mathrm{C}-\mathrm{F}}=10.0 \mathrm{~Hz}\right), 111.7\left(\mathrm{~d}, J_{\mathrm{C}-\mathrm{F}}=25.0 \mathrm{~Hz}\right), 103.7(\mathrm{~d}$, $\left.J_{\mathrm{C}-\mathrm{F}}=24.0 \mathrm{~Hz}\right), 66.9,54.7,44.6,36.5,32.4,21.8 .{ }^{19} \mathrm{~F} \mathrm{NMR}\left(376 \mathrm{MHz}, \mathrm{CDCl}_{3}\right) \delta-119.60$. HPLC: Chiracel IG column, elute: $n$-hexane/i-propanol $=90 / 10$, detector: $254 \mathrm{~nm}, 30{ }^{\circ} \mathrm{C}$, flow rate: 0.8 $\mathrm{mL} / \mathrm{min}$, retention time $29.4 \mathrm{~min}$ (major) and $35.6 \mathrm{~min}$; HRMS (EI-TOF) $\mathrm{m} / \mathrm{z}$ : $[\mathrm{M}]^{+} \mathrm{C}_{29} \mathrm{H}_{24} \mathrm{FNO}_{4} \mathrm{~S}$ Calcd for 501.1410; found 501.1417.

Benzyl (7R,10S)-4-fluoro-5-tosyl-6,10-dihydro-7,10-methanocyclohepta[b]indole-7(5H)carboxylate (3m): the reaction was conducted at $0.10 \mathrm{mmol} \mathrm{scale,} 0.0349 \mathrm{~g}, 70 \%$ yield, $91 \%$ ee,

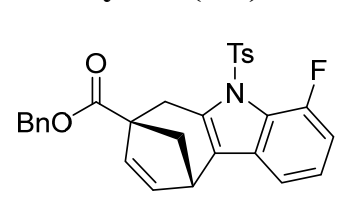
unknown compound, white foam solid, $[\alpha]^{20}{ }_{\mathrm{D}}=-122.23\left(\mathrm{c} 0.77, \mathrm{CHCl}_{3}\right)$, $\mathrm{R}_{f}=0.37$ (petroleum ether/ethyl acetate 9/1); ${ }^{1} \mathrm{H}$ NMR $(400 \mathrm{MHz}$, $\left.\mathrm{CDCl}_{3}\right) \delta 7.71(\mathrm{~d}, J=8.0 \mathrm{~Hz}, 2 \mathrm{H}), 7.46-7.31(\mathrm{~m}, 5 \mathrm{H}), 7.25-7.19(\mathrm{~m}$, $3 \mathrm{H}), 7.15-7.08(\mathrm{~m}, 1 \mathrm{H}), 6.92-6.83(\mathrm{~m}, 1 \mathrm{H}), 6.37(\mathrm{dd}, J=5.2,2.6 \mathrm{~Hz}$, $1 \mathrm{H}), 5.88(\mathrm{~d}, J=5.5 \mathrm{~Hz}, 1 \mathrm{H}), 5.28(\mathrm{~s}, 2 \mathrm{H}), 3.81-3.68(\mathrm{~m}, 2 \mathrm{H}), 3.34(\mathrm{~d}, J=18.4 \mathrm{~Hz}, 1 \mathrm{H}), 2.47(\mathrm{dd}$, $J=9.9,4.5 \mathrm{~Hz}, 1 \mathrm{H}), 2.36(\mathrm{~s}, 3 \mathrm{H}), 2.08(\mathrm{~d}, J=9.9 \mathrm{~Hz}, 1 \mathrm{H}) .{ }^{13} \mathrm{C} \mathrm{NMR}\left(100 \mathrm{MHz}, \mathrm{CDCl}_{3}\right) \delta 174.6$, $149.9\left(\mathrm{~d}, J_{\mathrm{C}-\mathrm{F}}=250.0 \mathrm{~Hz}\right), 144.9,140.6,136.5,136.1,134.5,132.1\left(\mathrm{~d}, J_{\mathrm{C}-\mathrm{F}}=4.0 \mathrm{~Hz}\right), 130.2,129.9$, 128.8, 128.5, 128.7, $127.3\left(\mathrm{~d}, J_{\mathrm{C}-\mathrm{F}}=3.0 \mathrm{~Hz}\right), 124.2\left(\mathrm{~d}, J_{\mathrm{C}-\mathrm{F}}=7.0 \mathrm{~Hz}\right), 123.5\left(\mathrm{~d}, J_{\mathrm{C}-\mathrm{F}}=2.0 \mathrm{~Hz}\right)$, $122.3\left(\mathrm{~d}, J_{\mathrm{C}-\mathrm{F}}=10.0 \mathrm{~Hz}\right), 113.7\left(\mathrm{~d}, J_{\mathrm{C}-\mathrm{F}}=3.0 \mathrm{~Hz}\right), 111.3\left(\mathrm{~d}, J_{\mathrm{C}-\mathrm{F}}=22.0 \mathrm{~Hz}\right), 66.9,55.0,44.4,36.7$, 33.0, 21.8. ${ }^{19} \mathrm{~F}$ NMR (376 MHz, $\left.\mathrm{CDCl}_{3}\right) \delta-117.76$. HPLC: Chiracel AD-H column, elute: $n$-hexane/i-propanol $=90 / 10$, detector: $254 \mathrm{~nm}, 30{ }^{\circ} \mathrm{C}$, flow rate: $0.8 \mathrm{~mL} / \mathrm{min}$, retention time 19.7 
min and 25.9 min (major); HRMS (EI-TOF) $\mathrm{m} / z$ : $[\mathrm{M}]^{+} \mathrm{C}_{29} \mathrm{H}_{24} \mathrm{FNO}_{4} \mathrm{~S}$ Calcd for 501.1410; found 501.1418 .

Benzyl (7R,10S)-3-chloro-5-tosyl-6,10-dihydro-7,10-methanocyclohepta[b]indole-7(5H)carboxylate (3n): the reaction was conducted at $0.10 \mathrm{mmol}$ scale, $0.0320 \mathrm{~g}, 62 \%$ yield, $80 \%$ ee,<smiles>Cn1c2ccc(C(=O)OCc3ccccc3)cc2c2ccc(Cl)cc21</smiles>
unknown compound, white foam solid, $[\alpha]^{20}{ }_{\mathrm{D}}=-154.99$ (c 0.60 , $\left.\mathrm{CHCl}_{3}\right), \mathrm{R}_{f}=0.20$ (petroleum ether/ethyl acetate $\left.15 / 1\right) ;{ }^{1} \mathrm{H}$ NMR $(400$ $\left.\mathrm{MHz}, \mathrm{CDCl}_{3}\right) \delta 8.16-8.10(\mathrm{~m}, 1 \mathrm{H}), 7.61(\mathrm{~d}, J=8.3 \mathrm{~Hz}, 2 \mathrm{H}), 7.43-7.29$ (m, 6H), 7.22-7.16 (m, 3H), $6.33(\mathrm{dd}, J=5.4,2.7 \mathrm{~Hz}, 1 \mathrm{H}), 5.81(\mathrm{~d}, J$ $=5.5 \mathrm{~Hz}, 1 \mathrm{H}), 5.28(\mathrm{~d}, J=12.8 \mathrm{~Hz}, 1 \mathrm{H}), 5.25(\mathrm{~d}, J=12.9 \mathrm{~Hz}, 1 \mathrm{H}), 3.68-3.62(\mathrm{~m}, 1 \mathrm{H}), 3.53(\mathrm{~d}, J$ $=18.3 \mathrm{~Hz}, 1 \mathrm{H}), 3.20(\mathrm{~d}, J=18.3 \mathrm{~Hz}, 1 \mathrm{H}), 2.43(\mathrm{dd}, J=9.9,4.6 \mathrm{~Hz}, 1 \mathrm{H}), 2.34(\mathrm{~s}, 3 \mathrm{H}), 1.96(\mathrm{~d}, J=$ $9.9 \mathrm{~Hz}, 1 \mathrm{H}) .{ }^{13} \mathrm{C}$ NMR $\left(100 \mathrm{MHz}, \mathrm{CDCl}_{3}\right) \delta 174.6,145.3,140.8,136.1,136.0,135.7,132.4,130.2$, 130.0, 130.0, 128.8, 128.5, 128.1, 126.7, 126.6, 124.1, 124.0, 118.6, 114.8, 66.9, 54.7, 44.6, 36.5, 32.2, 21.8. HPLC: Chiracel IG column, elute: $n$-hexane/i-propanol $=90 / 10$, detector: $254 \mathrm{~nm}, 30$ ${ }^{\circ} \mathrm{C}$, flow rate: $0.8 \mathrm{~mL} / \mathrm{min}$, retention time $29.5 \mathrm{~min}$ (major) and $35.7 \mathrm{~min}$; HRMS (EI-TOF) $\mathrm{m} / \mathrm{z}$ : $[\mathrm{M}]^{+} \mathrm{C}_{29} \mathrm{H}_{24} \mathrm{ClNO}_{4} \mathrm{~S}$ Calcd for 517.1115; found 517.1125.

Benzyl (7R,10S)-2-chloro-5-tosyl-6,10-dihydro-7,10-methanocyclohepta[b]indole-7(5H)carboxylate (3o): the reaction was conducted at $0.10 \mathrm{mmol}$ scale, $0.0276 \mathrm{~g}, 53 \%$ yield, $87 \%$ ee,

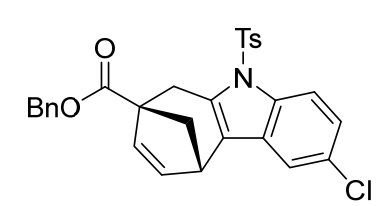
unknown compound, white foam solid, $[\alpha]^{20}{ }_{\mathrm{D}}=-161.47$ (c 0.54 , $\left.\mathrm{CHCl}_{3}\right), \mathrm{R}_{f}=0.20$ (petroleum ether/ethyl acetate $\left.15 / 1\right) ;{ }^{1} \mathrm{H}$ NMR $(400$ $\left.\mathrm{MHz}, \mathrm{CDCl}_{3}\right) \delta 8.01(\mathrm{~d}, J=8.9 \mathrm{~Hz}, 1 \mathrm{H}), 7.59(\mathrm{~d}, J=8.3 \mathrm{~Hz}, 2 \mathrm{H})$, 7.44-7.32 (m, 6H), 7.21-7.15 (m, 3H), $6.33(\mathrm{dd}, J=5.4,2.7 \mathrm{~Hz}, 1 \mathrm{H})$, $5.82(\mathrm{~d}, J=5.5 \mathrm{~Hz}, 1 \mathrm{H}), 5.28(\mathrm{~d}, J=12.6 \mathrm{~Hz}, 1 \mathrm{H}), 5.25(\mathrm{~d}, J=12.6 \mathrm{~Hz}, 1 \mathrm{H}), 3.67-3.59(\mathrm{~m}, 1 \mathrm{H})$, $3.54(\mathrm{~d}, J=18.4 \mathrm{~Hz}, 1 \mathrm{H}), 3.21$ (d, $J=18.4 \mathrm{~Hz}, 1 \mathrm{H}), 2.43$ (dd, $J=10.0,4.6 \mathrm{~Hz}, 1 \mathrm{H}), 2.33(\mathrm{~s}, 3 \mathrm{H})$, $1.96(\mathrm{~d}, J=10.0 \mathrm{~Hz}, 1 \mathrm{H}) .{ }^{13} \mathrm{C} \mathrm{NMR}\left(100 \mathrm{MHz}, \mathrm{CDCl}_{3}\right) \delta 174.5,145.2,140.8,136.1,135.7,134.0$, 133.3, 130.1, 130.0, 129.5, 129.4, 128.9, 128.5, 128.2, 126.5, 124.1, 123.9, 117.7, 115.7, 67.0, 54.7, 44.6, 36.5, 32.3, 21.8. HPLC: Chiracel IG column, elute: $n$-hexane/i-propanol $=90 / 10$, detector: $254 \mathrm{~nm}, 30{ }^{\circ} \mathrm{C}$, flow rate: $0.8 \mathrm{~mL} / \mathrm{min}$, retention time $27.6 \mathrm{~min}$ (major) and $34.4 \mathrm{~min}$; HRMS (EI-TOF) $\mathrm{m} / \mathrm{z}$ : $[\mathrm{M}]^{+} \mathrm{C}_{29} \mathrm{H}_{24} \mathrm{ClNO}_{4} \mathrm{~S}$ Calcd for 517.1115; found 517.1121.

Benzyl (7R,10S)-5-tosyl-2-(trifluoromethyl)-6,10-dihydro-7,10-methanocyclohepta[b]indole7(5H)-carboxylate (3p): the reaction was conducted at $0.10 \mathrm{mmol}$ scale, $0.0235 \mathrm{~g}, 43 \%$ yield,

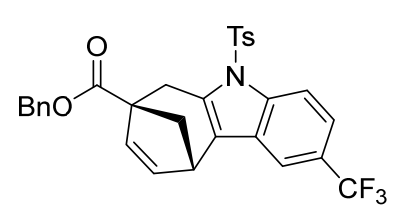

$74 \%$ ee, unknown compound, white foam solid, $[\alpha]^{20}{ }_{\mathrm{D}}=-106.80(\mathrm{c}$ $0.47, \mathrm{CHCl}_{3}$ ), $\mathrm{R}_{f}=0.20$ (petroleum ether/ethyl acetate $15 / 1$ ); ${ }^{1} \mathrm{H}$ NMR $\left(400 \mathrm{MHz}, \mathrm{CDCl}_{3}\right) \delta 8.20(\mathrm{~d}, J=8.7 \mathrm{~Hz}, 1 \mathrm{H}), 7.71(\mathrm{~s}, 1 \mathrm{H})$, $\mathrm{CF}_{3} 7.63(\mathrm{~d}, J=8.4 \mathrm{~Hz}, 2 \mathrm{H}), 7.51-7.46(\mathrm{~m}, 1 \mathrm{H}), 7.44-7.32(\mathrm{~m}, 5 \mathrm{H}), 7.20$ $(\mathrm{d}, J=8.3 \mathrm{~Hz}, 2 \mathrm{H}), 6.37(\mathrm{dd}, J=5.5,2.8 \mathrm{~Hz}, 1 \mathrm{H}), 5.84(\mathrm{~d}, J=5.5 \mathrm{~Hz}, 1 \mathrm{H}), 5.29(\mathrm{~d}, J=13.1 \mathrm{~Hz}$, 1H), $5.26(\mathrm{~d}, J=13.0 \mathrm{~Hz}, 1 \mathrm{H}), 3.77-3.69(\mathrm{~m}, 1 \mathrm{H}), 3.58(\mathrm{~d}, J=18.4 \mathrm{~Hz}, 1 \mathrm{H}), 3.24(\mathrm{~d}, J=18.4 \mathrm{~Hz}$, 1H), $2.47(\mathrm{dd}, J=10.0,4.6 \mathrm{~Hz}, 1 \mathrm{H}), 2.34(\mathrm{~s}, 3 \mathrm{H}), 1.98(\mathrm{~d}, J=10.0 \mathrm{~Hz}, 1 \mathrm{H}) .{ }^{13} \mathrm{C}$ NMR $(100 \mathrm{MHz}$, $\left.\mathrm{CDCl}_{3}\right) \delta 174.5,145.8,140.9,137.1,136.1,135.7,133.8,130.3,130.1,128.9,128.5,128.2,127.9$, 126.6, 126.2, 126.1, 125.7, 124.2, 120.7 (q, $\left.J_{\mathrm{C}-\mathrm{F}}=3.0 \mathrm{~Hz}\right), 115.4\left(\mathrm{q}, J_{\mathrm{C}-\mathrm{F}}=4.0 \mathrm{~Hz}\right), 114.8,67.0$, 54.7, 44.6, 36.5, 32.2, 21.8. ${ }^{19} \mathrm{~F}$ NMR (376 $\left.\mathrm{MHz}, \mathrm{CDCl}_{3}\right) \delta-61.17$. HPLC: Chiracel IG column, elute: $n$-hexane $/ \mathrm{i}$-propanol $=90 / 10$, detector: $254 \mathrm{~nm}, 30^{\circ} \mathrm{C}$, flow rate: $0.8 \mathrm{~mL} / \mathrm{min}$, retention time 
$17.1 \mathrm{~min}$ (major) and $20.8 \mathrm{~min}$; HRMS (EI-TOF) $\mathrm{m} / \mathrm{z}$ : $[\mathrm{M}]^{+} \mathrm{C}_{30} \mathrm{H}_{24} \mathrm{~F}_{3} \mathrm{NO}_{4} \mathrm{~S}$ Calcd for 551.1378; found 551.1375 .

Benzyl (7R,10S)-2-acetyl-5-tosyl-6,10-dihydro-7,10-methanocyclohepta[b]indole-7(5H)carboxylate (3q): the reaction was conducted at $0.10 \mathrm{mmol}$ scale, $0.0260 \mathrm{~g}, 49 \%$ yield, $78 \%$ ee,

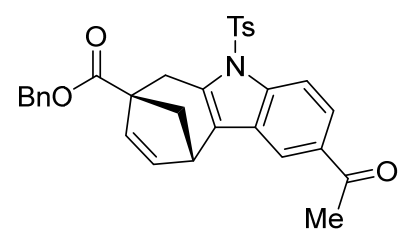
unknown compound, white foam solid, $[\alpha]^{20}{ }_{\mathrm{D}}=-141.66$ (c 0.42 , $\mathrm{CHCl}_{3}$ ), $\mathrm{R}_{f}=0.20$ (petroleum ether/ethyl acetate $4 / 1$ ); ${ }^{1} \mathrm{H}$ NMR $\left(400 \mathrm{MHz}, \mathrm{CDCl}_{3}\right) \delta 8.15(\mathrm{~d}, J=8.7 \mathrm{~Hz}, 1 \mathrm{H}), 8.08(\mathrm{~s}, 1 \mathrm{H})$, $7.90-7.83(\mathrm{~m}, 1 \mathrm{H}), 7.63(\mathrm{~d}, J=8.3 \mathrm{~Hz}, 2 \mathrm{H}), 7.48-7.31(\mathrm{~m}, 5 \mathrm{H})$, $7.19(\mathrm{~d}, J=8.1 \mathrm{~Hz}, 2 \mathrm{H}), 6.37(\mathrm{dd}, J=5.3,2.6 \mathrm{~Hz}, 1 \mathrm{H}), 5.83(\mathrm{~d}, J=$ $5.5 \mathrm{~Hz}, 1 \mathrm{H}), 5.29(\mathrm{~d}, J=13.1 \mathrm{~Hz}, 1 \mathrm{H}), 5.26(\mathrm{~d}, J=13.0 \mathrm{~Hz}, 1 \mathrm{H}), 3.84-3.69(\mathrm{~m}, 1 \mathrm{H}), 3.57$ (d, $J=$ $18.3 \mathrm{~Hz}, 1 \mathrm{H}), 3.23(\mathrm{~d}, J=18.3 \mathrm{~Hz}, 1 \mathrm{H}), 2.65(\mathrm{~s}, 3 \mathrm{H}), 2.47$ (dd, $J=10.0,4.6 \mathrm{~Hz}, 1 \mathrm{H}), 2.34(\mathrm{~s}, 3 \mathrm{H})$, $1.99(\mathrm{~d}, J=10.0 \mathrm{~Hz}, 1 \mathrm{H}) .{ }^{13} \mathrm{C} \mathrm{NMR}\left(100 \mathrm{MHz}, \mathrm{CDCl}_{3}\right) \delta 198.1,174.5,145.4,140.9,138.3,136.1$, 135.7, 133.5, 132.9, 130.2, 130.0, 128.9, 128.5, 128.2, 128.1, 126.6, 124.8, 124.4, 118.9, 114.3, 67.0, 54.7, 44.6, 36.5, 32.2, 27.0, 21.8. HPLC: Chiracel IG column, elute: $n$-hexane/i-propanol $=$ 80/20, detector: $254 \mathrm{~nm}, 30^{\circ} \mathrm{C}$, flow rate: $0.7 \mathrm{~mL} / \mathrm{min}$, retention time $57.0 \mathrm{~min}$ (major) and 73.1 $\min$; HRMS (EI-TOF) m/z: [M] ${ }^{+} \mathrm{C}_{31} \mathrm{H}_{27} \mathrm{NO}_{5} \mathrm{~S}$ Calcd for 525.1610; found 525.1606.

7-Benzyl 2-methyl (7R,10S)-5-tosyl-6,10-dihydro-7,10-methanocyclohepta[b]indole-2,7(5H)dicarboxylate (3r): the reaction was conducted at $0.10 \mathrm{mmol}$ scale, $0.0150 \mathrm{~g}, 28 \%$ yield, $74 \%$ ee,

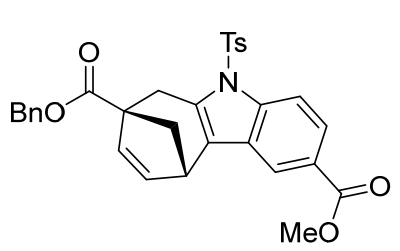
unknown compound, white foam solid, $[\alpha]^{20}{ }_{\mathrm{D}}=-64.33$ (c 0.30 , $\mathrm{CHCl}_{3}$ ), $\mathrm{R}_{f}=0.2$ (petroleum ether/ethyl acetate $\left.4 / 1\right) ;{ }^{1} \mathrm{H}$ NMR (400 $\left.\mathrm{MHz}, \mathrm{CDCl}_{3}\right) \delta 8.18-8.11(\mathrm{~m}, 2 \mathrm{H}), 7.96-7.91(\mathrm{~m}, 1 \mathrm{H}), 7.63(\mathrm{~d}, J=$ $8.4 \mathrm{~Hz}, 2 \mathrm{H}), 7.47-7.31(\mathrm{~m}, 5 \mathrm{H}), 7.18(\mathrm{~d}, J=8.1 \mathrm{~Hz}, 2 \mathrm{H}), 6.36(\mathrm{dd}, J$ $=5.5,2.7 \mathrm{~Hz}, 1 \mathrm{H}), 5.83(\mathrm{~d}, J=5.5 \mathrm{~Hz}, 1 \mathrm{H}), 5.29(\mathrm{~d}, J=12.5 \mathrm{~Hz}$, $1 \mathrm{H}), 5.25(\mathrm{~d}, J=12.7 \mathrm{~Hz}, 1 \mathrm{H}), 3.93(\mathrm{~s}, 3 \mathrm{H}), 3.78-3.72(\mathrm{~m}, 1 \mathrm{H}), 3.57$ (d, $J=18.3 \mathrm{~Hz}, 1 \mathrm{H}), 3.23(\mathrm{~d}$, $J=18.3 \mathrm{~Hz}, 1 \mathrm{H}), 2.46(\mathrm{dd}, J=10.0,4.6 \mathrm{~Hz}, 1 \mathrm{H}), 2.33(\mathrm{~s}, 3 \mathrm{H}), 1.99(\mathrm{~d}, J=10.0 \mathrm{~Hz}, 1 \mathrm{H}) .{ }^{13} \mathrm{C}$ NMR $\left(100 \mathrm{MHz}, \mathrm{CDCl}_{3}\right) \delta 174.6,167.5,145.4,141.0,138.3,136.1,135.7,133.2,130.2,130.0$, $128.9,128.5,128.2,128.0,126.6,125.5,125.2,124.6,120.2,114.3,67.0,54.7,52.4,44.6,36.5$, 32.2, 21.8. HPLC: Chiracel IG column, elute: $n$-hexane/i-propanol $=90 / 10$, detector: $254 \mathrm{~nm}, 30$ ${ }^{\circ} \mathrm{C}$, flow rate: $0.8 \mathrm{~mL} / \mathrm{min}$, retention time $71.0 \mathrm{~min}$ (major) and $79.8 \mathrm{~min}$; HRMS (EI-TOF) $\mathrm{m} / \mathrm{z}$ : $[\mathrm{M}]^{+} \mathrm{C}_{31} \mathrm{H}_{27} \mathrm{NO}_{6} \mathrm{~S}$ Calcd for 541.1559; found 541.1569.

Benzyl (7R,10S)-2,3-difluoro-5-tosyl-6,10-dihydro-7,10-methanocyclohepta[b]indole-7(5H)carboxylate (3s): the reaction was conducted at $0.10 \mathrm{mmol}$ scale, $0.0427 \mathrm{~g}, 82 \%$ yield, $86 \%$ ee,<smiles>CN1CC2=C(c3cc(F)c(F)cc31)C1C=CC(C(=O)OCc3ccccc3)(C2)C1</smiles>
unknown compound, white foam solid, $[\alpha]^{20}{ }_{\mathrm{D}}=-58.00$ (c 0.85 , $\left.\mathrm{CHCl}_{3}\right), \mathrm{R}_{f}=0.2$ (petroleum ether/ethyl acetate 15/1); ${ }^{1} \mathrm{H}$ NMR $(400$ $\left.\mathrm{MHz}, \mathrm{CDCl}_{3}\right) \delta 8.00-7.92(\mathrm{~m}, 1 \mathrm{H}), 7.59(\mathrm{~d}, J=8.4 \mathrm{~Hz}, 2 \mathrm{H}), 7.43-7.32$ (m, 5H), 7.23-7.11 (m, 3H), $6.33(\mathrm{dd}, J=5.5,2.7 \mathrm{~Hz}, 1 \mathrm{H}), 5.82(\mathrm{~d}, J=$ $5.5 \mathrm{~Hz}, 1 \mathrm{H}), 5.28(\mathrm{~d}, J=12.5 \mathrm{~Hz}, 1 \mathrm{H}), 5.25(\mathrm{~d}, J=12.6 \mathrm{~Hz}, 1 \mathrm{H}), 3.62-3.57(\mathrm{~m}, 1 \mathrm{H}), 3.52(\mathrm{~d}, J=$ $18.3 \mathrm{~Hz}, 1 \mathrm{H}), 3.19$ (d, $J=18.3 \mathrm{~Hz}, 1 \mathrm{H}), 2.43$ (dd, $J=10.0,4.7 \mathrm{~Hz}, 1 \mathrm{H}), 2.35$ (s, 3H), 1.95 (d, $J=$ $10.0 \mathrm{~Hz}, 1 \mathrm{H}) .{ }^{13} \mathrm{C}$ NMR $\left(100 \mathrm{MHz}, \mathrm{CDCl}_{3}\right) \delta 174.5,148.5\left(\mathrm{dd}, J_{\mathrm{C}-\mathrm{F}}=243.0,8.0 \mathrm{~Hz}\right), 148.4(\mathrm{dd}$, $\left.J_{\mathrm{C}-\mathrm{F}}=243.0,8.0 \mathrm{~Hz}\right), 145.4,140.7,136.1,135.5,133.1\left(\mathrm{~d}, J_{\mathrm{C}-\mathrm{F}}=5.0 \mathrm{~Hz}\right), 130.7\left(\mathrm{~d}, J_{\mathrm{C}-\mathrm{F}}=10.0 \mathrm{~Hz}\right)$, $130.2,130.1,128.9,128.5,128.2,126.6,124.02,124.0\left(\mathrm{~d}, J_{\mathrm{C}-\mathrm{F}}=1.0 \mathrm{~Hz}\right), 123.9\left(\mathrm{~d}, J_{\mathrm{C}-\mathrm{F}}=2.0 \mathrm{~Hz}\right)$, 
$105.1\left(\mathrm{~d}, J_{\mathrm{C}-\mathrm{F}}=20.0 \mathrm{~Hz}\right), 104.0\left(\mathrm{~d}, J_{\mathrm{C}-\mathrm{F}}=24.0 \mathrm{~Hz}\right), 67.0,54.6,44.6,36.5,32.3,21.8 .{ }^{19} \mathrm{~F}$ NMR $\left(376 \mathrm{MHz}, \mathrm{CDCl}_{3}\right) \delta-140.59(\mathrm{~d}, J=20.7 \mathrm{~Hz}),-142.45(\mathrm{~d}, J=20.8 \mathrm{~Hz})$. Chiracel IG column, elute: $n$-hexane/i-propanol $=90 / 10$, detector: $254 \mathrm{~nm}, 30{ }^{\circ} \mathrm{C}$, flow rate: $0.8 \mathrm{~mL} / \mathrm{min}$, retention time 25.4 min (major) and $30.1 \mathrm{~min}$; HRMS (EI-TOF) $\mathrm{m} / \mathrm{z}$ : $[\mathrm{M}]^{+} \mathrm{C}_{29} \mathrm{H}_{23} \mathrm{~F}_{2} \mathrm{NO}_{4} \mathrm{~S}$ Calcd for 519.1316; found 519.1317.

Benzyl (7R,10S)-2-fluoro-3-methyl-5-tosyl-6,10-dihydro-7,10-methanocyclohepta[b]indole$7(\mathbf{5 H})$-carboxylate (3t): the reaction was conducted at $0.10 \mathrm{mmol}$ scale, $0.0390 \mathrm{~g}, 76 \%$ yield,<smiles>Cc1cc2c(cc1F)C1C=CC2CC1C(=O)OCc1ccccc1</smiles>
$94 \%$ ee, unknown compound, white foam solid, $[\alpha]^{20}{ }_{D}=-169.04(c$ $\left.0.63, \mathrm{CHCl}_{3}\right), \mathrm{R}_{f}=0.30$ (petroleum ether/ethyl acetate $9 / 1$ ); ${ }^{1} \mathrm{H}$ $\operatorname{NMR}\left(400 \mathrm{MHz}, \mathrm{CDCl}_{3}\right) \delta 7.91(\mathrm{~d}, J=6.5 \mathrm{~Hz}, 1 \mathrm{H}), 7.57(\mathrm{~d}, J=8.4$ $\mathrm{Hz}, 2 \mathrm{H}), 7.46-7.28(\mathrm{~m}, 5 \mathrm{H}), 7.17(\mathrm{~d}, J=8.1 \mathrm{~Hz}, 2 \mathrm{H}), 7.01(\mathrm{~d}, J=$ $9.4 \mathrm{~Hz}, 1 \mathrm{H}), 6.32(\mathrm{dd}, J=5.5,2.7 \mathrm{~Hz}, 1 \mathrm{H}), 5.81(\mathrm{~d}, J=5.5 \mathrm{~Hz}, 1 \mathrm{H}), 5.28(\mathrm{~d}, J=12.5 \mathrm{~Hz}, 1 \mathrm{H})$, $5.24(\mathrm{~d}, J=12.5 \mathrm{~Hz}, 1 \mathrm{H}), 3.62-3.56(\mathrm{~m}, 1 \mathrm{H}), 3.51(\mathrm{~d}, J=18.3 \mathrm{~Hz}, 1 \mathrm{H}), 3.18(\mathrm{~d}, J=18.3 \mathrm{~Hz}, 1 \mathrm{H})$, $2.41(\mathrm{dd}, J=9.9,4.7 \mathrm{~Hz}, 1 \mathrm{H}), 2.36(\mathrm{~d}, J=1.9 \mathrm{~Hz}, 3 \mathrm{H}), 2.33(\mathrm{~s}, 3 \mathrm{H}), 1.96(\mathrm{~d}, J=9.9 \mathrm{~Hz}, 1 \mathrm{H}) .{ }^{13} \mathrm{C}$ NMR $\left(100 \mathrm{MHz}, \mathrm{CDCl}_{3}\right) \delta 174.6,158.8\left(\mathrm{~d}, J_{\mathrm{C}-\mathrm{F}}=238.0 \mathrm{~Hz}\right), 145.0,140.8,136.1,135.9,132.3$, $132.0,130.0,130.0,128.8,128.5,128.1,127.2\left(\mathrm{~d}, J_{\mathrm{C}-\mathrm{F}}=10.0 \mathrm{~Hz}\right), 126.4,124.4\left(\mathrm{~d}, J_{\mathrm{C}-\mathrm{F}}=4.0 \mathrm{~Hz}\right)$, $121.5\left(\mathrm{~d}, J_{\mathrm{C}-\mathrm{F}}=20.0 \mathrm{~Hz}\right), 116.7\left(\mathrm{~d}, J_{\mathrm{C}-\mathrm{F}}=6.0 \mathrm{~Hz}\right), 103.3\left(\mathrm{~d}, J_{\mathrm{C}-\mathrm{F}}=25.0 \mathrm{~Hz}\right), 66.9,54.7,44.6,36.6$, 32.4, 21.8, $15.7\left(\mathrm{~d}, J_{\mathrm{C}-\mathrm{F}}=4.0 \mathrm{~Hz}\right) .{ }^{19} \mathrm{~F}$ NMR $\left(376 \mathrm{MHz}, \mathrm{CDCl}_{3}\right) \delta-122.83$. HPLC: Chiracel IG column, elute: $n$-hexane/i-propanol $=90 / 10$, detector: $254 \mathrm{~nm}, 30{ }^{\circ} \mathrm{C}$, flow rate: $0.8 \mathrm{~mL} / \mathrm{min}$, retention time $24.9 \mathrm{~min}$ (major) and $31.1 \mathrm{~min}$; HRMS (EI-TOF) $\mathrm{m} / \mathrm{z}$ : [M] ${ }^{+} \mathrm{C}_{30} \mathrm{H}_{26} \mathrm{FNO}_{4} \mathrm{~S}$ Calcd for 515.1567 ; found 515.1577 .

Benzyl (7R,10S)-4-chloro-2-fluoro-5-tosyl-6,10-dihydro-7,10-methanocyclohepta[b]indole-

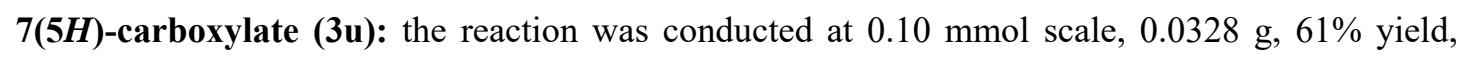<smiles></smiles>
$90 \%$ ee, unknown compound, white foam solid, $[\alpha]^{20}{ }_{D}=-133.38(c$ $\left.0.65, \mathrm{CHCl}_{3}\right), \mathrm{R}_{f}=0.20$ (petroleum ether/ethyl acetate $10 / 1$ ); ${ }^{1} \mathrm{H} \mathrm{NMR}$ $\left(400 \mathrm{MHz}, \mathrm{CDCl}_{3}\right) \delta 7.51(\mathrm{~d}, J=8.4 \mathrm{~Hz}, 2 \mathrm{H}), 7.44-7.29(\mathrm{~m}, 5 \mathrm{H}), 7.18$ $(\mathrm{d}, J=8.1 \mathrm{~Hz}, 2 \mathrm{H}), 7.01-6.94(\mathrm{~m}, 2 \mathrm{H}), 6.28(\mathrm{dd}, J=5.5,2.7 \mathrm{~Hz}, 1 \mathrm{H})$, $5.85(\mathrm{~d}, J=5.5 \mathrm{~Hz}, 1 \mathrm{H}), 5.27(\mathrm{~d}, J=12.8 \mathrm{~Hz}, 1 \mathrm{H}), 5.24(\mathrm{~d}, J=12.8 \mathrm{~Hz}, 1 \mathrm{H}), 3.70(\mathrm{~d}, J=18.6 \mathrm{~Hz}$, $1 \mathrm{H}), 3.61-3.56(\mathrm{~m}, 1 \mathrm{H}), 3.21(\mathrm{~d}, J=18.6 \mathrm{~Hz}, 1 \mathrm{H}), 2.43(\mathrm{dd}, J=10.0,4.6 \mathrm{~Hz}, 1 \mathrm{H}), 2.37(\mathrm{~s}, 3 \mathrm{H})$, $1.98(\mathrm{~d}, J=10.0 \mathrm{~Hz}, 1 \mathrm{H}) .{ }^{13} \mathrm{C} \mathrm{NMR}\left(100 \mathrm{MHz}, \mathrm{CDCl}_{3}\right) \delta 174.3,159.5\left(\mathrm{~d}, J_{\mathrm{C}-\mathrm{F}}=244.0 \mathrm{~Hz}\right), 144.8$, $140.1,138.1,136.5,136.1,133.6\left(\mathrm{~d}, J_{\mathrm{C}-\mathrm{F}}=10.0 \mathrm{~Hz}\right), 131.0\left(\mathrm{~d}, J_{\mathrm{C}-\mathrm{F}}=2.0 \mathrm{~Hz}\right), 130.7,129.7,128.9$, $128.5,128.2,126.7,126.3\left(\mathrm{~d}, J_{\mathrm{C}-\mathrm{F}}=4.0 \mathrm{~Hz}\right), 122.3\left(\mathrm{~d}, J_{\mathrm{C}-\mathrm{F}}=34.0 \mathrm{~Hz}\right), 114.8\left(\mathrm{~d}, J_{\mathrm{C}-\mathrm{F}}=27.0 \mathrm{~Hz}\right)$, $102.8\left(\mathrm{~d}, J_{\mathrm{C}-\mathrm{F}}=23.0 \mathrm{~Hz}\right), 67.0,55.0,44.2,36.6,33.6,21.8 .{ }^{19} \mathrm{~F} \mathrm{NMR}\left(376 \mathrm{MHz}, \mathrm{CDCl}_{3}\right) \delta-117.51$. HPLC: Chiracel IG column, elute: $n$-hexane/i-propanol $=90 / 10$, detector: $254 \mathrm{~nm}, 30{ }^{\circ} \mathrm{C}$, flow rate: $0.8 \mathrm{~mL} / \mathrm{min}$, retention time $39.4 \mathrm{~min}$ (major) and $42.6 \mathrm{~min}$; HRMS (EI-TOF) $\mathrm{m} / \mathrm{z}:[\mathrm{M}]^{+}$ $\mathrm{C}_{29} \mathrm{H}_{23} \mathrm{ClFNO}_{4} \mathrm{~S}$ Calcd for 535.1020; found 535.1030. 
Benzyl (7R,10S)-2-chloro-4-fluoro-5-tosyl-6,10-dihydro-7,10-methanocyclohepta[b]indole$7(5 \mathrm{H})$-carboxylate $(\mathbf{3 v})$ : the reaction was conducted at $0.10 \mathrm{mmol}$ scale, $0.0368 \mathrm{~g}, 69 \%$ yield, (1) $91 \%$ ee, unknown compound, white foam solid, $[\alpha]^{20}{ }_{\mathrm{D}}=-97.80$ (c 0.74 , $\left.\mathrm{CHCl}_{3}\right), \mathrm{R}_{f}=0.20$ (petroleum ether/ethyl acetate $\left.10 / 1\right) ;{ }^{1} \mathrm{H}$ NMR (400 $\left.\mathrm{MHz}, \mathrm{CDCl}_{3}\right) \delta 7.69(\mathrm{~d}, J=7.9 \mathrm{~Hz}, 2 \mathrm{H}), 7.44-7.31(\mathrm{~m}, 5 \mathrm{H}), 7.25-7.17$ $(\mathrm{m}, 3 \mathrm{H}), 6.93-6.83(\mathrm{~m}, 1 \mathrm{H}), 6.35(\mathrm{dd}, J=5.5,2.7 \mathrm{~Hz}, 1 \mathrm{H}), 5.89(\mathrm{~d}, J=$ $5.5 \mathrm{~Hz}, 1 \mathrm{H}), 5.29(\mathrm{~d}, J=12.6 \mathrm{~Hz}, 1 \mathrm{H}), 5.26(\mathrm{~d}, J=12.6 \mathrm{~Hz}, 1 \mathrm{H}), 3.74(\mathrm{~d}, J=18.6 \mathrm{~Hz}, 1 \mathrm{H})$, 3.69-3.64 (m, 1H), $3.33(\mathrm{~d}, J=18.5 \mathrm{~Hz}, 1 \mathrm{H}), 2.47$ (dd, $J=10.0,4.6 \mathrm{~Hz}, 1 \mathrm{H}), 2.38(\mathrm{~s}, 3 \mathrm{H}), 2.06(\mathrm{~d}$, $J=10.0 \mathrm{~Hz}, 1 \mathrm{H}) .{ }^{13} \mathrm{C}$ NMR $\left(100 \mathrm{MHz}, \mathrm{CDCl}_{3}\right) \delta 174.4,149.3\left(\mathrm{~d}, J_{\mathrm{C}-\mathrm{F}}=253.0 \mathrm{~Hz}\right), 145.2,140.5$, 136.2, 136.1, 136.0, $132.6\left(\mathrm{~d}, J_{\mathrm{C}-\mathrm{F}}=4.0 \mathrm{~Hz}\right), 130.4,130.0,129.1\left(\mathrm{~d}, J_{\mathrm{C}-\mathrm{F}}=10.0 \mathrm{~Hz}\right), 128.9,128.5$, $128.2,127.3\left(\mathrm{~d}, J_{\mathrm{C}-\mathrm{F}}=2.0 \mathrm{~Hz}\right), 123.1\left(\mathrm{~d}, J_{\mathrm{C}-\mathrm{F}}=2.0 \mathrm{~Hz}\right), 120.9\left(\mathrm{~d}, J_{\mathrm{C}-\mathrm{F}}=10.0 \mathrm{~Hz}\right), 113.7\left(\mathrm{~d}, J_{\mathrm{C}-\mathrm{F}}=\right.$ $4.0 \mathrm{~Hz}), 111.9\left(\mathrm{~d}, J_{\mathrm{C}-\mathrm{F}}=25.0 \mathrm{~Hz}\right), 67.0,54.9,44.3,36.6,32.9,21.8 .{ }^{19} \mathrm{~F} \mathrm{NMR}\left(376 \mathrm{MHz}, \mathrm{CDCl}_{3}\right)$ $\delta$-115.31. HPLC: Chiracel IG column, elute: $n$-hexane/i-propanol $=90 / 10$, detector: $254 \mathrm{~nm}, 30$ ${ }^{\circ} \mathrm{C}$, flow rate: $0.8 \mathrm{~mL} / \mathrm{min}$, retention time $30.7 \mathrm{~min}$ (major) and $33.7 \mathrm{~min}$; HRMS (EI-TOF) $\mathrm{m} / \mathrm{z}$ : $[\mathrm{M}]^{+} \mathrm{C}_{29} \mathrm{H}_{23} \mathrm{ClFNO}_{4} \mathrm{~S}$ Calcd for 535.1020; found 535.1014.

Benzyl (7R,10S)-2,4-difluoro-5-tosyl-6,10-dihydro-7,10-methanocyclohepta[b]indole-7(5H)carboxylate (3w): the reaction was conducted at $0.10 \mathrm{mmol}$ scale, $0.0416 \mathrm{~g}, 80 \%$ yield, $93 \%$ ee,

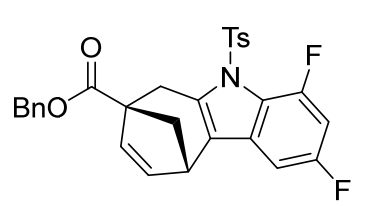
unknown compound, white foam solid, $[\alpha]^{20}{ }_{\mathrm{D}}=-126.70$ (c 0.88 , $\left.\mathrm{CHCl}_{3}\right), \mathrm{R}_{f}=0.20$ (petroleum ether/ethyl acetate $\left.15 / 1\right) ;{ }^{1} \mathrm{H}$ NMR (400 $\left.\mathrm{MHz}, \mathrm{CDCl}_{3}\right) \delta 7.68(\mathrm{~d}, J=8.1 \mathrm{~Hz}, 2 \mathrm{H}), 7.45-7.32(\mathrm{~m}, 5 \mathrm{H}), 7.26-7.21$ $(\mathrm{m}, 2 \mathrm{H}), 6.95-6.85(\mathrm{~m}, 1 \mathrm{H}), 6.70-6.60(\mathrm{~m}, 1 \mathrm{H}), 6.35(\mathrm{dd}, J=5.4,2.7$ $\mathrm{Hz}, 1 \mathrm{H}), 5.89$ (d, $J=5.5 \mathrm{~Hz}, 1 \mathrm{H}), 5.28(\mathrm{~s}, 2 \mathrm{H}), 3.74(\mathrm{~d}, J=18.6 \mathrm{~Hz}, 1 \mathrm{H}), 3.67-3.59(\mathrm{~m}, 1 \mathrm{H}), 3.33$ (d, $J=18.5 \mathrm{~Hz}, 1 \mathrm{H}), 2.47(\mathrm{dd}, J=10.0,4.6 \mathrm{~Hz}, 1 \mathrm{H}), 2.37(\mathrm{~s}, 3 \mathrm{H}), 2.06(\mathrm{~d}, J=10.0 \mathrm{~Hz}, 1 \mathrm{H}) .{ }^{13} \mathrm{C}$ $\operatorname{NMR}\left(100 \mathrm{MHz}, \mathrm{CDCl}_{3}\right) \delta 174.5,159.2\left(\mathrm{dd}, J_{\mathrm{C}-\mathrm{F}}=242.0,11.0 \mathrm{~Hz}\right), 149.4\left(\mathrm{dd}, J_{\mathrm{C}-\mathrm{F}}=252.0,13.0\right.$ $\mathrm{Hz}), 145.1,140.5,136.4,136.2,136.1,132.1\left(\mathrm{dd}, J_{\mathrm{C}-\mathrm{F}}=11.0,5.0 \mathrm{~Hz}\right), 130.4,130.0,128.9,128.5$, $128.2,127.3\left(\mathrm{~d}, J_{\mathrm{C}-\mathrm{F}}=2.0 \mathrm{~Hz}\right), 123.0\left(\mathrm{dd}, J_{\mathrm{C}-\mathrm{F}}=4.0,1.0 \mathrm{~Hz}\right), 119.1\left(\mathrm{dd}, J_{\mathrm{C}-\mathrm{F}}=9.0,2.0 \mathrm{~Hz}\right), 100.3$ $\left(\mathrm{dd}, J_{\mathrm{C}-\mathrm{F}}=29.0,26.0 \mathrm{~Hz}\right), 99.6\left(\mathrm{dd}, J_{\mathrm{C}-\mathrm{F}}=24.0,4.0 \mathrm{~Hz}\right), 67.0,54.9,44.4,36.6,33.0,21.8 .{ }^{19} \mathrm{~F}$ NMR $\left(376 \mathrm{MHz}, \mathrm{CDCl}_{3}\right) \delta-113.79(\mathrm{~d}, J=5.6 \mathrm{~Hz}),-116.79(\mathrm{~d}, J=5.5 \mathrm{~Hz})$. HPLC: Chiracel AD-H column, elute: $n$-hexane/i-propanol $=90 / 10$, detector: $254 \mathrm{~nm}, 30{ }^{\circ} \mathrm{C}$, flow rate: $0.8 \mathrm{~mL} / \mathrm{min}$, retention time $18.1 \mathrm{~min}$ and $24.2 \mathrm{~min}$ (major); HRMS (EI-TOF) $\mathrm{m} / \mathrm{z}$ : $[\mathrm{M}]^{+} \mathrm{C}_{29} \mathrm{H}_{23} \mathrm{~F}_{2} \mathrm{NO}_{4} \mathrm{~S}$ Calcd for 519.1316; found 519.1326.

Methyl (7R,10S)-5-tosyl-6,10-dihydro-7,10-methanocyclohepta[b]indole-7(5H)-carboxylate (3x): the reaction was conducted at $0.10 \mathrm{mmol}$ scale, $0.0316 \mathrm{~g}, 78 \%$ yield, $90 \%$ ee, unknown<smiles>[3H]n1c2c(c3ccccc31)C1C3CCC(C(=O)OC)(CC3)C1C2</smiles>
compound, white foam solid, $[\alpha]^{20}{ }_{\mathrm{D}}=-141.60\left(\mathrm{c} 0.56, \mathrm{CHCl}_{3}\right), \mathrm{R}_{f}=0.15$ (petroleum ether/ethyl acetate 10/1); ${ }^{1} \mathrm{H}$ NMR $\left(400 \mathrm{MHz}, \mathrm{CDCl}_{3}\right) \delta$ 8.14-8.09 (m, 1H), $7.65(\mathrm{~d}, J=8.3 \mathrm{~Hz}, 2 \mathrm{H}), 7.48-7.43(\mathrm{~m}, 1 \mathrm{H})$, $7.29-7.25(\mathrm{~m}, 2 \mathrm{H}), 7.18(\mathrm{~d}, J=8.1 \mathrm{~Hz}, 2 \mathrm{H}), 6.35(\mathrm{dd}, J=5.4,2.7 \mathrm{~Hz}$, $1 \mathrm{H}), 5.79(\mathrm{~d}, J=5.5 \mathrm{~Hz}, 1 \mathrm{H}), 3.83(\mathrm{~s}, 3 \mathrm{H}), 3.73-3.67(\mathrm{~m}, 1 \mathrm{H}), 3.55(\mathrm{~d}, J=18.3 \mathrm{~Hz}, 1 \mathrm{H}), 3.21(\mathrm{~d}$, $J=18.2 \mathrm{~Hz}, 1 \mathrm{H}), 2.42(\mathrm{dd}, J=9.9,4.6 \mathrm{~Hz}, 1 \mathrm{H}), 2.32(\mathrm{~s}, 3 \mathrm{H}), 1.98(\mathrm{~d}, J=9.9 \mathrm{~Hz}, 1 \mathrm{H}) .{ }^{13} \mathrm{C} \mathrm{NMR}$ $\left(100 \mathrm{MHz}, \mathrm{CDCl}_{3}\right) \delta 175.4,144.9,140.9,136.0,135.7,131.7,130.0,129.9,128.3,126.5,124.5$, 124.1, 123.5, 117.9, 114.7, 54.7, 52.5, 44.6, 36.5, 32.4, 21.7. HPLC: Chiracel IC column, elute: $n$-hexane $/ i$-propanol $=90 / 10$, detector: $254 \mathrm{~nm}, 30{ }^{\circ} \mathrm{C}$, flow rate: $0.8 \mathrm{~mL} / \mathrm{min}$, retention time 23.4 
min (major) and $27.1 \mathrm{~min}$; HRMS (EI-TOF) $\mathrm{m} / \mathrm{z}$ : $[\mathrm{M}]^{+} \mathrm{C}_{23} \mathrm{H}_{21} \mathrm{NO}_{4} \mathrm{~S}$ Calcd for 407.1191; found 407.1197.

Ethyl (7R,10S)-5-tosyl-6,10-dihydro-7,10-methanocyclohepta[b]indole-7(5H)-carboxylate (3y): the reaction was conducted at $0.10 \mathrm{mmol}$ scale, $0.0319 \mathrm{~g}, 76 \%$ yield, $89 \%$ ee, unknown<smiles>CCOC(=O)C12CCC(C1)c1c2c2ccccc2n1C</smiles>
compound, white foam solid, $[\alpha]^{20}{ }_{\mathrm{D}}=-183.71\left(\mathrm{c} 0.52, \mathrm{CHCl}_{3}\right), \mathrm{R}_{f}=0.15$ (petroleum ether/ethyl acetate 10/1); ${ }^{1} \mathrm{H}$ NMR $\left(400 \mathrm{MHz}, \mathrm{CDCl}_{3}\right) \delta$ 8.12-8.06 (m, 1H), $7.62(\mathrm{~d}, J=8.3 \mathrm{~Hz}, 2 \mathrm{H}), 7.46-7.40(\mathrm{~m}, 1 \mathrm{H})$, $7.27-7.21(\mathrm{~m}, 2 \mathrm{H}), 7.17(\mathrm{~d}, J=8.1 \mathrm{~Hz}, 2 \mathrm{H}), 6.34(\mathrm{dd}, J=5.4,2.7 \mathrm{~Hz}$, $1 \mathrm{H}), 5.80(\mathrm{~d}, J=5.5 \mathrm{~Hz}, 1 \mathrm{H}), 4.28(\mathrm{q}, J=7.1 \mathrm{~Hz}, 2 \mathrm{H}), 3.72-3.66(\mathrm{~m}, 1 \mathrm{H}), 3.54(\mathrm{~d}, J=18.3 \mathrm{~Hz}$, $1 \mathrm{H}), 3.20(\mathrm{~d}, J=18.2 \mathrm{~Hz}, 1 \mathrm{H}), 2.41(\mathrm{dd}, J=9.9,4.6 \mathrm{~Hz}, 1 \mathrm{H}), 2.32(\mathrm{~s}, 3 \mathrm{H}), 1.97(\mathrm{~d}, J=9.9 \mathrm{~Hz}$, $1 \mathrm{H}), 1.34(\mathrm{t}, J=7.1 \mathrm{~Hz}, 3 \mathrm{H}) .{ }^{13} \mathrm{C}$ NMR $\left(100 \mathrm{MHz}, \mathrm{CDCl}_{3}\right) \delta 175.0,144.9,140.8,136.0,135.7$, 131.8, 130.1, 130.0, 128.3, 126.5, 124.5, 124.0, 123.5, 117.9, 114.7, 61.3, 54.7, 44.6, 36.5, 32.4, 21.7, 14.5. HPLC: Chiracel IC column, elute: $n$-hexane/i-propanol $=90 / 10$, detector: $254 \mathrm{~nm}, 30$ ${ }^{\circ} \mathrm{C}$, flow rate: $0.8 \mathrm{~mL} / \mathrm{min}$, retention time $21.5 \mathrm{~min}$ (major) and $23.8 \mathrm{~min}$; HRMS (EI-TOF) $\mathrm{m} / \mathrm{z}$ : $[\mathrm{M}]^{+} \mathrm{C}_{24} \mathrm{H}_{23} \mathrm{NO}_{4} \mathrm{~S}$ Calcd for 421.1348; found 421.1356 .

\section{Isopropyl}

(7R,10S)-5-tosyl-6,10-dihydro-7,10-methanocyclohepta[b]indole-7(5H)carboxylate (3z): the reaction was conducted at $0.10 \mathrm{mmol}$ scale, $0.0306 \mathrm{~g}, 70 \%$ yield, $89 \%$ ee,

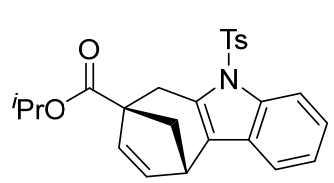
unknown compound, white foam solid, $[\alpha]^{20}{ }_{\mathrm{D}}=-214.64$ (c 0.43, $\left.\mathrm{CHCl}_{3}\right)$, $\mathrm{R}_{f}=0.27$ (petroleum ether/ethyl acetate $\left.15 / 1\right) ;{ }^{1} \mathrm{H}$ NMR $(400 \mathrm{MHz}$, $\left.\mathrm{CDCl}_{3}\right) \delta 8.12-8.07(\mathrm{~m}, 1 \mathrm{H}), 7.62(\mathrm{~d}, J=8.3 \mathrm{~Hz}, 2 \mathrm{H}), 7.45-7.41(\mathrm{~m}, 1 \mathrm{H})$, $7.25-7.21(\mathrm{~m}, 2 \mathrm{H}), 7.18(\mathrm{~d}, J=8.2 \mathrm{~Hz}, 2 \mathrm{H}), 6.33(\mathrm{dd}, J=5.4,2.7 \mathrm{~Hz}$, $1 \mathrm{H}), 5.79$ (d, $J=5.5 \mathrm{~Hz}, 1 \mathrm{H}), 5.14$ (hept, $J=6.2 \mathrm{~Hz}, 1 \mathrm{H}) .3 .74-3.61(\mathrm{~m}, 1 \mathrm{H}), 3.52$ (d, $J=18.3 \mathrm{~Hz}$, 1H), $3.18(\mathrm{~d}, J=18.3 \mathrm{~Hz}, 1 \mathrm{H}), 2.38(\mathrm{dd}, J=9.9,4.6 \mathrm{~Hz}, 1 \mathrm{H}), 2.33(\mathrm{~s}, 3 \mathrm{H}), 1.96(\mathrm{~d}, J=9.9 \mathrm{~Hz}$, $1 \mathrm{H}), 1.33(\mathrm{~d}, J=6.2,3 \mathrm{H}), 1.32(\mathrm{~d}, J=6.2,3 \mathrm{H}) .{ }^{13} \mathrm{C} \mathrm{NMR}\left(100 \mathrm{MHz}, \mathrm{CDCl}_{3}\right) \delta 174.5,144.9$, 140.6, 136.1, 135.7, 131.9, 130.2, 130.0, 128.3, 126.6, 124.6, 124.0, 123.5, 117.9, 114.7, 68.6, 54.8, 44.6, 36.5, 32.3, 22.0, 22.0, 21.8. HPLC: Chiracel IC column, elute: $n$-hexane/i-propanol = 90/10, detector: $254 \mathrm{~nm}, 30{ }^{\circ} \mathrm{C}$, flow rate: $0.8 \mathrm{~mL} / \mathrm{min}$, retention time $15.7 \mathrm{~min}$ (major) and 17.8 min; HRMS (EI-TOF) m/z: [M] ${ }^{+} \mathrm{C}_{25} \mathrm{H}_{25} \mathrm{NO}_{4} \mathrm{~S}$ Calcd for 435.1504; found 435.1511.

tert-Butyl (7R,10S)-5-tosyl-6,10-dihydro-7,10-methanocyclohepta[b]indole-7(5H)-carboxylate (3aa): the reaction was conducted at $0.10 \mathrm{mmol}$ scale, $0.0290 \mathrm{~g}, 65 \%$ yield, $83 \%$ ee, unknown

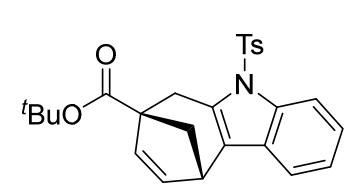
compound, white foam solid, $[\alpha]_{\mathrm{D}}^{20}=-155.10\left(\right.$ c $\left.0.47, \mathrm{CHCl}_{3}\right), \mathrm{R}_{f}=$ 0.20 (petroleum ether/ethyl acetate 30/1); ${ }^{1} \mathrm{H}$ NMR $\left(400 \mathrm{MHz}, \mathrm{CDCl}_{3}\right) \delta$ 8.15-8.05 $(\mathrm{m}, 1 \mathrm{H}), 7.62(\mathrm{~d}, J=8.4 \mathrm{~Hz}, 2 \mathrm{H}), 7.45-7.40(\mathrm{~m}, 1 \mathrm{H})$, $7.25-7.15(\mathrm{~m}, 4 \mathrm{H}), 6.31(\mathrm{dd}, J=5.5,2.7 \mathrm{~Hz}, 1 \mathrm{H}), 5.78(\mathrm{~d}, J=5.5 \mathrm{~Hz}$, 1H), 3.72-3.62 (m, 1H), $3.50(\mathrm{~d}, J=18.3 \mathrm{~Hz}, 1 \mathrm{H}), 3.16(\mathrm{~d}, J=18.3 \mathrm{~Hz}, 1 \mathrm{H}), 2.35$ (dd, $J=10.0$, $4.8 \mathrm{~Hz}, 1 \mathrm{H}), 2.32(\mathrm{~s}, 2 \mathrm{H}), 1.92(\mathrm{~d}, J=9.9 \mathrm{~Hz}, 1 \mathrm{H}), 1.54(\mathrm{~s}, 9 \mathrm{H}) .{ }^{13} \mathrm{C} \mathrm{NMR}\left(100 \mathrm{MHz}, \mathrm{CDCl}_{3}\right) \delta$ $174.3,144.8,140.4,136.0,135.7,132.1,130.5,130.0,128.4,126.6,124.7,124.0,123.5,117.9$, 114.7, 81.3, 55.6, 44.6, 36.5, 32.4, 28.3, 21.8. HPLC: Chiracel IG column, elute: $n$-hexane/i-propanol $=90 / 10$, detector: $254 \mathrm{~nm}, 30{ }^{\circ} \mathrm{C}$, flow rate: $0.8 \mathrm{~mL} / \mathrm{min}$, retention time 13.4 min (major) and $15.0 \mathrm{~min}$; HRMS (EI-TOF) $\mathrm{m} / \mathrm{z}$ : $[\mathrm{M}]^{+} \mathrm{C}_{26} \mathrm{H}_{27} \mathrm{NO}_{4} \mathrm{~S}$ Calcd for 449.1661; found 449.1665 . 
Morpholino((7R,10S)-5-tosyl-6,10-dihydro-7,10-methanocyclohepta[b]indol-7(5H)-yl)-metha none (3ab): the reaction was conducted at $0.10 \mathrm{mmol}$ scale, $0.0329 \mathrm{~g}, 71 \%$ yield, $87 \%$ ee, (1) unknown compound, white solid, $[\alpha]_{\mathrm{D}}^{20}=-178.79$ (c 0.50, $\left.\mathrm{CHCl}_{3}\right), \mathrm{R}_{f}$ $=0.30$ (petroleum ether/ethyl acetate $2 / 1) ;{ }^{1} \mathrm{H}$ NMR $(400 \mathrm{MHz}$, $\left.\mathrm{CDCl}_{3}\right) \delta 8.18-8.06(\mathrm{~m}, 1 \mathrm{H}), 7.65(\mathrm{~d}, J=8.3 \mathrm{~Hz}, 2 \mathrm{H}), 7.46-7.39(\mathrm{~m}$, $1 \mathrm{H}), 7.25-7.13(\mathrm{~m}, 4 \mathrm{H}), 6.30(\mathrm{dd}, J=5.2,2.6 \mathrm{~Hz}, 1 \mathrm{H}), 5.85(\mathrm{~d}, J=$ $5.5 \mathrm{~Hz}, 1 \mathrm{H}), 3.94-3.58(\mathrm{~m}, 10 \mathrm{H}), 3.15(\mathrm{~d}, J=18.6 \mathrm{~Hz}, 1 \mathrm{H}), 2.47(\mathrm{dd}, J=10.3,4.7 \mathrm{~Hz}, 1 \mathrm{H}), 2.31$ (s, 3H), $2.04(\mathrm{~d}, J=10.4 \mathrm{~Hz}, 1 \mathrm{H}) .{ }^{13} \mathrm{C} \mathrm{NMR}\left(100 \mathrm{MHz}, \mathrm{CDCl}_{3}\right) \delta 172.4,144.9,139.6,135.9$, 135.7, 132.4, 130.3, 130.0, 128.0, 126.5, 124.0, 124.0, 123.5, 117.8, 114.6, 67.0, 66.9, 54.9, 45.0, 36.3, 32.7, 21.7. HPLC: Chiracel AS-H column, elute: $n$-hexane/i-propanol $=80 / 20$, detector: 254 $\mathrm{nm}, 30{ }^{\circ} \mathrm{C}$, flow rate: $0.65 \mathrm{~mL} / \mathrm{min}$, retention time $38.1 \mathrm{~min}$ (major) and $45.4 \mathrm{~min}$; HRMS (EI-TOF) $\mathrm{m} / \mathrm{z}$ : [M] ${ }^{+} \mathrm{C}_{26} \mathrm{H}_{26} \mathrm{~N}_{2} \mathrm{O}_{4} \mathrm{~S}$ Calcd for 462.1613; found 462.1618 .

\section{(7R,10S)- $N, N$-diethyl-5-tosyl-6,10-dihydro-7,10-methanocyclohepta[b]indole-7(5H)-carboxa} mide (3ac): the reaction was conducted at $0.10 \mathrm{mmol}$ scale, $0.0303 \mathrm{~g}, 68 \%$ yield, $81 \%$ ee, (1) unknown compound, white foam solid, $[\alpha]^{20}{ }_{\mathrm{D}}=-140.32\left(\mathrm{c} 0.60, \mathrm{CHCl}_{3}\right)$, $\mathrm{R}_{f}=0.20$ (petroleum ether/ethyl acetate $\left.4 / 1\right) ;{ }^{1} \mathrm{H}$ NMR $(400 \mathrm{MHz}$, $\left.\mathrm{CDCl}_{3}\right) \delta 8.32-7.93(\mathrm{~m}, 1 \mathrm{H}), 7.64(\mathrm{~d}, J=8.2 \mathrm{~Hz}, 2 \mathrm{H}), 7.48-7.37(\mathrm{~m}$, $1 \mathrm{H}), 7.25-7.13(\mathrm{~m}, 4 \mathrm{H}), 6.30(\mathrm{dd}, J=5.2,2.6 \mathrm{~Hz}, 1 \mathrm{H}), 5.92(\mathrm{~d}, J=5.5$ $\mathrm{Hz}, 1 \mathrm{H}), 3.75-3.61(\mathrm{~m}, 3 \mathrm{H}), 3.58-3.34(\mathrm{~m}, 3 \mathrm{H}), 3.21(\mathrm{~d}, J=18.5 \mathrm{~Hz}, 1 \mathrm{H}), 2.49$ (dd, $J=10.3,4.7$ $\mathrm{Hz}, 1 \mathrm{H}), 2.32$ (s, 3H), $2.05(\mathrm{~d}, J=10.3 \mathrm{~Hz}, 1 \mathrm{H}), 1.30-1.15$ (m, 6H) ${ }^{13} \mathrm{C} \mathrm{NMR}\left(100 \mathrm{MHz}, \mathrm{CDCl}_{3}\right)$ $\delta 172.8,144.9,139.5,136.0,135.9,132.5,130.8,130.0,128.2$, 126.6, 124.5, 123.9, 123.5, 117.8, 114.7, 55.6, 45.3, 41.8, 41.2, 36.1, 33.1, 21.7, 14.7, 13.0. HPLC: Chiracel AS-H column, elute: $n$-hexane/i-propanol $=90 / 10$, detector: $254 \mathrm{~nm}, 30{ }^{\circ} \mathrm{C}$, flow rate: $0.65 \mathrm{~mL} / \mathrm{min}$, retention time 22.2 min (major) and 25.6 min; HRMS (EI-TOF) $\mathrm{m} / z$ : $[\mathrm{M}]^{+} \mathrm{C}_{26} \mathrm{H}_{28} \mathrm{~N}_{2} \mathrm{O}_{3} \mathrm{~S}$ Calcd for 448.1821; found 448.1827.

((7R,10S)-5-tosyl-6,10-dihydro-7,10-methanocyclohepta[b]indol-7(5H)-yl)methyl

acetate

(3ad): the reaction was conducted at $0.10 \mathrm{mmol}$ scale, $0.0239 \mathrm{~g}, 57 \%$ yield, $80 \%$ ee, unknown

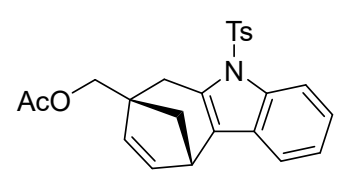
compound, white foam solid, $[\alpha]^{20}{ }_{\mathrm{D}}=-139.99\left(\mathrm{c} 0.48, \mathrm{CHCl}_{3}\right), \mathrm{R}_{f}=0.20$ (petroleum ether/ethyl acetate 9/1); ${ }^{1} \mathrm{H}$ NMR $\left(400 \mathrm{MHz}, \mathrm{CDCl}_{3}\right) \delta$ 8.13-8.03 (m, 1H), $7.60(\mathrm{~d}, J=8.3 \mathrm{~Hz}, 2 \mathrm{H}), 7.48-7.41(\mathrm{~m}, 1 \mathrm{H})$, 7.25-7.12 (m, 4H), $6.30(\mathrm{dd}, J=5.4,2.7 \mathrm{~Hz}, 1 \mathrm{H}), 5.60(\mathrm{~d}, J=5.5 \mathrm{~Hz}$, $1 \mathrm{H}), 4.34(\mathrm{~d}, J=11.1 \mathrm{~Hz}, 1 \mathrm{H}), 4.24(\mathrm{~d}, J=11.1 \mathrm{~Hz}, 1 \mathrm{H}), 3.71-3.62(\mathrm{~m}, 1 \mathrm{H}), 3.16(\mathrm{~d}, J=18.1 \mathrm{~Hz}$, 1H), $2.96(\mathrm{~d}, J=18.1 \mathrm{~Hz}, 1 \mathrm{H}), 2.33(\mathrm{~s}, 3 \mathrm{H}), 2.14$ (s, 3H), 2.05 (dd, $J=9.7,4.6 \mathrm{~Hz}, 1 \mathrm{H}), 1.68$ (d, $J$ $=9.7 \mathrm{~Hz}, 1 \mathrm{H}) \cdot{ }^{13} \mathrm{C} \mathrm{NMR}\left(100 \mathrm{MHz}, \mathrm{CDCl}_{3}\right) \delta 171.5,144.8,140.7,136.1,135.8,132.6,131.2$, 130.0, 128.5, 126.5, 125.2, 123.9, 123.5, 117.9, 114.7, 69.5, 49.0, 44.2, 36.7, 32.4, 21.8, 21.2. HPLC: Chiracel IC column, elute: $n$-hexane/i-propanol $=90 / 10$, detector: $254 \mathrm{~nm}, 30{ }^{\circ} \mathrm{C}$, flow rate: $0.8 \mathrm{~mL} / \mathrm{min}$, retention time $38.0 \mathrm{~min}$ and $51.4 \mathrm{~min}$ (major); HRMS (EI-TOF) $\mathrm{m} / \mathrm{z}$ : $[\mathrm{M}]^{+}$ $\mathrm{C}_{24} \mathrm{H}_{23} \mathrm{NO}_{4} \mathrm{~S}$ Calcd for 421.1348; found 421.1357 . 
(7R,10S)-7-((benzyloxy)methyl)-5-tosyl-5,6,7,10-tetrahydro-7,10-methanocyclohepta[b]indole

(3ae): the reaction was conducted at $0.10 \mathrm{mmol}$ scale, $0.0400 \mathrm{~g}, 85 \%$ yield, $91 \%$ ee, unknown

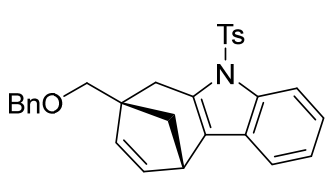
compound, white foam solid, $[\alpha]^{20}{ }_{\mathrm{D}}=-152.52\left(c 0.75, \mathrm{CHCl}_{3}\right), \mathrm{R}_{f}=0.50$ (petroleum ether/ethyl acetate/triethylamine 15/1/1); ${ }^{1} \mathrm{H}$ NMR $(400 \mathrm{MHz}$, $\left.\mathrm{CDCl}_{3}\right) \delta 8.11-8.05(\mathrm{~m}, 1 \mathrm{H}), 7.60(\mathrm{~d}, J=8.3 \mathrm{~Hz}, 2 \mathrm{H}), 7.44-7.36(\mathrm{~m}, 5 \mathrm{H})$, 7.34-7.28 (m, 1H), 7.23-7.18 (m, 2H), 7.13 (d, J=8.1 Hz, 2H), 6.27 (dd, $J=5.4,2.7 \mathrm{~Hz}, 1 \mathrm{H}), 5.64(\mathrm{~d}, J=5.5 \mathrm{~Hz}, 1 \mathrm{H}), 4.65(\mathrm{~d}, J=12.6 \mathrm{~Hz}, 1 \mathrm{H}), 4.62(\mathrm{~d}, J=12.7 \mathrm{~Hz}, 1 \mathrm{H})$, 3.67-3.61 (m, 3H), $3.22(\mathrm{~d}, J=18.2 \mathrm{~Hz}, 1 \mathrm{H}), 2.95(\mathrm{~d}, J=18.2 \mathrm{~Hz}, 1 \mathrm{H}), 2.30(\mathrm{~s}, 3 \mathrm{H}), 2.08(\mathrm{dd}, J=$ 9.7, 4.6 Hz, 1H), $1.68(\mathrm{~d}, J=9.7 \mathrm{~Hz}, 1 \mathrm{H}) .{ }^{13} \mathrm{C}$ NMR $\left(100 \mathrm{MHz}, \mathrm{CDCl}_{3}\right) \delta 144.7,140.0,138.8$, 136.2, 135.7, 133.3, 132.3, 129.9, 128.6, 128.6, 127.7 126.5, 125.3, 123.7, 123.4, 117.8, 114.6, 75.8, 73.6, 50.1, 44. 6, 36.8, 32.6, 21.7. HPLC: Chiracel AD-H column, elute: $n$-hexane/i-propanol $=90 / 10$, detector: $254 \mathrm{~nm}, 30{ }^{\circ} \mathrm{C}$, flow rate: $0.8 \mathrm{~mL} / \mathrm{min}$, retention time $9.1 \mathrm{~min}$ and $10.5 \mathrm{~min}$ (major); HRMS (EI-TOF) m/z: [M] ${ }^{+} \mathrm{C}_{29} \mathrm{H}_{27} \mathrm{NO}_{3} \mathrm{~S}$ Calcd for 469.1712; found 469.1714.

(7R,10S)-7-(((tert-butyldimethylsilyl)oxy)methyl)-5-tosyl-5,6,7,10-tetrahydro-7,10-methanocy clohepta[b]indole (3af): the reaction was conducted at $0.083 \mathrm{mmol}$ scale, $0.0382 \mathrm{~g}, 93 \%$ yield, TBSO $96 \%$ ee, unknown compound, white foam solid, $[\alpha]^{20}{ }_{D}=-169.20(c$ 0.76, $\left.\mathrm{CHCl}_{3}\right), \mathrm{R}_{f}=0.62$ (petroleum ether/ethyl acetate 30/1); ${ }^{1} \mathrm{H}$ NMR (400 $\left.\mathrm{MHz}, \mathrm{CDCl}_{3}\right) \delta 8.18-8.05(\mathrm{~m}, 1 \mathrm{H}), 7.61(\mathrm{~d}, J=8.3 \mathrm{~Hz}, 2 \mathrm{H}), 7.49-7.35$ (m, 1H), 7.25-7.18 (m, 2H), $7.15(\mathrm{~d}, J=8.2 \mathrm{~Hz}, 2 \mathrm{H}), 6.25(\mathrm{dd}, J=5.4$, $2.7 \mathrm{~Hz}, 1 \mathrm{H}), 5.56(\mathrm{~d}, J=5.5 \mathrm{~Hz}, 1 \mathrm{H}), 3.81(\mathrm{~d}, J=9.8 \mathrm{~Hz}, 1 \mathrm{H}), 3.76(\mathrm{~d}, J=9.9 \mathrm{~Hz}, 1 \mathrm{H}), 3.68-3.57$ (m, 1H), 3.23 (d, $J=18.2 \mathrm{~Hz}, 1 \mathrm{H}), 2.85(\mathrm{~d}, J=18.2 \mathrm{~Hz}, 1 \mathrm{H}), 2.32(\mathrm{~s}, 3 \mathrm{H}), 1.98-1.92(\mathrm{~m}, 1 \mathrm{H})$, $1.65(\mathrm{~d}, J=9.6 \mathrm{~Hz}, 1 \mathrm{H}), 0.94(\mathrm{~s}, 9 \mathrm{H}), 0.11(\mathrm{~s}, 3 \mathrm{H}), 0.10(\mathrm{~s}, 3 \mathrm{H}) .{ }^{13} \mathrm{C}$ NMR $\left(100 \mathrm{MHz}, \mathrm{CDCl}_{3}\right) \delta$ 144.6, 139.9, 136.3, 135.9, 133.7, 132.3, 129.9, 128.7, 126.5, 125.3, 123.6, 123.4, 117.8, 114.7, 68.7, 51.5, 44.5, 36.8, 32.1, 26.1, 21.7, 18.5, 5.1, 5.2. HPLC: Chiracel OD-H column, elute: $n$-hexane/i-propanol $=98 / 2$, detector: $254 \mathrm{~nm}, 30{ }^{\circ} \mathrm{C}$, flow rate: $0.6 \mathrm{~mL} / \mathrm{min}$, retention time 7.8 min (major) and $8.4 \mathrm{~min}$; HRMS (EI-TOF) m/z: $[\mathrm{M}]^{+} \mathrm{C}_{28} \mathrm{H}_{35} \mathrm{NO}_{3} \mathrm{SSi}$ Calcd for 493.2107; found 493.2117.

\section{4-(((7R,10S)-5-tosyl-6,10-dihydro-7,10-methanocyclohepta[b]indol-7(5H)-yl)methyl)morphol} ine (3ag): the reaction was conducted at $0.10 \mathrm{mmol}$ scale, $0.0309 \mathrm{~g}, 69 \%$ yield, $91 \%$ ee, unknown

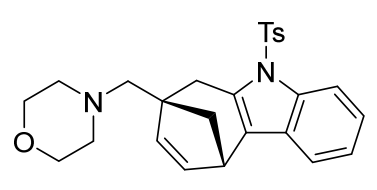
compound, white foam solid, $[\alpha]^{20}{ }_{\mathrm{D}}=-166.99\left(\right.$ c $\left.0.60, \mathrm{CHCl}_{3}\right), \mathrm{R}_{f}=$ 0.13 (petroleum ether/ethyl acetate 10/1); ${ }^{1} \mathrm{H}$ NMR $\left(400 \mathrm{MHz}, \mathrm{CDCl}_{3}\right)$

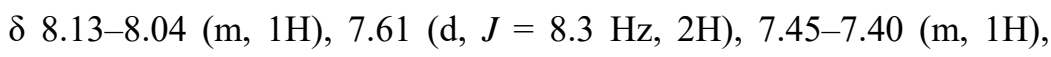
$7.25-7.14(\mathrm{~m}, 4 \mathrm{H}), 6.20(\mathrm{dd}, J=5.4,2.7 \mathrm{~Hz}, 1 \mathrm{H}), 5.53(\mathrm{~d}, J=5.5 \mathrm{~Hz}$, $1 \mathrm{H}), 3.77-3.66(\mathrm{~m}, 4 \mathrm{H}), 3.66-3.58(\mathrm{~m}, 1 \mathrm{H}), 3.25(\mathrm{~d}, J=18.2 \mathrm{~Hz}, 1 \mathrm{H}), 2.84(\mathrm{~d}, J=18.2 \mathrm{~Hz}, 1 \mathrm{H})$, 2.73-2.58 (m, 4H), 2.57-2.48 (m, 2H), $2.33(\mathrm{~s}, 3 \mathrm{H}), 2.11$ (dd, $J=9.6,4.6 \mathrm{~Hz}, 1 \mathrm{H}), 1.63(\mathrm{~d}, J=9.6$ $\mathrm{Hz}, 1 \mathrm{H}) .{ }^{13} \mathrm{C}$ NMR $\left(100 \mathrm{MHz}, \mathrm{CDCl}_{3}\right) \delta 144.7,139.1,136.3,135.8,134.1,133.6,129.9,128.6$, 126.5, 125.3, 123.7, 123.4, 117.8, 114.7, 67.5, 66.0, 55.8, 50.0, 45.7, 37.0, 33.6, 21.8. HPLC: Chiracel AS-H column, elute: $n$-hexane/i-propanol $=90 / 10$, detector: $254 \mathrm{~nm}, 30^{\circ} \mathrm{C}$, flow rate: 0.7 $\mathrm{mL} / \mathrm{min}$, retention time $18.8 \mathrm{~min}$ and $23.8 \mathrm{~min}$ (major); HRMS (EI-TOF) m/z: [M] ${ }^{+} \mathrm{C}_{26} \mathrm{H}_{28} \mathrm{NO}_{3} \mathrm{~S}$ Calcd for 448.1821; found 448.1829 . 
Benzyl 1-((1-tosyl-1H-indol-2-yl)methyl)cyclopent-3-ene-1-carboxylate (5a): the reaction was conducted at $0.10 \mathrm{mmol}$ scale (Table1, entry 1), $0.0076 \mathrm{~g}, 16 \%$ yield. unknown compound, white<smiles>[13CH3]n1c(CC2(C(=O)OCc3ccccc3)CC=CC2)cc2ccccc21</smiles>
foam solid, $\mathrm{R}_{f}=0.14$ (petroleum ether/ethyl acetate 20/1); ${ }^{1} \mathrm{H}$ NMR (400 $\left.\mathrm{MHz}, \mathrm{CDCl}_{3}\right) \delta 8.12(\mathrm{~d}, J=8.4 \mathrm{~Hz}, 1 \mathrm{H}), 7.56(\mathrm{~d}, J=8.4 \mathrm{~Hz}, 2 \mathrm{H})$, $7.31-7.11(\mathrm{~m}, 8 \mathrm{H}), 7.06(\mathrm{~d}, J=8.1 \mathrm{~Hz}, 2 \mathrm{H}), 6.20(\mathrm{~s}, 1 \mathrm{H}), 5.65(\mathrm{~d}, J=8.2$ $\mathrm{Hz}, 1 \mathrm{H}), 5.63(\mathrm{~d}, J=8.2 \mathrm{~Hz}, 1 \mathrm{H}), 5.08(\mathrm{~s}, 2 \mathrm{H}), 3.56(\mathrm{~s}, 2 \mathrm{H}), 3.07$ (d, $J=$ $14.8 \mathrm{~Hz}, 2 \mathrm{H}), 2.56(\mathrm{~d}, J=14.8 \mathrm{~Hz}, 2 \mathrm{H}), 2.28(\mathrm{~s}, 3 \mathrm{H}) .{ }^{13} \mathrm{C} \mathrm{NMR}\left(100 \mathrm{MHz}, \mathrm{CDCl}_{3}\right) \delta 176.9,144.8$, 138.8, 137.0, 136.1, 136.0, 129.9, 128.6, 128.4, 128.2, 128.1, 126.5, 124.3, 123.7, 120.6, 115.0, 109.7, 66.9, 51.8, 43.7, 37.9, 21.7. HRMS (EI-TOF) $\mathrm{m} / \mathrm{z}$ : $[\mathrm{M}]^{+} \mathrm{C}_{29} \mathrm{H}_{27} \mathrm{NO}_{4} \mathrm{~S}$ Calcd for 485.1661; found 485.1659 .

\section{Determination of the absolute configuration of product $3 \mathrm{k}$.}<smiles>O=C(OCc1ccccc1)C12C=CC(CC1)C1c3c(c4ccc(F)cc4n3[13F])C12</smiles>

(S)

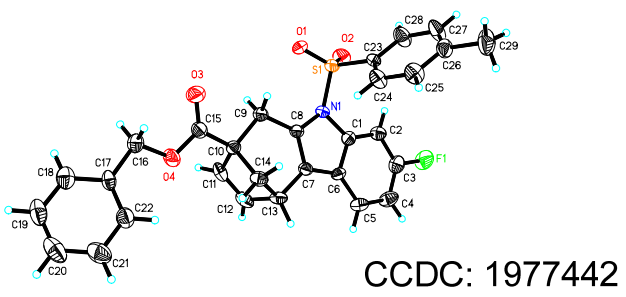

Figure S1. X-ray Single Crystal Structure of 3k

Procedure for recrystallization of (-)-3k: The hexane was slowly added into the solution of (-)-3k in dichloromethane, then the solution was slowly cooled down to $0{ }^{\circ} \mathrm{C}$, and the single crystal of (-)-3k was obtained after one night. The structure showed the absolute configuration of (-)-3k is benzyl (7R,10S)-3-fluoro-5-tosyl-6,10-dihydro-7,10-methanocyclohepta[b]indole-7(5H)carboxylate. The CCDC number is 1977442. These details can be obtained free of charge from the Cambridge Crystallographic Data Centre, whose website is https://www.ccdc.cam.ac.uk/structures/.

Thermal Ellipsoid Plot for (7R,10S)-(-)-3k (30\% probability level)

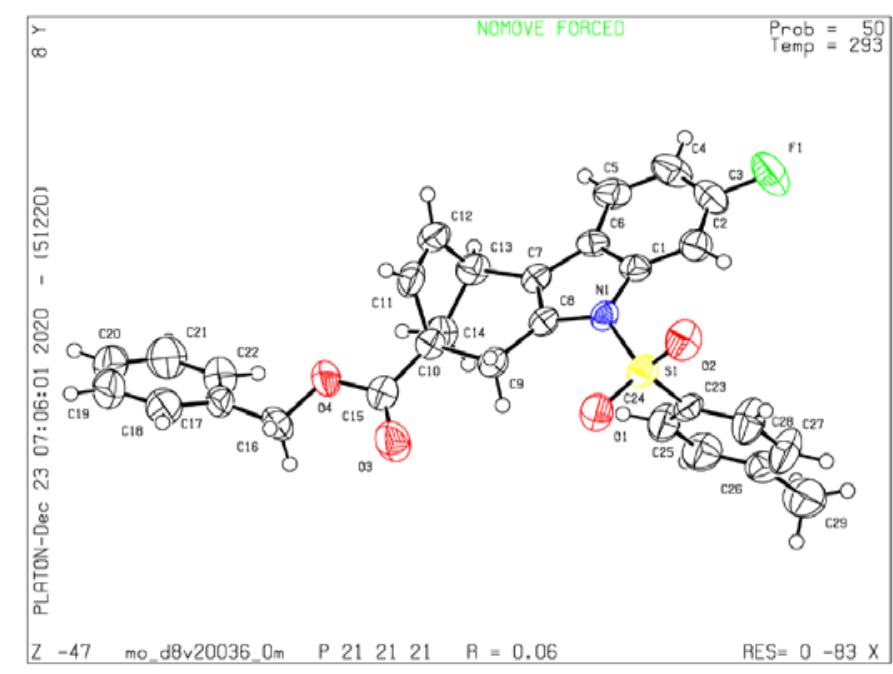


Crystal data and structure refinement for mo_d8v20036_0m [(7R,10S)-(-)-3k].

Identification code

Empirical formula

Formula weight

Temperature

Wavelength

Crystal system

Space group

Unit cell dimensions

Volume

Z

Density (calculated)

Absorption coefficient

$\mathrm{F}(000)$

Crystal size

Theta range for data collection

Index ranges

Reflections collected

Independent reflections

Completeness to theta $=25.242^{\circ}$

Absorption correction

Max. and min. transmission

Refinement method

Data / restraints / parameters

Goodness-of-fit on $\mathrm{F}^{2}$

Final $\mathrm{R}$ indices [I $>2 \operatorname{sigma}(\mathrm{I})]$

$\mathrm{R}$ indices (all data)

Absolute structure parameter

Extinction coefficient

Largest diff. peak and hole mo_d8v20036_0m

C29 H24 F N O4 S

501.55

293(2) K

$0.71073 \AA$

Orthorhombic

P 212121

$\mathrm{a}=9.4567(17) \AA$

$\alpha=90^{\circ}$.

$\mathrm{b}=11.542(2) \AA$

$\beta=90^{\circ}$.

$\mathrm{c}=22.277(4) \AA$

$\gamma=90^{\circ}$.

2431.4(8) $\AA^{3}$

4

$1.370 \mathrm{Mg} / \mathrm{m}^{3}$

$0.178 \mathrm{~mm}^{-1}$

1048

$0.150 \times 0.120 \times 0.060 \mathrm{~mm}^{3}$

2.340 to $25.494^{\circ}$.

$-11<=\mathrm{h}<=11,-13<=\mathrm{k}<=12,-23<=\mathrm{l}<=26$

11600

$4496[\mathrm{R}($ int $)=0.0771]$

$99.9 \%$

Semi-empirical from equivalents

0.7456 and 0.6153

Full-matrix least-squares on $\mathrm{F}^{2}$

4496 / 0 / 327

0.996

$\mathrm{R} 1=0.0553, \mathrm{wR} 2=0.0835$

$\mathrm{R} 1=0.1354, \mathrm{wR} 2=0.1093$

$-0.07(10)$

$0.0054(8)$

0.193 and -0.188 e. $\AA^{-3}$ 


\section{Control experiments.}

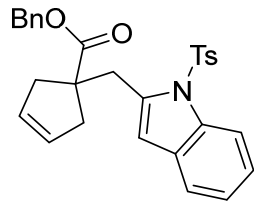

$5 a$

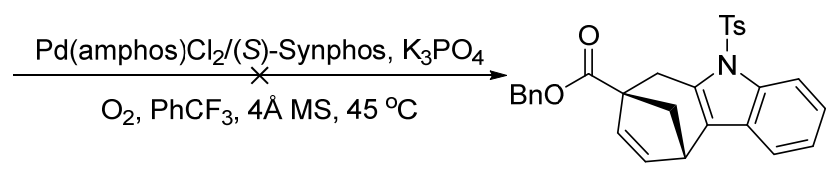

3a

In the glovebox, a Schlenk tube was charged with $5 \mathrm{a}(48.6 \mathrm{mg}, 0.10 \mathrm{mmol}), \mathrm{Pd}(\mathrm{amphos}) \mathrm{Cl}_{2}$ (7.1 mg, $0.01 \mathrm{mmol}),(S)$-Synphos (7.0 mg, $0.011 \mathrm{mmol}), \mathrm{K}_{3} \mathrm{PO}_{4}(31.8 \mathrm{mg}, 0.15 \mathrm{mmol})$, and $4 \AA$ MS $(100 \mathrm{mg})$ in the $\mathrm{PhCF}_{3}(2.0 \mathrm{~mL})$. Then, the Schlenk tube was removed from glovebox and the nitrogen atmosphere was replaced with the oxygen using an oxygen balloon. The mixture was stirred in $45^{\circ} \mathrm{C}$ oil bath. After 48 hours, no target product is observed.

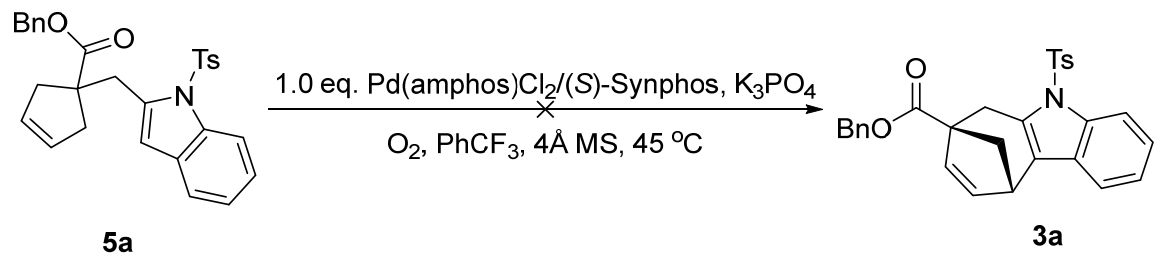

In the glovebox, a Schlenk tube was charged with 5a (48.6 mg, $0.10 \mathrm{mmol}), \mathrm{Pd}(\mathrm{amphos}) \mathrm{Cl}_{2}$ (71.0 mg, $0.10 \mathrm{mmol}),(S)-S y n p h o s(0.0700 \mathrm{~g}, 0.11 \mathrm{mmol}), \mathrm{K}_{3} \mathrm{PO}_{4}(31.8 \mathrm{mg}, 0.15 \mathrm{mmol})$, and $4 \AA$ MS $(100 \mathrm{mg})$ in the $\mathrm{PhCF}_{3}(2.0 \mathrm{~mL})$. Then, the Schlenk tube was removed from glovebox and the nitrogen atmosphere was replaced with the oxygen using an oxygen balloon. The mixture was stirred in $45^{\circ} \mathrm{C}$ oil bath. After 48 hours, no target product is observed.

\section{Scale-up experiment for enantioselective synthesis of Indole-Fused Bicyclo[3.2.1]octane 3i.}<smiles>Cc1ccc(C#CCC2(C(=O)OCc3ccccc3)CC=CC2)c(N[As])c1</smiles>

4i: $0.500 \mathrm{~g}, 1.0 \mathrm{mmol}$<smiles>Cc1ccc2c(c1)c1c(n2[13CH3])C[C@@]2(C(=O)OCc3ccccc3)C=C[C@@H]1C2</smiles>

3i: $0.353 \mathrm{~g}, 71 \%$ yield, $92 \%$ ee

In the glovebox, a Schlenk tube was charged with $4 \mathbf{i}(0.5000 \mathrm{~g}, 1.0 \mathrm{mmol}), \mathrm{Pd}(\mathrm{amphos}) \mathrm{Cl}_{2}$ (0.0710 g, $0.10 \mathrm{mmol}),(S)$-Synphos (0.0700 g, $0.11 \mathrm{mmol}), \mathrm{K}_{3} \mathrm{PO}_{4}(0.3180 \mathrm{~g}, 1.50 \mathrm{mmol})$, and $4 \AA$ MS $(1.0000 \mathrm{~g})$ in the $\mathrm{PhCF}_{3}(20.0 \mathrm{~mL})$. Then, the Schlenk tube was removed from glovebox and the nitrogen atmosphere was replaced with the oxygen using an oxygen balloon. The mixture was stirred in $45{ }^{\circ} \mathrm{C}$ oil bath. After completion of the reaction (monitored by TLC), the resulting mixture was concentrated under reduced pressure. The resulting crude material was purified by flash column chromatography to afford the desired product $\mathbf{3 i}$ as a white foam solid $(0.3530 \mathrm{~g}$, $71 \%$ yield, $92 \%$ ee). 


\section{Further synthetic transformations of product $3 i$}

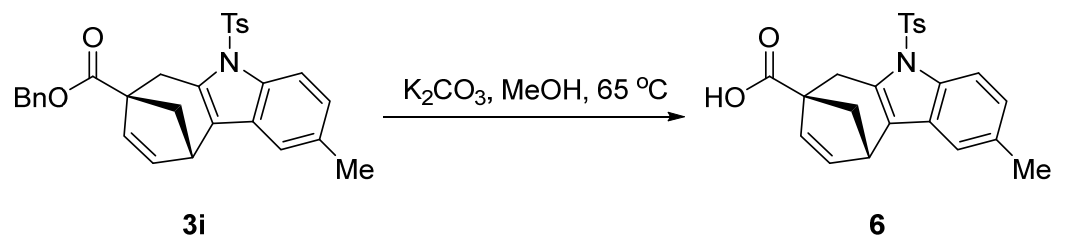

Under nitrogen atmosphere, the mixture of $3 \mathbf{i}(50.0 \mathrm{mg}, 0.1 \mathrm{mmol})$ and $\mathrm{K}_{2} \mathrm{CO}_{3}(55.3 \mathrm{mg}, 0.4$ $\mathrm{mmol})$ in the methanol $(2.0 \mathrm{~mL})$ was stirred overnight in $65{ }^{\circ} \mathrm{C}$ oil bath. After completion of the reaction (monitored by TLC), the reaction was cooled to room temperature and quenched by $1 \mathrm{M}$ $\mathrm{HCl}$, then extracted with ethyl acetate $(3 \times 10 \mathrm{~mL})$. The combined organic layers were dried over sodium sulfate, filtered and concentrated under reduced pressure. The resulting crude material was purified by flash column chromatography (petroleum ether/ethyl acetate $=3 / 1$ ) to afford the desired compound as a white solid $\left(37.1 \mathrm{mg}, 94 \%\right.$ yield, $93 \%$ ee). Unknown compound, $[\alpha]^{20}{ }_{D}=$ -188.09 ( c $\left.0.58, \mathrm{CHCl}_{3}\right), \mathrm{R}_{f}=0.20$ (petroleum ether/ethyl acetate 4/1). ${ }^{1} \mathrm{H} \mathrm{NMR}\left(400 \mathrm{MHz}, \mathrm{CDCl}_{3}\right)$ $\delta 7.97(\mathrm{~d}, J=8.5 \mathrm{~Hz}, 1 \mathrm{H}), 7.62(\mathrm{~d}, J=8.3 \mathrm{~Hz}, 2 \mathrm{H}), 7.22(\mathrm{~s}, 1 \mathrm{H}), 7.18(\mathrm{~d}, J=8.2 \mathrm{~Hz}, 2 \mathrm{H}), 7.07(\mathrm{~d}$, $J=8.5 \mathrm{~Hz}, 1 \mathrm{H}), 6.38(\mathrm{dd}, J=5.3,2.7 \mathrm{~Hz}, 1 \mathrm{H}), 5.83(\mathrm{~d}, J=5.5 \mathrm{~Hz}, 1 \mathrm{H}), 3.75-3.67(\mathrm{~m}, 1 \mathrm{H}), 3.59$ (d, $J=18.2 \mathrm{~Hz}, 1 \mathrm{H}), 3.23(\mathrm{~d}, J=18.2 \mathrm{~Hz}, 1 \mathrm{H}), 2.47$ (dd, $J=9.8,4.6 \mathrm{~Hz}, 1 \mathrm{H}), 2.41(\mathrm{~s}, 3 \mathrm{H}), 2.32(\mathrm{~s}$, $3 \mathrm{H}), 2.01(\mathrm{~d}, J=9.9 \mathrm{~Hz}, 1 \mathrm{H}) .{ }^{13} \mathrm{C} \mathrm{NMR}\left(100 \mathrm{MHz}, \mathrm{CDCl}_{3}\right) \delta 181.0,144.8,141.3,136.1,134.0$, 133.2, 131.6, 130.0, 129.5, 128.5, 126.5, 125.5, 124.3, 117.9, 114.5, 54.6, 44.7, 36.7, 32.2, 21.8, 21.5. HPLC: Chiracel IG column, elute: $n$-hexane/i-propanol $=80 / 20$, detector: $254 \mathrm{~nm}, 30{ }^{\circ} \mathrm{C}$, flow rate: $0.7 \mathrm{~mL} / \mathrm{min}$, retention time $34.1 \mathrm{~min}$ (major) and $52.4 \mathrm{~min}$; HRMS (EI-TOF) $\mathrm{m} / \mathrm{z}:[\mathrm{M}]^{+}$ $\mathrm{C}_{23} \mathrm{H}_{21} \mathrm{NO}_{4} \mathrm{~S}$ Calcd for 407.1191; found 407.1200.

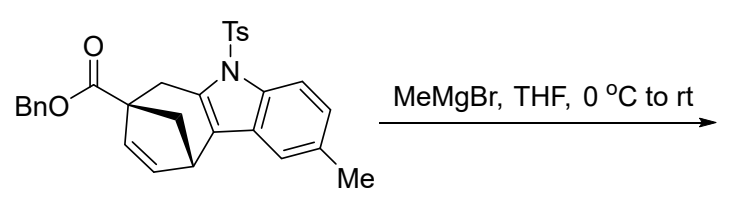

$3 \mathbf{i}$

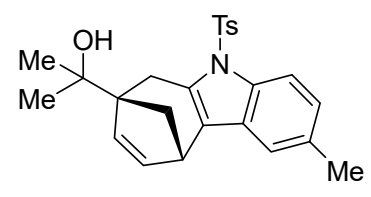

7

In a nitrogen-filled Schlenk tube, to a solution of the $3 \mathbf{i}(50.0 \mathrm{mg}, 0.1 \mathrm{mmol})$ in THF $(2.0 \mathrm{~mL})$ was added dropwise a solution of $\mathrm{MeMgBr}$ in diethyl ether $(3.0 \mathrm{M}, 0.13 \mathrm{~mL}, 0.4 \mathrm{mmol})$ at $0{ }^{\circ} \mathrm{C}$. After the addition, the mixture was warmed to room temperature and stirred for overnight. The reaction was quenched by aq. $\mathrm{NH}_{4} \mathrm{Cl}(5.0 \mathrm{~mL})$ and extracted with ethyl acetate $(3 \times 10 \mathrm{~mL})$. The combined organic layers were dried over sodium sulfate, filtered and concentrated under reduced pressure. The resulting crude material was purified by flash column chromatography (petroleum ether/ethyl acetate $=10 / 1)$ to afford the desired compound as a white foam solid $(37.7 \mathrm{mg}, 89 \%$ yield, $92 \%$ ee). Unknown compound, $[\alpha]^{20}{ }_{\mathrm{D}}=-158.77$ (c $\left.0.82, \mathrm{CHCl}_{3}\right), \mathrm{R}_{f}=0.35$ (petroleum ether/ethyl acetate 4/1). ${ }^{1} \mathrm{H}$ NMR $\left(400 \mathrm{MHz}, \mathrm{CDCl}_{3}\right) \delta 7.94(\mathrm{~d}, J=8.5 \mathrm{~Hz}, 1 \mathrm{H}), 7.57$ (d, $J=8.2$ $\mathrm{Hz}, 2 \mathrm{H}), 7.21(\mathrm{~s}, 1 \mathrm{H}), 7.15$ (d, $J=8.1 \mathrm{~Hz}, 2 \mathrm{H}), 7.03$ (d, $J=8.4 \mathrm{~Hz}, 1 \mathrm{H}), 6.25$ (dd, $J=5.1,2.3 \mathrm{~Hz}$, $1 \mathrm{H}), 5.67(\mathrm{~d}, J=5.5 \mathrm{~Hz}, 1 \mathrm{H}), 3.66-3.61(\mathrm{~m}, 1 \mathrm{H}), 3.25(\mathrm{~d}, J=18.1 \mathrm{~Hz}, 1 \mathrm{H}), 2.88(\mathrm{~d}, J=18.1 \mathrm{~Hz}$, $1 \mathrm{H}), 2.40(\mathrm{~s}, 3 \mathrm{H}), 2.30(\mathrm{~s}, 3 \mathrm{H}), 2.18(\mathrm{dd}, J=9.6,4.7 \mathrm{~Hz}, 1 \mathrm{H}), 1.59(\mathrm{~d}, J=9.7 \mathrm{~Hz}, 1 \mathrm{H}), 1.38(\mathrm{~s}$, $3 \mathrm{H}), 1.35(\mathrm{~s}, 3 \mathrm{H}) .{ }^{13} \mathrm{C}$ NMR $\left(100 \mathrm{MHz}, \mathrm{CDCl}_{3}\right) \delta 144.6,140.1,136.1,134.1,134.0,133.1,131.0$, 129.9, 128.8, 126.4, 125.4, 125.1, 117.9, 114.4, 73.1, 57.3, 41.7, 36.8, 30.8, 26.6, 25.8, 21.7, 21.5. HPLC: Chiracel IG column, elute: $n$-hexane/i-propanol $=80 / 20$, detector: $254 \mathrm{~nm}, 30{ }^{\circ} \mathrm{C}$, flow 
rate: $0.7 \mathrm{~mL} / \mathrm{min}$, retention time $18.8 \mathrm{~min}$ and $23.3 \mathrm{~min}$ (major); HRMS (EI-TOF) $\mathrm{m} / \mathrm{z}:[\mathrm{M}]^{+}$ $\mathrm{C}_{25} \mathrm{H}_{27} \mathrm{NO}_{3} \mathrm{~S}$ Calcd for 421.1712; found 421.1715.

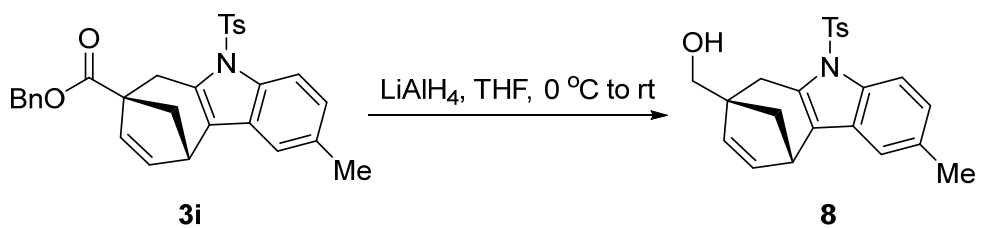

In a nitrogen-filled Schlenk tube, the $\mathrm{LiAlH}_{4}(5.7 \mathrm{mg}, 0.15 \mathrm{mmol})$ was added slowly to a solution of the $3 \mathbf{i}(50.0 \mathrm{mg}, 0.1 \mathrm{mmol})$ in THF $(2.0 \mathrm{~mL})$ at $0{ }^{\circ} \mathrm{C}$. The mixture was warmed to room temperature and stirred for 2 hours. Subsequently, the reaction was quenched by a saturated aqueous solution of sodium tartrate $(5.0 \mathrm{~mL})$ and extracted with ethyl acetate $(4 \times 10 \mathrm{~mL})$. The combined organic layers were dried over sodium sulfate, filtered and concentrated under reduced pressure. The resulting crude material was purified by flash column chromatography (petroleum ether/ethyl acetate $=4 / 1)$ to afford the desired compound as a white foam solid $(35.0 \mathrm{mg}, 92 \%$ yield, $92 \%$ ee). Unknown compound, $[\alpha]^{20}{ }_{\mathrm{D}}=-136.61\left(\mathrm{c} 0.74, \mathrm{CHCl}_{3}\right), \mathrm{R}_{f}=0.2$ (petroleum ether/ethyl acetate 4/1). ${ }^{1} \mathrm{H}$ NMR $\left(400 \mathrm{MHz}, \mathrm{CDCl}_{3}\right) \delta 7.93(\mathrm{~d}, J=8.5 \mathrm{~Hz}, 1 \mathrm{H}), 7.58(\mathrm{~d}, 8.4 \mathrm{~Hz}$, 2H), $7.22(\mathrm{~s}, 1 \mathrm{H}), 7.15(\mathrm{~d}, J=8.0 \mathrm{~Hz}, 2 \mathrm{H}), 7.06-7.00(\mathrm{~m}, 1 \mathrm{H}), 6.30(\mathrm{dd}, J=5.5,2.7 \mathrm{~Hz}, 1 \mathrm{H}), 5.62$ $(\mathrm{d}, J=5.5 \mathrm{~Hz}, 1 \mathrm{H}), 3.90$ (d, $J=10.8 \mathrm{~Hz}, 1 \mathrm{H}), 3.81(\mathrm{~d}, J=10.9 \mathrm{~Hz}, 1 \mathrm{H}), 3.68-3.62$ (dd, $J=4.3$, $2.9 \mathrm{~Hz}, 1 \mathrm{H}), 3.15$ (d, $J=18.1 \mathrm{~Hz}, 1 \mathrm{H}), 2.90$ (d, $J=18.1 \mathrm{~Hz}, 1 \mathrm{H}), 2.40$ (s, 3H), 2.30 (s, 3H), 2.09 $(\mathrm{dd}, J=9.6,4.6 \mathrm{~Hz}, 1 \mathrm{H}), 1.64(\mathrm{~d}, J=9.6 \mathrm{~Hz}, 1 \mathrm{H}) .{ }^{13} \mathrm{C} \mathrm{NMR}\left(100 \mathrm{MHz}, \mathrm{CDCl}_{3}\right) \delta 144.6,140.9$, 136.1, 134.0, 133.1, 133.1, 131.6, 129.9, 128.8, 126.4, 125.3, 125.1, 117.9, 114.4, 68.5, 51.4, 43.8, 36.9, 32.2, 21.7, 21.5. HPLC: Chiracel IG column, elute: $n$-hexane/i-propanol $=80 / 20$, detector: $254 \mathrm{~nm}, 30{ }^{\circ} \mathrm{C}$, flow rate: $0.7 \mathrm{~mL} / \mathrm{min}$, retention time $21.0 \mathrm{~min}$ and $23.1 \mathrm{~min}$ (major); HRMS (EI-TOF) $\mathrm{m} / z$ : $[\mathrm{M}]^{+} \mathrm{C}_{23} \mathrm{H}_{23} \mathrm{NO}_{3} \mathrm{~S}$ 393.1399; found 393.1396.

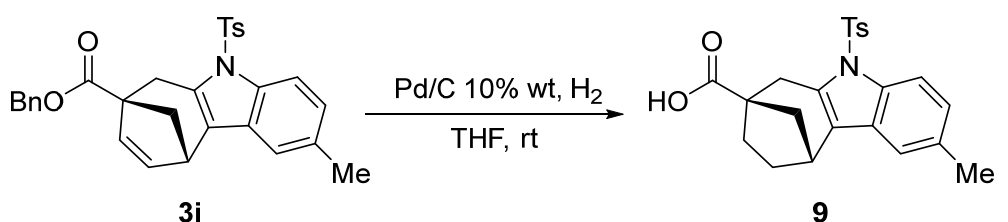

Under hydrogen atmosphere, the mixture of $3 \mathbf{i}(50.0 \mathrm{mg}, 0.1 \mathrm{mmol})$ and $10 \% \mathrm{Pd} / \mathrm{C}(42.5 \mathrm{mg}$, $0.4 \mathrm{mmol})$ in THF $(2.0 \mathrm{~mL})$ was stirred at room temperature for $12 \mathrm{~h}$. Subsequently, the reaction was concentrated under reduced pressure. The resulting crude material was purified by flash column chromatography (petroleum ether/ethyl acetate $=1 / 1$ ) to afford the desired compound as a white solid (38.1 mg, 96\% yield, 92\% ee). Unknown compound, $[\alpha]^{20}{ }_{\mathrm{D}}=-37.27$ (c 0.77, $\mathrm{CHCl}_{3}$ ), $\mathrm{R}_{f}=0.20$ (petroleum ether/ethyl acetate 1/1); ${ }^{1} \mathrm{H}$ NMR $\left(400 \mathrm{MHz}, \mathrm{CDCl}_{3}\right) \delta 8.00(\mathrm{~d}, J=8.5 \mathrm{~Hz}$, 1H), $7.65(\mathrm{~d}, J=8.3 \mathrm{~Hz}, 2 \mathrm{H}), 7.22-7.16(\mathrm{~m}, 3 \mathrm{H}), 7.09$ (d, $J=8.5 \mathrm{~Hz}, 1 \mathrm{H}), 3.49(\mathrm{~d}, J=17.6 \mathrm{~Hz}$, 1H), 3.35-3.24 (m, 2H), 2.42 (s, 3H), 2.39-2.29 (m, 4H), 2.26-2.15 (m, 1H), 2.10-1.99 (m, 1H), 1.94-1.78 (m, 2H), 1.76-1.61 (m, 1H). ${ }^{13} \mathrm{C}$ NMR (100 MHz, $\left.\mathrm{CDCl}_{3}\right) \delta 183.5,144.8,136.1,135.0$, 133.2, 131.8, 130.0, 128.6, 126.5, 125.6, 125.4, 118.0, 114.4, 49.6, 40.0, 38.2, 35.7, 34.5, 33.7, 21.8, 21.5. HPLC: Chiracel IG column, elute: $n$-hexane/i-propanol $=70 / 30$, detector: $254 \mathrm{~nm}, 30$ ${ }^{\circ} \mathrm{C}$, flow rate: $0.7 \mathrm{~mL} / \mathrm{min}$, retention time $17.7 \mathrm{~min}$ and $21.3 \mathrm{~min}$ (major); HRMS (EI-TOF) $\mathrm{m} / \mathrm{z}$ : $[\mathrm{M}]^{+} \mathrm{C}_{23} \mathrm{H}_{23} \mathrm{NO}_{4} \mathrm{~S}$ Calcd for 409.1348; found 409.1350. 


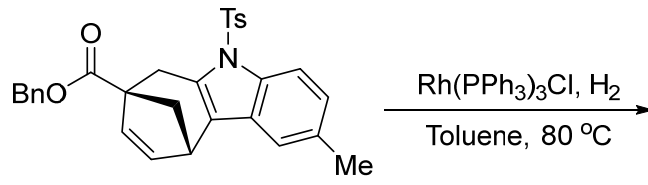

$3 \mathbf{i}$

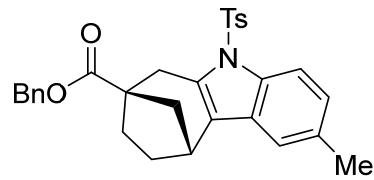

10

Under hydrogen atmosphere, the mixture of $3 \mathbf{i}(50.0 \mathrm{mg}, 0.1 \mathrm{mmol}), \mathrm{Rh}\left(\mathrm{PPh}_{3}\right)_{3} \mathrm{Cl}(4.7 \mathrm{mg}$, $0.005 \mathrm{mmol})$ and toluene $(2.0 \mathrm{~mL})$ was stirred in $80{ }^{\circ} \mathrm{C}$ oil bath for $14 \mathrm{~h}$. Then the reaction was concentrated under reduced pressure. The resulting crude material was purified by flash column chromatography (petroleum ether/ethyl acetate $=10 / 1$ ) to afford the desired compound as a white foam solid (23.7 mg, 95\% yield, 92\% ee). Unknown compound, $[\alpha]^{20}{ }_{\mathrm{D}}=-36.56$ (c 0.51, $\mathrm{CHCl}_{3}$ ), $\mathrm{R}_{f}=0.35$ (petroleum ether/ethyl acetate 10/1). ${ }^{1} \mathrm{H}$ NMR $\left(400 \mathrm{MHz}, \mathrm{CDCl}_{3}\right) \delta 7.98(\mathrm{~d}, J=8.5 \mathrm{~Hz}$, $1 \mathrm{H}), 7.61(\mathrm{~d}, J=8.3 \mathrm{~Hz}, 2 \mathrm{H}), 7.48-7.29(\mathrm{~m}, 5 \mathrm{H}), 7.19-7.12(\mathrm{~m}, 3 \mathrm{H}), 7.10-7.04(\mathrm{~m}, 1 \mathrm{H}), 5.25(\mathrm{~d}$, $J=12.5 \mathrm{~Hz}, 1 \mathrm{H}), 5.21(\mathrm{~d}, J=12.6 \mathrm{~Hz}, 1 \mathrm{H}), 3.45(\mathrm{~d}, J=17.6 \mathrm{~Hz}, 1 \mathrm{H}), 3.30(\mathrm{~d}, J=17.6 \mathrm{~Hz}, 1 \mathrm{H})$, 3.27-3.23 (m, 1H), $2.40(\mathrm{~s}, 3 \mathrm{H}), 2.32(\mathrm{~s}, 3 \mathrm{H}), 2.29-2.23(\mathrm{~m}, 1 \mathrm{H}), 2.21-2.14(\mathrm{~m}, 1 \mathrm{H}), 2.05-1.93(\mathrm{~m}$, $1 \mathrm{H}), 1.88(\mathrm{~d}, J=11.8 \mathrm{~Hz}, 1 \mathrm{H}), 1.86-1.78(\mathrm{~m}, 1 \mathrm{H}), 1.70-1.60(\mathrm{~m}, 1 \mathrm{H}) .{ }^{13} \mathrm{C}$ NMR $(100 \mathrm{MHz}$, $\left.\mathrm{CDCl}_{3}\right) \delta 176.7,144.7,136.4,136.2,135.1,133.2,132.1,132.0,130.0,128.8,128.6,128.4,128.1$, 126.5, 125.5, 118.0, 114.4, 66.7, 49.8, 39.9, 38.5, 35.6, 34.3, 33.5, 21.7, 21.5. HPLC: Chiracel IG column, elute: $n$-hexane/i-propanol $=80 / 20$, detector: $254 \mathrm{~nm}, 30{ }^{\circ} \mathrm{C}$, flow rate: $0.7 \mathrm{~mL} / \mathrm{min}$, retention time $33.4 \mathrm{~min}$ (major) and $35.5 \mathrm{~min}$; HRMS (EI-TOF) m/z: [M] ${ }^{+} \mathrm{C}_{30} \mathrm{H}_{29} \mathrm{NO}_{4} \mathrm{~S}$ Calcd for 499.1817; found 499.1825 .

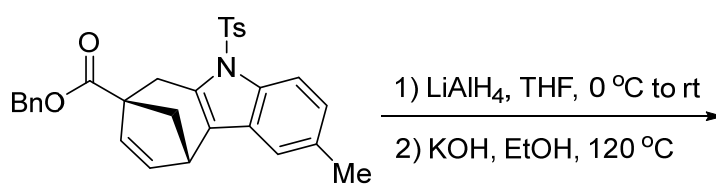

$3 \mathbf{i}$

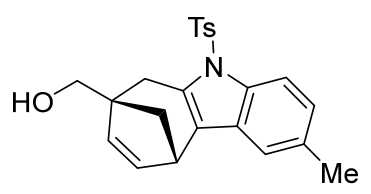

11

In a nitrogen-filled Schlenk tube, the $\mathrm{LiAlH}_{4}(3.1 \mathrm{mg}, 0.083 \mathrm{mmol})$ was added slowly to a solution of the $3 \mathbf{i}(27.5 \mathrm{mg}, 0.055 \mathrm{mmol})$ in THF $(1.0 \mathrm{~mL})$ at $0{ }^{\circ} \mathrm{C}$. The mixture was warmed to room temperature and stirred for 2 hours. Subsequently, the reaction was quenched by a saturated aqueous solution of sodium tartrate $(5.0 \mathrm{~mL})$ and extracted with ethyl acetate $(4 \times 10 \mathrm{~mL})$. The combined organic layers were dried over sodium sulfate, filtered and concentrated under reduced pressure. The resulting crude material was used directly for next step.

Under nitrogen atmosphere, crude material and $\mathrm{KOH}(30.0 \mathrm{mg}, 0.53 \mathrm{mmol})$ in ethanol $(2.0 \mathrm{~mL})$ was stirred overnight in $120{ }^{\circ} \mathrm{C}$ oil bath. After completion of the reaction (monitored by TLC), the reaction was cooled to room temperature and quenched by water, then extracted with ether $(3 \times 10$ $\mathrm{mL}$ ). The combined organic layers were dried over sodium sulfate, filtered and concentrated under reduced pressure. The resulting crude material was purified by flash column chromatography (petroleum ether/ethyl acetate $=3 / 1)$ to afford the desired compound as a white foam solid $(8.7 \mathrm{mg}$, $63 \%$ yield for two steps, $92 \%$ ee). Unknown compound, $[\alpha]^{20}{ }_{\mathrm{D}}=-461.65$ (c 0.17 , Ether), $\mathrm{R}_{f}=0.25$ (petroleum ether/ethyl acetate 3/1). ${ }^{1} \mathrm{H}$ NMR $\left(400 \mathrm{MHz}, \mathrm{CDCl}_{3}\right) \delta 7.62($ br s, $1 \mathrm{H}), 7.32(\mathrm{~s}, 1 \mathrm{H})$, $7.15(\mathrm{~d}, J=8.2 \mathrm{~Hz}, 1 \mathrm{H}), 6.93-6.86(\mathrm{~m}, 1 \mathrm{H}), 6.44(\mathrm{dd}, J=5.4,2.7 \mathrm{~Hz}, 1 \mathrm{H}), 5.55(\mathrm{~d}, J=5.5 \mathrm{~Hz}$, 1H), 3.89-3.75 (m, 3H), 2.91 (d, $J=16.5 \mathrm{~Hz}, 1 \mathrm{H}), 2.55$ (d, $J=16.5 \mathrm{~Hz}, 1 \mathrm{H}), 2.44(\mathrm{~s}, 3 \mathrm{H}), 2.12$ $(\mathrm{dd}, J=9.5,4.8 \mathrm{~Hz}, 1 \mathrm{H}), 1.76(\mathrm{~d}, J=9.4 \mathrm{~Hz}, 1 \mathrm{H}) .{ }^{13} \mathrm{C} \mathrm{NMR}\left(100 \mathrm{MHz}, \mathrm{CDCl}_{3}\right) \delta 142.8,133.4$, 
132.0, 129.9, 128.6, 126.0, 122.2, 117.2, 116.1, 110.6, 68.9, 51.5, 45.2, 37.3, 30.0, 21.7. HPLC: Chiracel IC column, elute: $n$-hexane/i-propanol $=90 / 10$, detector: $254 \mathrm{~nm}, 30{ }^{\circ} \mathrm{C}$, flow rate: 0.8 $\mathrm{mL} / \mathrm{min}$, retention time $9.7 \mathrm{~min}$ and $10.7 \mathrm{~min}$ (major); HRMS (EI-TOF) $\mathrm{m} / \mathrm{z}$ : $[\mathrm{M}]^{+} \mathrm{C}_{16} \mathrm{H}_{17} \mathrm{NO}$ Calcd for 239.1310; found 239.1311.

\section{References}

1. Tamanini, E.; Watkinson, M.; Todd, M. H. Improved synthesis of the valuable peptidomimetic intermediate 3-azido-4-hydroxy cyclopentanoic acid. Tetrahedron: Asymmetry 2006, 17, 2235-2239.

2. Liu, S.; Zhou, J. Desymmetrization of cyclic olefins via asymmetric Heck reaction and hydroarylation. Chem. Commun. 2013, 49, 11758-11760.

3. Stafford, J. A.; Gonzales, S. S.; Barrett, D. G.; Suh, E. M.; Feldman, P. L. Degradative Rearrangements of $\mathrm{N}$-(t-Butyloxycarbonyl)-O-methanesulfonyl-hydroxamic Acids: A Novel, Reagent-Based Alternative to the Lossen Rearrangement. J. Org. Chem. 1998, 63, 10040-10044.

4. Gui, Q.; Wang, J.; Ng, S.; Dancevic, A.; Wright, T. B.; Evans, P. A. Copper-catalyzed desymmetrization of prochiral 4,4-disubstituted cyclopentenes via a site-selective allylic oxidation: a concise total synthesis of untenone A. Chem. Commun. 2019, 55, 12368-12371.

5. Mohamed, A.; Mohamed, S.; Sekiguchi, A.; Masui, K.; Mori, A. Aqueous Ammonia as a New Activator for Sonogashira Coupling. Bull. Chem. Soc. Jpn. 2005, 78, 160-168.

6. Jang, S. S.; Youn, S. W. The cooperative $\mathrm{FeCl}_{3} / \mathrm{DDQ}$ system for the regioselective synthesis of 3-arylindoles from $\beta$-monosubstituted 2-alkenylanilines. Org. Biom. Chem. 2016, 14, 2200-2204.

7. Akhtar, W. M.; Armstrong, R. J.; Frost, J. R.; Stevenson, N. G.; Donohoe, T. J. Stereoselective Synthesis of Cyclohexanes via an Iridium Catalyzed $(5+1)$ Annulation Strategy. J. Am. Chem. Soc. 2018, 140, 11916-11920.

8. Balduzzi, S.; Brook, M. A. Alkoxyallylsilanes: Functional Protecting Groups. Tetrahedron 2000, 56, 1617-1622.

9. Chakraborti, A. K.; Chankeshwara, S. V. Counterattack Mode Differential Acetylative Deprotection of Phenylmethyl Ethers: Applications to Solid Phase Organic Reactions. J. Org. Chem. 2009, 74, 1367-1370.

10. Malik, H.; Kohlen, W.; Jamil, M.; Rutjes, F. P. J. T.; Zwanenburg, B. Aromatic A-ring analogues of orobanchol, new germination stimulants for seeds of parasitic weeds. Org. Biomol. Chem. 2011, 9, 2286-2293. 
10. Copy of NMR and HPLC
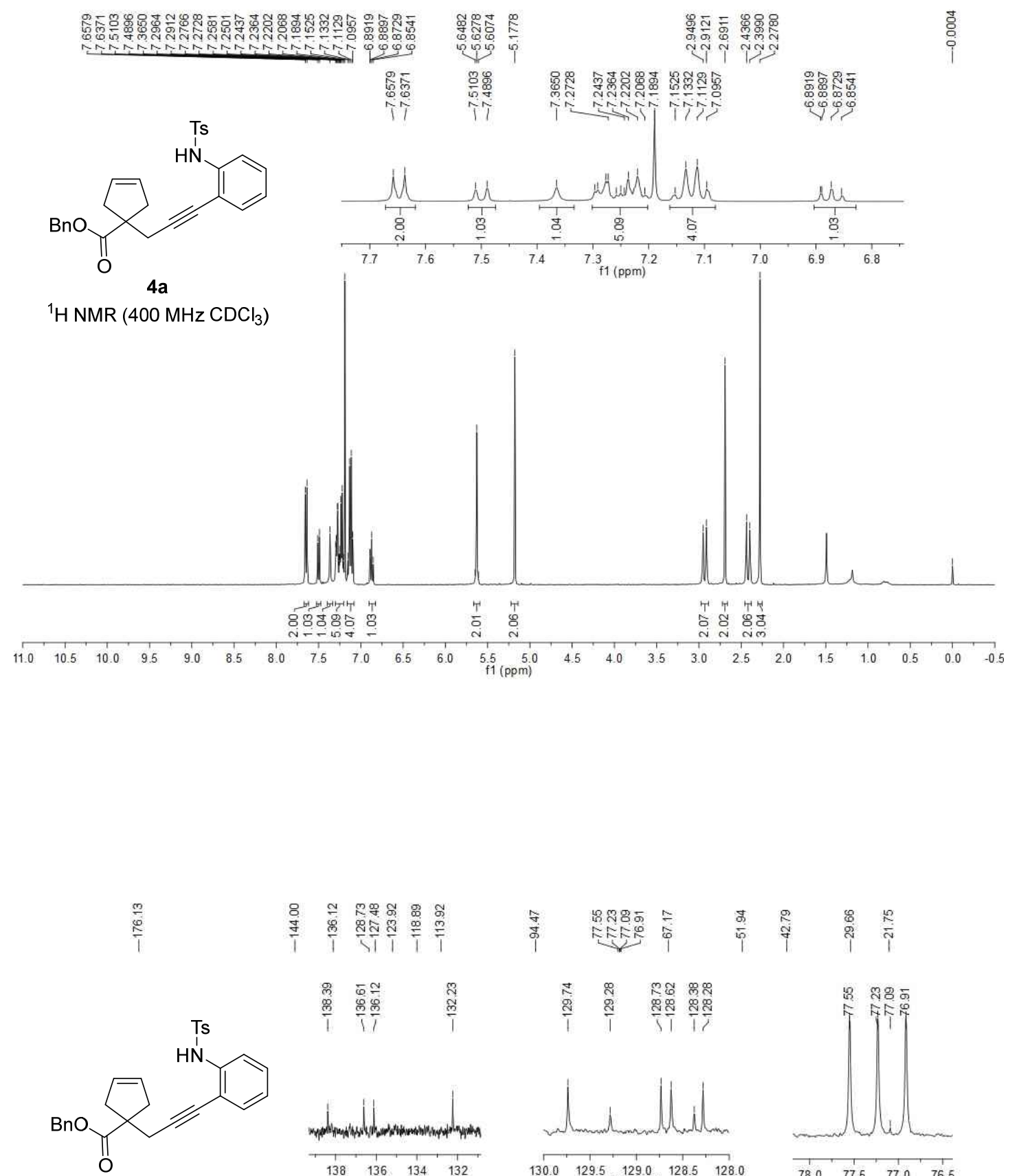

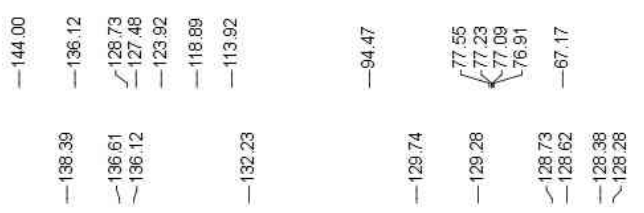

4a
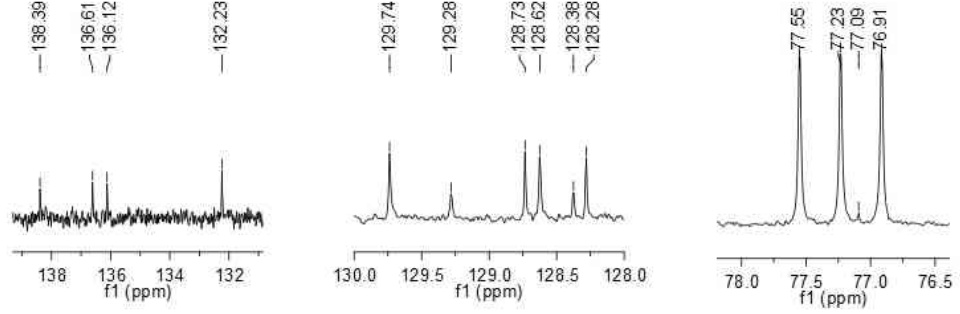

$\left.{ }^{13} \mathrm{C} \mathrm{NMR} \mathrm{(100} \mathrm{MHz} \mathrm{CDCl}_{3}\right)$ 

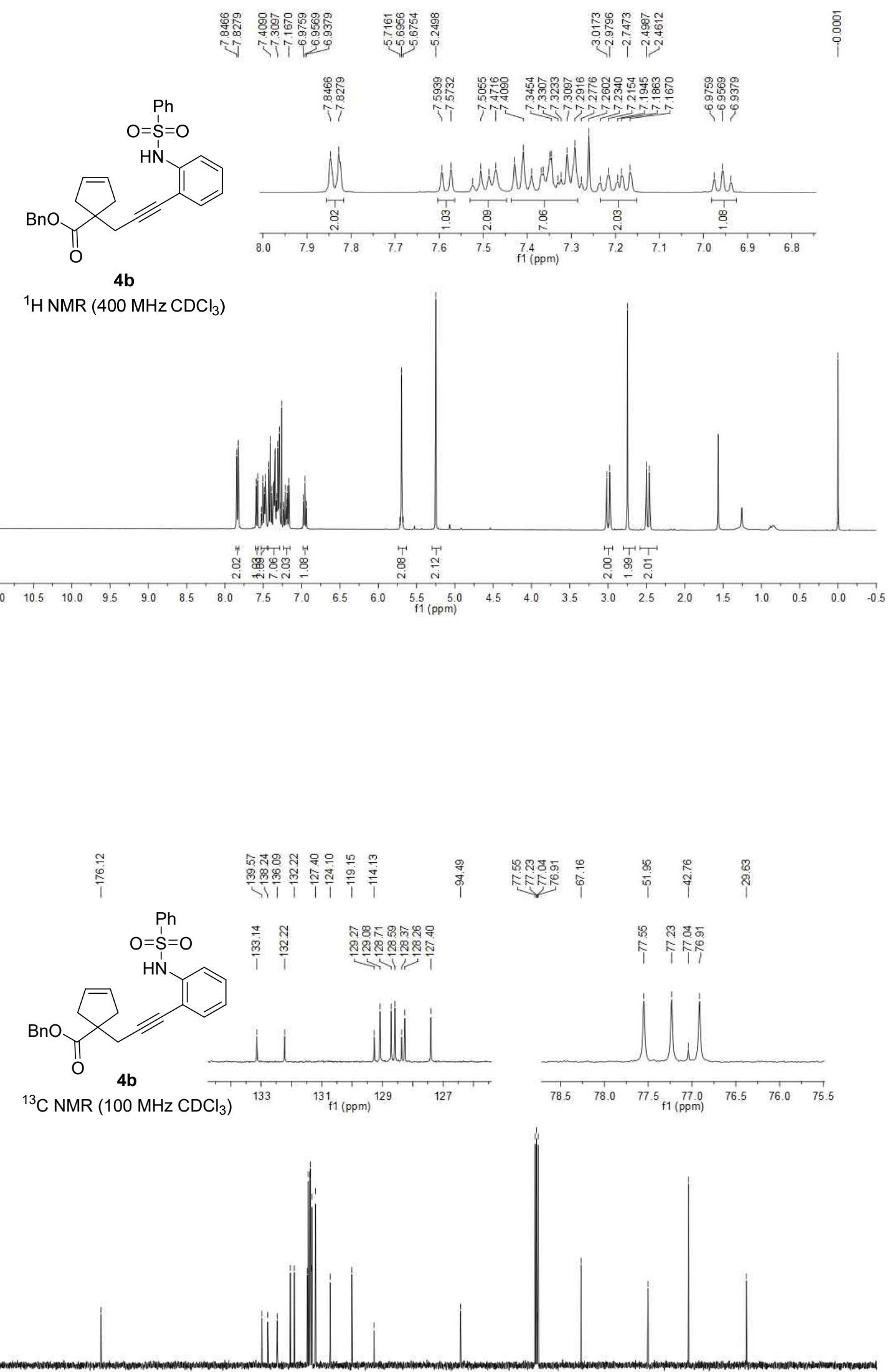

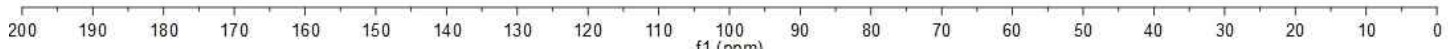


<smiles>COc1ccc(S(=O)(=O)Nc2ccccc2C#CCC2(C(=O)Cc3ccccc3)CC=CC2)cc1</smiles>

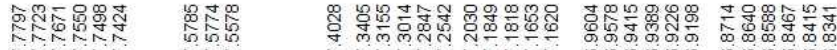

aris कर

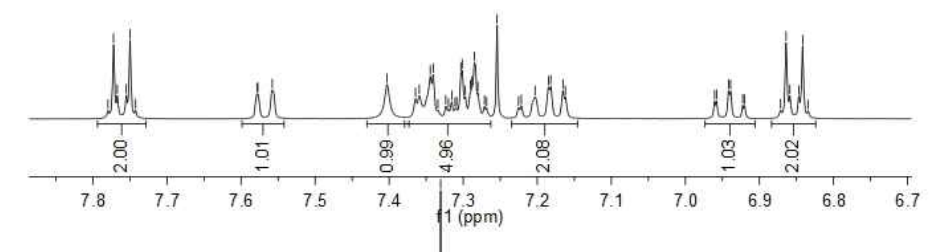

${ }^{1} \mathrm{H} \mathrm{NMR}\left(400 \mathrm{MHz} \mathrm{CDCl}_{3}\right)$
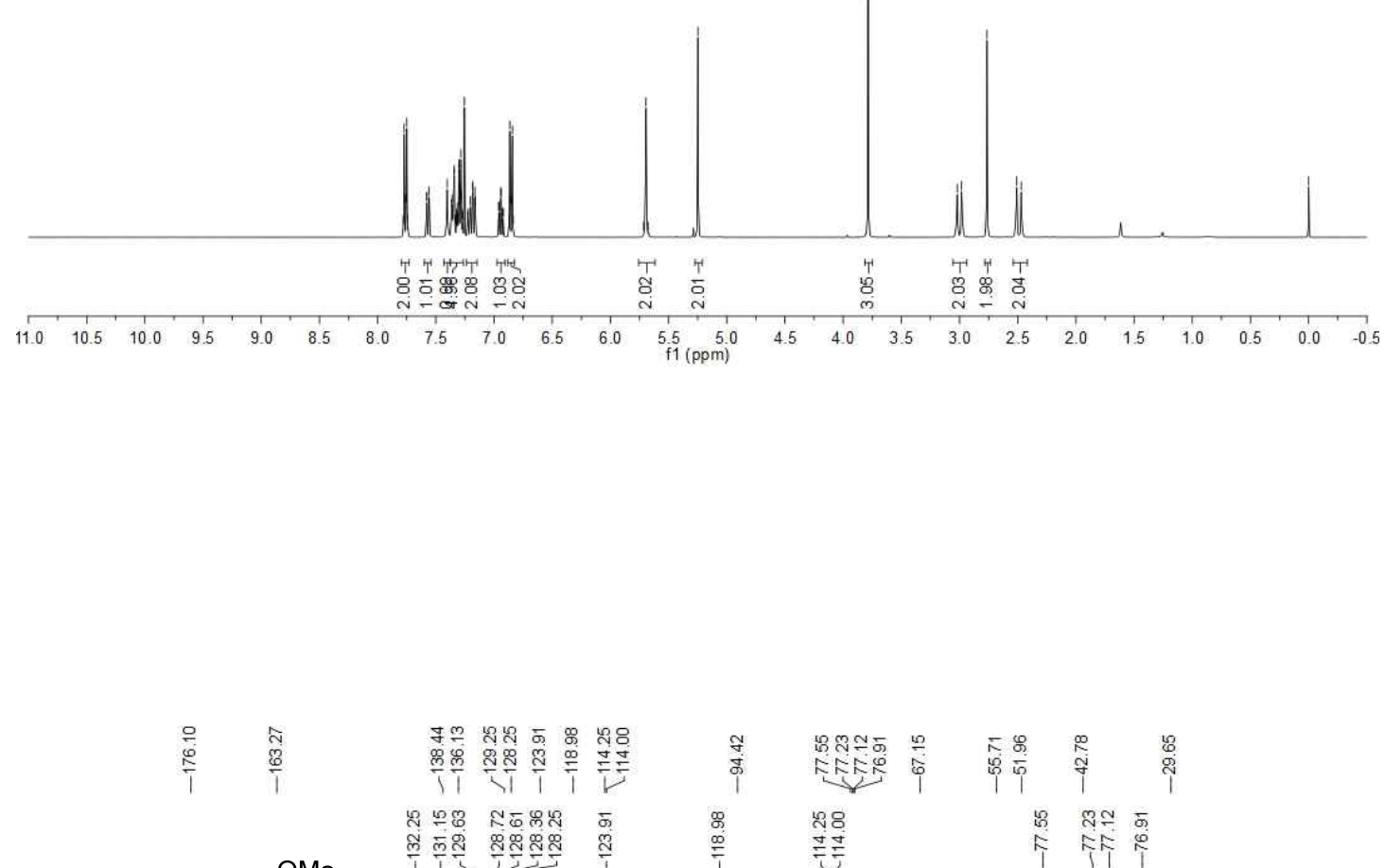

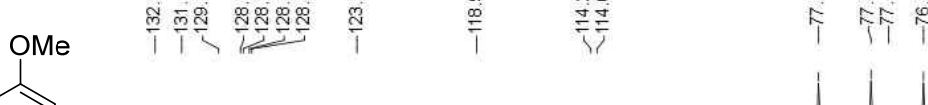

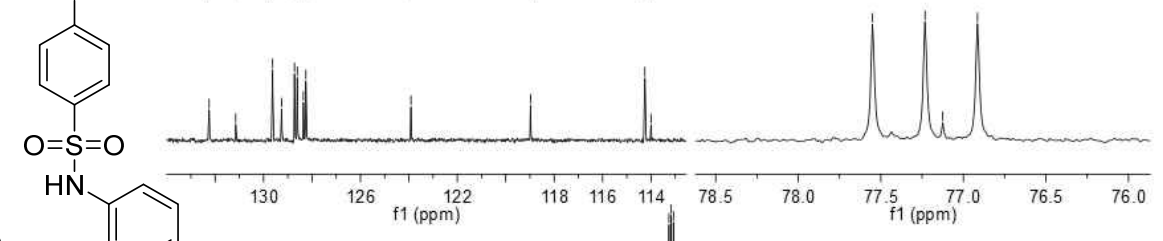<smiles>Nc1ccccc1C#CCC1(C(=O)Cc2ccccc2)CC=CC1</smiles>

${ }^{13} \mathrm{C} \mathrm{NMR}\left(100 \mathrm{MHz} \mathrm{CDCl}_{3}\right)$

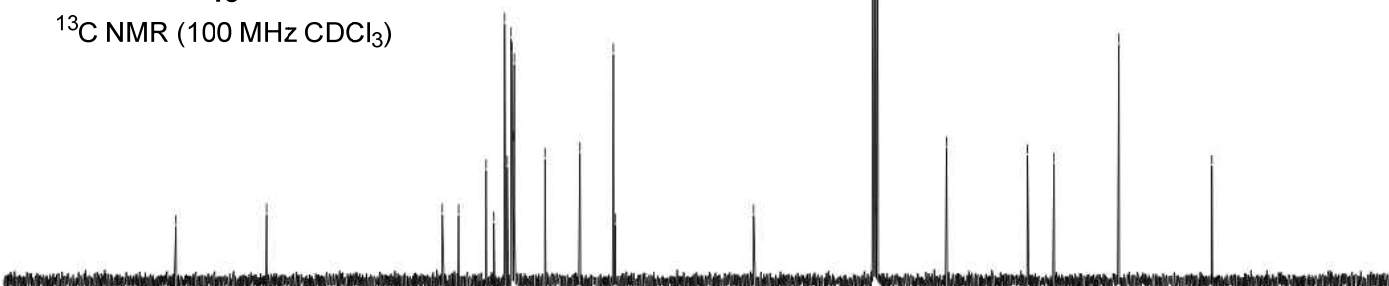

200

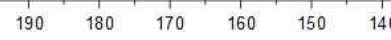

$130 \quad 120$

$10 \begin{gathered}100 \\ \mathrm{f} 1(\mathrm{ppm})\end{gathered}$ 

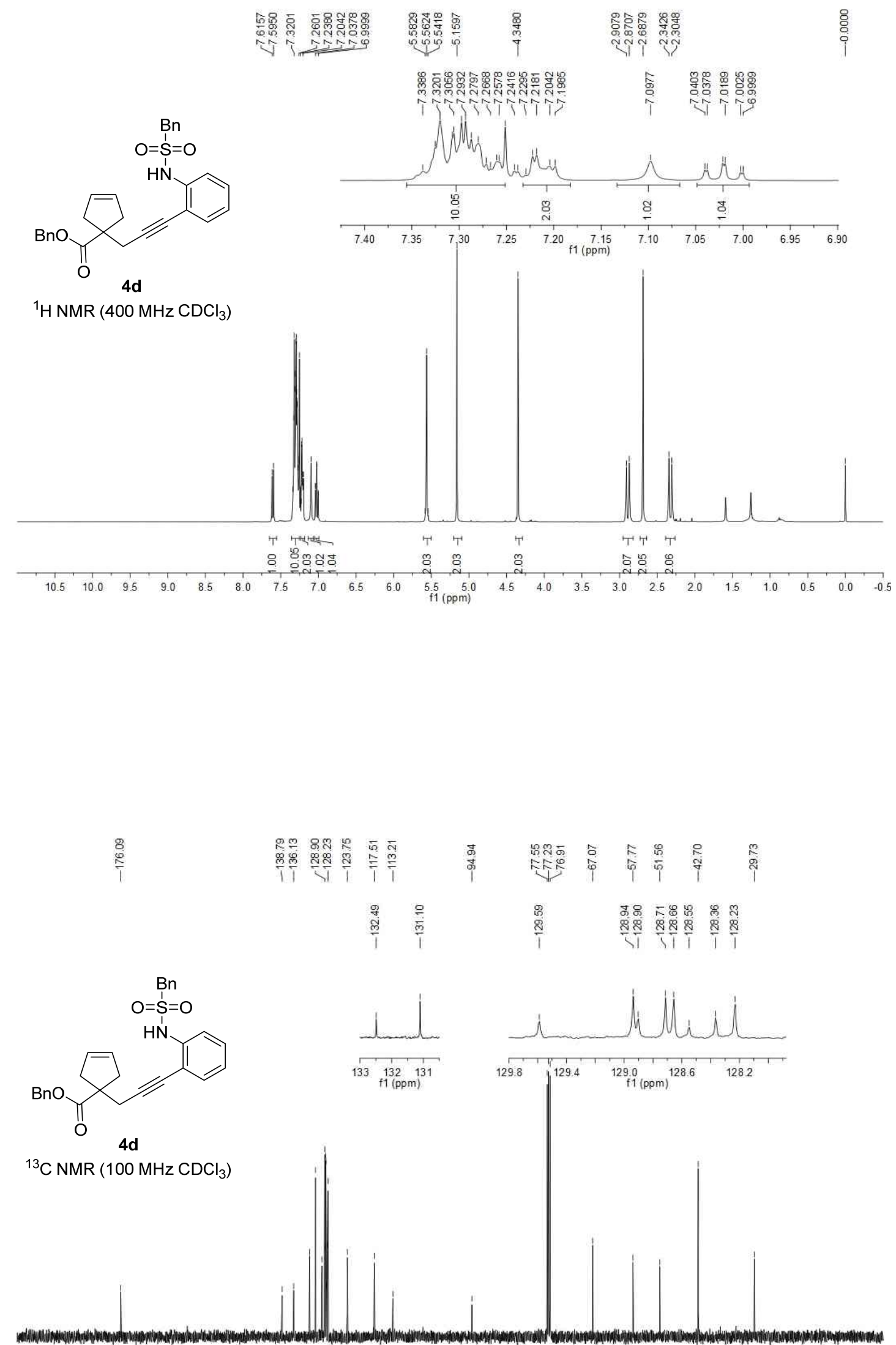

\begin{tabular}{llllllllllllllllllllll}
\hline 200 & 190 & 180 & 170 & 160 & 150 & 140 & 130 & 120 & 110 & 100 & 10 & 80 & 70 & 60 & 50 & 40 & 30 & 20 & 10 & 1
\end{tabular} 

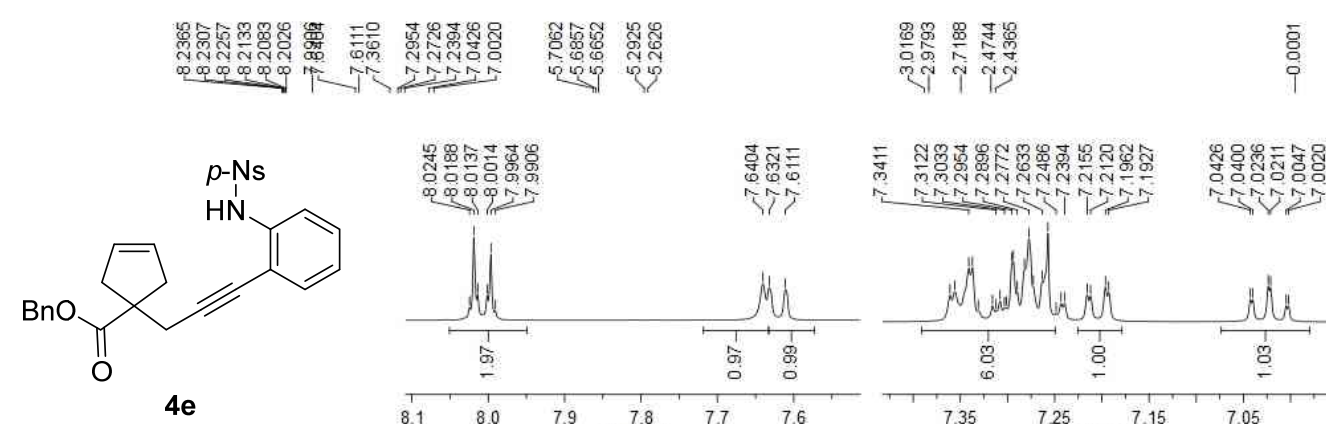

${ }^{1} \mathrm{H}$ NMR (400 MHz $\left.\mathrm{CDCl}_{3}\right)$
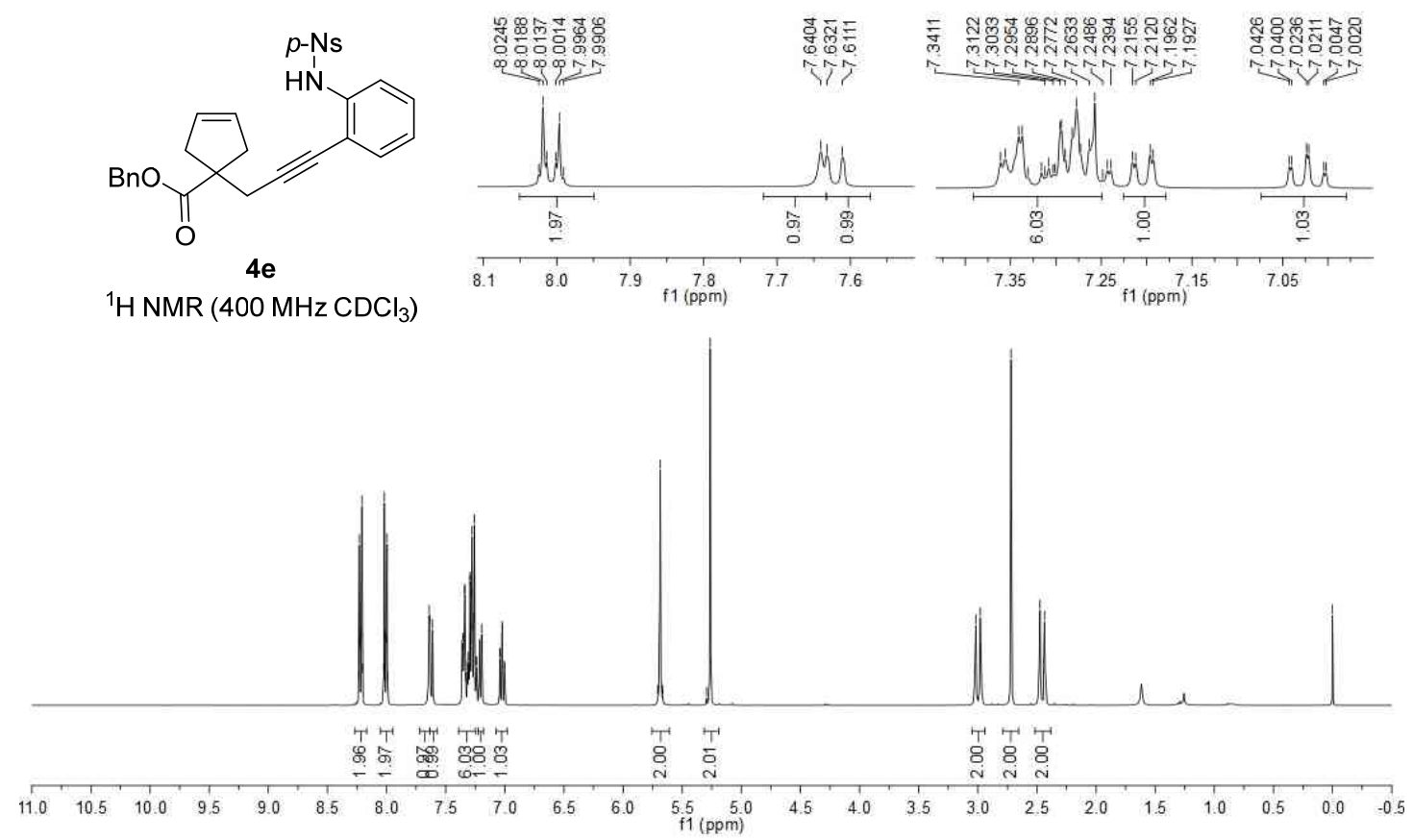

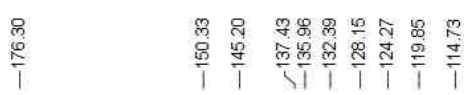

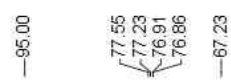

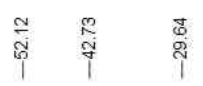

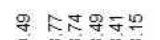

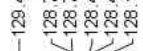

市 ते

(1)

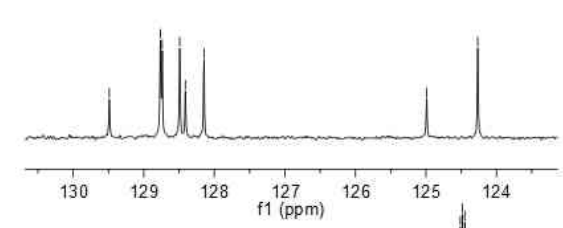

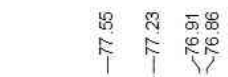

${ }^{13} \mathrm{C} \mathrm{NMR}\left(100 \mathrm{MHz} \mathrm{CDCl}_{3}\right)$

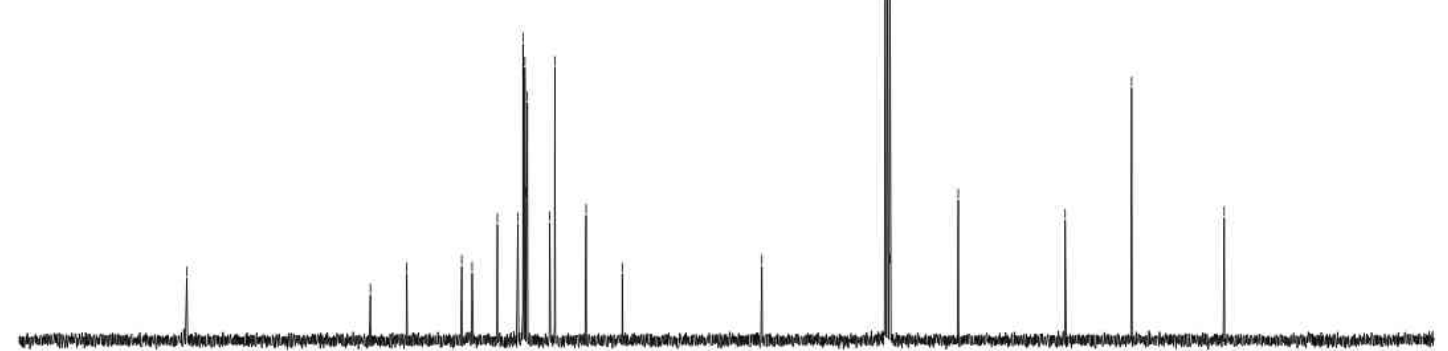

200

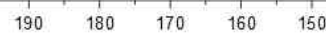

$140 \quad 130 \quad 120$

100
$\mathrm{f} 1(\mathrm{ppm})$ 


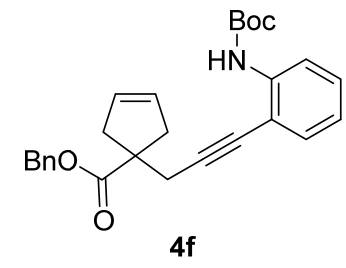

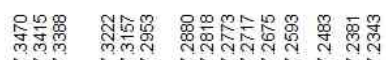

${ }^{1} \mathrm{H}$ NMR $\left(400 \mathrm{MHz} \mathrm{CDCl}_{3}\right)$

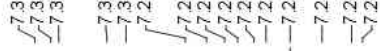

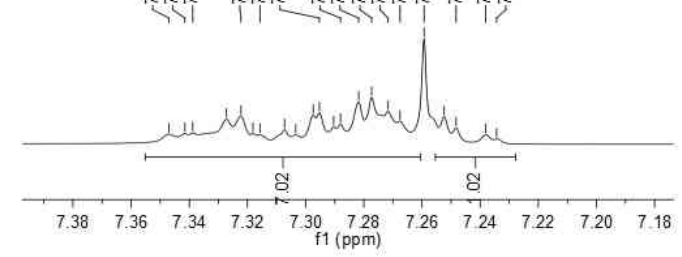

1

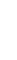

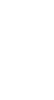
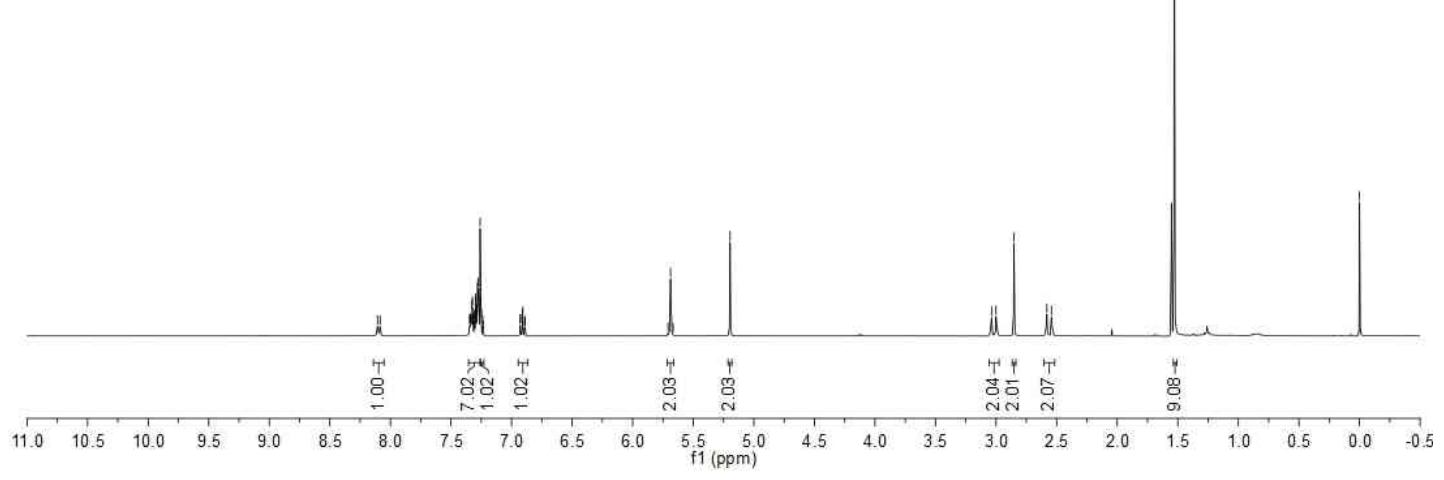

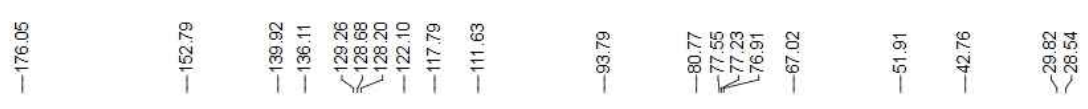

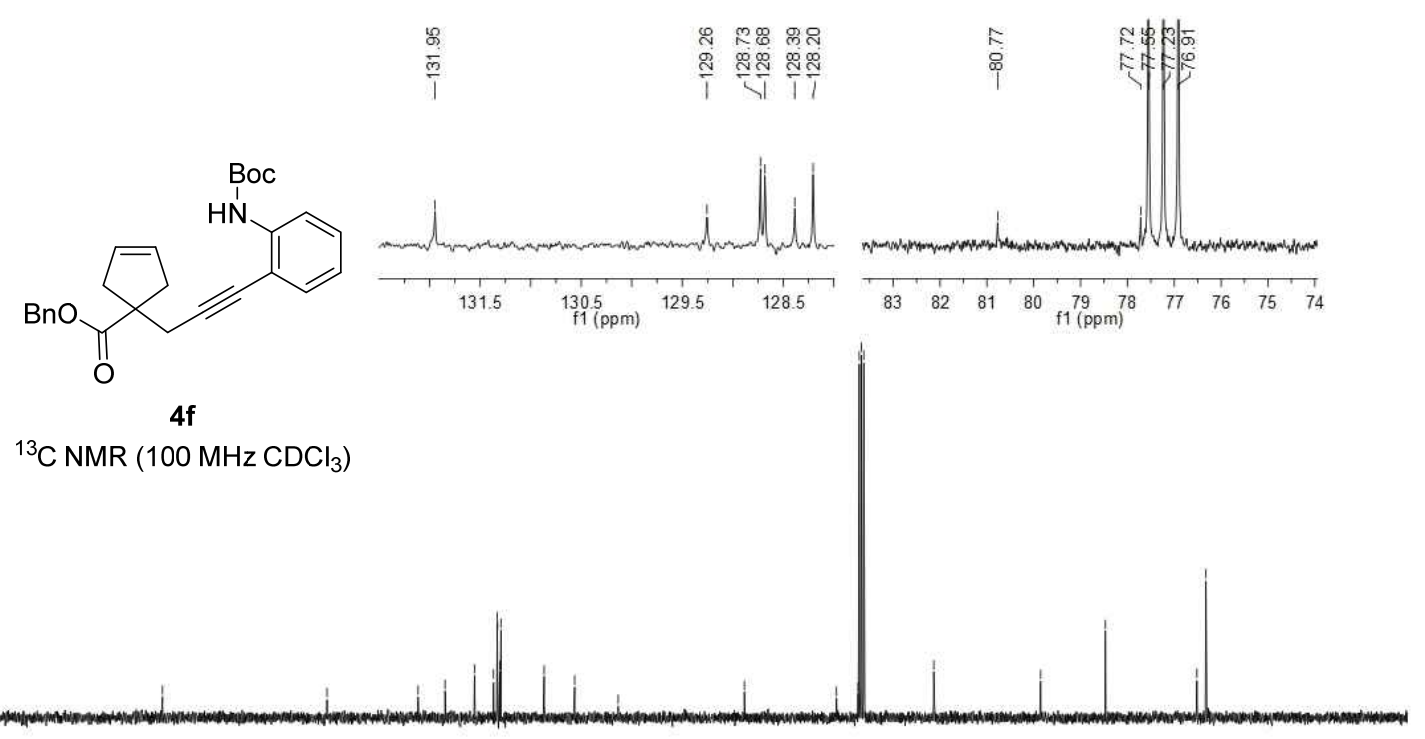

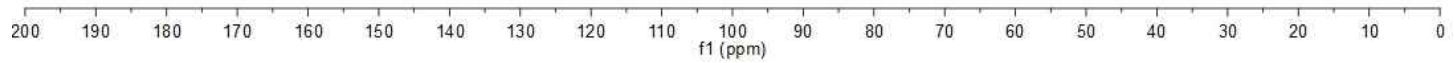




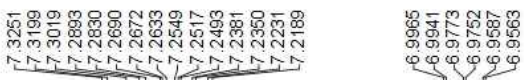

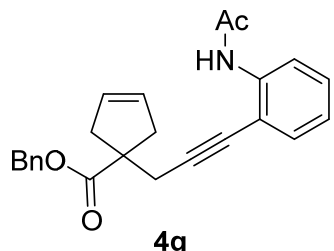

${ }^{1} \mathrm{H}$ NMR $\left(400 \mathrm{MHz} \mathrm{CDCl}_{3}\right)$
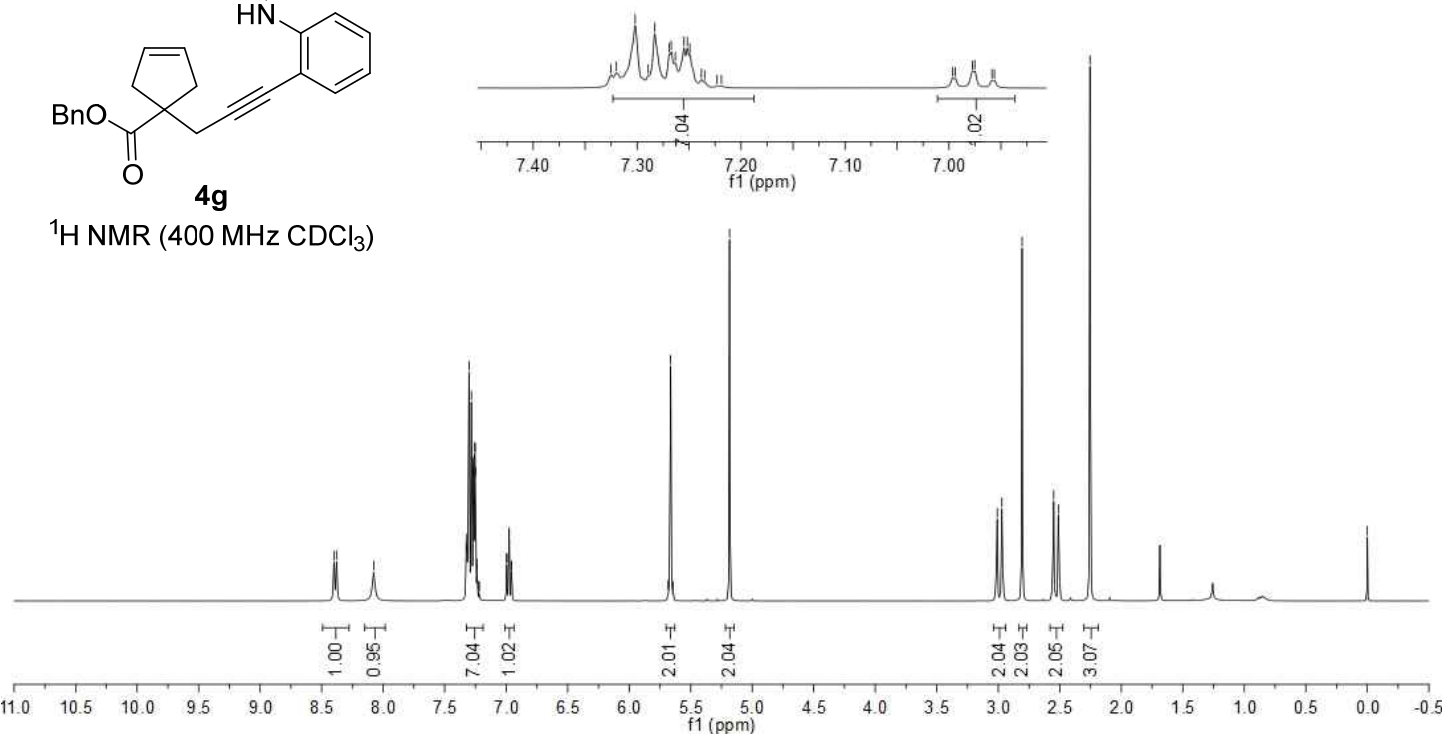

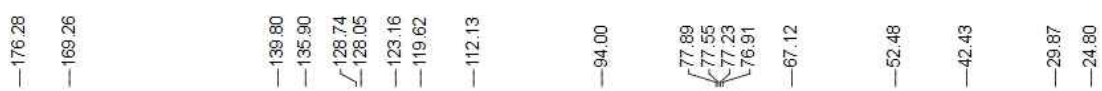
鲜

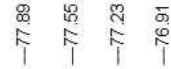
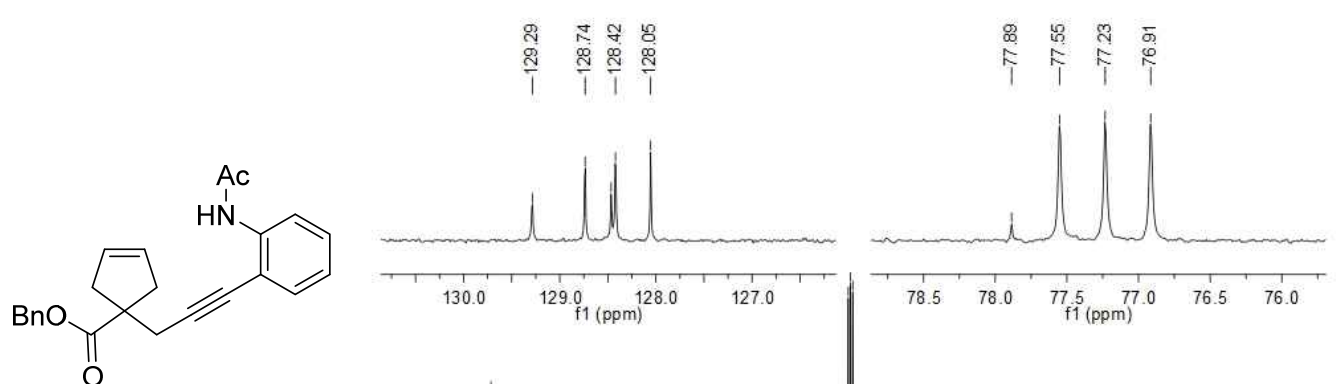

$4 \mathrm{~g}$

${ }^{13} \mathrm{C}$ NMR $\left(100 \mathrm{MHz} \mathrm{CDCl}_{3}\right)$

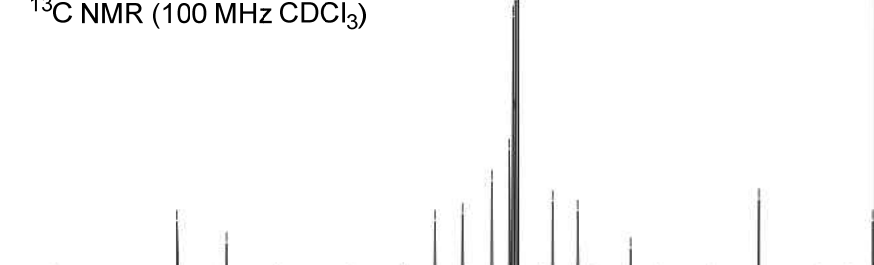

200

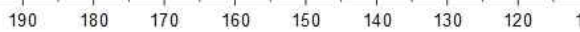

$100 \quad 90$

1
70
60 


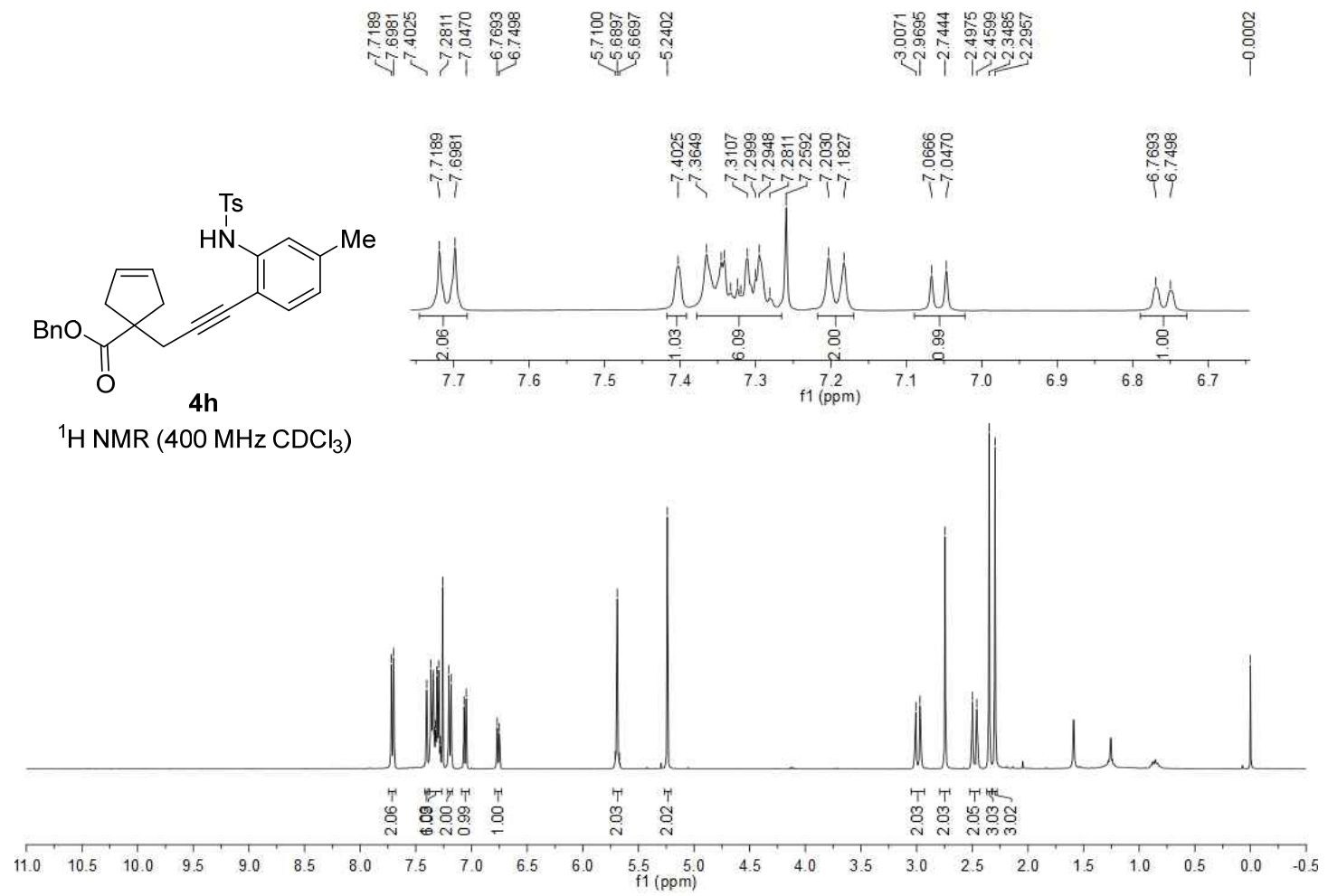

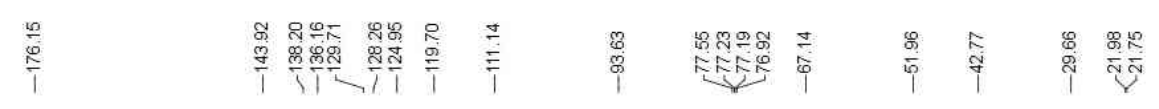

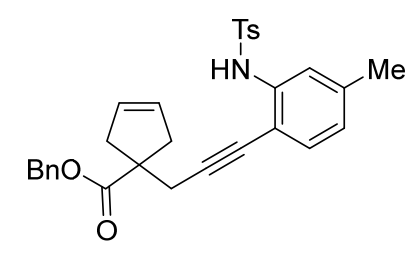

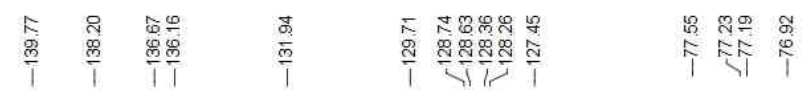

$4 \mathrm{~h}$

${ }^{13} \mathrm{C} \mathrm{NMR}\left(100 \mathrm{MHz}^{\mathrm{CDCl}} 3\right)$

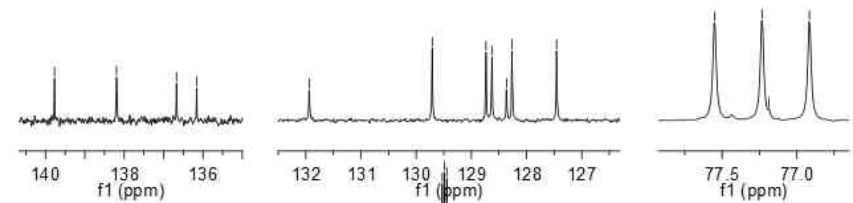

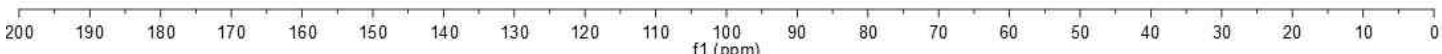



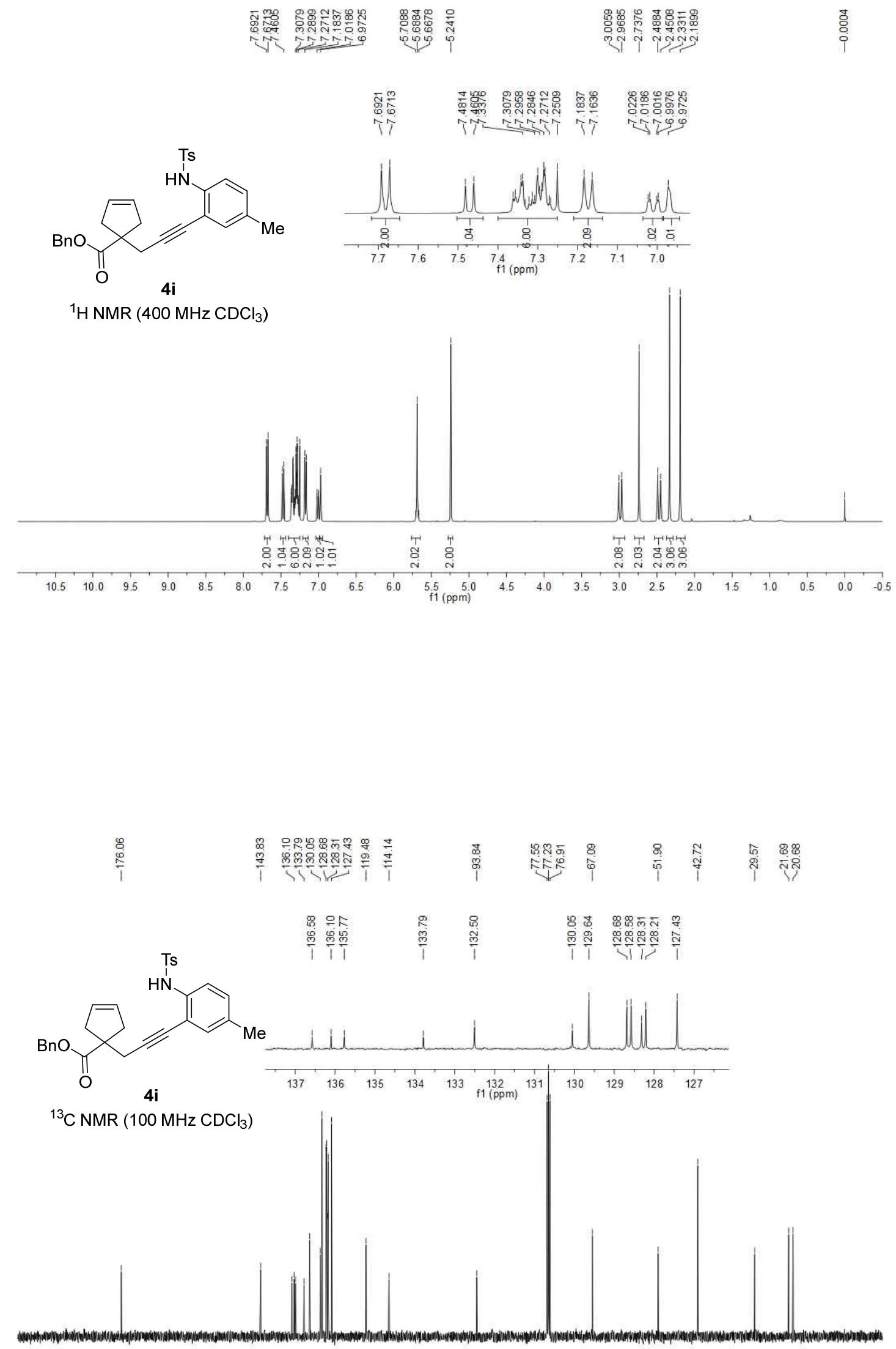

\begin{tabular}{lllllllllllllllllllllll}
\hline 200 & 190 & 180 & 170 & 160 & 150 & 140 & 130 & 120 & 110 & 100 & 90 & 80 & 70 & 60 & 50 & 40 & 30 & 20 & 10 & 0
\end{tabular} 


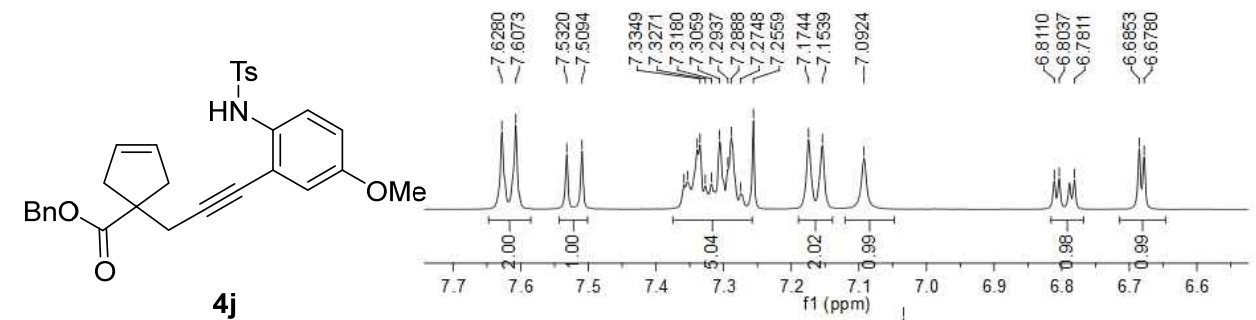

${ }^{1} \mathrm{H}$ NMR $\left(400 \mathrm{MHz} \mathrm{CDCl}_{3}\right)$

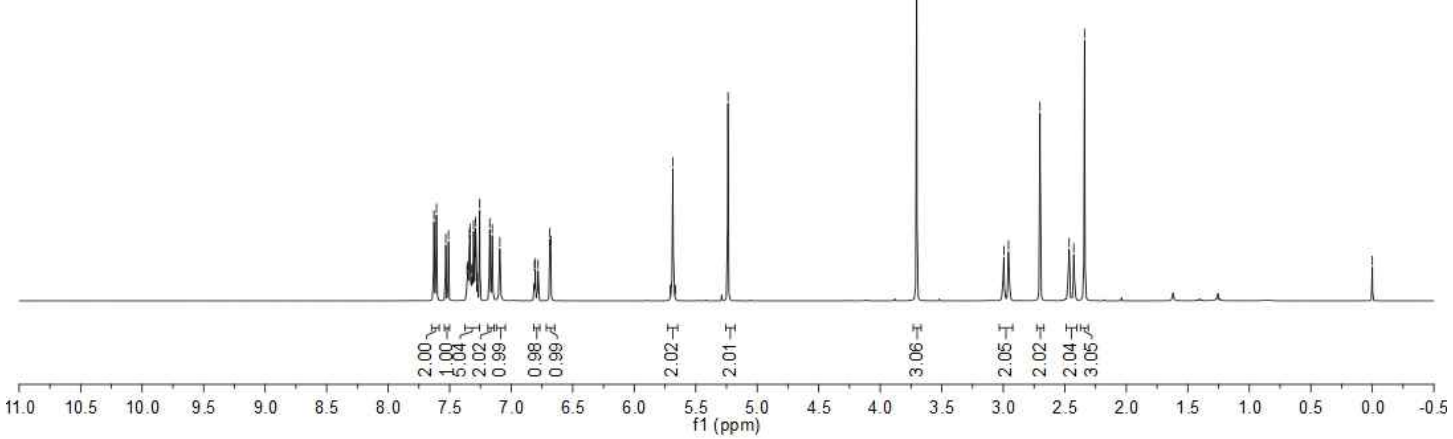

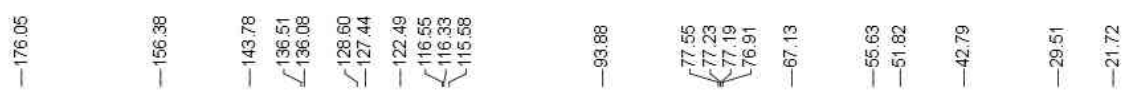<smiles>CNc1ccc(OC)cc1C#CCC1(C(=O)OCc2ccccc2)CC=CC1</smiles>

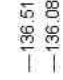

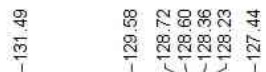

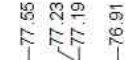

$4 j$

${ }^{13} \mathrm{C} \mathrm{NMR}\left(100 \mathrm{MHz} \mathrm{CDCl}_{3}\right)$
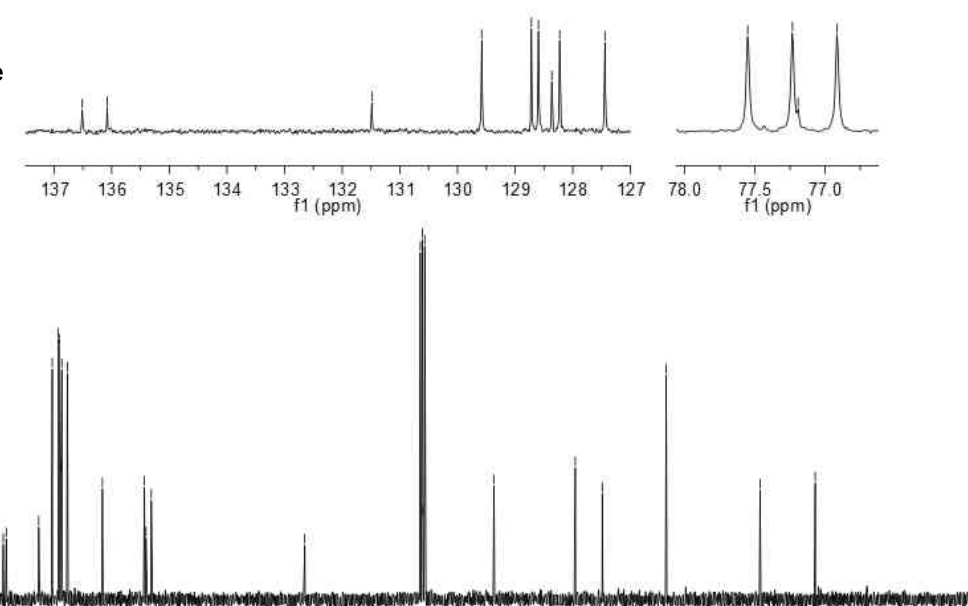

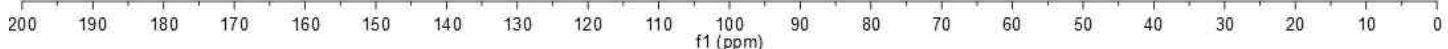



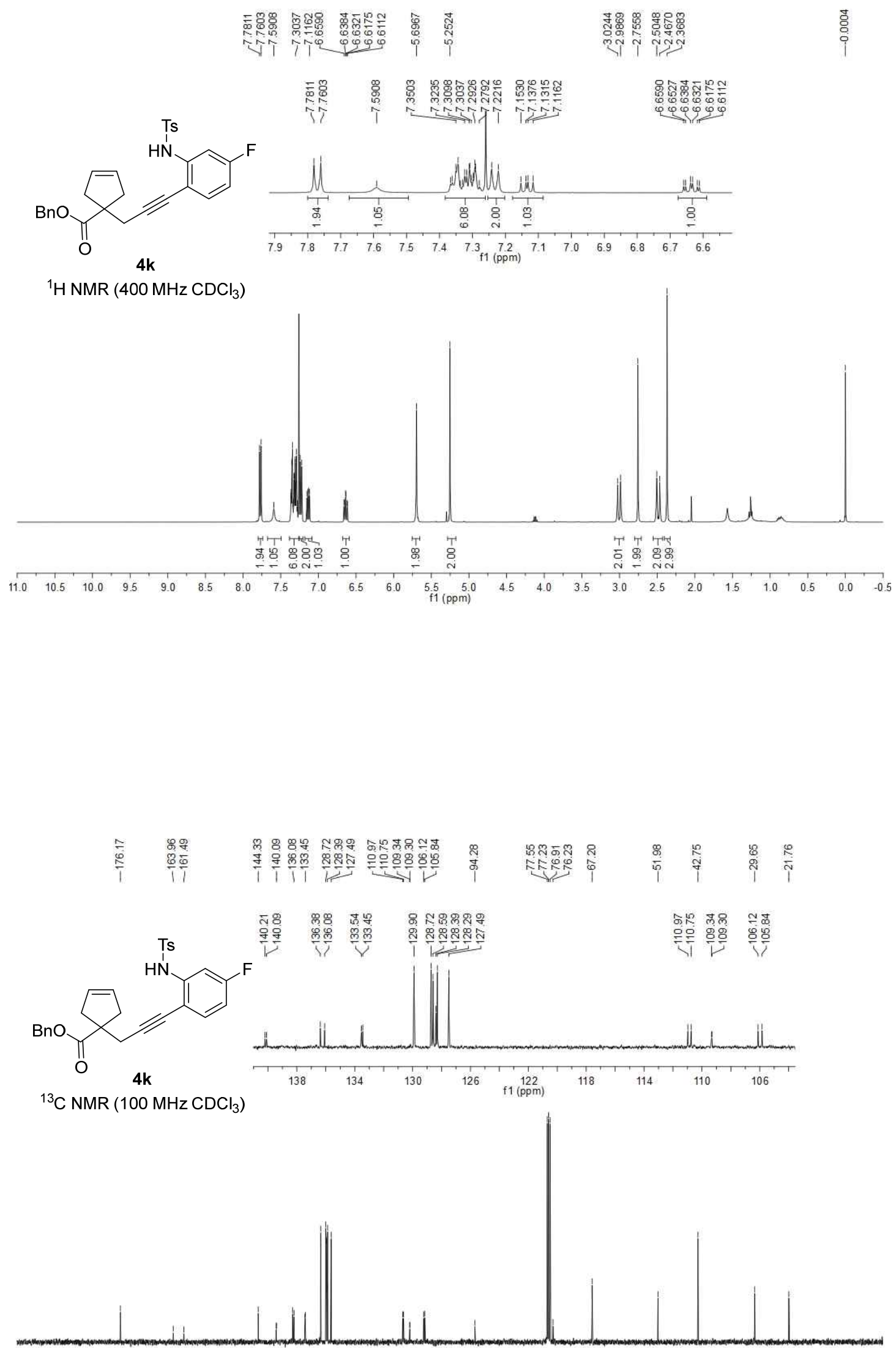

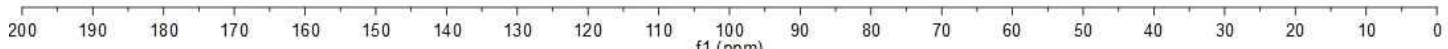




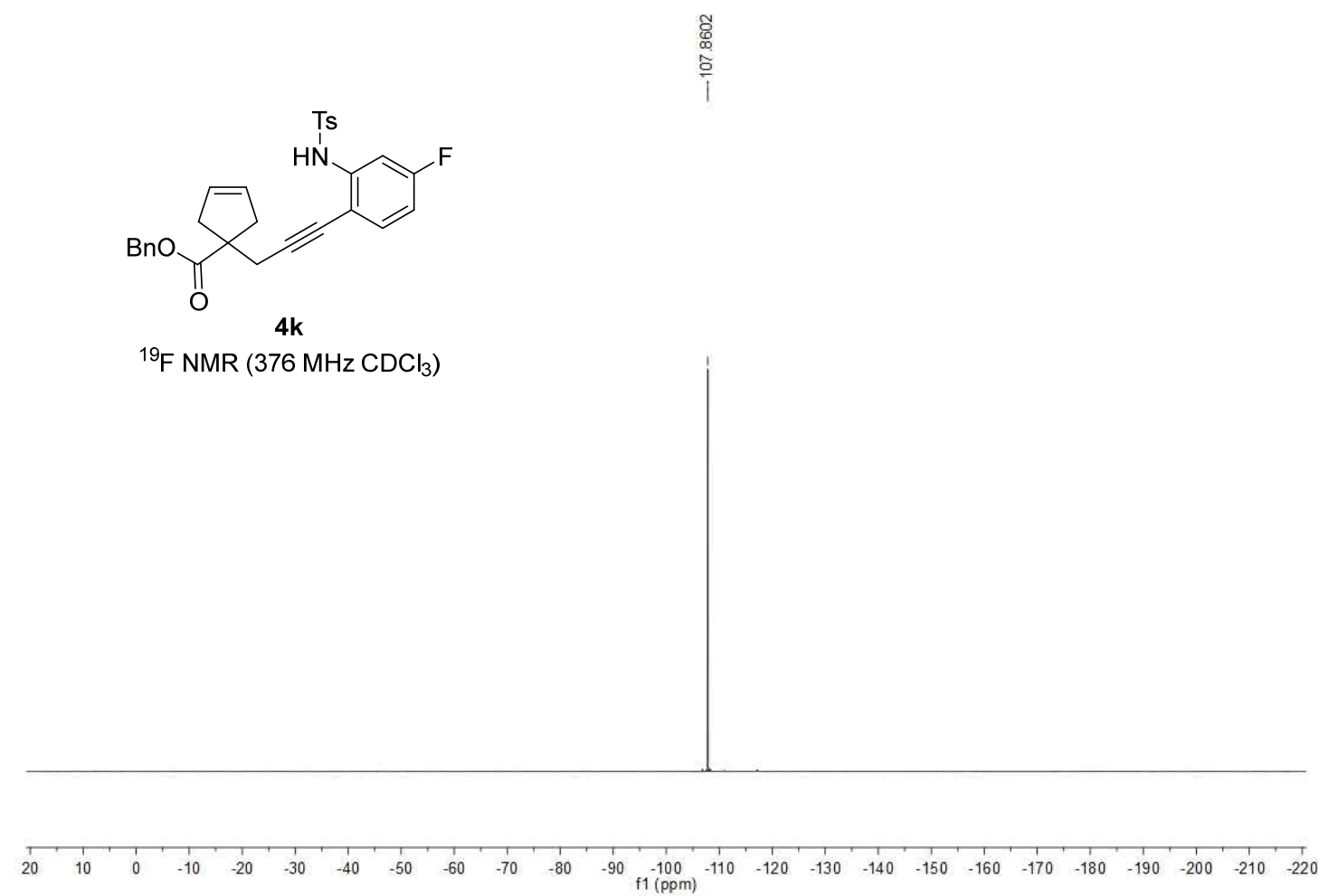



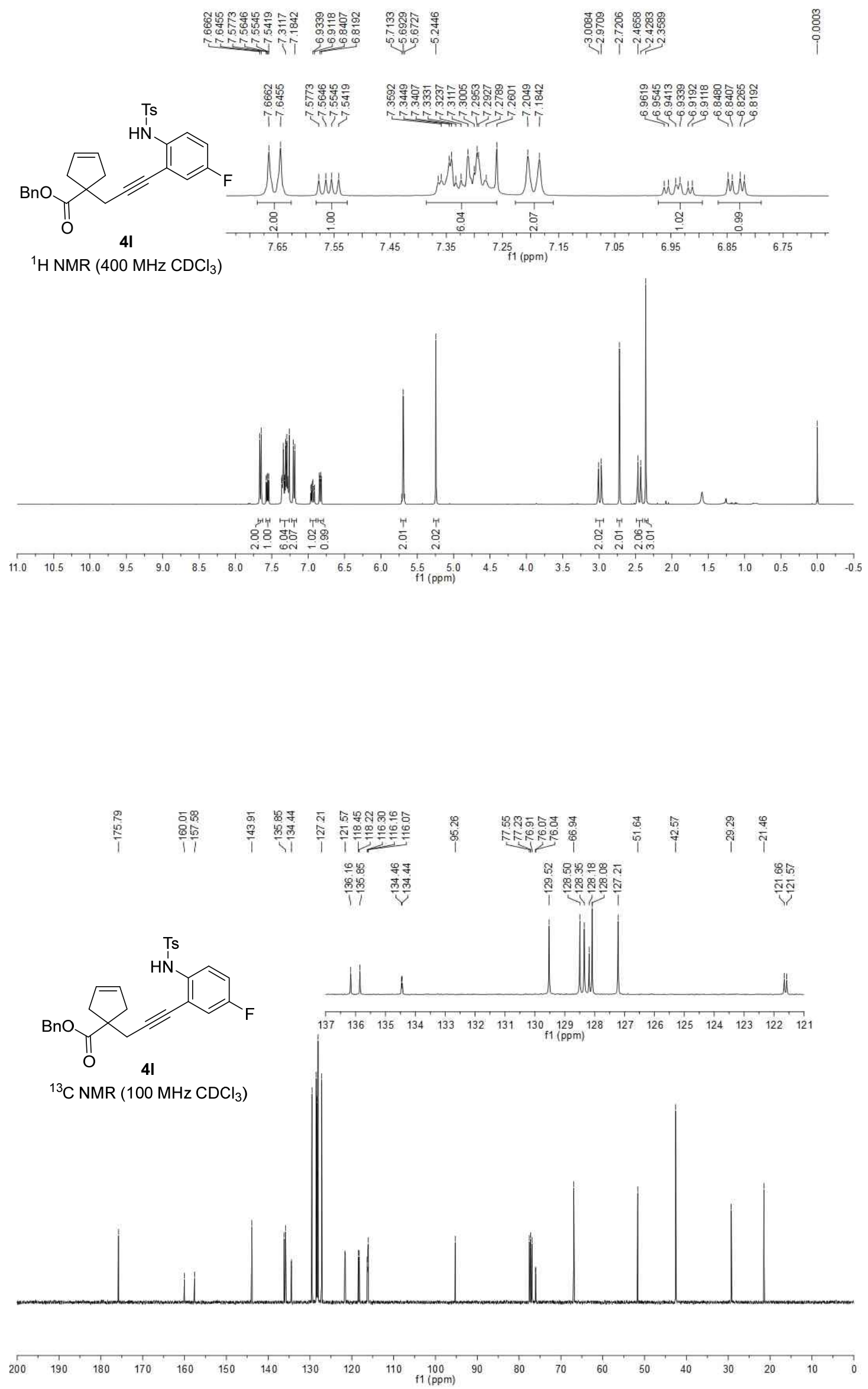


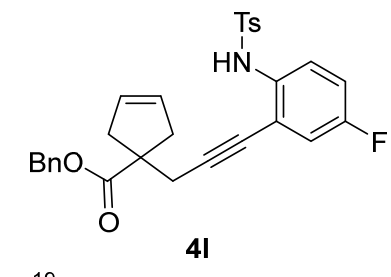

$\left.{ }^{19} \mathrm{~F} \mathrm{NMR} \mathrm{(376} \mathrm{MHz} \mathrm{CDCl}_{3}\right)$

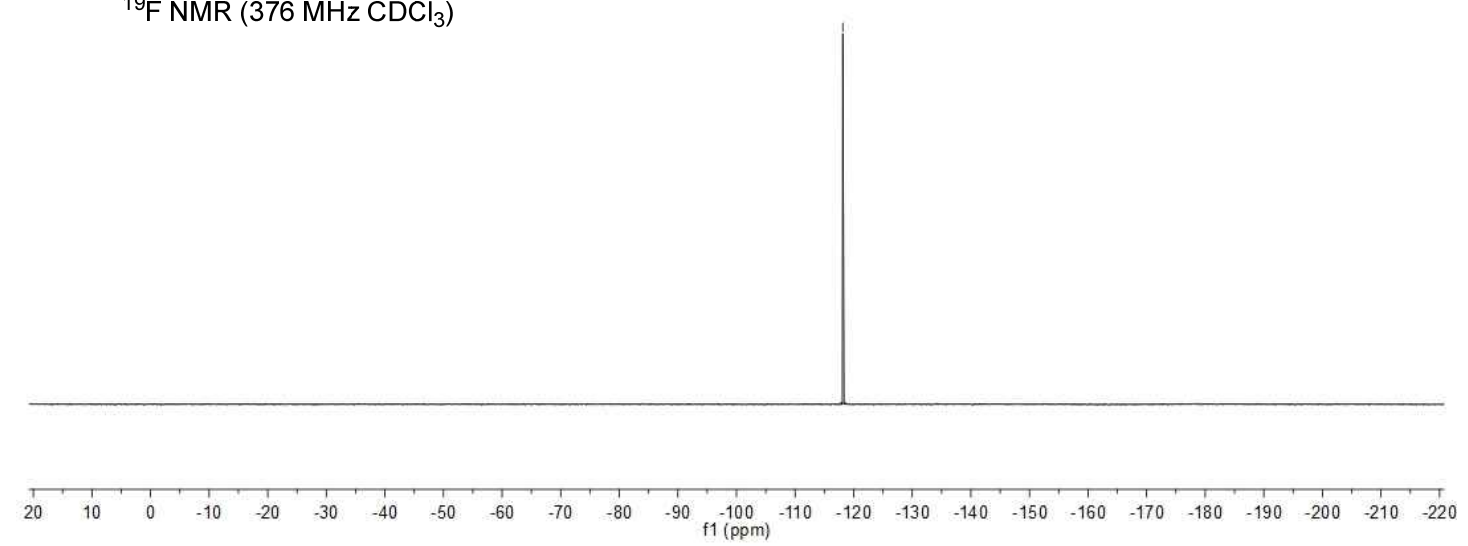




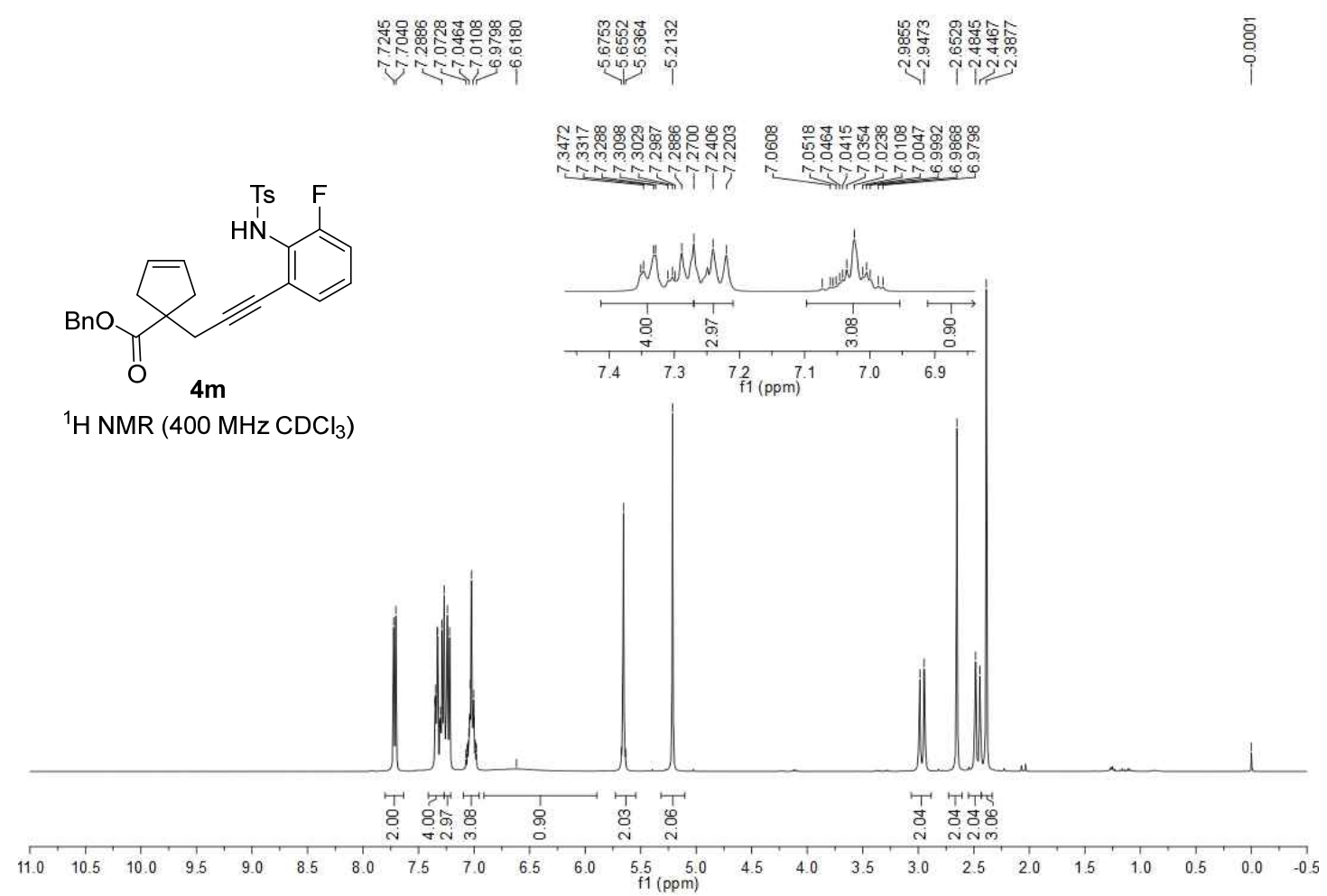

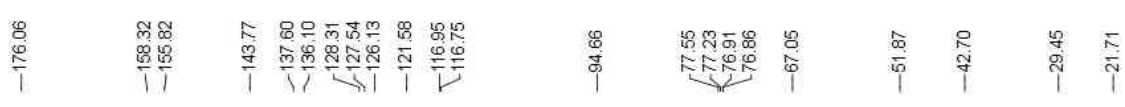

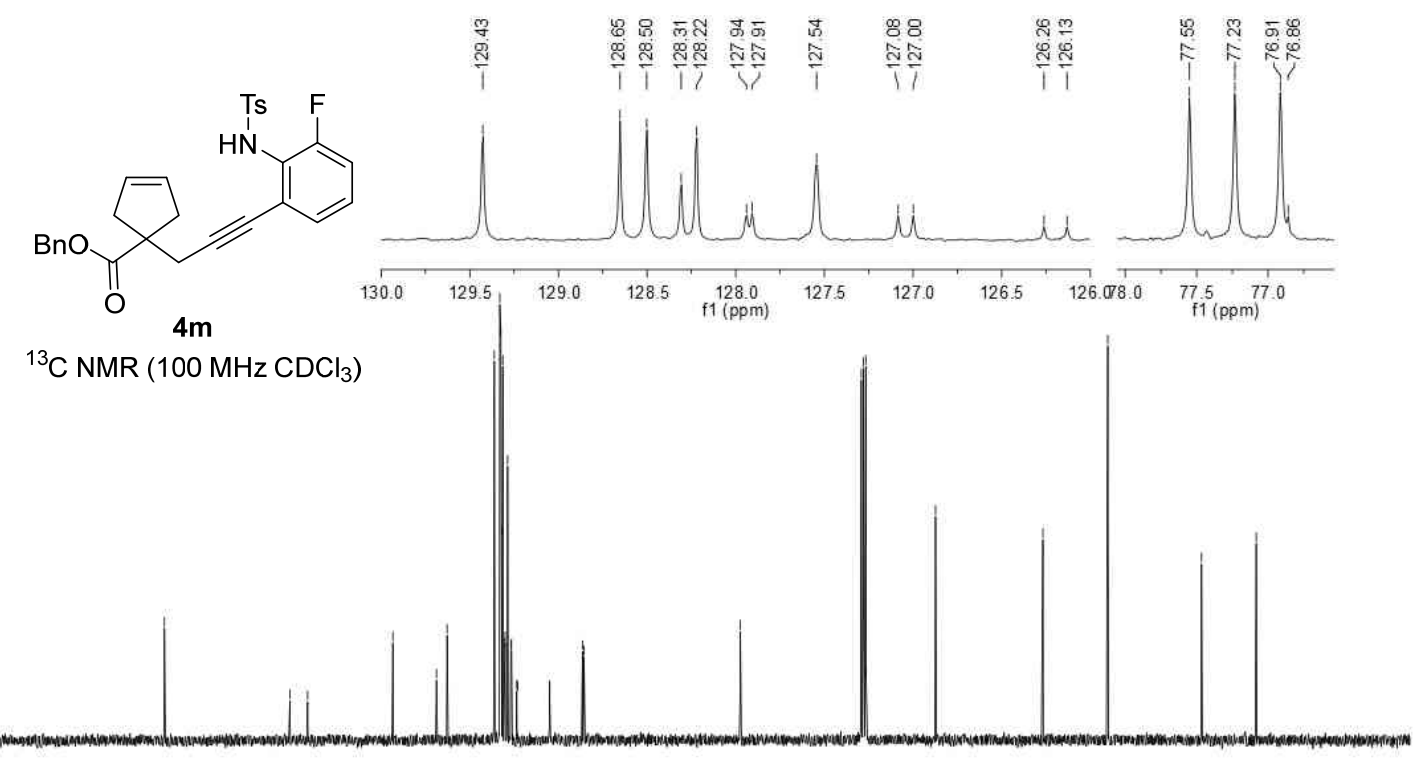

\begin{tabular}{lllllllllllllllllllllllll}
\hline & 190 & 190 & 180 & 170 & 160 & 150 & 140 & 130 & 120 & 110 & 100 & 90 & 80 & 70 & 60 & 50 & 40 & 30 & 20 & 10
\end{tabular} 


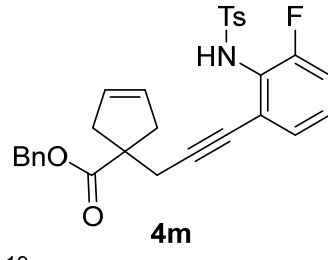

$\left.{ }^{19} \mathrm{~F} \mathrm{NMR} \mathrm{(376} \mathrm{MHz} \mathrm{CDCl}_{3}\right)$

$\begin{array}{lllllllllllllllllllllllll}20 & 10 & 0 & -10 & -20 & -30 & -40 & -50 & -60 & -70 & -80 & -90 & -100 & -110 & -120 & -130 & -140 & -150 & -160 & -170 & -180 & -190 & -200 & -210 & -220\end{array}$ 

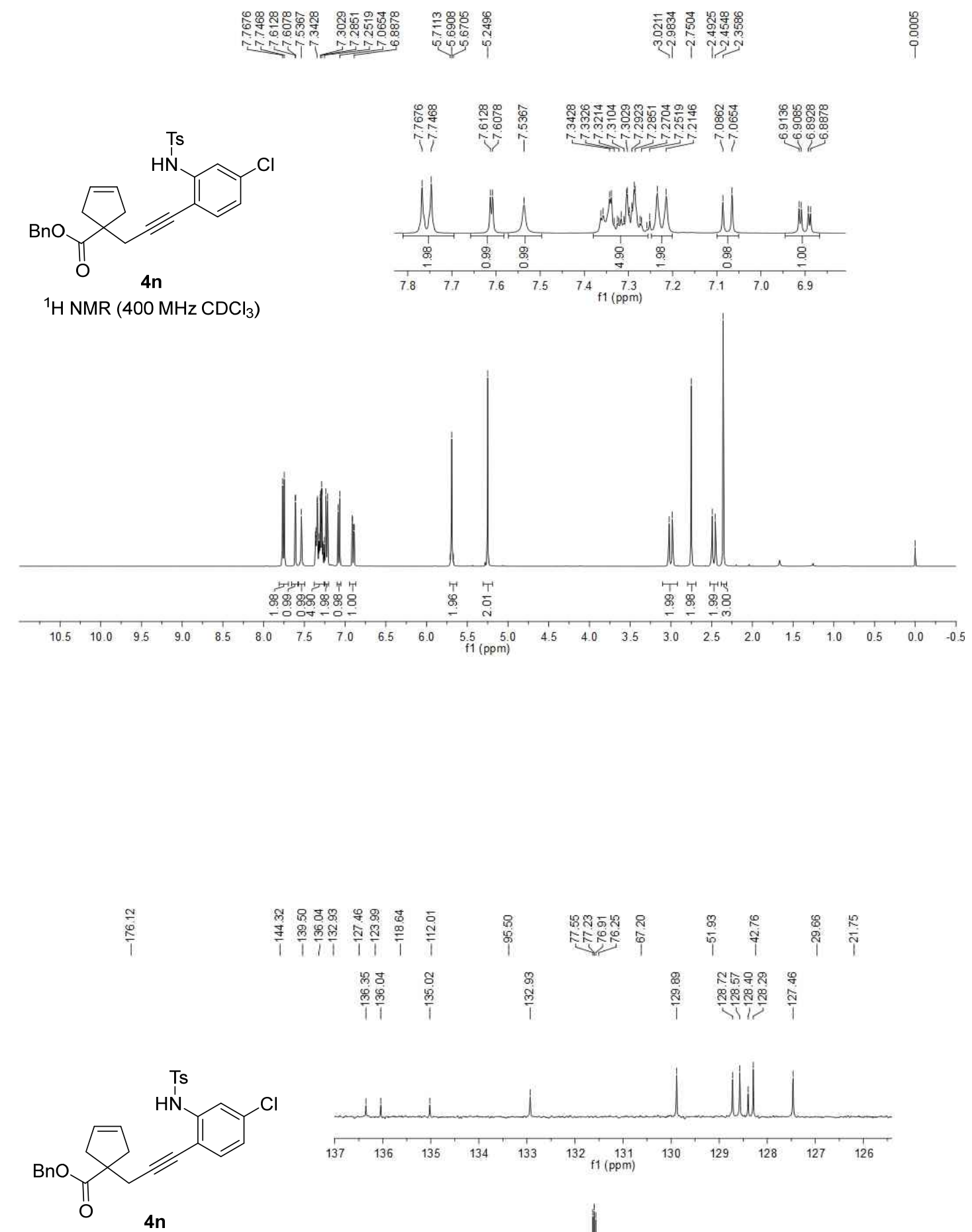

${ }^{13} \mathrm{C} \mathrm{NMR}\left(100 \mathrm{MHz} \mathrm{CDCl}_{3}\right)$

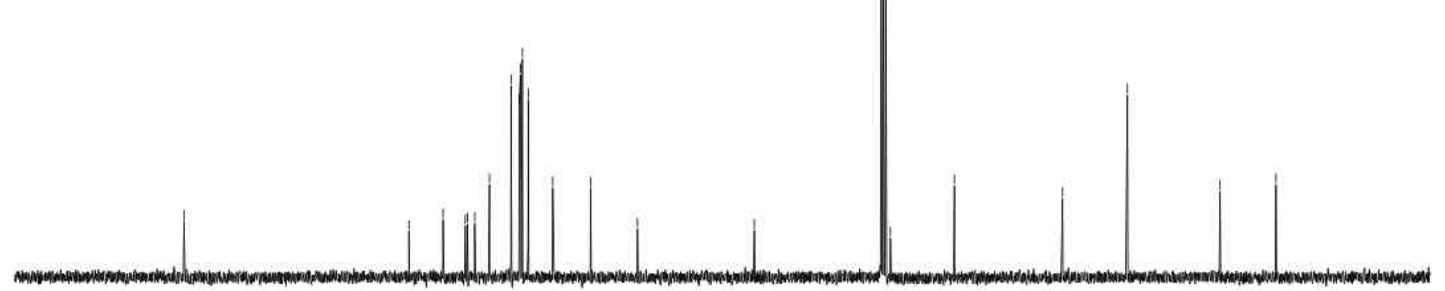

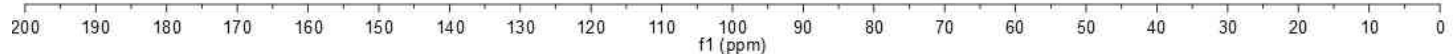



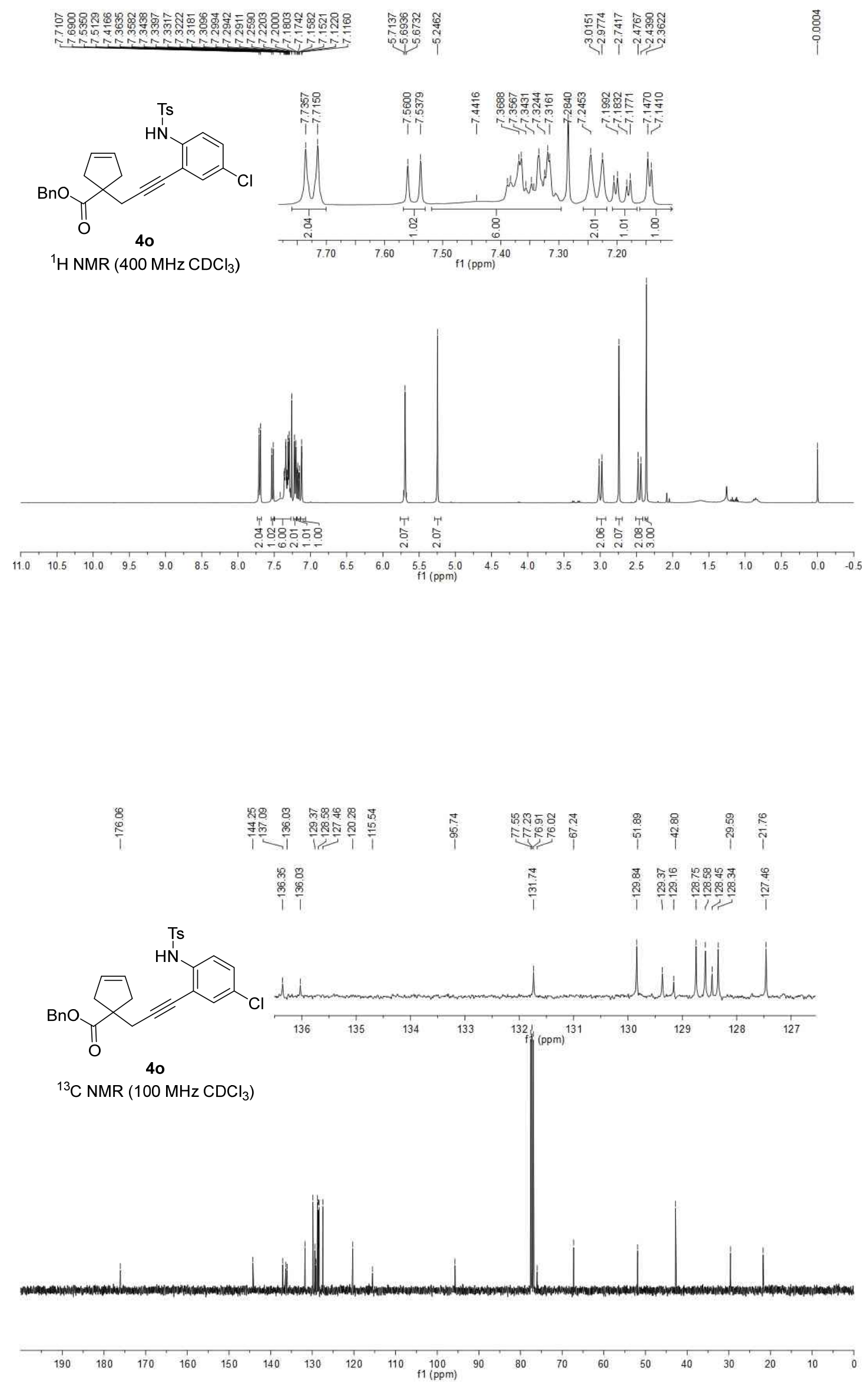

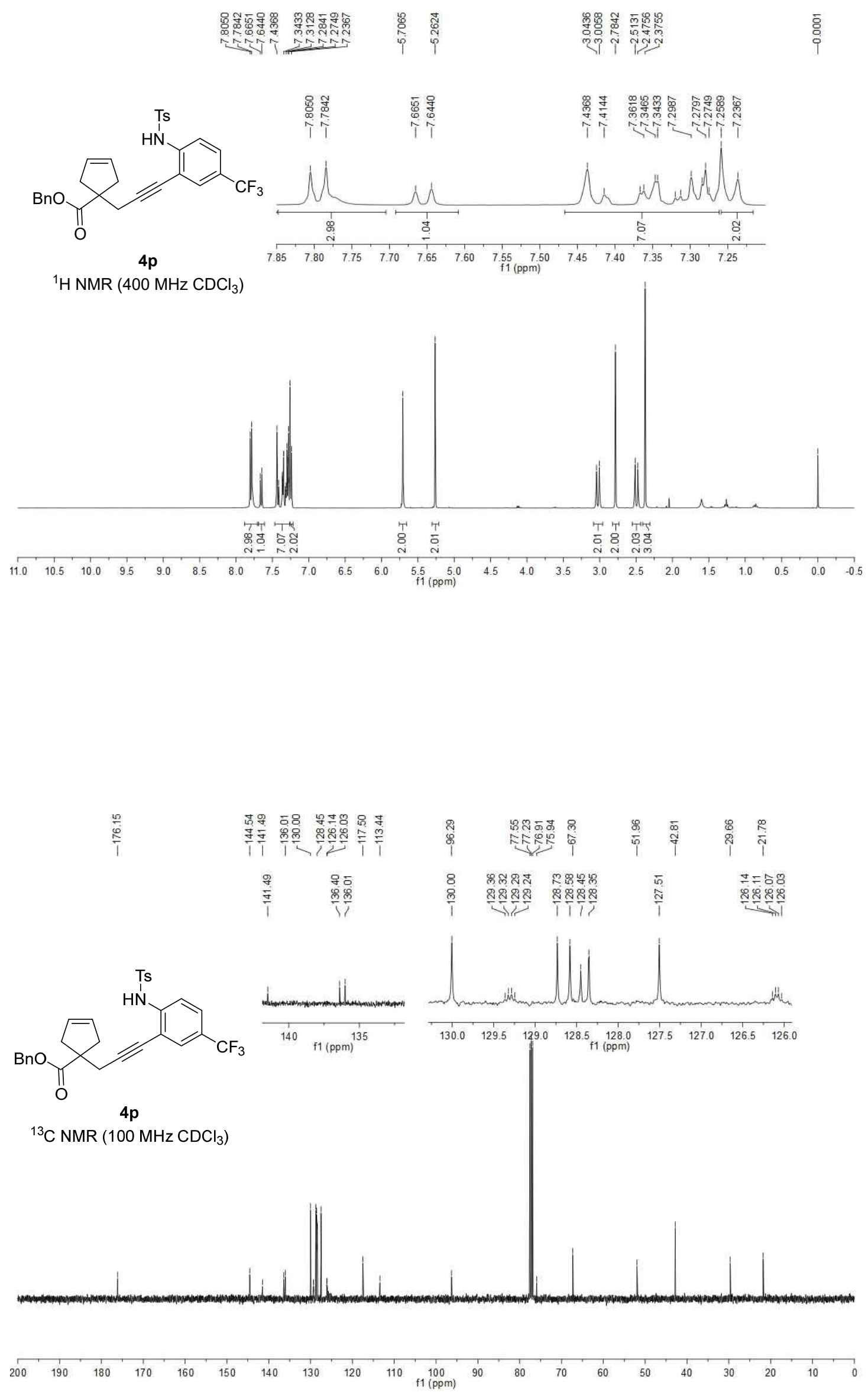


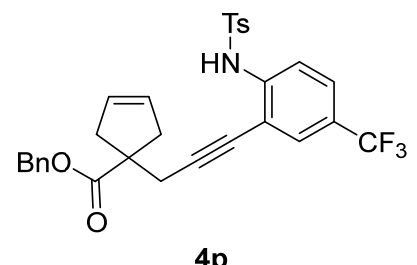

${ }^{19} \mathrm{~F} \mathrm{NMR}\left(376 \mathrm{MHz} \mathrm{CDCl}_{3}\right)$

$\begin{array}{lllllllllllllllllllllllllllllll}20 & 10 & 0 & -10 & -20 & -30 & -40 & -50 & -60 & -70 & -80 & -90 & -100 & -110 & -120 & -130 & -140 & -150 & -160 & -170 & -180 & -190 & -200 & -210 & -220\end{array}$ 


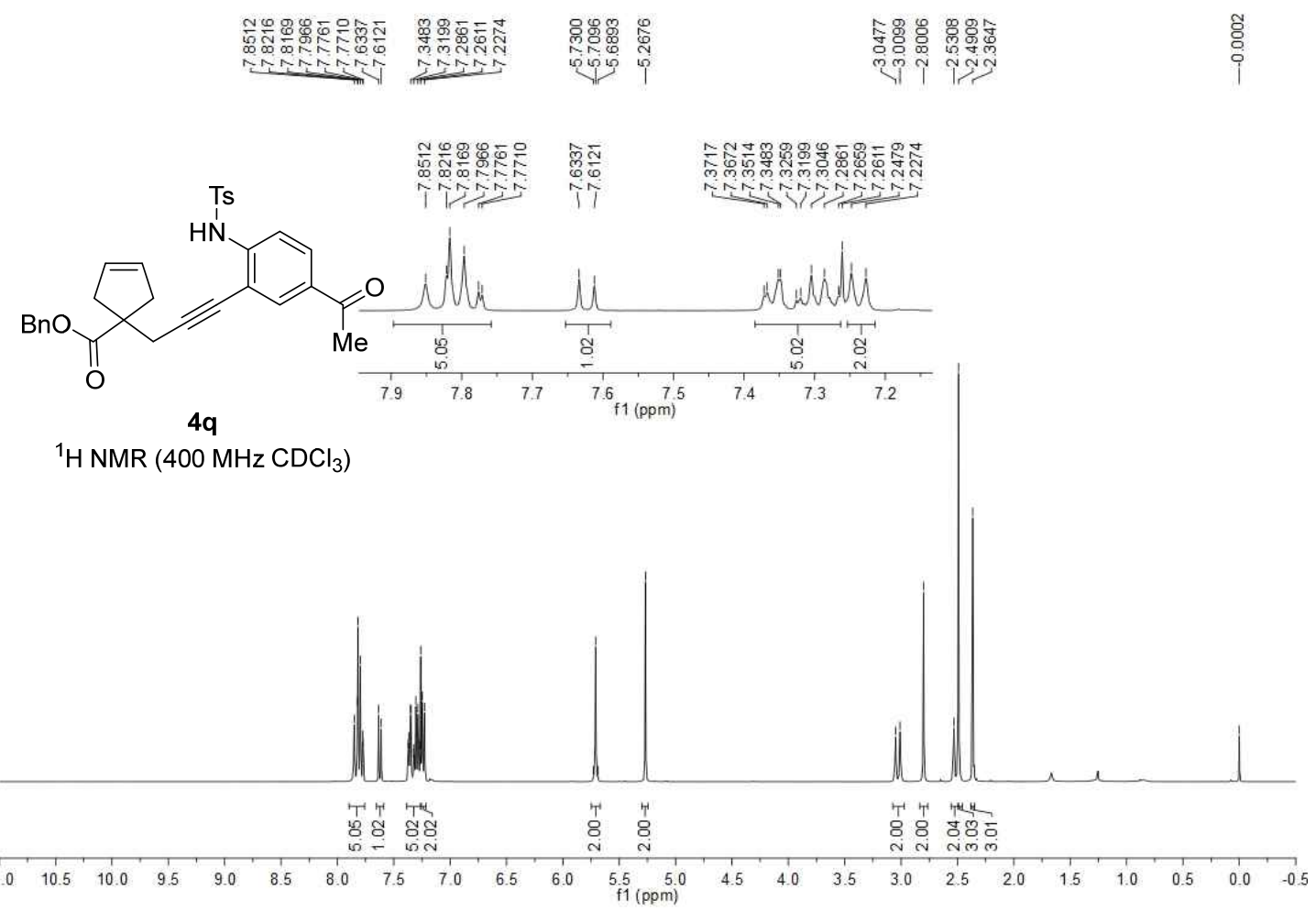

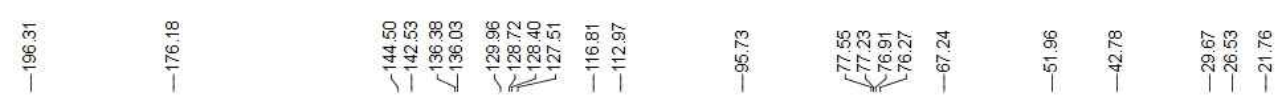<smiles>CNc1ccc(C(C)=O)cc1C#CCC1(C(=O)OCc2ccccc2)CC=CC1</smiles>

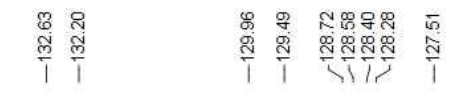

${ }^{13} \mathrm{C}$ NMR $\left(100 \mathrm{MHz} \mathrm{CDCl}_{3}\right)$
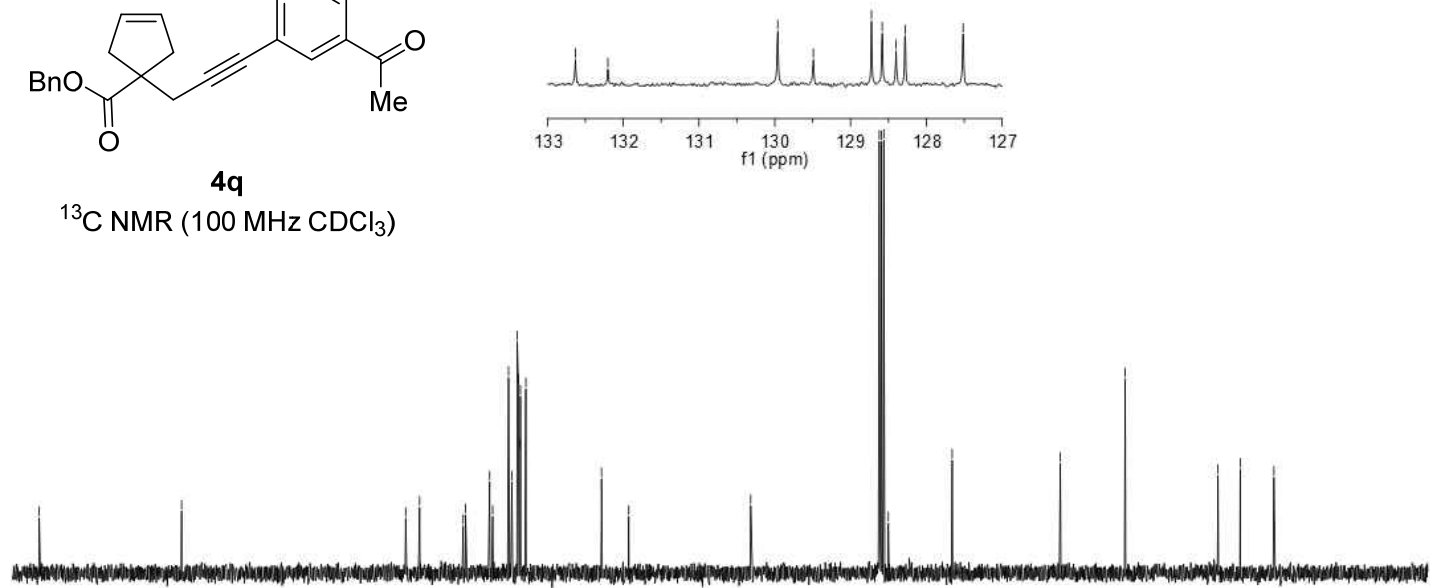

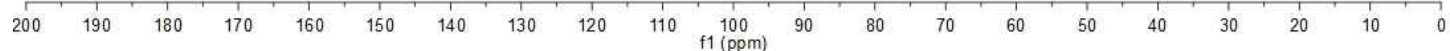



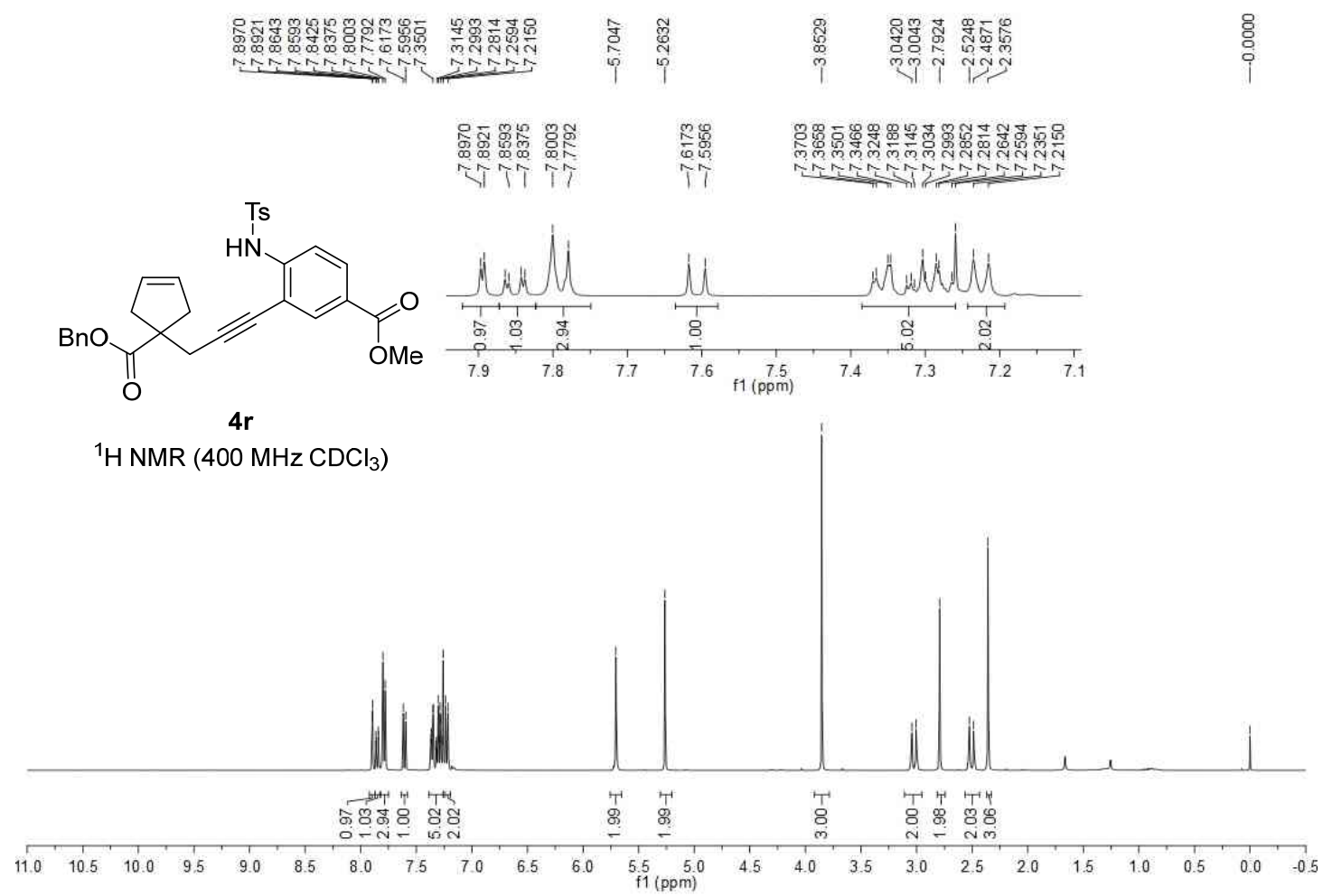

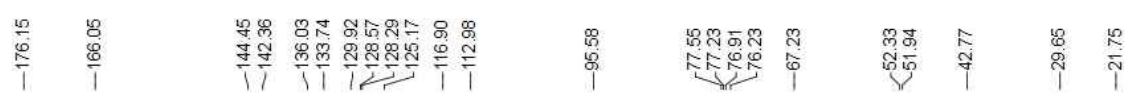
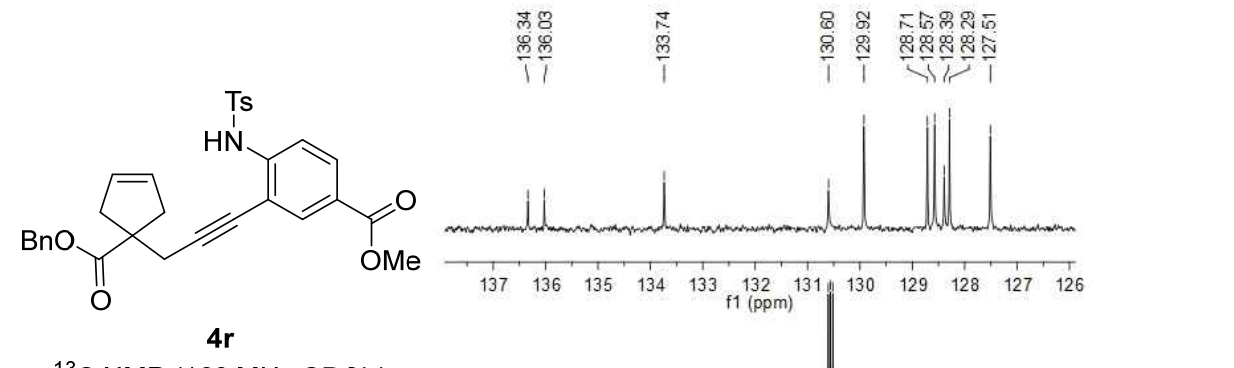

${ }^{13} \mathrm{C} \mathrm{NMR} \mathrm{(100} \mathrm{MHz} \mathrm{CDCl} 3$ )

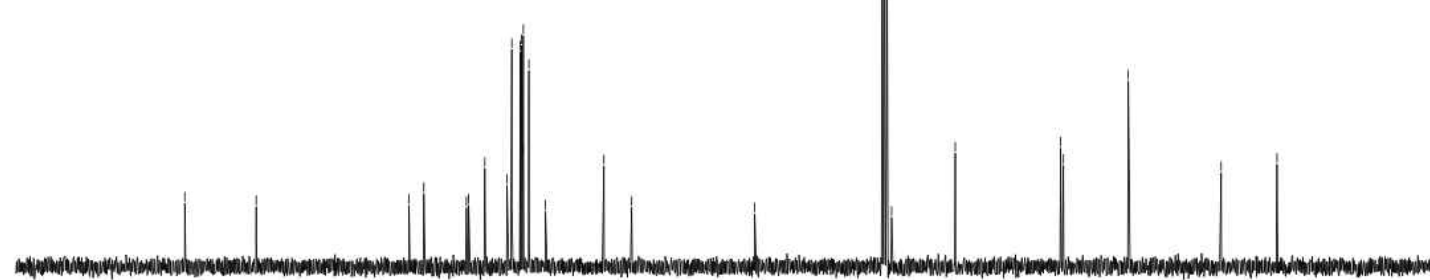

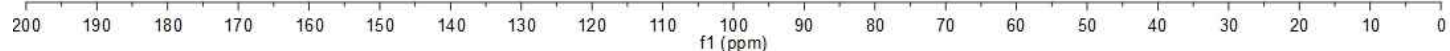



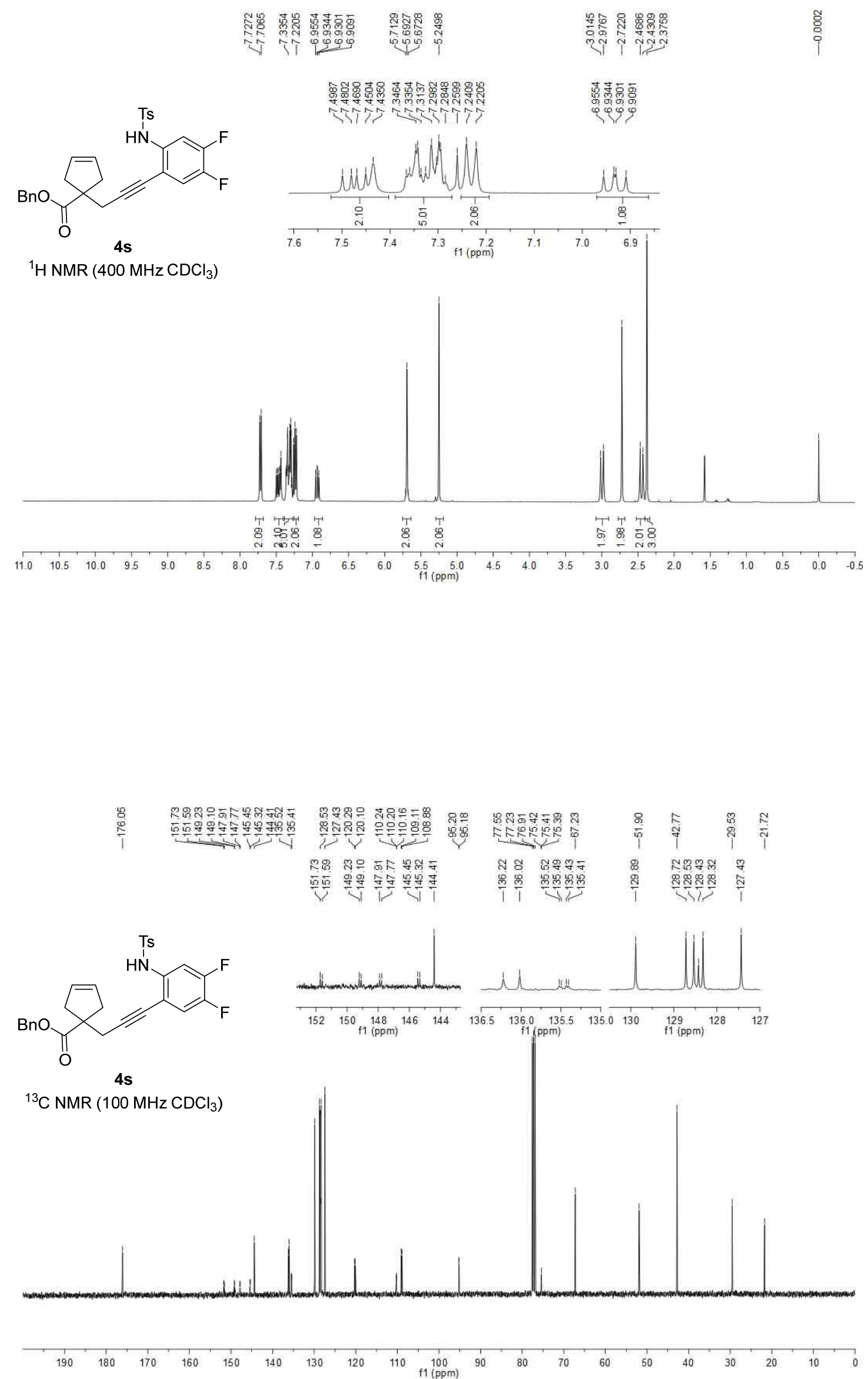


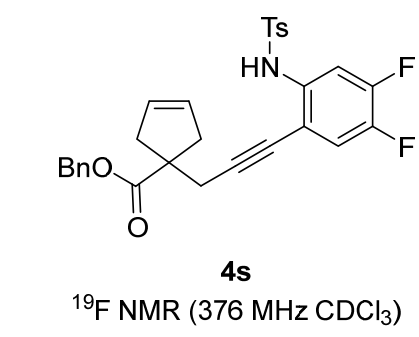

$\left.{ }^{19} \mathrm{~F} \mathrm{NMR} \mathrm{(376} \mathrm{MHz} \mathrm{CDCl}_{3}\right)$

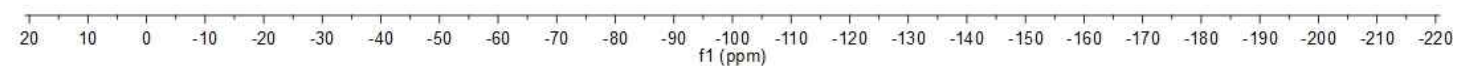



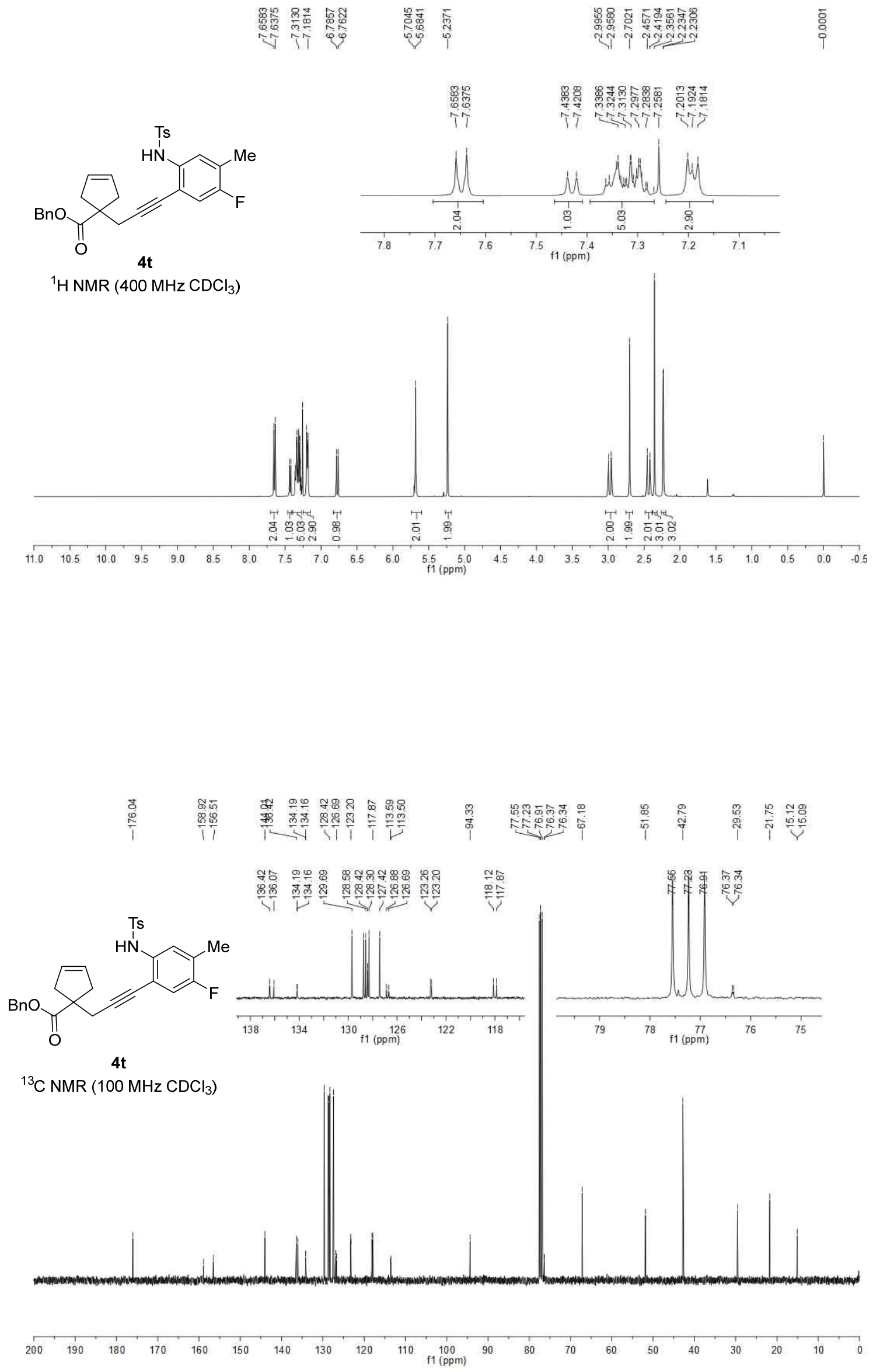


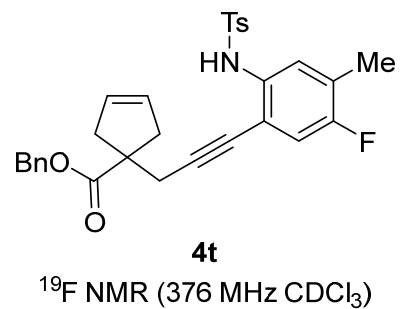

${ }^{19} \mathrm{~F} \mathrm{NMR}\left(376 \mathrm{MHz} \mathrm{CDCl}_{3}\right)$

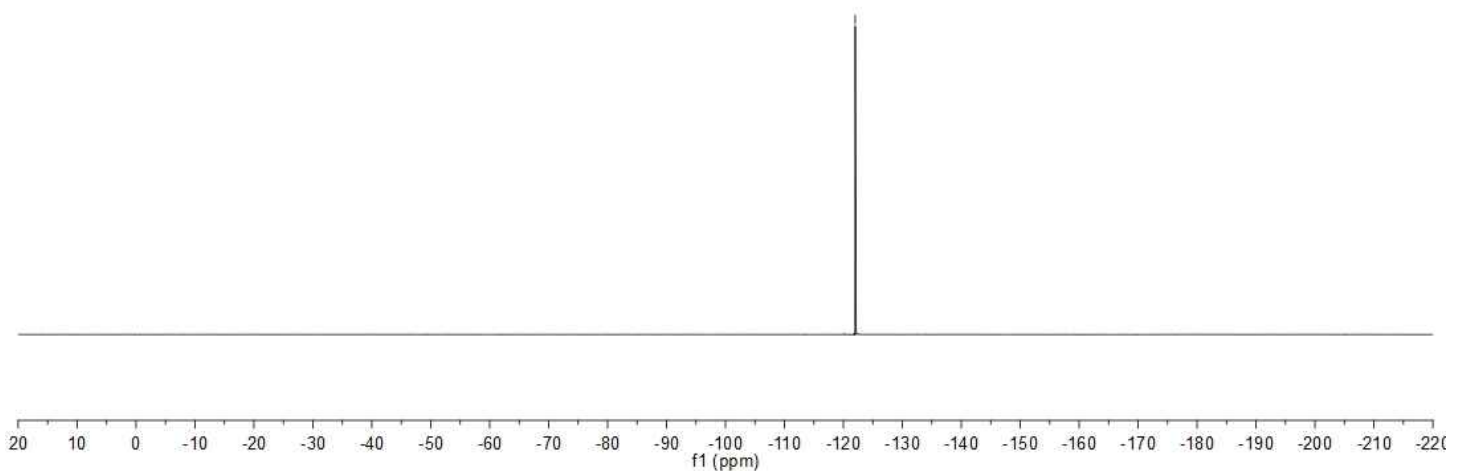



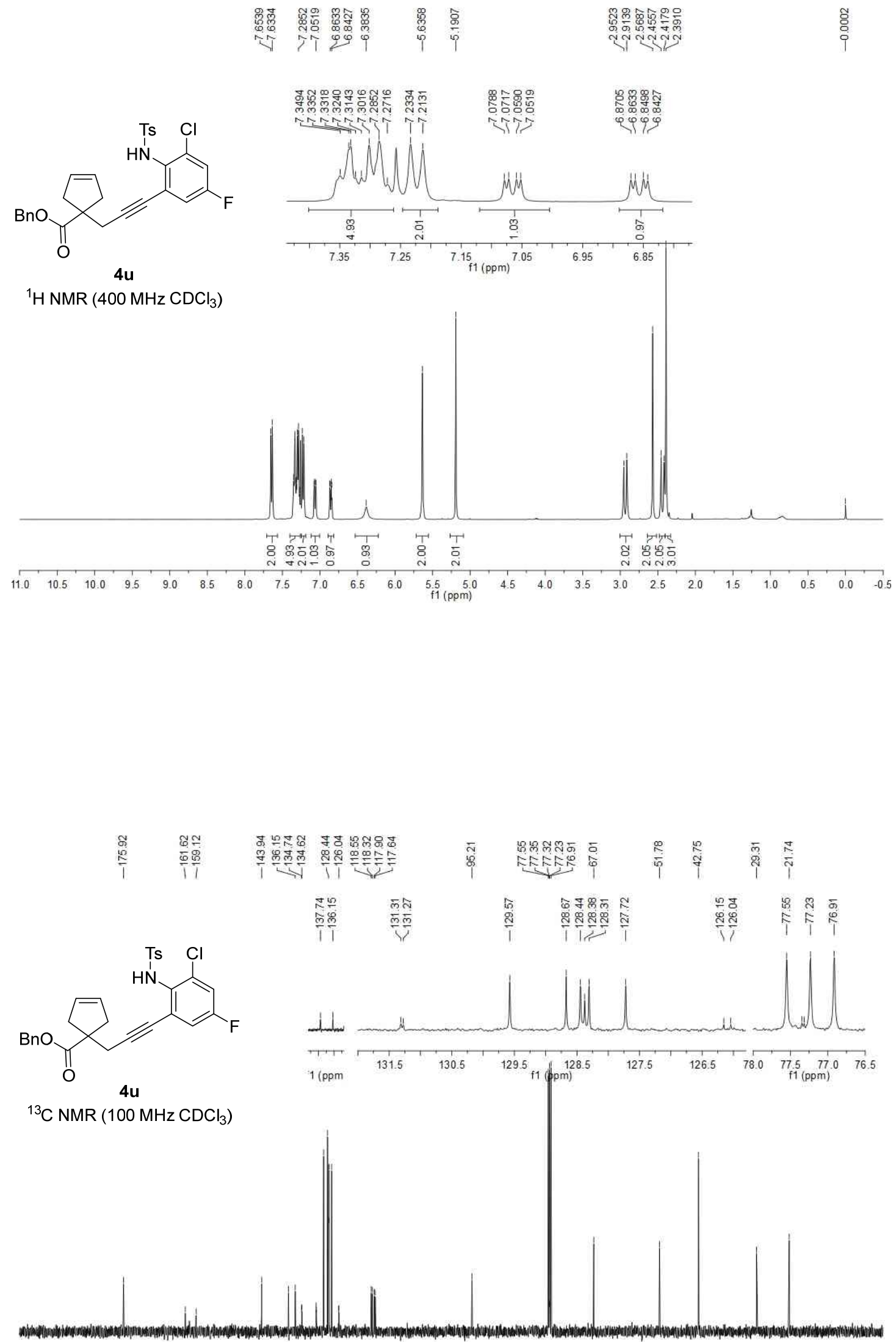

$\begin{array}{lllllllllllllllllllll}200 & 190 & 180 & 170 & 160 & 150 & 140 & 130 & 120 & 110 & 100 & 90 & 80 & 70 & 60 & 50 & 40 & 30 & 20 & 10 & 0\end{array}$ 


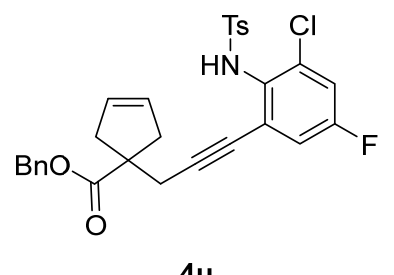

$\left.{ }^{19} \mathrm{~F} \mathrm{NMR} \mathrm{(376} \mathrm{MHz} \mathrm{CDCl}_{3}\right)$

$\begin{array}{lllllllllllllllllllllllllllllllll}20 & 10 & 0 & -10 & -20 & -30 & -40 & -50 & -60 & -70 & -80 & -90 & -100 & -110 & -120 & -130 & -140 & -150 & -160 & -170 & -180 & -190 & -200 & -210 & -220\end{array}$ 

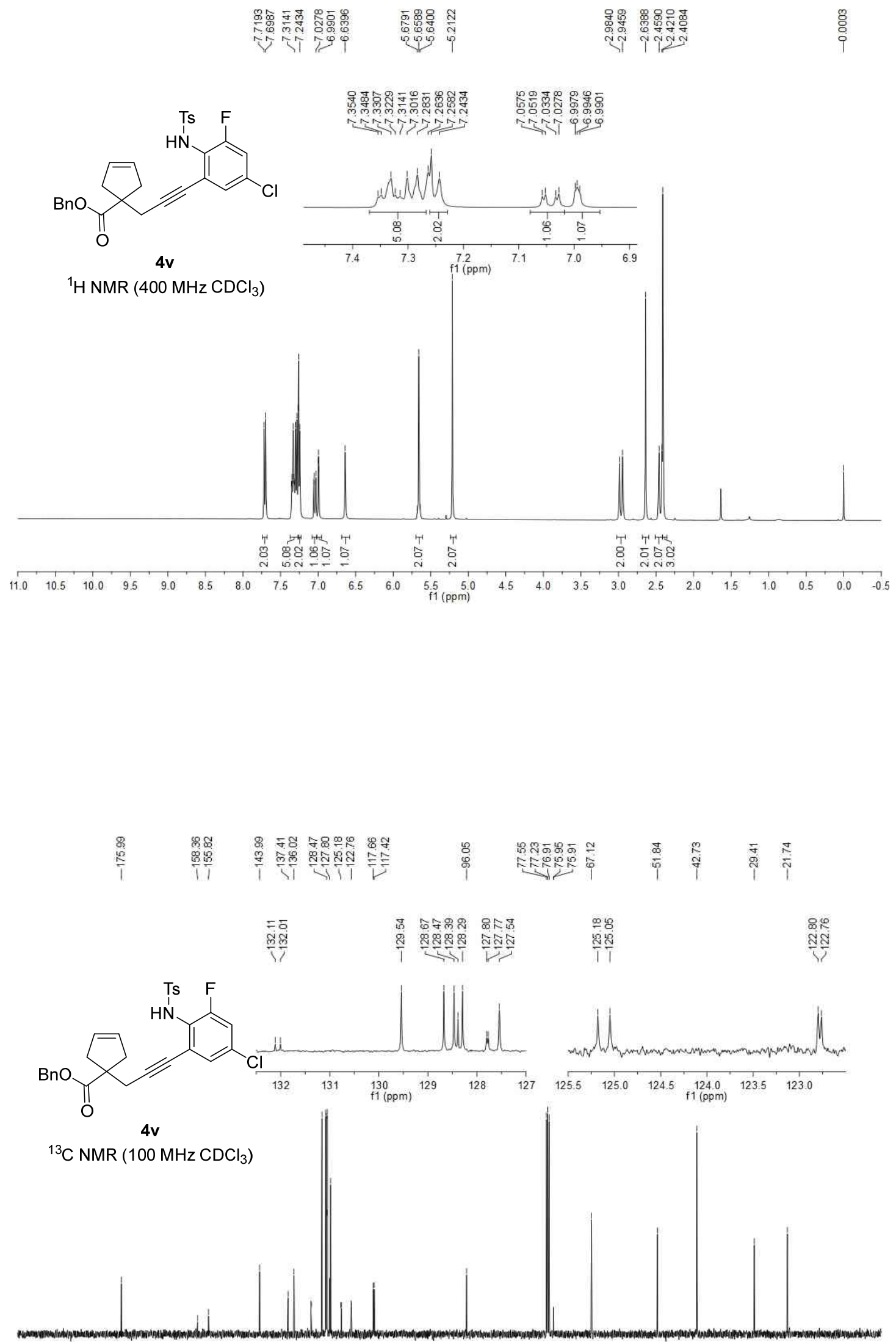

$\begin{array}{lllllllllllllllllllll}200 & 190 & 180 & 170 & 160 & 150 & 140 & 130 & 120 & 110 & 100 & 90 & 80 & 70 & 60 & 50 & 40 & 30 & 20 & 10 & 0\end{array}$ 


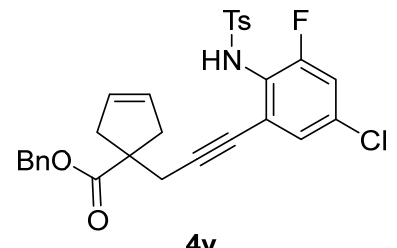

${ }^{19} \mathrm{~F} \mathrm{NMR} \mathrm{(376} \mathrm{MHz} \mathrm{CDCl}_{3}$ )

$\begin{array}{llllllllllllllllllllllllllllll}20 & 10 & 0 & -10 & -20 & -30 & -40 & -50 & -60 & -70 & -80 & -90 & -100 & -110 & -120 & -130 & -140 & -150 & -160 & -170 & -180 & -190 & -200 & -210 & -220\end{array}$ 


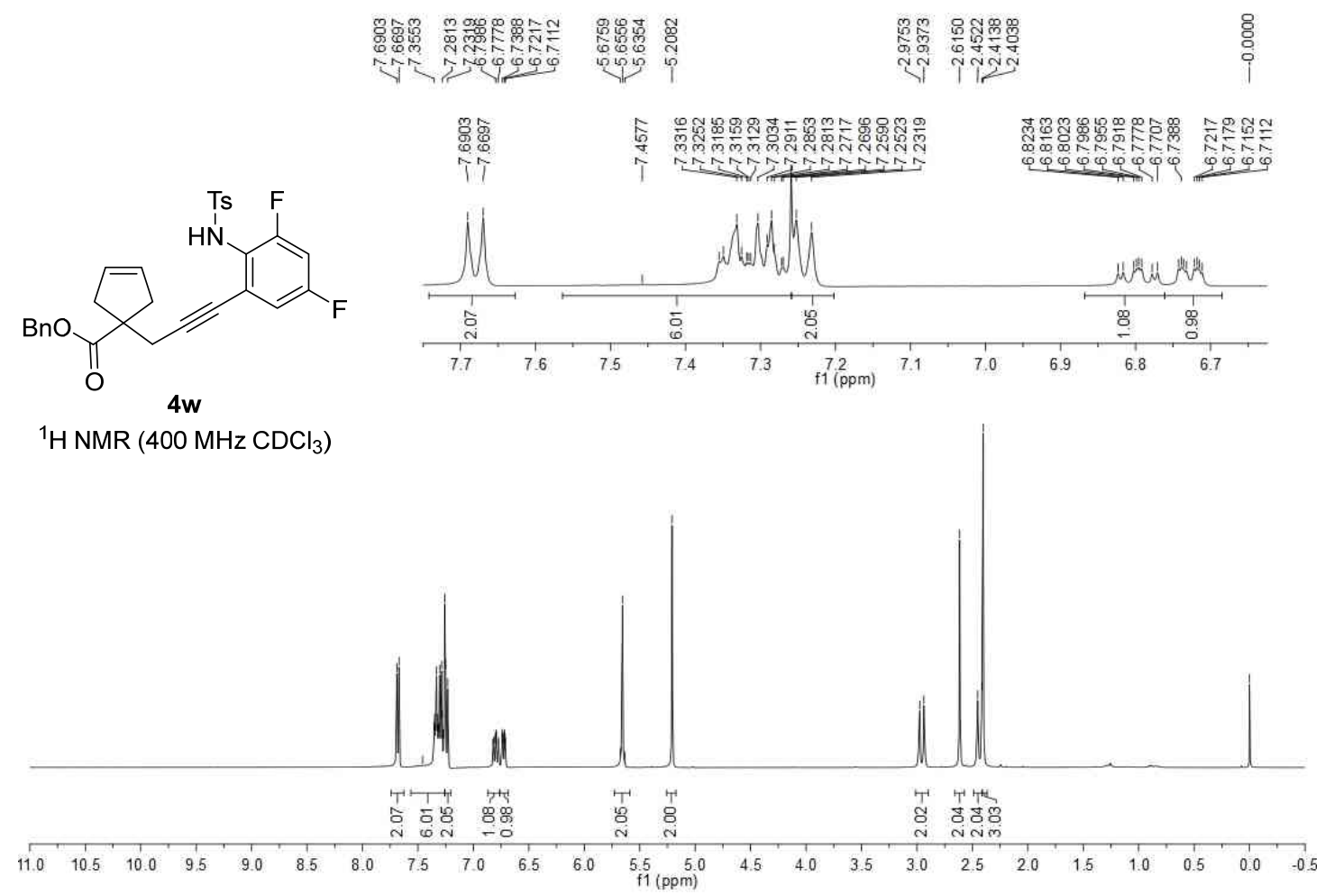

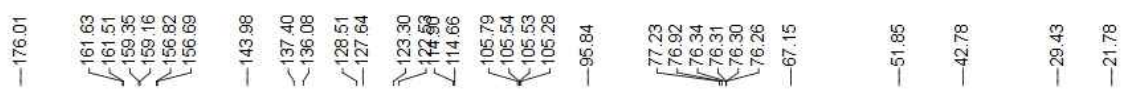

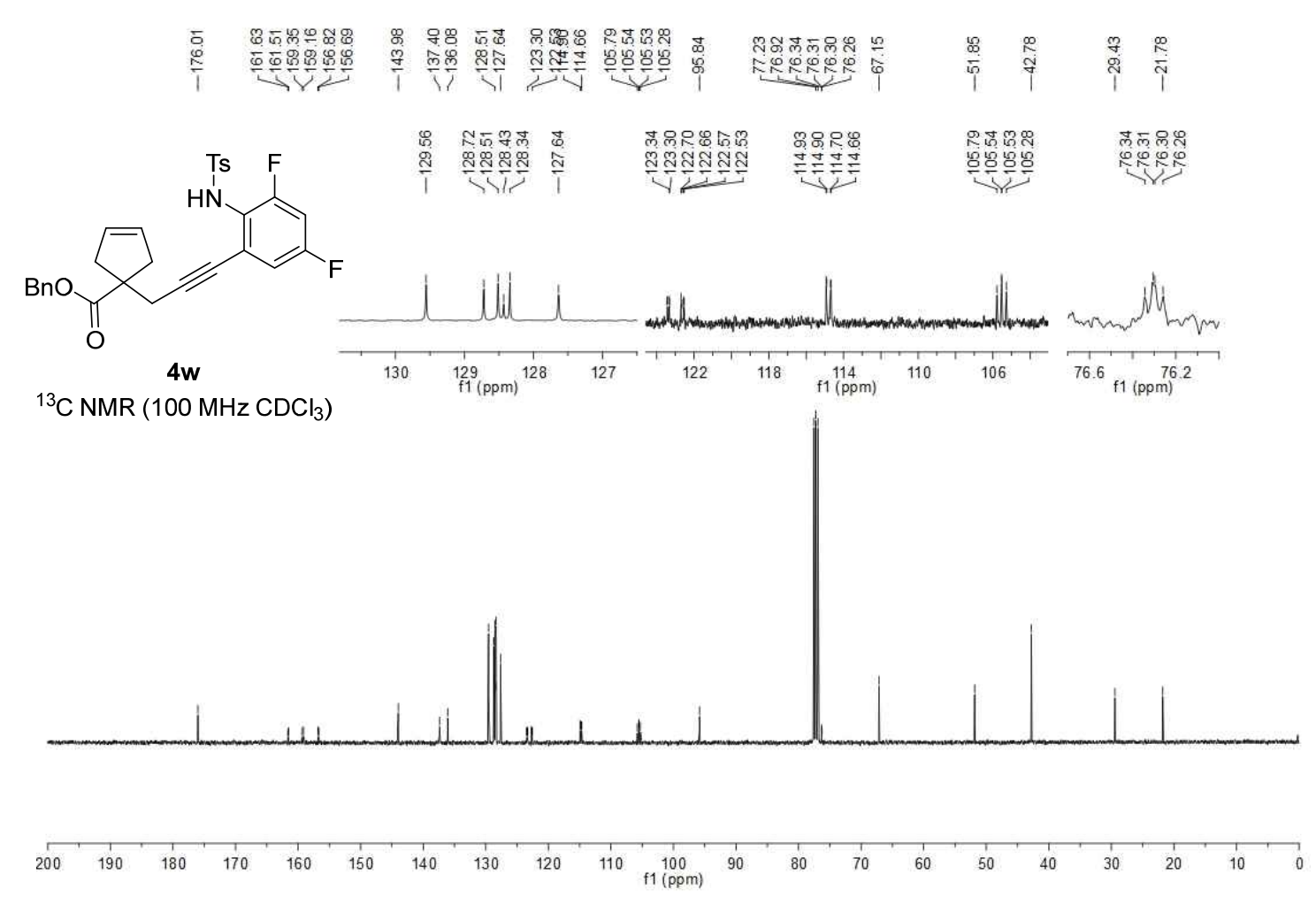

$\left.{ }^{13} \mathrm{C} \mathrm{NMR} \mathrm{(100} \mathrm{MHz} \mathrm{CDCl}_{3}\right)$ 


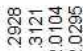

튼둘둔

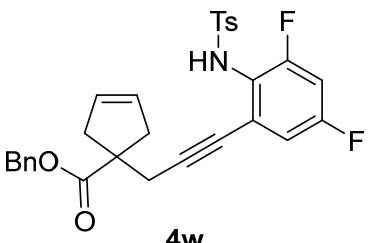

${ }^{19} \mathrm{~F} \mathrm{NMR}\left(376 \mathrm{MHz} \mathrm{CDCl}_{3}\right)$

$\begin{array}{lllllllllllllllllllllllll}20 & 10 & 0 & -10 & -20 & -30 & -40 & -50 & -60 & -70 & -80 & -90 & -100 & -110 & -120 & -130 & -140 & -150 & -160 & -170 & -180 & -190 & -200 & -210 & -220\end{array}$ 

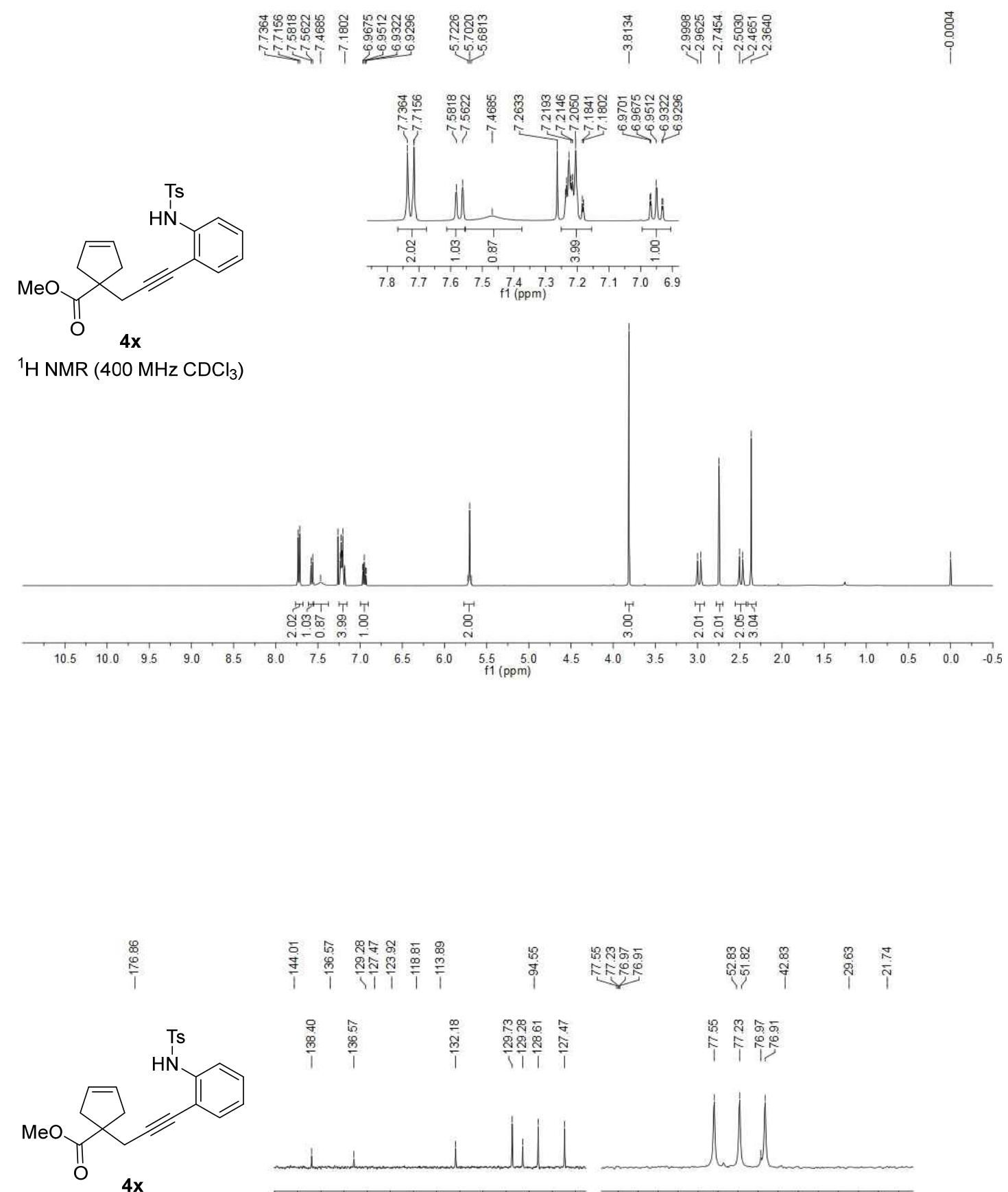

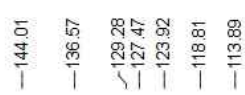

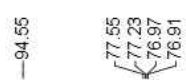

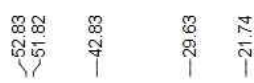

${ }^{13} \mathrm{C}$ NMR $\left(100 \mathrm{MHz} \mathrm{CDCl}_{3}\right)$
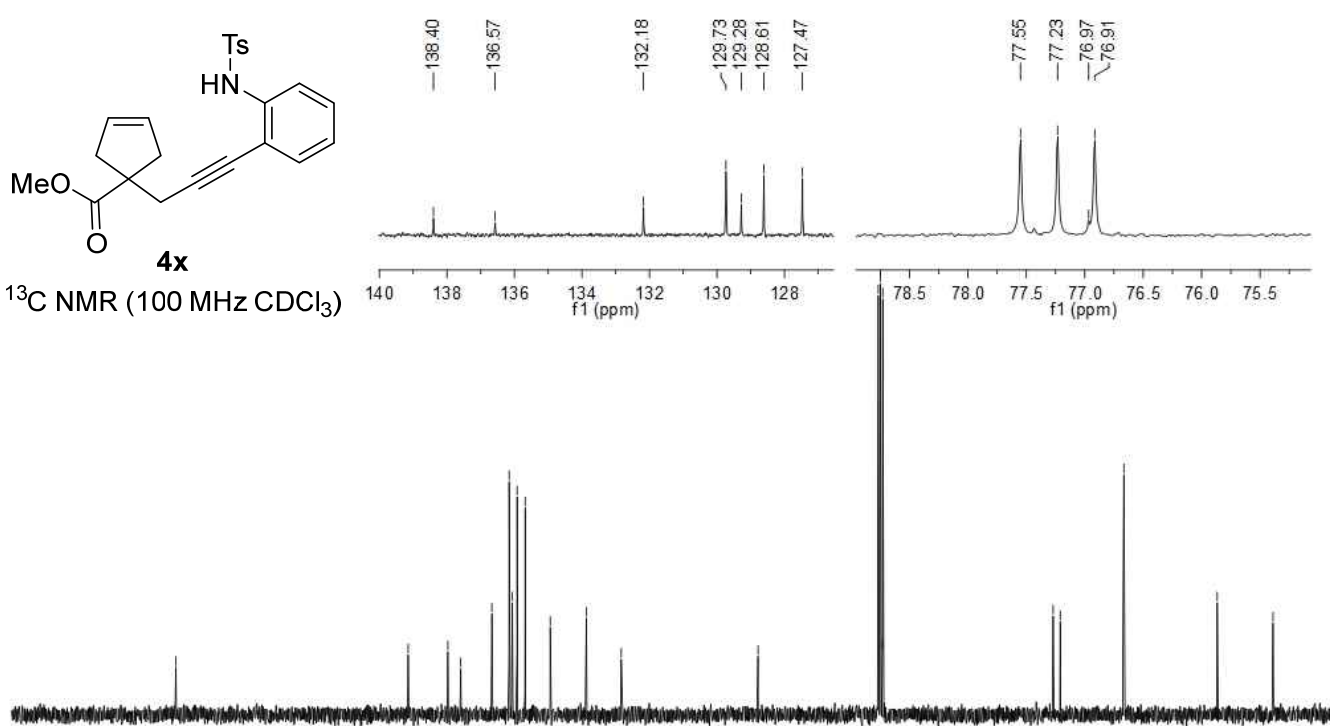

\begin{tabular}{llllllllllllllllllllllll}
\hline 200 & 190 & 180 & 170 & 160 & 150 & 140 & 130 & 120 & 110 & 100 & 90 & 80 & 70 & 60 & 50 & 40 & 30 & 20 & 10 & 1
\end{tabular} 

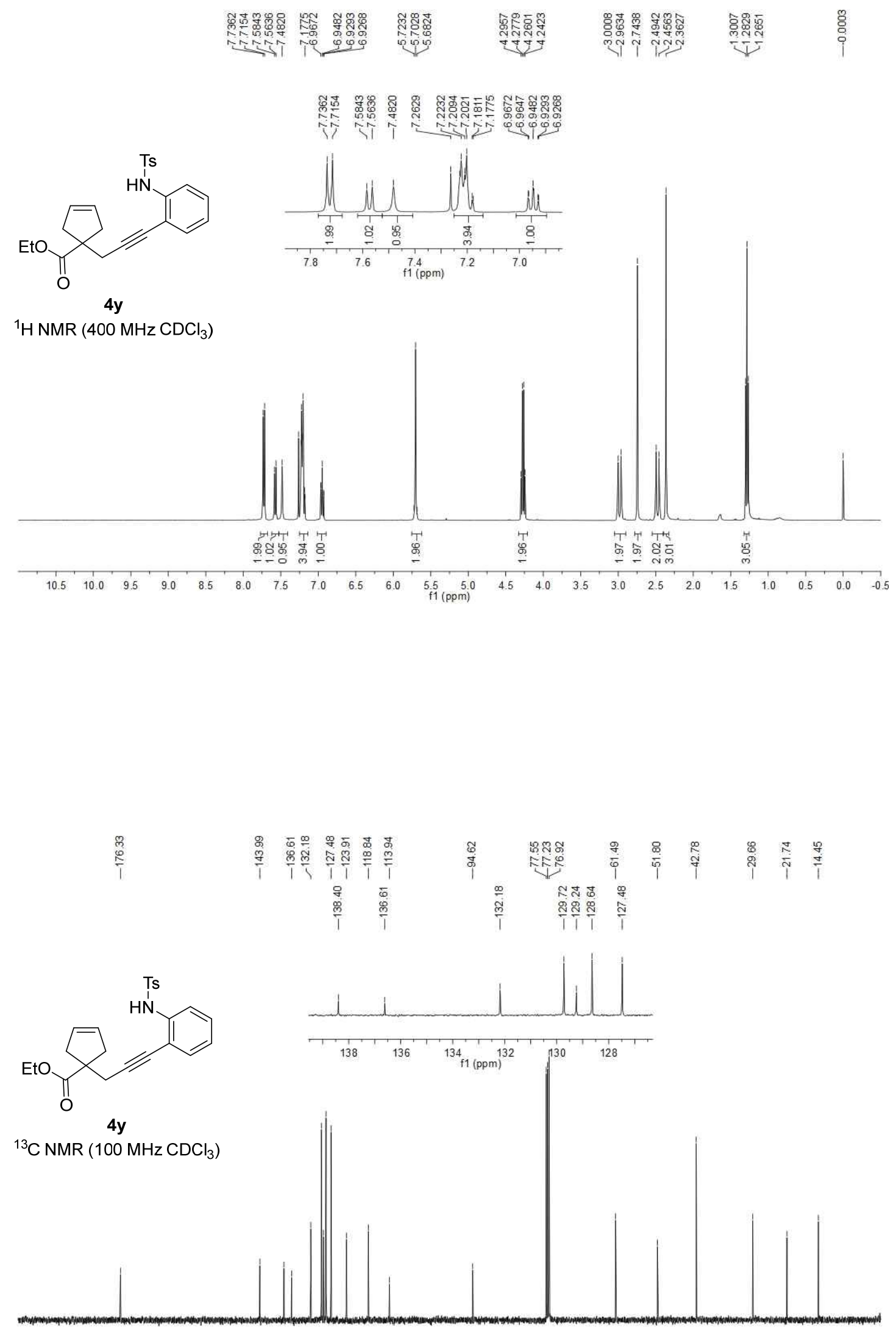

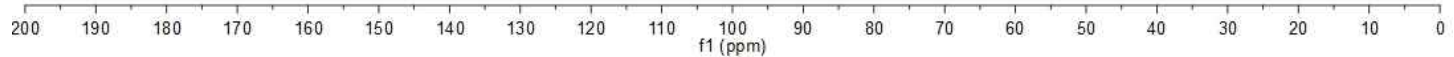



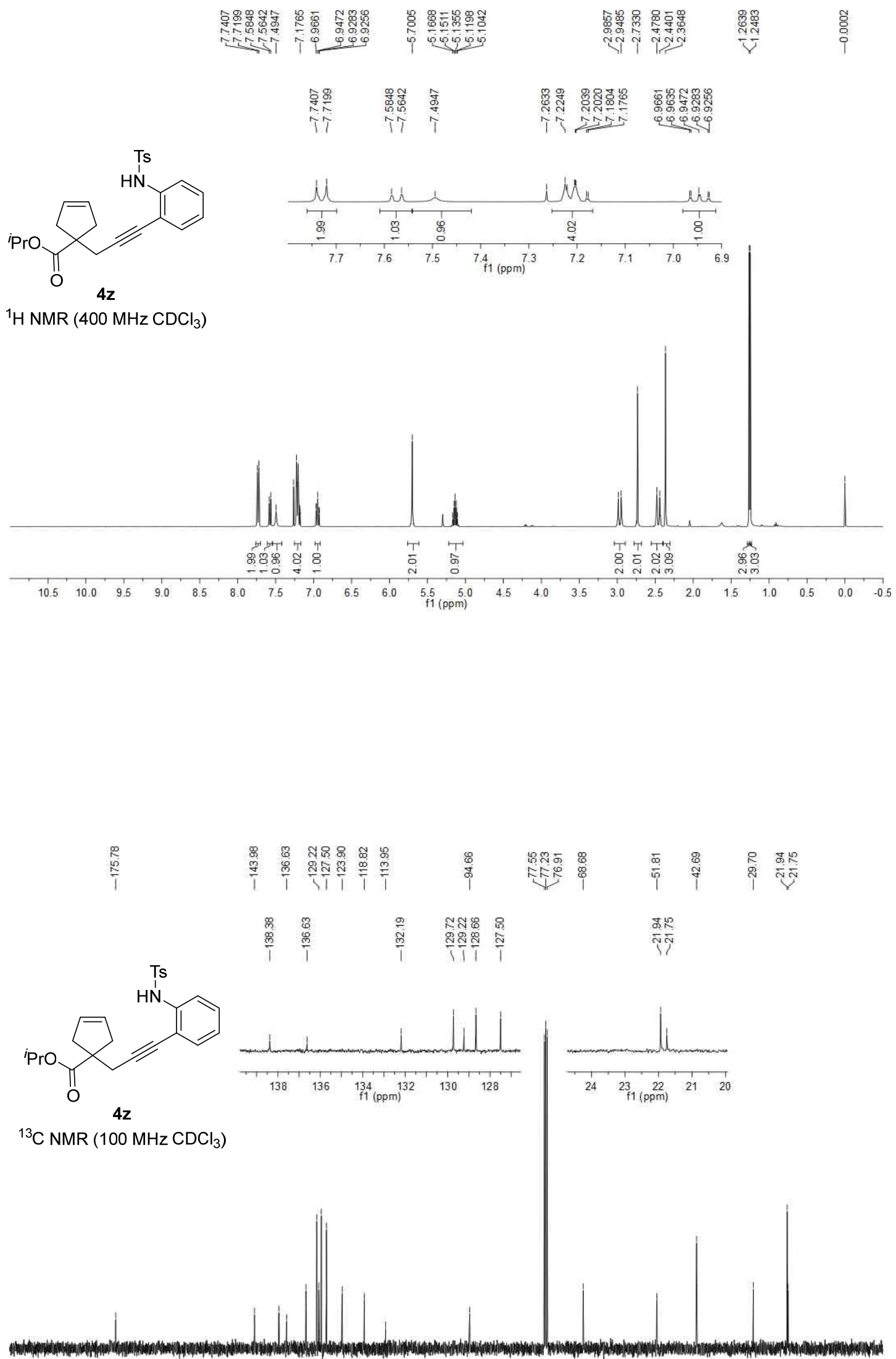

\begin{tabular}{lllllllllllllllllllllllll}
\hline 200 & 190 & 180 & 170 & 160 & 150 & 140 & 130 & 120 & 110 & 100 & 90 & 80 & 70 & 60 & 50 & 40 & 30 & 20 & 10 & 0
\end{tabular} 

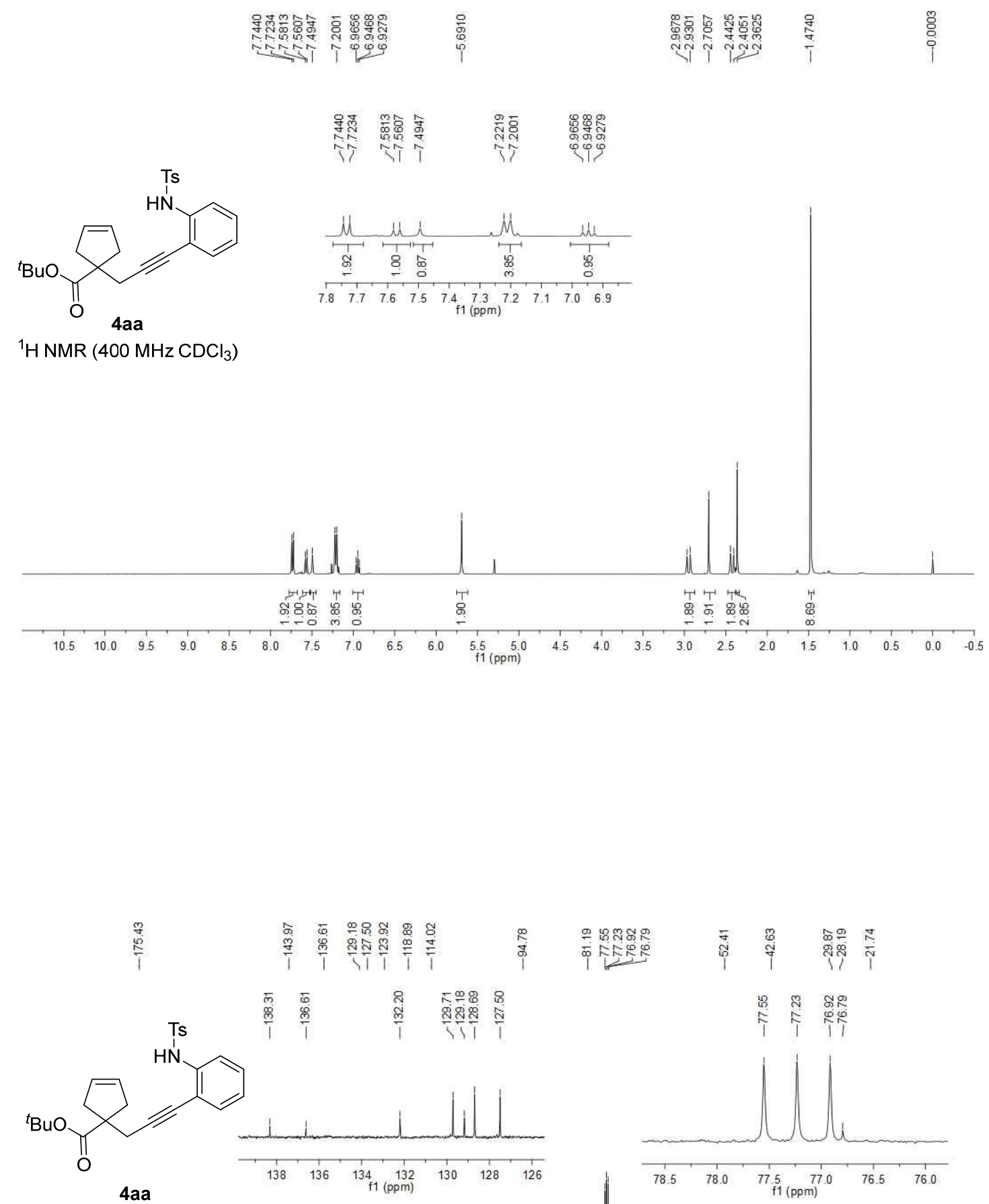

${ }^{13} \mathrm{C} \mathrm{NMR}\left(100 \mathrm{MHz} \mathrm{CDCl}_{3}\right)$

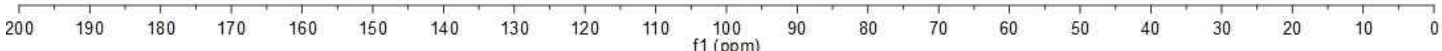



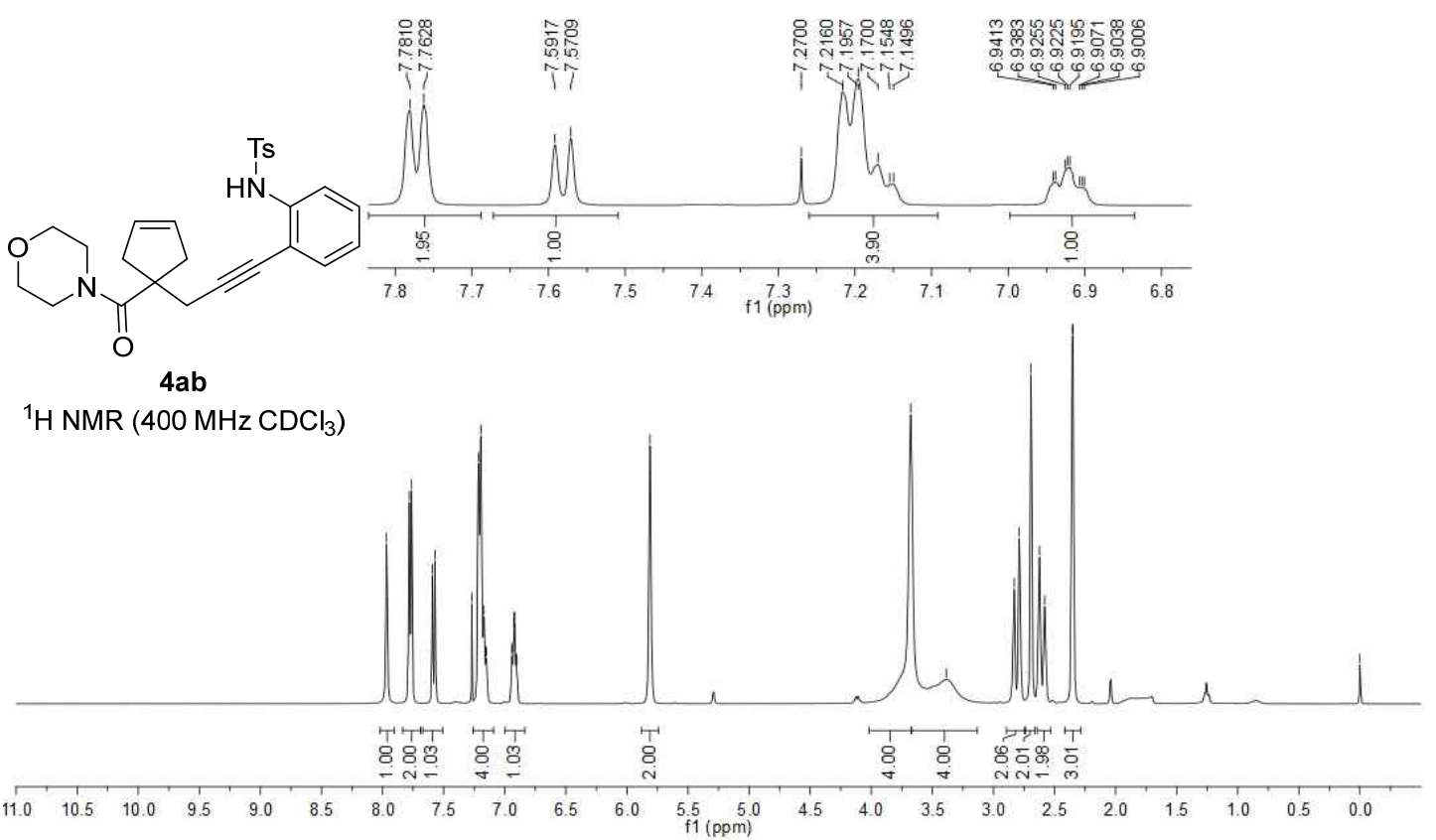

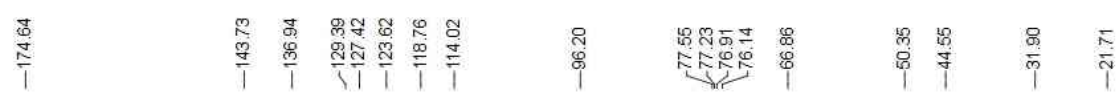

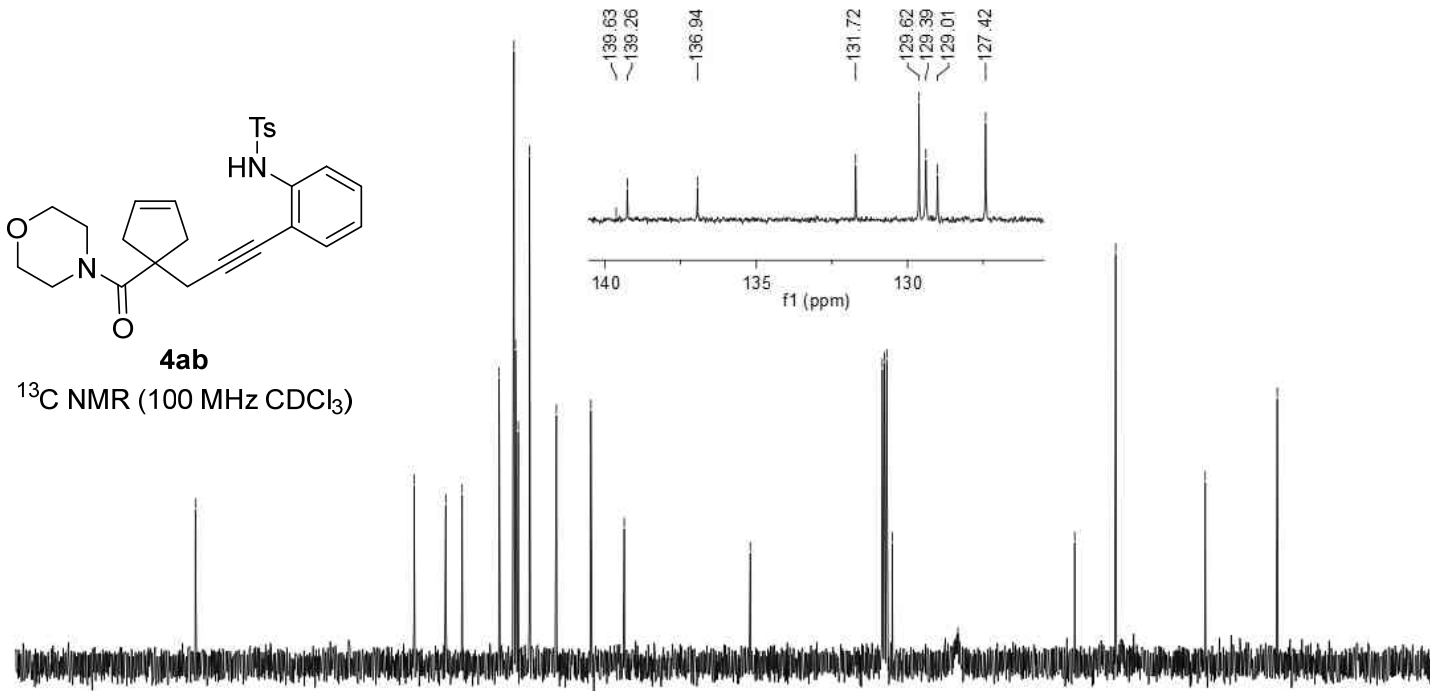

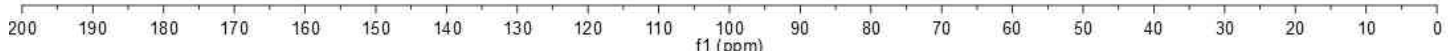



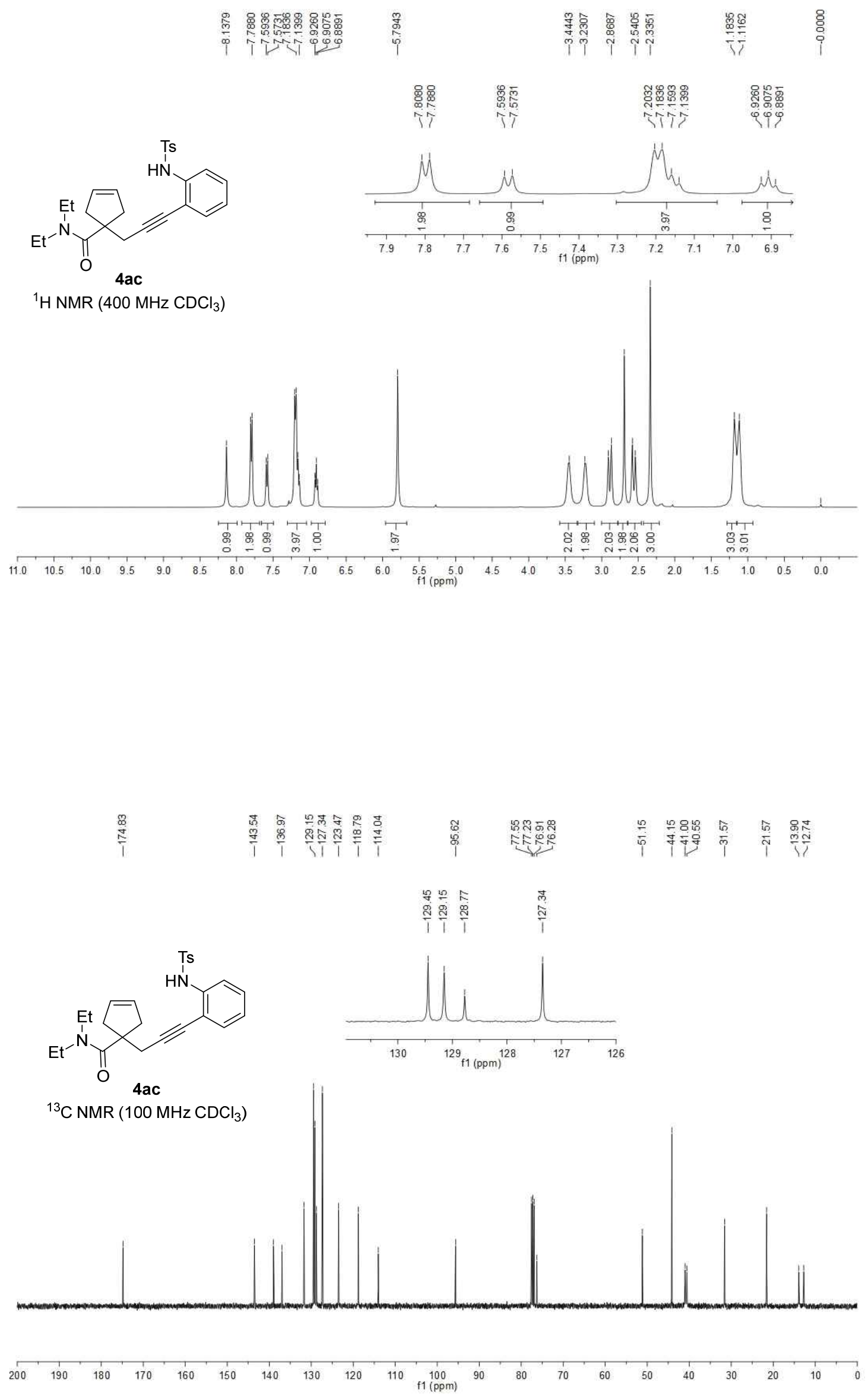

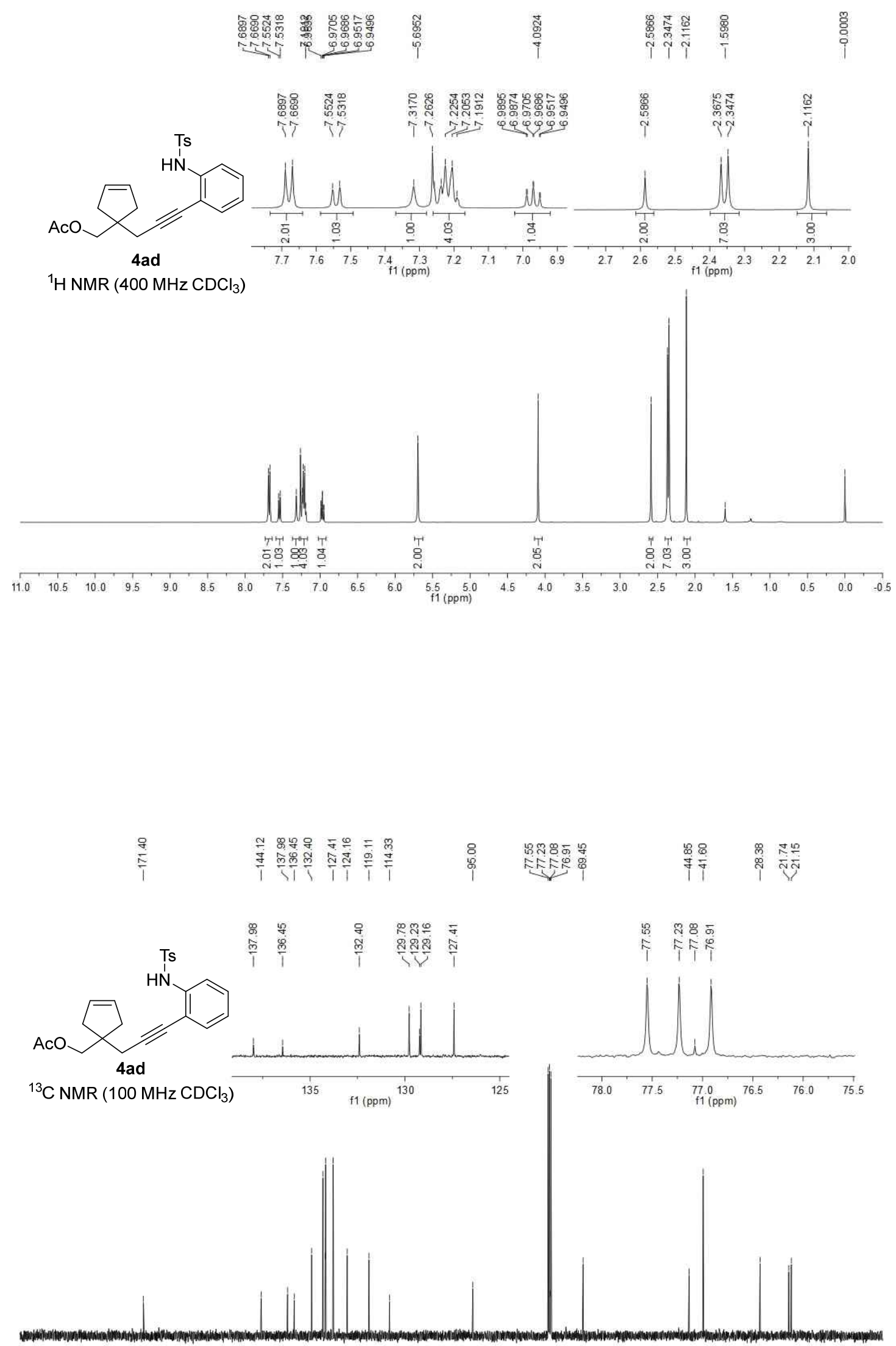

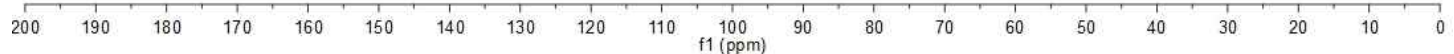



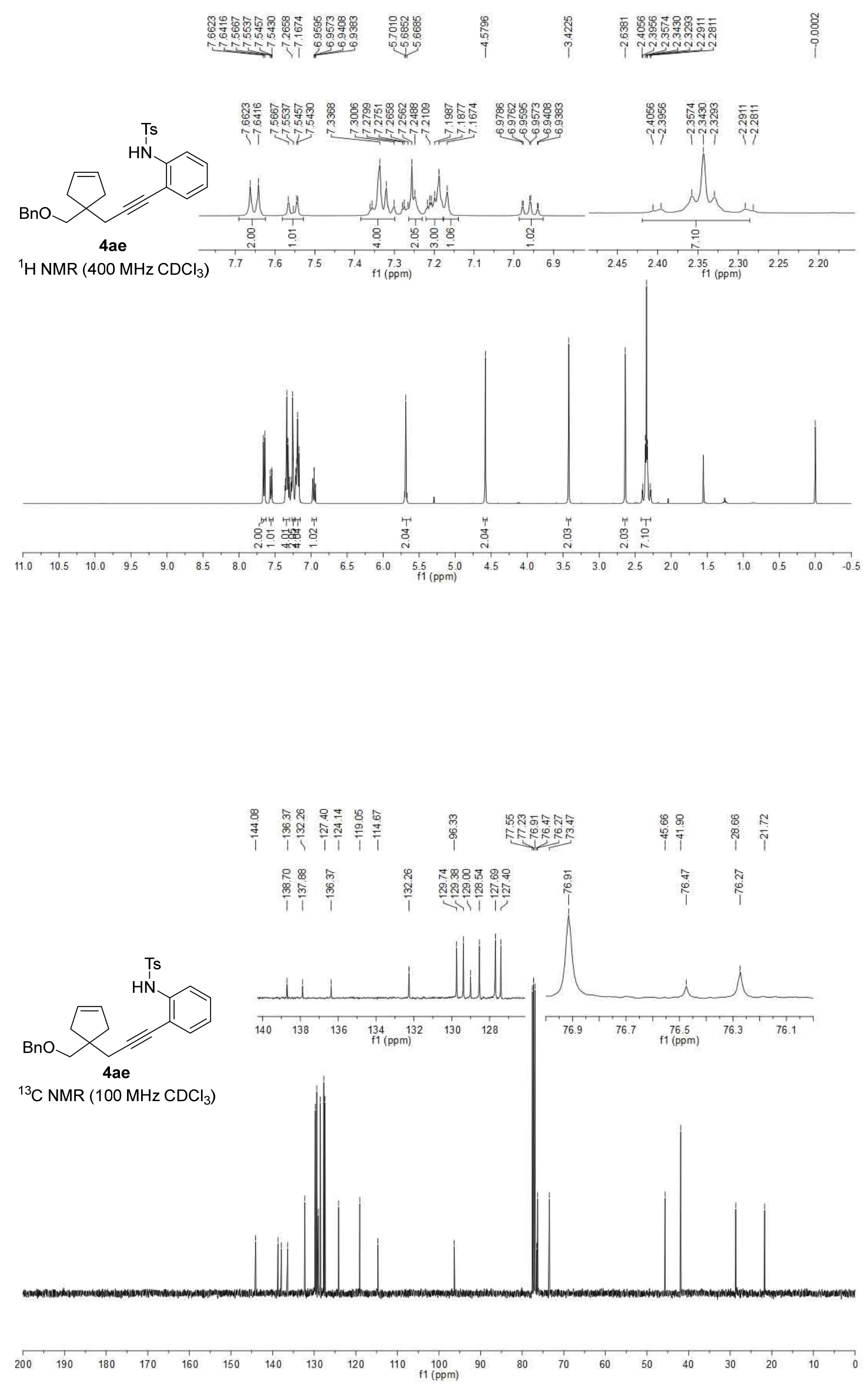

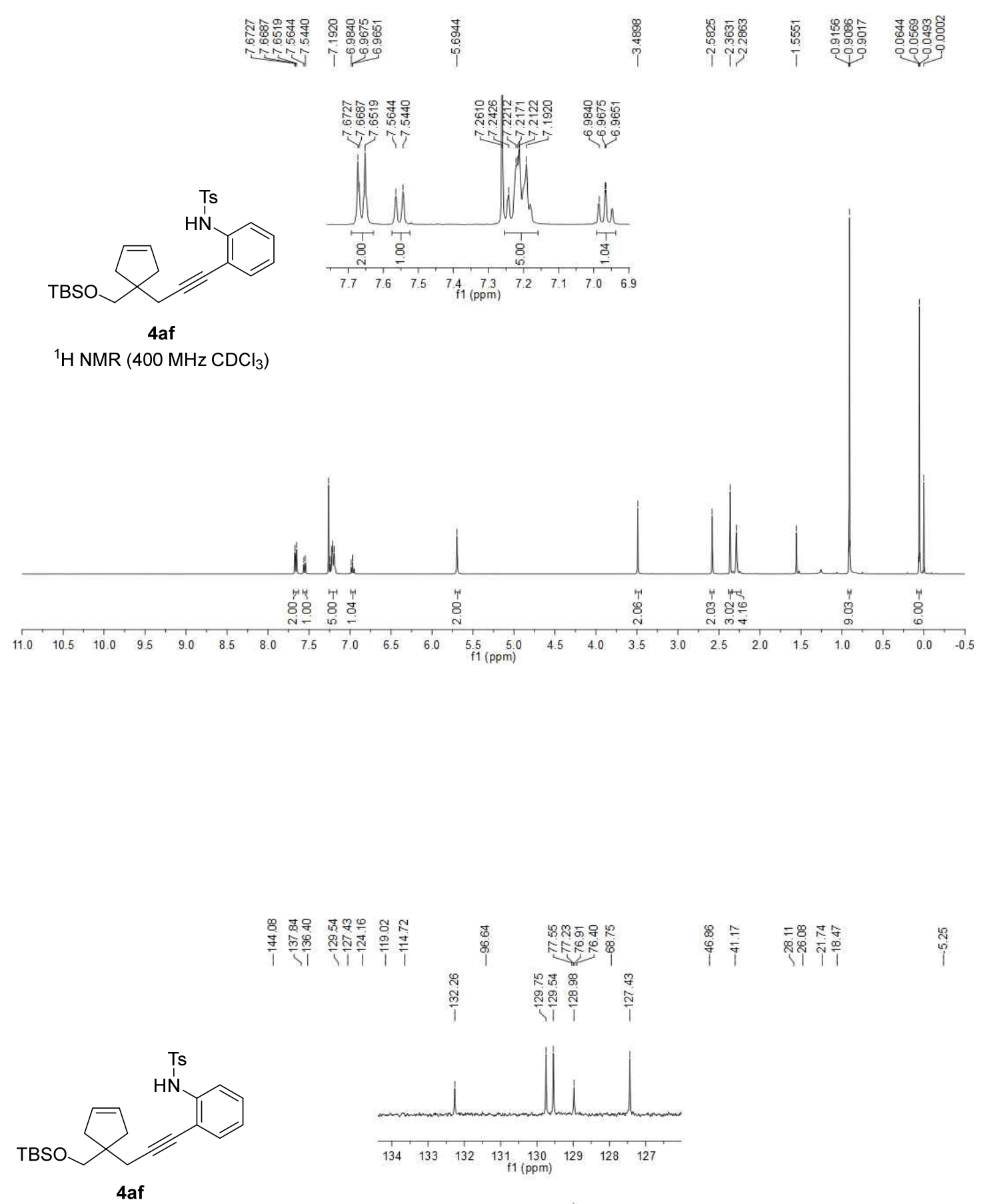

${ }^{13} \mathrm{C}$ NMR $\left(100 \mathrm{MHz} \mathrm{CDCl}_{3}\right)$

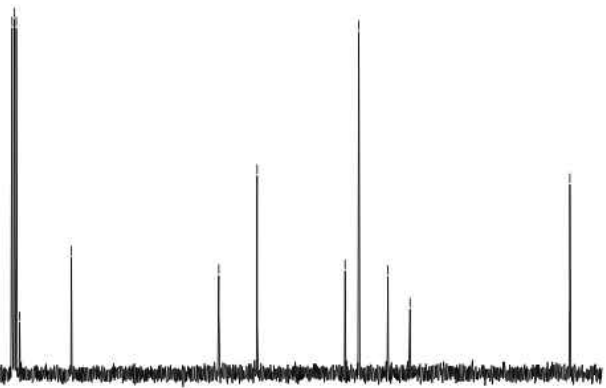

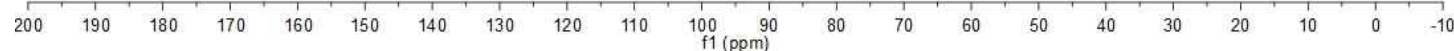



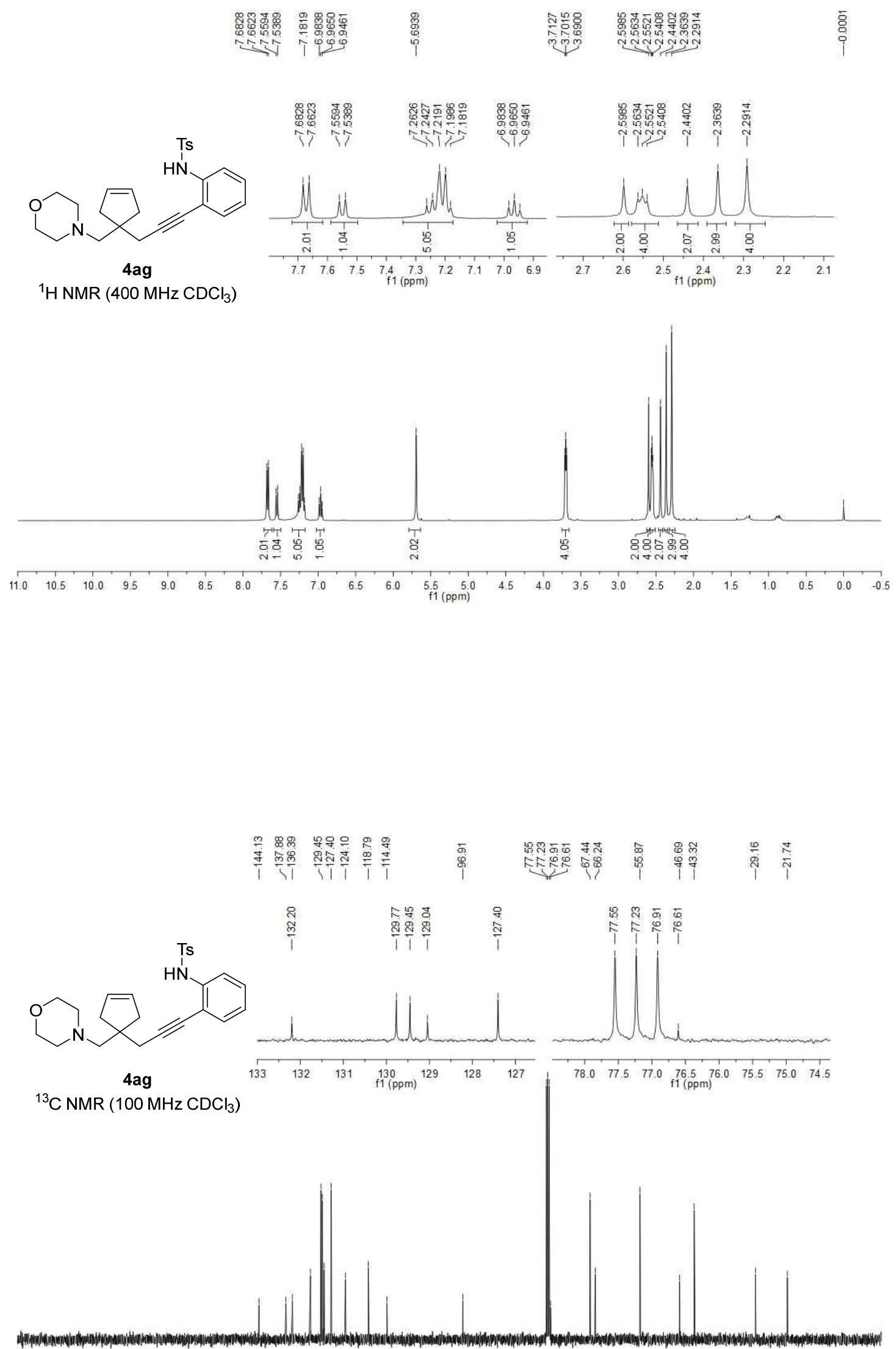

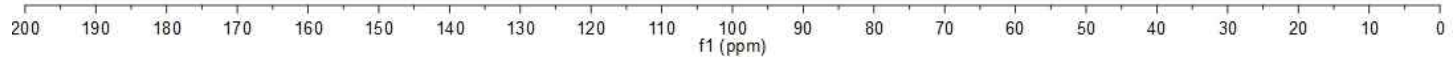


<smiles>O=C(Cc1ccccc1)C12C=CC(C1)c1c2c2ccccc2n1[AsH3]</smiles>

3a

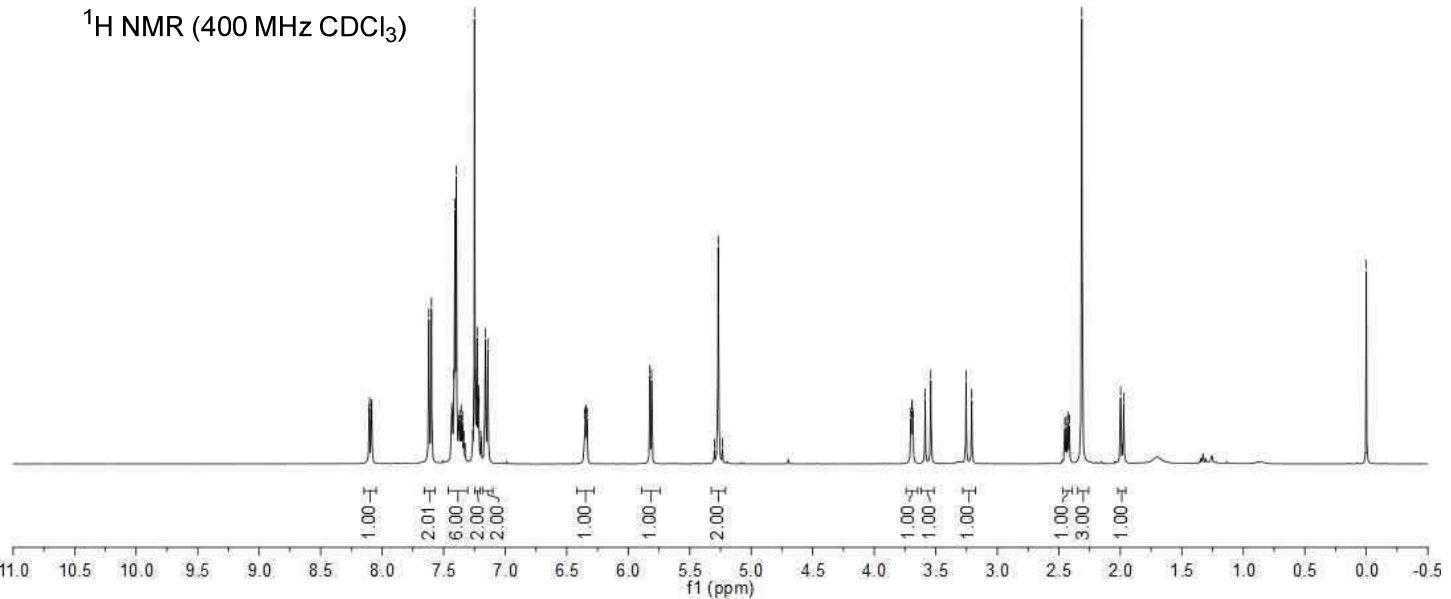

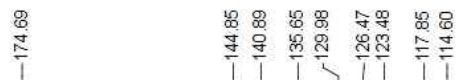

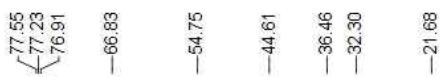<smiles></smiles>

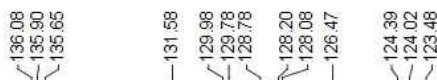

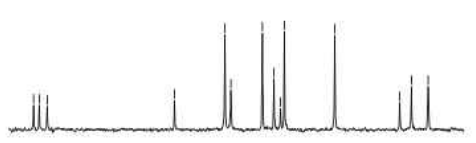

${ }^{13} \mathrm{C} \mathrm{NMR}\left(100 \mathrm{MHz} \mathrm{CDCl}_{3}\right)$
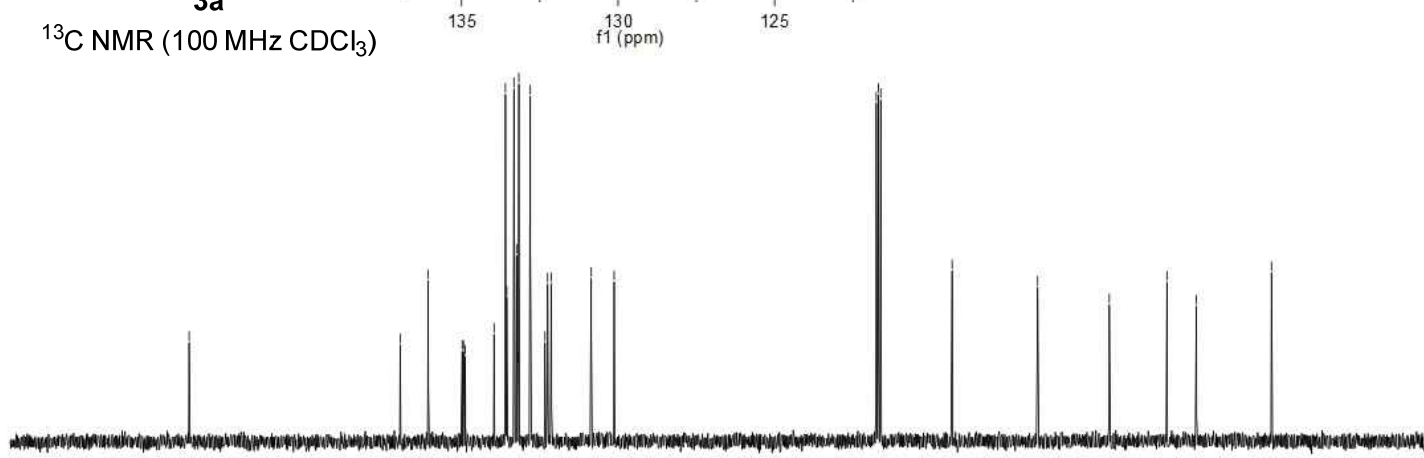

200

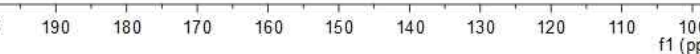




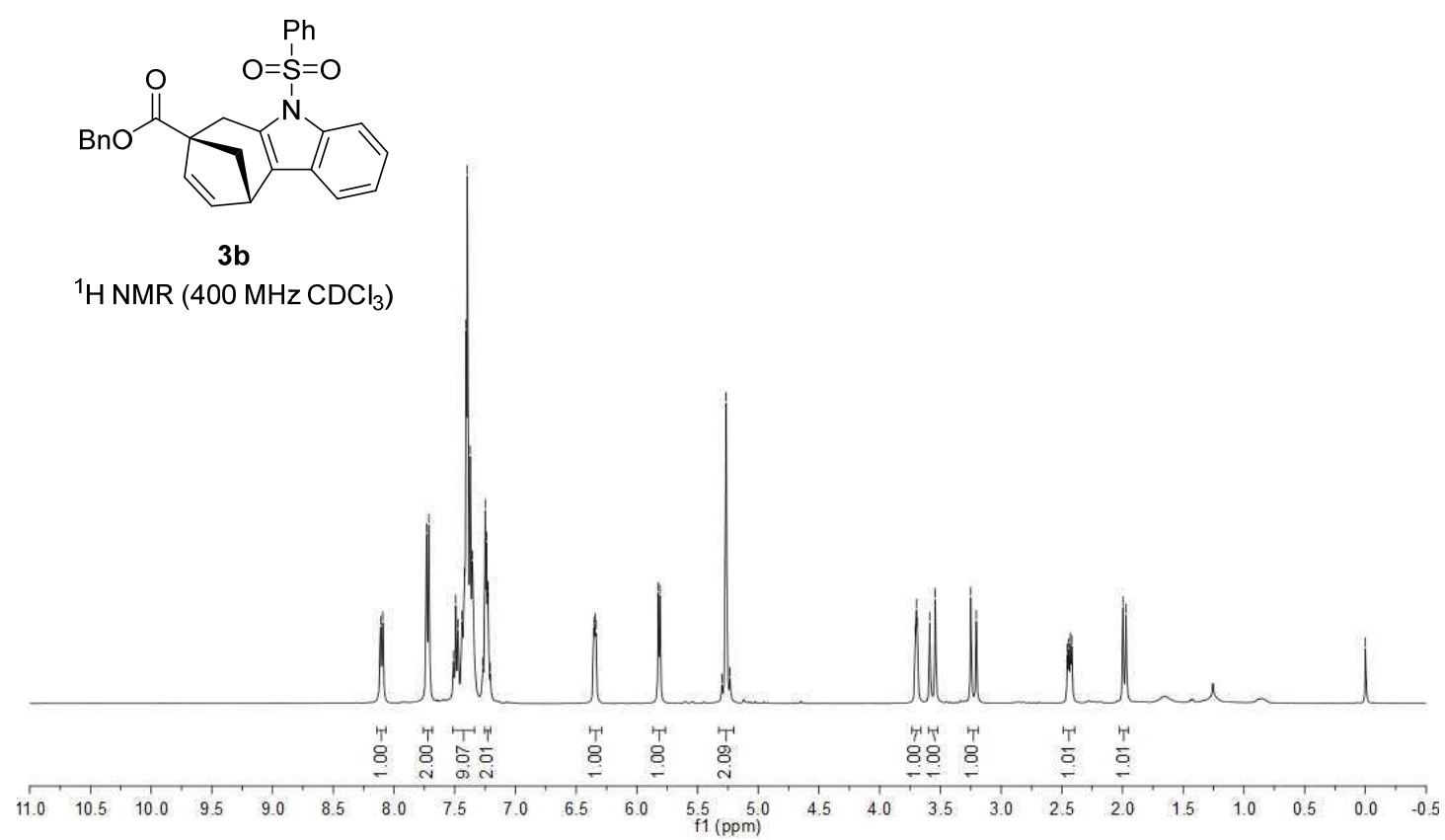

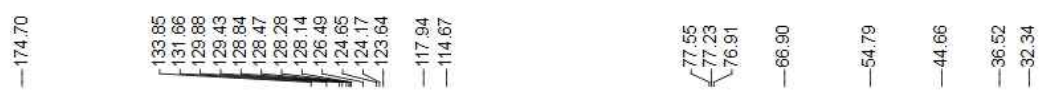<smiles>O=C(OCc1ccccc1)C12C=CC(C1)c1c2n(S(=O)(=O)c2ccccc2)c2ccccc12</smiles>

$3 b$

${ }^{13} \mathrm{C}$ NMR $\left(100 \mathrm{MHz} \mathrm{CDCl}_{3}\right)$

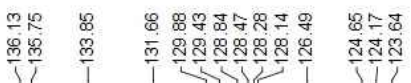

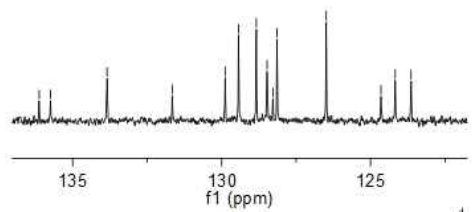



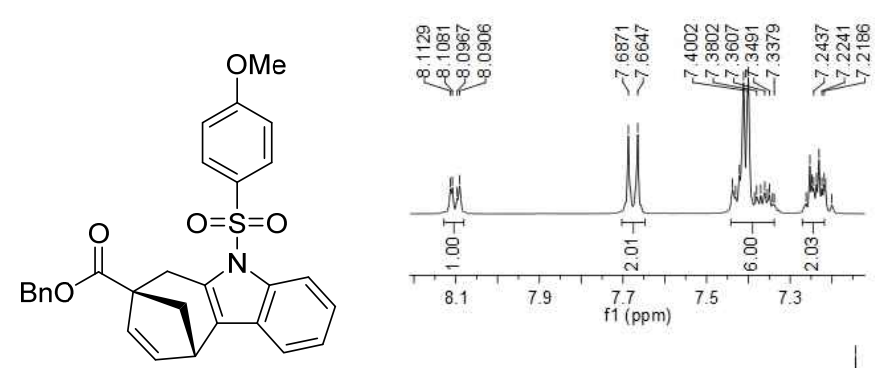

${ }^{1} \mathrm{H}$ NMR $\left(400 \mathrm{MHz} \mathrm{CDCl}_{3}\right)$

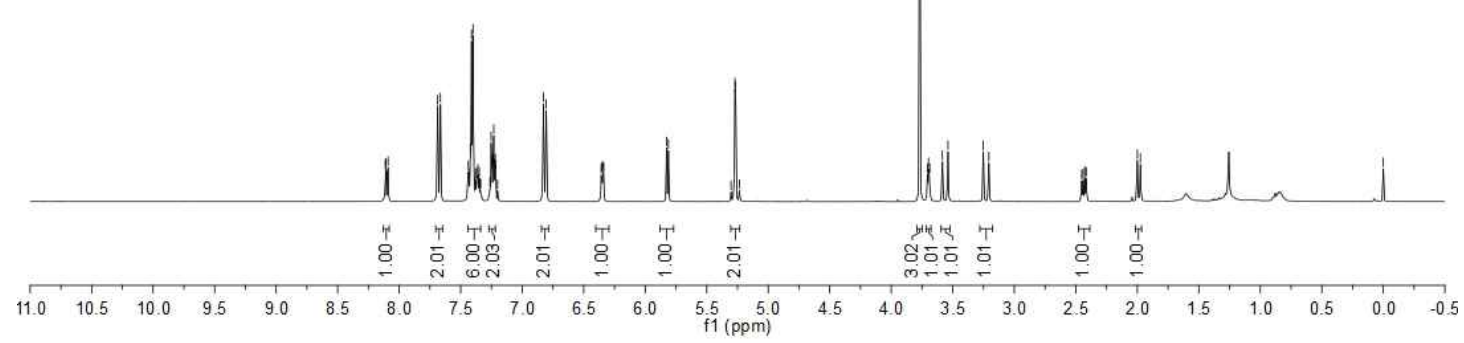

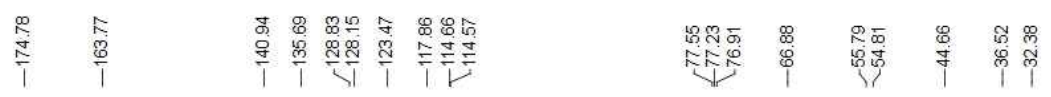

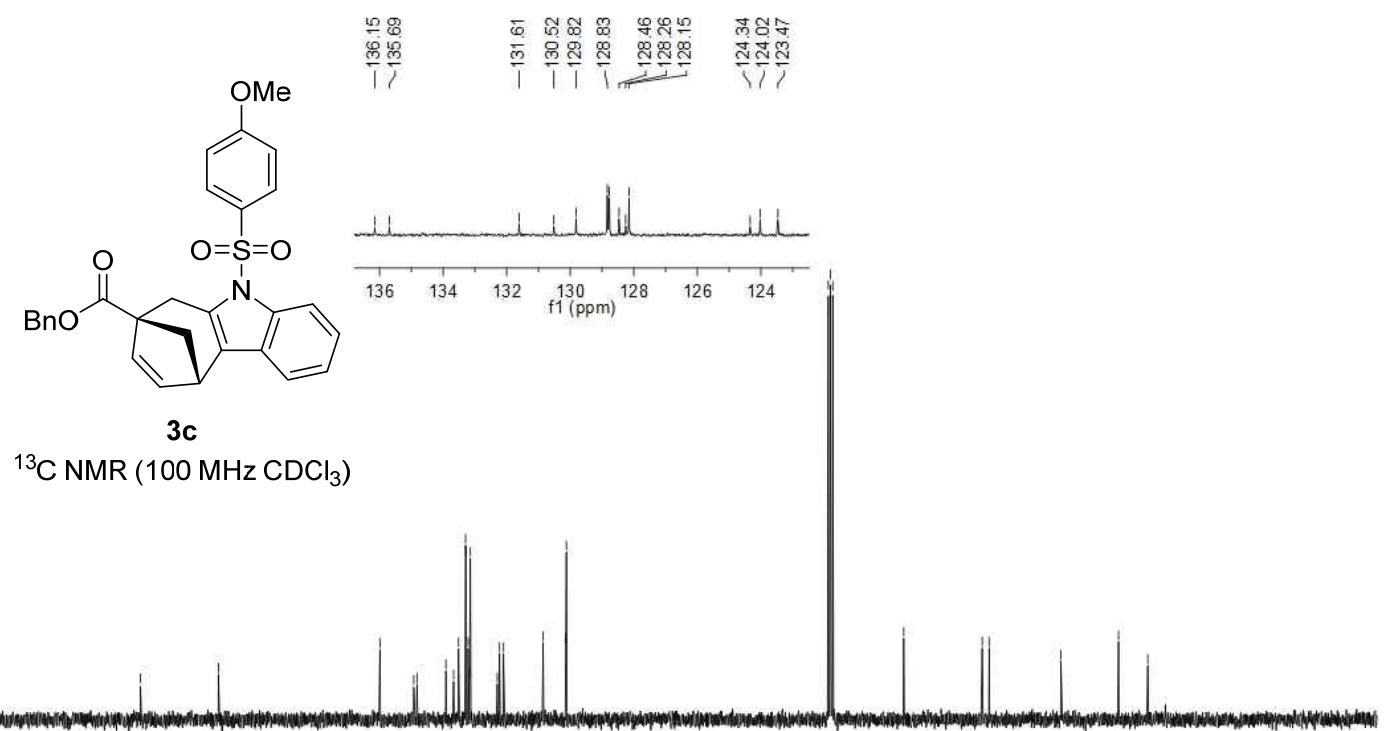

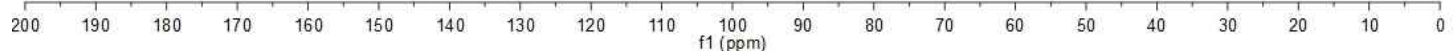




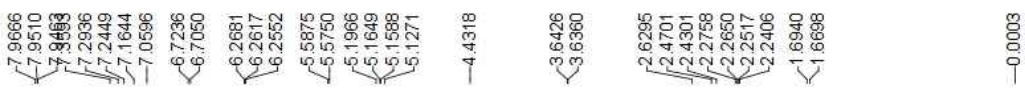

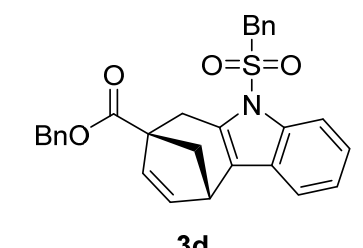

${ }^{1} \mathrm{H}$ NMR $\left(400 \mathrm{MHz} \mathrm{CDCl}_{3}\right)$
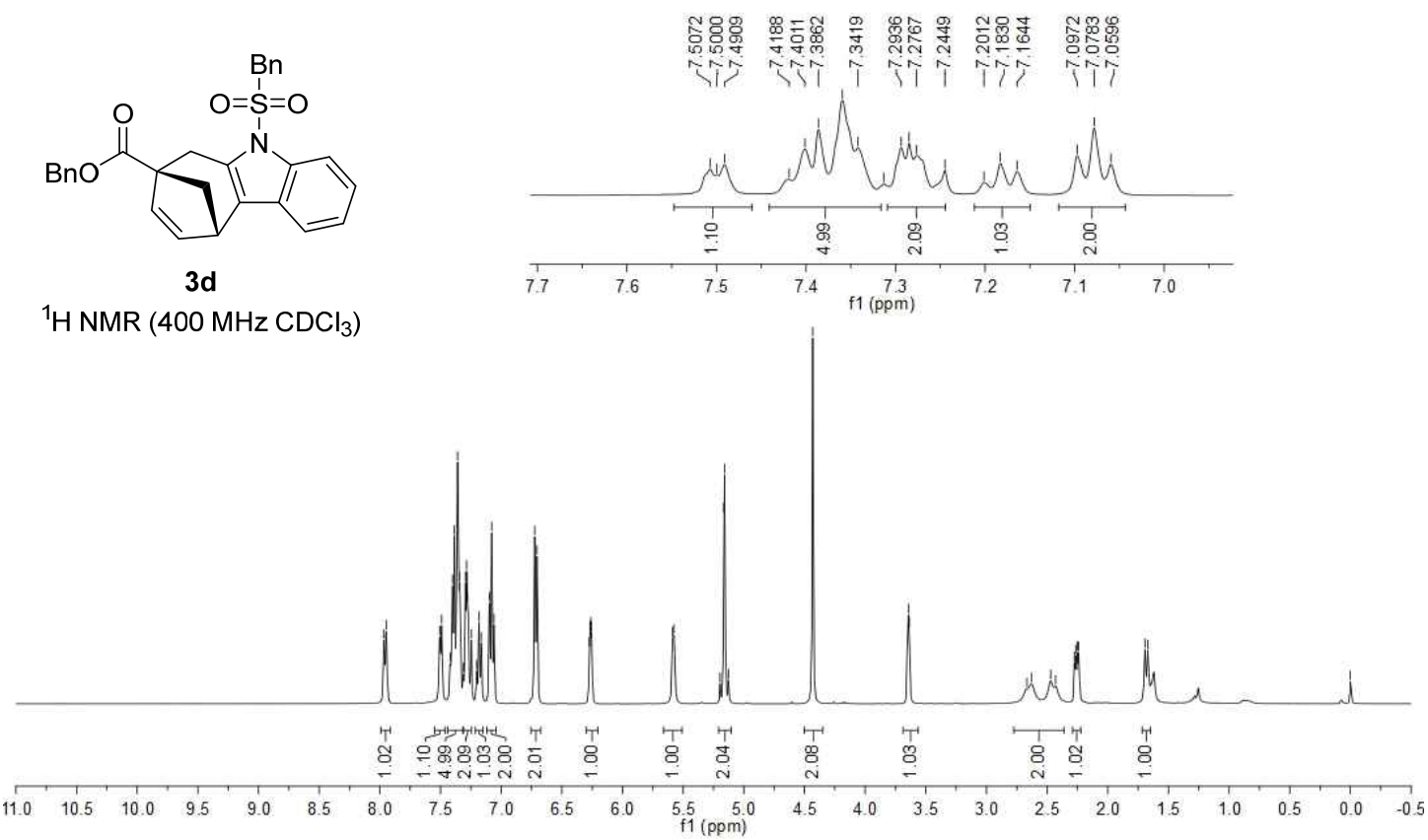

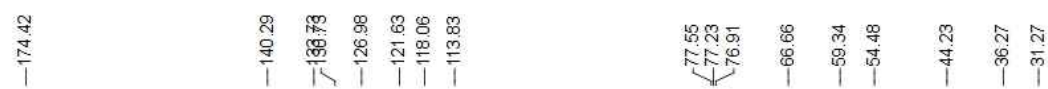

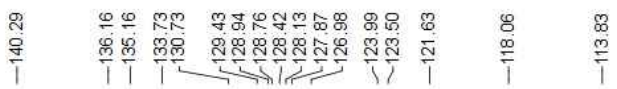

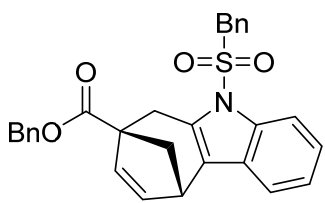

3d

${ }^{13} \mathrm{C}$ NMR $\left(100 \mathrm{MHz} \mathrm{CDCl}_{3}\right)$
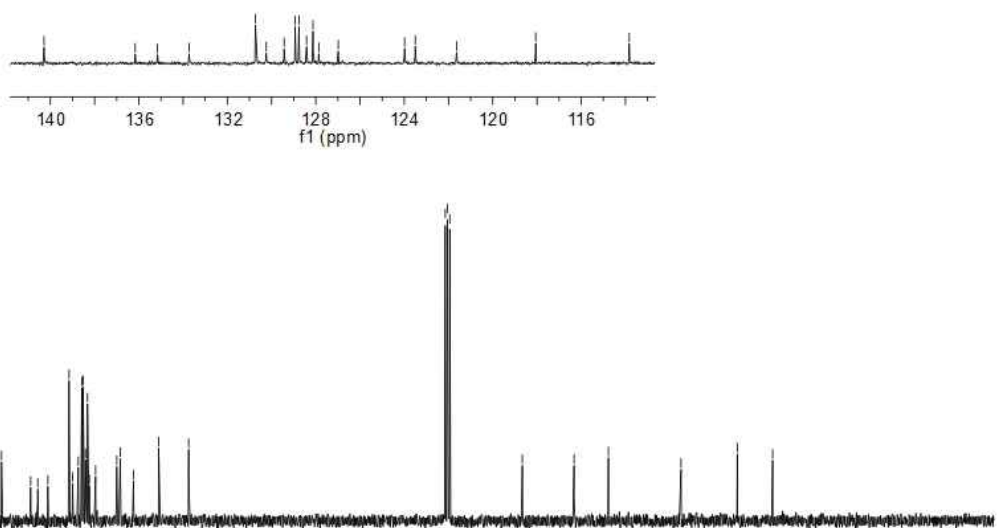

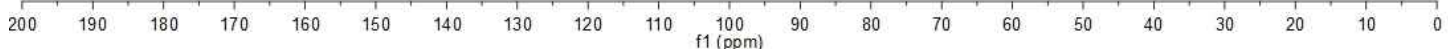



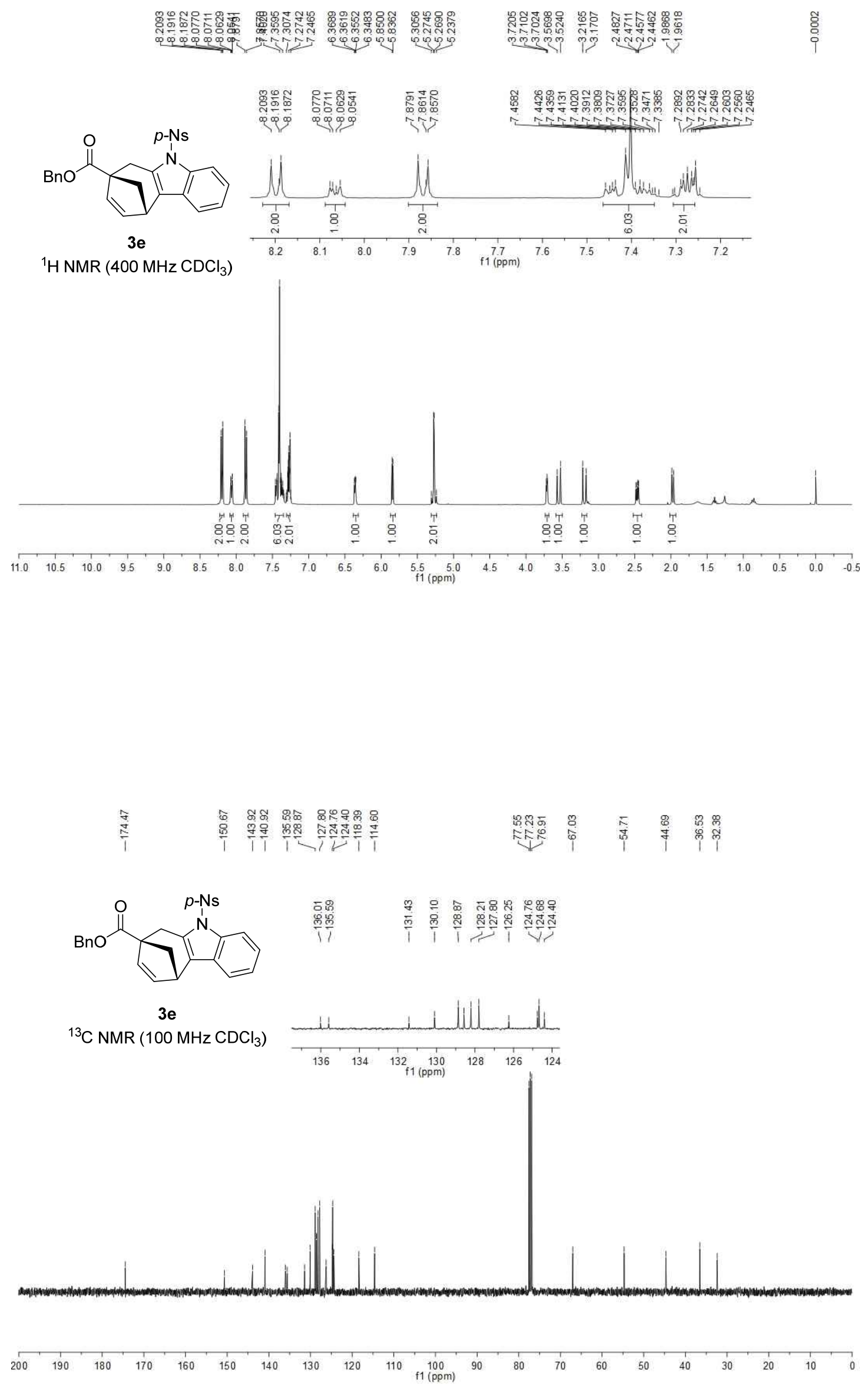

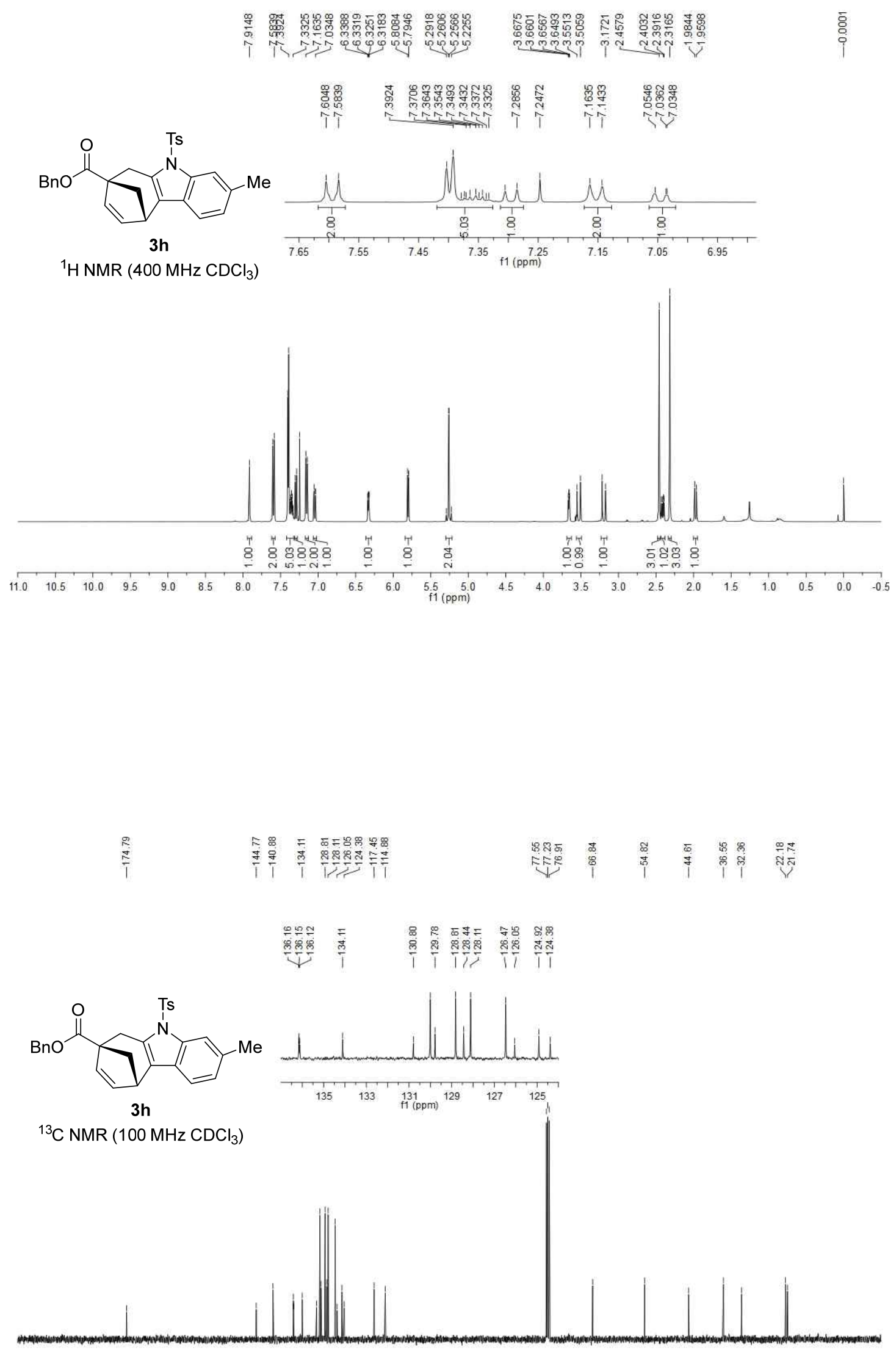

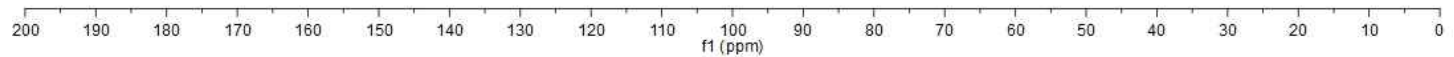




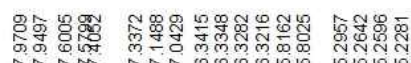

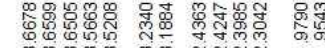

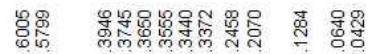

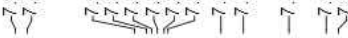<smiles>Cc1ccc2c(c1)c1c(n2[13CH3])CC2(C(=O)OCc3ccccc3)C=CC1C2</smiles>

3i

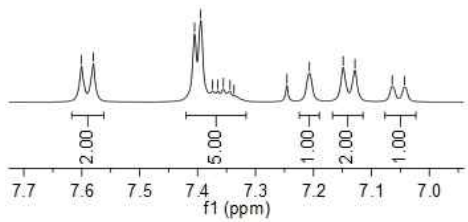

${ }^{1} \mathrm{H} \mathrm{NMR}\left(400 \mathrm{MHz} \mathrm{CDCl}_{3}\right)$

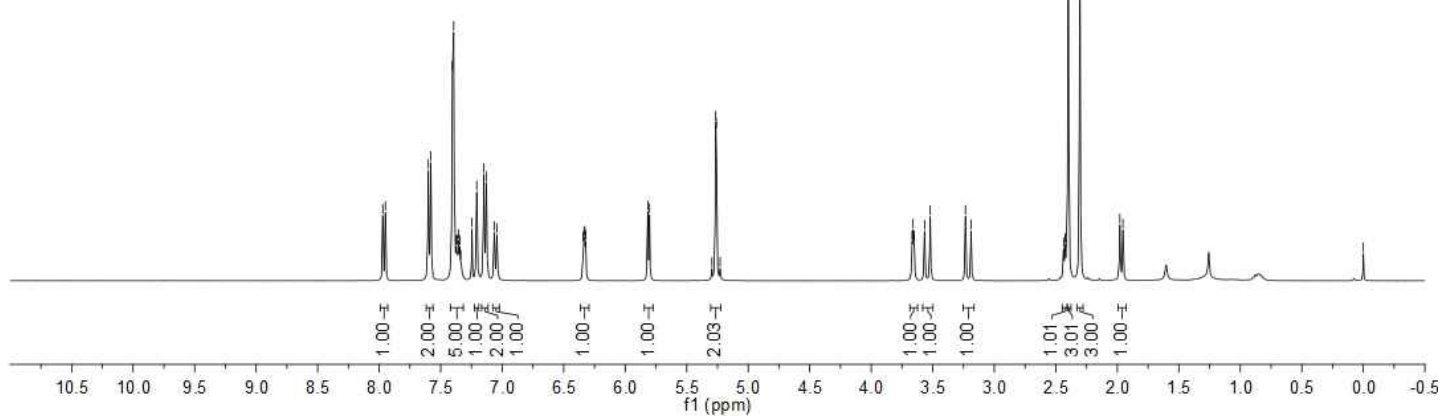

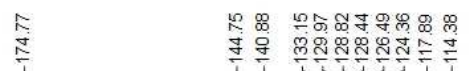

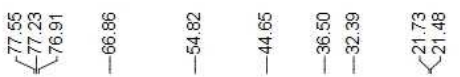

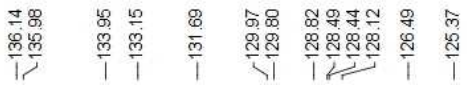

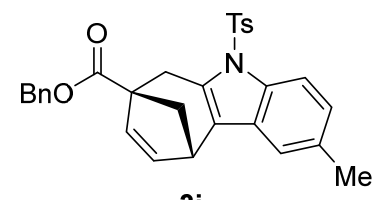

3i

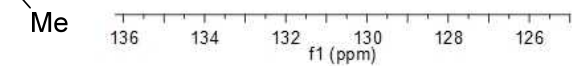

${ }^{13} \mathrm{C}$ NMR (100 MHz CDCl$\left.)_{3}\right)$

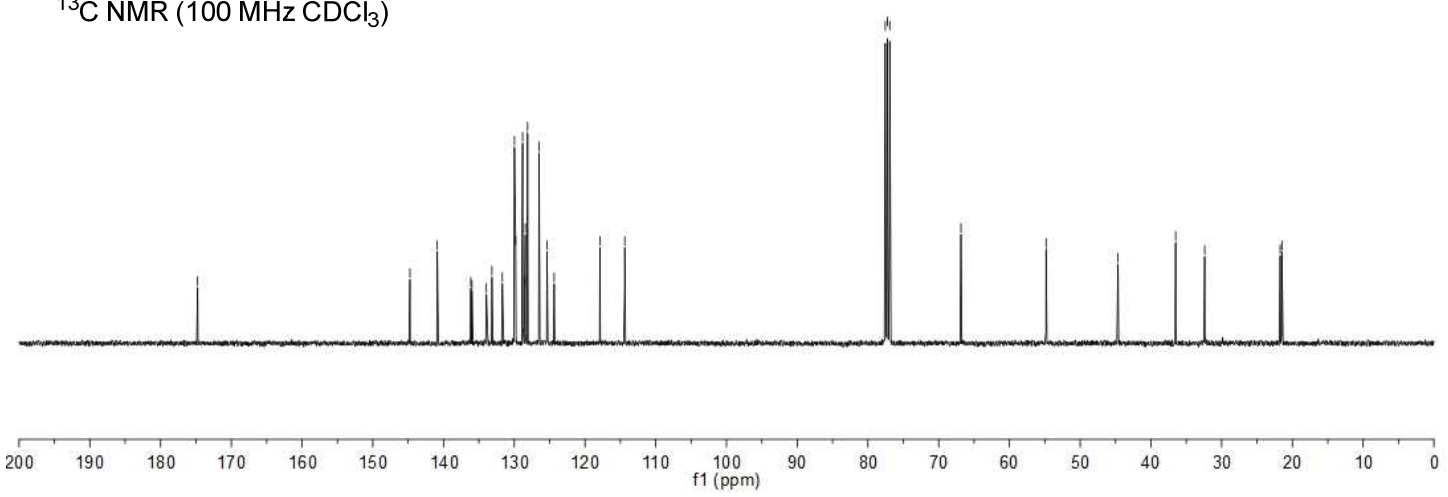




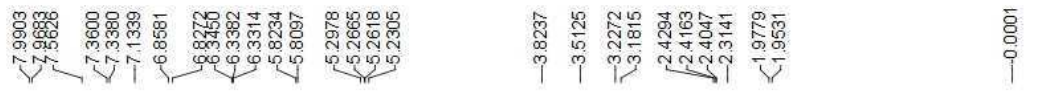

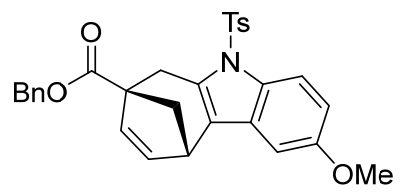

3j

${ }^{1} \mathrm{H} \mathrm{NMR}\left(400 \mathrm{MHz} \mathrm{CDCl}_{3}\right)$

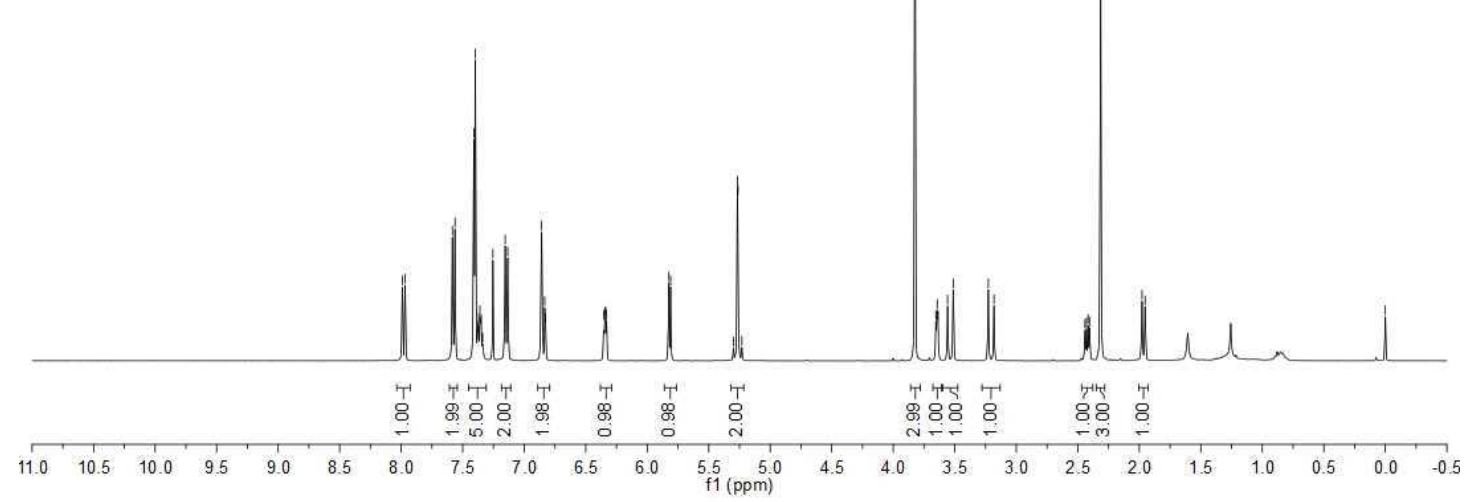

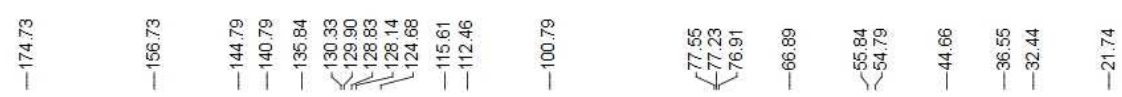
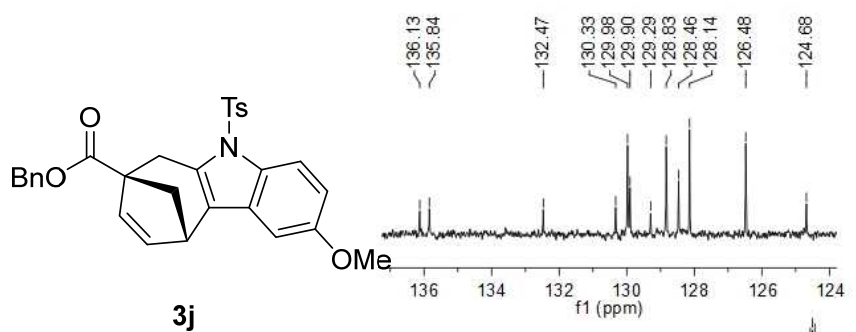

${ }^{13} \mathrm{C}$ NMR $\left(100 \mathrm{MHz} \mathrm{CDCl}_{3}\right)$

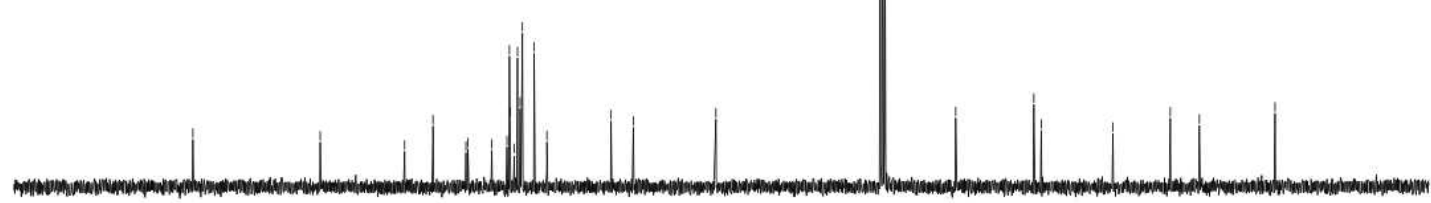

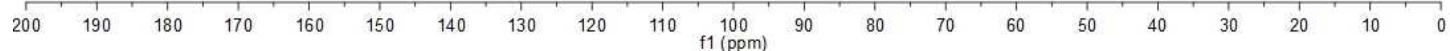




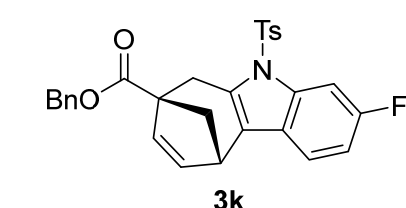

${ }^{1} \mathrm{H}$ NMR $\left(400 \mathrm{MHz} \mathrm{CDCl}_{3}\right)$

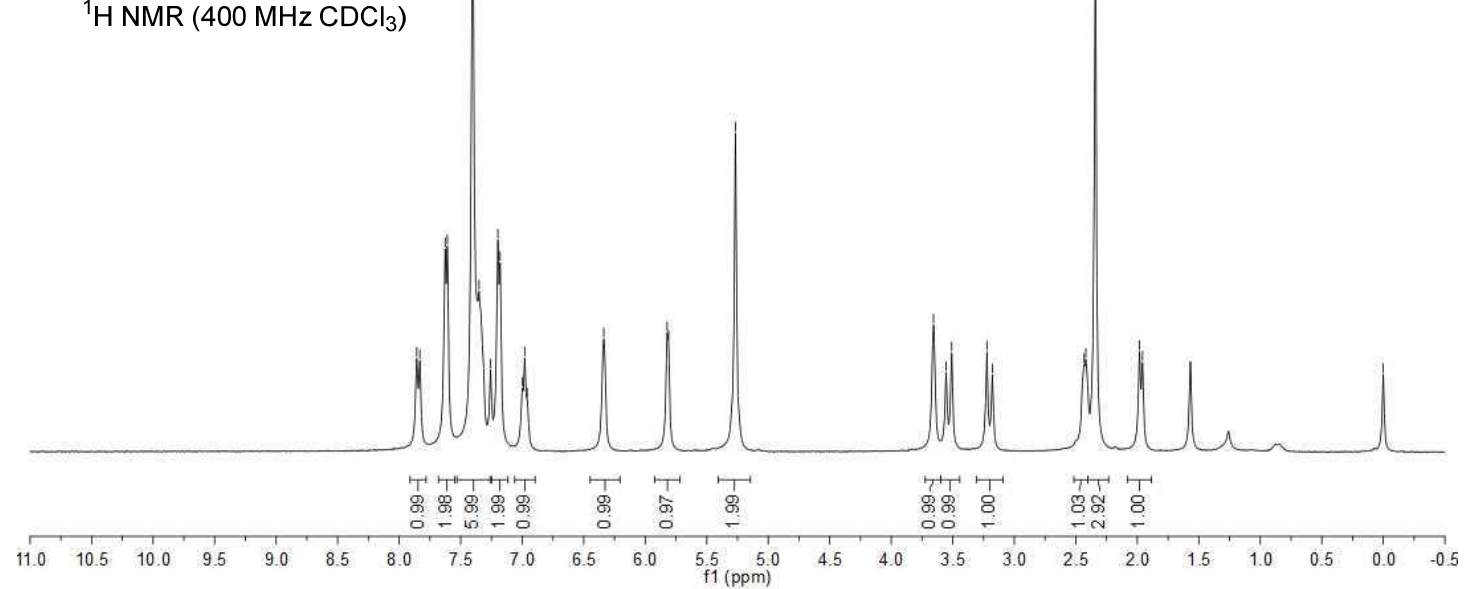

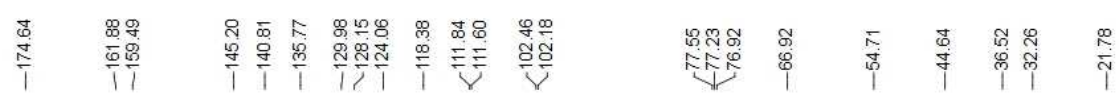

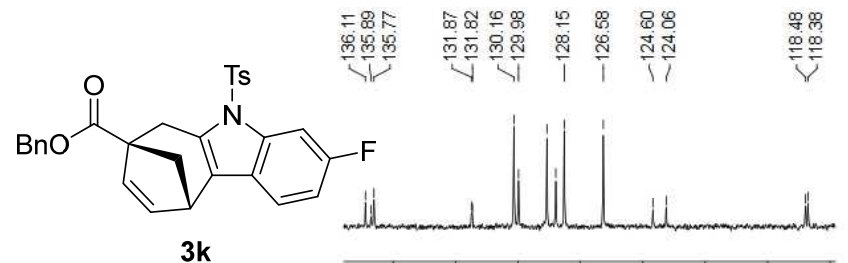

$\left.{ }^{13} \mathrm{C} \mathrm{NMR}\left(100 \mathrm{MHz}^{\mathrm{CDCl}}\right)_{3}\right)^{135} \quad{ }^{130}{ }_{\mathrm{f} 1(\mathrm{ppm})}^{125} \quad{ }^{120}$

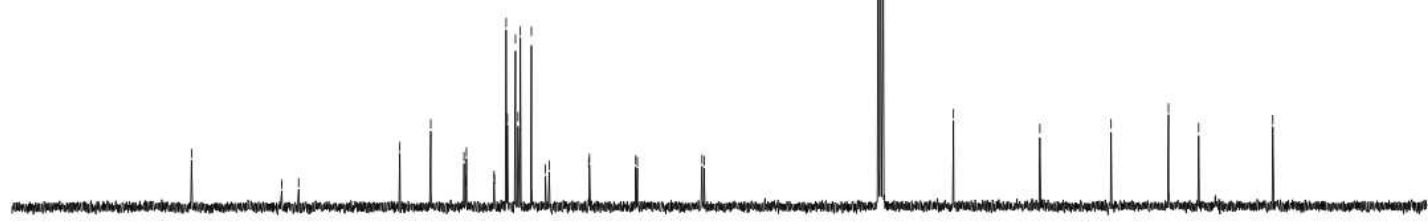

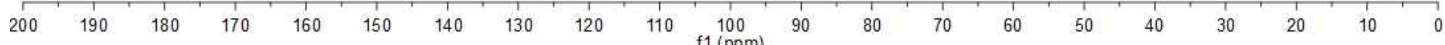




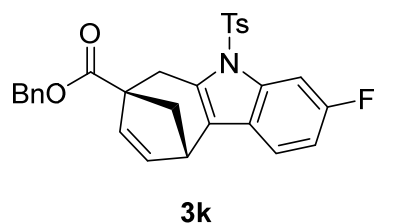

$\frac{i}{\frac{1}{2}}$

${ }^{19} \mathrm{~F} \mathrm{NMR}\left(376 \mathrm{MHz} \mathrm{CDCl}_{3}\right)$

$\begin{array}{lllllllllllllllllllllllllllllllll}20 & 10 & 0 & -10 & -20 & -30 & -40 & -50 & -60 & -70 & -80 & -90 & -100 & -110 & -120 & -130 & -140 & -150 & -160 & -170 & -180 & -190 & -200 & -210 & -226\end{array}$ 


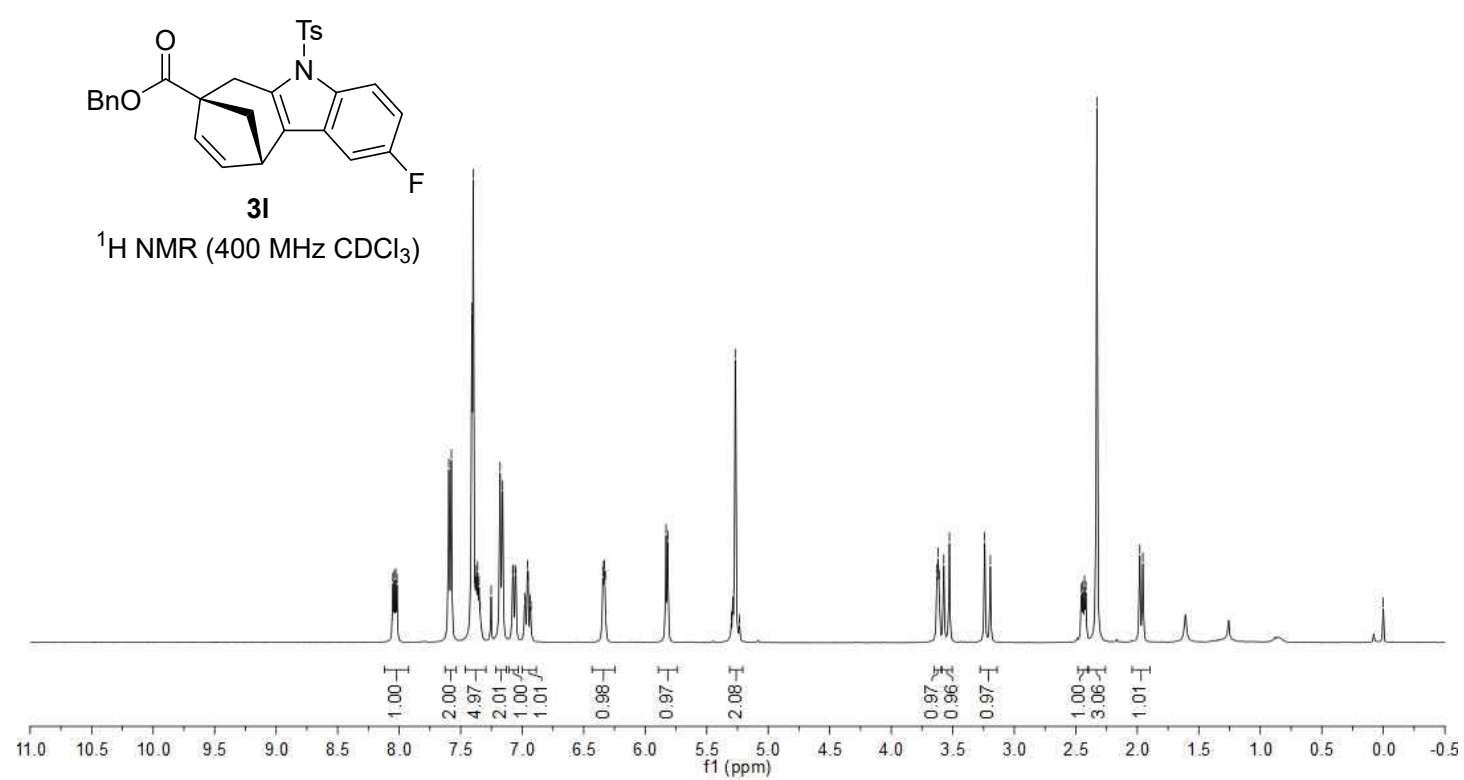

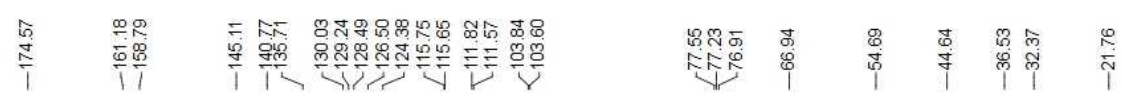

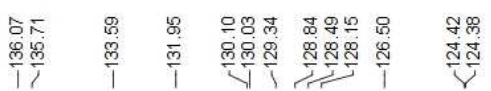

(s)

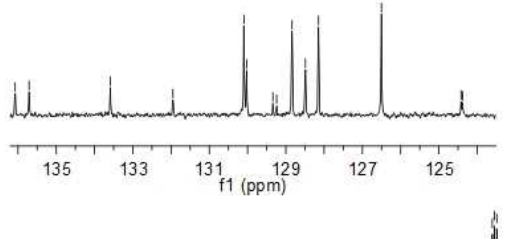

${ }^{13} \mathrm{C} \mathrm{NMR}\left(100 \mathrm{MHz}^{\mathrm{CDCl}}{ }_{3}\right)$

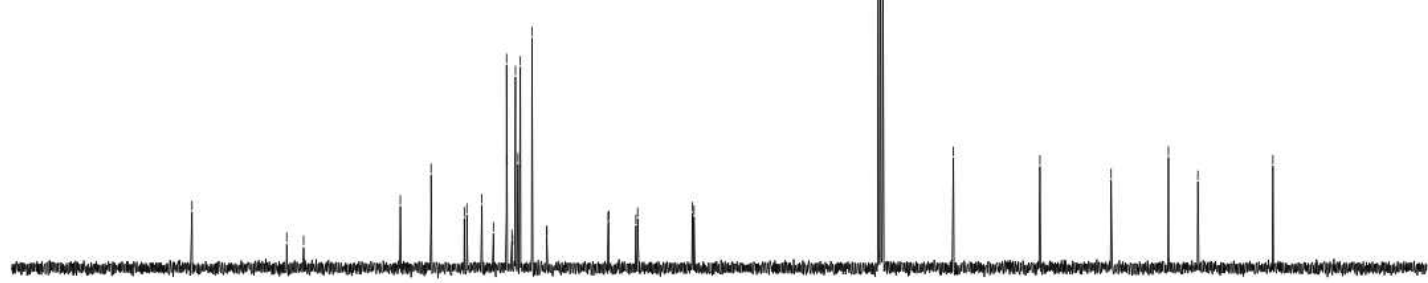

200

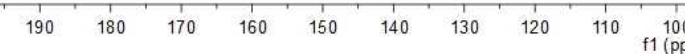




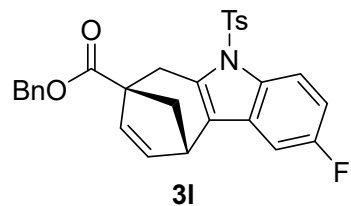

$\left.{ }^{19} \mathrm{~F} \mathrm{NMR} \mathrm{(376} \mathrm{MHz} \mathrm{CDCl}_{3}\right)$

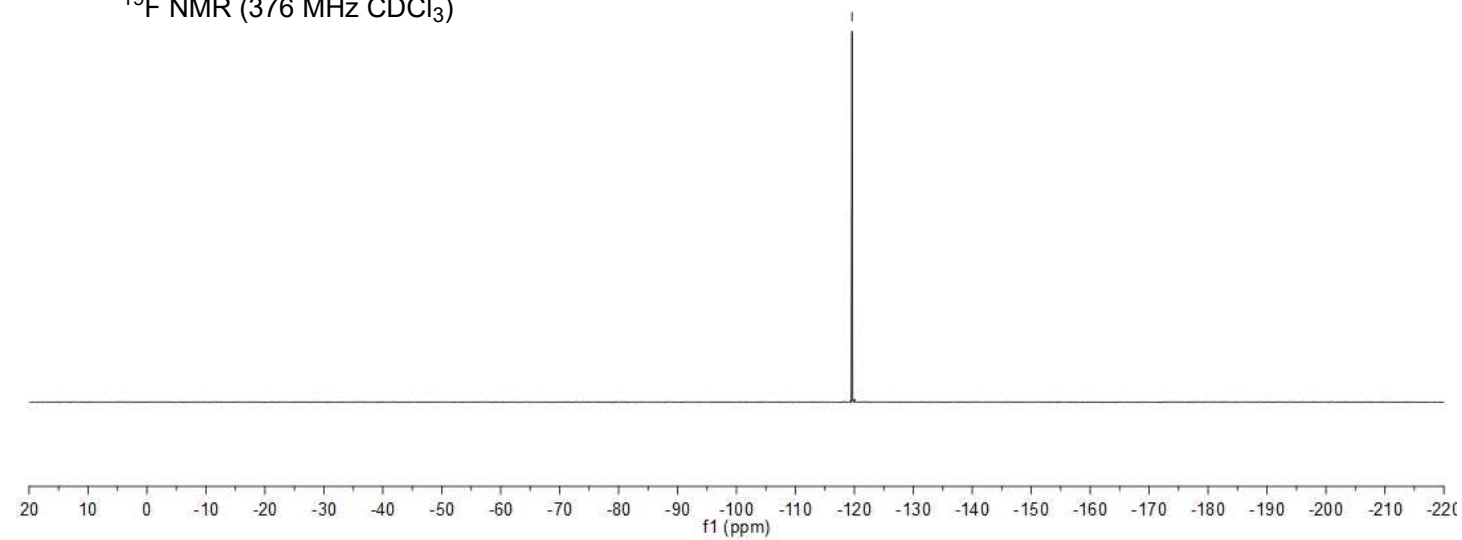




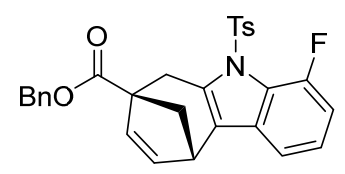

$3 \mathrm{~m}$

${ }^{1} \mathrm{H}$ NMR $\left(400 \mathrm{MHz} \mathrm{CDCl}_{3}\right)$

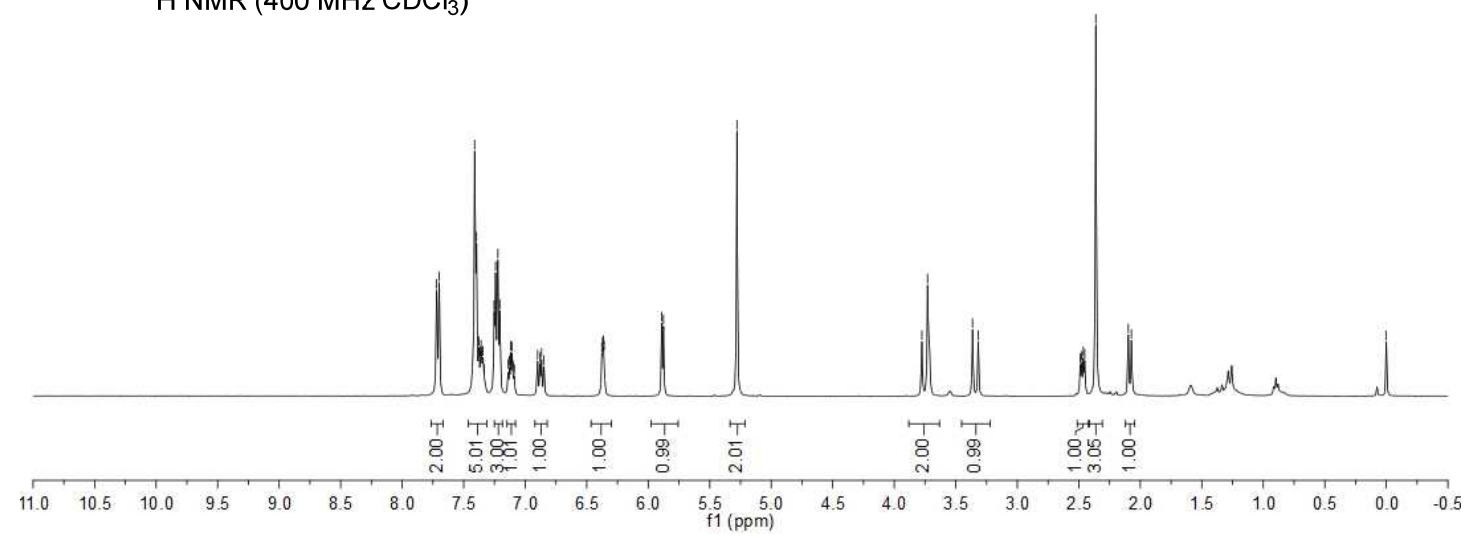

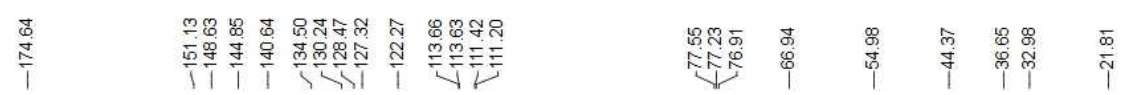

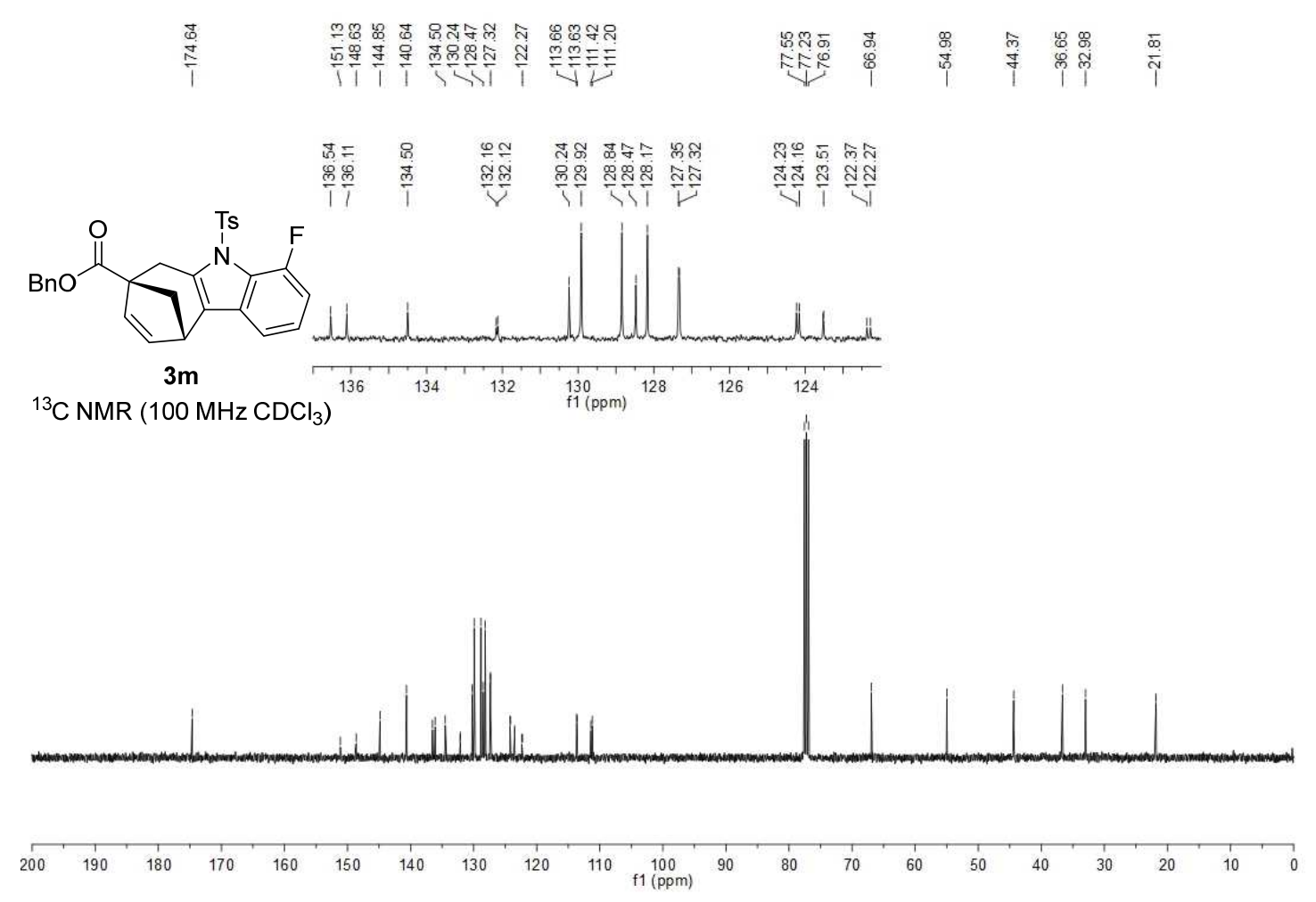

${ }^{13} \mathrm{C}$ NMR $\left(100 \mathrm{MHz} \mathrm{CDCl}_{3}\right)$ 


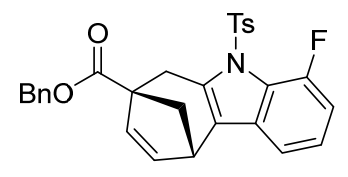

$3 \mathrm{~m}$

$\left.{ }^{19} \mathrm{~F} \mathrm{NMR} \mathrm{(376} \mathrm{MHz} \mathrm{CDCl}_{3}\right)$

$\begin{array}{llllllllllllllllllllllllllllll}20 & 10 & 0 & -10 & -20 & -30 & -40 & -50 & -60 & -70 & -80 & -90 & -100 & -110 & -120 & -130 & -140 & -150 & -160 & -170 & -180 & -190 & -200 & -210 & -226\end{array}$ 


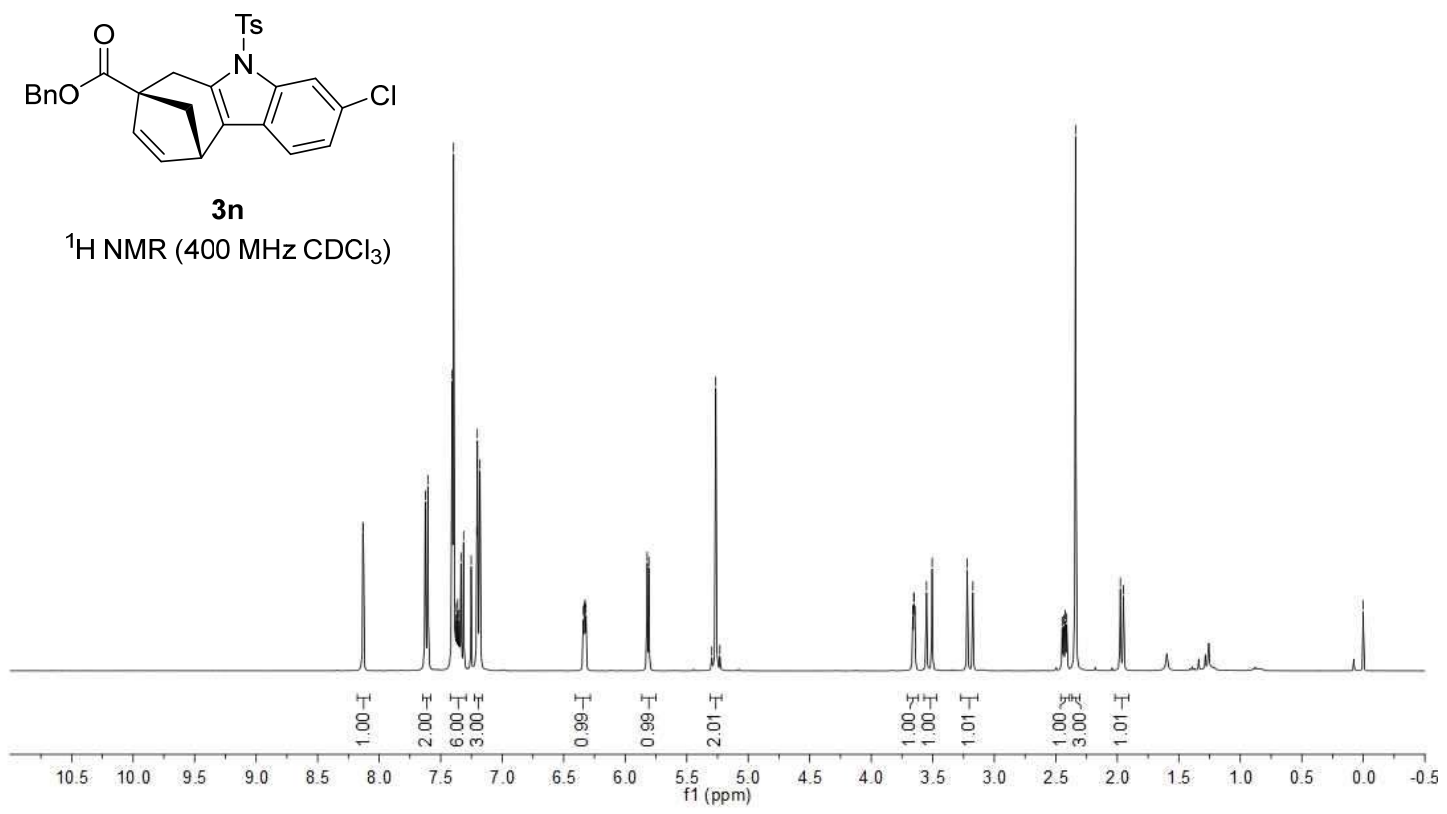

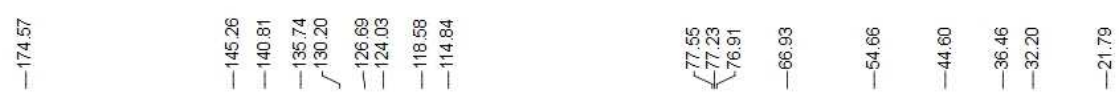

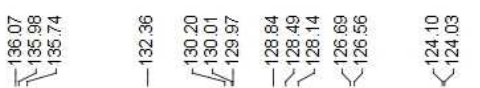<smiles></smiles>

$3 n$

${ }^{13} \mathrm{C}$ NMR $\left(100 \mathrm{MHz} \mathrm{CDCl}_{3}\right)$
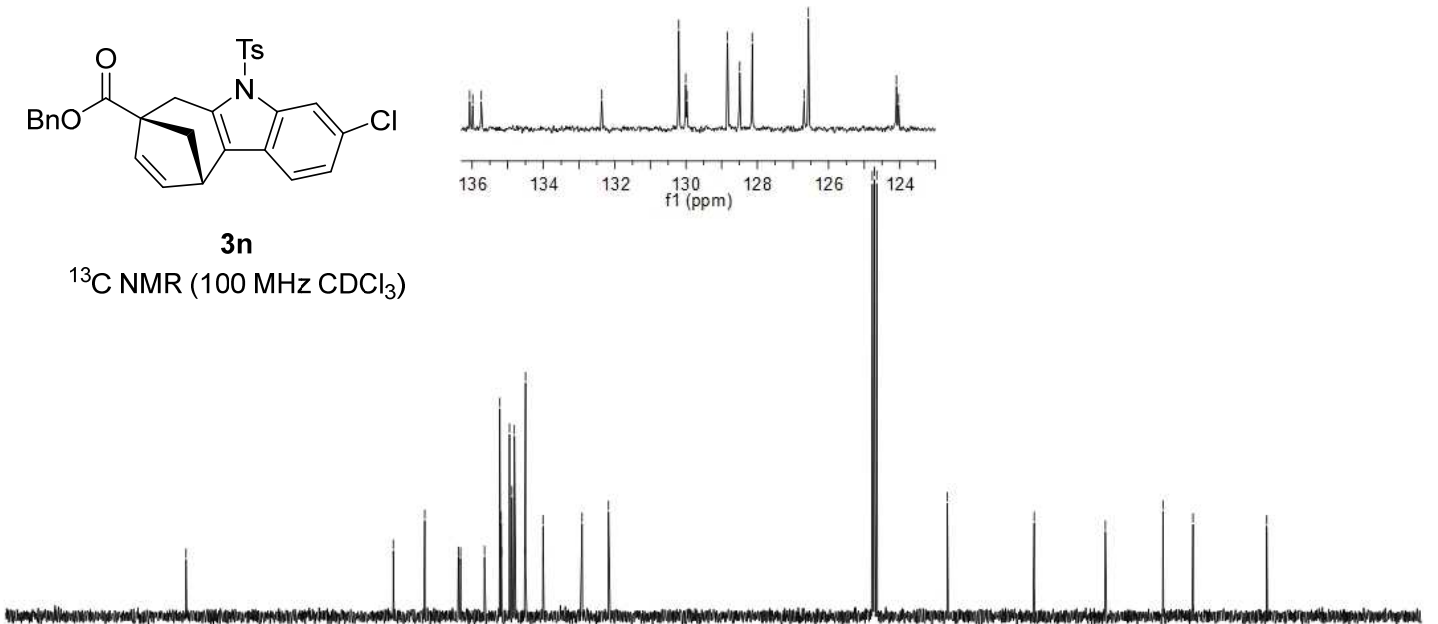

\begin{tabular}{lllllllllllllllllllllll}
1 & 180 & 170 & 160 & 150 & 140 & 130 & 120 & 110 & 100 & 1 & 1 & 1 & 1 & 1 & 1 & 1 & 1 & 1 & 1 & 1 & 1 & 1 \\
\hline
\end{tabular} 


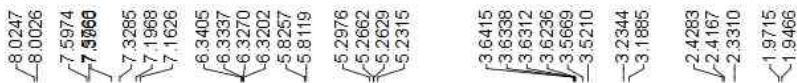<smiles>Cn1c2c(c3cc(Cl)ccc31)C1C=CC2(C(=O)OCc2ccccc2)CC1</smiles>

30 ${ }^{1} \mathrm{H} \mathrm{NMR}\left(400 \mathrm{MHz} \mathrm{CDCl}_{3}\right)$
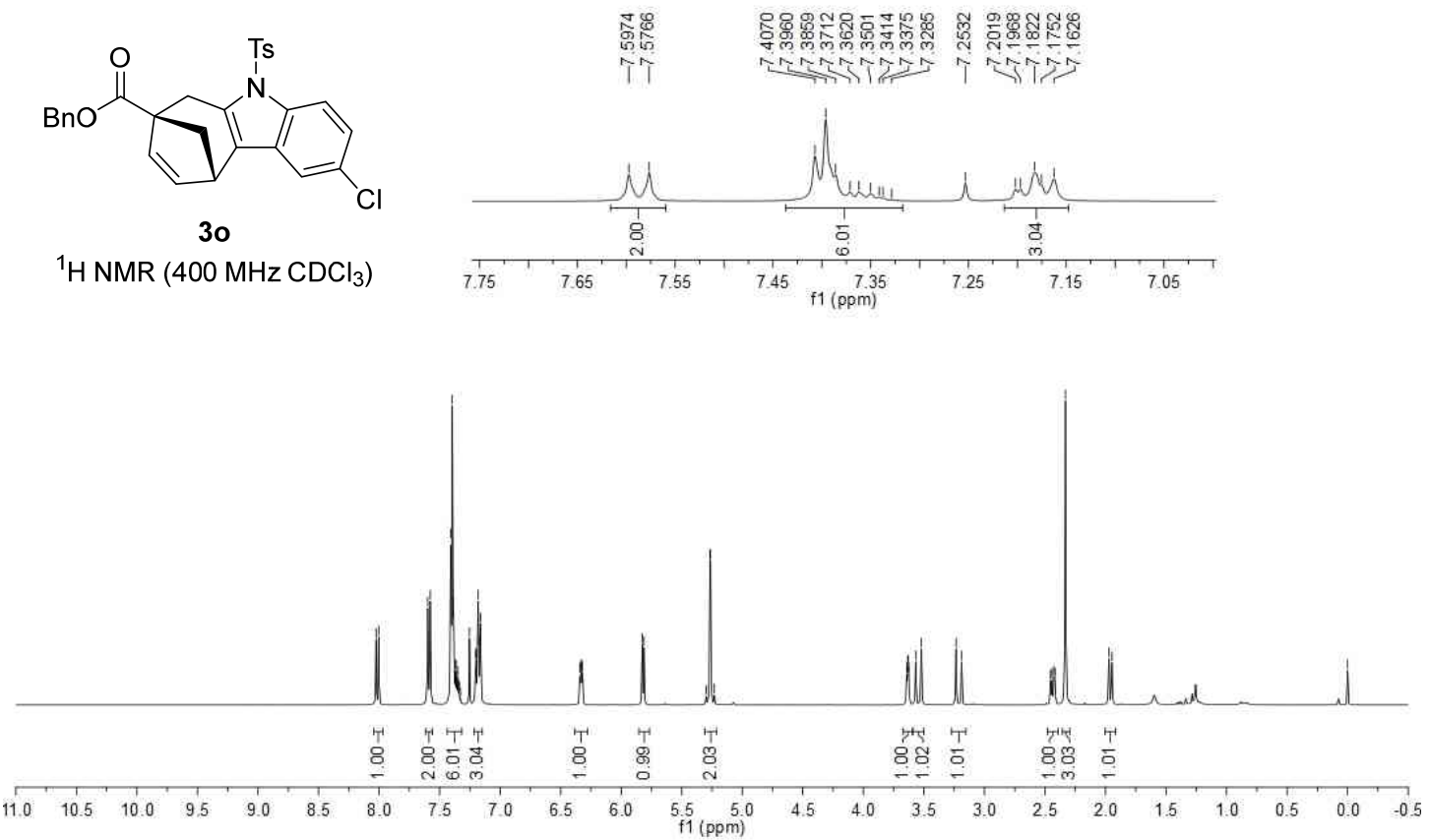

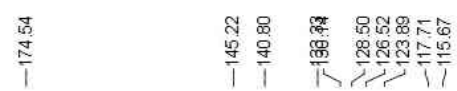

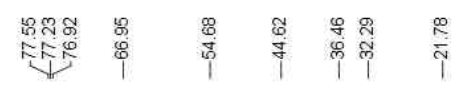

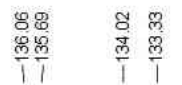<smiles>[Y5]C1=C2C3=C(C=CC3=C1)C1(C(=O)OCc3ccccc3)C=CC2CC1</smiles>

30

$\left.{ }^{13} \mathrm{C} \mathrm{NMR} \mathrm{(100} \mathrm{MHz} \mathrm{CDCl}_{3}\right)$

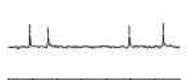
$136 \underset{f 1(\mathrm{ppm})}{135} 134$
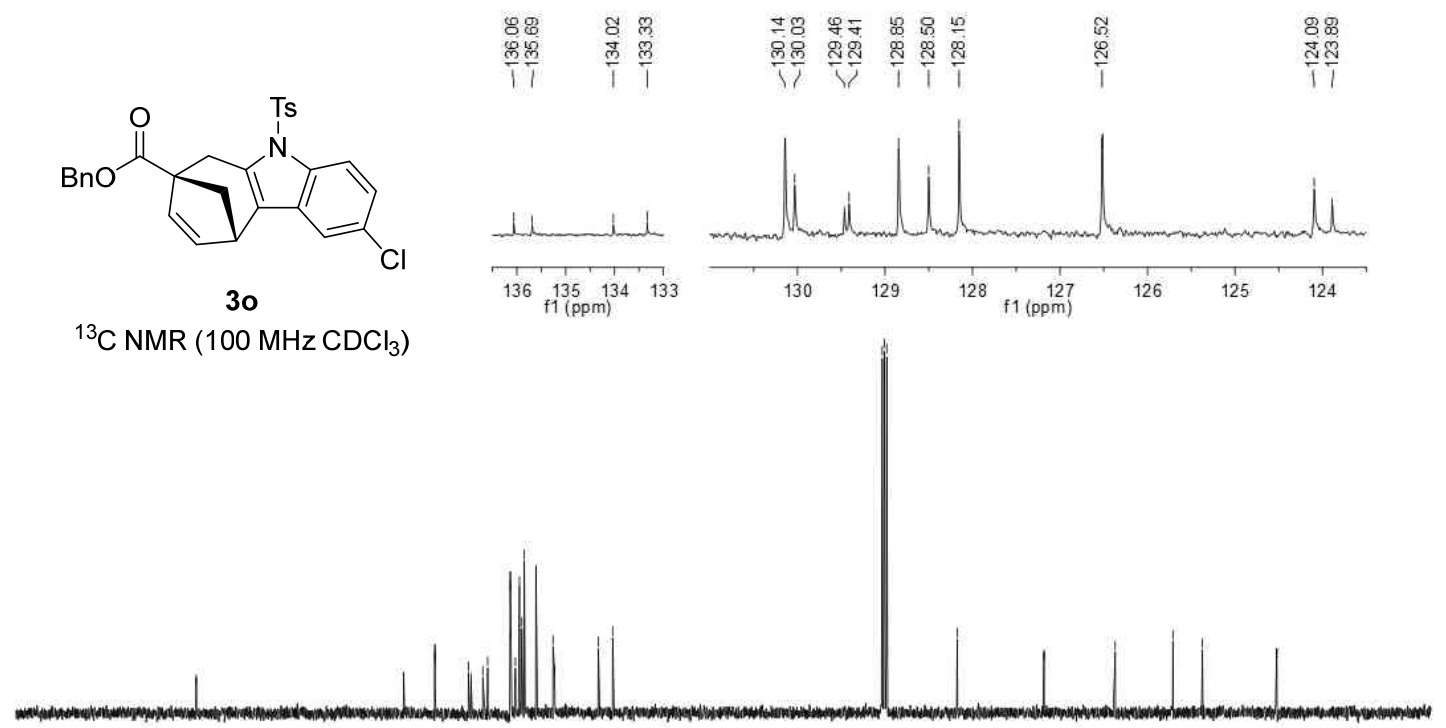

200 $\begin{array}{llllllllll}190 & 180 & 170 & 160 & 150 & 140 & 130 & 120 & 110 & 100 \\ \mathrm{f} 1(\mathrm{ppm})\end{array}$ 


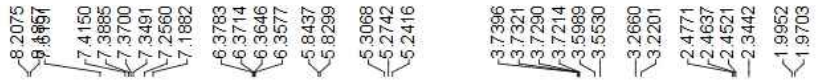
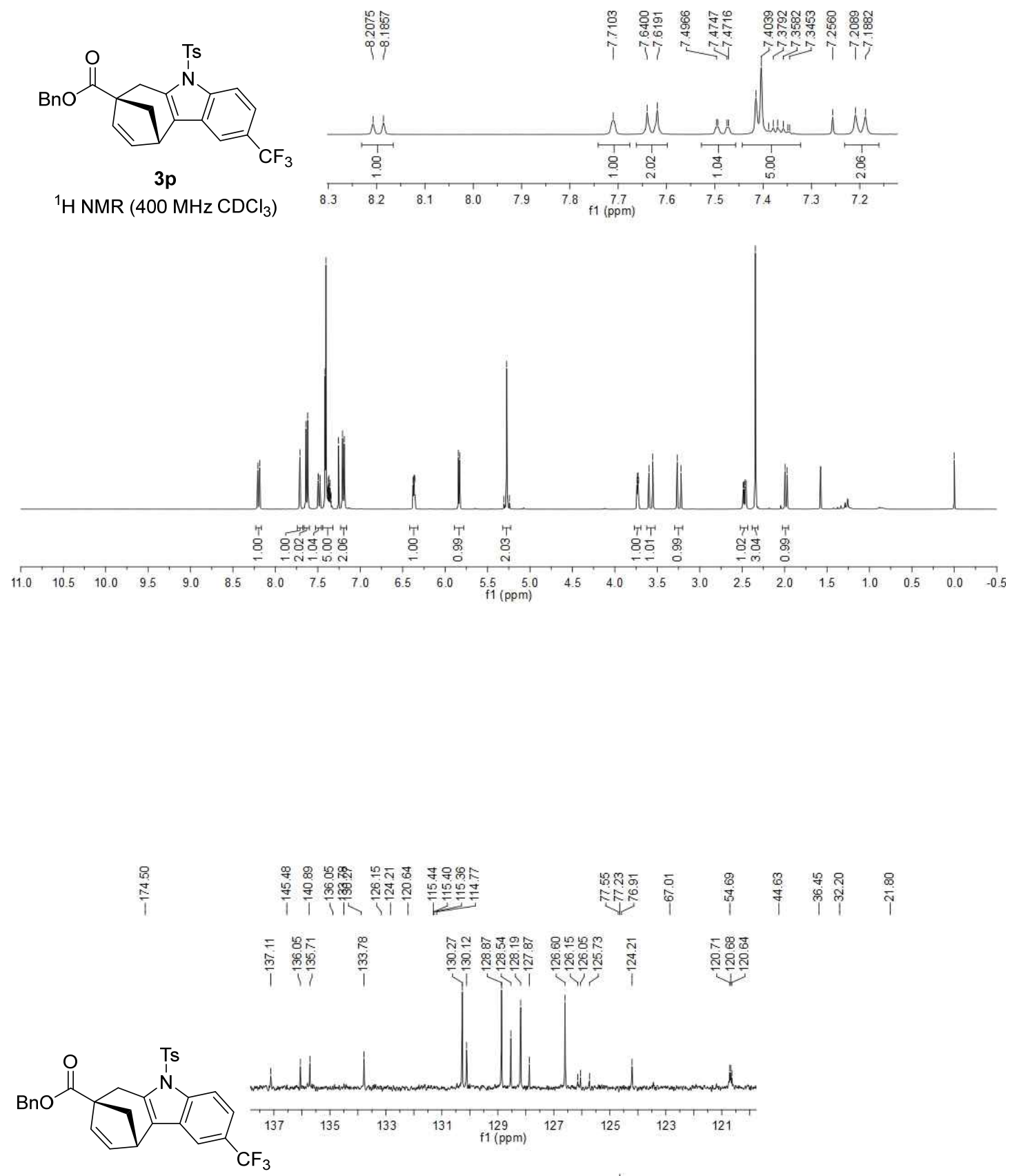

$3 p$

${ }^{13} \mathrm{C} \mathrm{NMR}\left(100 \mathrm{MHz} \mathrm{CDCl}_{3}\right)$

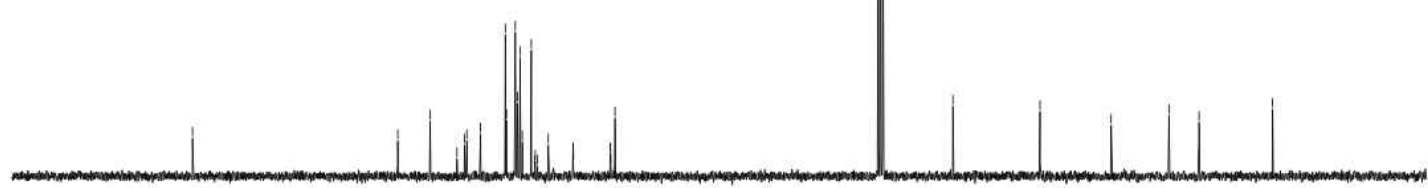

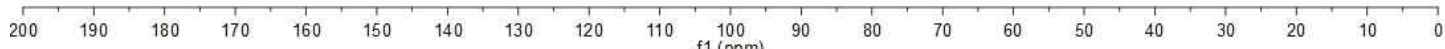




$$
\frac{g}{\frac{8}{\overline{0}}}
$$<smiles>O=C(OCc1ccccc1)C12C=CC(C1)c1c2n([135I])c2ccc(C(F)(F)F)cc12</smiles>

$3 p$

${ }^{19} \mathrm{~F} \mathrm{NMR}\left(376 \mathrm{MHz} \mathrm{CDCl}_{3}\right)$

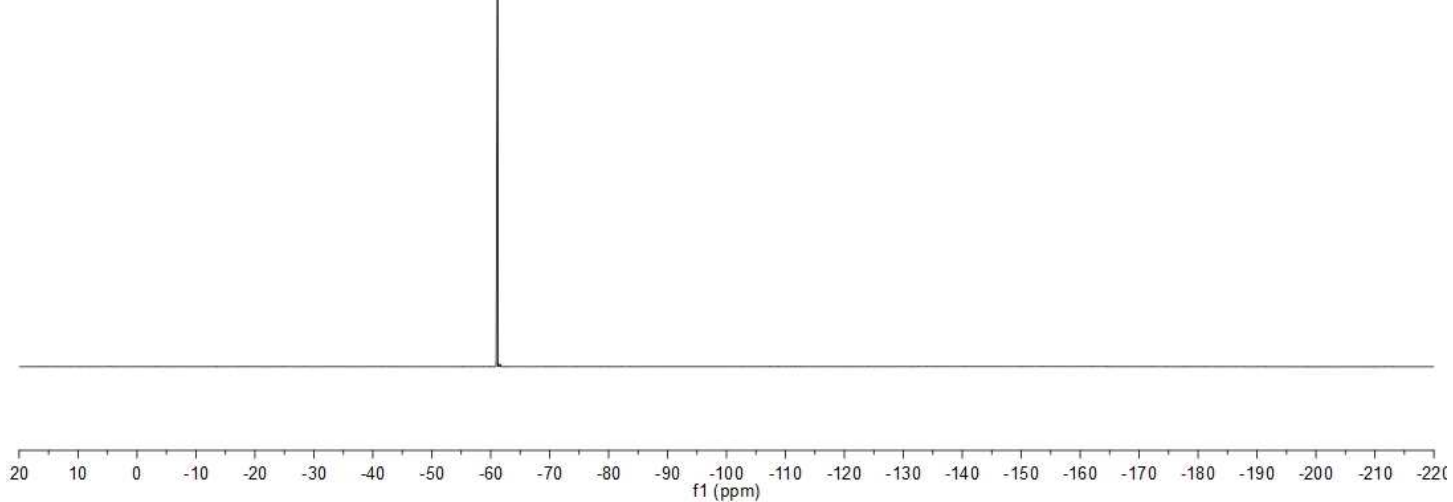



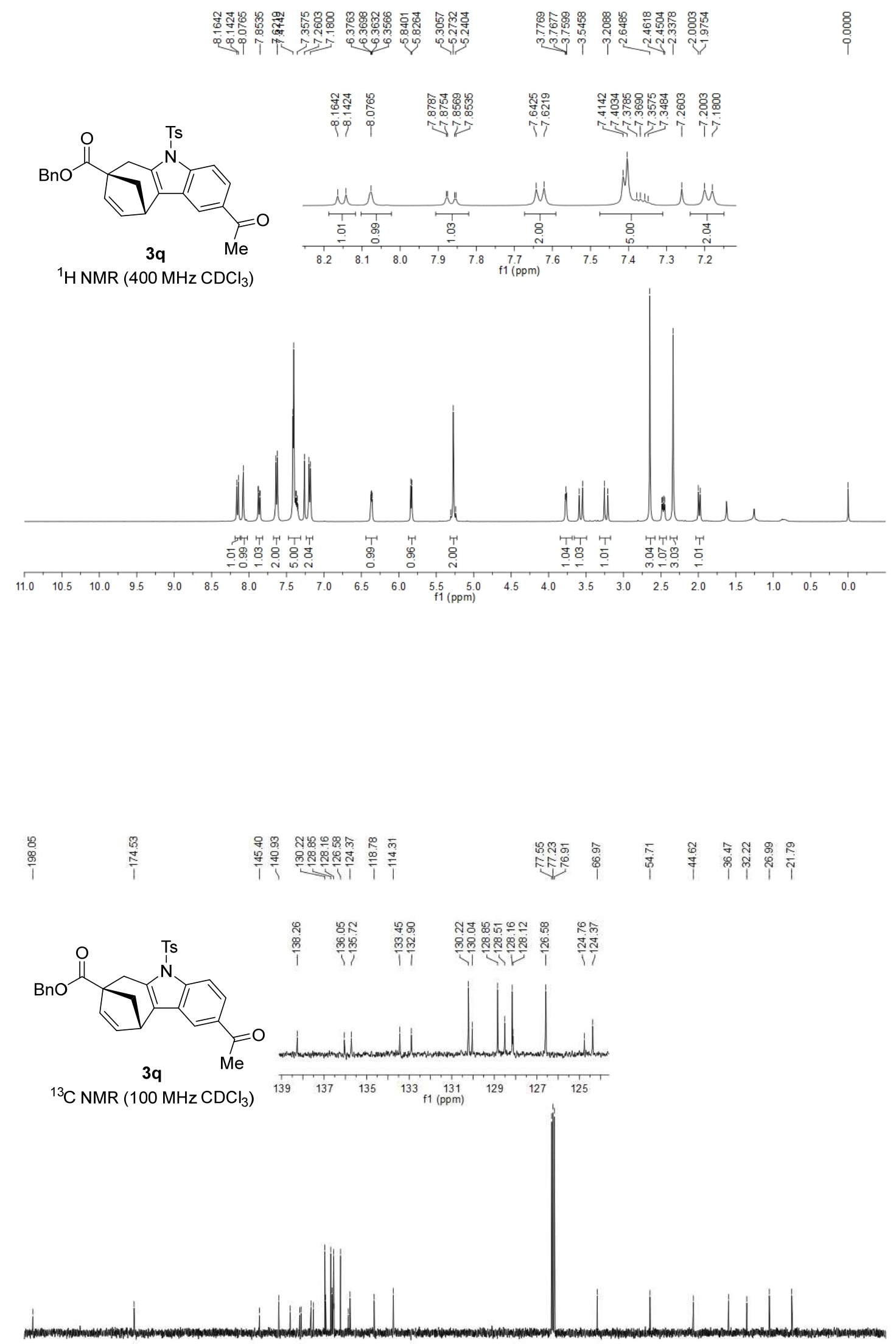

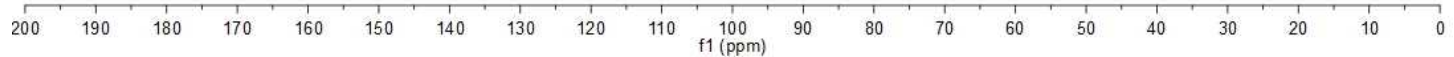




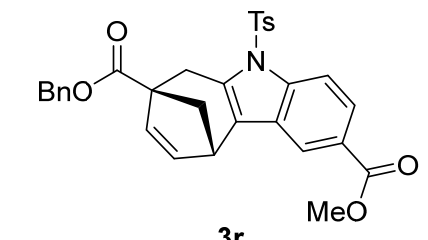

${ }^{1} \mathrm{H}$ NMR (400 $\mathrm{MHz} \mathrm{CDCl}_{3}$ )

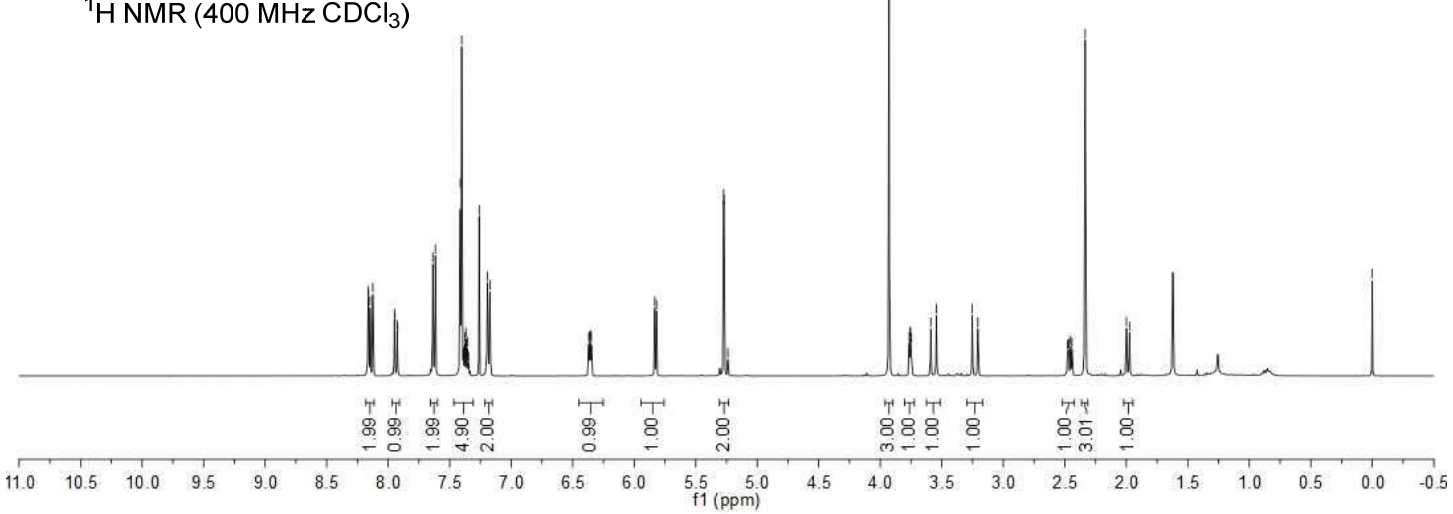

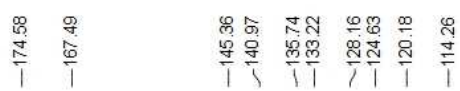
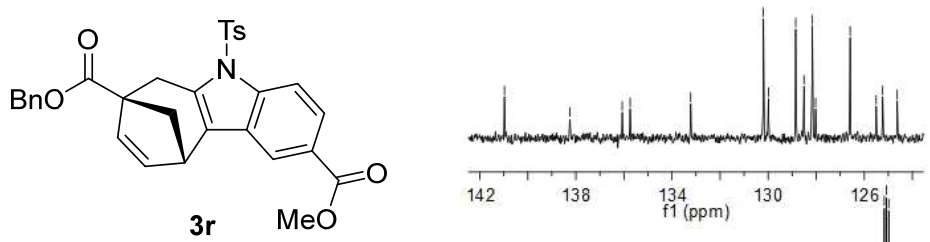

${ }^{13} \mathrm{C} \mathrm{NMR}\left(100 \mathrm{MHz} \mathrm{CDCl}_{3}\right)$

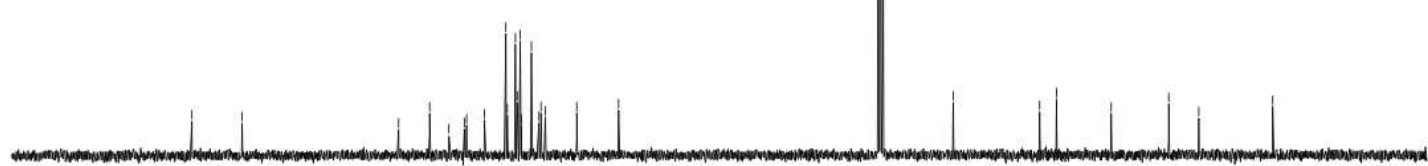

200

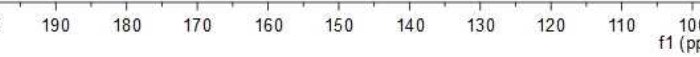


<smiles>Cn1c2c(c3cc(F)c(F)cc31)C1(C(=O)OCc3ccccc3)C=CC2CC1</smiles>

3s

${ }^{1} \mathrm{H} \mathrm{NMR}\left(400 \mathrm{MHz} \mathrm{CDCl}_{3}\right)$

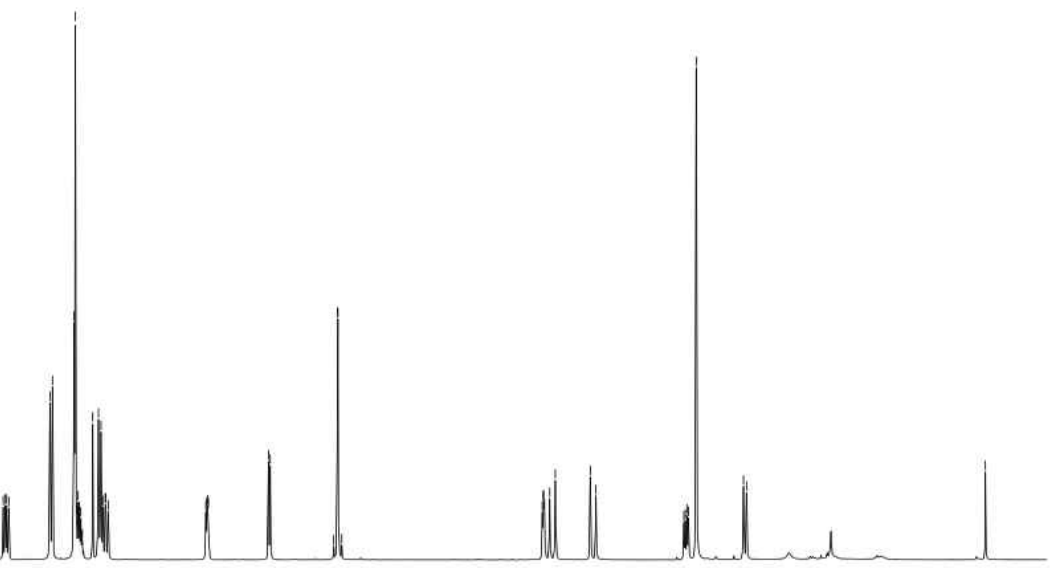

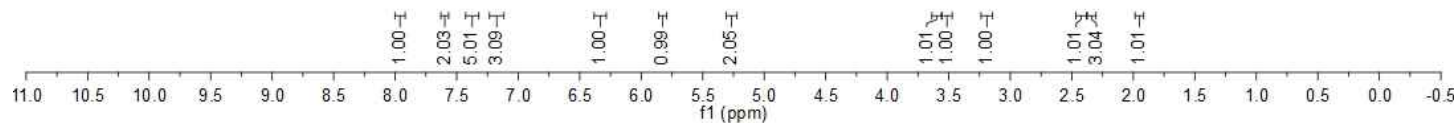

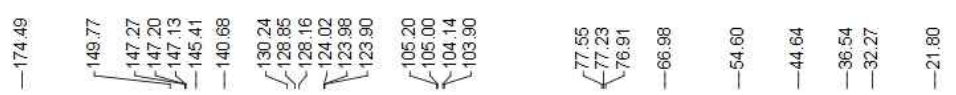

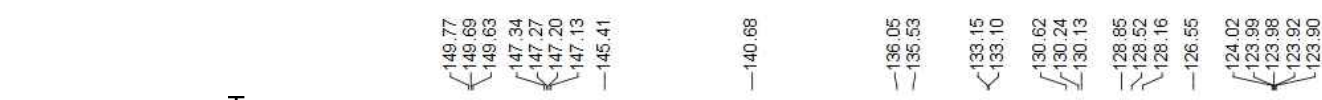<smiles></smiles>

3s

${ }^{13} \mathrm{C}$ NMR $\left(100 \mathrm{MHz} \mathrm{CDCl}_{3}\right)$
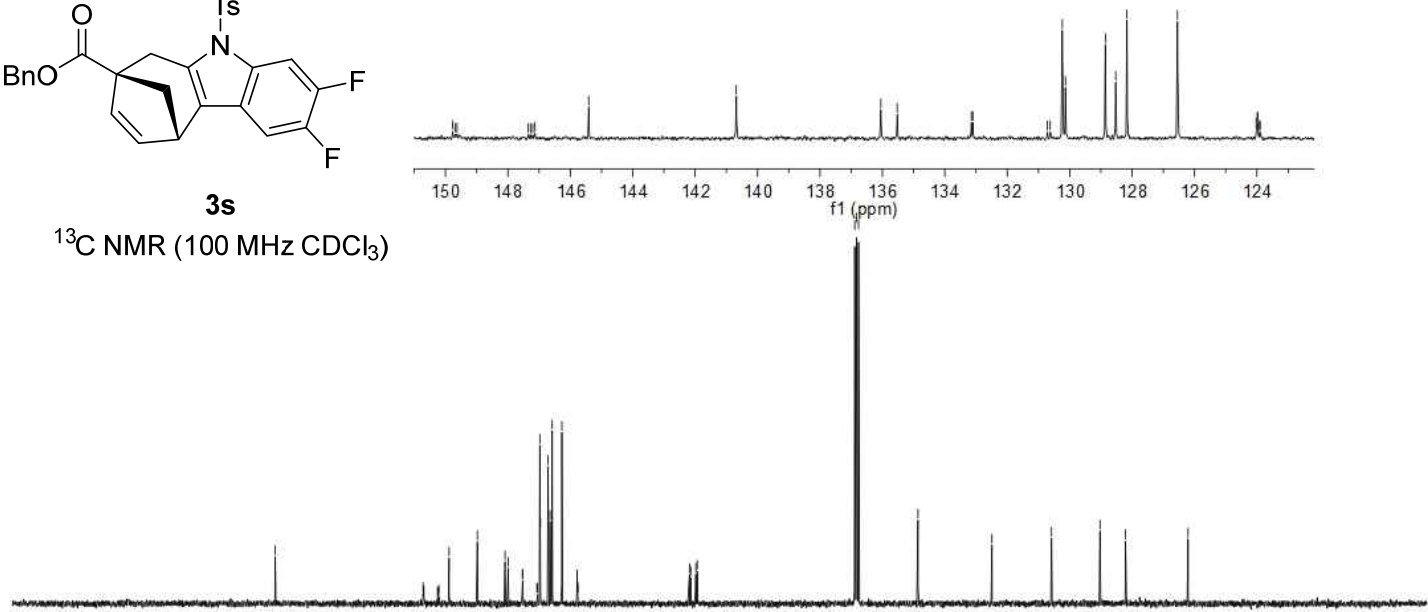

$\begin{array}{llllllllllllllllllllllllllll} & 1 & 200 & 190 & 180 & 170 & 160 & 150 & 140 & 130 & 120 & 110 & \begin{array}{c}100 \\ \mathrm{f} 1(\mathrm{pom})\end{array} & 90 & 80 & 70 & 60 & 50 & 40 & 30 & 20 & 10 & 0 & -10\end{array}$ 


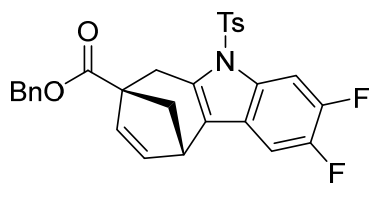

3s

$\left.{ }^{19} \mathrm{~F} \mathrm{NMR} \mathrm{(376} \mathrm{MHz} \mathrm{CDCl}_{3}\right)$

$\begin{array}{lllllllllllllllllllllllllll}20 & 10 & 0 & -10 & -20 & -30 & -40 & -50 & -60 & -70 & -80 & -90 & -100 & -110 & -120 & -130 & -140 & -150 & -160 & -170 & -180 & -190 & -200 & -210 & -22\end{array}$ 
<smiles>Cc1cc2c(cc1F)c1c(n2[13CH3])C[C@@]2(C(=O)OCc3ccccc3)C=C[C@H]1C2</smiles>

${ }^{1} \mathrm{H}$ NMR (400 $\left.\mathrm{MHz} \mathrm{CDCl}_{3}\right)$

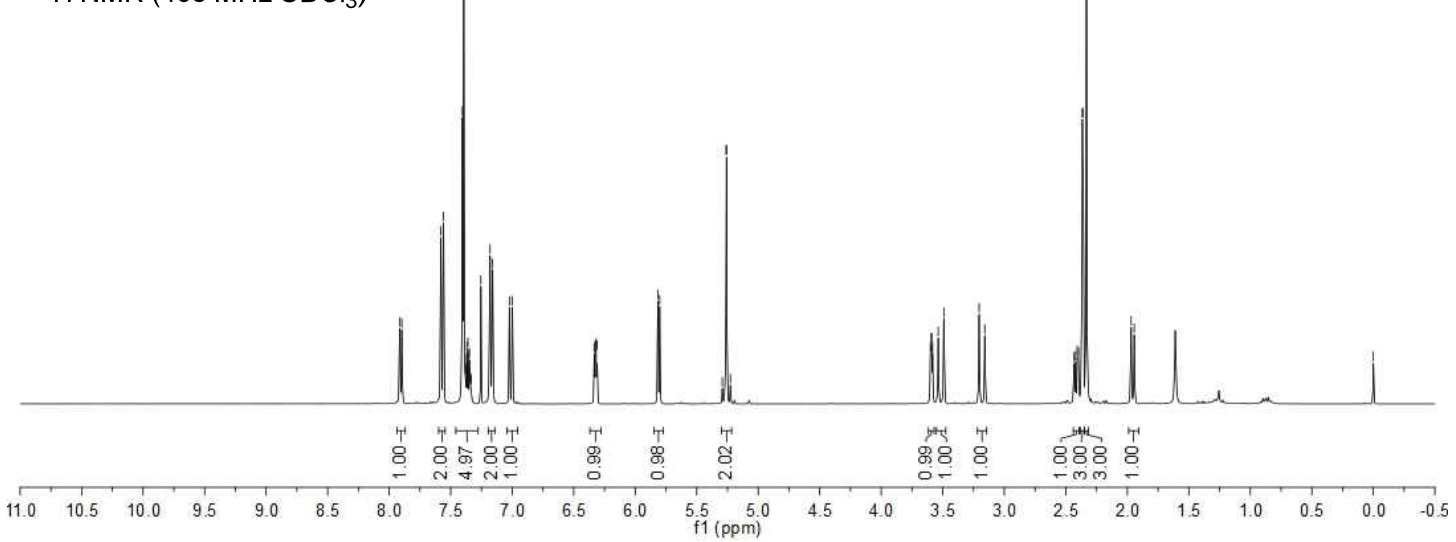

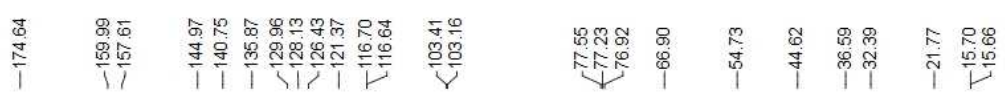

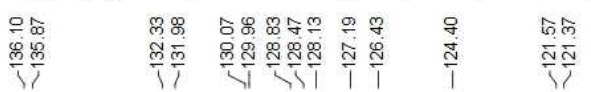<smiles>Cc1cc2c(cc1F)c1c(n2[13CH3])CC2(C(=O)OCc3ccccc3)C=CC3CC2CC13</smiles>

3t

${ }^{13} \mathrm{C} \mathrm{NMR}\left(100 \mathrm{MHz}_{\mathrm{CDCl}}\right)$
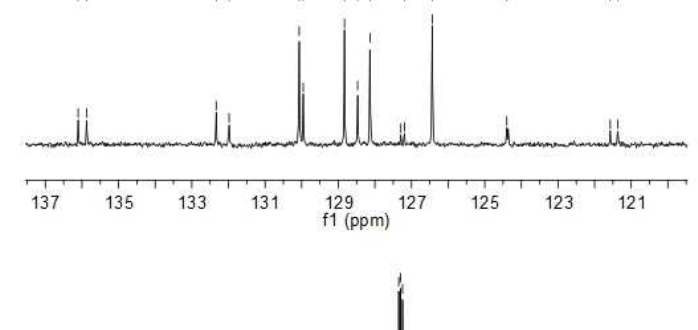

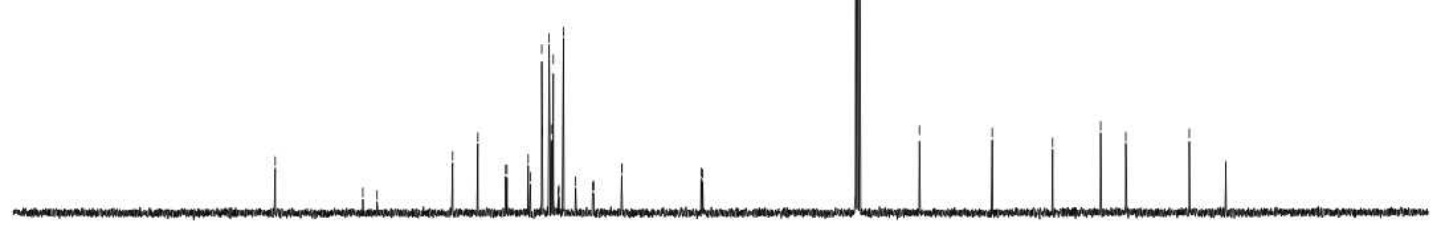

\begin{tabular}{|lllllllllllllllllllllllllllll} 
& 110 & 200 & 190 & 180 & 170 & 160 & 150 & 140 & 130 & 120 & 110 & 100 & 90 & 80 & 70 & 60 & 50 & 40 & 30 & 20 & 10 & 0 & -10
\end{tabular} 


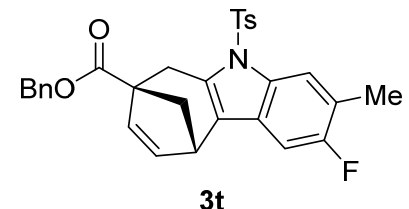

${ }^{19} \mathrm{~F}$ NMR (376 $\mathrm{MHz} \mathrm{CDCl}_{3}$ )

$\begin{array}{lllllllllllllllllllllllll}20 & 10 & 0 & -10 & -20 & -30 & -40 & -50 & -60 & -70 & -80 & -90 & -100 & -110 & -120 & -130 & -140 & -150 & -160 & -170 & -180 & -190 & -200 & -210 & -22\end{array}$ 


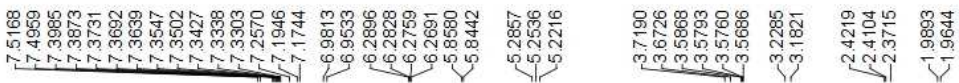

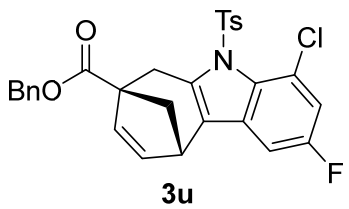

${ }^{1} \mathrm{H}$ NMR $\left(400 \mathrm{MHz} \mathrm{CDCl}_{3}\right)$

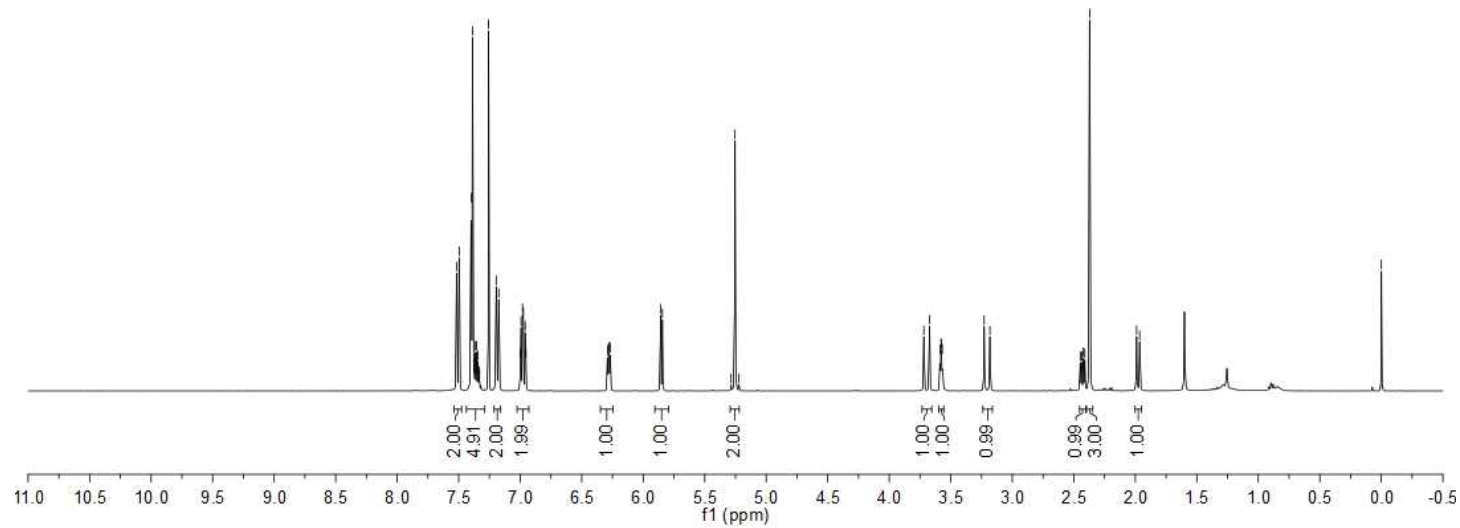

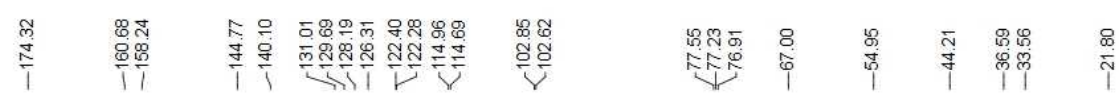

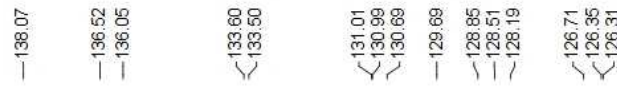<smiles>[13CH3]n1c2c(c3cc(F)cc(Cl)c31)C1(C(=O)OCc3ccccc3)C=CC2C1</smiles>

3u

${ }^{13} \mathrm{C} \mathrm{NMR}\left(100 \mathrm{MHz} \mathrm{CDCl}_{3}\right)$

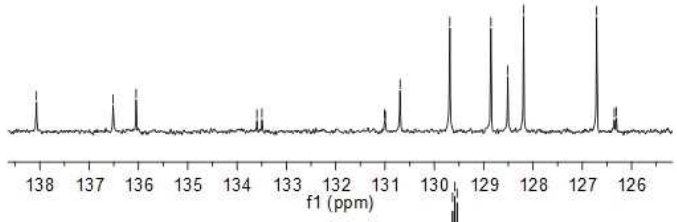

$\begin{array}{lllllllllllll}138 & 137 & 136 & 135 & 134 & 133 & 132 & 131 & 130 & 129 & 128 & 127 & 126\end{array}$

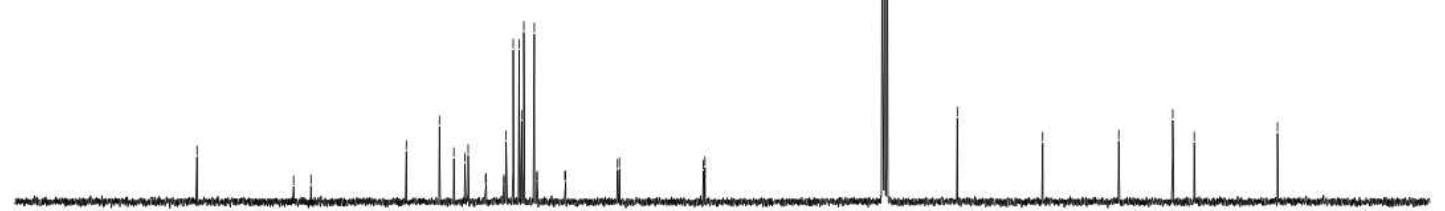

200 $\begin{array}{llllllllll}190 & 180 & 170 & 160 & 150 & 140 & 130 & 120 & 110 & 100 \\ \mathrm{f} 1(\mathrm{ppm})\end{array}$ 


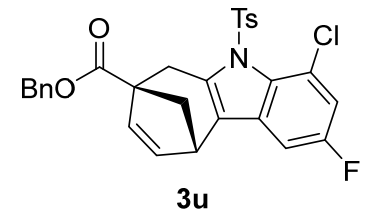

$\left.{ }^{19} \mathrm{~F} \mathrm{NMR} \mathrm{(376} \mathrm{MHz} \mathrm{CDCl}_{3}\right)$

$\begin{array}{llllllllllllllllllllllllllllll}20 & 10 & 0 & -10 & -20 & -30 & -40 & -50 & -60 & -70 & -80 & -90 & -100 & -110 & -120 & -130 & -140 & -150 & -160 & -170 & -180 & -190 & -200 & -210 & -220\end{array}$ 
<smiles>Cn1c2c(c3cc(Cl)cc(F)c31)C1C=CC2CC1(C)C(=O)OCc1ccccc1</smiles>

${ }^{1} \mathrm{H} \mathrm{NMR}\left(400 \mathrm{MHz} \mathrm{CDCl}_{3}\right)$

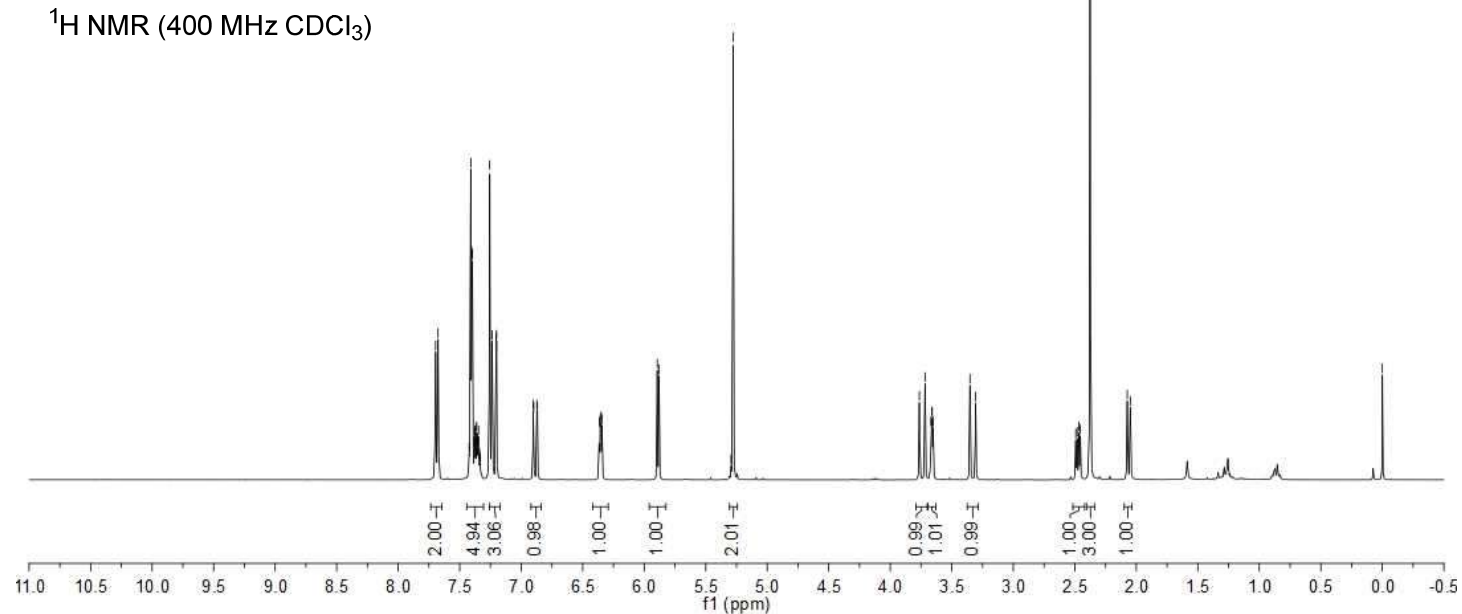

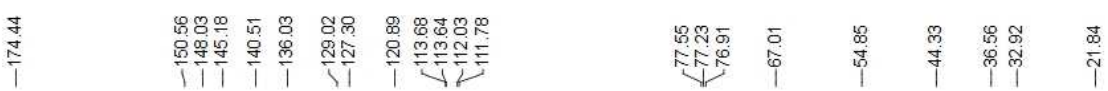

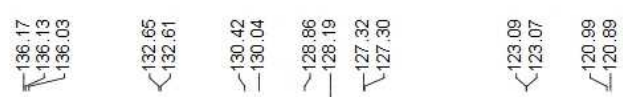<smiles></smiles>

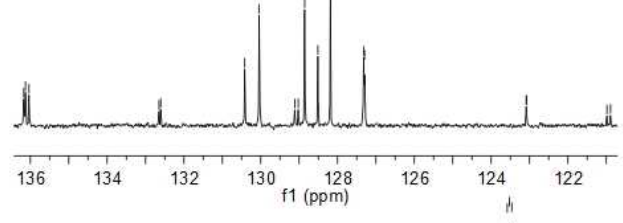

${ }^{13} \mathrm{C}$ NMR $\left(100 \mathrm{MHz} \mathrm{CDCl}_{3}\right)$

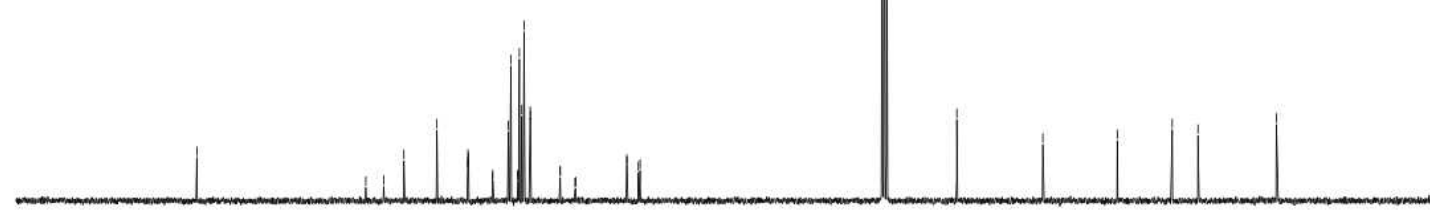

200

\begin{tabular}{llllllllll}
\hline 190 & 180 & 170 & 160 & 150 & 140 & 130 & 120 & 110 & 10
\end{tabular} 
<smiles>Cn1c2c(c3cc(Cl)cc(F)c31)C1C=CC2CC1(CC(=O)O)C(=O)OCc1ccccc1</smiles>

${ }^{19} \mathrm{~F} \mathrm{NMR}\left(376 \mathrm{MHz} \mathrm{CDCl}_{3}\right)$

$\begin{array}{llllllllllllllllllllllllllll}20 & 10 & 0 & -10 & -20 & -30 & -40 & -50 & -60 & -70 & -80 & -90 & -100 & -110 & -120 & -130 & -140 & -150 & -160 & -170 & -180 & -190 & -200 & -210 & -22\end{array}$ 
<smiles>[Y8]n1c2c(c3cc(F)cc(F)c31)C1C=CC2(C(=O)OCc2ccccc2)CC1</smiles>

$3 w$

$\left.\left.{ }^{1} \mathrm{H} \mathrm{NMR} \mathrm{(400} \mathrm{MHz} \mathrm{CDCl}\right)_{3}\right)$

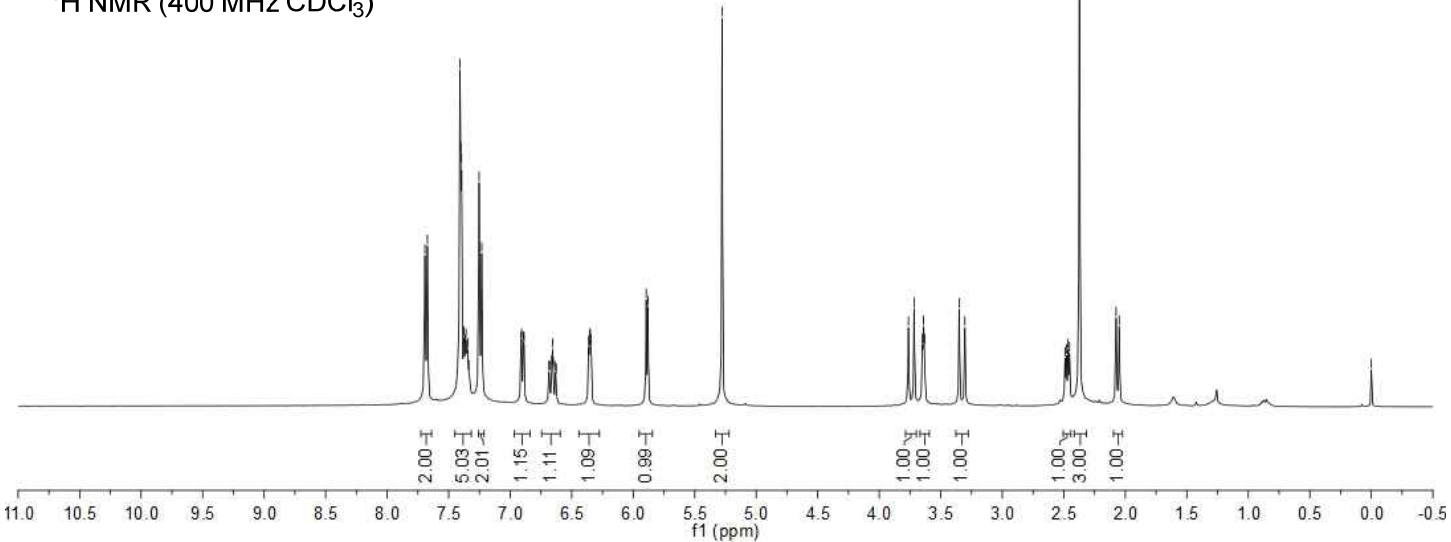

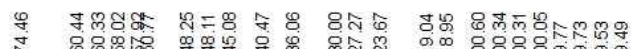

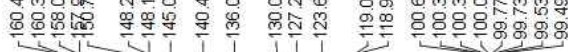

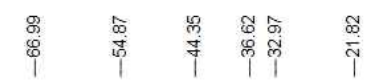

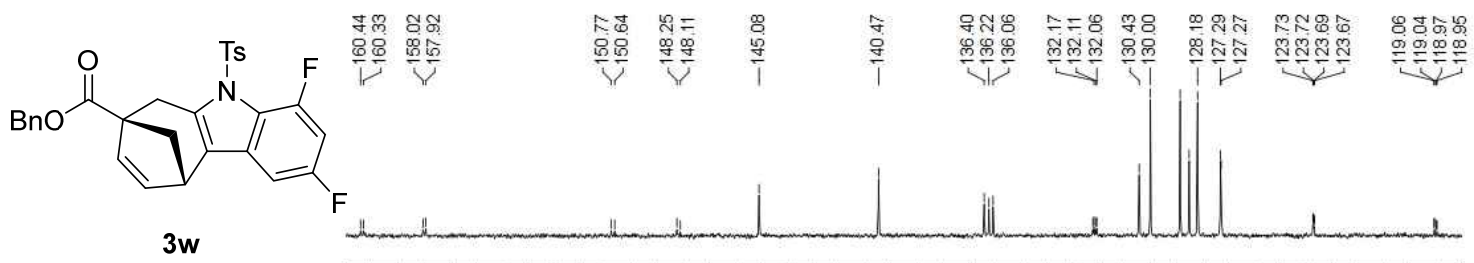

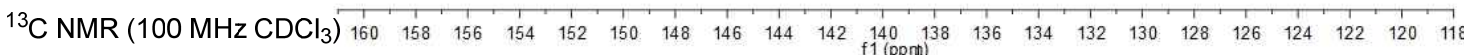

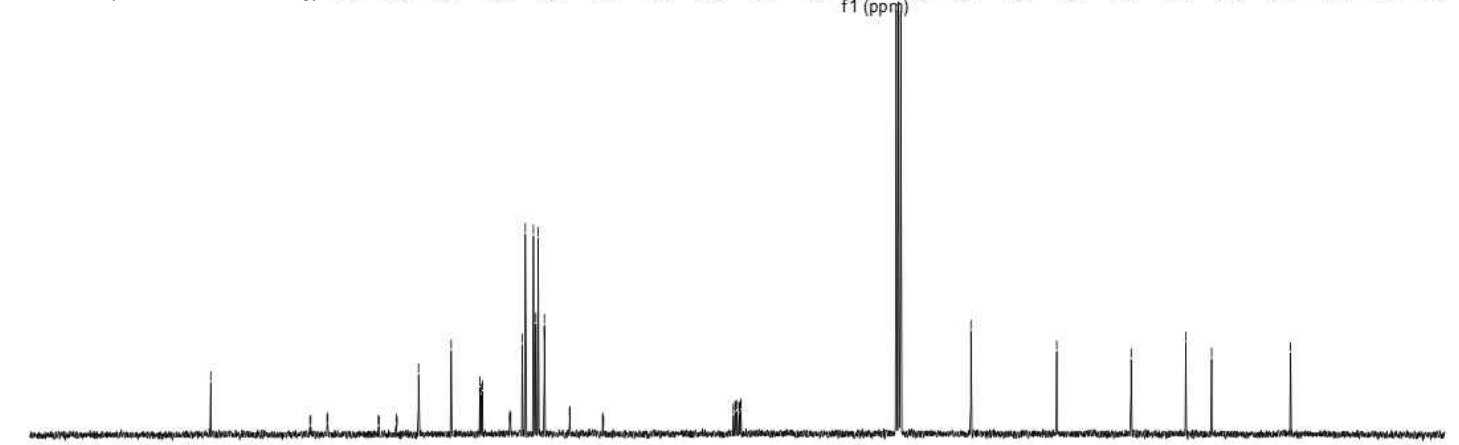

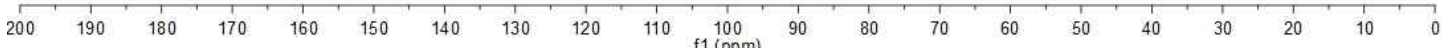




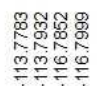

(n)

$3 w$

$\left.{ }^{19} \mathrm{~F} \mathrm{NMR} \mathrm{(376} \mathrm{MHz} \mathrm{CDCl}_{3}\right)$

$\begin{array}{lllllllllllllllllllllllll}20 & 10 & 0 & -10 & -20 & -30 & -40 & -50 & -60 & -70 & -80 & -90 & -100 & -110 & -120 & -130 & -140 & -150 & -160 & -170 & -180 & -190 & -200 & -210 & -22\end{array}$ 
<smiles>COC(=O)C12C=CC(CC1)c1c2c2ccccc2n1C</smiles>

$3 \mathrm{x}$

${ }^{1} \mathrm{H}$ NMR $\left(400 \mathrm{MHz} \mathrm{CDCl}_{3}\right)$

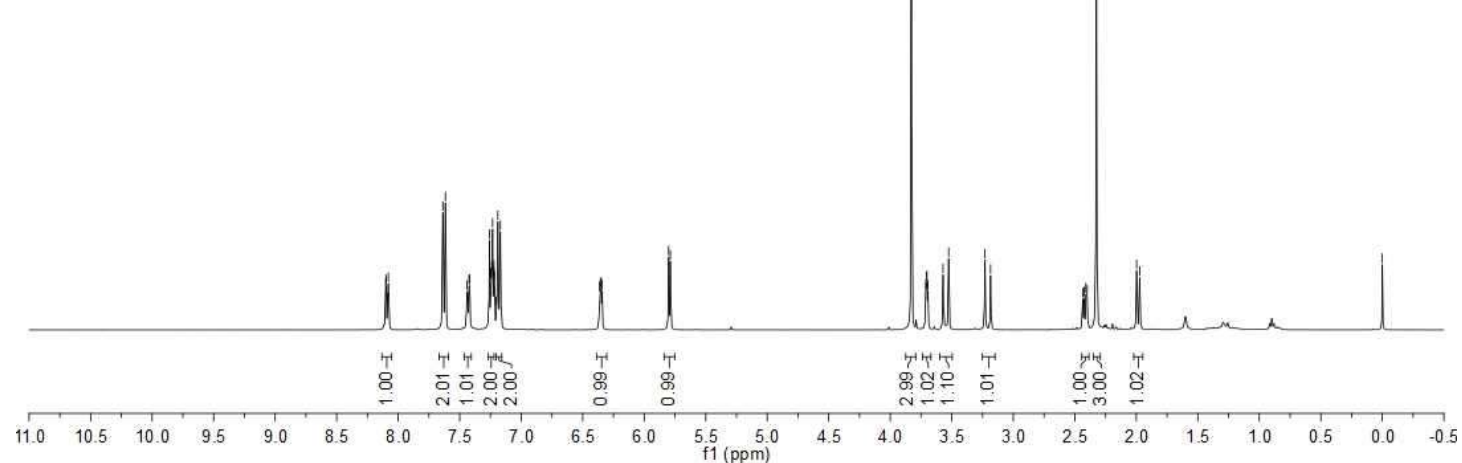

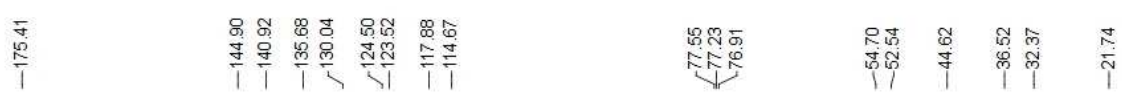<smiles>COC(=O)C12CCC(CC1)c1c2n([125I])c2ccccc12</smiles>

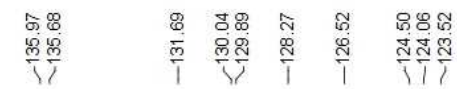

$3 \mathbf{x}$

${ }^{13} \mathrm{C} \mathrm{NMR}\left(100 \mathrm{MHz} \mathrm{CDCl}_{3}\right)$
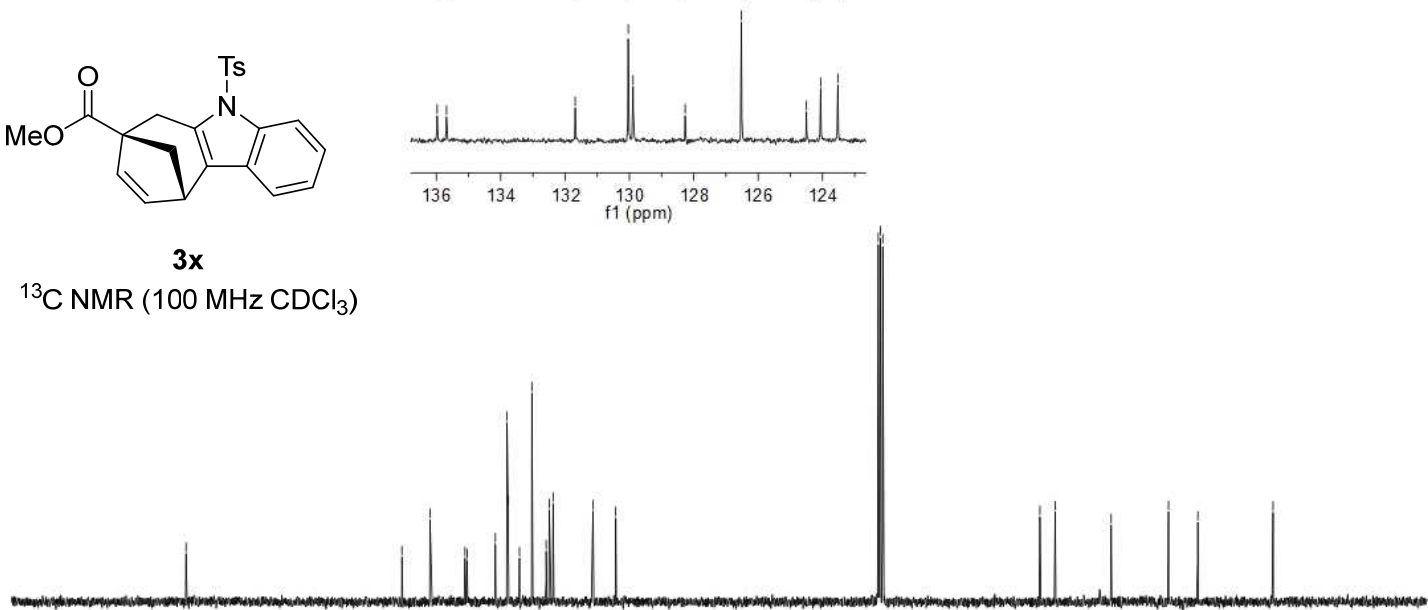

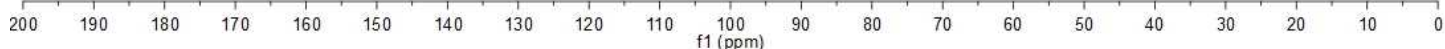


<smiles>CCOC(=O)C12C=CC(CC1)c1c2n([AsH3])c2ccccc12</smiles>

$3 y$

${ }^{1} \mathrm{H} \mathrm{NMR}\left(400 \mathrm{MHz} \mathrm{CDCl}_{3}\right)$
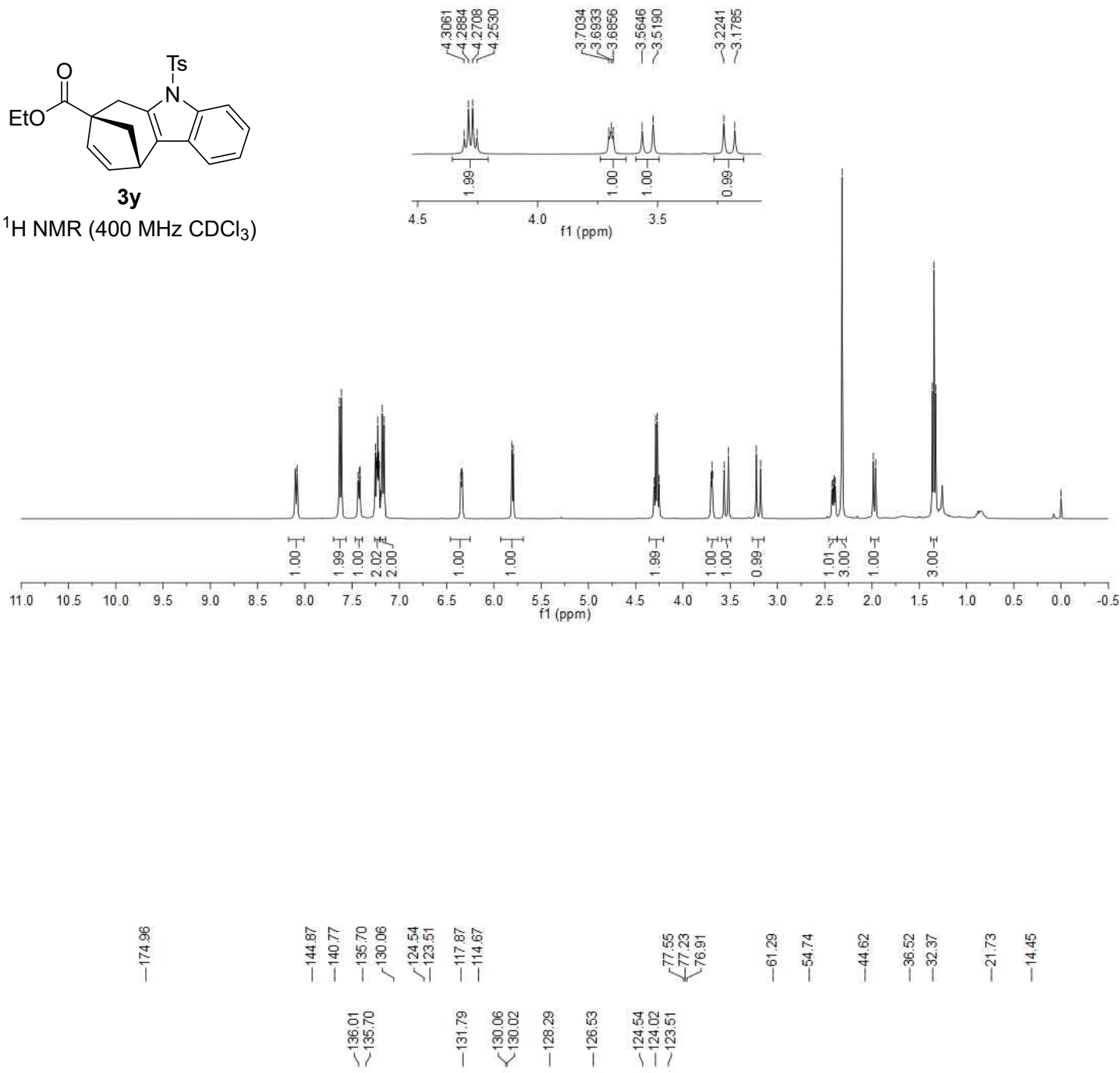<smiles>CCOC(=O)C12C=CC(CC1)c1c2n([135I])c2ccccc12</smiles>

$3 y$

${ }^{13} \mathrm{C}$ NMR $\left(100 \mathrm{MHz} \mathrm{CDCl}_{3}\right)$

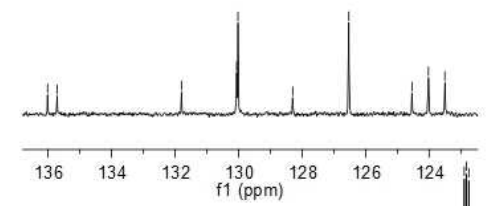

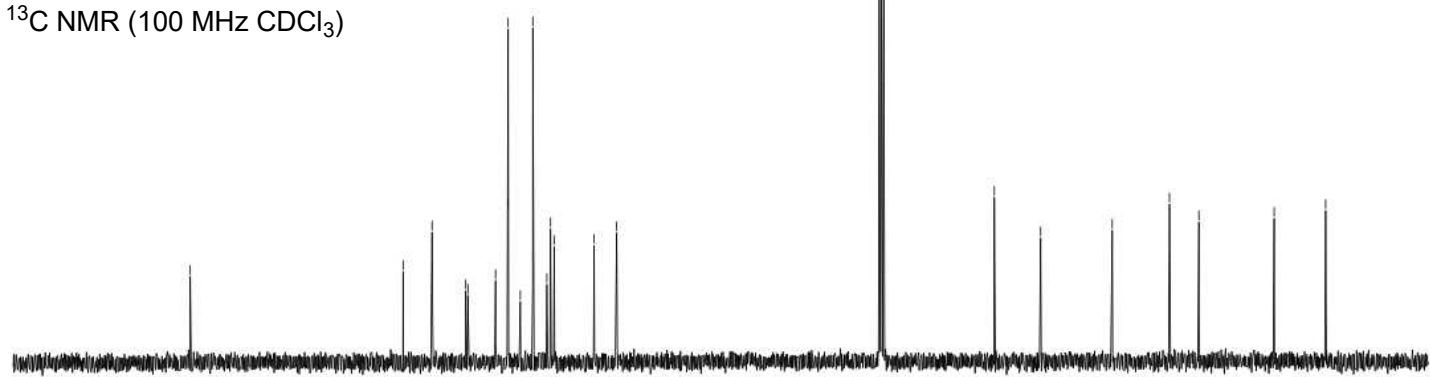

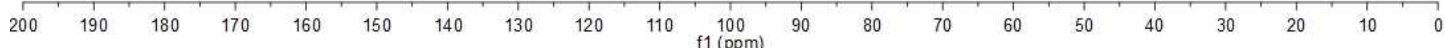




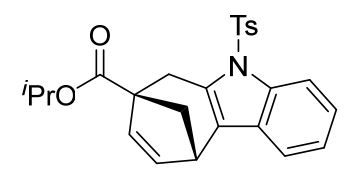

$3 z$

${ }^{1} \mathrm{H}$ NMR (400 $\left.\mathrm{MHz} \mathrm{CDCl}_{3}\right)$
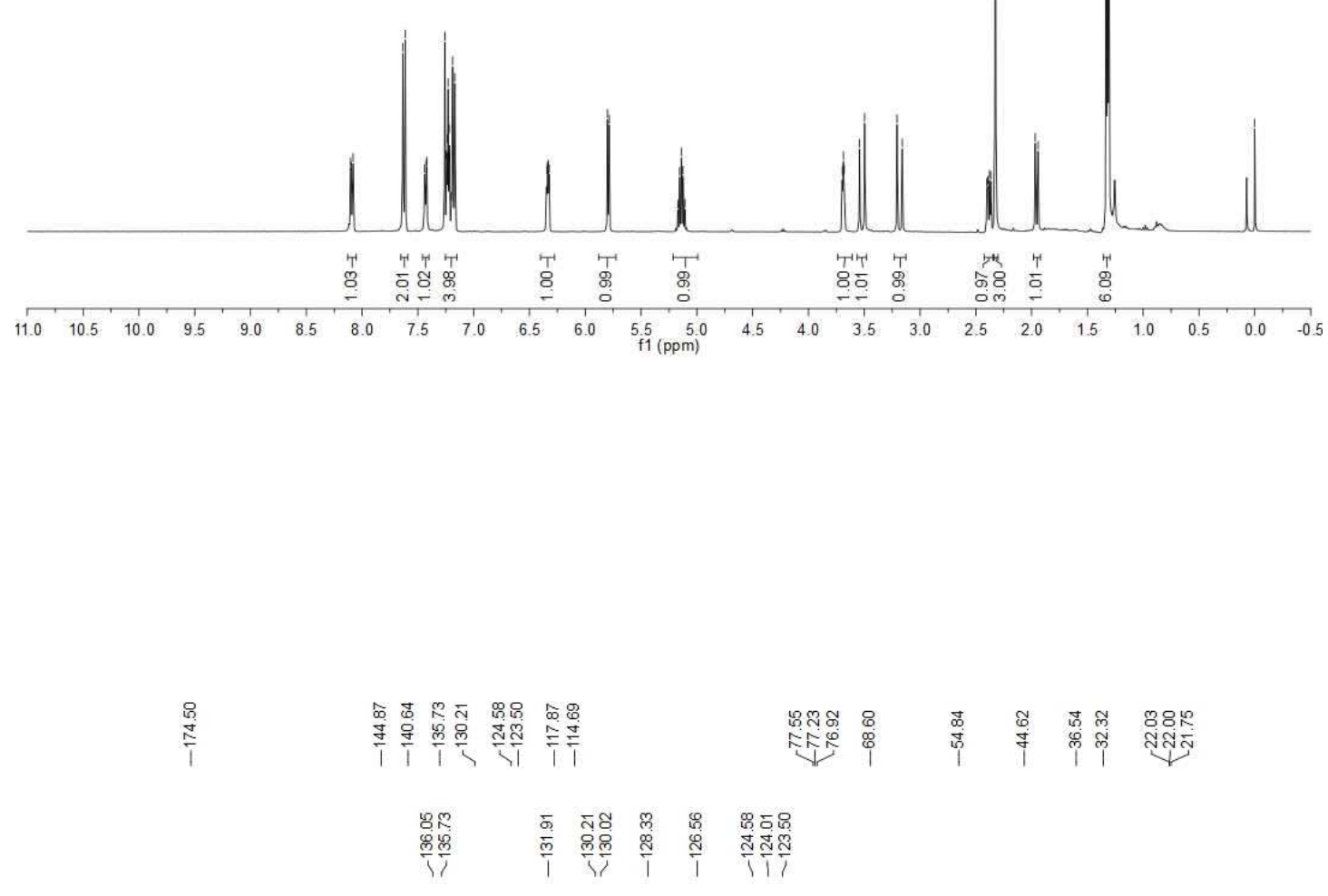<smiles></smiles>

$3 z$

${ }^{13} \mathrm{C} \mathrm{NMR}\left(100 \mathrm{MHz} \mathrm{CDCl}_{3}\right)$
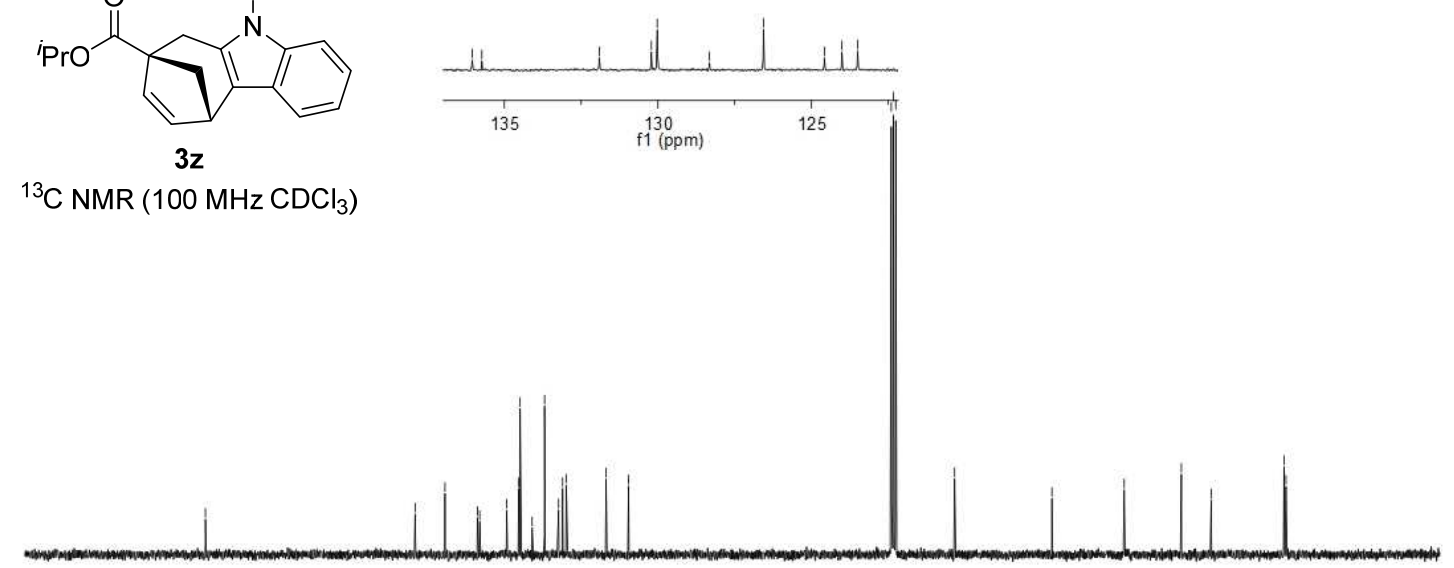

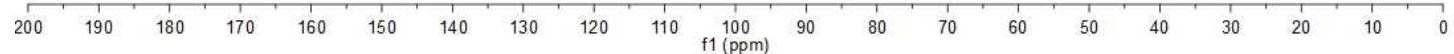




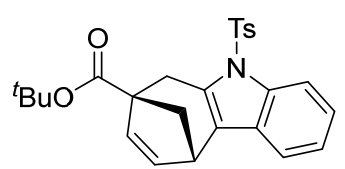

3aa

${ }^{1} \mathrm{H}$ NMR (400 MHz $\mathrm{CDCl}_{3}$ )

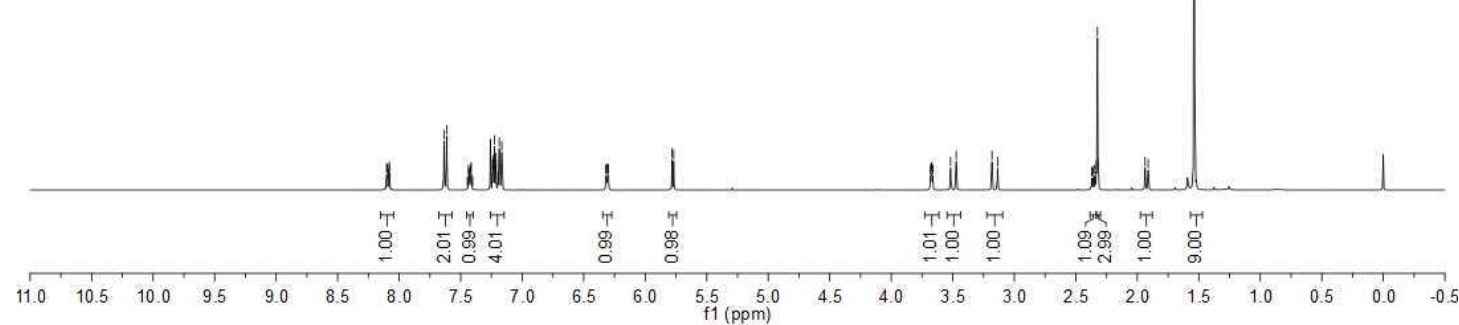

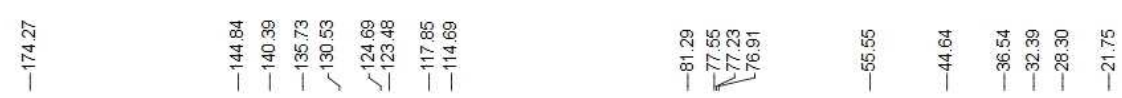

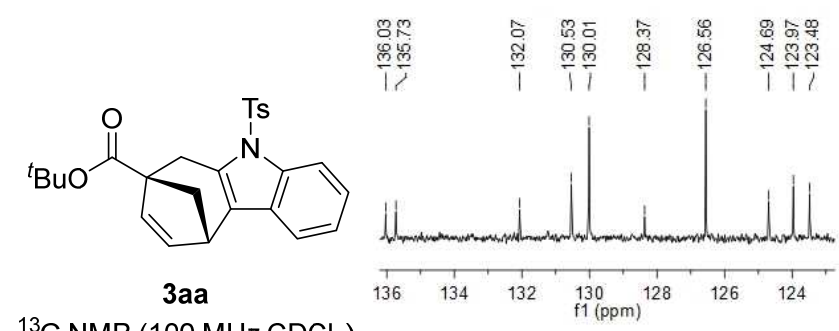

${ }^{13} \mathrm{C}$ NMR (100 $\mathrm{MHz} \mathrm{CDCl}_{3}$ )

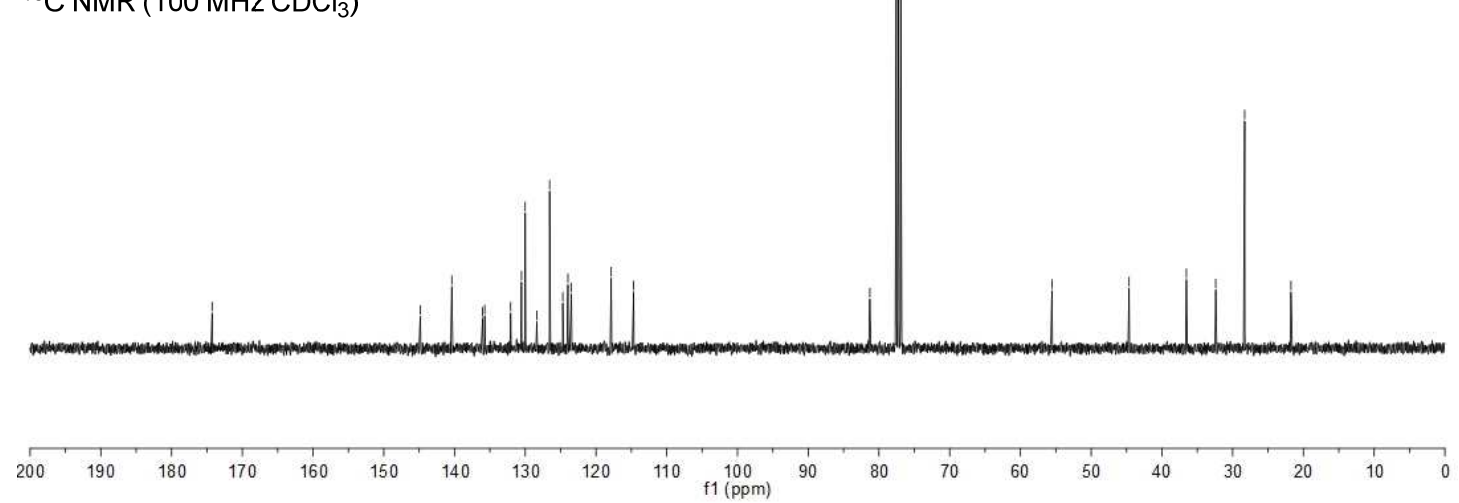




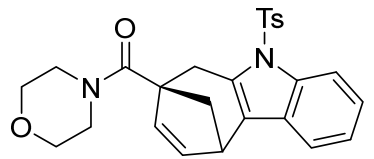

$3 a b$

${ }^{1} \mathrm{H}$ NMR $\left(400 \mathrm{MHz} \mathrm{CDCl}_{3}\right)$
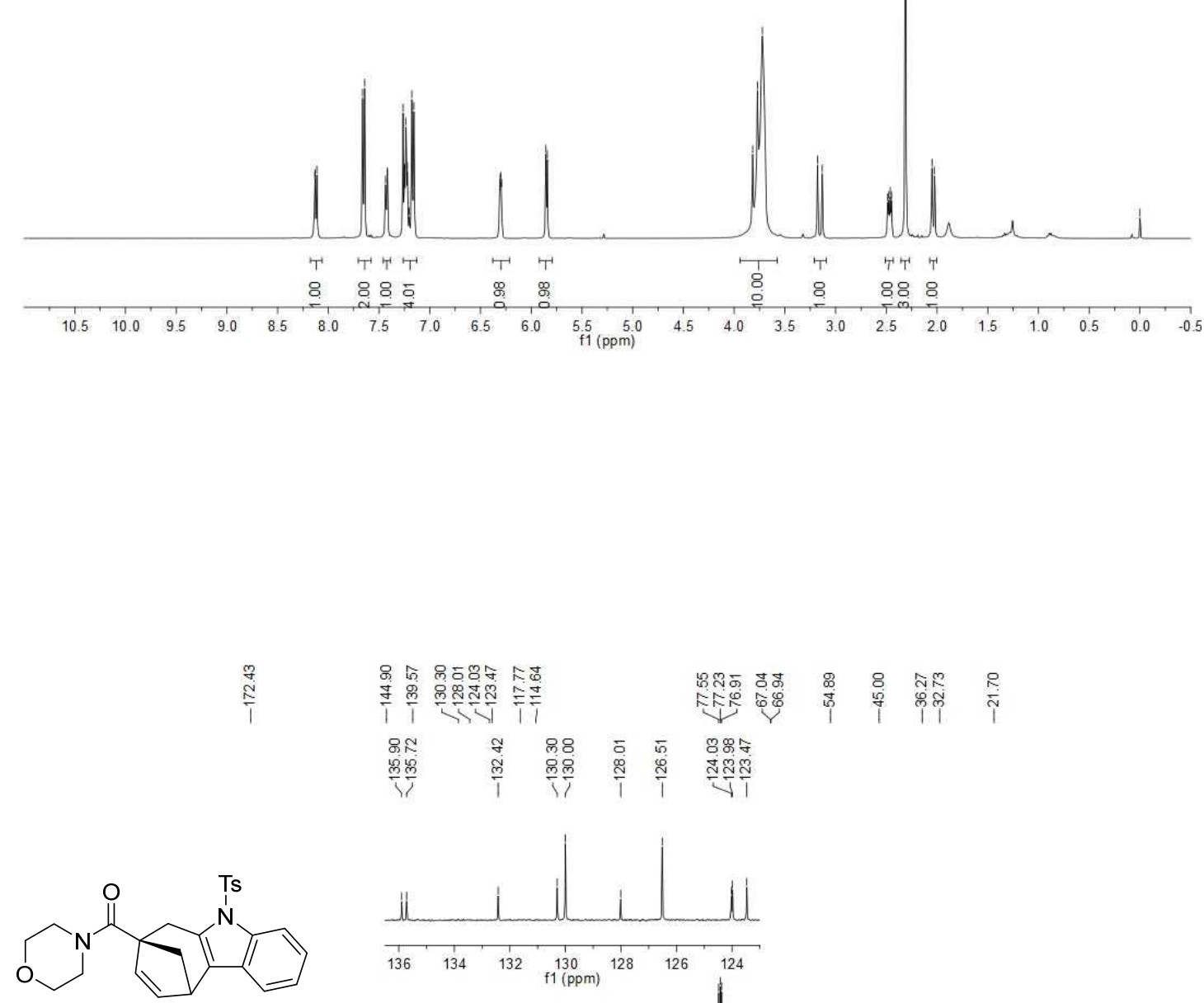

$3 a b$

${ }^{13} \mathrm{C}$ NMR (100 $\left.\mathrm{MHz} \mathrm{CDCl}_{3}\right)$

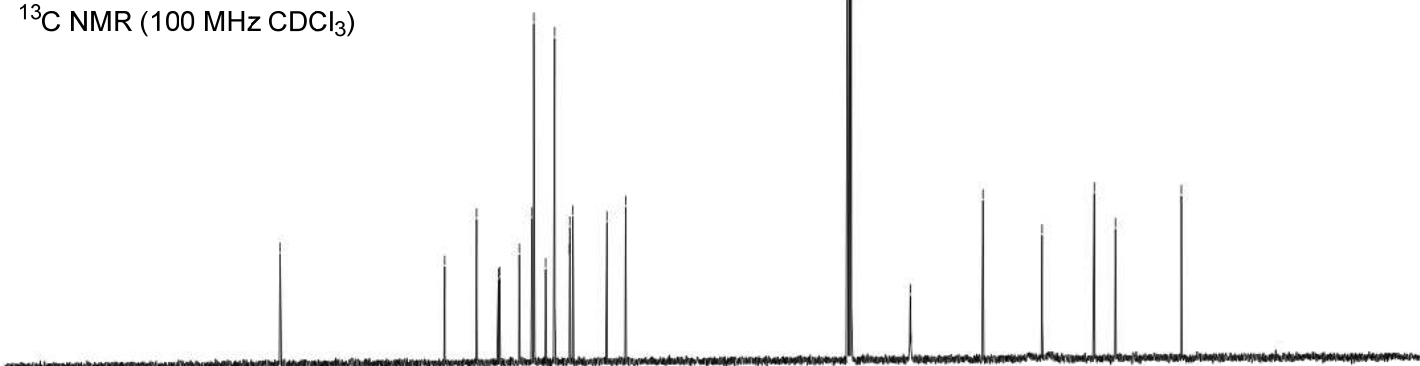

$\begin{array}{llllllllllllllllllllllll} & 1 & 200 & 190 & 180 & 170 & 160 & 150 & 140 & 130 & 120 & 110 & 100 & 90 & 80 & 70 & 60 & 50 & 40 & 30 & 20 & 10 & 0 & -10\end{array}$ 

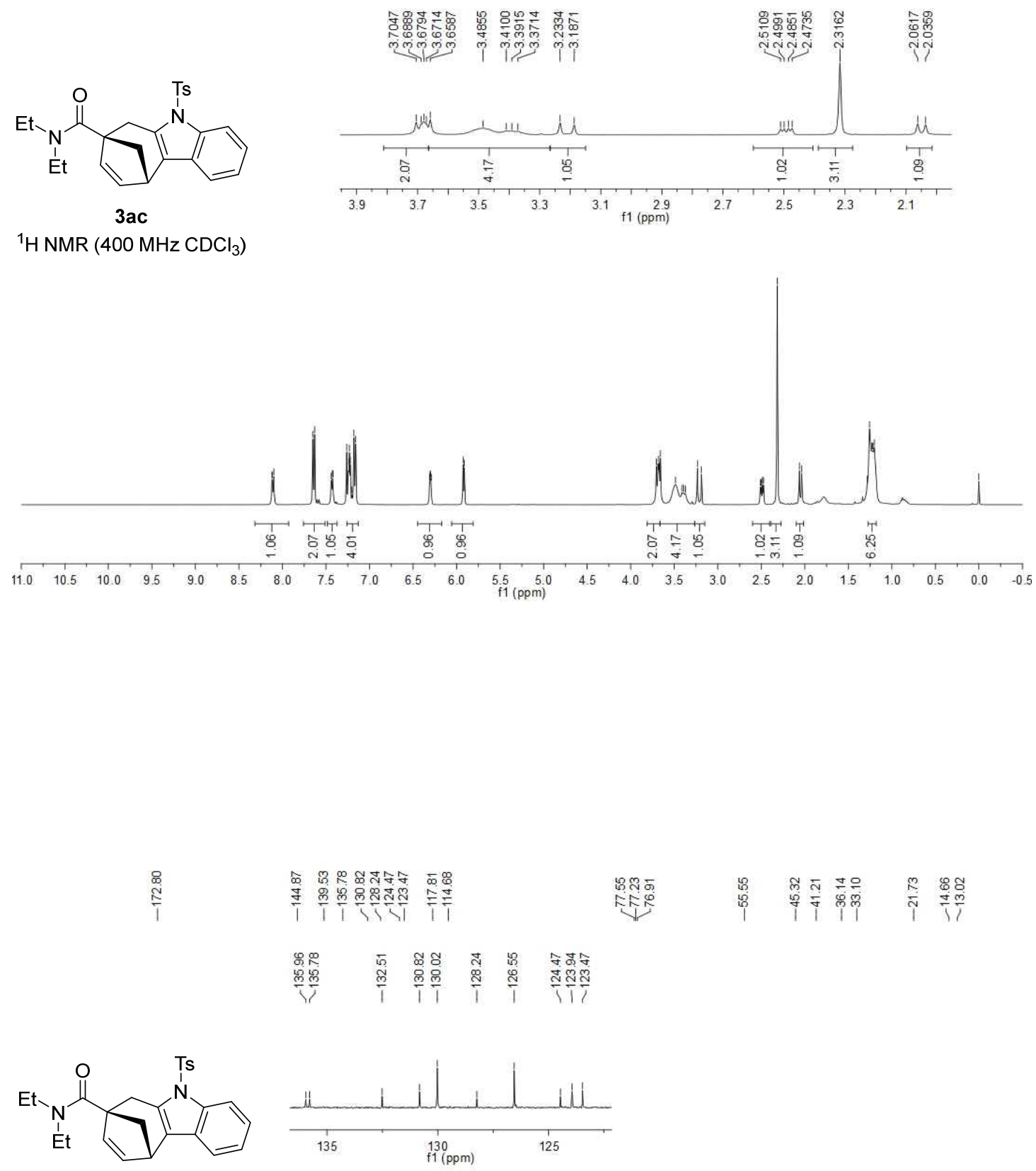

${ }^{13} \mathrm{C} \mathrm{NMR}\left(100 \mathrm{MHz} \mathrm{CDCl}_{3}\right)$

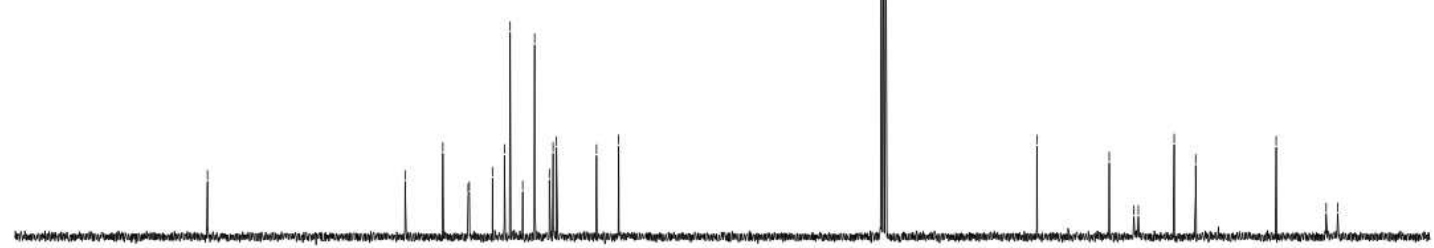

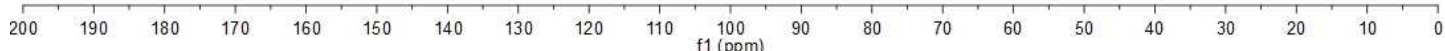




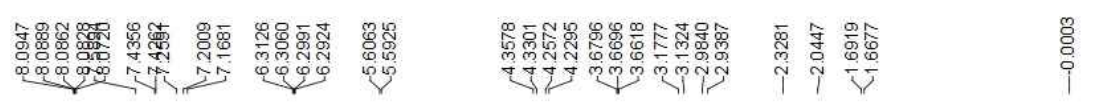
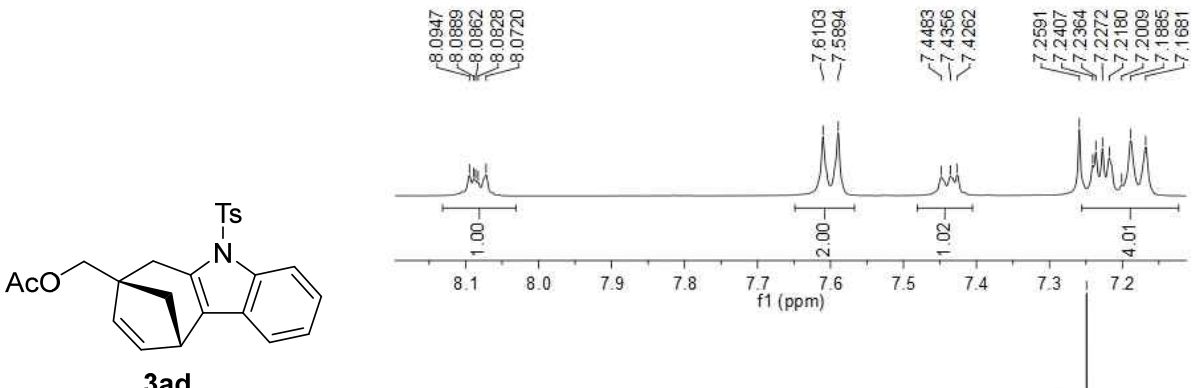

${ }^{1} \mathrm{H}$ NMR $\left(400 \mathrm{MHz} \mathrm{CDCl}_{3}\right)$
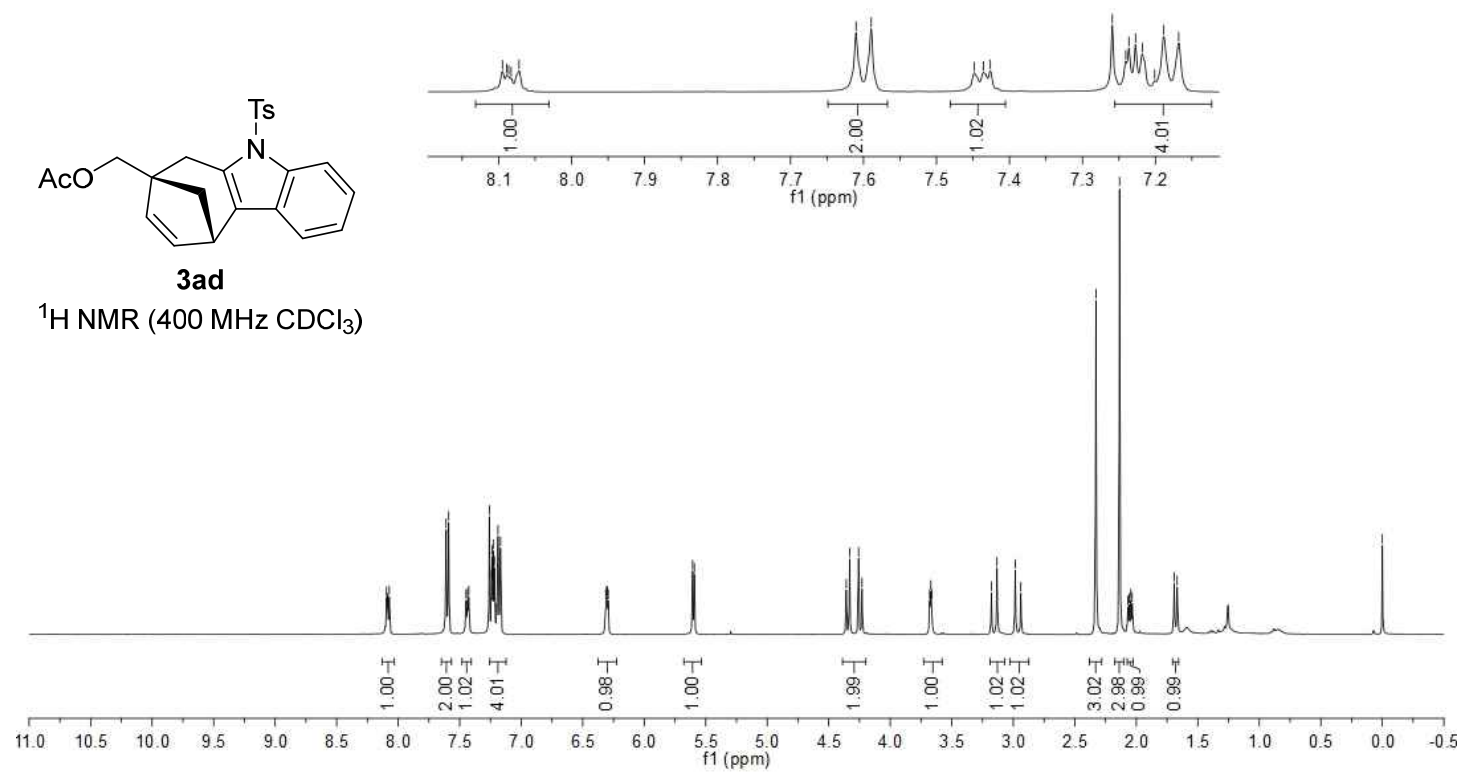

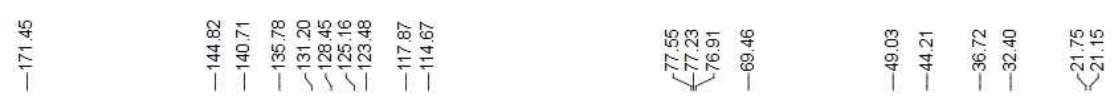

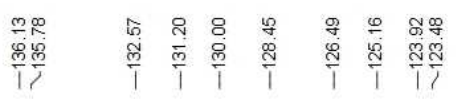<smiles>CC(=O)OCC12C=CC(CC1)c1c2c2ccccc2n1C</smiles>

3ad

${ }^{13} \mathrm{C} \mathrm{NMR}\left(100 \mathrm{MHz}_{\mathrm{CDCl}}\right)$
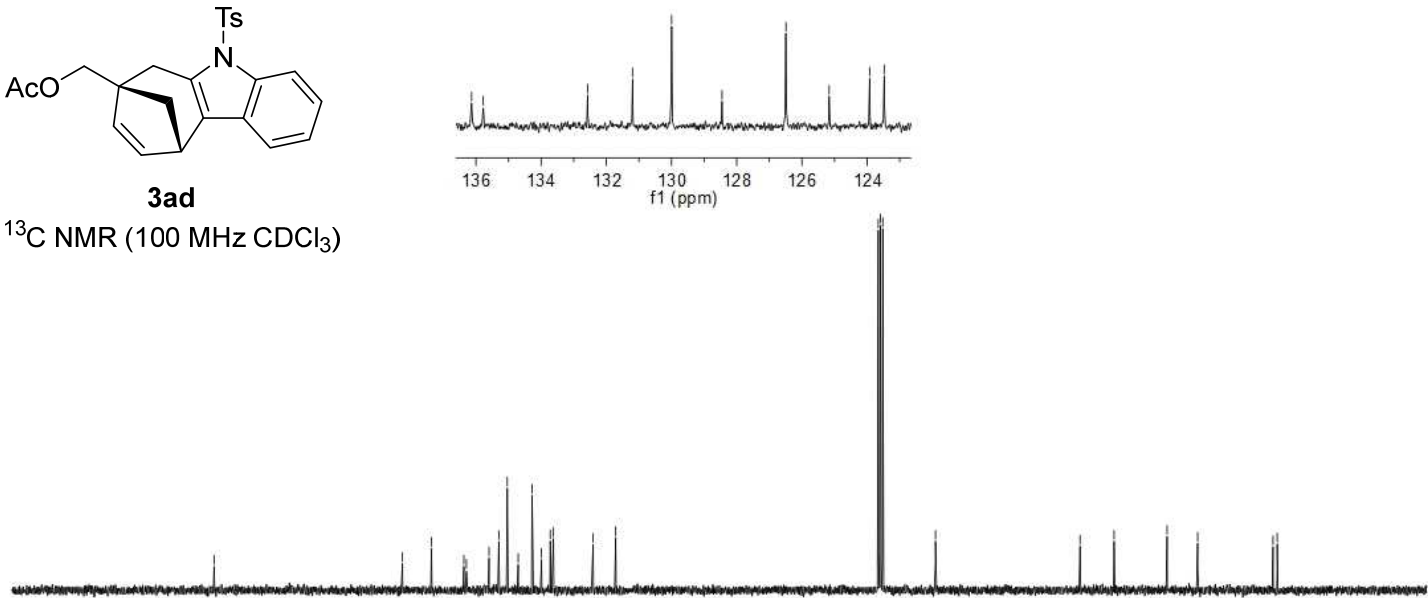

\begin{tabular}{llllllllllllllllllllllllll}
\hline & 200 & 190 & 180 & 170 & 160 & 150 & 140 & 130 & 120 & 110 & 100 & 90 & 80 & 70 & 60 & 50 & 40 & 30 & 20 & 10 & 0
\end{tabular} 


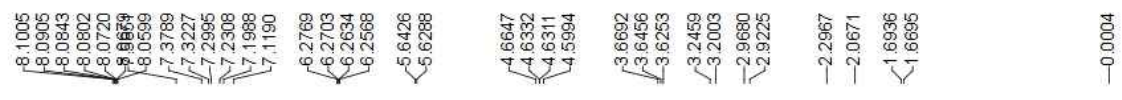

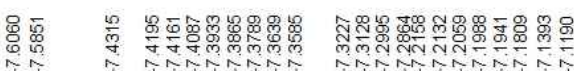

(1)

3ae

${ }^{1} \mathrm{H}$ NMR (400 $\left.\mathrm{MHz} \mathrm{CDCl}_{3}\right)$

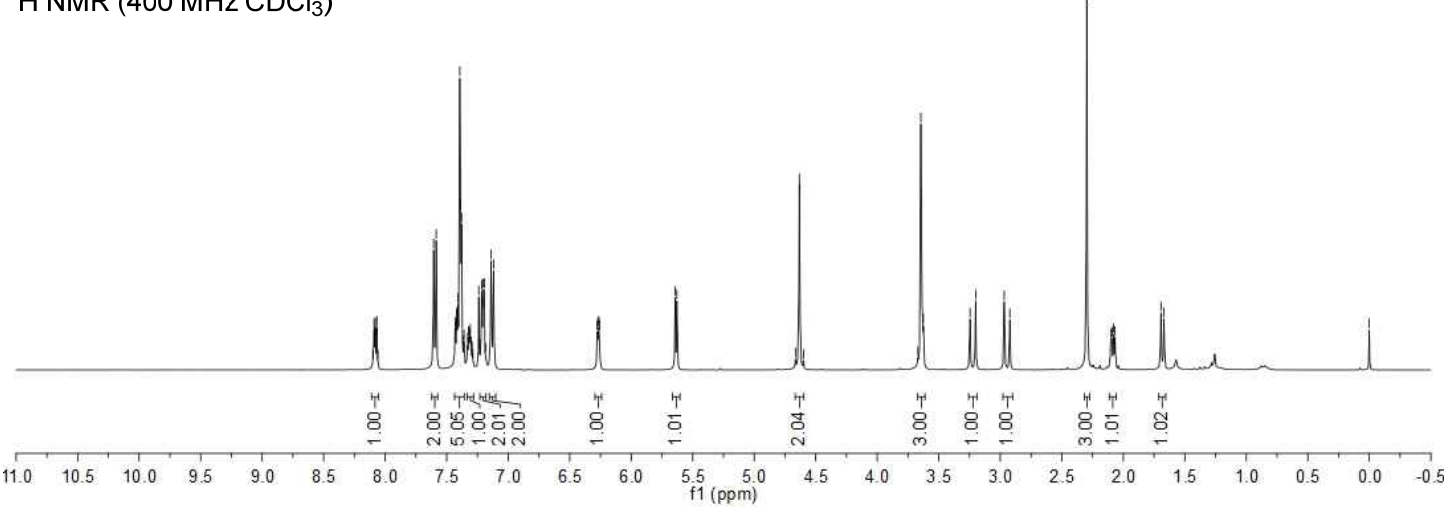

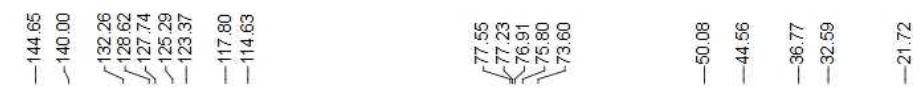

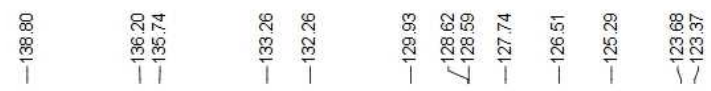

$\overbrace{1}^{\mathrm{T}}$

3 ae

${ }^{13} \mathrm{C} \mathrm{NMR}\left(100 \mathrm{MHz} \mathrm{CDCl}_{3}\right)$

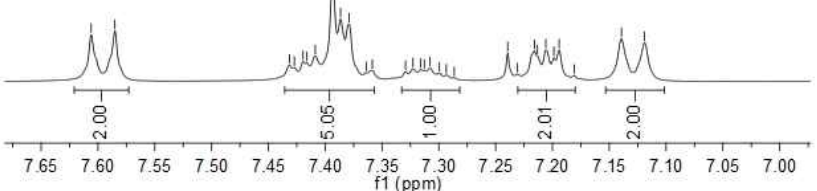

$\begin{array}{llllllll}65 & 7.60 & 7.55 & 7.50 & 7.45 & 7.40 & 7.35 & 7.30 \\ f 1(\mathrm{ppm})\end{array}$

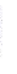

. 


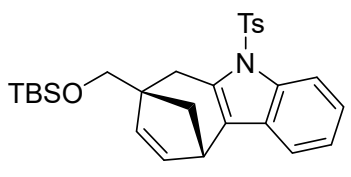

3af

${ }^{1} \mathrm{H}$ NMR $\left(400 \mathrm{MHz} \mathrm{CDCl}_{3}\right)$

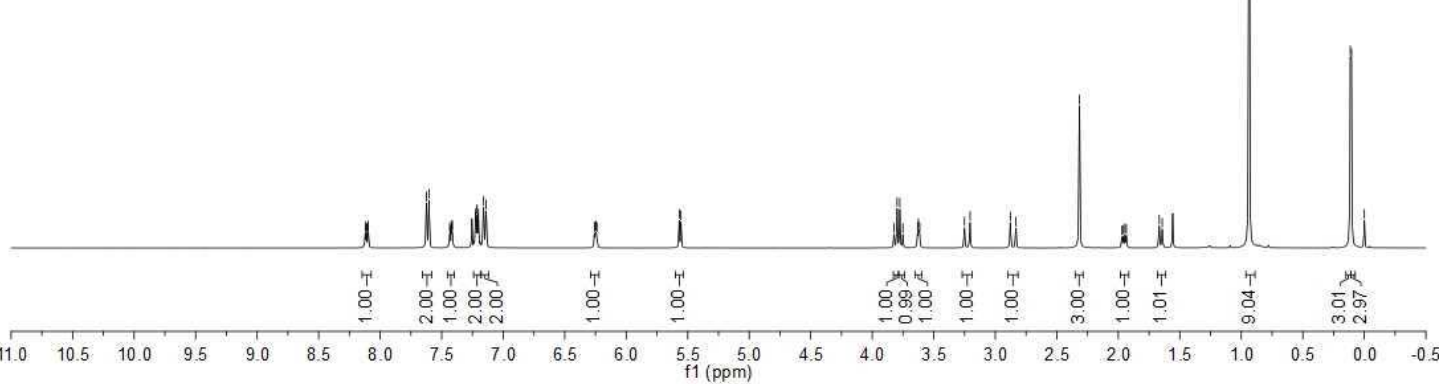

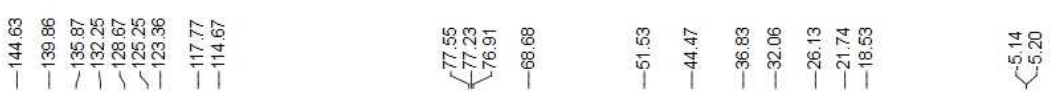

TBSO

3af

${ }^{13} \mathrm{C}$ NMR $\left(100 \mathrm{MHz} \mathrm{CDCl}_{3}\right)$

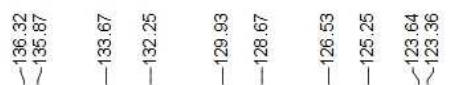

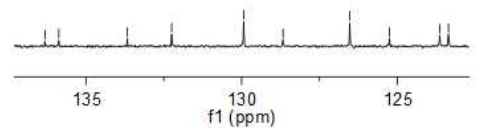

$\begin{array}{llllllllllll}200 & 190 & 180 & 170 & 160 & 150 & 140 & 130 & 120 & 110 & \underset{f 1}{100} 90 \\ \mathrm{f} 1(\mathrm{ppm})\end{array}$ 


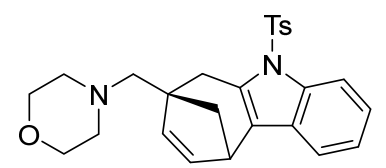

$3 \mathrm{ag}$

${ }^{1} \mathrm{H} \mathrm{NMR}\left(400 \mathrm{MHz} \mathrm{CDCl}_{3}\right)$

H NMR (400 $\left.\mathrm{MHz} \mathrm{CDCl}_{3}\right)$

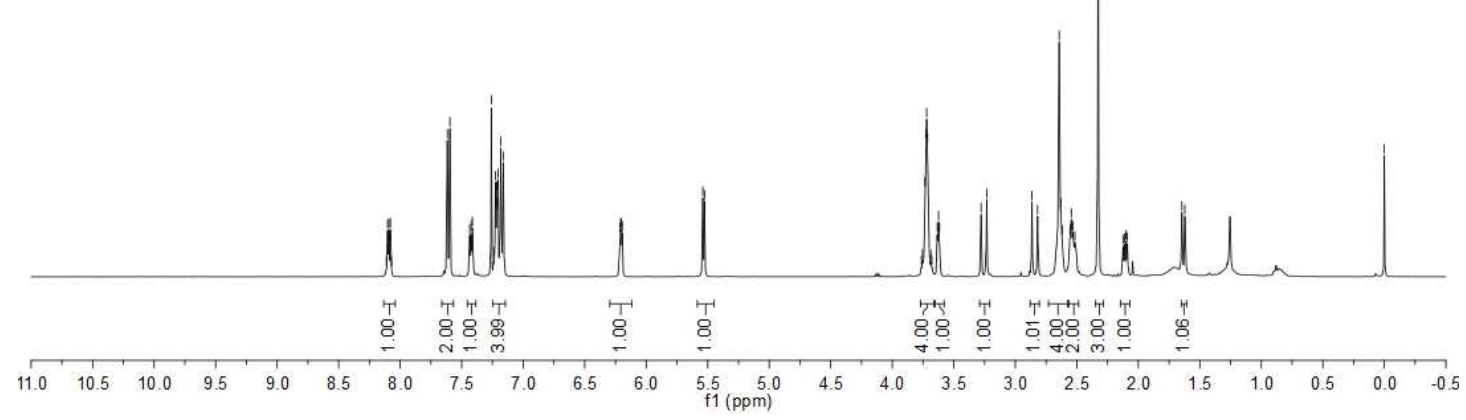

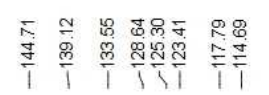

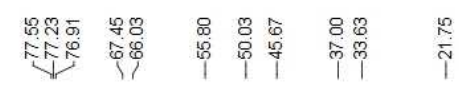

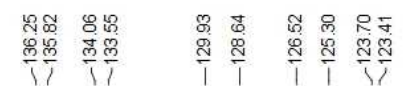
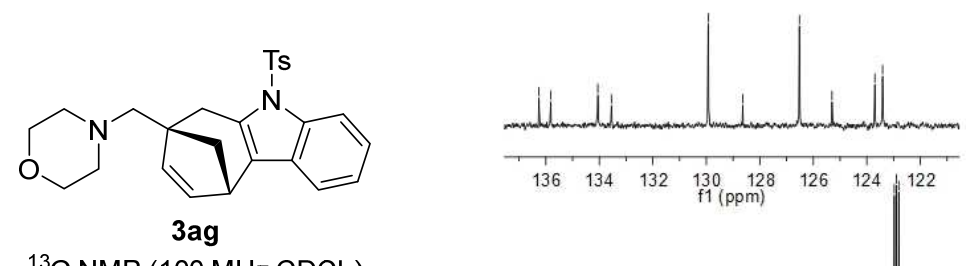

${ }^{13} \mathrm{C} \mathrm{NMR}\left(100 \mathrm{MHz} \mathrm{CDCl}_{3}\right)$

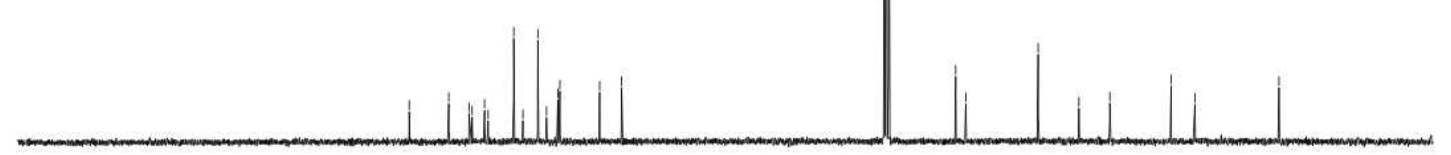

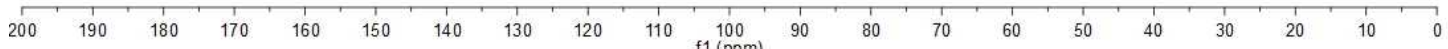



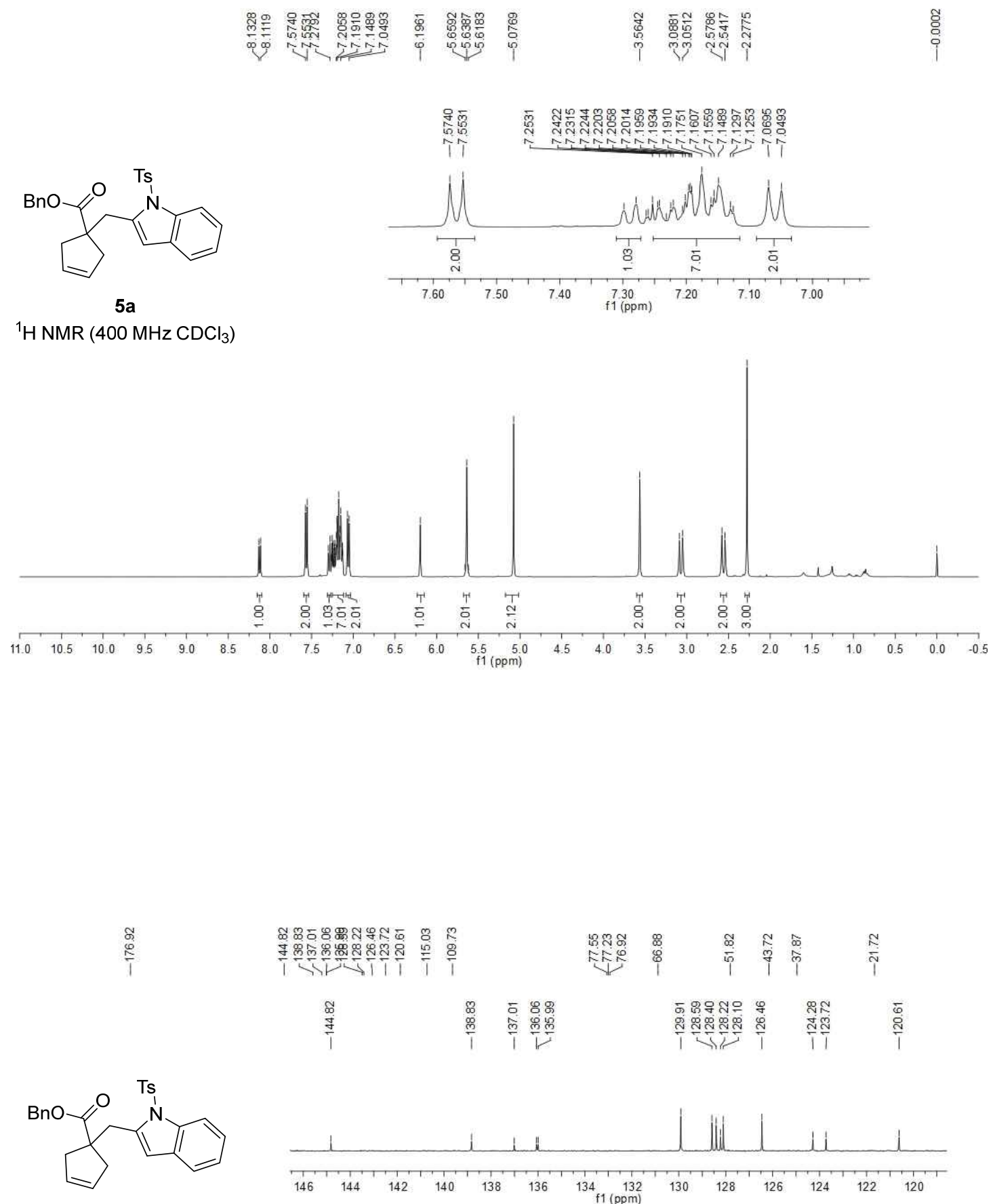

$5 \mathbf{a}$

${ }^{13} \mathrm{C}$ NMR $\left(100 \mathrm{MHz} \mathrm{CDCl}_{3}\right)$
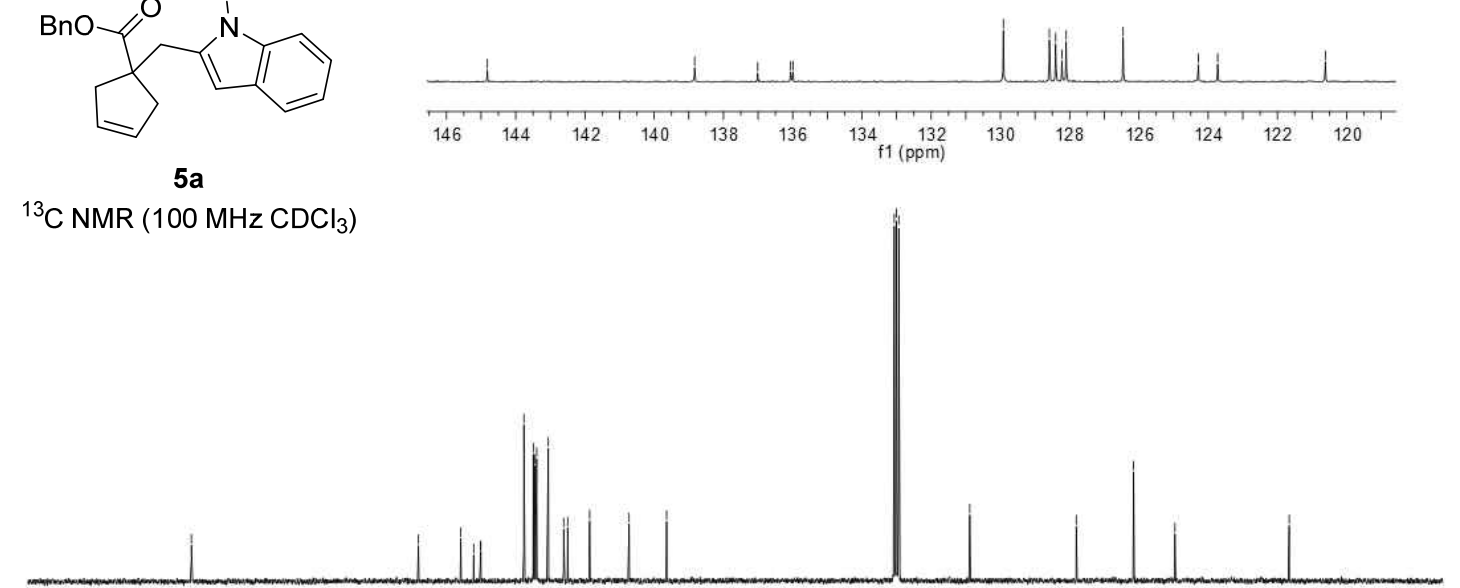

\begin{tabular}{lllllllllllllllllllllll}
\hline 200 & 190 & 180 & 170 & 160 & 150 & 140 & 130 & 120 & 110 & 100 & 90 & 80 & 70 & 60 & 50 & 40 & 30 & 20 & 10 & 0
\end{tabular} 
<smiles>Cc1ccc2c(c1)c1c(n2[13CH3])C[C@@]2(C(=O)O)C=C[C@H]1CC21CC1</smiles>

${ }^{1} \mathrm{H} \mathrm{NMR}\left(400 \mathrm{MHz} \mathrm{CDCl}_{3}\right)$

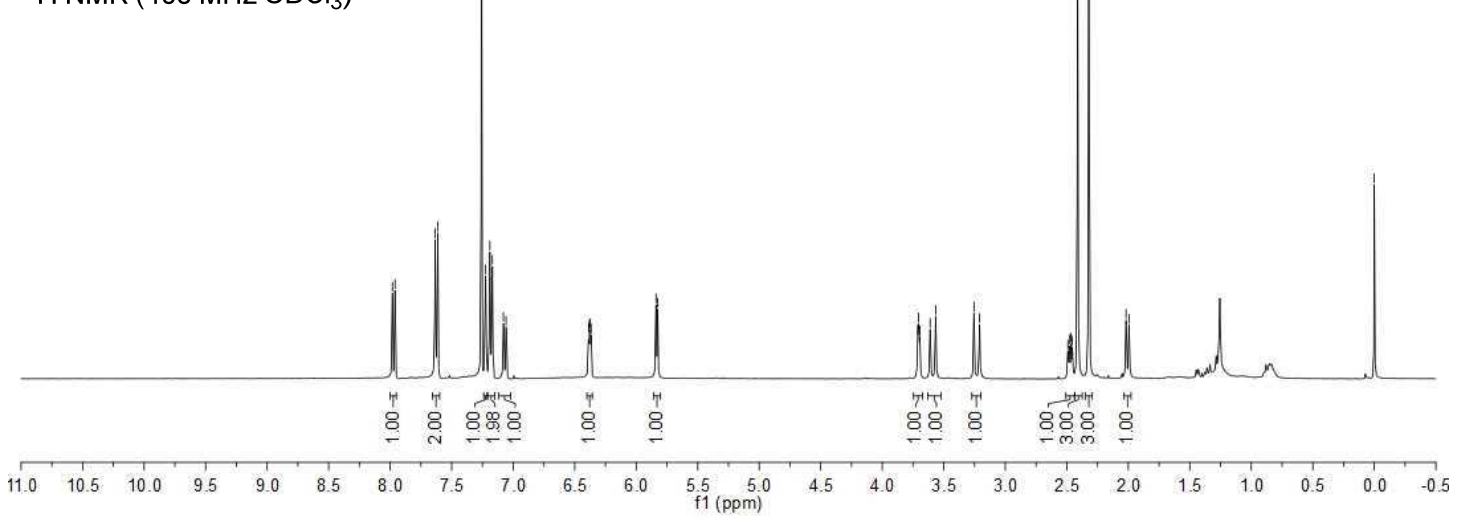

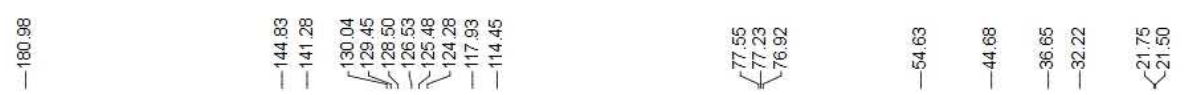

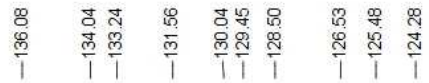<smiles>Cc1ccc2c(c1)c1c(n2[13CH3])C2CCC1(C(=O)O)CC2</smiles>

${ }^{13} \mathrm{C} \mathrm{NMR}\left(100 \mathrm{MHz} \mathrm{CDCl}_{3}\right)$
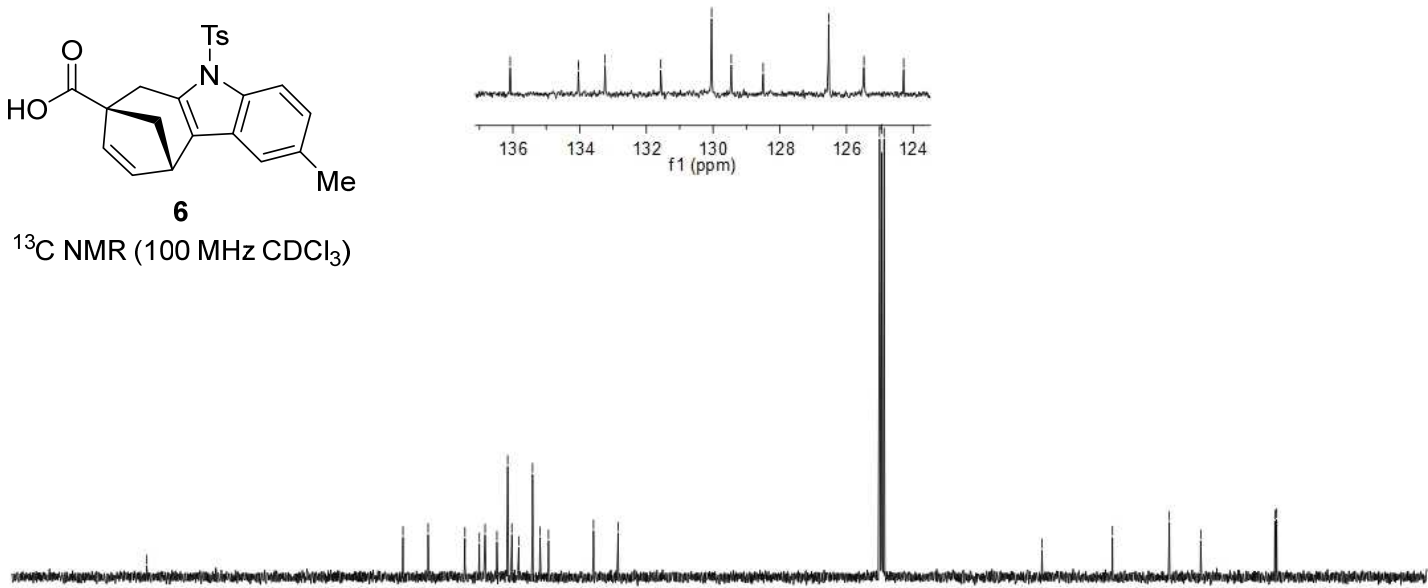

200

190

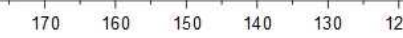

$20 \quad 110 \quad 100$ 


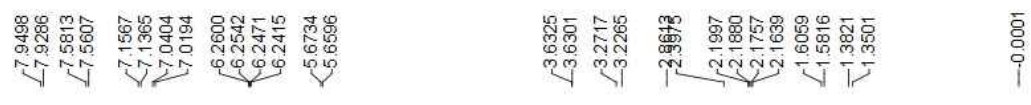

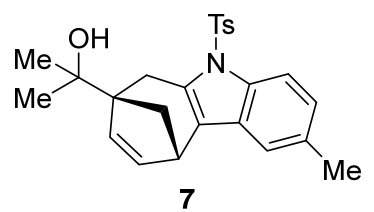

${ }^{1} \mathrm{H} \mathrm{NMR}\left(400 \mathrm{MHz} \mathrm{CDCl}_{3}\right)$
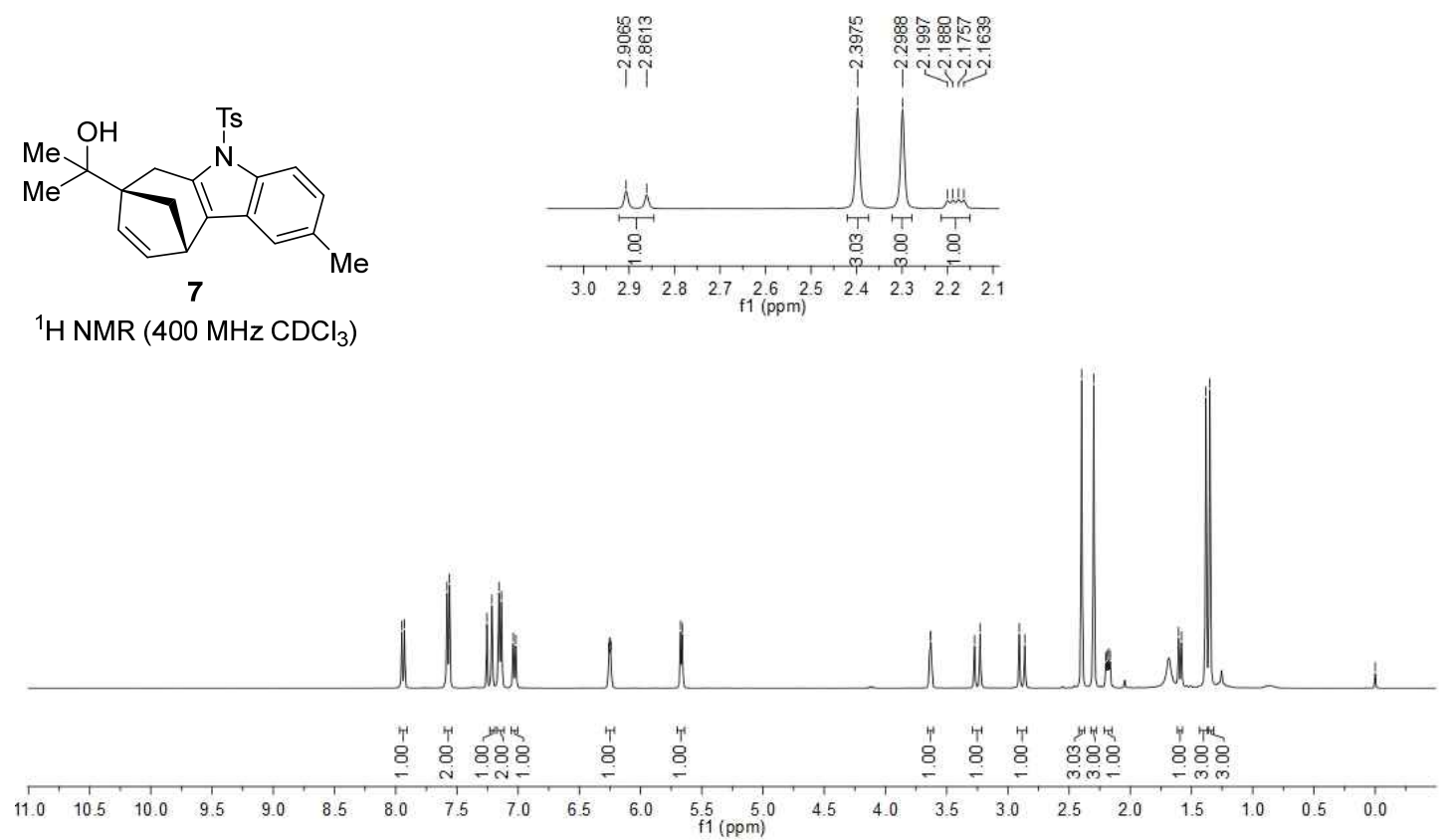

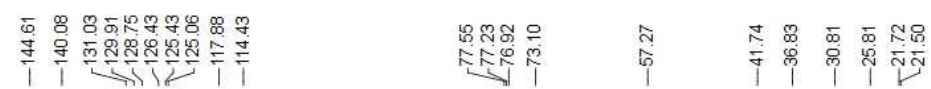

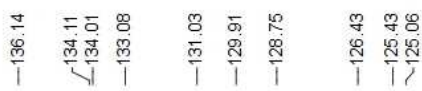
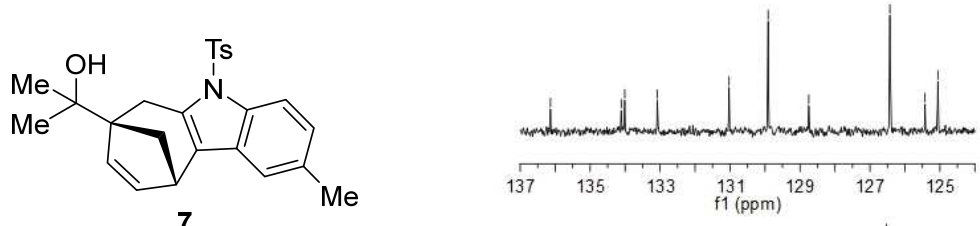

${ }^{13} \mathrm{C} \mathrm{NMR}\left(100 \mathrm{MHz} \mathrm{CDCl}_{3}\right)$

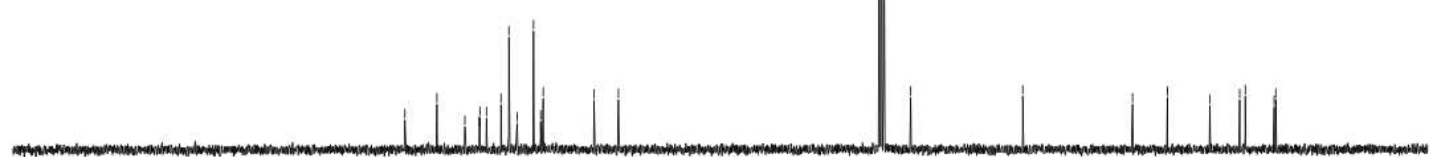

\begin{tabular}{llllllllllllllllllllllllll}
\hline & 200 & 190 & 180 & 170 & 160 & 150 & 140 & 130 & 120 & 110 & 100 & 90 & 80 & 70 & 60 & 50 & 40 & 30 & 20 & 10 & 0
\end{tabular} 


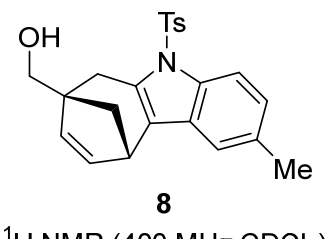

${ }^{1} \mathrm{H} \mathrm{NMR}\left(400 \mathrm{MHz} \mathrm{CDCl}_{3}\right)$

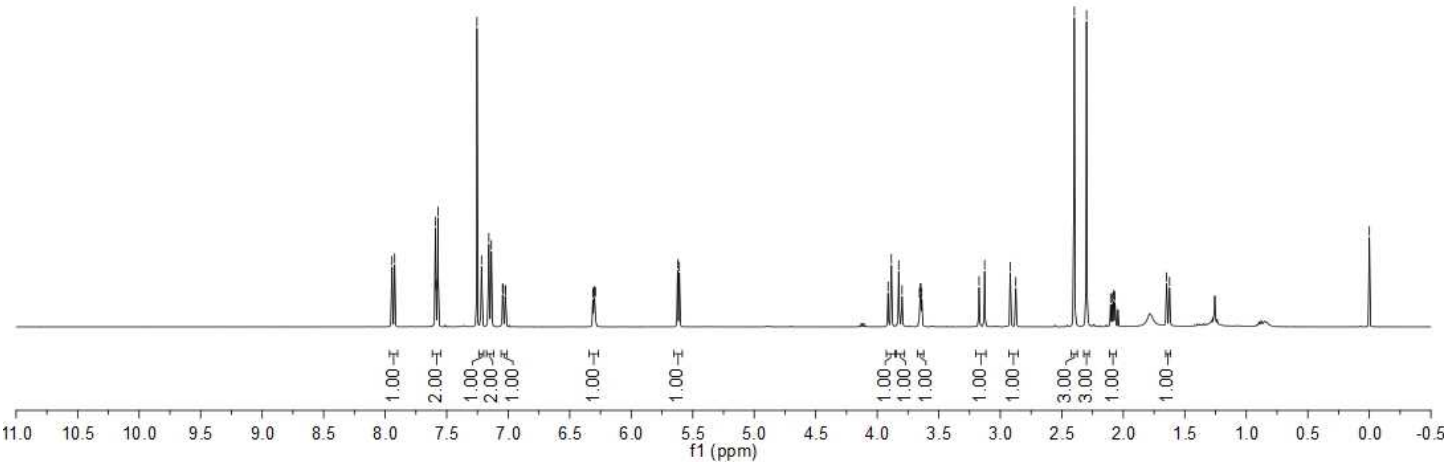

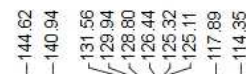

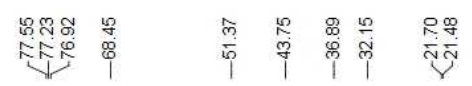

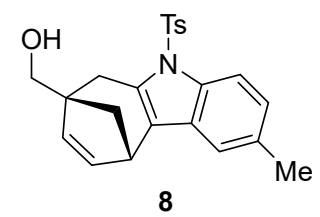

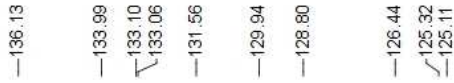

${ }^{13} \mathrm{C}$ NMR $\left(100 \mathrm{MHz} \mathrm{CDCl}_{3}\right)$
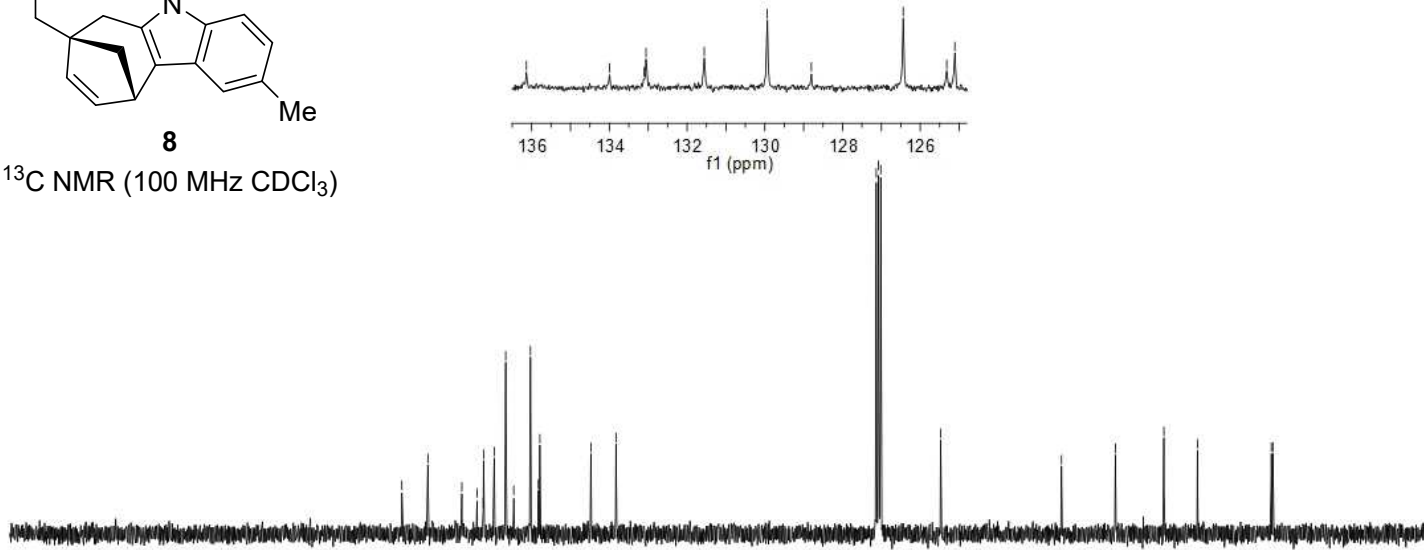

$\begin{array}{lllllllllllllllllllllllllllllll}200 & 190 & 180 & 170 & 160 & 150 & 140 & 130 & 120 & 110 & 100 & 90 & 80 & 70 & 60 & 50 & 40 & 30 & 20 & 10 & 0\end{array}$ 


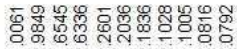

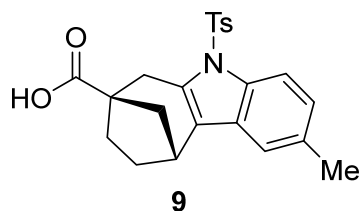

${ }^{1} \mathrm{H} \mathrm{NMR}\left(400 \mathrm{MHz} \mathrm{CDCl}_{3}\right)$

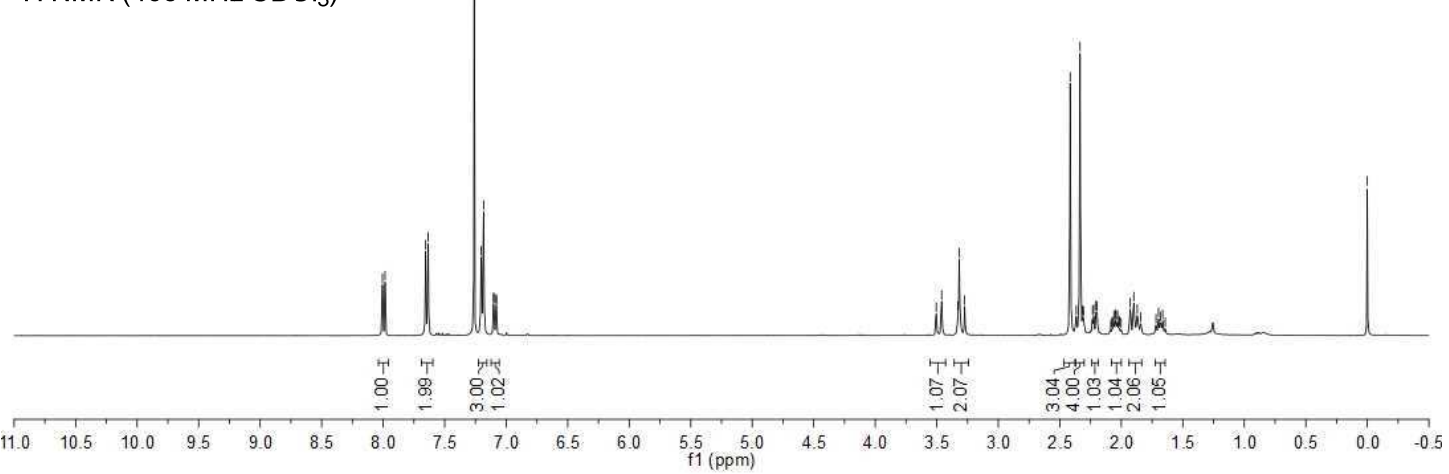

尔

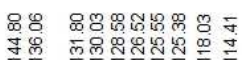

桨色

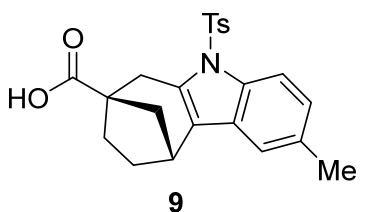

${ }^{13} \mathrm{C} \mathrm{NMR}\left(100 \mathrm{MHz} \mathrm{CDCl}_{3}\right)$
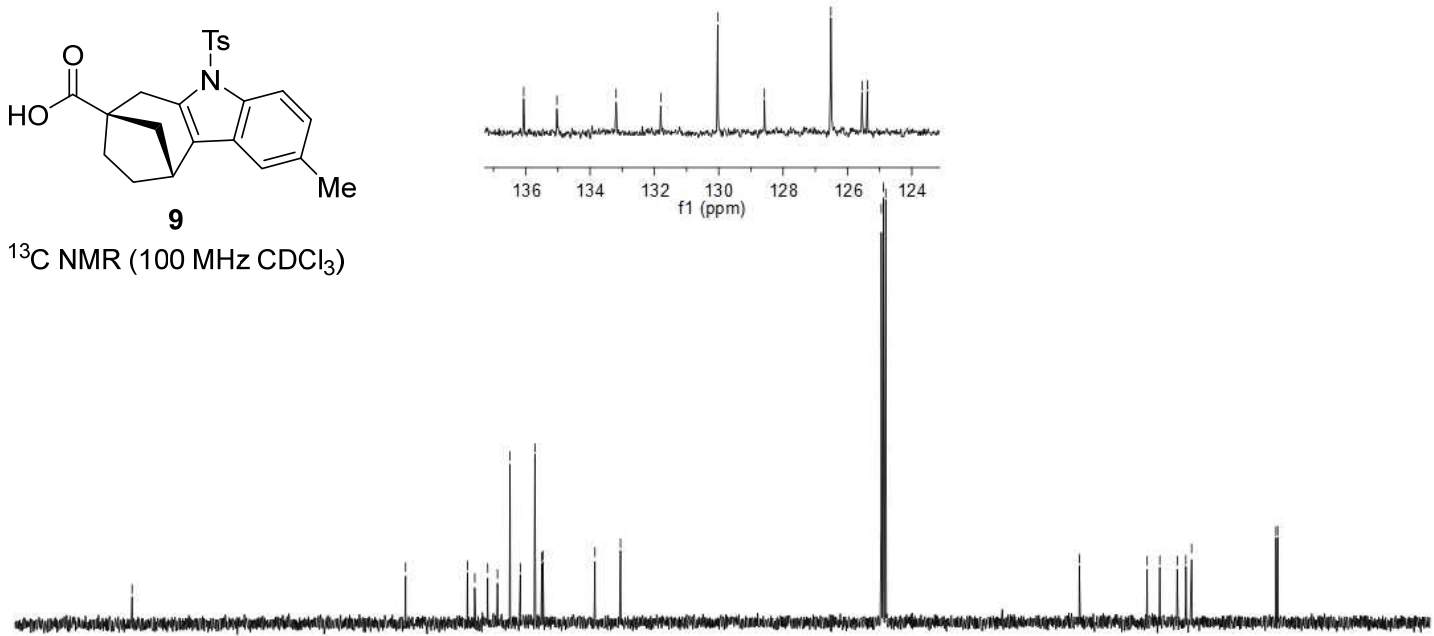

200

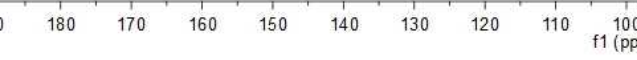



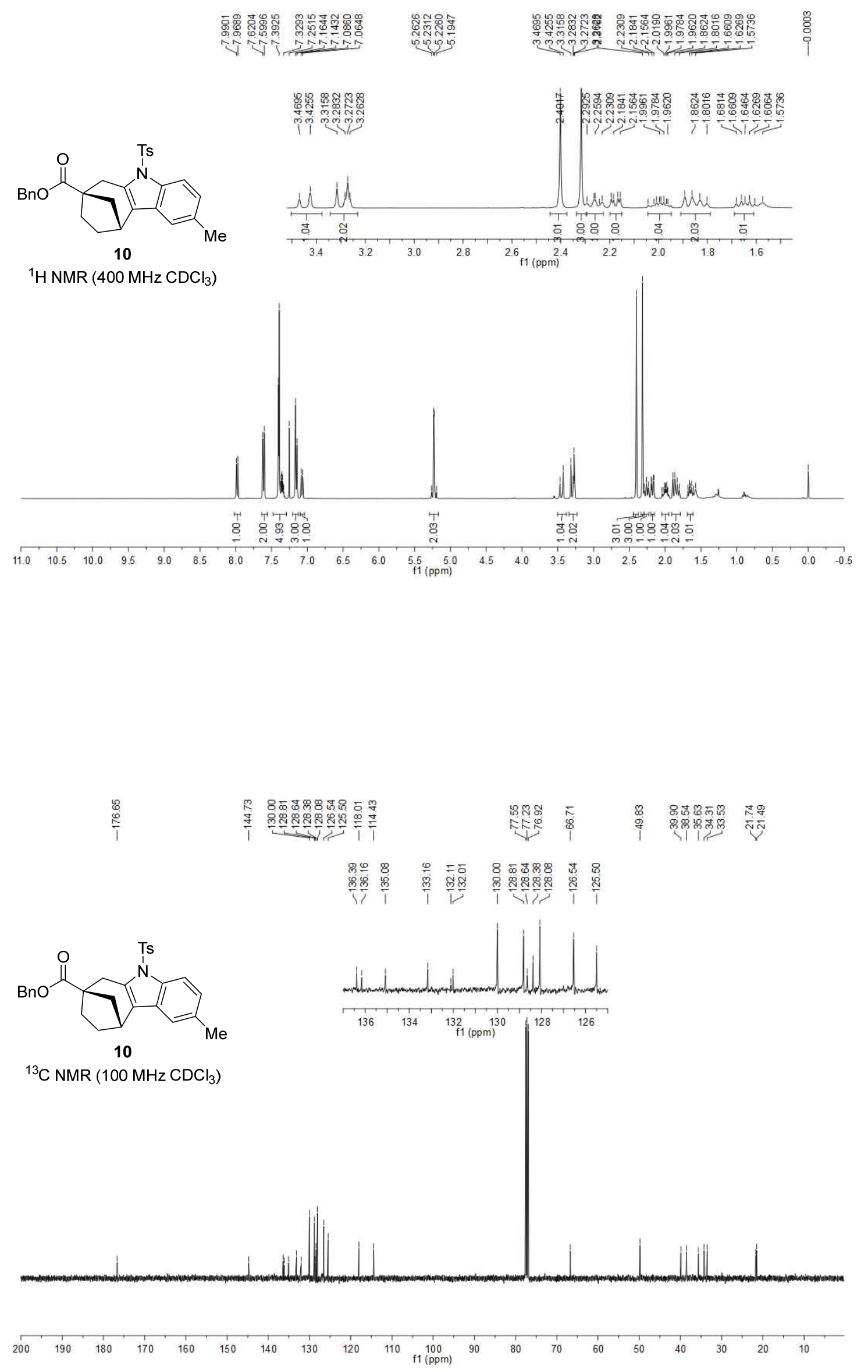


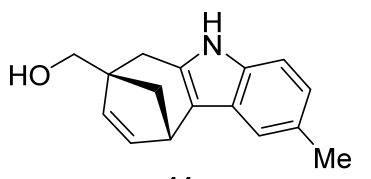

${ }^{1} \mathrm{H}$ NMR $\left(400 \mathrm{MHz} \mathrm{CDCl}_{3}\right)$
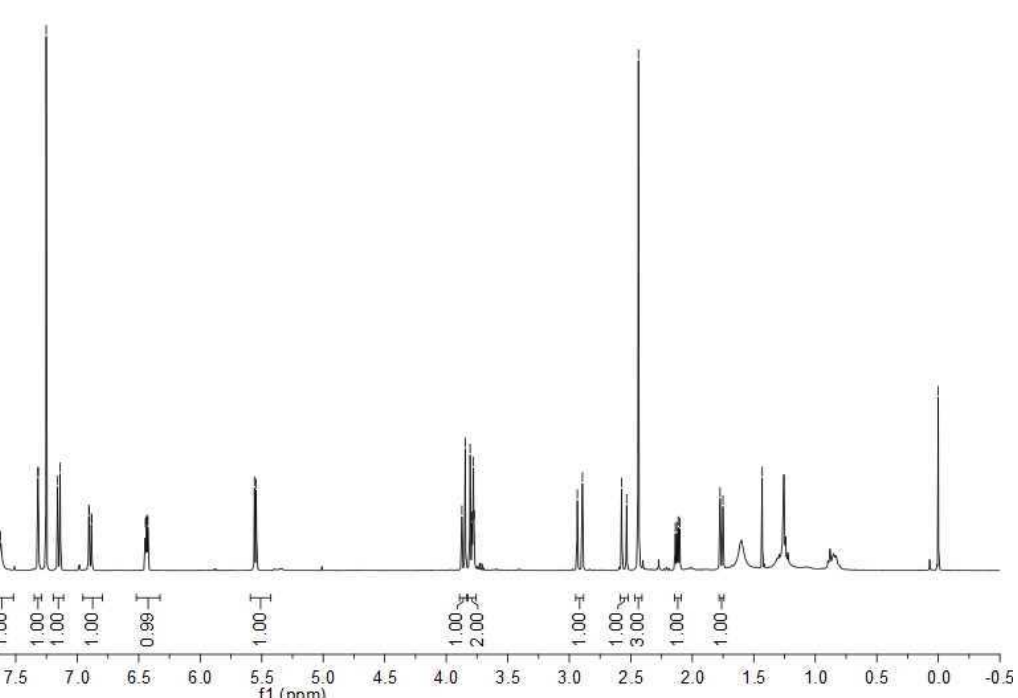

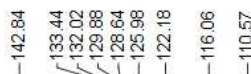

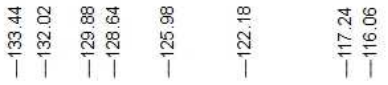
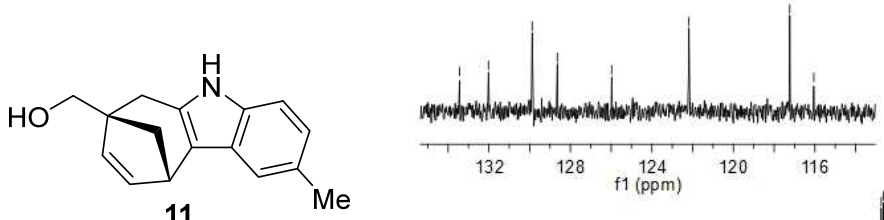

${ }^{13} \mathrm{C}$ NMR $\left(100 \mathrm{MHz} \mathrm{CDCl}_{3}\right)$

200

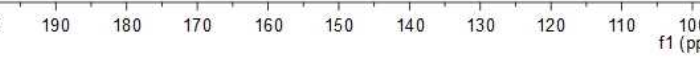




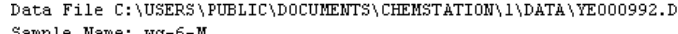
Sample Name: wg-6-M

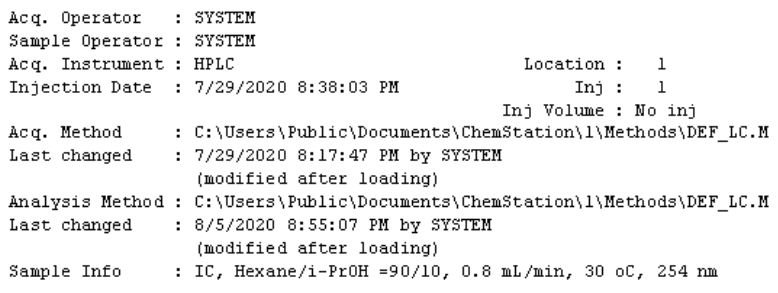

Anj Volume : No inj

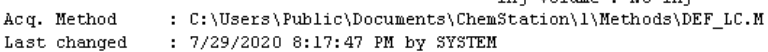
Last changed : 7/29/2020 $8: 17: 47$ fH by sister

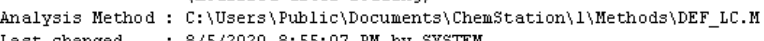
Last changed : 8/5/2020 8:55:07 PII bY sYTTEM

Sample Info : Ic, Hexane/1-Prof $=90 \% 110,0.8 \mathrm{~mL} / \mathrm{min}, 30$ oc, $254 \mathrm{~mm}$
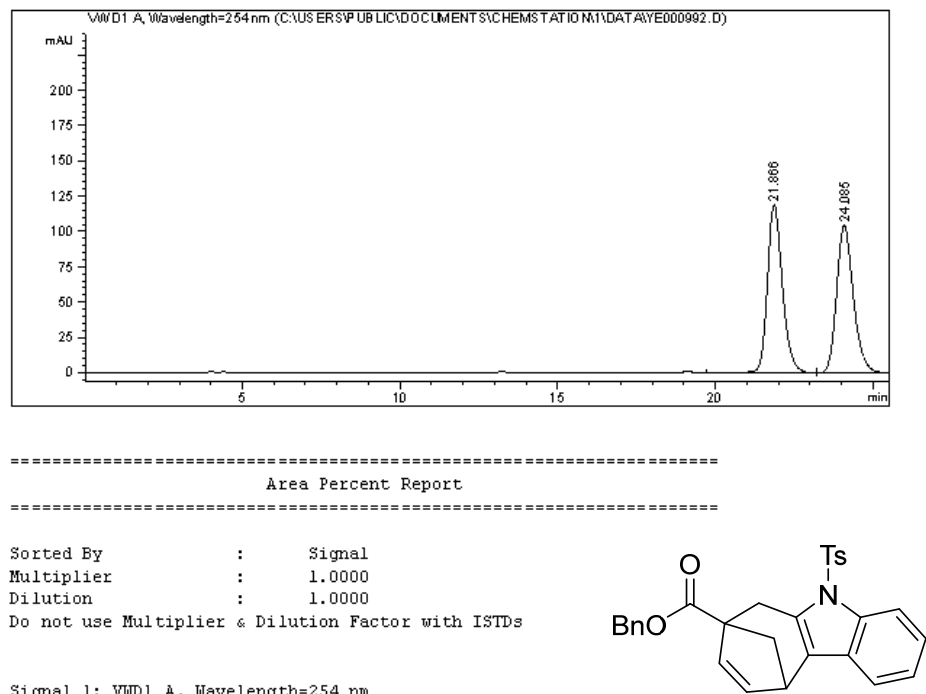

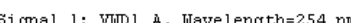

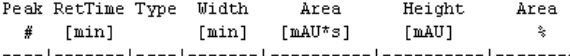

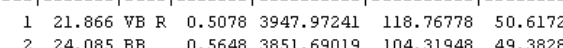

Totals :

$$
7799.66260 \quad 223.06726
$$

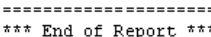

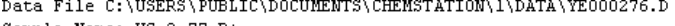
Sample Wäme: WiG-3-77-B

\section{eq. Operator : : FYSTEM \\ Sample operator : รTSTEM}

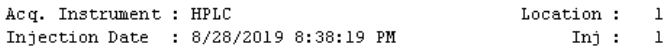

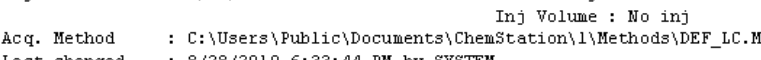

Lasst changed : 8/28/2019 6:33:44 FII bY SYSTE

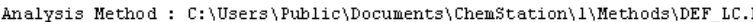

Last changed : 8/5/2020 8:50:59 PM by sพsTEM

Sample Info: : Ic, Hexane $/ 1$-Proff $=90 / 10,0.8 \mathrm{~mL} / \mathrm{min}, 30$ oc, $254 \mathrm{~mm}$

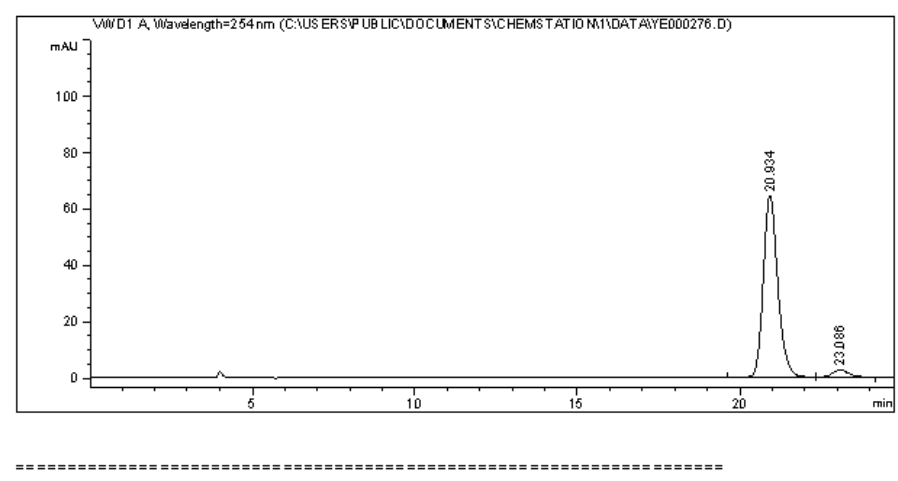

Mrea Percent. Report.

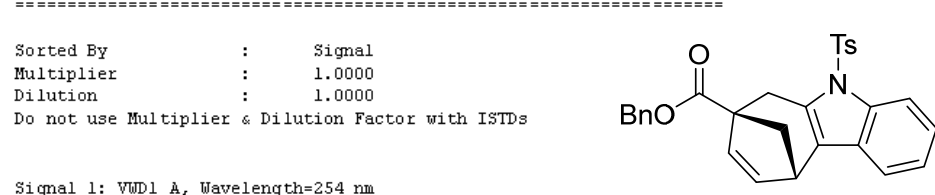

Si gral 1: WU⿴囗十 A, Wavelength $=254$ nII

$(-)-3 a$

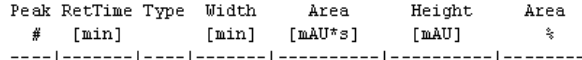

$$
\begin{aligned}
& \begin{array}{rrrrrr}
1 & 20.934 \mathrm{~EB} & 0.4726 & 1992.07666 & 64.44283 & 95.7992 \\
2 & 23.086 \mathrm{~EB} & 0.5284 & 87.35310 & 2.53255 & 4.2008
\end{array} \\
& \text { Totals } \\
& 2079.42976 \quad 66.97539
\end{aligned}
$$

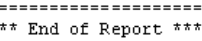




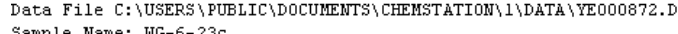
Sample Name: hic-6-zac

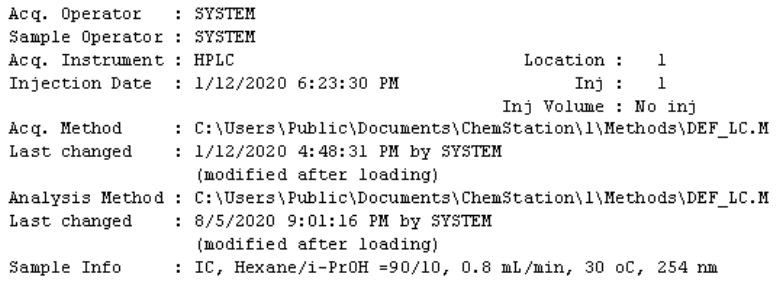

Inj Volume: : No inj

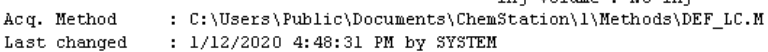
Last changed : 1/12/2020 4: 46:31 Fin by STS1EM

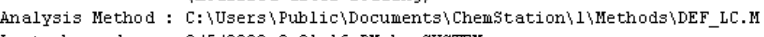
Last changed : 8/5/2020 9:01:16 PII bY 5YTTEM

Sample Info : IC, Hexane $/ 1$-ProH $=90 / 10,0.8 \mathrm{~mL} / \mathrm{min}, 30$ oC, $254 \mathrm{~mm}$

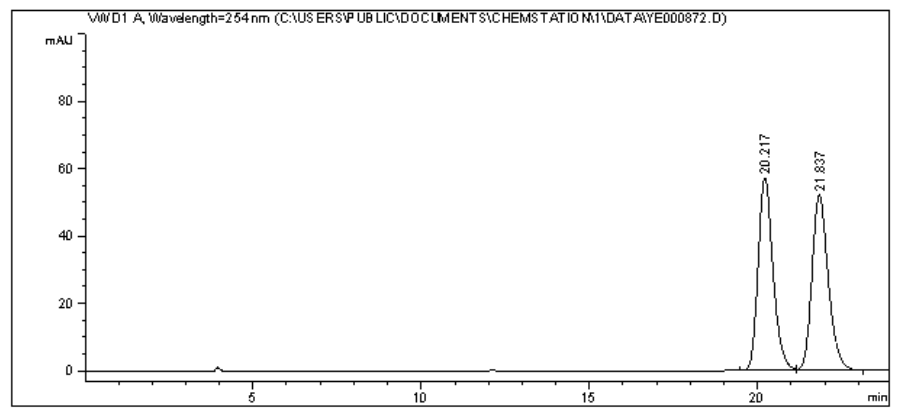

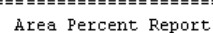

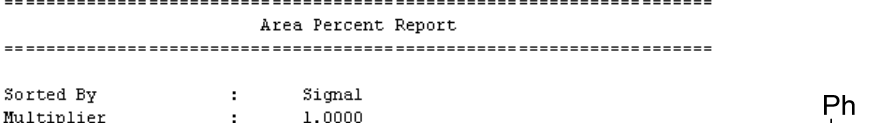

\section{flultiplier : 1.0000}

Do not use Multiplier \& Dilution Factor with IsTDs

S1 gnal 1: vili 1 , wavelengthe254 no

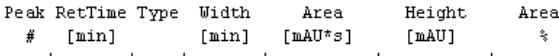

$\begin{array}{llllll}1 & 20.217 \mathrm{BE} & 0.4683 & 1745.37830 & 56.98553 & 50.0526 \\ 2 & 21.837 \mathrm{BE} & 0.5133 & 1741.70654 & 51.92323 & 49.9474\end{array}$

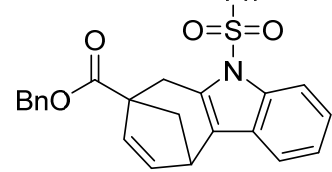

Totals : $\quad 3487.08484 \quad 108.9087$

$(+/-)--3 b$

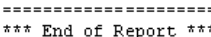

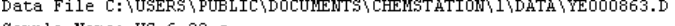
Sample Wame:

\section{Acq. Operator : : FYSTEM
Sample operator : : 2 TSTEM}

Sample operator: : FrsTEM

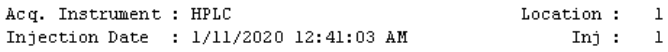

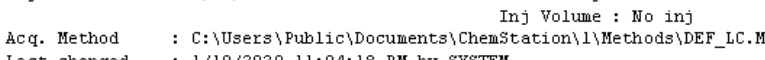

Last changed : 1/10/2020 11:04:18 PM bY SYSTEM

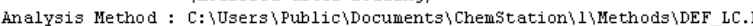

Last changed : 8/5/2020 9:00:15 PM by sพsTEM

Sample Info : (nodified after loading)

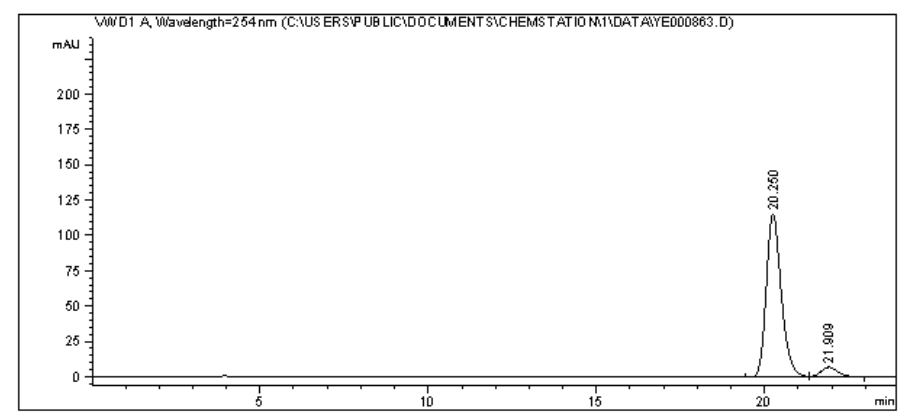

Area

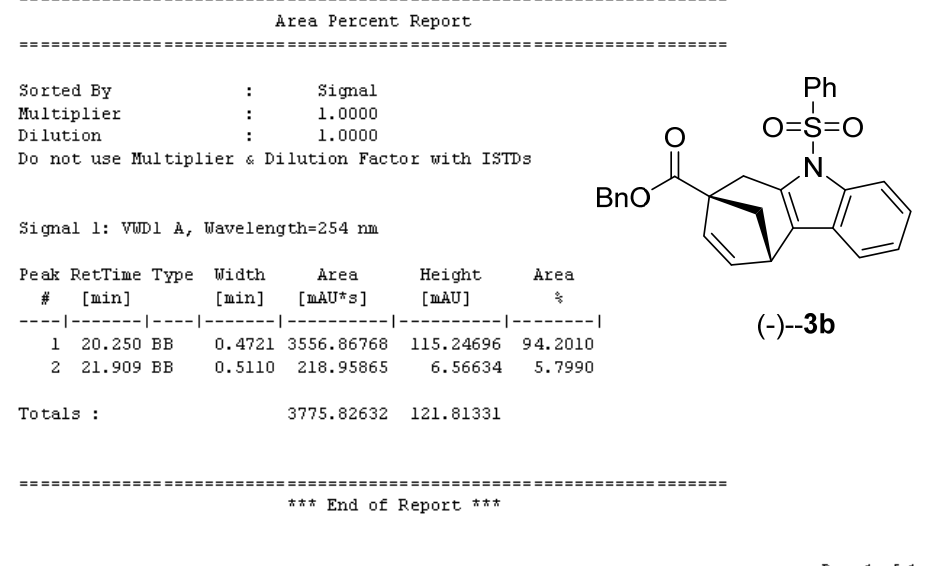

HPIC 8/5/2020 9:00:20 PII SYSTEM 


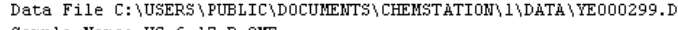
ample Name: hiG-6-17-E-0NE
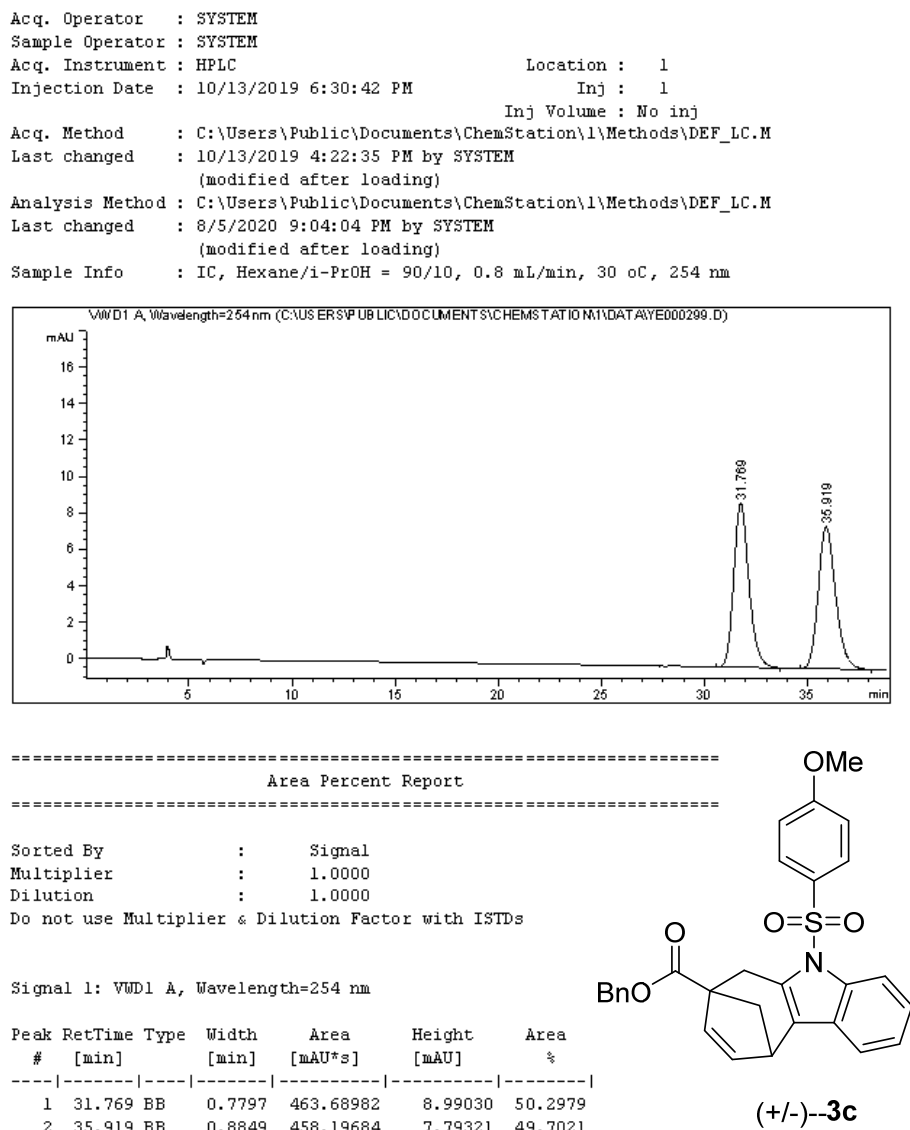

Totals :

$921.80665 \quad 16.7835$

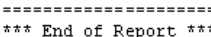

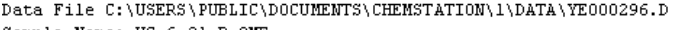
Sample Hame: hiG-6-21-B-0ME

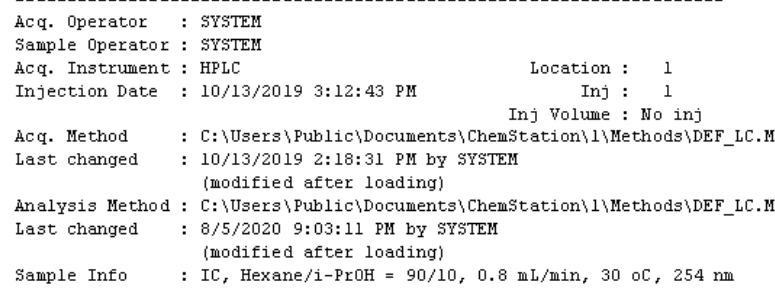

Inj volume : No inj

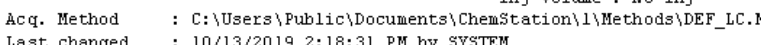
be

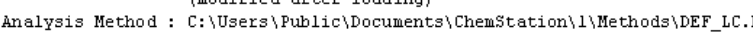
Last changed : 8/5/2020 9:03:11 PM bY FYTTEM

Sample Info: : Ic, Hexane $/ \mathrm{i}-\mathrm{PrOH}=90 / 10,0.8 \mathrm{~mL} / \mathrm{min}, 30$ oc, $254 \mathrm{~mm}$
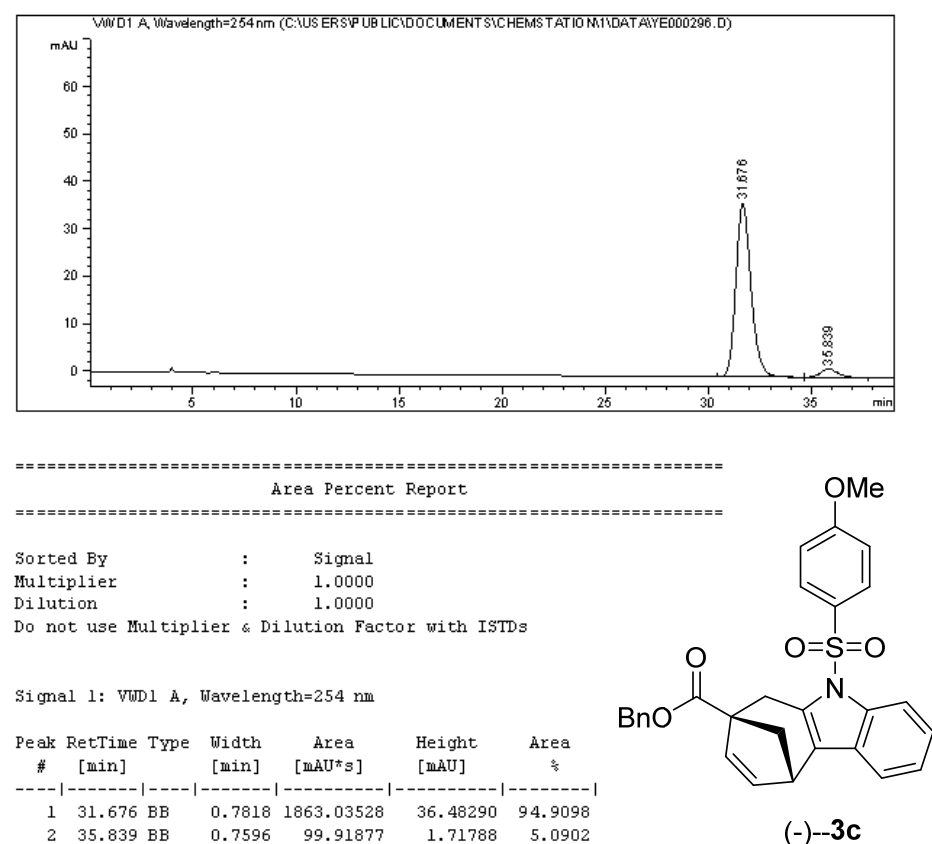

Totals:

$1962.95405 \quad 38.20077$

$(-)-3 c$

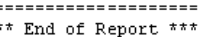




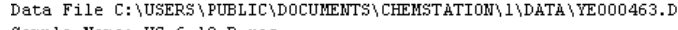
Nample Wäne: hí-6-18-B-rac
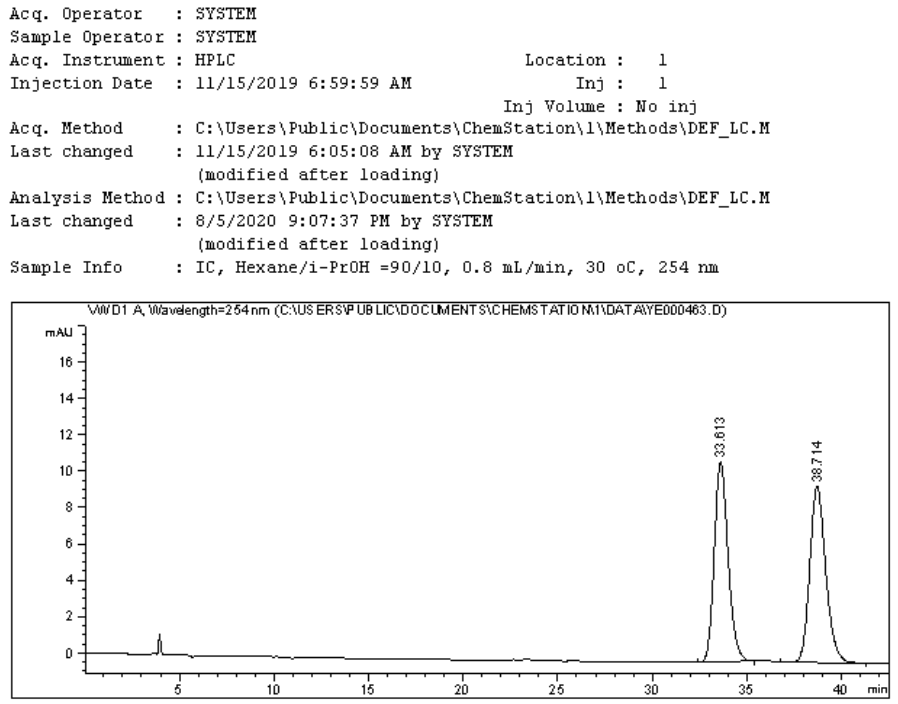

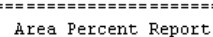

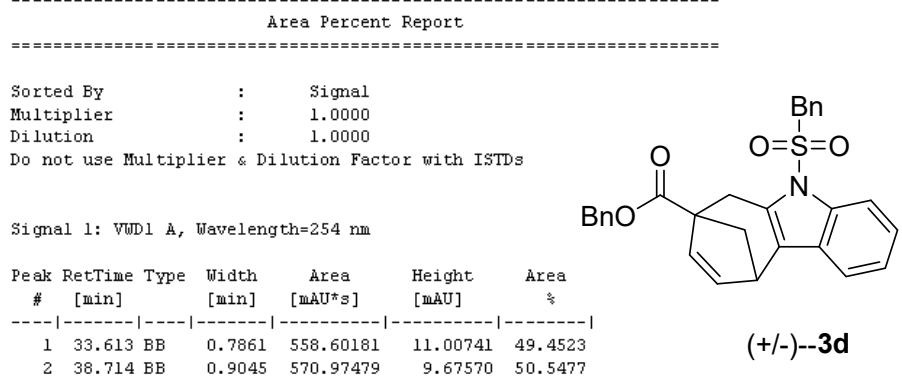

Totals :

$1129.57660 \quad 20.6031$

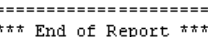

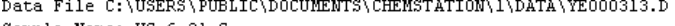
ample Name: $\mathrm{i}[\mathrm{G}-6-21-$

Acq. Operator : : FYSTEMI
Sample Operatox : FrsTEM

Sample operator : Frst
heq. Instruwent : HPIC

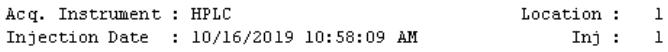

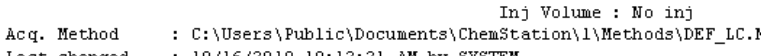

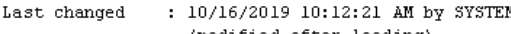

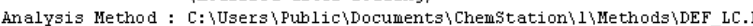

Last changed : 8/5/2020 9:07:37 PM by SYSTEM

Sample Info : IC, Hexane $/ 1-\mathrm{PrOH}=90 / 10,0.8 \mathrm{~mL} / \mathrm{min}, 30 \mathrm{oC}, 254 \mathrm{~mm}$

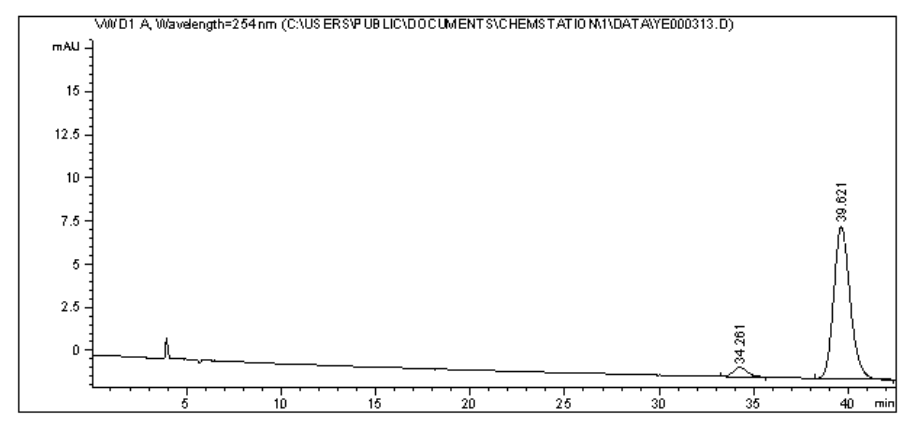

M

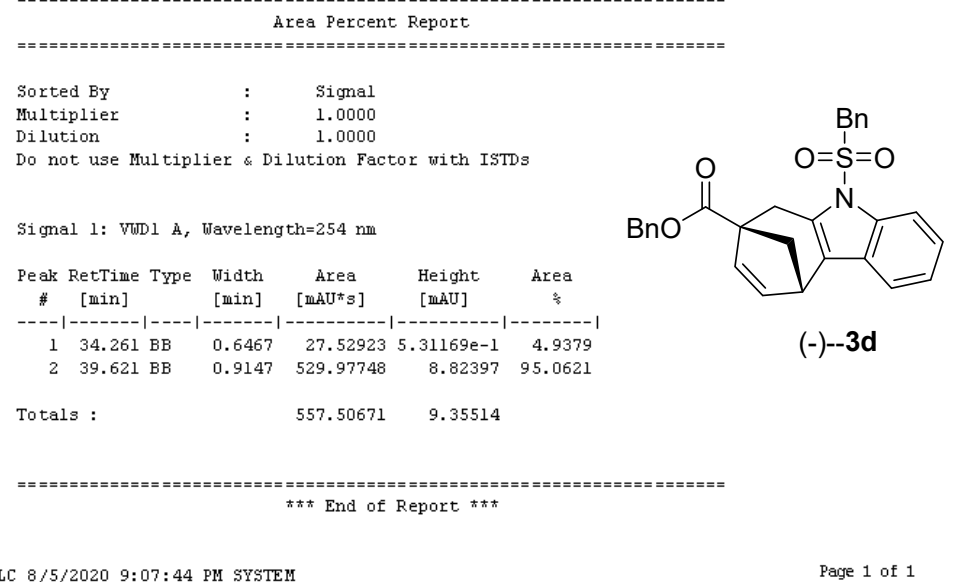




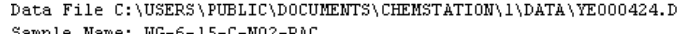
Sample hame: hig-6-15-L-N02-RAC
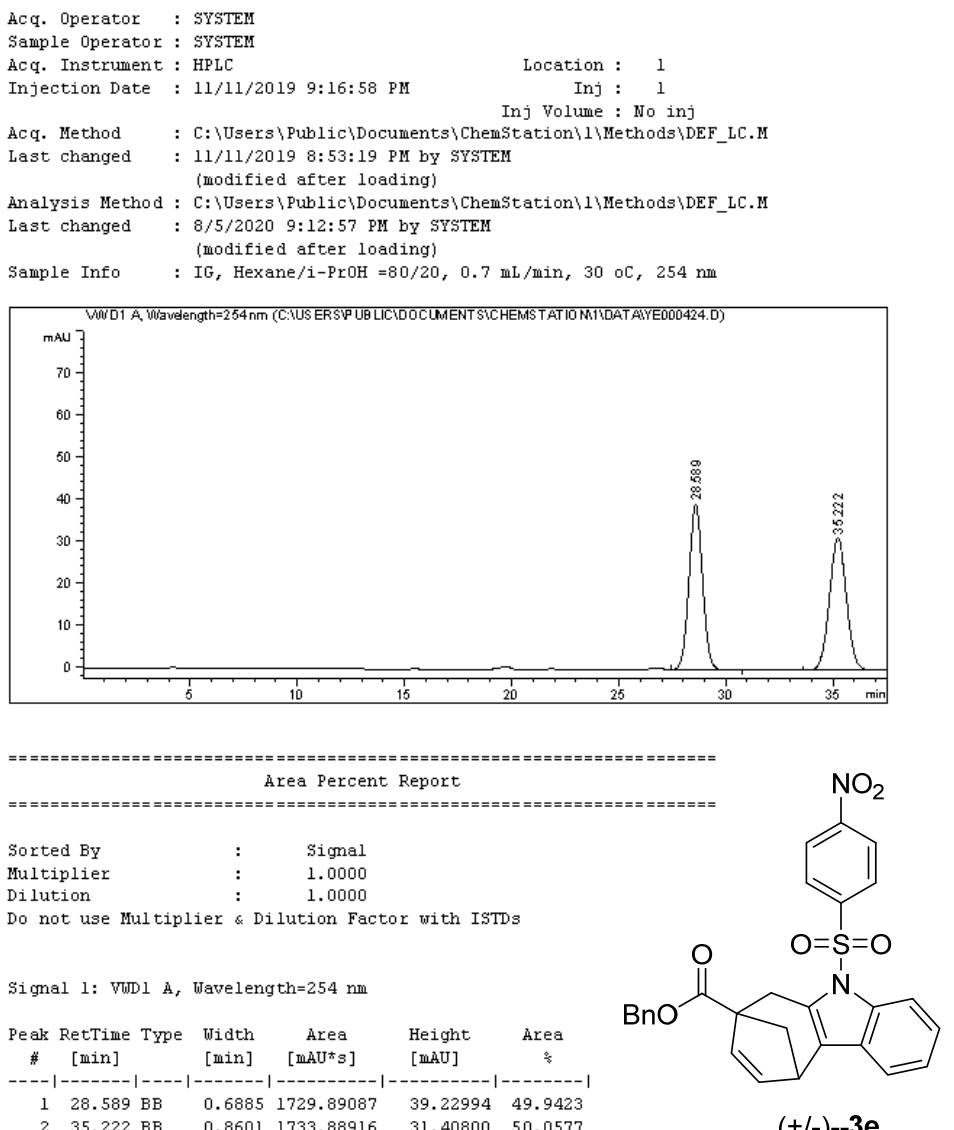

Sorted BY

mitcipise

\section{Sigmal
1.0000}

Do not use Hultiplier \& Dilution Factor with IsTD

S1 gnal 1: VWid 1 A, wavelengthe254 nm

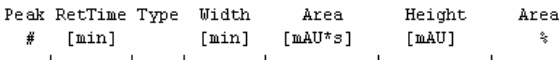

$\begin{array}{llllll}1 & 28.589 \mathrm{~EB} & 0.6885 & 1729.89087 & 39.22994 & 49.9423 \\ 2 & 35.222 \mathrm{BE} & 0.8601 & 1733.88916 & 31.40800 & 50.057\end{array}$

$(+/-)--3 e$

Tatâls

$3463.78003 \quad 70.6379$

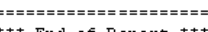

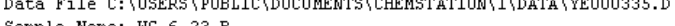
Sample Name: hiG-6-23-B

\section{Acq. Operator : : FYSTEM
Sample Operator : : FrsTEM}

Sample Operator: : FrsTrM
Aeq. Instrument : HPIC

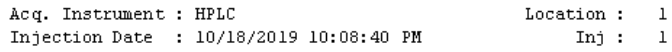

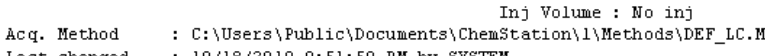

Last changed : 10/18/2019 g:51:50 PM by SYSTEM

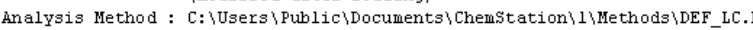

Last changed : 8/5/2020 9:11:05 PMI bY SYSTEM

Sample Info: : IFodified Hexane $/ 1-\mathrm{Pr}$ -

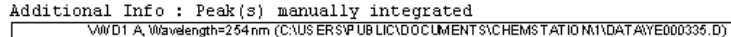

man
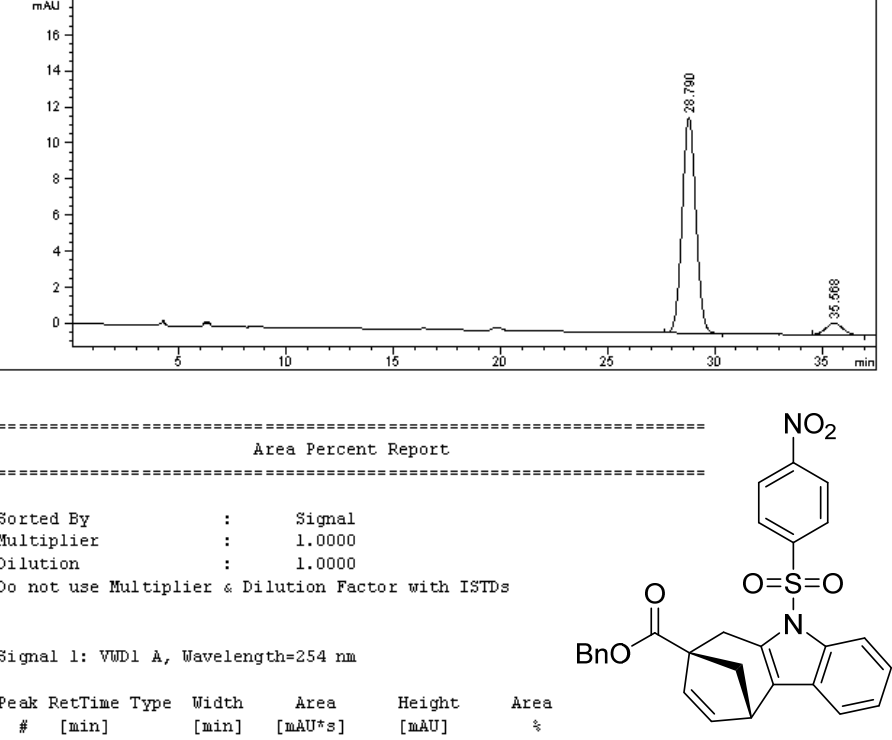

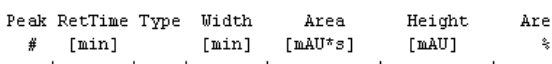

$\begin{array}{rrrrrr}1 & 28.790 \mathrm{BE} & 0.6920 & 533.68744 & 11.97304 & 93.5788 \\ 2 & 35.568 \mathrm{EE} & 0.6695 & 36.62072 & 6.68132 \mathrm{e}-1 & 6.4212\end{array}$

$(-)-3 e$

Totâls :

$570.30016 \quad 12.64117$

$\pi x+$ End of Report $\pi m \pi$

HPIC 8/5/2020 9:11:14 PII SYSTEM 


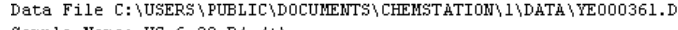
Sample Name: if $6-6-29-\mathrm{E}(-\beta+)$
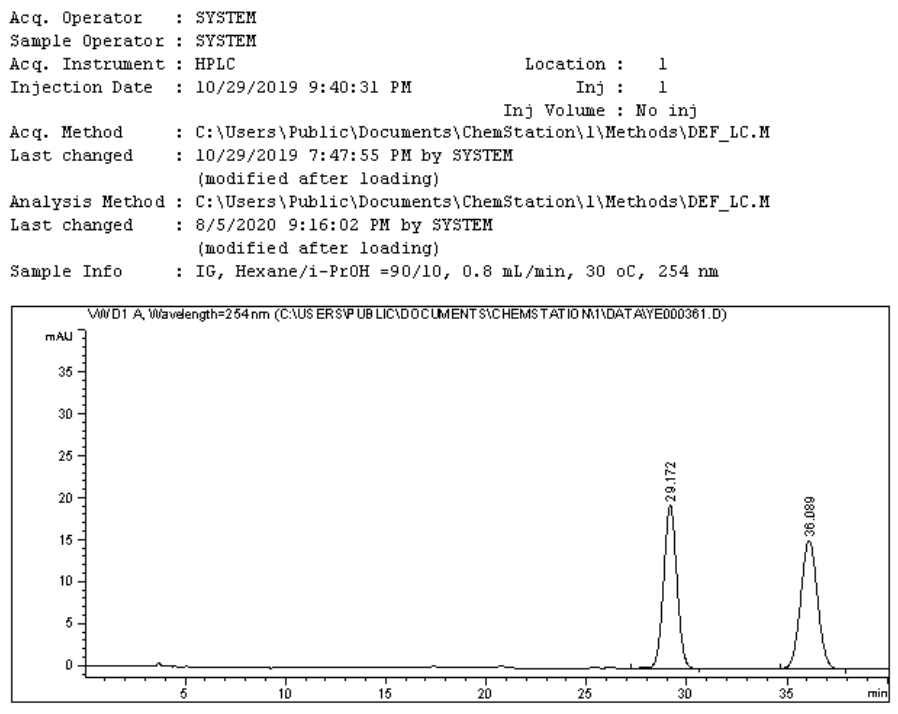

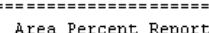

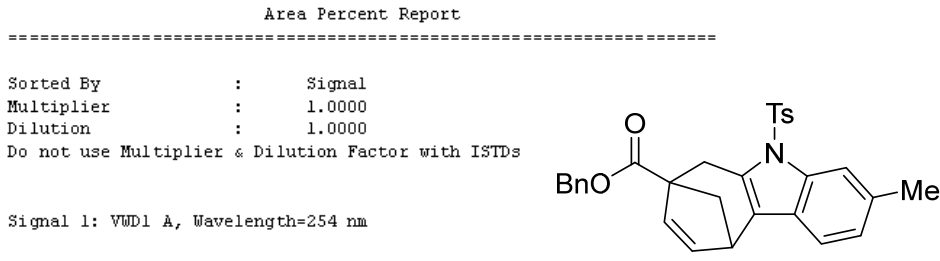

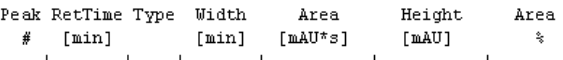

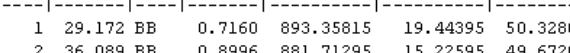

$(+/-)--3 h$

Totals :

$1775.07111 \quad 34.6699$

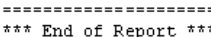

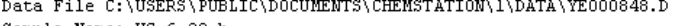
sample Name: $1 \mathrm{i}(\mathrm{s}-6-29-1$

\section{Acq. Operator : FTSTEM
Sample operatox : FrTEM}

Sample operator: इFSTEM

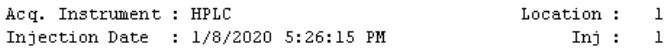

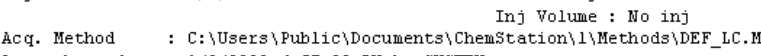

Last changed : 1/8/2020 4:57:20 PII bY SYSTEM

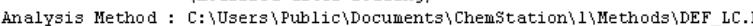

Last changed : 8/5/2020 9:14:20 PII by sพsTEM

Sample Info : (noditied after loading)

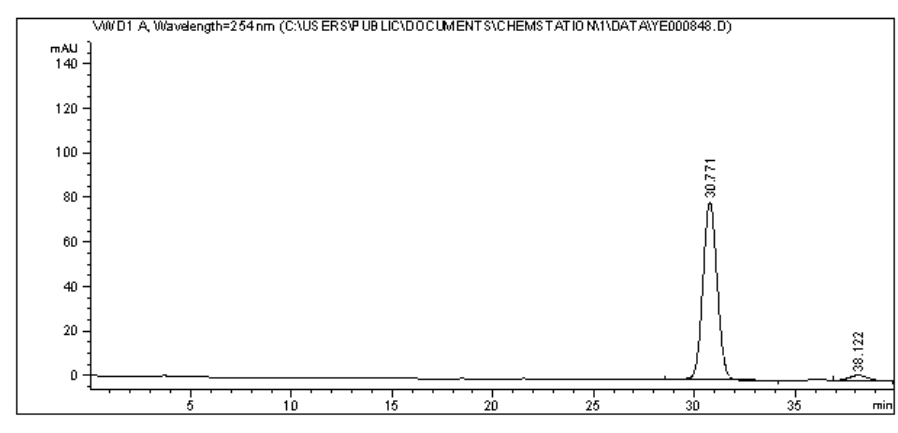

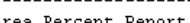

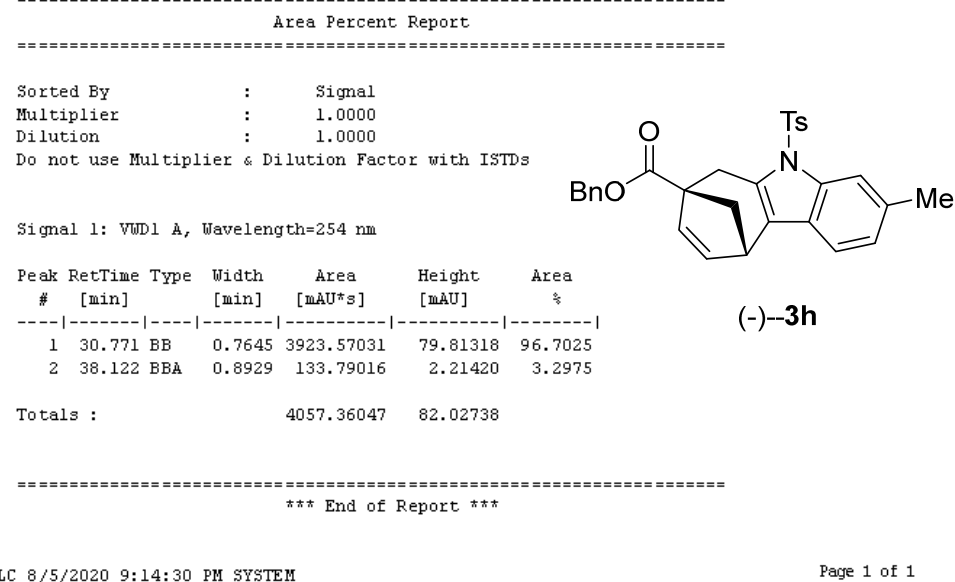




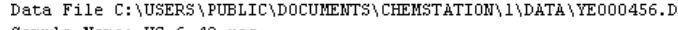
Sample Name: $\mathrm{k}[\mathbf{G}-6-43-\mathrm{rac}$
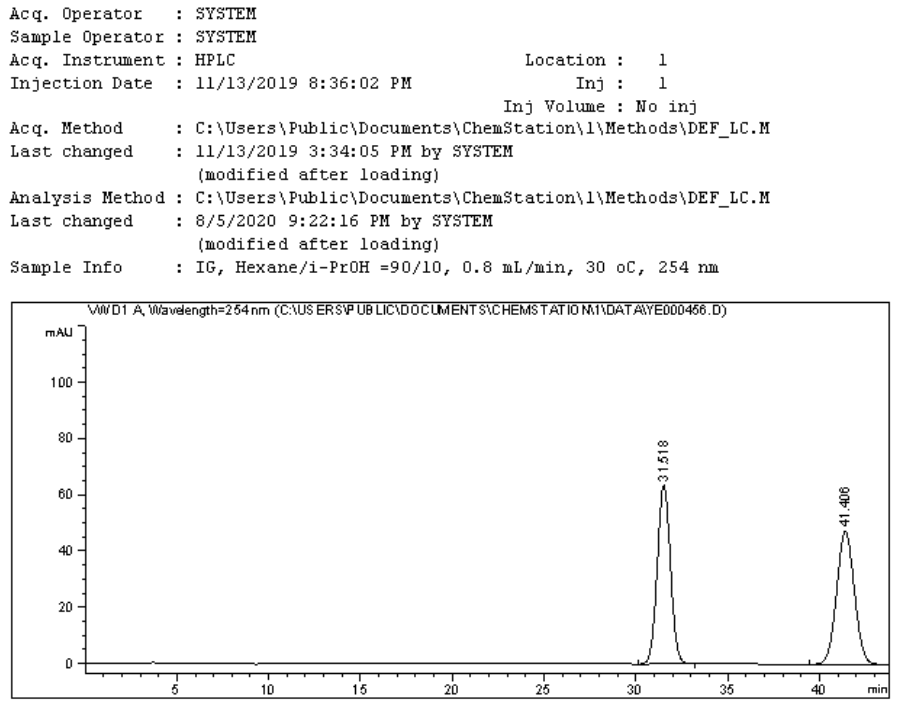

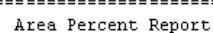

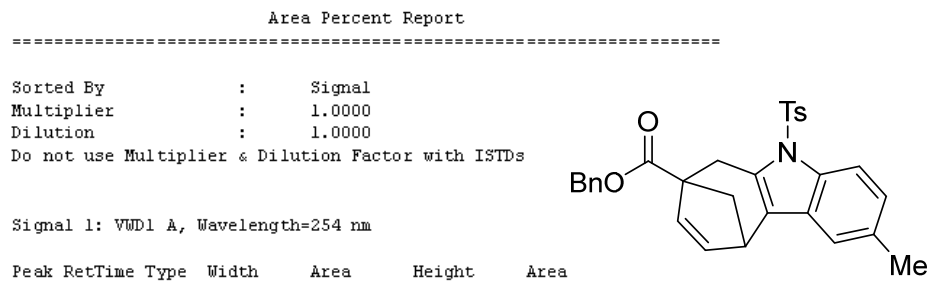

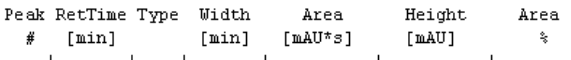

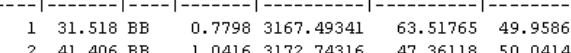

Tatâls

$6340.23657 \quad 110.8780$

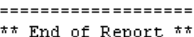

HPIC 8/5/2020 9:22:20 PII SYSTEM

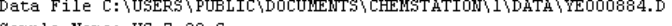
ample Name: $\mathrm{hG}-7-3 \overline{\mathrm{a}}-\mathrm{C}$

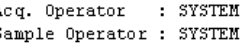

Sample operator: : FrsTrR

$\begin{array}{lll}\text { Lec. Instrument : } & \text { Location }: & 1 \\ \text { Injection Date }: 6 / 29 / 2020 \text { 8:23:58 PII } & \text { Inj: } & 1\end{array}$

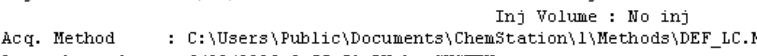

Last changed : 6/29/2020 $3: 55: 51$ PII by SYSTE

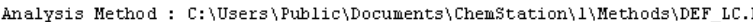

Last changed : 8/5/2020 9:20:53 PM by sพsTEM

Sample Info : IG, Hexane $/ 1-\mathrm{Fr} 0 \mathrm{H}=90 / 10,0.8 \mathrm{~mL} / \mathrm{min}, 30$ oc, $254 \mathrm{~m}$

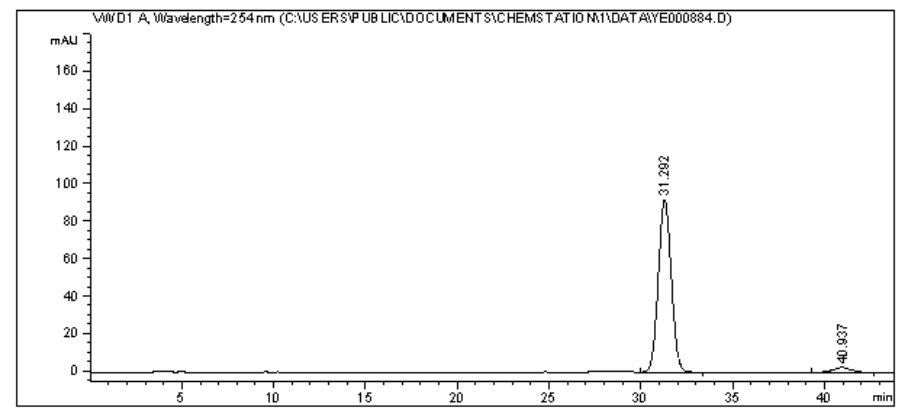

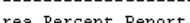

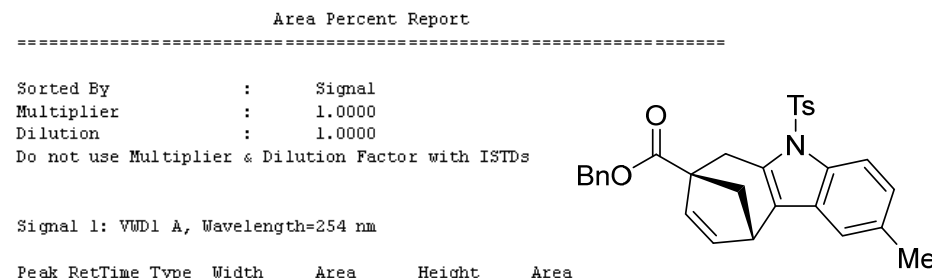

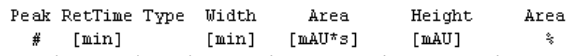

$\begin{array}{rrrrrr}1 & 31.292 \mathrm{BE} & 0.7600 & 4494.82520 & 92.00378 & 96.6462 \\ 2 & 40.937 \mathrm{BE} & 1.0064 & 155.97833 & 2.39313 & 3.3538\end{array}$

$(-)-3 \mathbf{i}$

Totáls:

$4650.80353 \quad 94.39691$

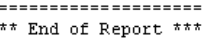

HPIC 8/5/2020 9:20:59 PII SYSTEM

Page 1 of 1 


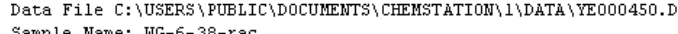
Sample Name: WhG-6-3a-rac
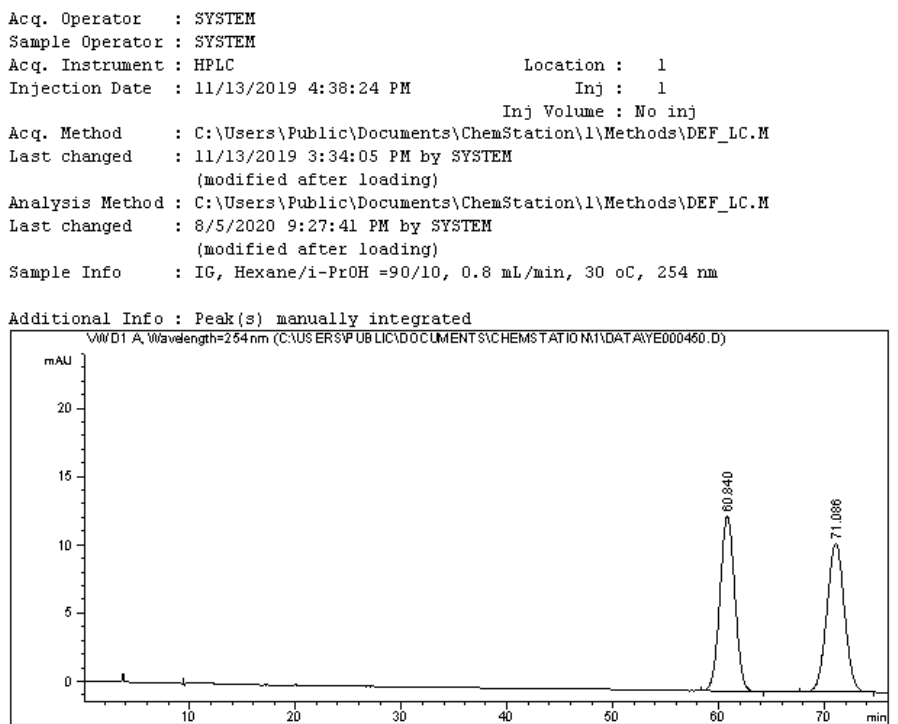

A rea Percent Report

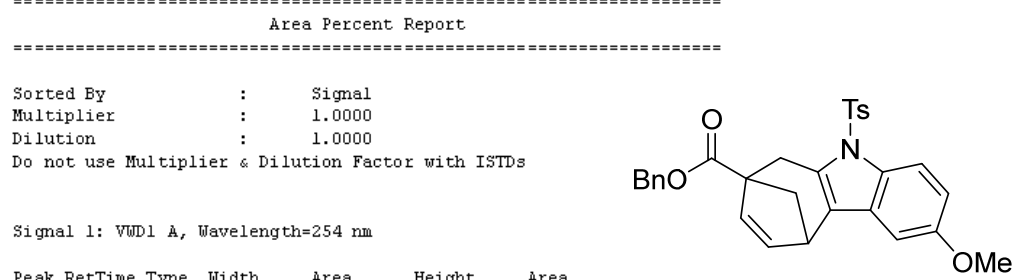

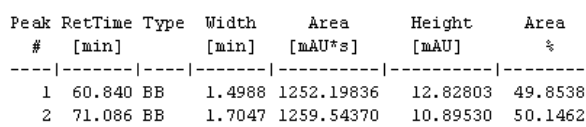

$(+/-)--3 \mathbf{j}$
Tatals :
$2511.74207 \quad 23.72333$

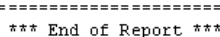

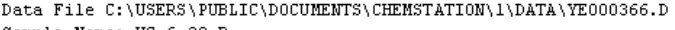
Sample Name: 10

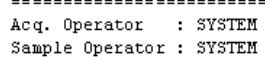

Sample Operator : FrsTEM
Aeq. Instrument : HPIC

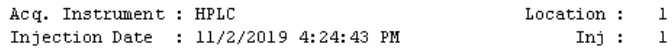

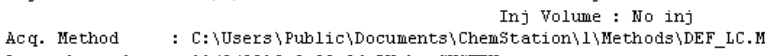

Lasst changed : 11/2/2019 3:33:34 FII bY STSTE

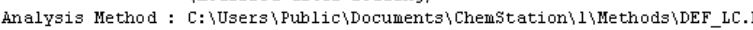

Last changed : 8/5/2020 9:25:16 PMI bY sYSTEM

Sample Info : IF, Hexane $/ 1-\mathrm{Pr}$ - lof $=90 / 10,0.8 \mathrm{mI} / \mathrm{min}, 30$ oc, $254 \mathrm{~mm}$

Addi tional Info: : Peak is) manually integrated

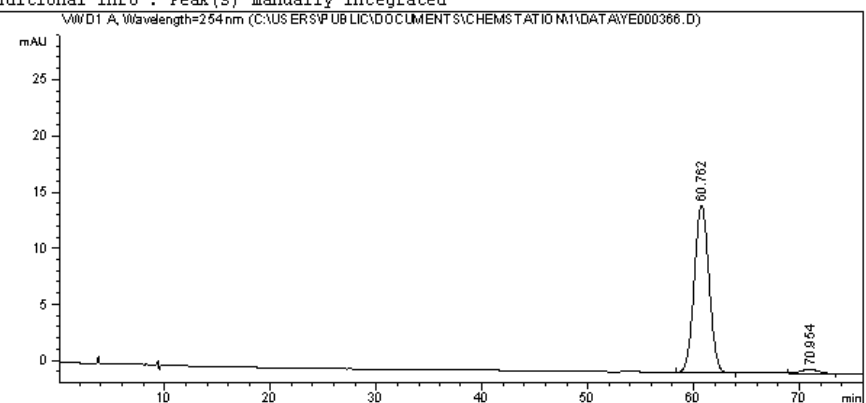

Ärea Percent Report

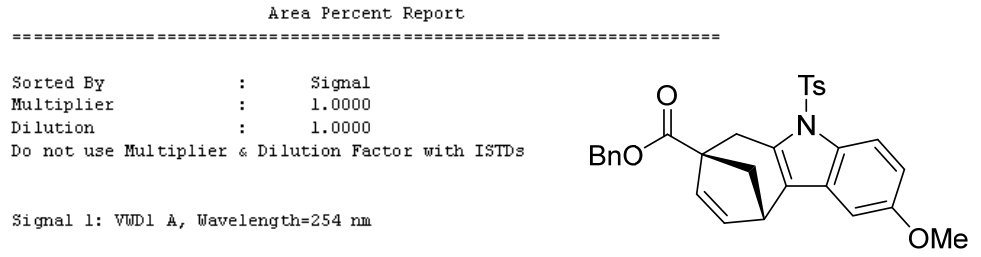

Peak RetTime Type Width hirea Height area

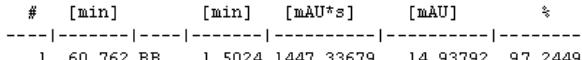
$\begin{array}{rrrrrr}1 & 60.762 \mathrm{EE} & 1.5024 & 1447.33679 & 14.93792 & 97.2449 \\ 2 & 70.954 \mathrm{EE} & 1.3127 & 41.00587 & 3.67799 \mathrm{e}-1 & 2.7551\end{array}$
Tatâls :
$1486.34266 \quad 15.30572$

$\pi x+$ End of Report $\pi * \pi$

HPIC 8/5/2020 9:25:32 PII SYSTEM

Page 1 of 1 


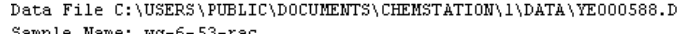
Jample Name: wg-6-53-rac
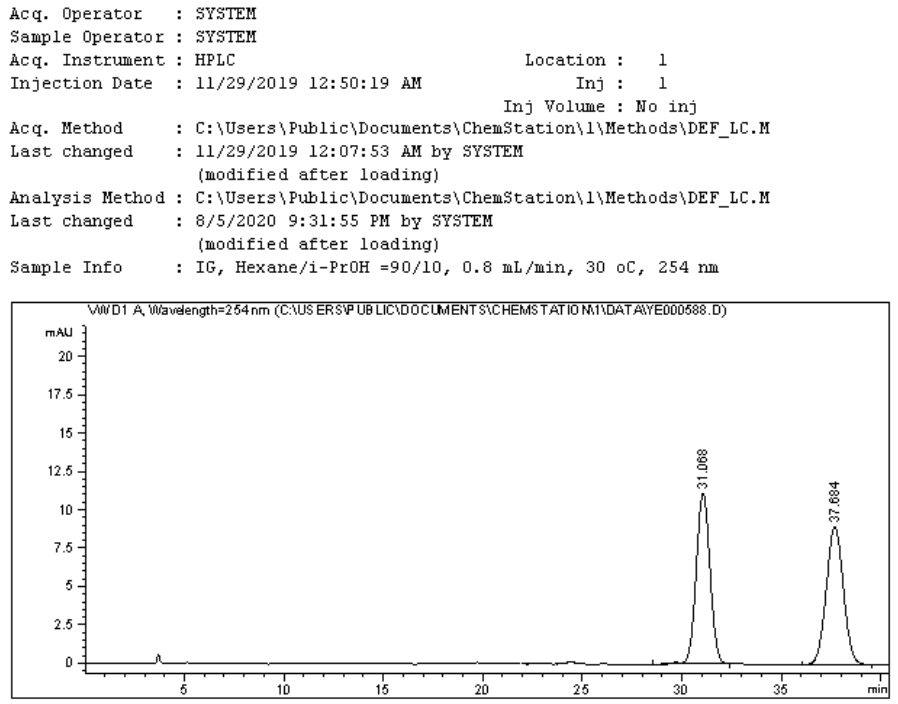

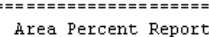

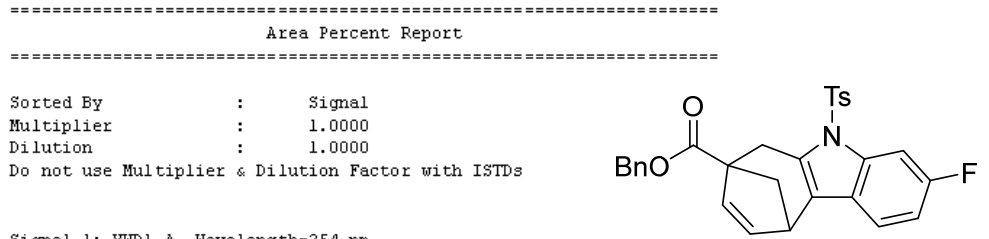

G1 gral 1: Vill 1 A, wavelengthe254

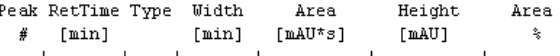

$$
\begin{aligned}
& \begin{array}{rrrrrrr}
1 & 31.068 \text { FB R } & 0.7536 & 537.54065 & 11.12799 & 50.0253 \\
2 & 37.684 \mathrm{BE} & 0.9146 & 536.99591 & 8.99422 & 49.9747
\end{array} \\
& \text { Totals : } \\
& 1074.53656 \quad 20.1222
\end{aligned}
$$

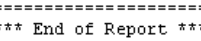

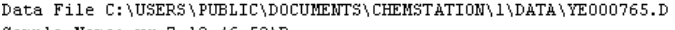
Sample Wame: wg-7-13-(6-53)

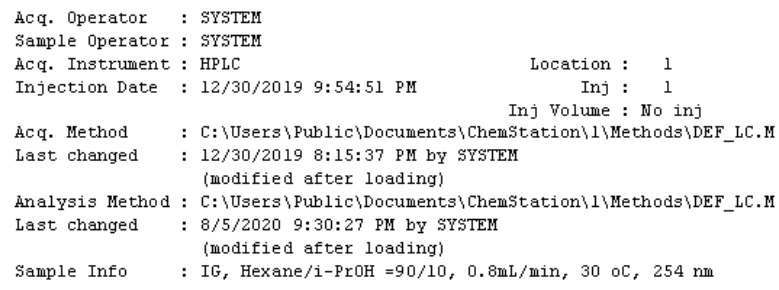

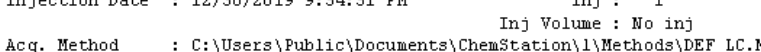

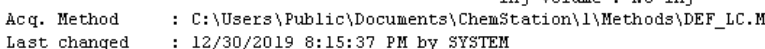
be

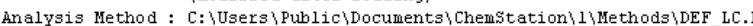
Last changed : 8/5/2020 9:30:27 PM bY SYSTTM

Sample Info : If, Hexane $/ 1-\mathrm{PrOH}=90 / 10,0.8 \mathrm{~mL} / \mathrm{min}, 30$ oc, $254 \mathrm{~m}$

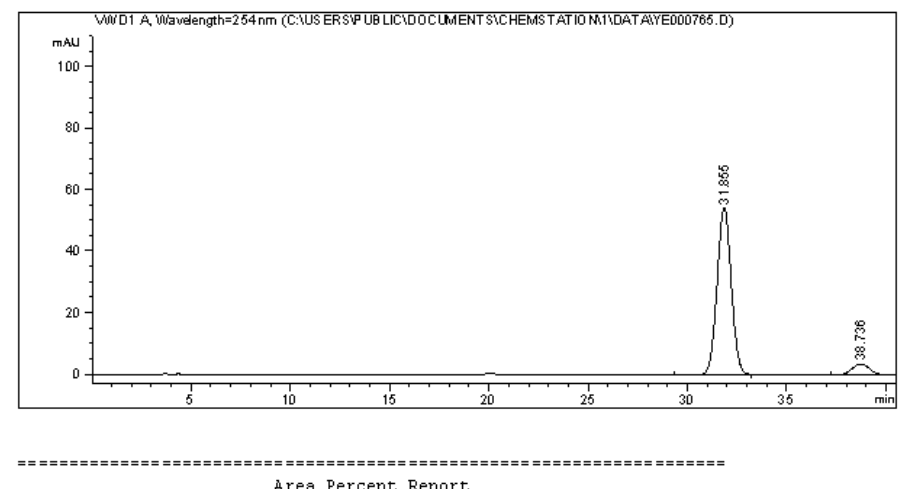

(1)

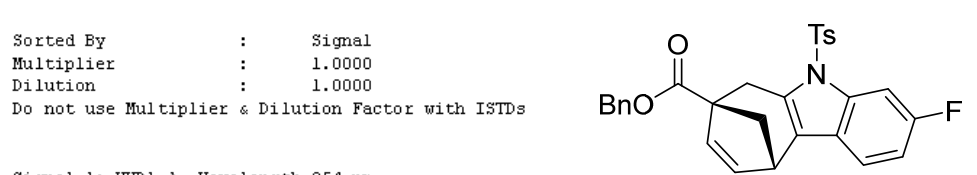

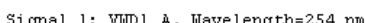

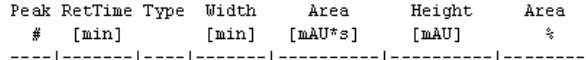

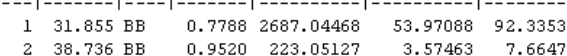

$$
\begin{aligned}
& \text { Totals: } \\
& 2910.09595 \quad 57.54551
\end{aligned}
$$

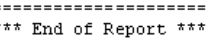




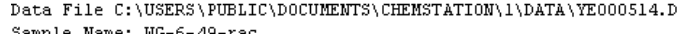
Sample Name: hic-6-49-rac
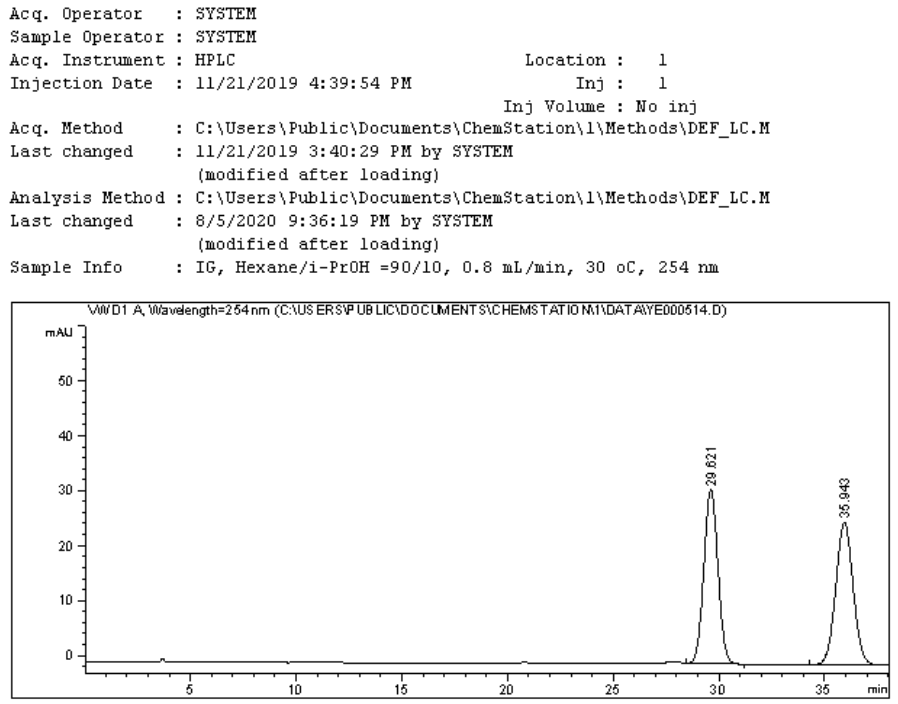

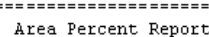

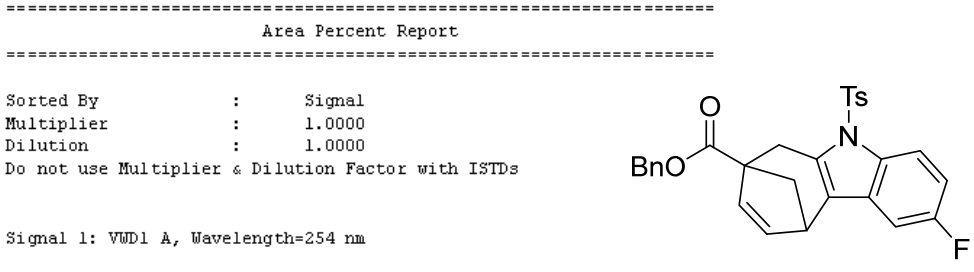

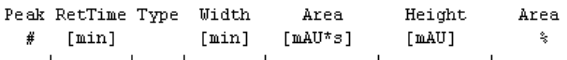

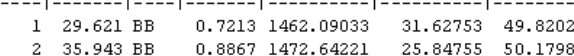

Totals :

$2934.73254 \quad 57.47507$

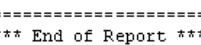

HPIC 8/5/2020 9:36:22 PII SYSTEH

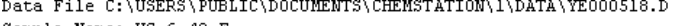
sample Name: irG-6-40-r

\section{Acq. Operator : : FYSTEM
Sample operator : FTSTEM}

Sample operator: : FrSTEM

$\begin{array}{lll}\text { Lec. Instrument : Hprt } & \text { Loation : } & 1 \\ \text { Injection Date : } 11 / 21 / 20196: 07: 01 \mathrm{PM} & \text { Inj : } & 1\end{array}$

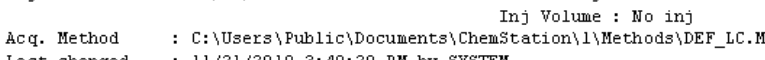

Last changed : 11/21/2019 3:40:29 PM bY SYSTEM

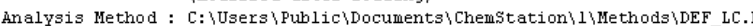

Last changed : 8/5/2020 9:35:08 PM by sพsTEM

Sample Info : (nodified after loading)

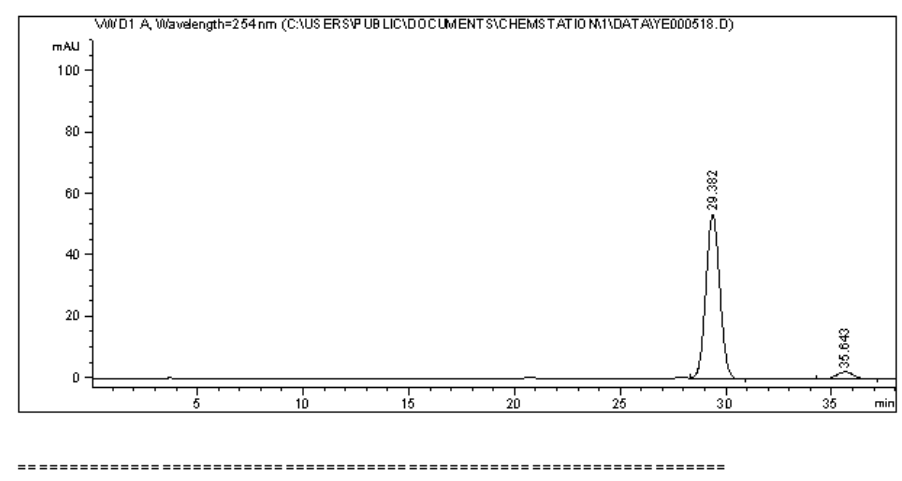

(1)

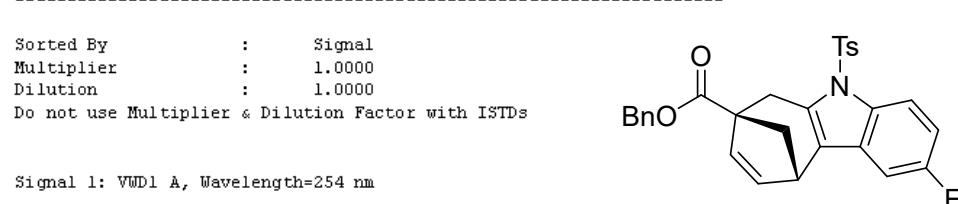

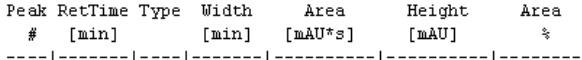

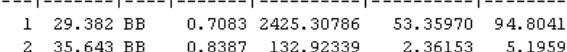

$$
\begin{aligned}
& 2558.23125 \quad 55.72123
\end{aligned}
$$

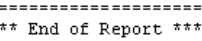

HPIC 8/5/2020 9:35:13 PII SYSTEM

Page 1 of 1 


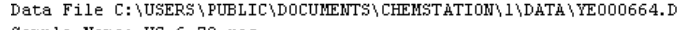
Sample Wame: hig-6-70-rac
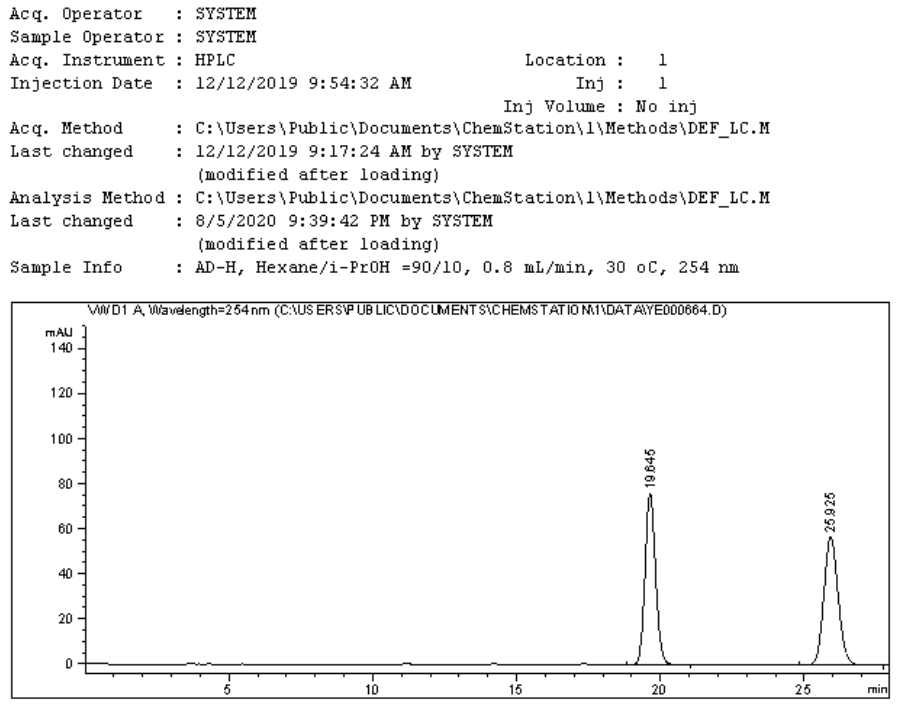

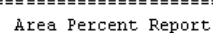

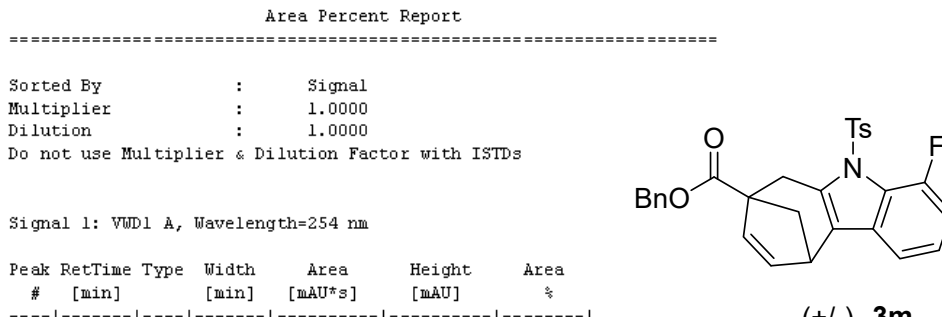

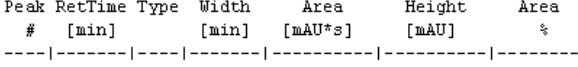

$\begin{array}{llllll}1 & 19.645 \mathrm{BE} & 0.3940 & 1937.90808 & 75.87404 & 49.962 \\ 2 & 25.925 \mathrm{BE} & 0.5310 & 1940.79309 & 56.74994 & 50.0372\end{array}$

Tatáls

$3676.70117 \quad 132.62397$

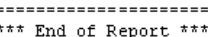

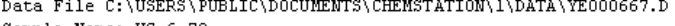
sample Name: iG $-6-7$

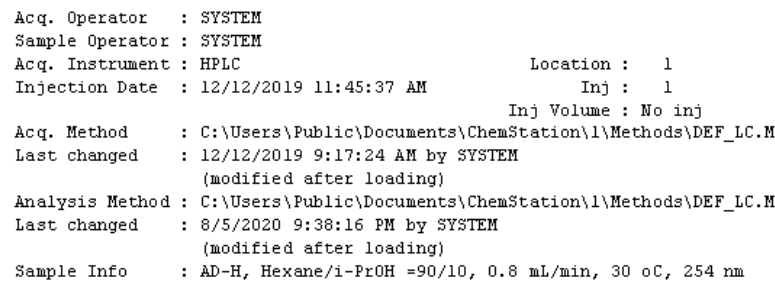

Inj Yolume : : No inj
The

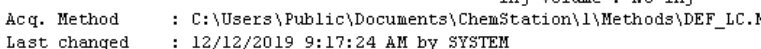

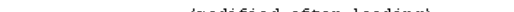

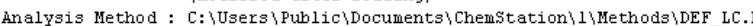
Last changed : 8/5/2020 9:38:16 PMI bY SYSTEM

Sample Info : : $\mathrm{HD}-\mathrm{H}$, Hexant $/ \mathrm{i}-\mathrm{PrOH}=90 / 10,0.8 \mathrm{~mL} / \mathrm{min}, 30 \mathrm{oC}, 254 \mathrm{~mm}$

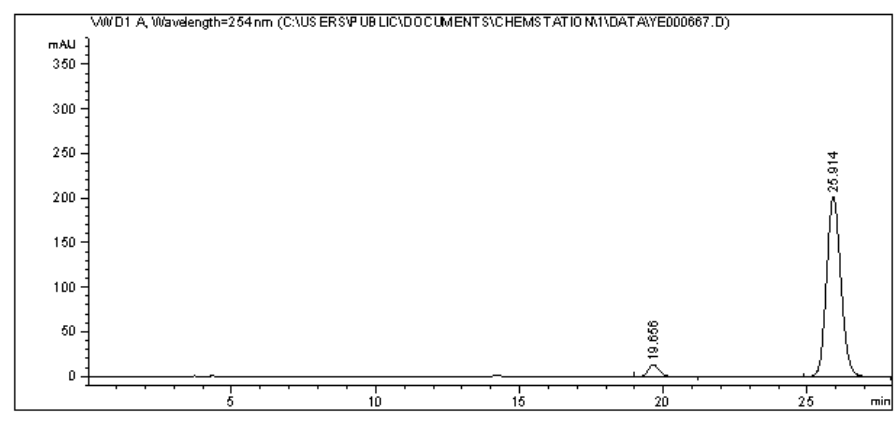

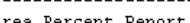

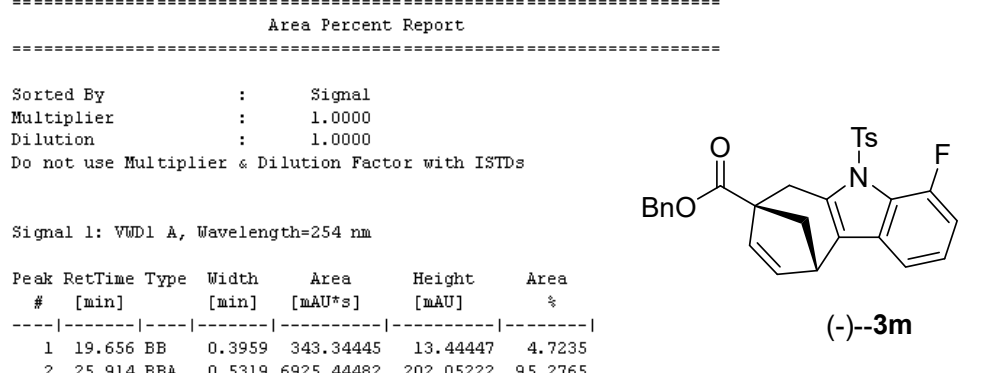

Totals

$7268.79928 \quad 215.49669$

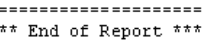

HPIC 8/5/2020 9:38:26 PII SYSTEM

Page 1 of 1 


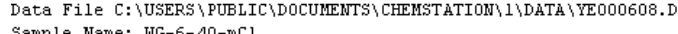

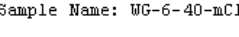
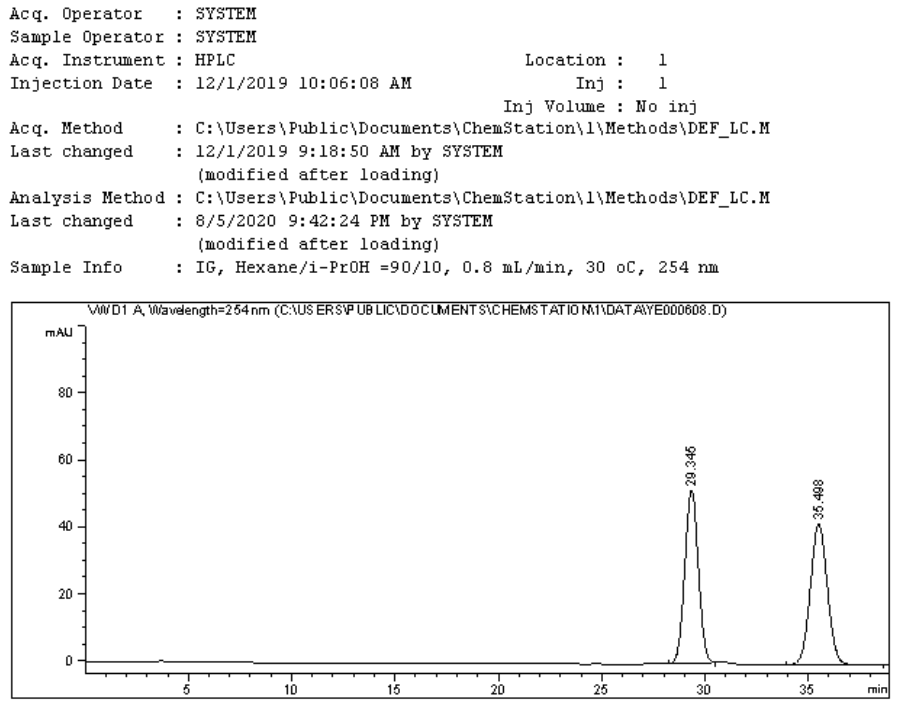
M
in rea Percent Report

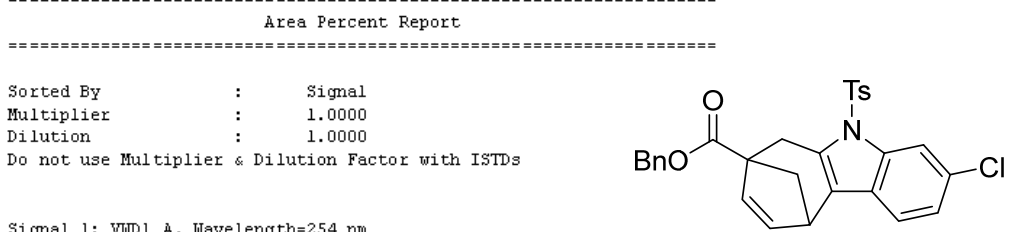

S1 gral 1: Vill 1 A, wavelengthe254 nm

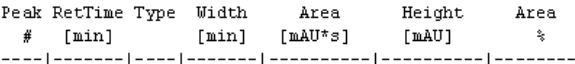

$$
\begin{aligned}
& \begin{array}{llllll}
1 & 29.345 \mathrm{BE} & 0.7111 & 2349.25391 & 51.60397 & 49.5827 \\
2 & 35.498 \mathrm{BE} & 0.8868 & 2388.80078 & 41.927272 & 50.4173
\end{array} \\
& \begin{array}{lll}
\text { Totals : } & 4738.05469 & 93.52669
\end{array}
\end{aligned}
$$

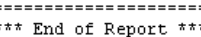

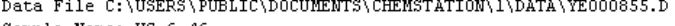
Sample Name: ing- $6-4$

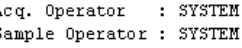

Sample operator : FrsTEM
Aeq. Instrument : HPIC

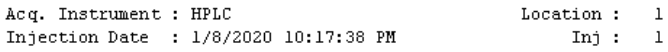

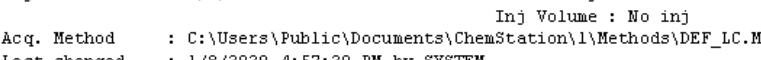

Last changed : 1/8/2020 4:57:20 PII bY SYSTEM

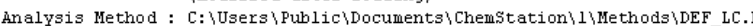

Last changed : 8/5/2020 9:41:24 FII by srsteM

Sample Info : (nodified after loading)

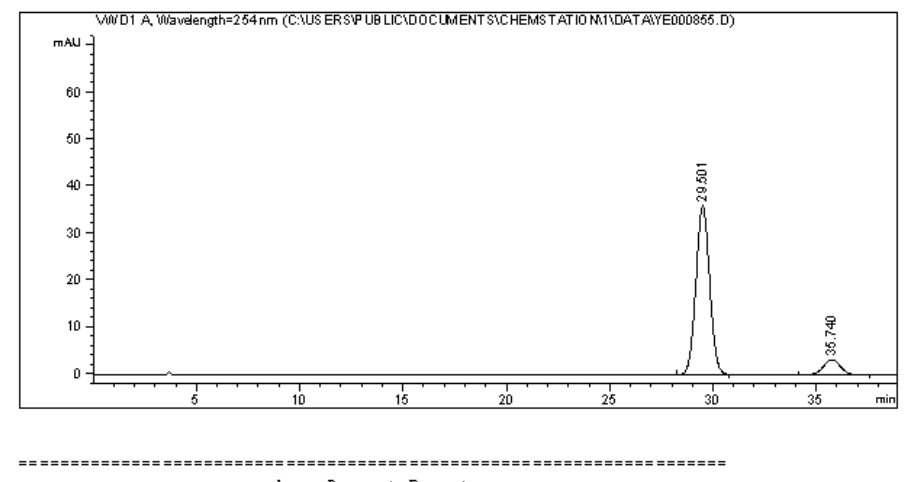

Area

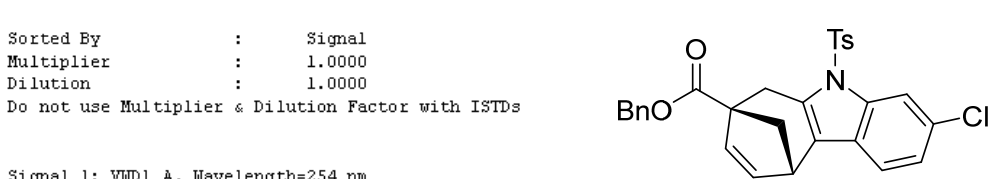

Signal 1: MUI 1 A, Wavelength $=254$ mil

$(-)-3 n$$$
1843.73064 \quad 39.20070
$$

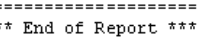




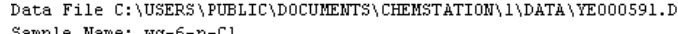
Sample Name: wog-6-p-ci
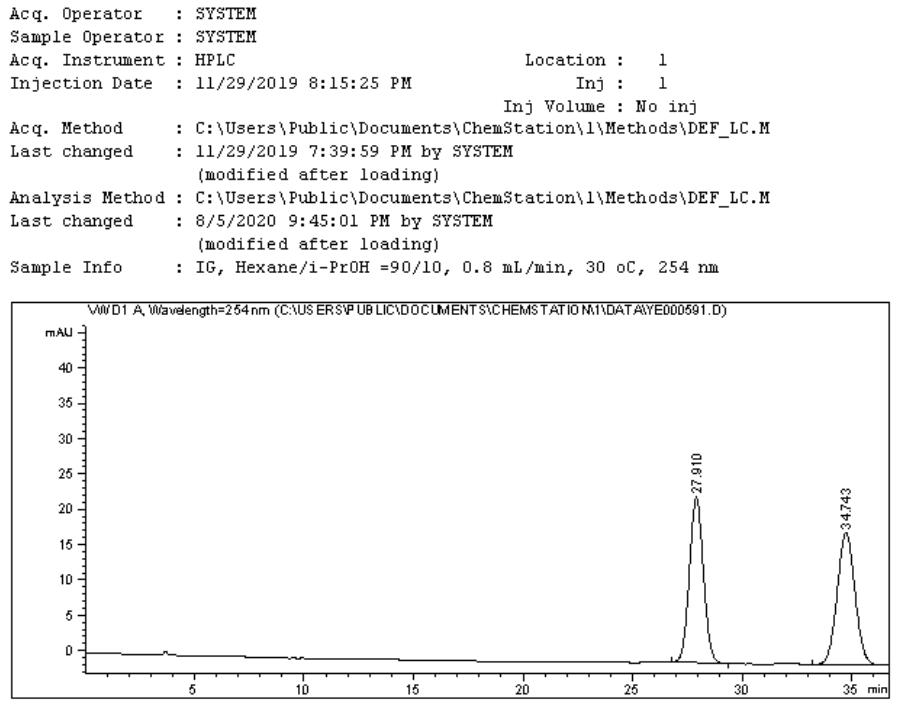

M
in rea Percent Report

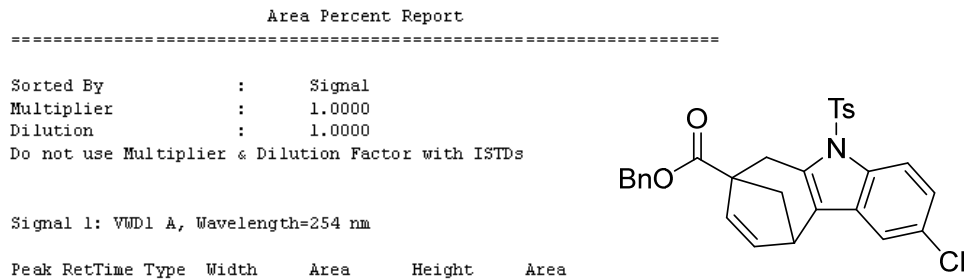

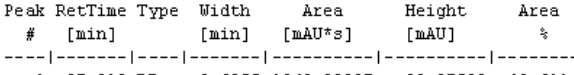

$(+/-)--30$

$\begin{array}{llllll}1 & 27.910 \mathrm{BB} & 0.6955 & 1040.83337 & 23.37539 & 49.611 \\ 2 & 34.743 \mathrm{EBA} & 0.8793 & 1057.14087 & 18.64975 & 50.388\end{array}$
Totals :
$2097.97424 \quad 42.02514$

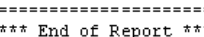

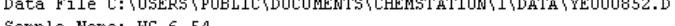
Sample Mane:

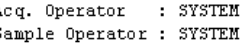

Sample operator: : FrsTEM

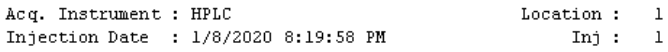

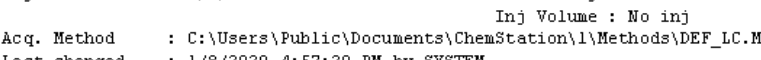

Last changed : 1/8/2020 4:57:20 PII bY SYSTEM

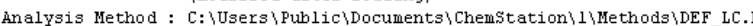

Last changed : 8/5/2020 9:43:48 PM by srsteM

Sample Info : (nodified after loading)

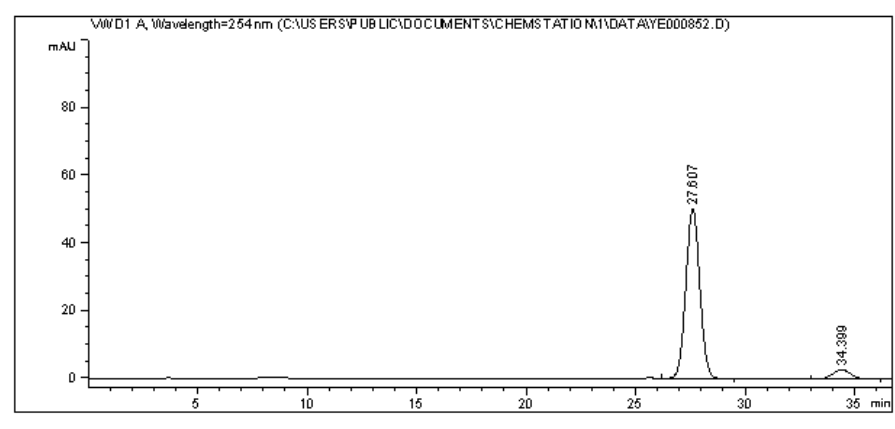

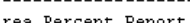

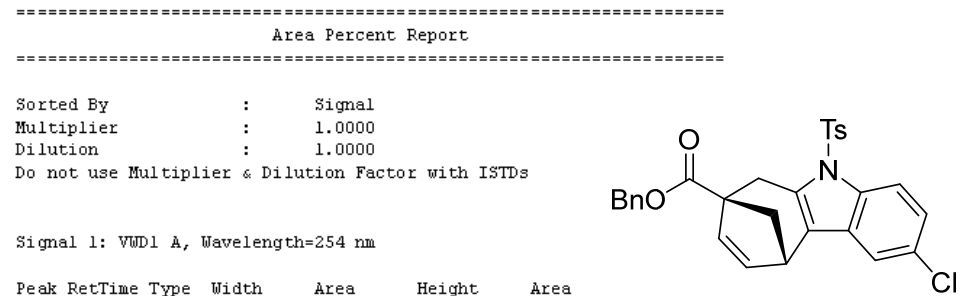

al 1: VIIII $A$,

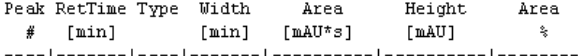

$$
\begin{aligned}
& \begin{array}{llllll}
1 & 27.607 \mathrm{EE} & 0.6896 & 2227.52466 & 50.21128 & 93.5516 \\
2 & 34.399 \mathrm{BE} & 0.8511 & 153.54054 & 2.74286 & 6.4484
\end{array} \\
& 2361.06520 \quad 52.95414
\end{aligned}
$$

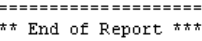

HPIC 8/5/2020 9:44:02 PII SYSTEM 


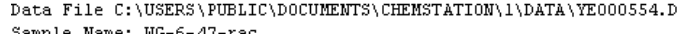
Sample Name: hiG-6-47-rac
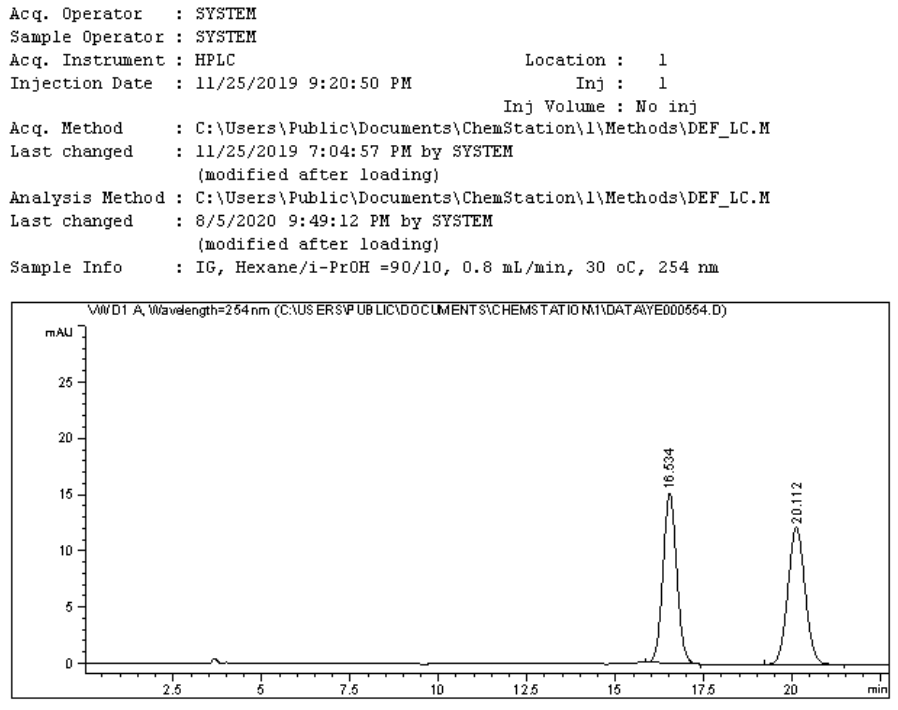

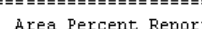

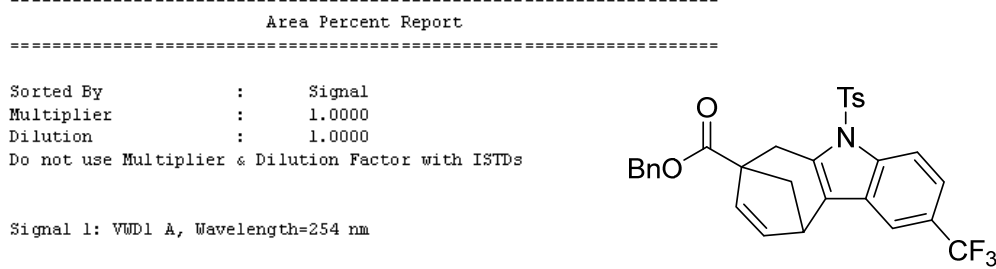

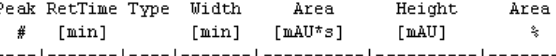

$$
\begin{aligned}
& \begin{array}{llllll}
1 & 16.534 \mathrm{BE} & 0.4186 & 405.98483 & 15.05459 & 49.7649 \\
2 & 20.112 \mathrm{FE} & 0.5264 & 409.82034 & 12.18374 & 50.2351
\end{array} \\
& \text { Tota13 : } \quad 815.00518 \quad 27.23839
\end{aligned}
$$

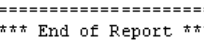

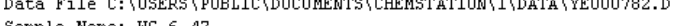
Sample Name: ing- $6-47$

$$
\begin{array}{ll}
\text { Acq. Operator } & : \text { SYSTEM } \\
\text { Sample Operator } & \text { : FrSTEII } \\
\text { ACq. Instrwient } & \text { HPLC }
\end{array}
$$

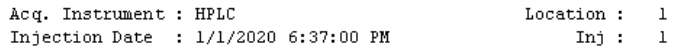

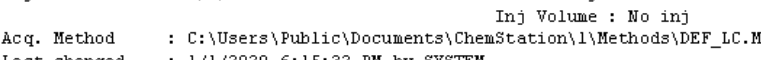
La ät changed : 1/1/2020 6:15:32 PII bY STSTEM

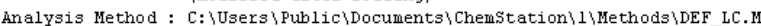

Last changed : 8/5/2020 9:47:19 PM by srsteM

Sample Info : (nodified after loading)

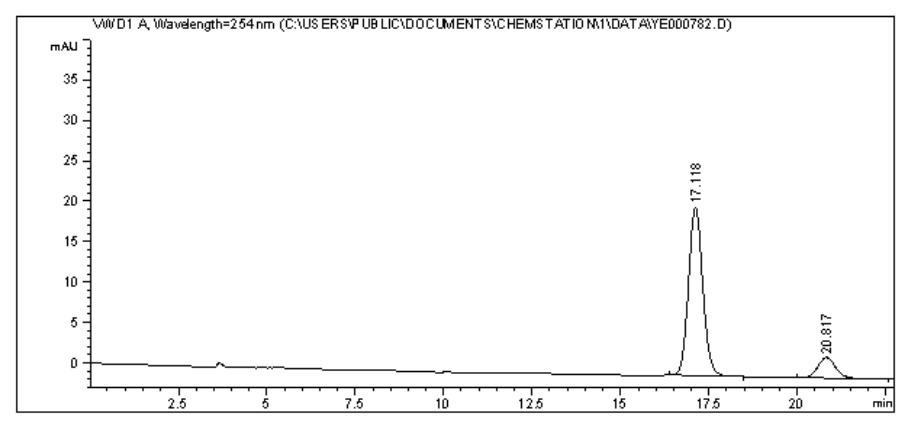

Area

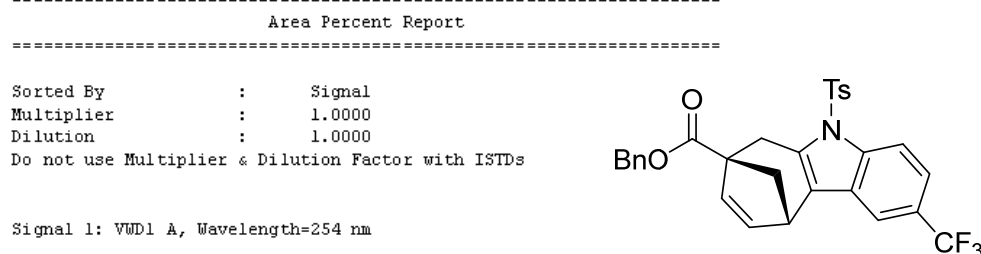

Si gnal 1: WhD $1 \mathrm{~A}$, Wavelength=254

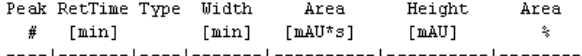

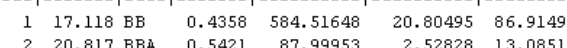

$$
\begin{aligned}
& \text { Totáls: } \\
& 672.51601 \quad 23.33324
\end{aligned}
$$$$
(-)-3 p
$$

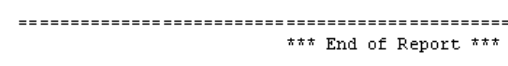




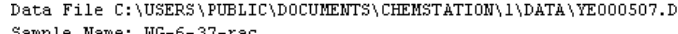
ample Name:
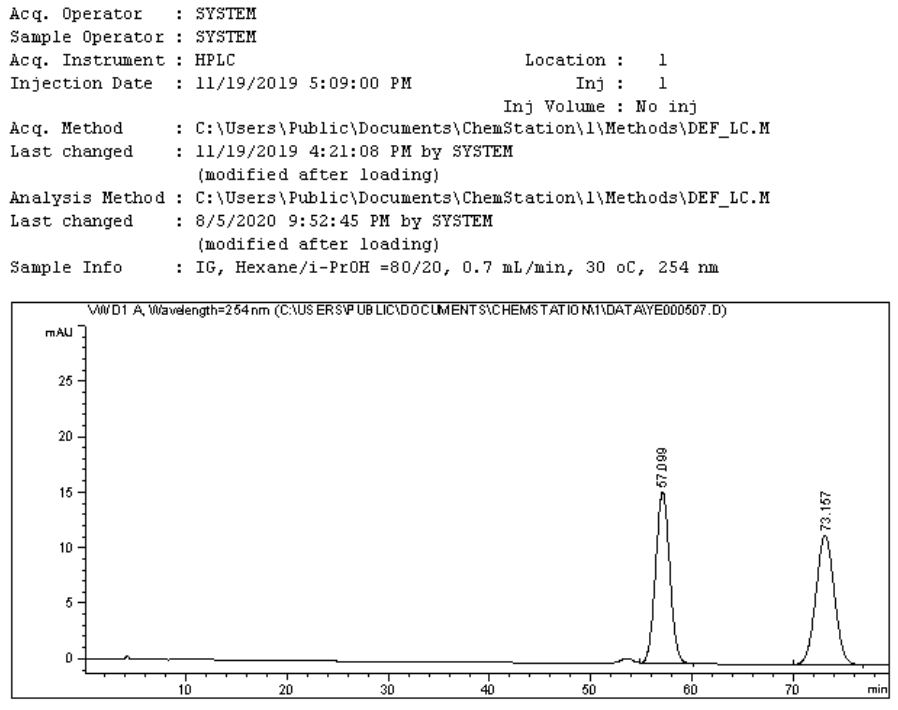

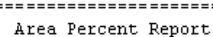

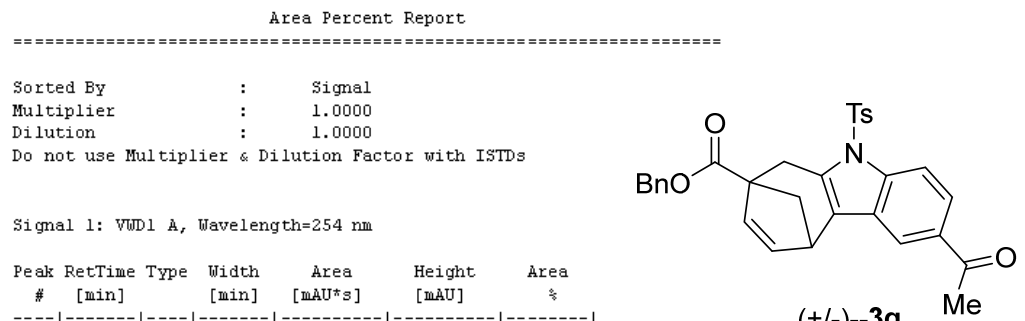

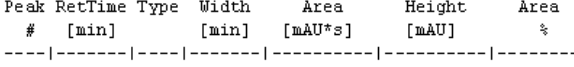

$(+/-)--3 q$

$\begin{array}{llllll}1 & 57.099 \mathrm{BE} & 1.5088 & 1493.91870 & 15.41278 & 50.480 \\ 2 & 73.157 \mathrm{BE} & 1.9276 & 1465.49097 & 11.60261 & 49.5197\end{array}$

Totals : $\quad 2959.40967 \quad 27.01540$

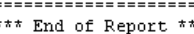

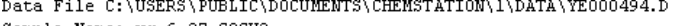
Sample Wame: wg-6-37-coch

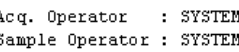

Sample operator : : STSTEM
Acq. Instrument : HPIC

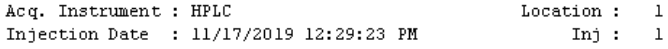

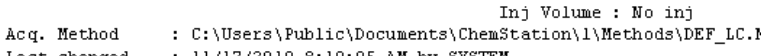

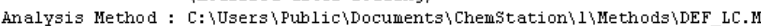

Last changed : 8/5/2020 9:53:52 PM by sพsTEM

Sample Info : (nodified after loadmg)

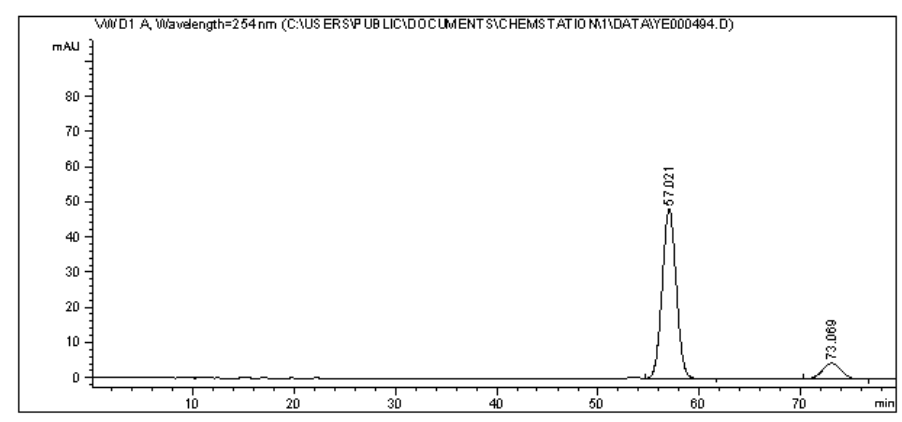

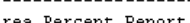

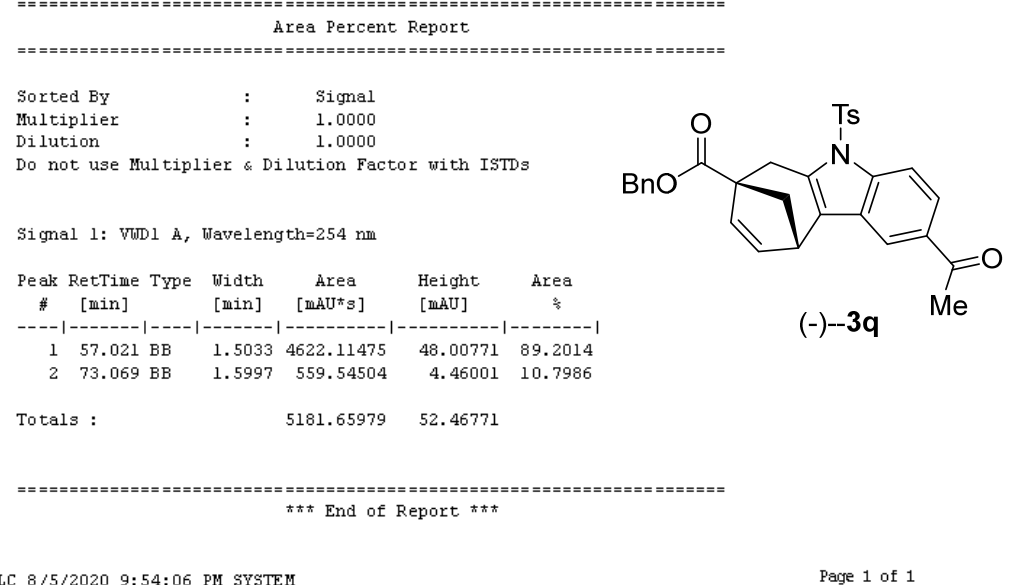




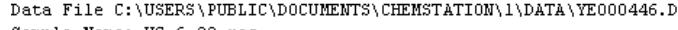
Gample Name: wiG-6-33-rac
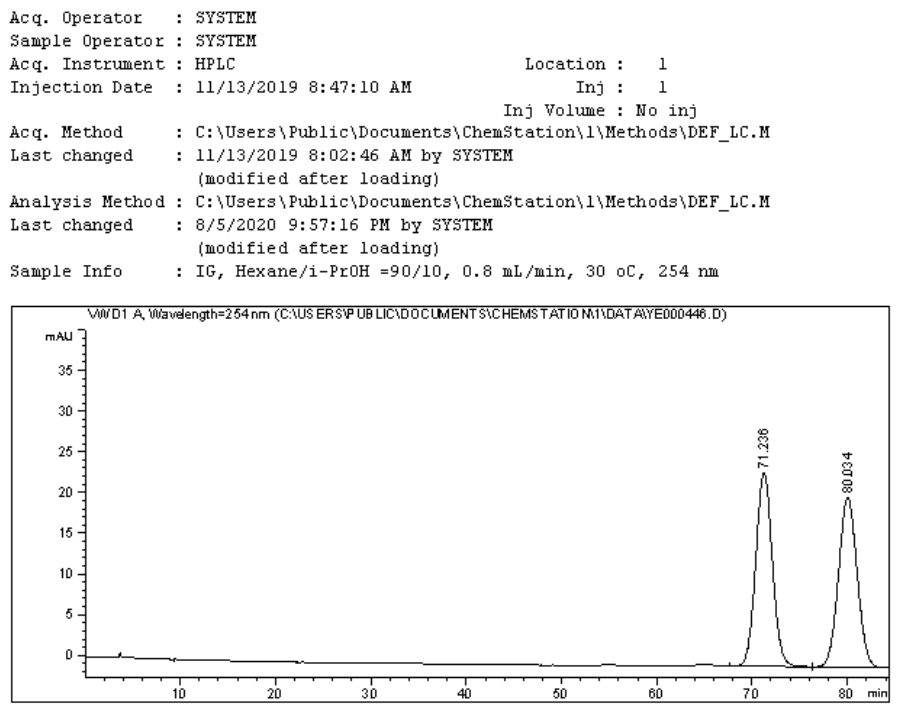

Area Percent. Report

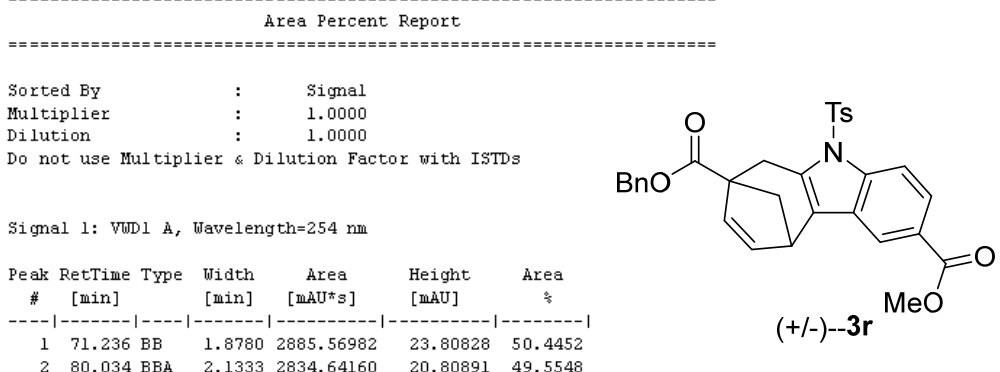

$\begin{array}{llllll}1 & 71.236 \mathrm{BE} & 1.8780 & 2885.56982 & 23.80828 & 50.4452 \\ 2 & 80.034 \mathrm{BEA} & 2.1333 & 2834.64160 & 20.80891 & 49.554\end{array}$

$(+/-)-3 \mathbf{r}$

Totals : $\quad 5720.21143 \quad 44.61719$

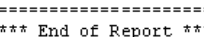

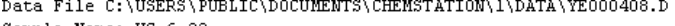
atuple Näie: hiG-6-3

\section{Acq. Operator : FTSTEM
Sample operatox : FrTEM}

Sample operator: : FrsTrR

$\begin{array}{lll}\text { Lec. Instrument : Hprt } & \text { Loation : } & 1 \\ \text { Injection Date : } 11 / 9 / 2019 \text { 8:45:04 All } & \text { Inj : } & 1\end{array}$

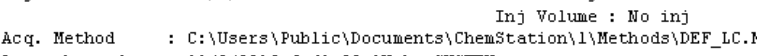
Läst changed : 11/9/2019 6:41:02 All bY STST

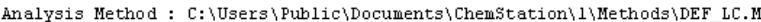

Last changed : 8/5/2020 9:56:26 PM by sพsTEM

Sample Info : IG, Hexane $/ 1-\mathrm{FrOH}=90 / 10,0.8 \mathrm{~mL} / \mathrm{min}, 30$ oc, $254 \mathrm{~m}$

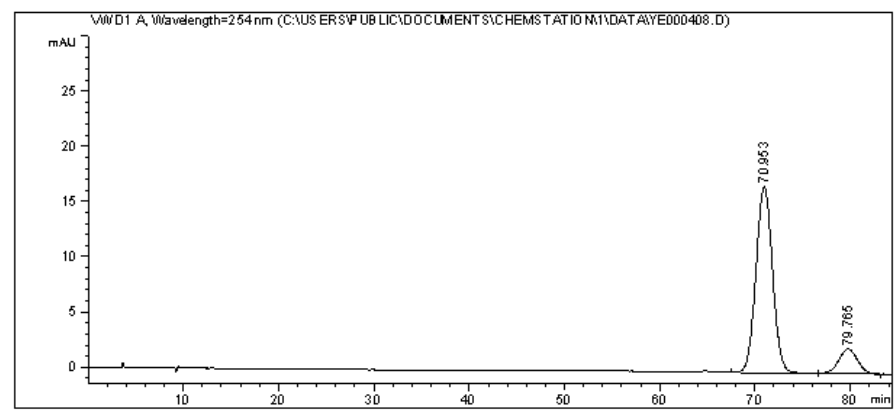

Area Percent. Report

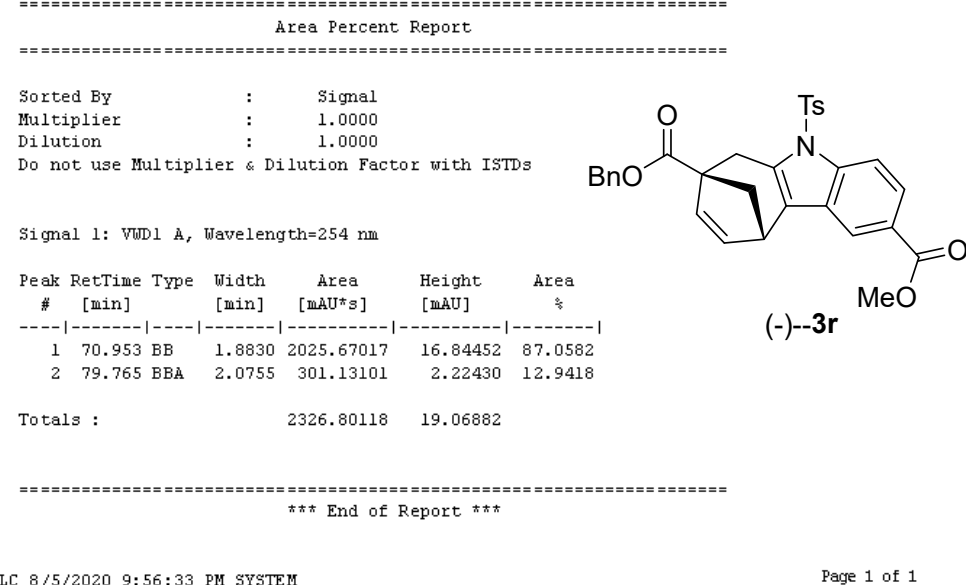




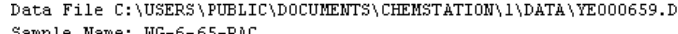
Sample Name:
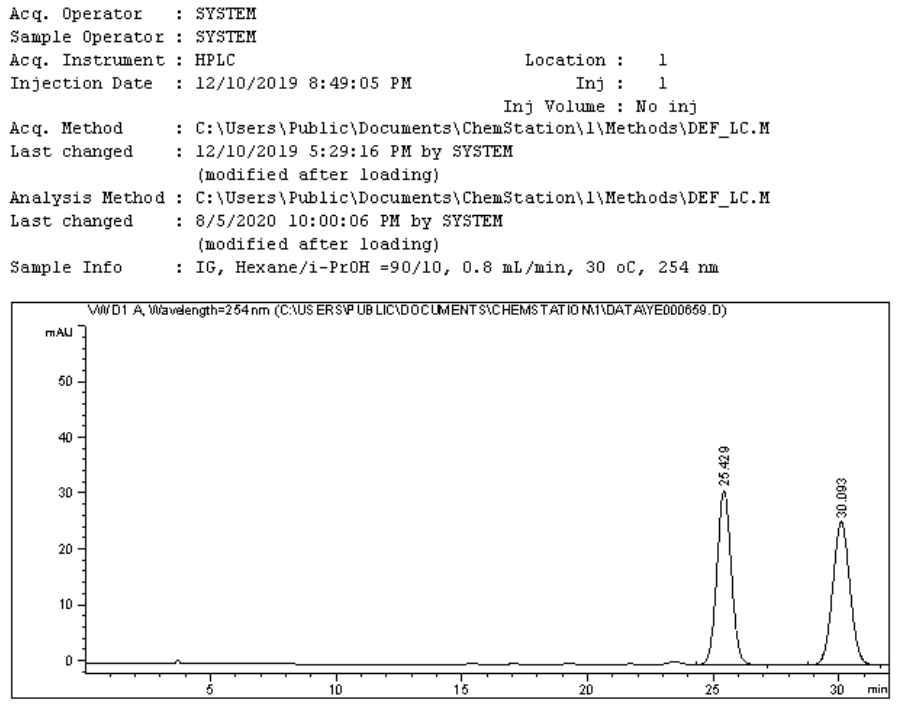

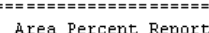

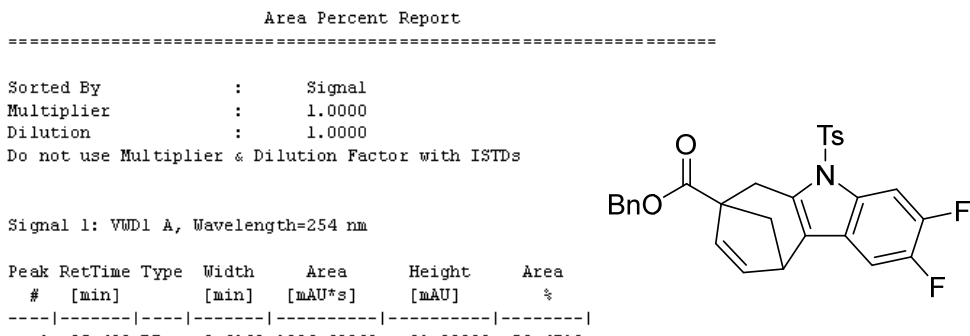

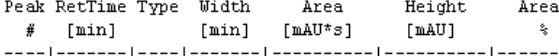

\begin{tabular}{ll|llll}
1 & $25.429 \mathrm{EE}$ & 0.6162 & 1236.63062 & 31.08088 & 50.4718 \\
2 & $30.093 \mathrm{FB}$ & 0.7371 & 1213.5111 & 25.55222 & 49.5282
\end{tabular}

$(+/-)--3 \mathbf{s}$

Totals :

$2450.14172 \quad 56.63310$

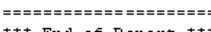

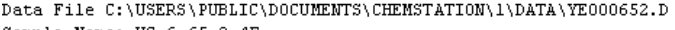
Sample Name: wiG-6-65-3,4F

\section{Acq. Operator : : FYSTEM
Sample operator : : 2 TSTEM}

Sample operator: इFSTEM

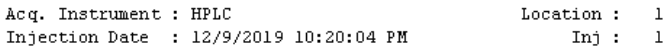

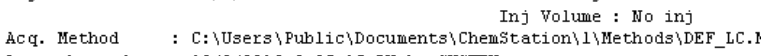
Last changed : 12/9/2019 8:25:15 PII by STSTH

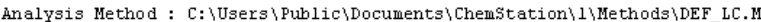

Last changed : 8/5/2020 9:58:50 PM by sพsTEM

Sample Info : IG, Hexane $/ 1-\mathrm{FrOH}=90 / 10,0.8 \mathrm{~mL} / \mathrm{min}, 30$ oc, $254 \mathrm{~m}$

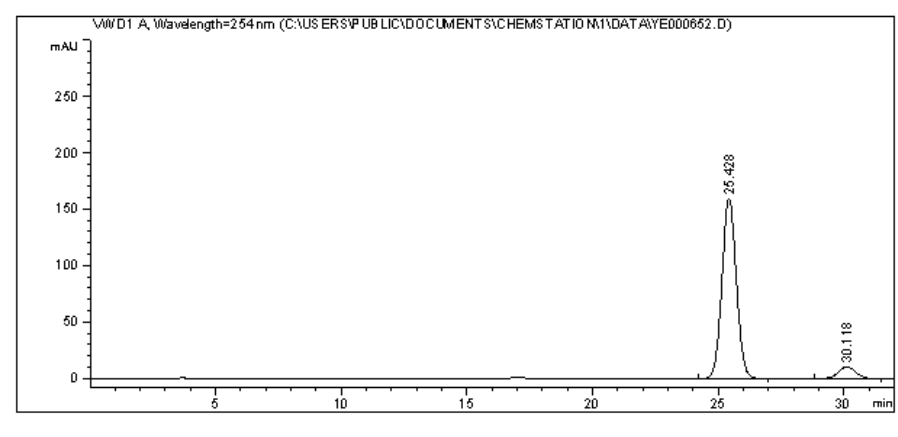

4rea Percent. Report.

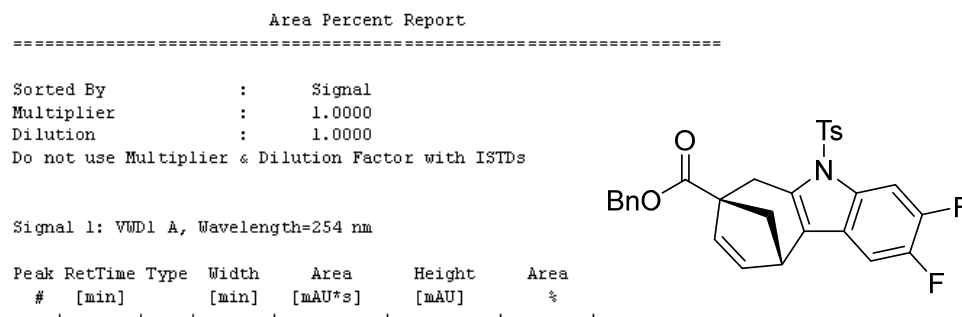

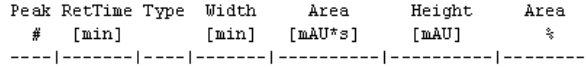

$\begin{array}{rrrrrr}1 & 25.428 \mathrm{BE} & 0.6180 & 6325.43652 & 159.39877 & 92.8958 \\ 2 & 30.118 \mathrm{EE} & 0.7367 & 483.73474 & 10.17334 & 7.1042\end{array}$

$(-)-3 \mathbf{s}$

Totals:

$$
6809.17126 \quad 169.57212
$$

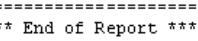

APIC 8/5/2020 9:58:55 PII SYSTEM 


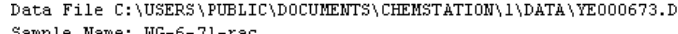
ample Name:
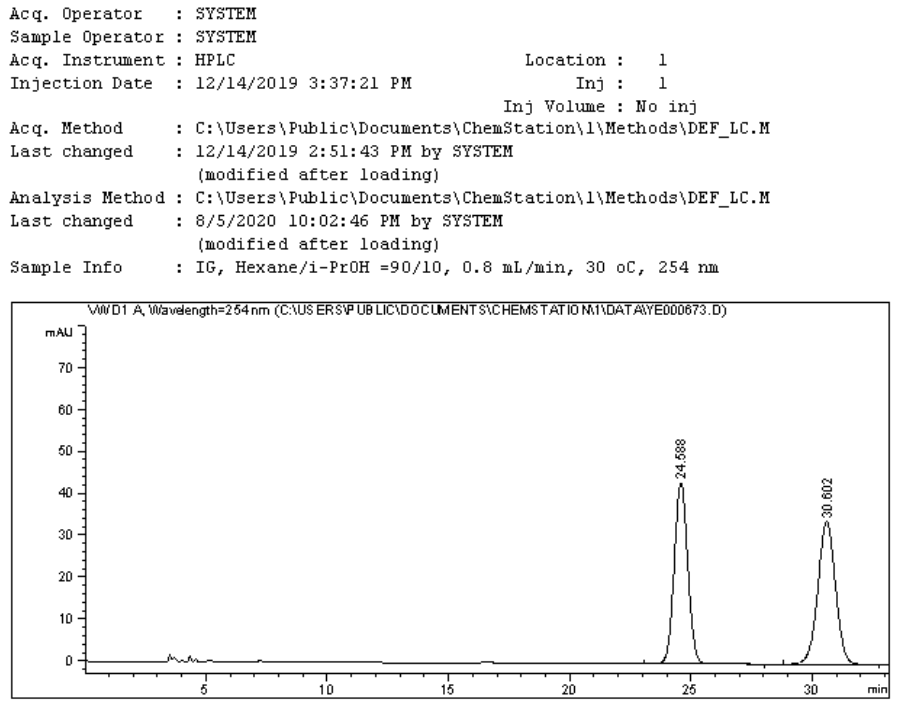

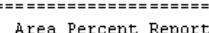

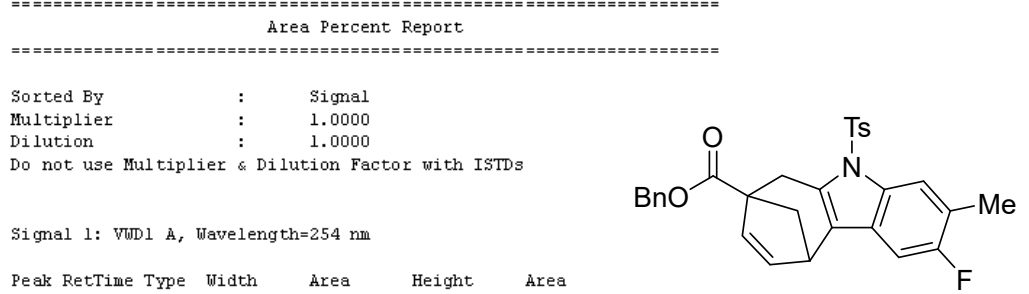

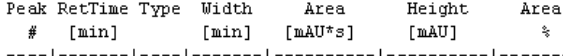

$\begin{array}{llllll}1 & 24.588 \mathrm{BE} & 0.6122 & 1669.98901 & 43.08283 & 49.3808 \\ 2 & 30.602 \mathrm{FEA} & 0.7802 & 1711.86658 & 33.95417 & 50.6192\end{array}$

$(+/-)--3 t$

Tatâls

$3361.85559 \quad 77.03700$

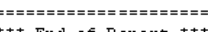

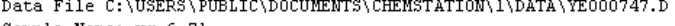
sample Name: wg-6-7

\section{Acq. Operator : : FYSTEM
Sample Operator : FTSTEM}

Sample operator : STSTEM
Acq. Instrument : HPIC

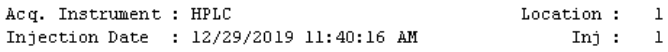

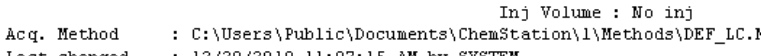
Last changed : : 12/29/2019 11:07:15 AM bY SYSTEM

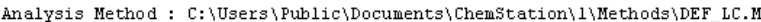

Last changed : 8/5/2020 10:01:58 PII by SYSTEM

Sample Info : IG, Hexane $/ 1$-FroH $=90 / 10$, 0. $8 \mathrm{~m} \mathrm{~m} / \mathrm{min}, 30$ oc, $254 \mathrm{~mm}$

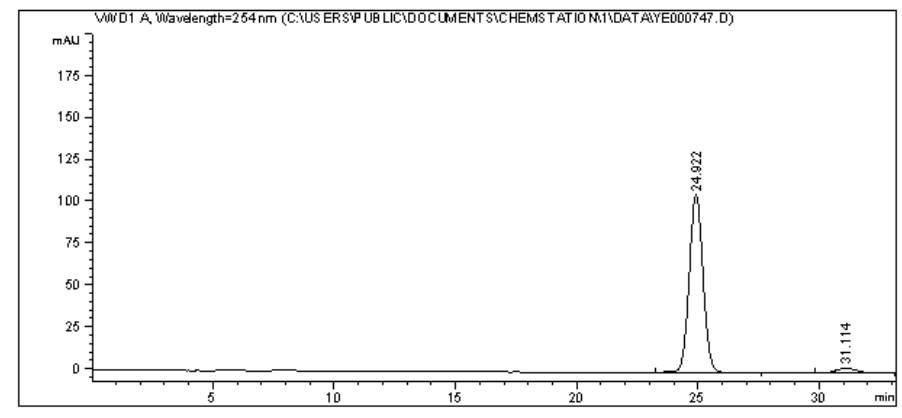

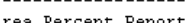

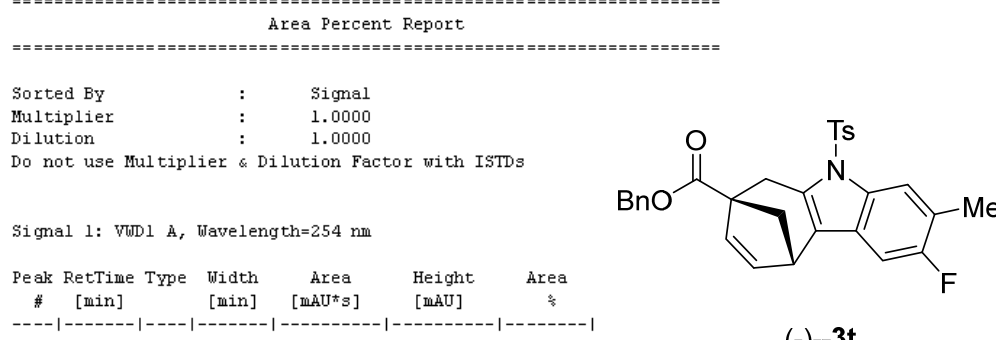

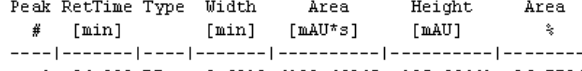

$\begin{array}{llllrr}1 & 24.922 \mathrm{BE} & 0.6218 & 4182.46045 & 105.66441 & 96.7784 \\ 2 & 31.114 \mathrm{EBA} & 0.8002 & 139.22830 & 2.74296 & 3.2216\end{array}$

$(-)-3 t$

Totals:

$$
\begin{array}{ll}
4321.68975 \quad 108.40737
\end{array}
$$

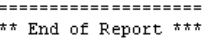

HPIC 8/5/2020 10:02:03 PM SYSTE 


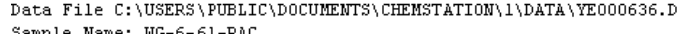
Gample Name: hig-6-61-Ric

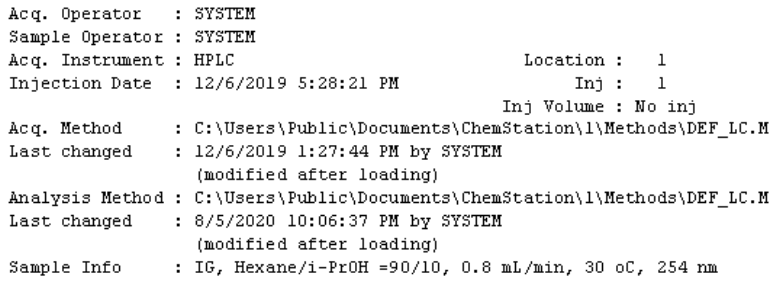

Inj Volume: : No inj

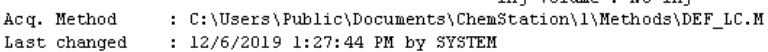

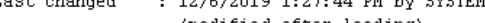

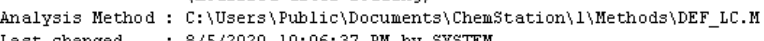
Last changed : 8/5/2020 10:06:37 PII by sFsTrm

Sample Info : (wG, Hexane/1-ProH $=90 / 10,0.6 \mathrm{mI} / \mathrm{min}, 30$ oc, $254 \mathrm{~mm}$

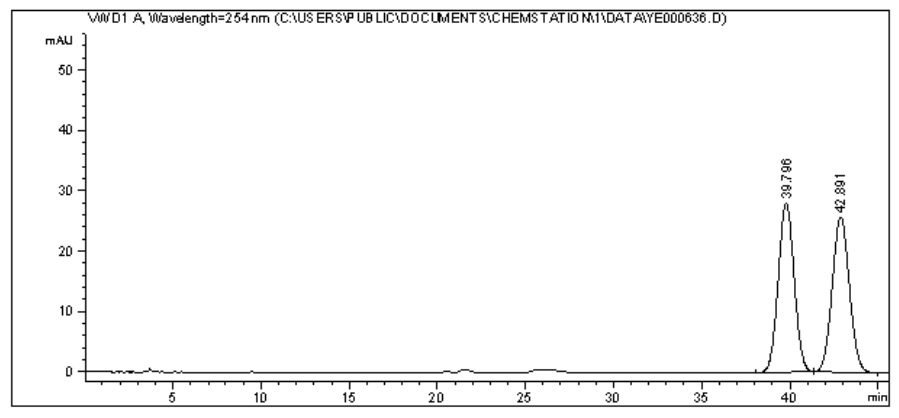

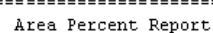

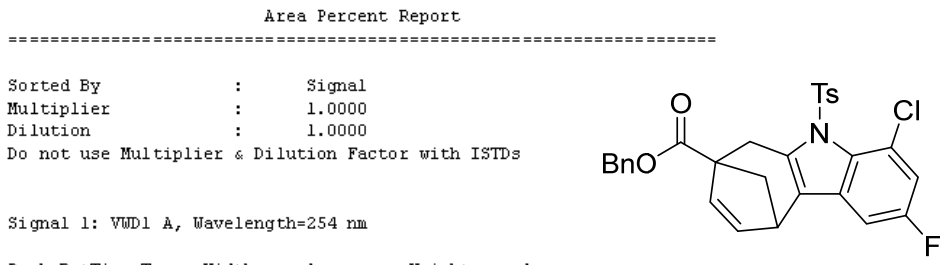

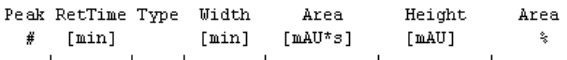

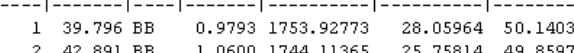

Totals :

$3498.04138 \quad 53.81777$

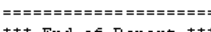

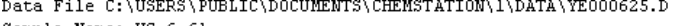
sample Name: iG $-6-6$

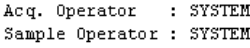

Sample operator: : FrsTrI
Aeq. Instrument : HPLC

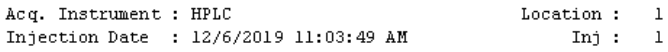

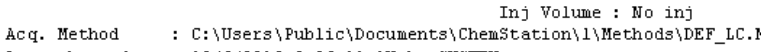
Läst changed : 12/6/2019 8:26:11 All bY SYSTEM

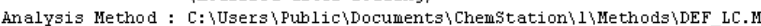

Last changed : 8/5/2020 10:05:48 $\mathrm{FII}$ by STSTEM

Sample Info : IG, Hexane $/ 1-\mathrm{Fr} 0 \mathrm{H}=90 / 10,0.8 \mathrm{~mL} / \mathrm{min}, 30$ oc, $254 \mathrm{~m}$

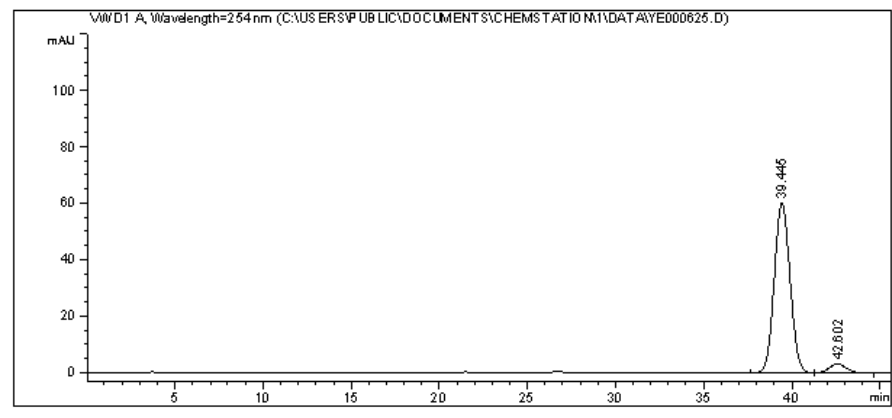

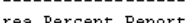

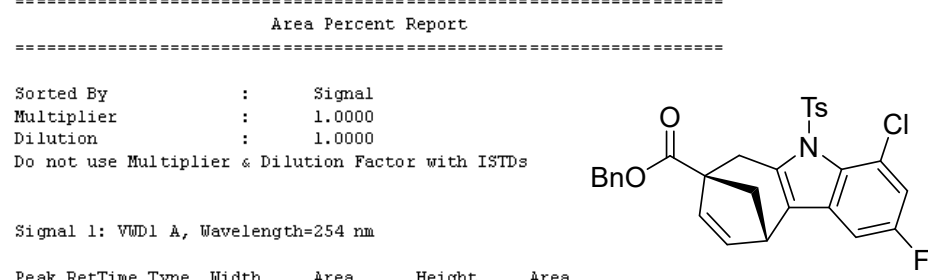

signal 1: Widl $\mathrm{A}$, Wave

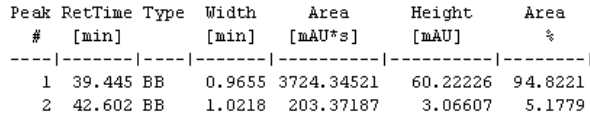

Totals:

$3927.71709 \quad 63.28032$

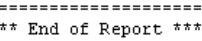

HPIC 8/5/2020 10:05:58 PM SYSTEI 


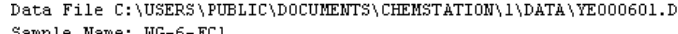
Sample Name: WTG-6-FC1

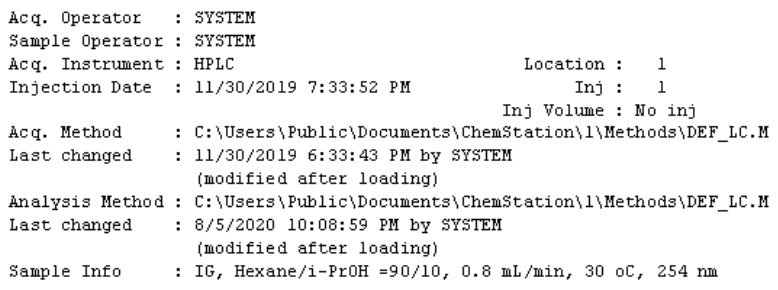

ACr. Wethod Inj Volume: : Ho inj

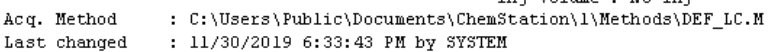
Last changed : $11 / 30 / 2019$ 6:33:43 PM GY STIT

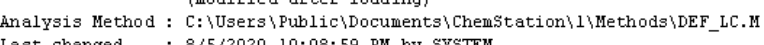
Last changed : 8/5/2020 10:08:59 PII by srsTrm

Sample Info : IG, Hexane $/ 1$-FroH $=90 \% 10,0.8 \mathrm{~mL} / \mathrm{min}, 30$ oc, $254 \mathrm{~mm}$

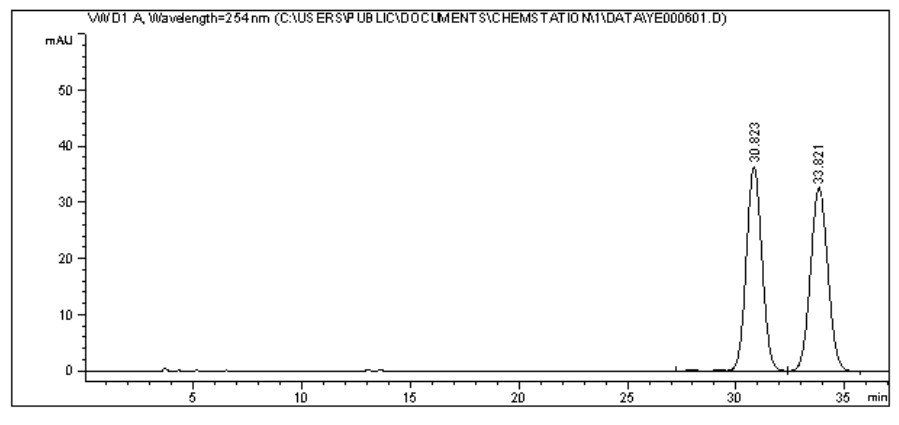

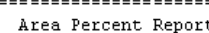

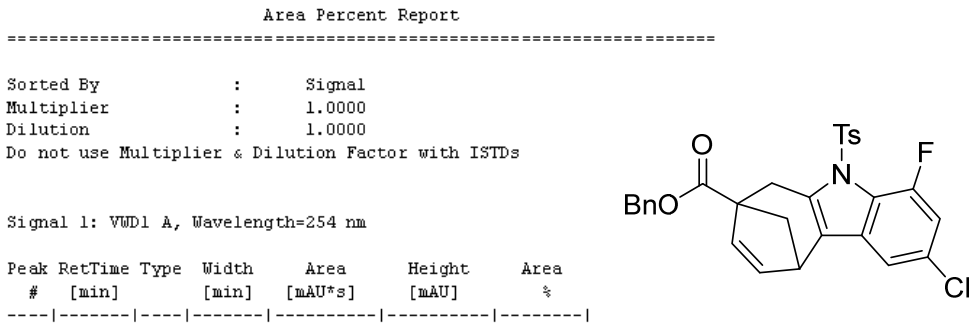

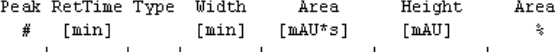

$\begin{array}{llllll}1 & 30.823 \text { FE R } & 0.7817 & 1815.44751 & 36.28884 & 50.0924 \\ 2 & 33.821 \mathrm{FE} & 0.8691 & 1808.74890 & 32.60997 & 49.9076\end{array}$

$(+/-)--3 v$

Totals :

$3624.19641 \quad 68.8980$

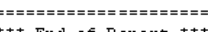

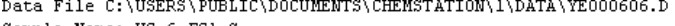
Sample Name: hiG-6-FC1-5

Acq. Operator : : FYSTEM
Sample Operator : : FrSTEII

Sample Operator : FrsTeM
Aeq. Instrument : HPLC

$\begin{array}{lll}\text { Lec. Instrument : HptC } & \text { Loation : } & 1 \\ \text { Injection Date : } 11 / 30 / 2019 \text { 9:40:10 PMI } & \text { Inj : } & 1\end{array}$

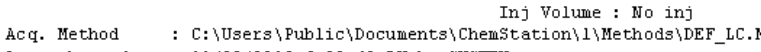
Last changed : 11/30/2019 6:33:43 PII bY SYSTEK

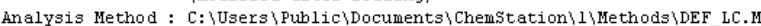

Last changed : 8/5/2020 10:08:59 PII by SYSTEM

Sample Info : IG, Hexane $/ 1-\mathrm{Fr} 0 \mathrm{H}=90 / 10,0.8 \mathrm{~mL} / \mathrm{min}, 30$ oc, $254 \mathrm{mb}$

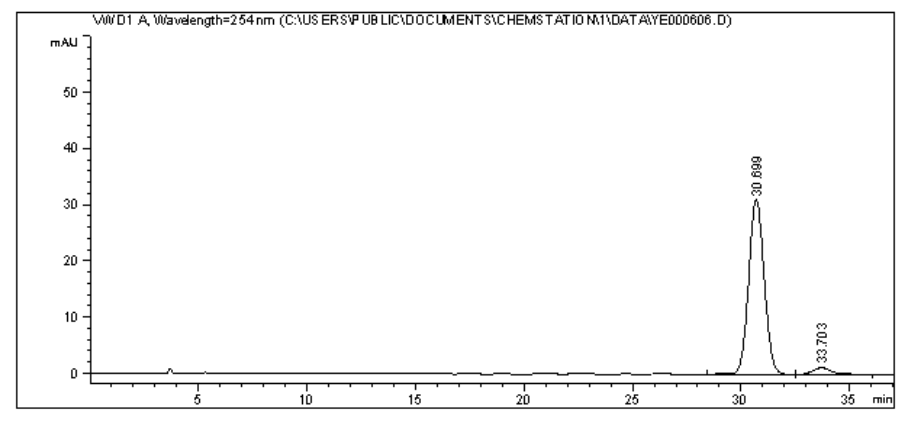

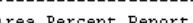

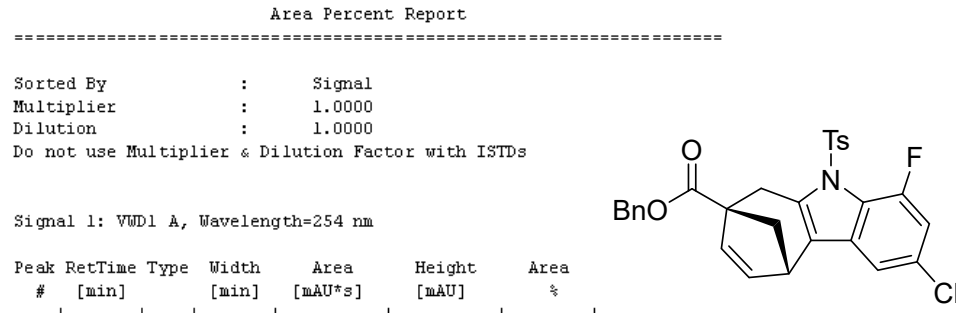

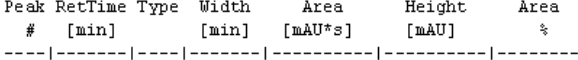

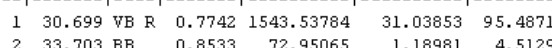

$(-)--3 v$

Totals:

$1616.49849 \quad 32.22834$

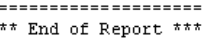

HPIC 8/5/2020 10:09:02 PM SYSTEI

Page 1 of 1 


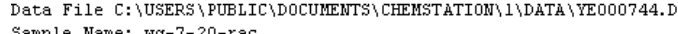
Dample Name: wog-7-zo-rac
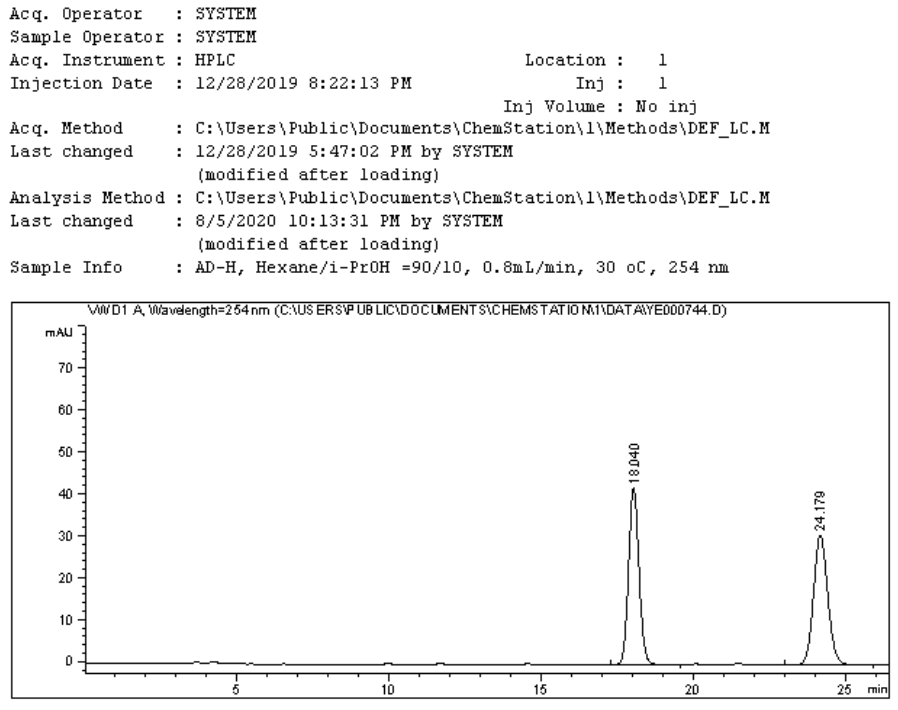

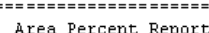

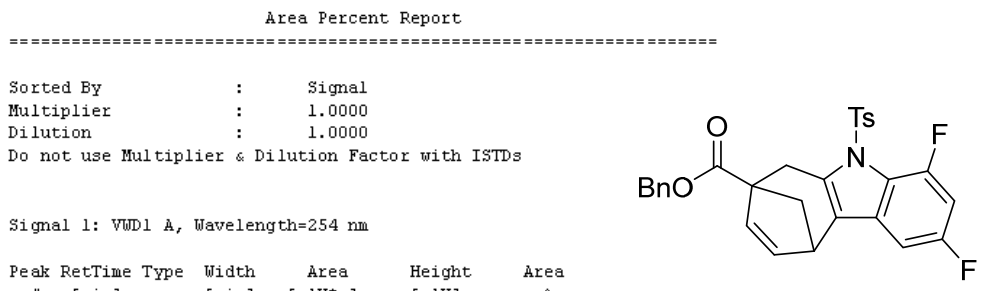

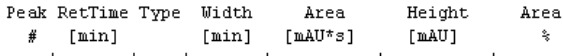

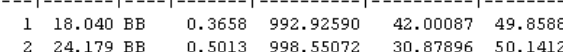

$(+/-)--3 w$

Tatâls

$1991.47662 \quad 72.87969$

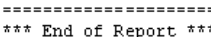

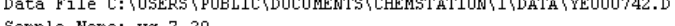
Sample Name: wg $-7-20$

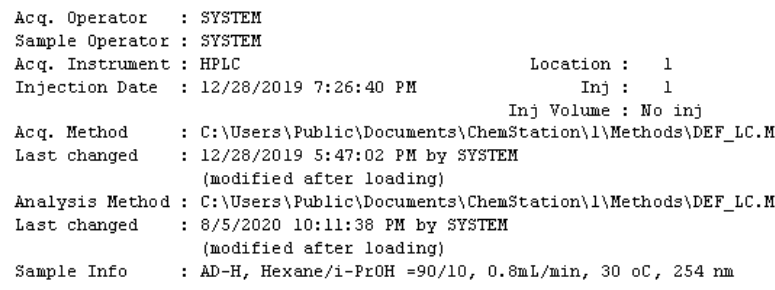

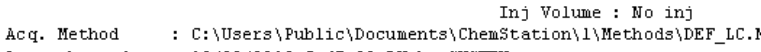
Last changed : 12/28/2019 5:47:02 PM bY STSTEM

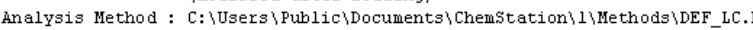

Last changed : 8/5/2020 10:11:38 PII bY STSTEM

Sample Info: : MD -H, Hexame $/ \mathrm{i}-\mathrm{FrOH}=90 / 10,0.8 \mathrm{~mL} / \mathrm{min}, 30$ oc, $254 \mathrm{~mm}$

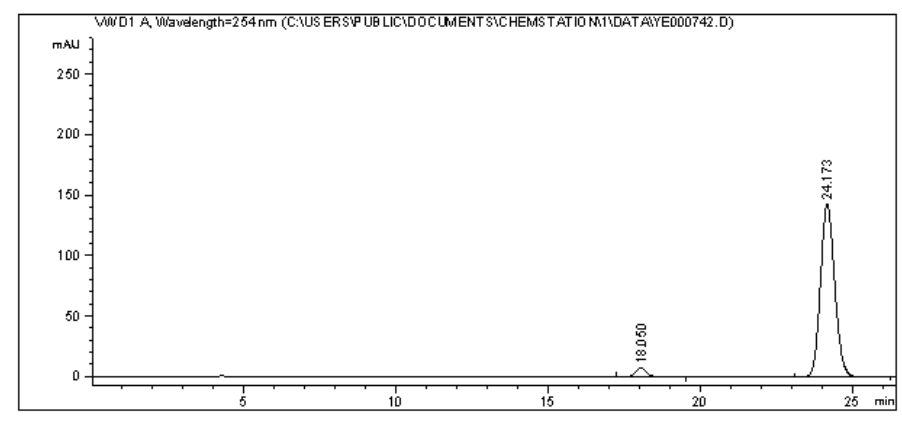

M

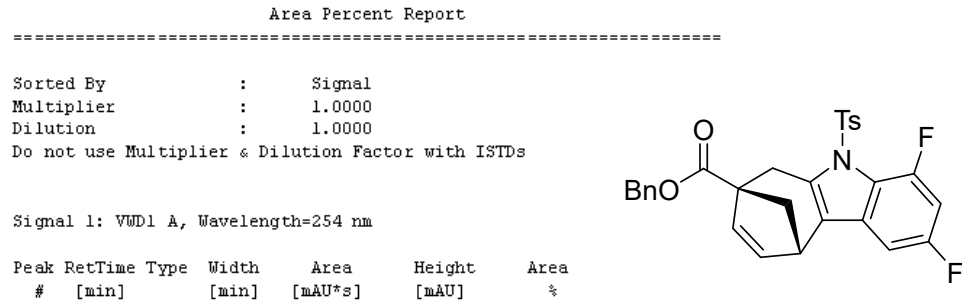

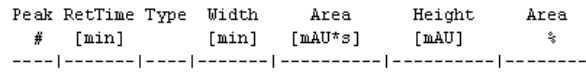

$\begin{array}{rrrrrr}1 & 18.050 \mathrm{BE} & 0.3690 & 172.78246 & 7.22389 & 3.5986 \\ 2 & 24.173 \mathrm{EE} & 0.5021 & 4628.59326 & 142.81302 & 96.4014\end{array}$

$(-)-3 \mathbf{w}$

Totáls:

$4801.37572 \quad 150.03691$

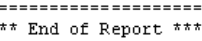

APIC 8/5/2020 10:12:15 PM SYSTEM

Favge 1 of 1 


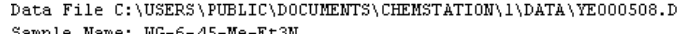
Gample Name: hig-6-45-rie-Etal
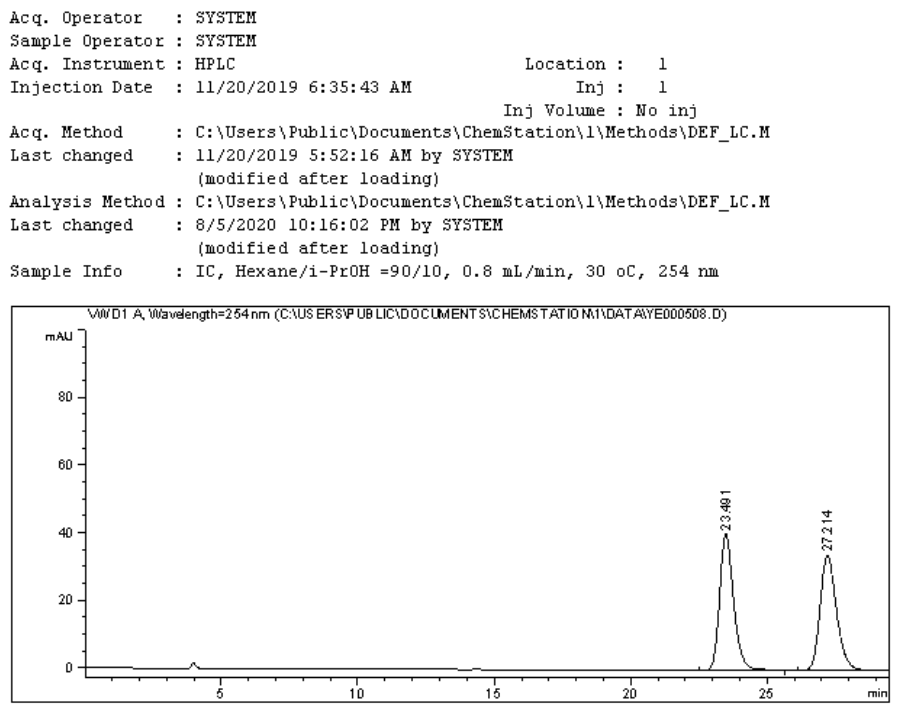

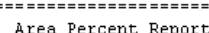
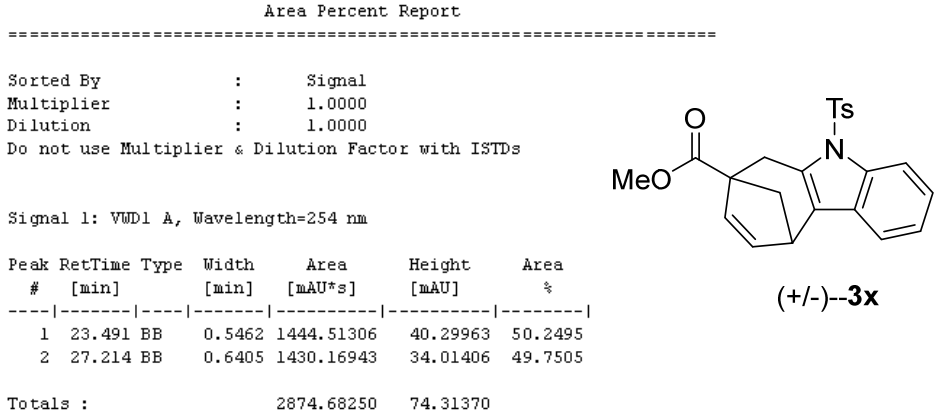

$(+/-)--3 x$

Totals

$2674.66250 \quad 74.31370$

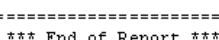

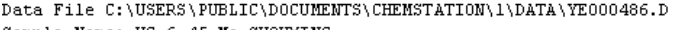
Sample Name: WrG-6-45-Me-SHotxi

\section{Acq. Operator : : इYSTEM
Sample Operator : : FTSTEM}

Sample Operator: : FrSTEM

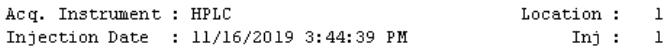

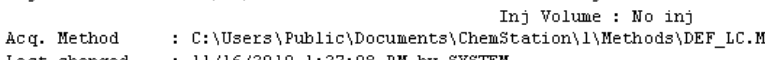

Last changed : 11/16/2019 1:27:06 PII bY SYSTEK

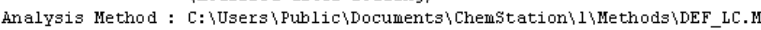

Last changed : 8/5/2020 10:14:50 PII by SYSTEM

Sample Info : Ic, Hexane $/ 1$-ProH $=90 / 10,0.8 \mathrm{~mL} / \mathrm{min}, 30$ oc, $254 \mathrm{~m}$

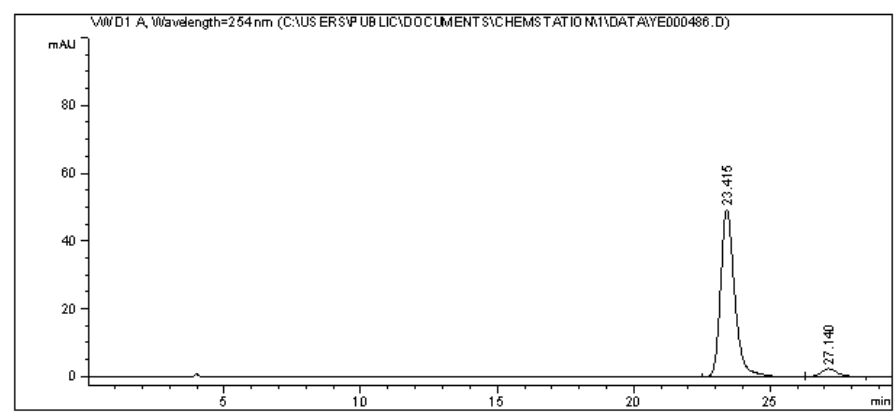

M

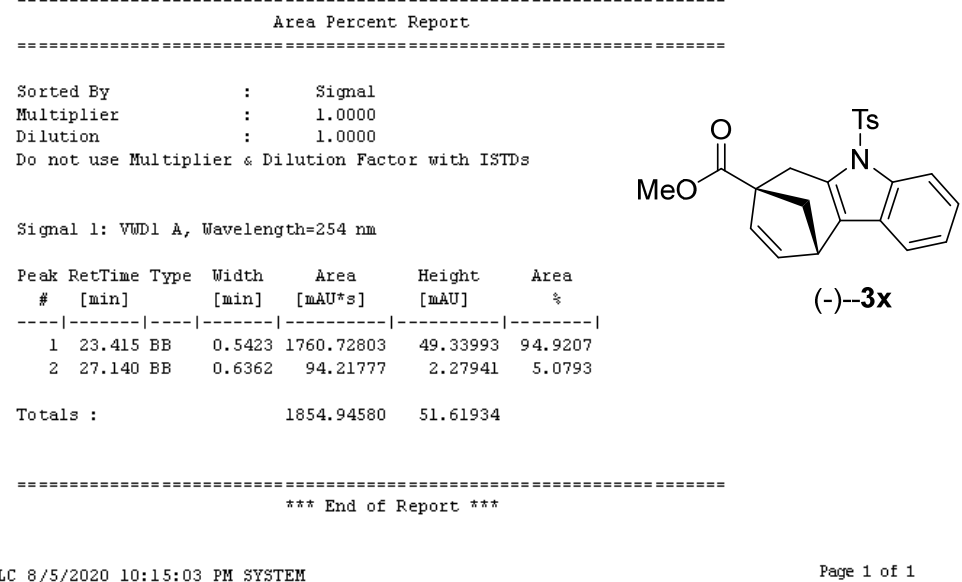




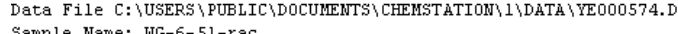
Gample Name: wiG-6-51-rac
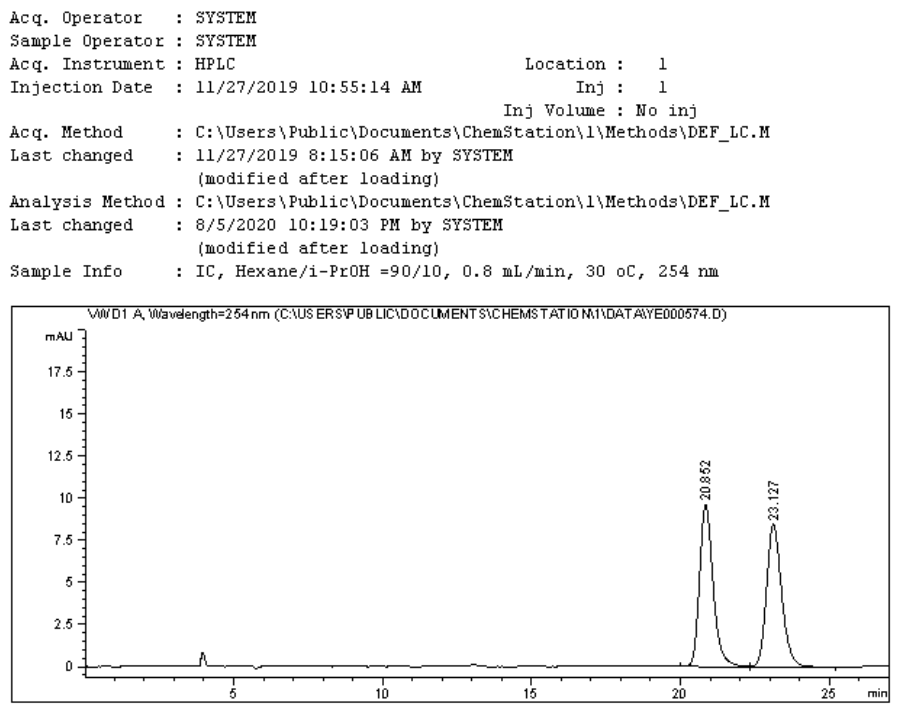

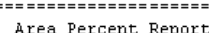

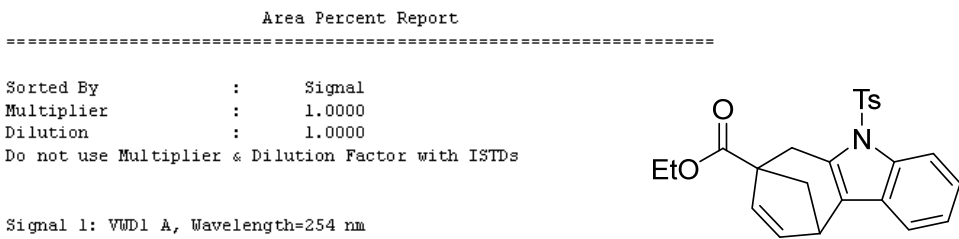

S1 gral 1: Vill 1 A, wavelengthe254 nm

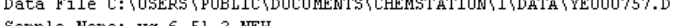
Sample Hame: wg-6-51-2-NE

\section{Acq. Operator : : FYSTEM
Sample Operator : FrsTEM}

Sample operator: : FrsTrR
Aeq. Instrument : HPLC

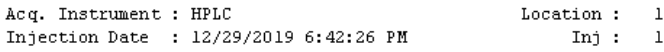

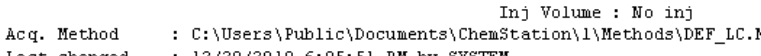
Last changed : : 12/29/2019 b.as:51 PM bY STSTER

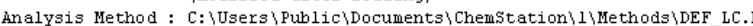

Last changed : 8/5/2020 10:17:52 PII by SYSTEM

Sample Info : IC, Hexane $/ 1-\mathrm{FrOH}=90 / 10,0.8 \mathrm{~mL} / \mathrm{min}, 30$ oc, $254 \mathrm{~m}$

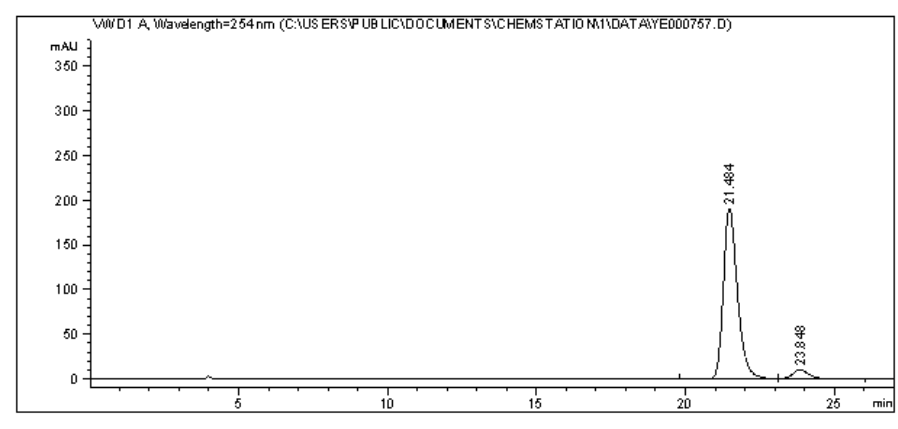

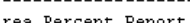

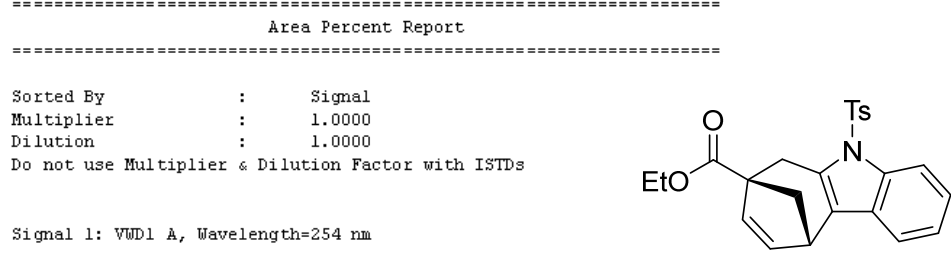

Signal 1. Thid 1 A, Wavelength $=254$

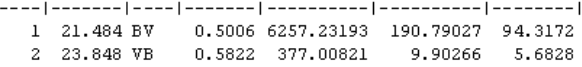

Totals : $\quad 6634.24014 \quad 200.69293$

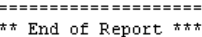




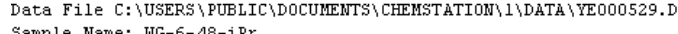
Sample Name: irg-6-40-i
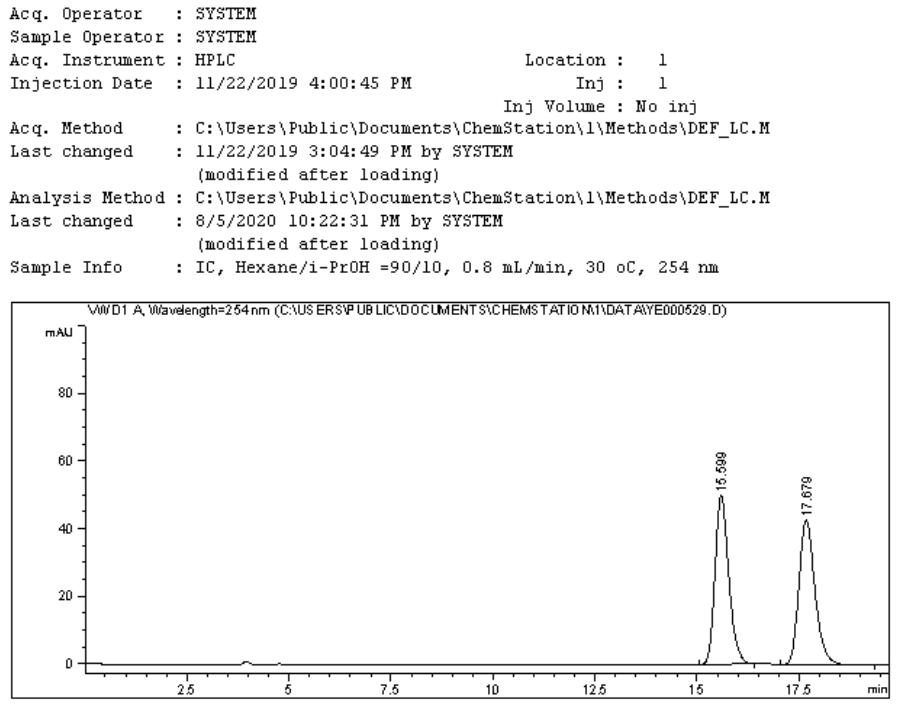
M
in rea Percent Report

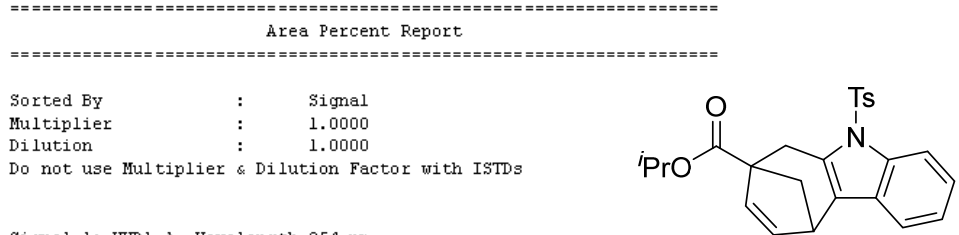

G1 gral 1: Vill 1 A, wavelengthe254

$(+/-)--3 z$

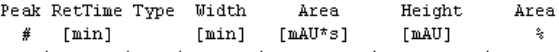

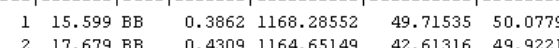

$$
\begin{aligned}
& \text { Tota13 : } \quad 2332.93701 \quad 92.3285
\end{aligned}
$$

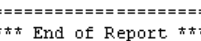

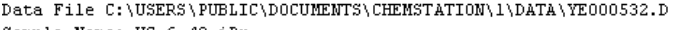
Sample Name: hiG-6-48-1

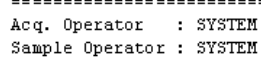

sample Operator : Frsto
Aeq. Instrument : HPLC

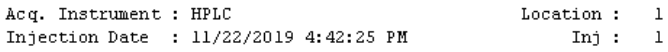

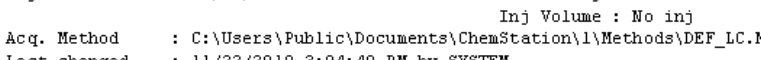
Last changed : 11/22/2019 3:04:49 PII bY SYSTEKI

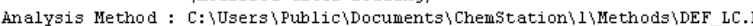

Last changed : 8/5/2020 10:21:10 PII by SYSTEM

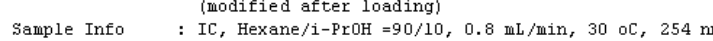

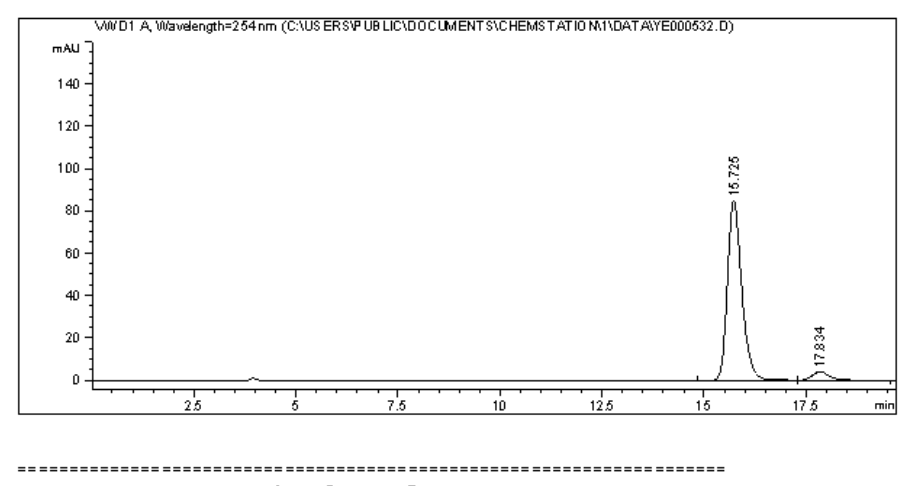

Area

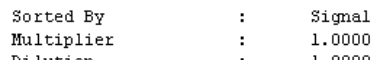

Do not use Multiplier \& Dilution Factor with IstT

Signal 1: VWD1 A, wavelength=254 nm

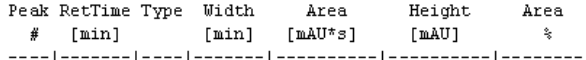

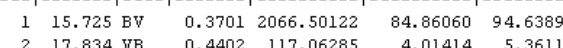

$$
\begin{aligned}
& \text { totals : } \\
& 2183.56407 \quad 88.87475
\end{aligned}
$$

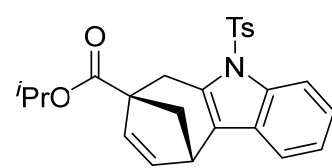

$(-)-3 z$

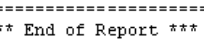




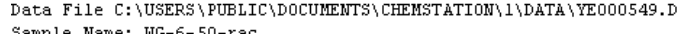
ample Name:

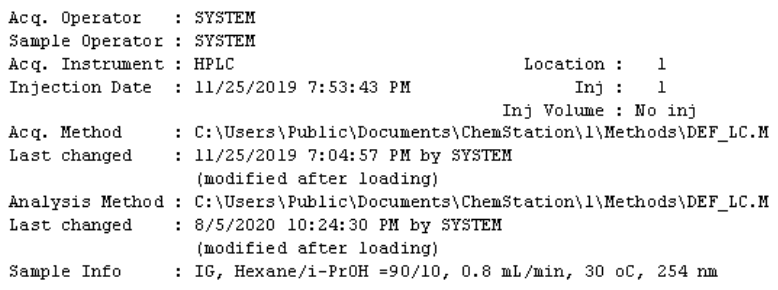

Acr. Wethod : Inj Volume : No inj

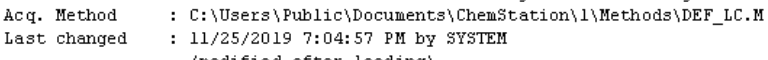

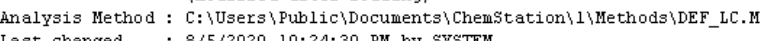
Last changed : 8/5/2020 10:24:30 PII by STSTEM

Sample Info : IG, Hexame $/ 1$ - Prof $=90 / 10,0.6 \mathrm{mI} / \mathrm{min}, 30$ oc, $254 \mathrm{~mm}$

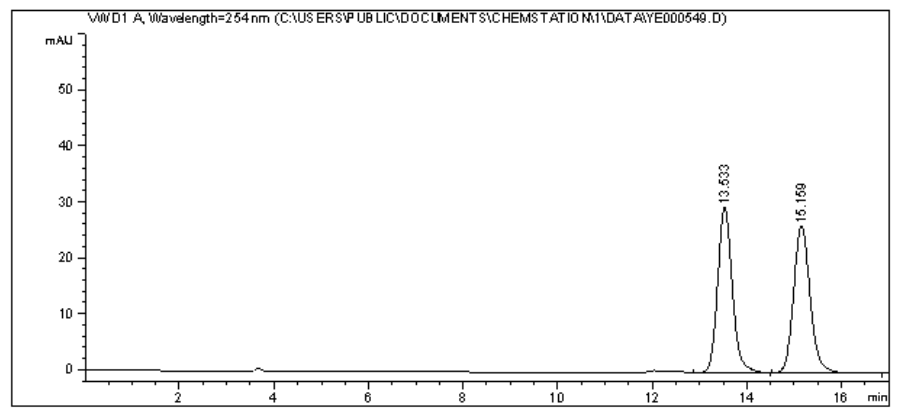

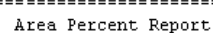

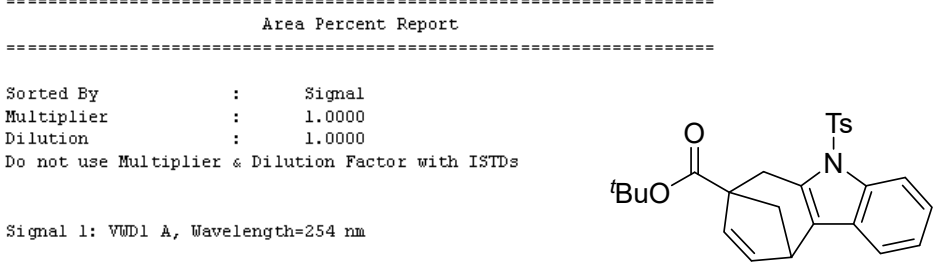

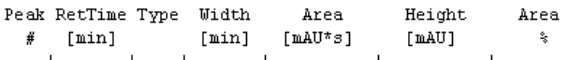

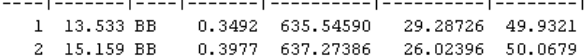

$(+/-)--3 a a$

Totals :

$1272.81976 \quad 55.3112$

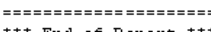

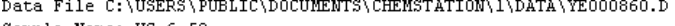
ample Näie: hig-6-50

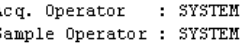

Sample operator : FrsTEM
ACq. Instrument : HPIC

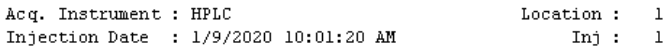

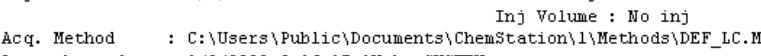

Lašt changed : 1/9/2020 8:16:17 AM bY SYSTEM

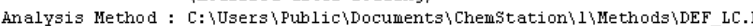

Last changed : 8/5/2020 10:23:37 PII by SYSTEM

Sample Info : (noditied atter 10ading)

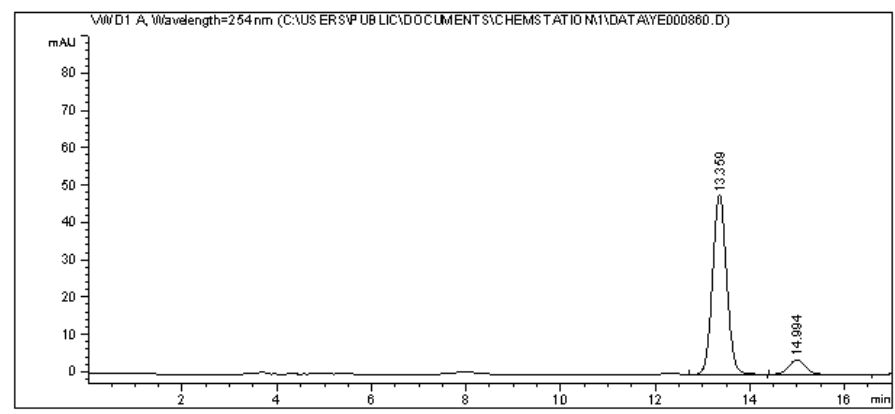

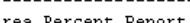

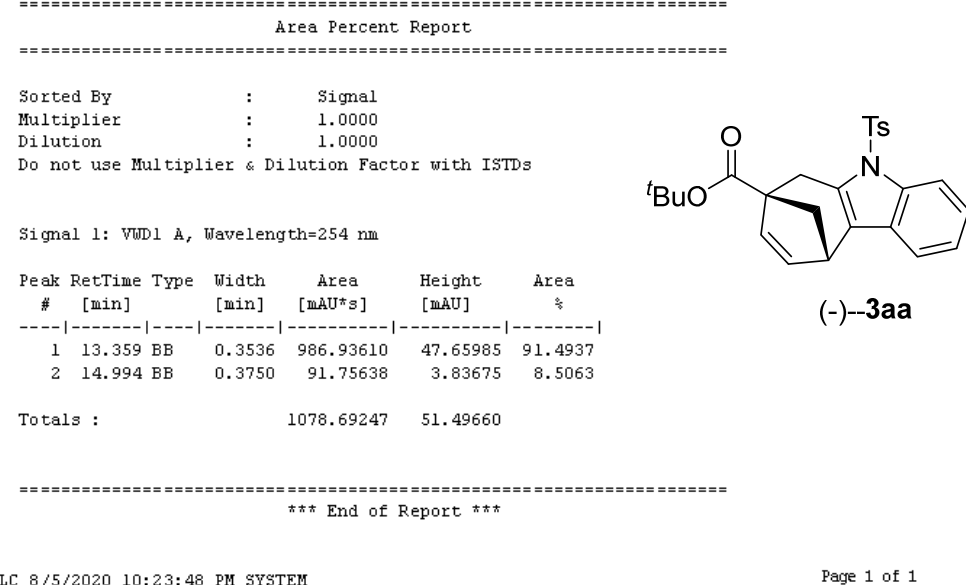




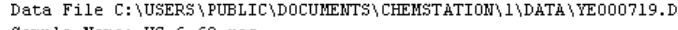
Sample Name: $\mathrm{h}[\mathrm{G}-6-69-\mathrm{rac}$
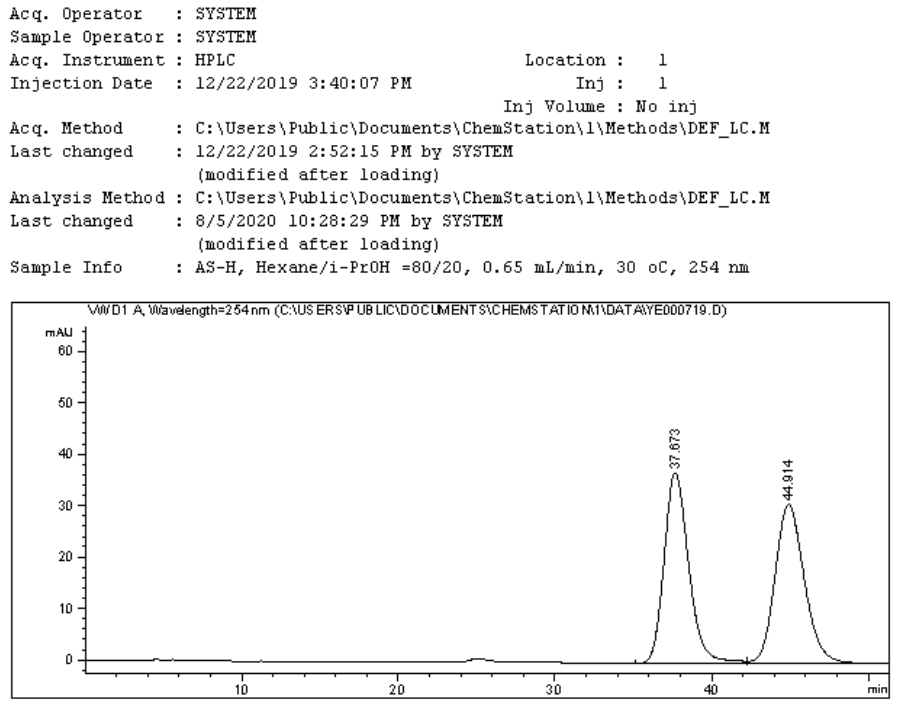

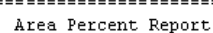

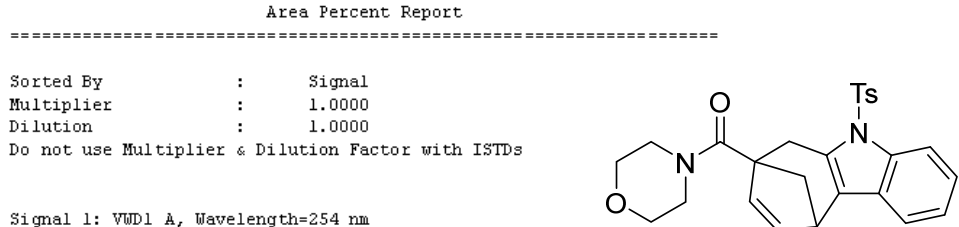

S1 gral 1: Vill 1 A, wavelengthe254 nm

$(+/-)--3 a b$

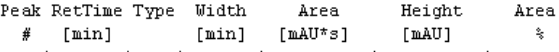

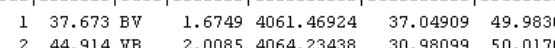

$$
\begin{aligned}
& 8125.70361 \quad 66.0300
\end{aligned}
$$$$
\text { Totals : }
$$

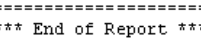

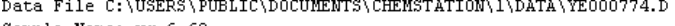
ample Narie: wrg-6-69

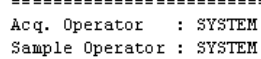

Sample Operator : FrsTEM
Aeq. Instrument : HPLC

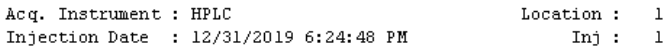

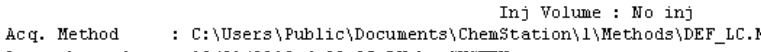

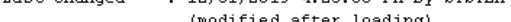

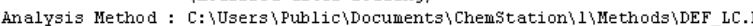

Last changed : 8/5/2020 10:27:23 PII by STSTEM

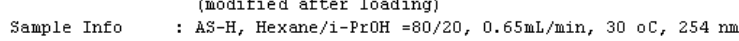

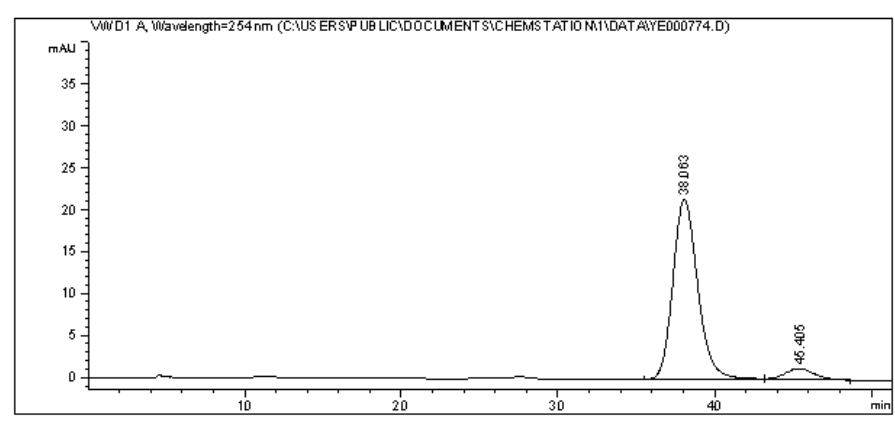

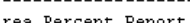

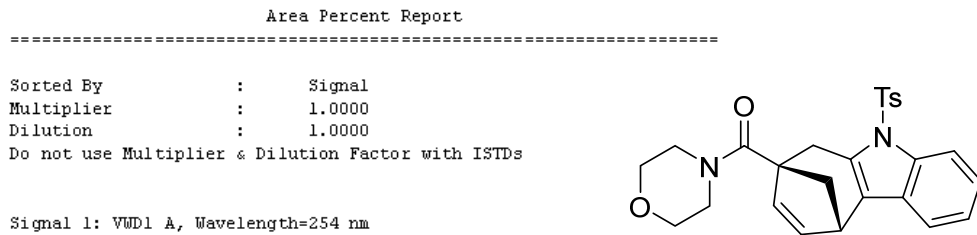

Signal 1: MU⿴囗十 1, Wavelength $=254$ nat

$(-)-3 a b$ 


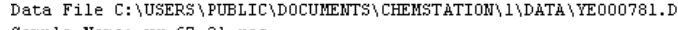
Aample Name: wog-67-21-rac

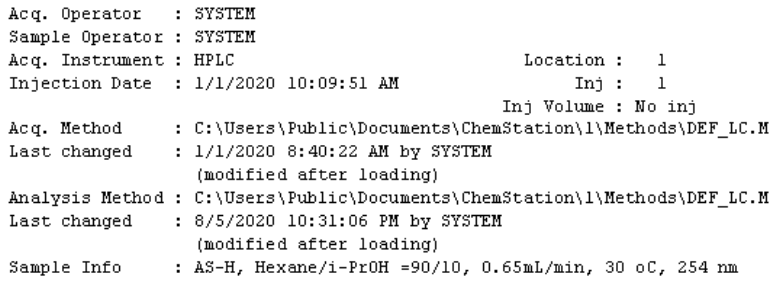

Anj Volume: : No inj

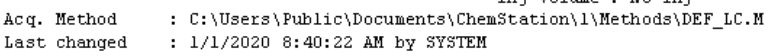
Last changed : $1 / 172020$ s:40:2z All by sisting

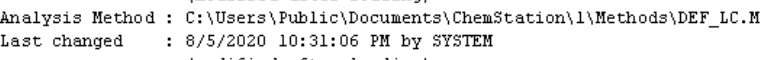

Sample Info : : $\mathrm{As}-\mathrm{H}$, Hexame $/ \mathrm{i}-\mathrm{PrOH}=90 / 10,0.65 \mathrm{~mL} / \mathrm{min}, 30$ oC, $254 \mathrm{~mm}$

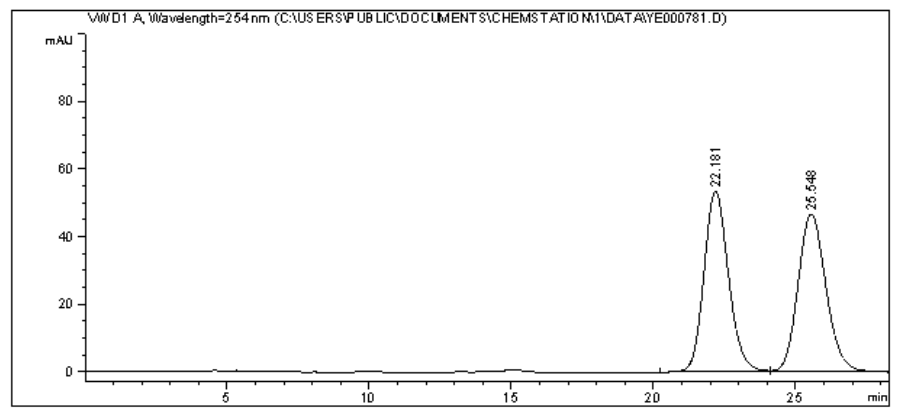

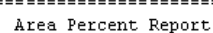

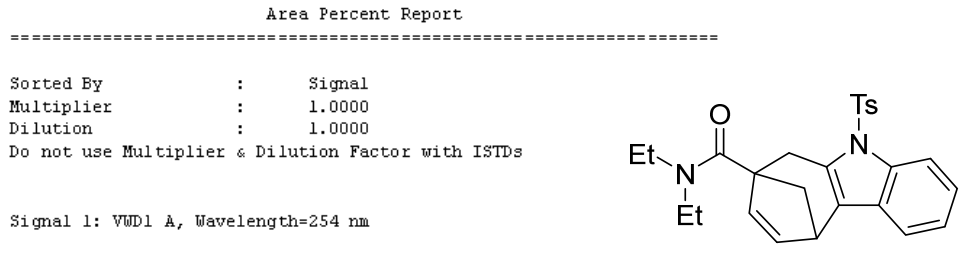

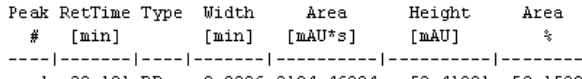

$(+/-)-3 a c$

$\begin{array}{llllll}1 & 22.181 \mathrm{BE} & 0.9226 & 3184.46094 & 53.41021 & 50.1539 \\ 2 & 25.548 \mathrm{~EB} & 1.0305 & 3164.92334 & 46.53031 & 49.8461\end{array}$

Totals : $\quad 6349.30428 \quad 99.94052$

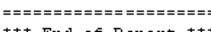

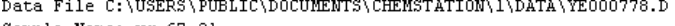
Sample Name: wg-67-21

Acq. Operator : : FYSTEM
Sample Operator : : FrSTEII

sample operator : Frst
Aeq. Instrument : HPIC

$\begin{array}{lll}\text { Lec. Instrument : } & \text { Location : } & 1 \\ \text { Injection Date }: 12 / 31 / 20199: 28: 39 \mathrm{PM} & \text { Inj : } & 1\end{array}$

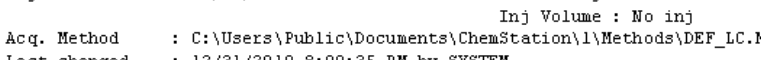

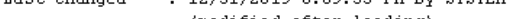

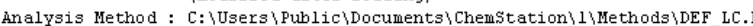

Last changed : 8/5/2020 10:29:58 PII bY STSTEM

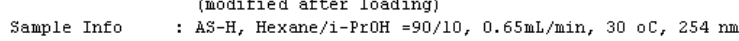

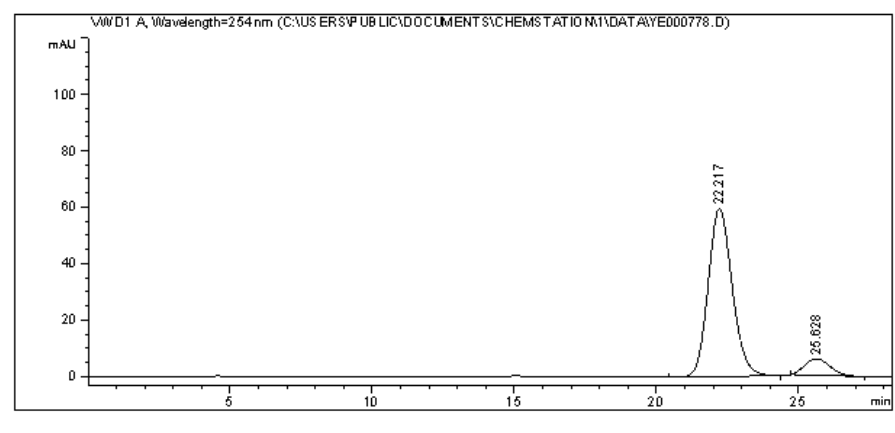

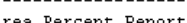

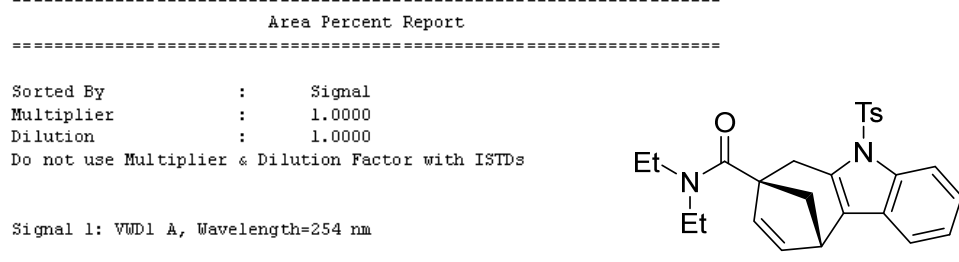

al 1: Whis $A$, Wave

$(-)-3 a c$

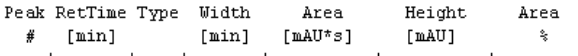

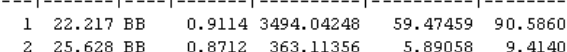

$$
\begin{aligned}
& 3857.15604 \quad 65.36517
\end{aligned}
$$

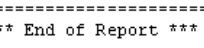

HPIC 8/5/2020 10:30:00 PM SYSTEM 


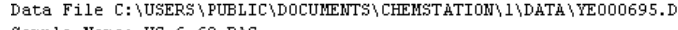
Sample Name:
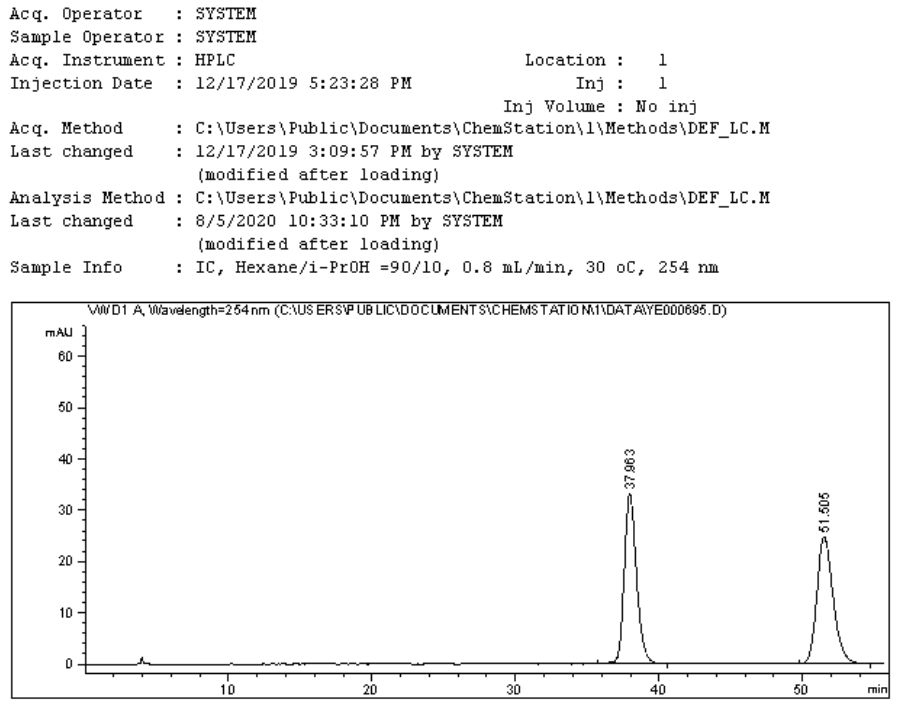

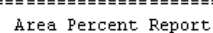
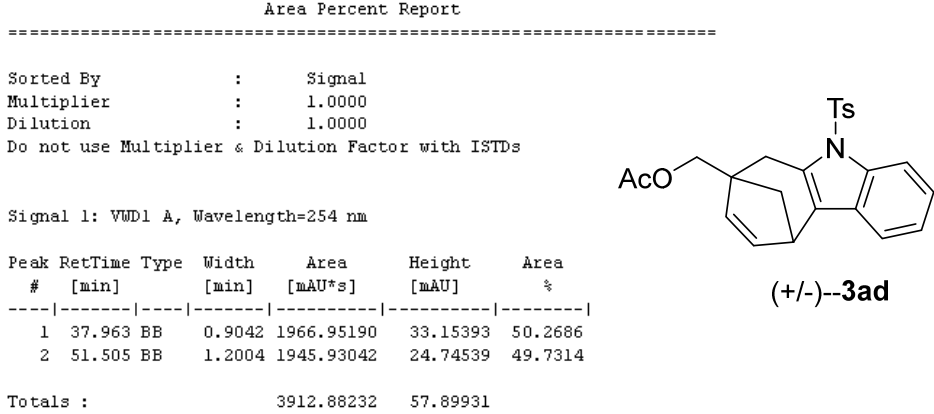

$(+/-)--3 a d$

Totals

$3912.80232 \quad 57.8993$

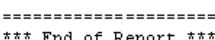

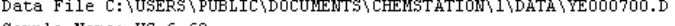
sample Name: ing-6-60

Acq. Operator : : FYSTEMI
Sample Operatox : FrsTEM

Sample operator : Frst
heq. Instruwent : HPIC

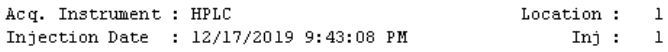

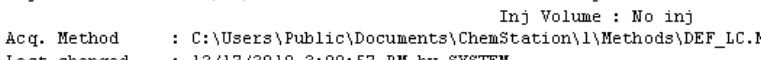
Last changed : : 12/17/2019 3:09:57 PII bY SYSTEM

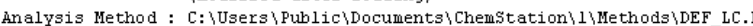

Last changed : 8/5/2020 10:32:30 PII by SYSTEM

Sample Info : In, Hexane $/ 1-\mathrm{Fr} 0 \mathrm{H}=90 / 10,0.8 \mathrm{~mL} / \mathrm{min}, 30 \mathrm{oc}, 254 \mathrm{~mm}$

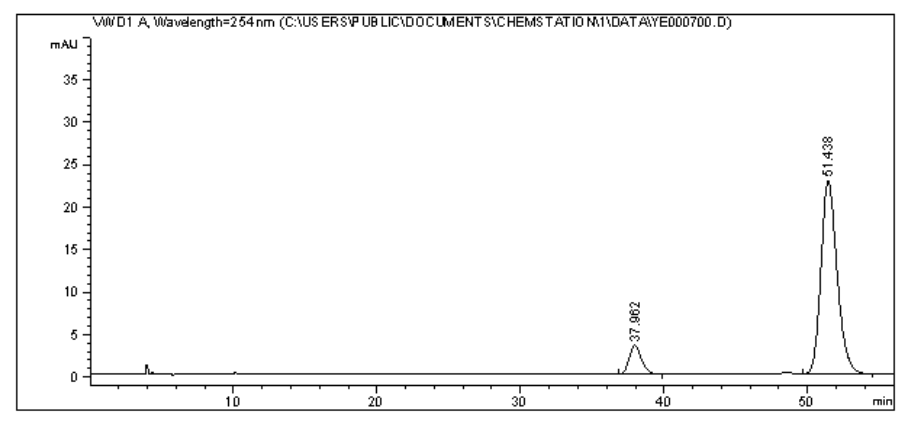

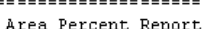

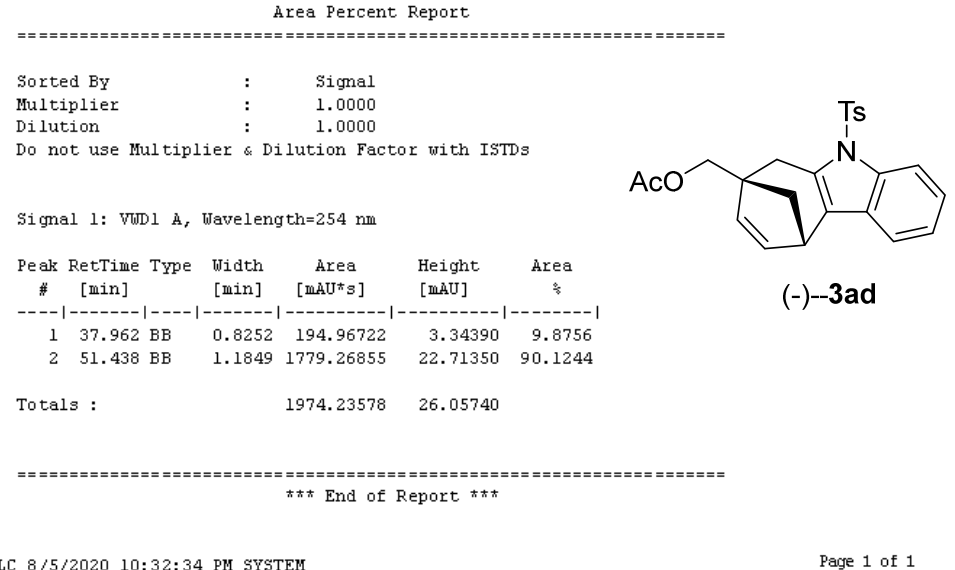




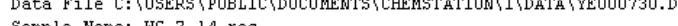
Sample Wame: wiG-7-14-rac
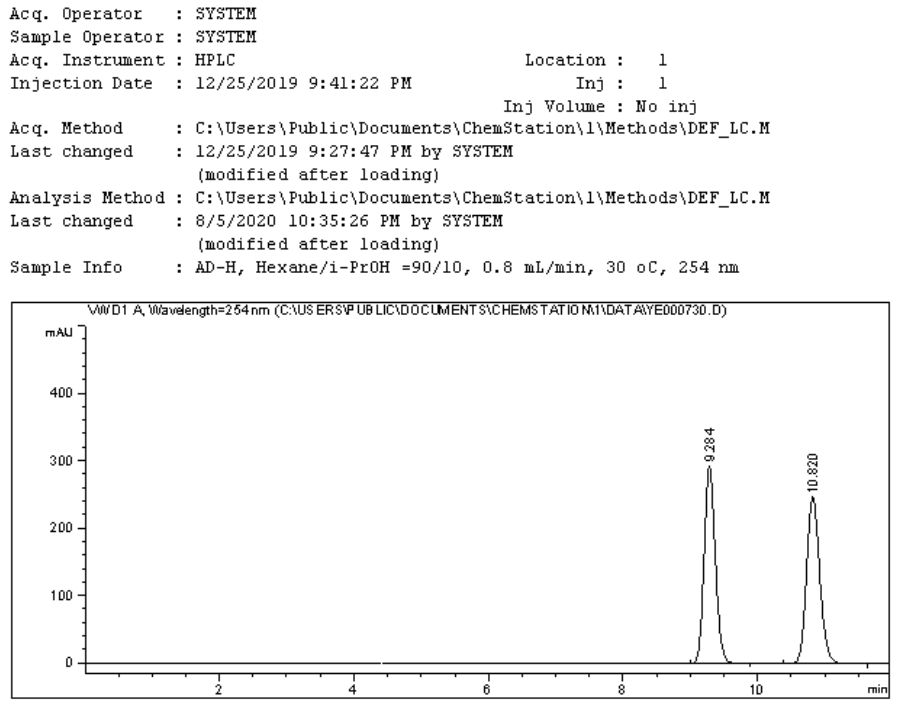

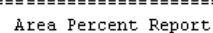

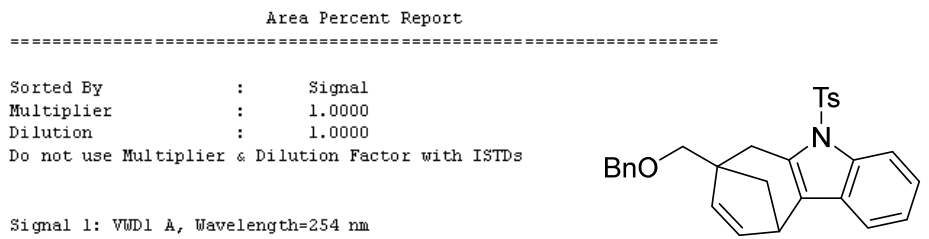

S1 gral 1: Vill 1 A, wavelengthe254 nm

$(+/-)--3 a e$

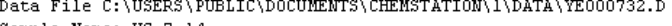
Sample Name: ing-7-14

$$
\begin{aligned}
& \text { Acq. Operator : : SYSTEMI } \\
& \text { Sample Operator : ITSTEMI }
\end{aligned}
$$

Sample Gperator : : TTSTEI

Injection Date : $12 / 25 / 2019$ 10:30:04 pw Location: 1

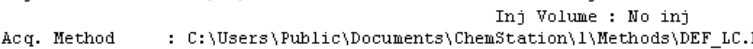
Last changed $\quad: \quad 12 / 25 / 20199: 9: 27: 47$ PM bY STSTEM

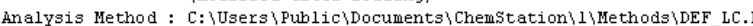

Last changed : 8/5/2020 10:34:48 PII bY STSTEM

Sample Info : : $\mathrm{MD}-\mathrm{H}$, Hexant $/ \mathrm{i}$-Prof $=90 / 10,0.8 \mathrm{~mL} / \mathrm{min}, 30 \mathrm{oC}, 254 \mathrm{~mm}$

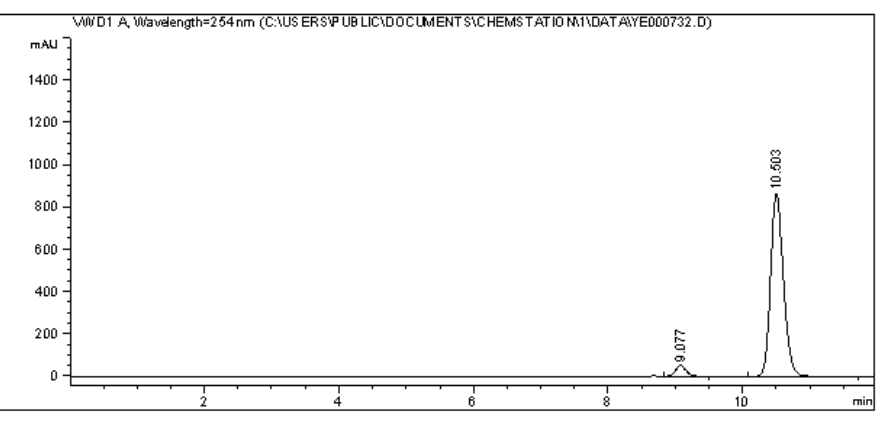

M

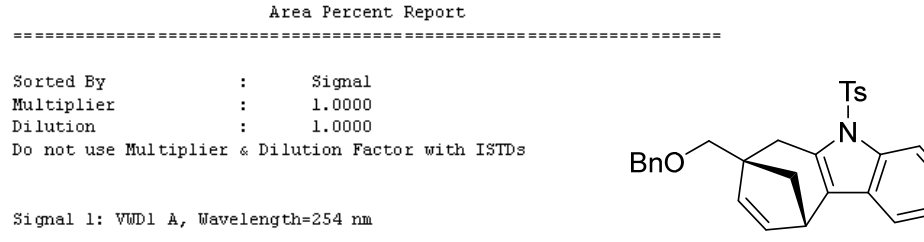

Si gmal 1: Virl 1 A, Tovelengthe254

$(-)-3 a e$

$\begin{array}{llllll}1 & 9.284 \mathrm{BB} & 0.1778 & 3351.50586 & 292.27463 & 49.9194 \\ 2 & 10.820 \mathrm{BB} & 0.2110 & 3562.32471 & 246.73080 & 50.0005\end{array}$

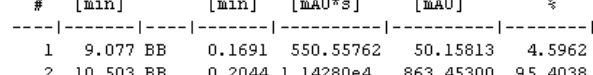

Totals:

$1.1978554 \quad 913.61113$

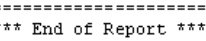

HPIC 8/5/2020 10:34:51 PM SYSTEI

Page 1 of 1 


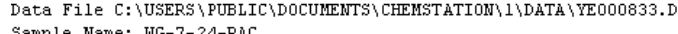
Saluple Name: $\mathrm{WG}-7-24-\mathrm{RhC}$

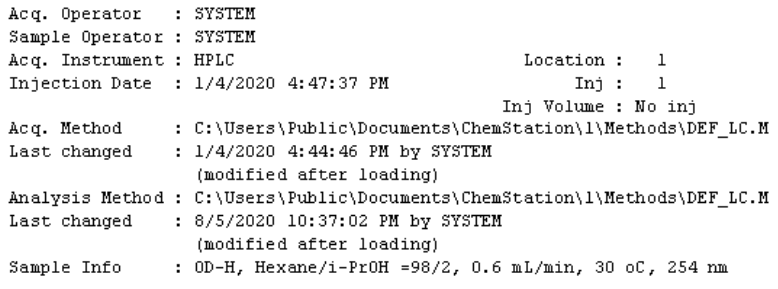

Inj Volume : No inj

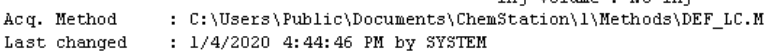
Last changed : $1 / 4 / 20204: 44: 46$ PII by STST

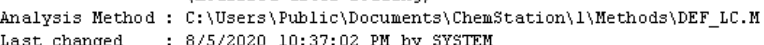
Last changed : 8/5/2020 10:37:02 PII by STSTEM

Sample Info: : OD-H, Hexame/i-ProH $=98 / 2,0.6 \mathrm{~mL} / \mathrm{min}, 30 \mathrm{oC}, 254 \mathrm{~mm}$

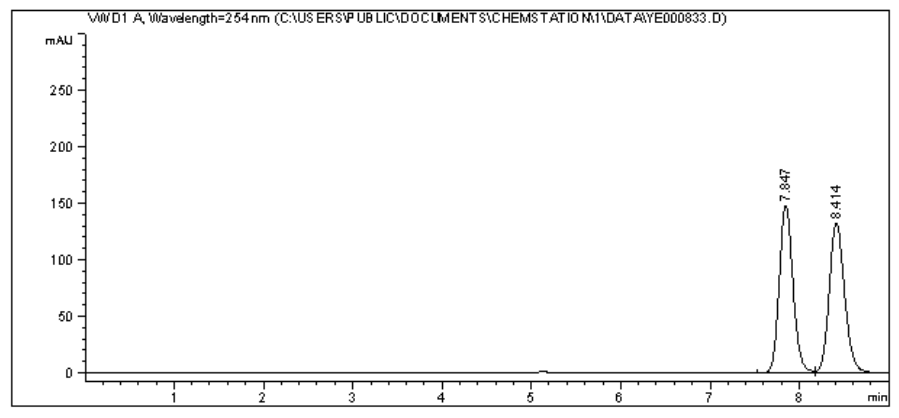

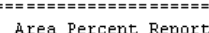

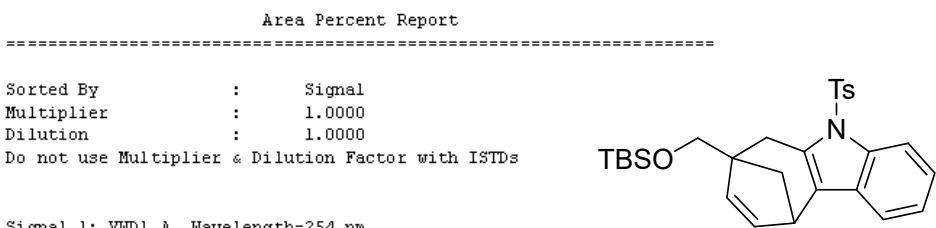

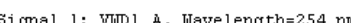

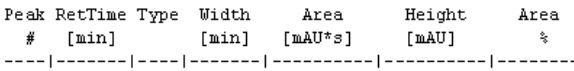

$\begin{array}{lllllll}1 & 7.847 \mathrm{BV} & 0.1644 & 1581.73450 & 148.37338 & 49.8963 \\ 2 & 8.414 \mathrm{WEA} & 0.1849 & 1588.31042 & 132.41850 & 50.1037\end{array}$

Totals : $\quad 3170.04492 \quad 280.79189$

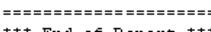

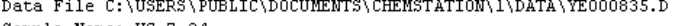
ample Name: wis-7-24

Acq. Operator : : FYSTEM
Sauple Operator : FrTEIII

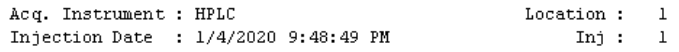

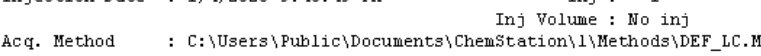

Last changed : 1/4/2020 9:15:16 PII bY SYSTEM

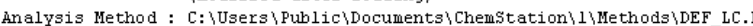

Last changed : 8/5/2020 10:36:23 PII by SYSTEM

Sample Info: : DD-H, Hexame $/ \mathrm{i}-\mathrm{PrOH}=96 / 2,0.6 \mathrm{~mL} / \mathrm{min}, 30$ oc, $254 \mathrm{~mm}$

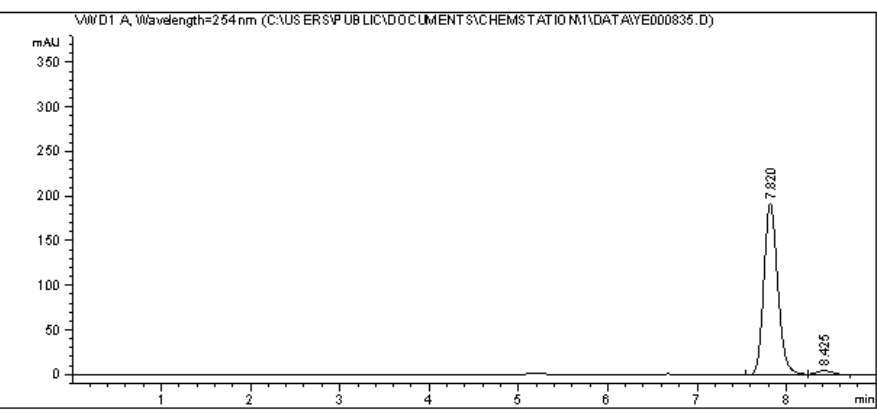

area Percent Report

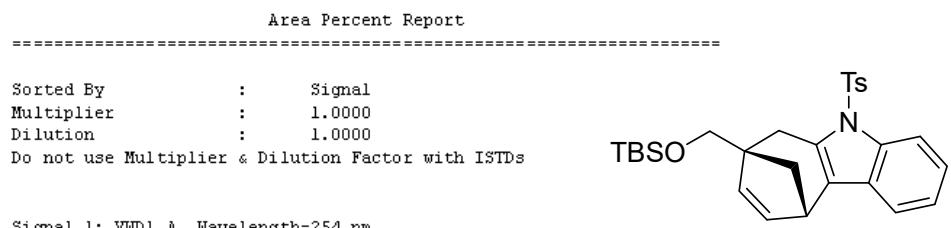

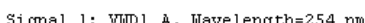

$(-)--3 a f$

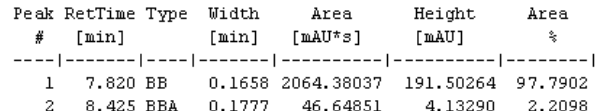

Totals

$2111.02898 \quad 195.63554$

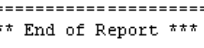

HPIC 8/5/2020 10:36:26 PM SYSTEI 


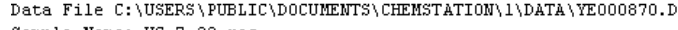
Sample Name: irg-7-23-rac

\section{heq. Operator : STSTEM \\ Sample Operator: : รTsT \\ Aeq. Instrument : HPIC
Tnjection Dare : 1/12/2020 4:15:40 FII}

Inj Volume $:$ No inj
Act. W

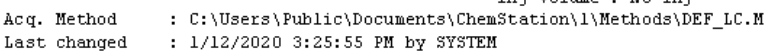

wast changed : 1/12/2020 j:25:5s Hin by SYSTEM

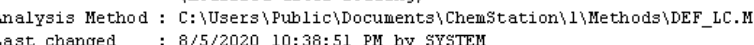

: 8/5/2020 10:38:51 FII bY STSTEM

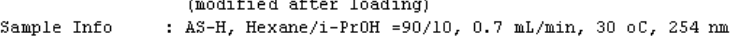

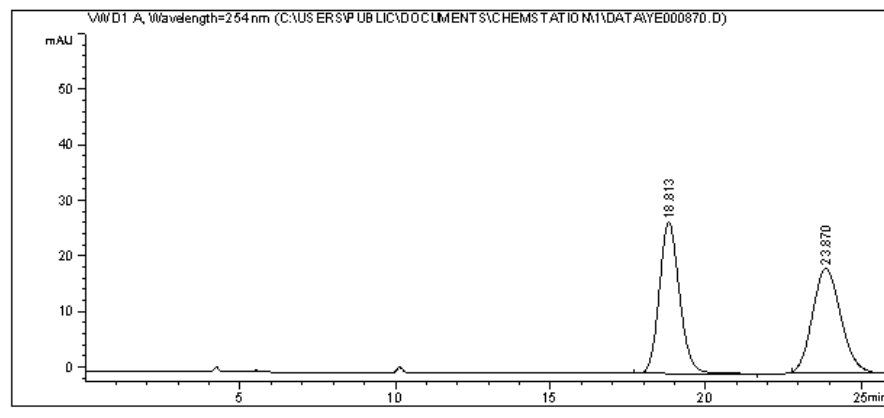

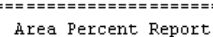

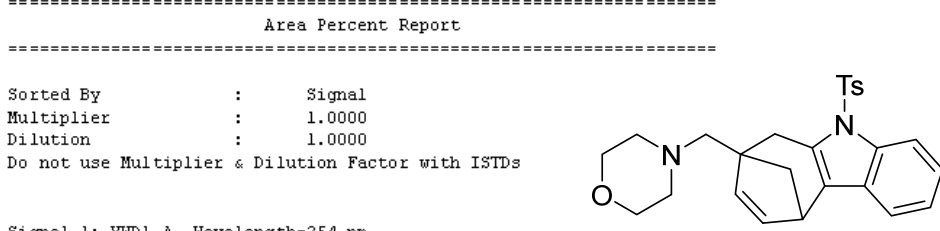

G1 gral 1: Vill 1 A, Wavelengthe254

$(+/-)--3 a g$

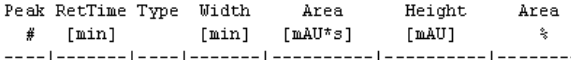

$$
\begin{aligned}
& \begin{array}{lllllll}
1 & 18.813 \mathrm{BV} R & 0.6973 & 1230.07715 & 27.21554 & 50.8654 \\
2 & 23.8700 & \text { FEA } & 0.9260 & 1188.22144 & 18.75798 & 49.1345
\end{array}
\end{aligned}
$$

Totals :

$2418.29658 \quad 45.9735$

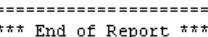

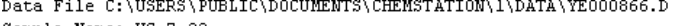
Sample Name: this-7-z3-

$$
\begin{array}{ll}
\text { Acq. Operator } & : \text { SYSTEM } \\
\text { Sauple operator : } & \text { FTSTEII }
\end{array}
$$

Sample dperator : รพรTEI

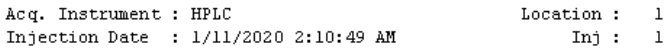

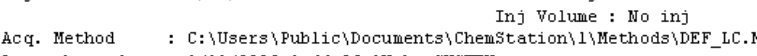

Last changed : 1/11/2020 1:44:26 AII bY SYSTE

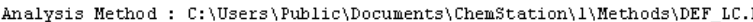

Last changed : 8/5/2020 10:38:01 PII by SYSTEM

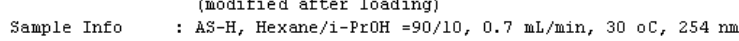

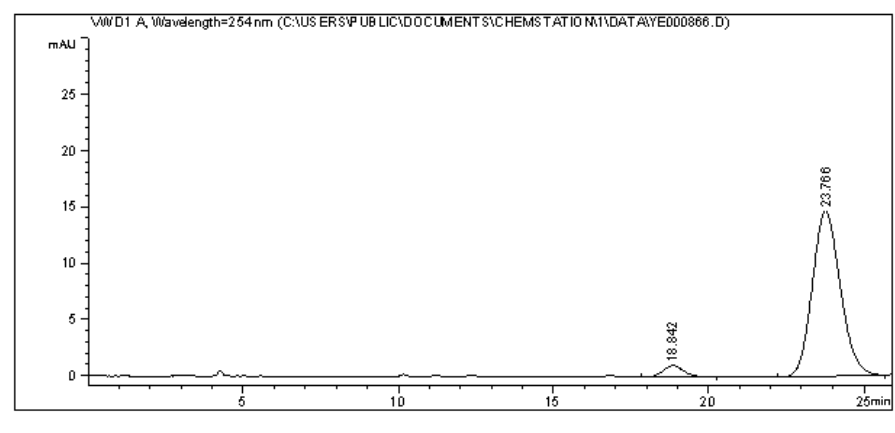

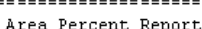

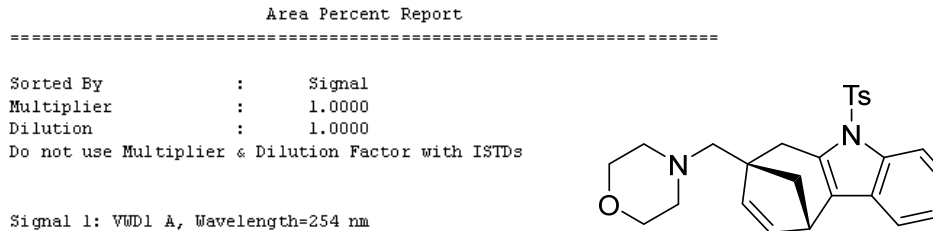

Signal 1: MU⿴囗十 1, Wavelength $=254$ nm

$963.32779 \quad 15.60991$

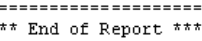




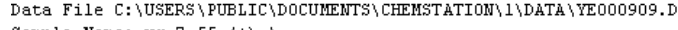
sample wäme: $\operatorname{mog}-7-55-(+)-1$

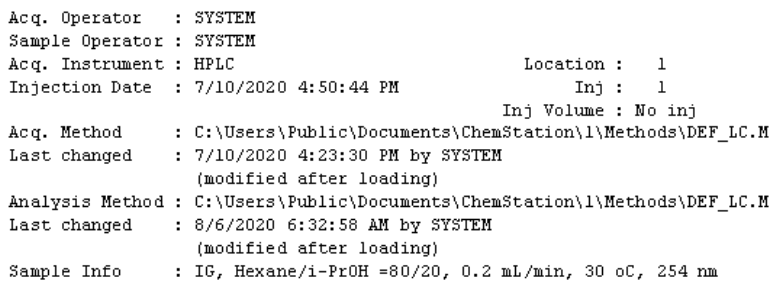

Inj Volume : No inj

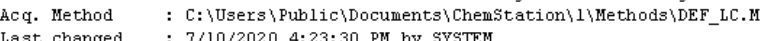
Last changed : : 7/10/2020 4:23:30 phi by shon

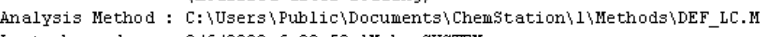
Last changed : 8/6/2020 6:32:58 AM bY sYTTEM

Sample Info : IG, Hexane/1-Prof =80/20, $0.2 \mathrm{~mL} / \mathrm{min}, 30$ oc, $254 \mathrm{~mm}$

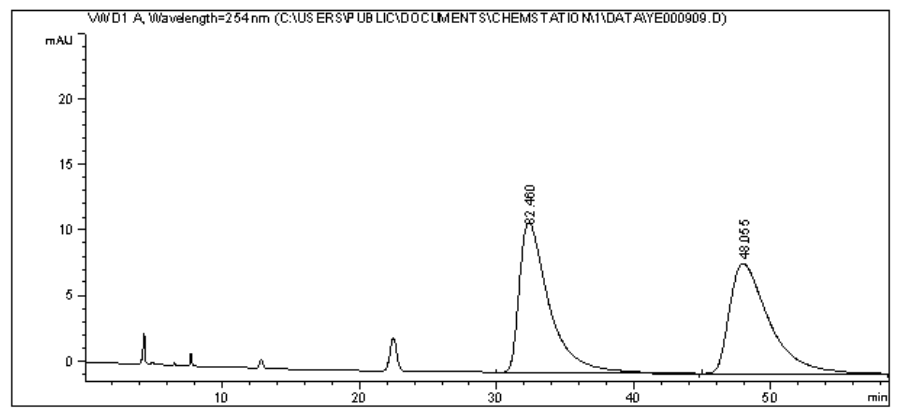

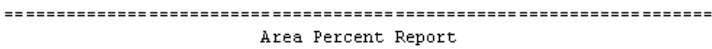

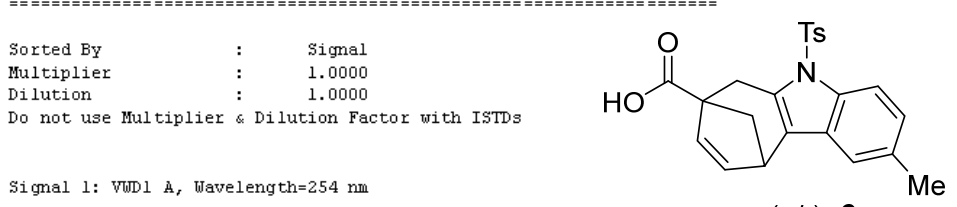

Si gral 1: Vurl 1 A, Wavelengthe254

$(+/-)-6$

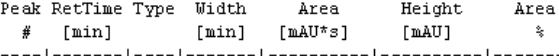

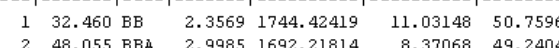

$$
\begin{aligned}
& \text { Totals : } \\
& 3436.64233 \quad 19.4021
\end{aligned}
$$

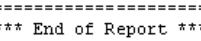

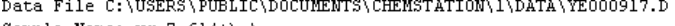
Sample Wame: wrg-7-61(+i-1)

\section{Acq. Operator : : FYSTEM
Sample Operator : FrsTEM}

Sample Operator: FrsTEM

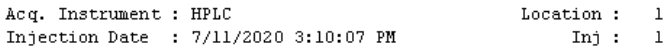

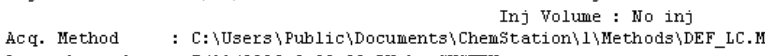

Last changed : $7 / 11 / 2020$ 2:28:22 FII bY STSTH

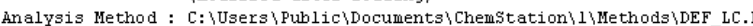

Last changed : 8/6/2020 6:34:06 AM by srstem

Sample Info : (nodified after loading)
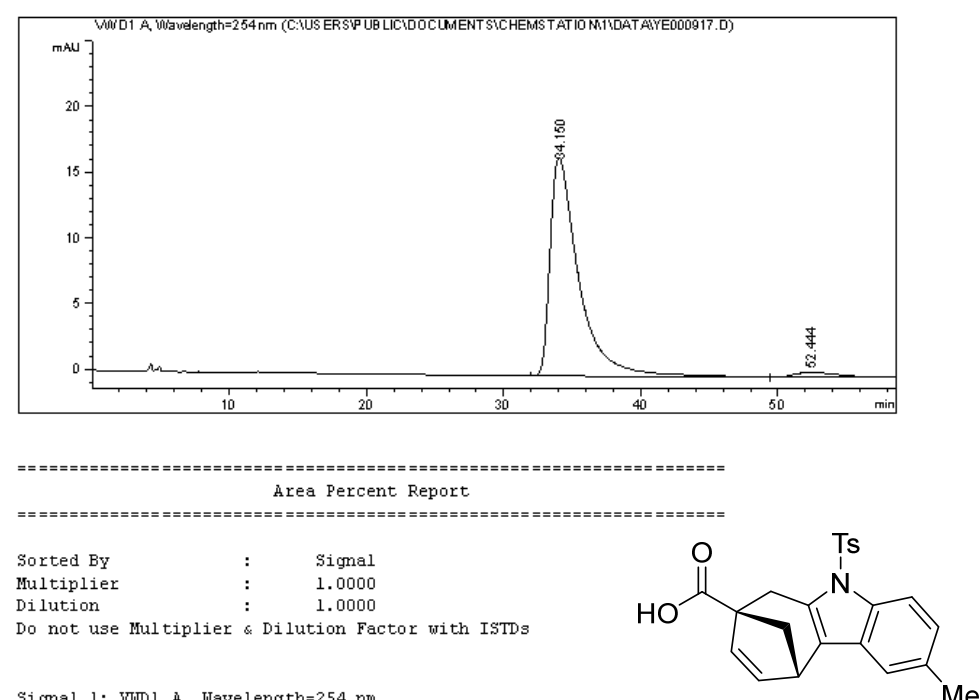

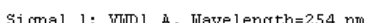

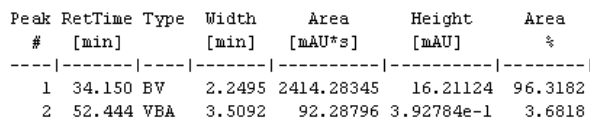

$(-)-6$

Totals : $\quad 2506.57140 \quad 16.60402$

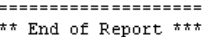

HPLC 8/6/2020 6:34:08 AMI SYSTEM

Page 1 of 1 


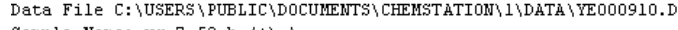
Jample Name: wgig-7-53-b- $(+)-1$

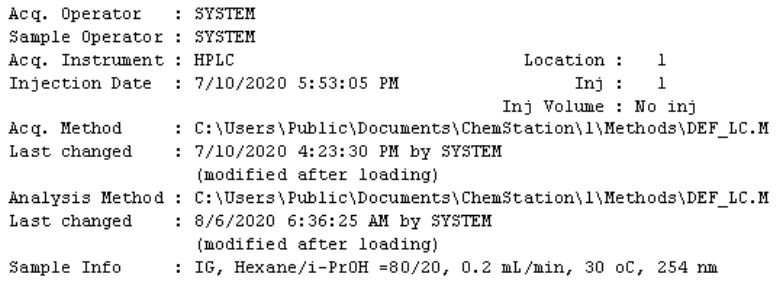

Inj Volume : : No inj
Anct.

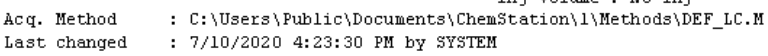

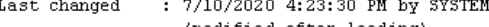

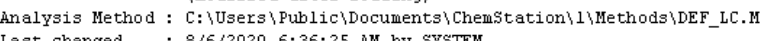
Last changed : 8/6/2020 6:36:25 AM bY sYTTEM

Sample Info : IG, Hexane $/ 1-\mathrm{PrOH}=60 / 20,0.2 \mathrm{~mL} / \mathrm{min}, 30$ oc, $254 \mathrm{~mm}$

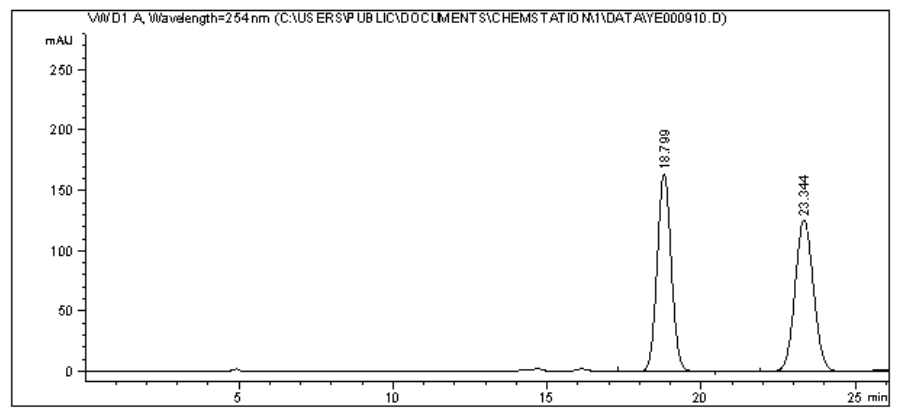

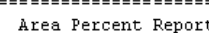

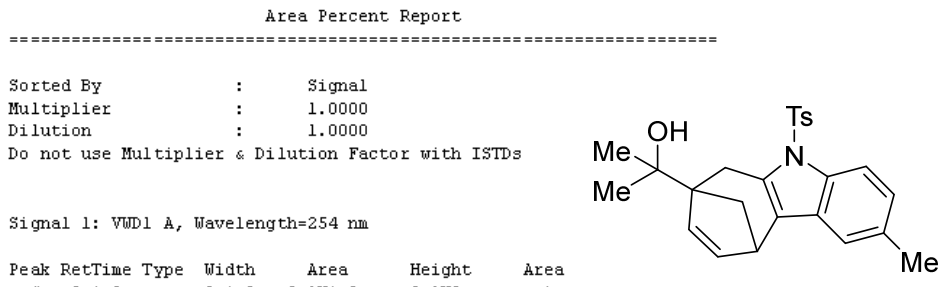

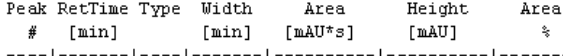

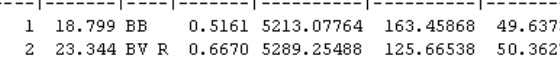

Totals :

$1.0502354 \quad 269.1240$

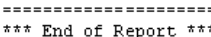

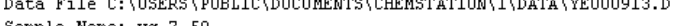
sample Name: wrg-7-5

\section{Acq. Operator : : FYSTEM
Sauple Operator : : STSTEM}

Sample operator: : FrsTEM
ACq. Instrument : HPLC

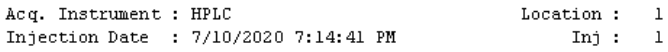

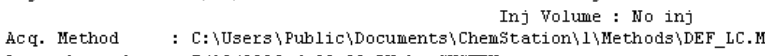

Lasest changed : $7 / 10 / 20204: 23: 30$ FII bY SYSTE

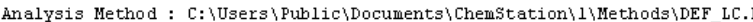

Last changed : 8/6/2020 6:35:17 AM by srstem

Sample Info : IG, Hexane $/ 1-\mathrm{FrOH}=00 / 20,0.7 \mathrm{~mL} / \mathrm{min}, 30$ oc, $254 \mathrm{~m}$

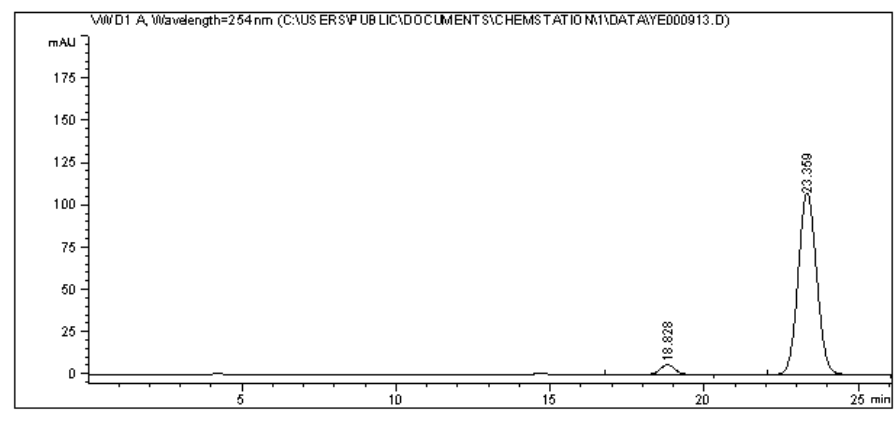

4rea Percent. Report.

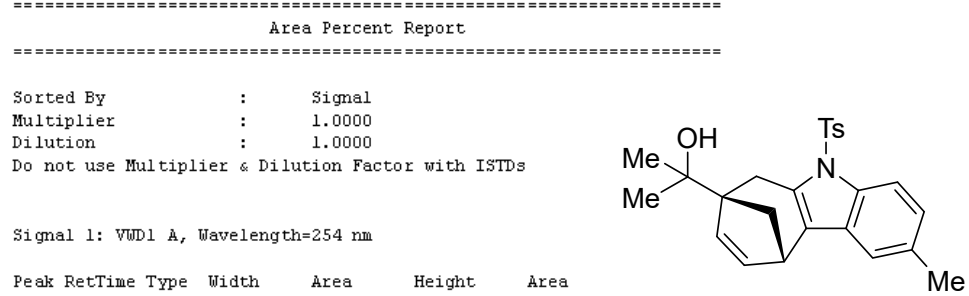

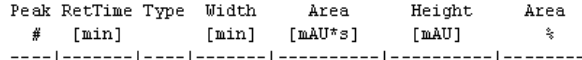

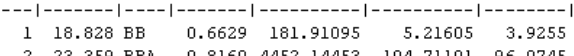

Totals:

$4634.05548 \quad 109.92705$

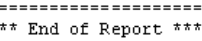

HPIC 8/6/2020 6:35:26 AMII SYSTE

Page 1 of 1 


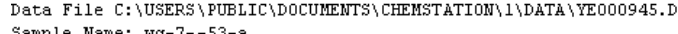
ample Wame: wog-7--53-a
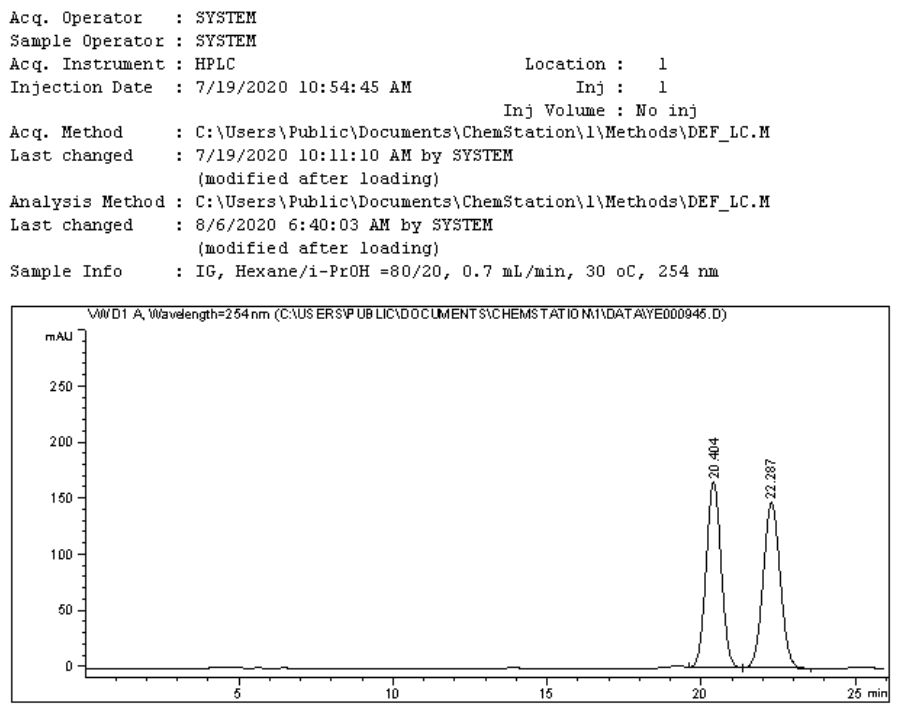

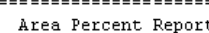
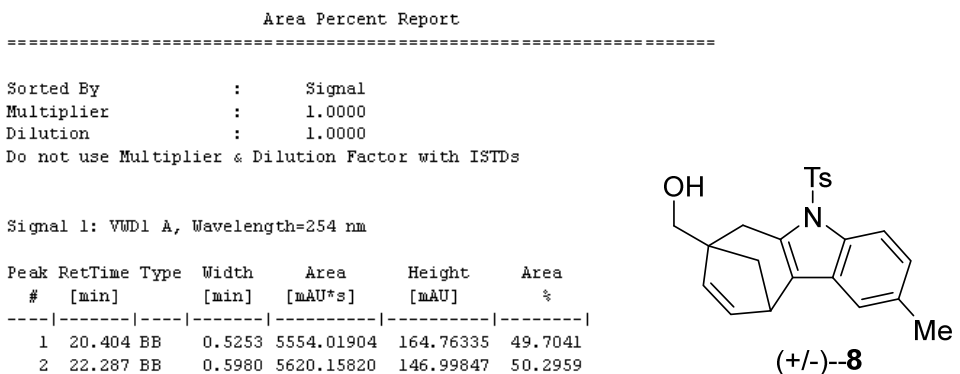

Tatâls

$1.11742 € 4 \quad 311.7618$

$(+/-)-8$

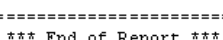

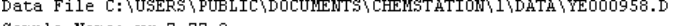
Sample Name: wog-7-77-2

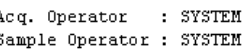

Sample operator : FrsTEM
Aeq. Instrument : HPIC

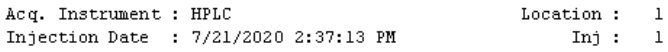

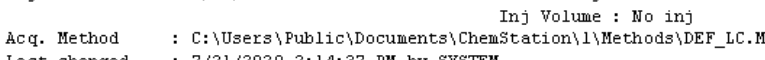

Last changed : 7/21/2020 $2: 14: 37$ FII bY SYSTE

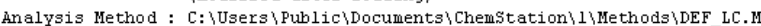

Last changed : 8/6/2020 6:38:27 MM by srstem

Sample Info : (nodified after loading)

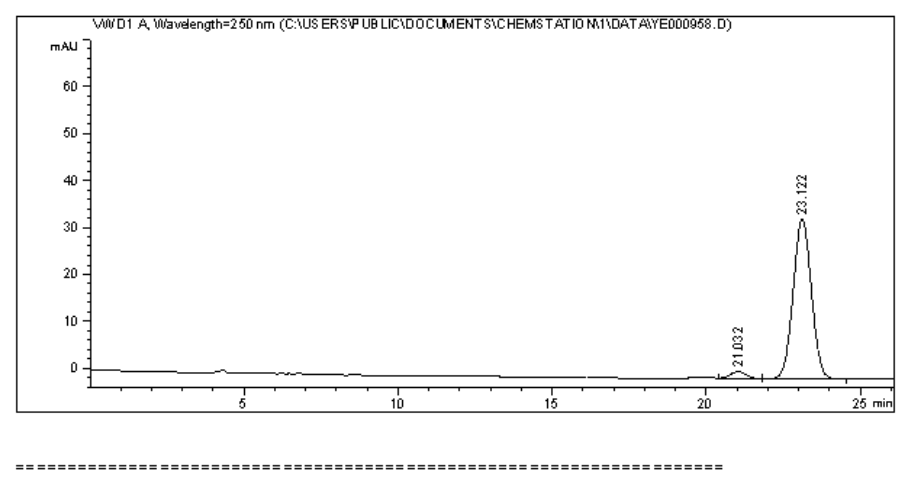

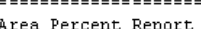

$\begin{array}{lll}\text { Sorted } \mathrm{EY} & : & \text { Sigmal } \\ \text { Multiplier } & : & 1.0000 \\ & 10000\end{array}$

Do not use Multiplier \& Dilution Factor with IstD

Signal 1: VWD1 A, wavelength=250 mu

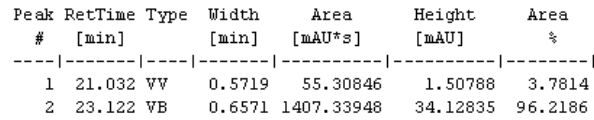

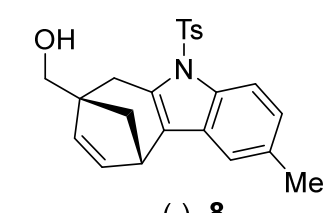

Totals

$1462.64794 \quad 35.63622$

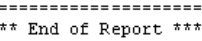

HPLC 8/6/2020 6:38:32 AMII SYSTEM

Page 1 of 1 


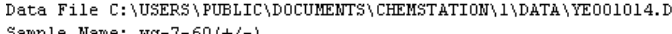
sample Name: wog $-7-60(+/-)$

Acq. Operator : : FYSTEM
Sample Operator : FTSTEM

Injection Date : 8/9/2020 4:11:04 PII

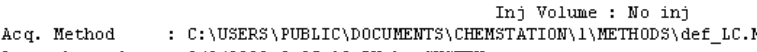
Last changed : 8/9/2020 3:05:10 PH bY SYST

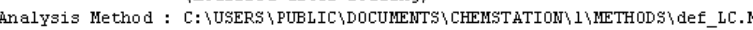
Last changed : 8/9/2020 4:52:50 PII by sYSTTM

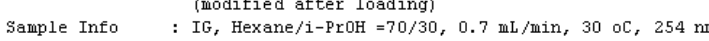

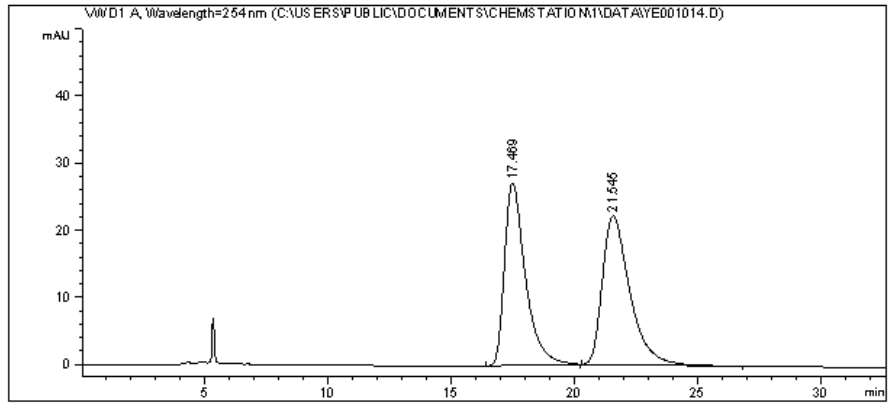

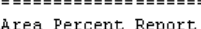

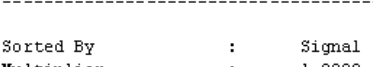

$\begin{array}{lll}\text { Multiplier } & \vdots & 1.0000 \\ \text { Dilution } & \vdots & 1.0000\end{array}$

Do not use Mul tiplier \& Dilution Factor with ISTT

Signal 1: WWI $\mathrm{A}$, wavelengthe=254 nm

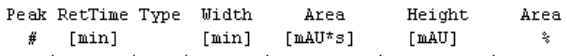

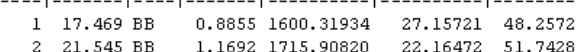

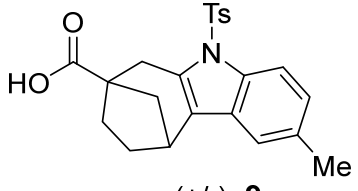

Totals:

$3316.22754 \quad 49.32193$

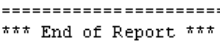

HPIC 8/9/2020 4:52:57 PII SYSTEM

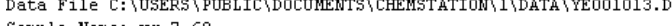
Gample Nâie: wịg-7-60

Acq. Operator : FTSTEM
Sauple operator : FrTEII

Acq. Instrument : HPLC
Tnjection Date : 8/9/2020 3:36:02 PH

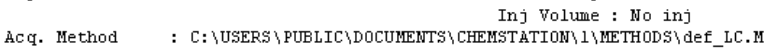
Lašt changed : 8/9/2020 3:05:10 PII bY 5YSTEM

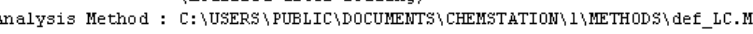
Last changed : 8/9/2020 4:49:46 PII bY 5YSTEM

Sample Info : IGo, Hexane $/ 1$ - $-\mathrm{PrOH}=70 / 30,0.7 \mathrm{nI} / \mathrm{min}, 30$ oc, $254 \mathrm{~mm}$
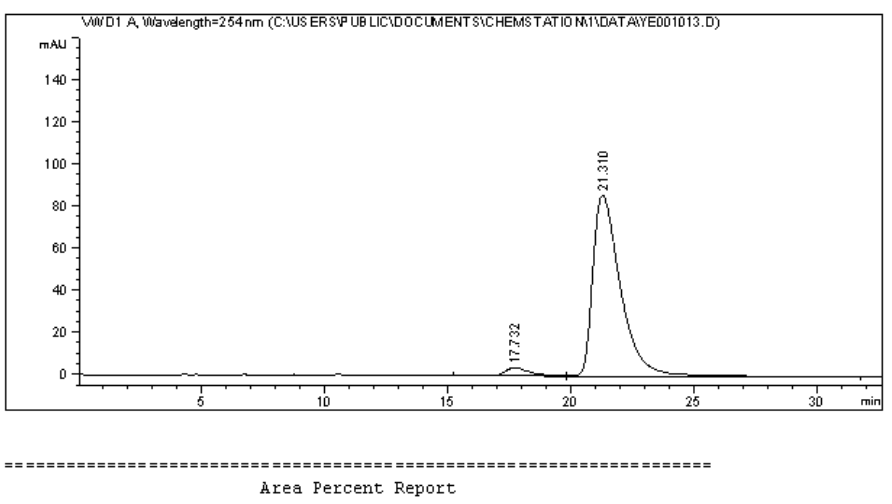

Sorted BY : Sigmal

$\begin{array}{lll}\text { Multiplier } & \vdots & 1.0000 \\ \text { Dilution } & : & 1.0000\end{array}$

Do not use Multiplier \& Dilution Factor with ISTD

Signal 1: val1 $\mathrm{A}$, wavelengthe=254 na

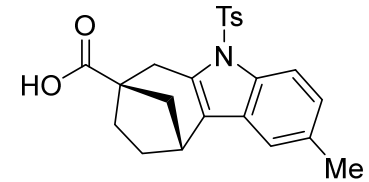

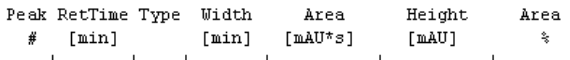

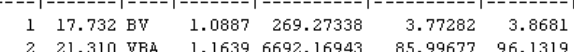

Totals : $\quad 6961.44281 \quad 89.76960$

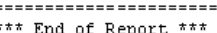

APIC 8/9/2020 4:49:50 PII SYSTEM 


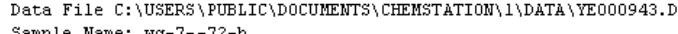
5ample Naim: wig-7--72-b

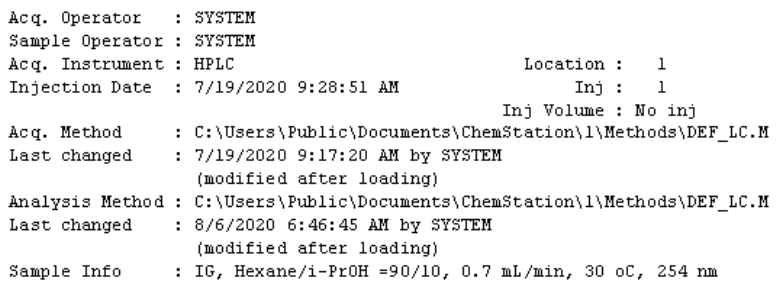

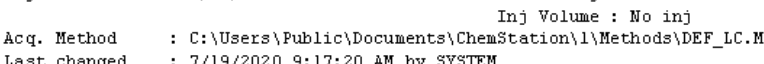

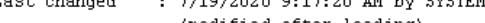

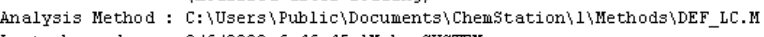
Last changed : 8/6/2020 6:46:45 AM bY sYTTEM

Sample Info: : IG, Hexane $/ 1$ - FrOH $=90,10,0.7 \mathrm{~mL} / \mathrm{min}, 30$ oC, $254 \mathrm{~mm}$

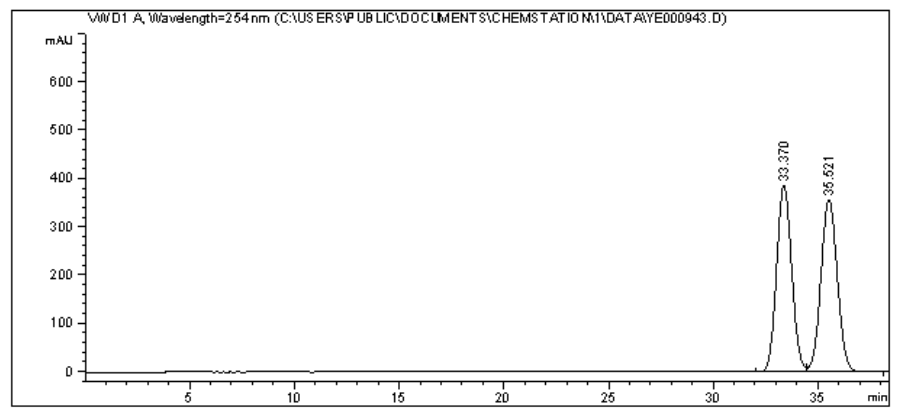

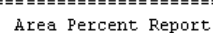

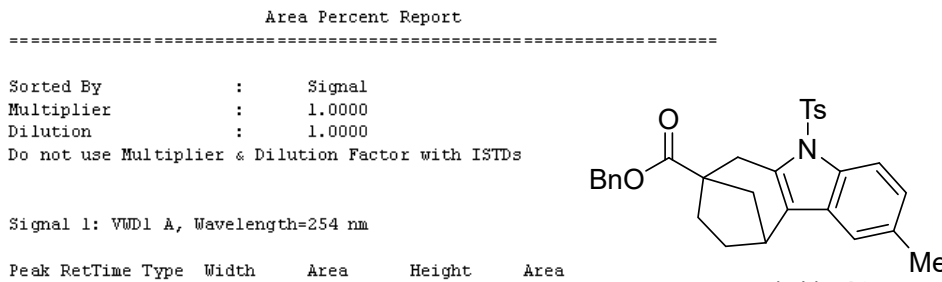

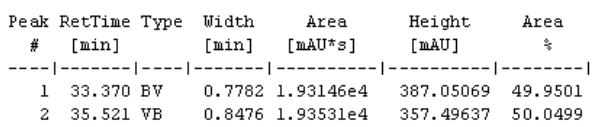

$(+/-)--10$

$\mathrm{Me}$

Totals : $\quad 3.66677 \mathrm{e} \quad 744.54706$

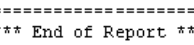

HPIC 8/6/2020 6:46:48 AII SYSTEM

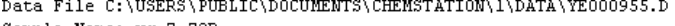
Sample Mame: wg-7-73B

Acq. Operator : : FTSTEM
Sample Operator :

Sample Operator: : FrSTEM
Acq. Instrument : HPIC

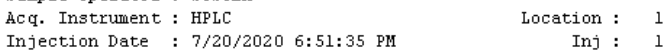

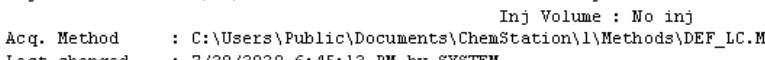

Lasst changed : 7/20/2020 6:45:13 PII bY STSTEM

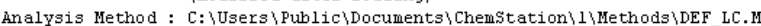

Last changed : 8/6/2020 6:45:56 AM by srstem

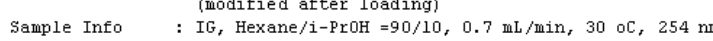

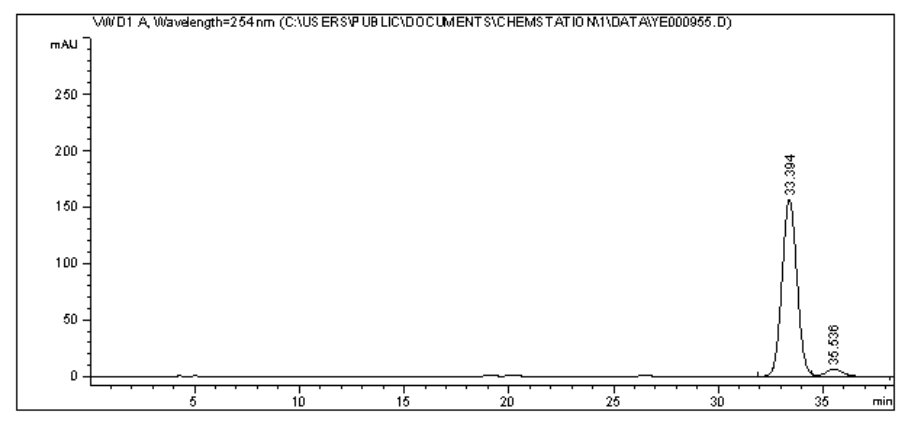

4rea Percent. Report.

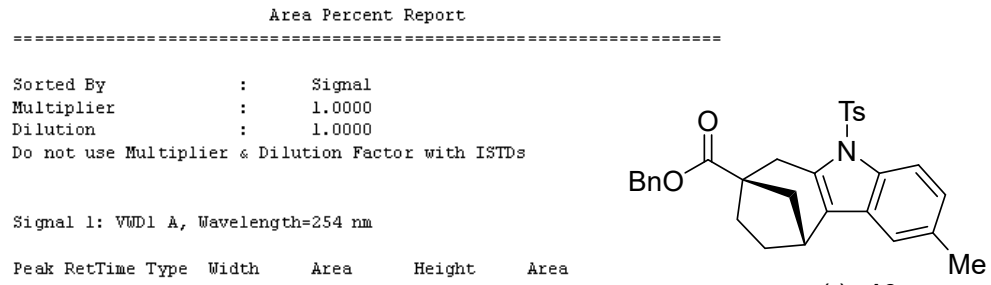

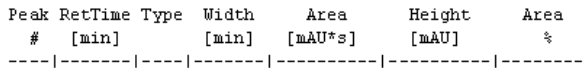

$(-)-10$

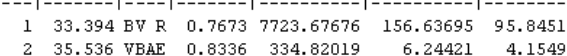

Totals:

$8058.49695 \quad 162.00116$

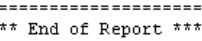

HPIC 8/6/2020 6:46:10 AII SYSTE

Page 1 of 1 


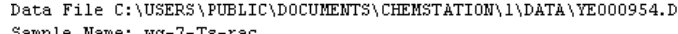
Sample Name: wog-7-Ts-rac

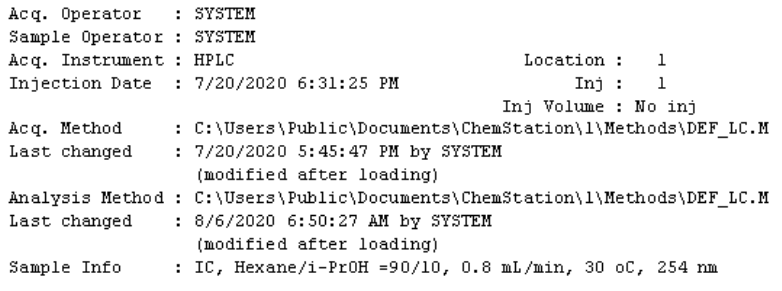

Anj Yolume: : No inj

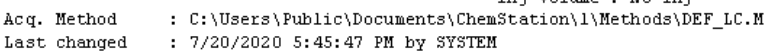
Last changed : T/20/2020 5: 4s:47 Fin by srocen

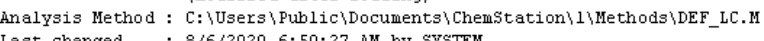
Last changed : 8/6/2020 6:50:27 AM by sTSTEM

Sample Info : IC, Hexane $/ 1$-ProH $=90 / 10,0.8 \mathrm{~mL} / \mathrm{min}, 30$ oC, $254 \mathrm{~mm}$

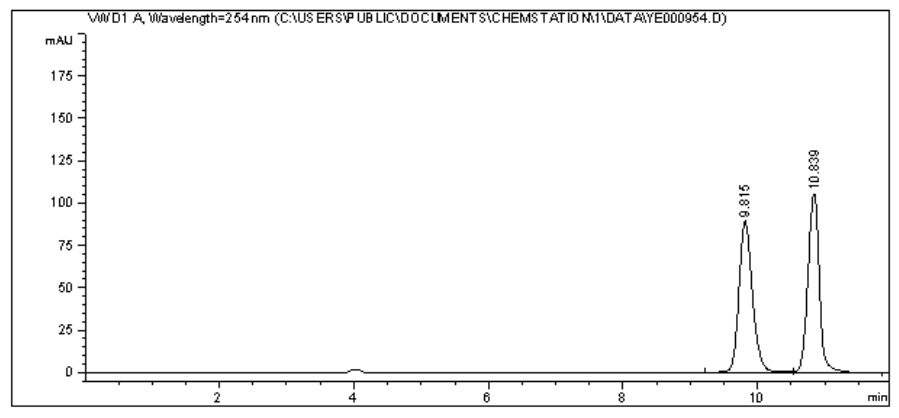

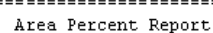

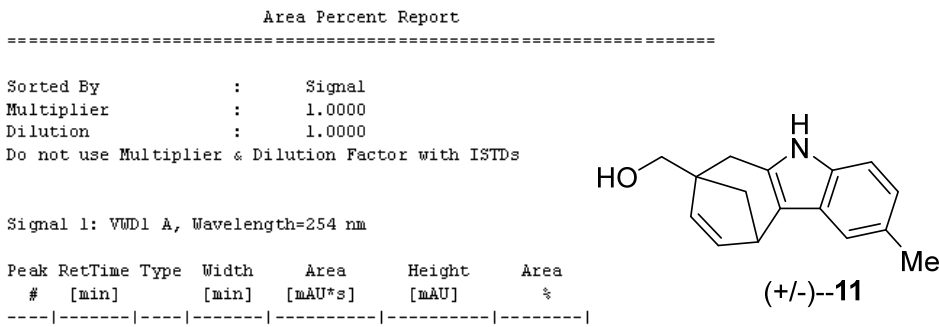

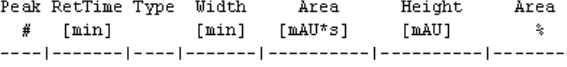

$(+/-)--11$

$\begin{array}{lrlllll}1 & 9.815 \mathrm{BV} R & 0.2160 & 1254.97229 & 89.28270 & 50.272 \\ 2 & 10.839 \mathrm{WEA} & 0.1853 & 1241.37622 & 105.46098 & 49.727\end{array}$

Totals : $\quad 2496.34851 \quad 194.74368$

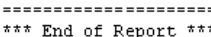

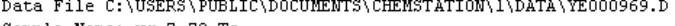
Sample Wame: wrg-7-78-T

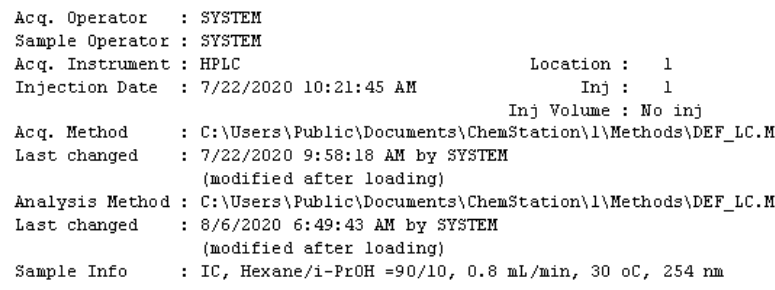

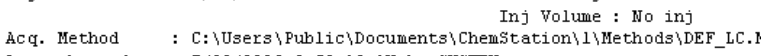
Lasst changed : 7/22/2020 9:58:18 AII bY STSTEM

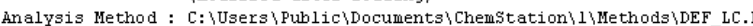

Last chenged : 8/6/2020 6:49:43 $\mathrm{AM}$ bY SYSTEM

Sample Info : (ncodified after loading)

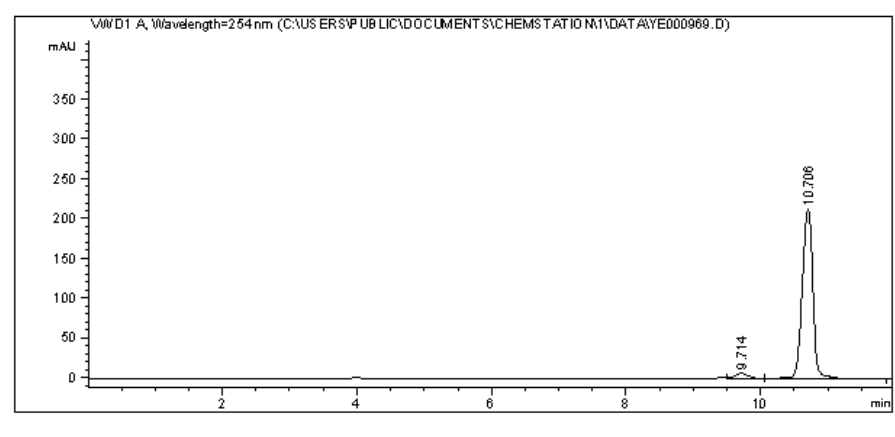

hea Percent. Renort.

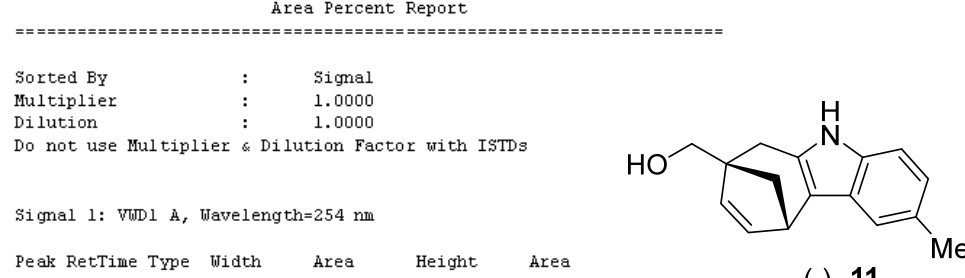

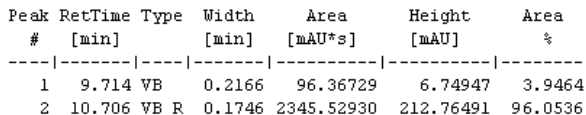

$(-)-11$

$\begin{array}{lll}2441.89658 & 219.51437\end{array}$

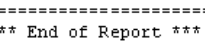

HPIC 8/6/2020 6:49:55 AMII SYSTEM

Page 1 of 1 